\title{
EXPERIMENTAL AND NUMERICAL STUDY ON THE THERMAL PERFORMANCE OF A VERTICAL PCM PANEL
}

\author{
by \\ Fabio Almeida \\ B.Eng., Ryerson University, 2006 \\ M.A.Sc., Ryerson University, 2008 \\ A dissertation \\ presented to Ryerson University \\ in partial fulfillment of the \\ requirements for the degree of \\ Doctor of Philosophy \\ in the Program of \\ Mechanical and Industrial Engineering
}

Toronto, Ontario, Canada, 2018

(C) Fabio Almeida, 2018 


\section{AUTHOR'S DECLARATION}

I hereby declare that I am the sole author of this dissertation. This is a true copy of the dissertation, including any required final revisions, as accepted by my examiners.

I authorize Ryerson University to lend this dissertation to other institutions or individuals for the purpose of scholarly research.

I further authorize Ryerson University to reproduce this dissertation by photocopying or by other means, in total or in part, at the request of other institutions or individuals for the purpose of scholarly research.

I understand that my dissertation may be made electronically available to the public. 


\title{
Experimental and Numerical Study on the Thermal Performance of a Vertical PCM Panel Doctor of Philosophy, 2018, Fabio Almeida Mechanical and Industrial Engineering, Ryerson University
}

\begin{abstract}
Due to an increased awareness of climate change and other environmental issues, methods to reduce the energy consumption of buildings has become of great importance. One way to improve the efficiency of a building is to use thermal storage material. A recent thermal storage strategy is to use phase change material (PCM) which allows for the storage and release of thermal energy. One of the main advantages of using PCM over traditional thermal storage (like concrete) is that PCM can achieve the same level of thermal storage as concrete while using less material. Using PCM can also reduce and delay peak load, improve the thermal comfort, and reduce the overall energy consumption of a building.
\end{abstract}

One of the main parameters that affect the performance and effectiveness of PCM in buildings is the convective heat transfer between a PCM wall and room air. Current convective heat transfer coefficients used in whole building simulation and in building codes (such as ASHRAE) may not be adequate for PCM applications. The present study investigates thermal performance of a vertical PCM panel. The investigation includes experiments using laser MachZehnder Interferometry (MZI) and a comparative numerical study using computational fluid dynamics (CFD). The study focuses on a vertical flat plate filled with PCM (soy wax) undergoing transient convective heat transfer by natural convection while the PCM solidifies. A novel method was developed to make interferometric surface temperature measurements using partial fringes as a reference temperature. The experimental results show a deviation from predicted heat transfer coefficients from established correlation and significant subcooling was observed as a temperature jump. The subcooling effect was reproduced using CFD by implementing the speed of crystallization within the PCM cavity. 


\section{ACKNOWLEDGEMENTS}

I would like to acknowledge Dr. Wey H. Leong and Dr. Alan S. Fung for their guidance and advice through the duration of this research and during the writing of this thesis.

I would also like to thank the Engineering support staff, especially Alan Machin and Joseph Amenkrah, for their time and effort to help build the experimental apparatus and for their willingness to teach their knowledge throughout the duration of this project.

I would also like to acknowledge the support of the Ontario Graduate Scholarship, Ryerson University's Centre for Urban Energy, and Mitacs for the funding of this research.

I want to thank my colleagues at Morrison Hershfield for their support, motivation, and enthusiasm for my work and specifically Sophie Mercier and Patrick Roppel for giving me that final push to finish my doctorate during moments of doubt.

I finally I would like to thank my wife, Nicole Almeida, for her tireless support and my son, Lucas Almeida, whom was born during the writing of this thesis for motiving me to finish and for occasionally taking me away from the keyboard. 


\section{TABLE OF CONTENTS}

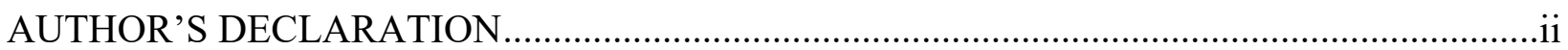

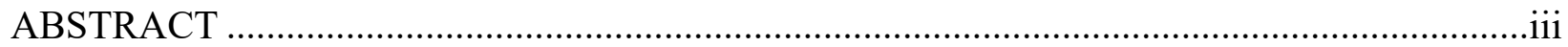

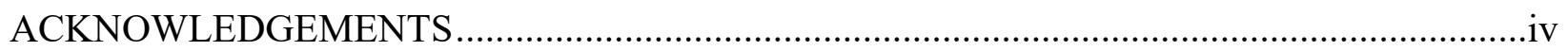

LIST OF FIGURES ……………………………..................................................

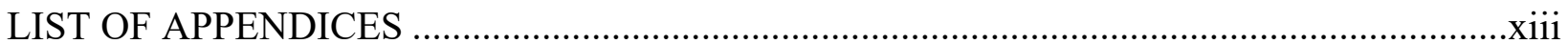

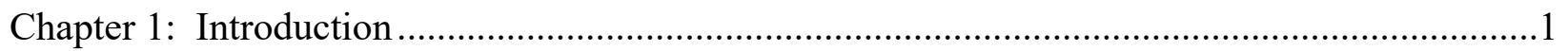

1.1 Introduction ...........................................................................................................

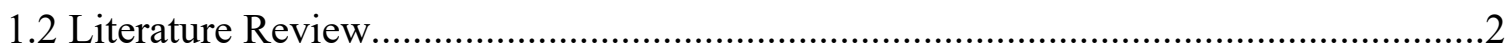

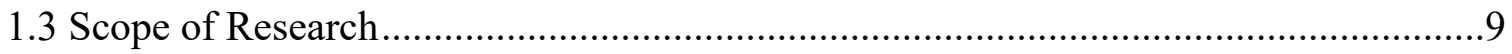

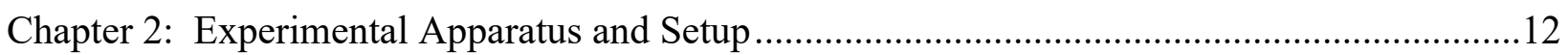

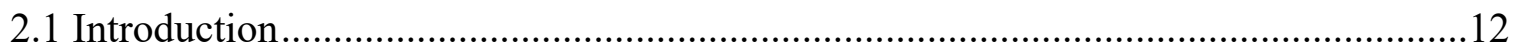

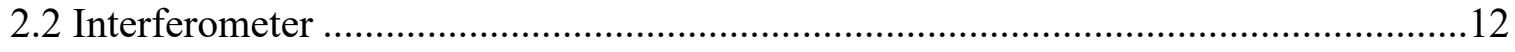

2.3 Differential Scanning Calorimetry ........................................................................

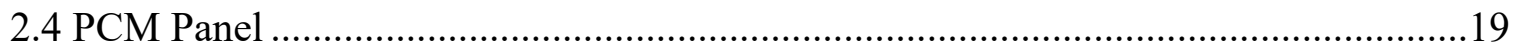

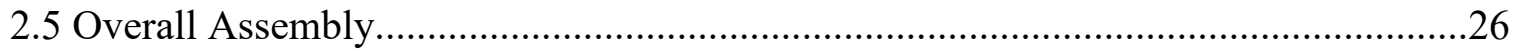

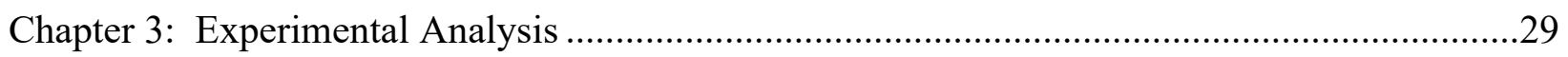

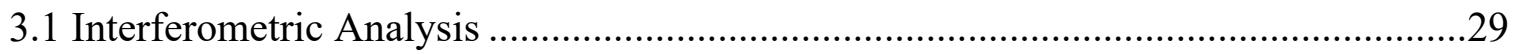

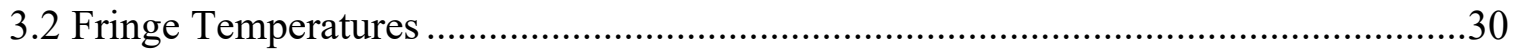

3.3 Local and Average Convective Heat Transfer..............................................................33

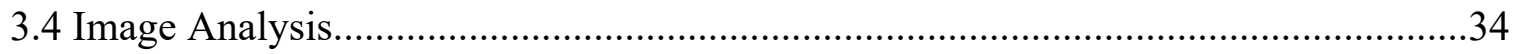

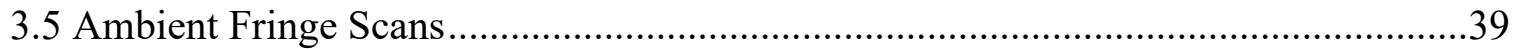

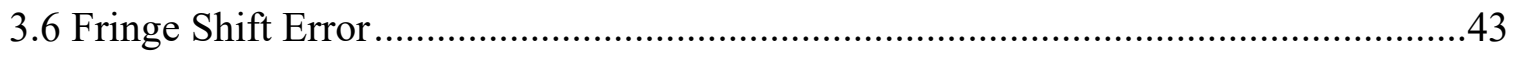

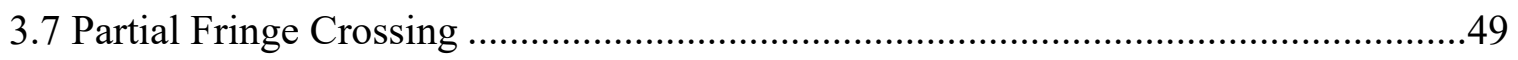

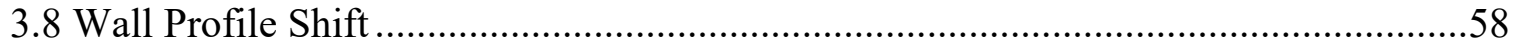




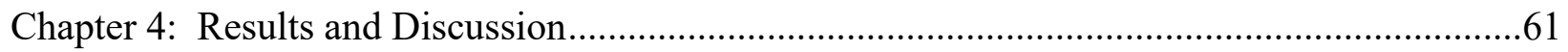

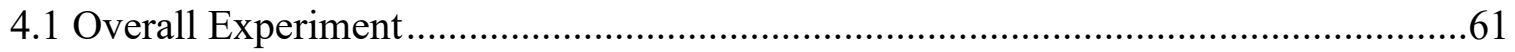

4.2 High Temperature Liquid Phase ................................................................................67

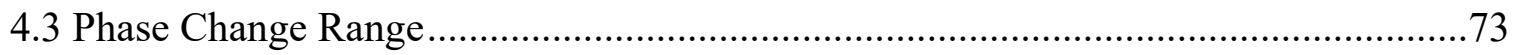

4.4 Low Temperature Solid Phase ..............................................................................99

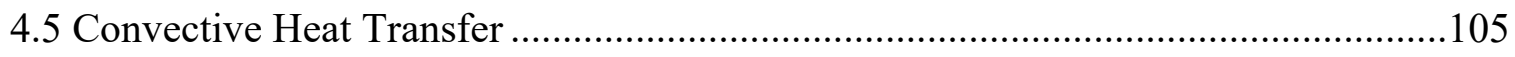

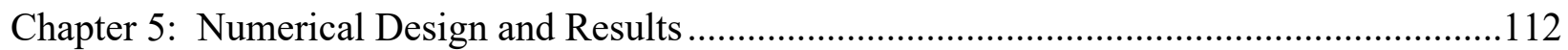

5.1 Governing Equations ...................................................................................112

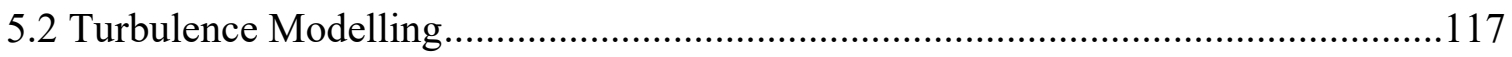

5.3 k- - Turbulence Model................................................................................118

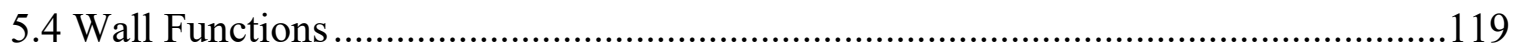

5.5 Solidification and Melting .........................................................................120

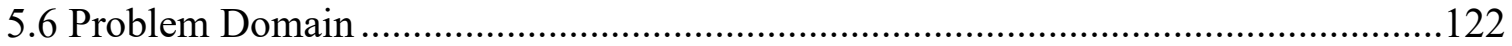

5.7 Boundary Conditions and Initial Conditions ......................................................125

5.8 Comparison of Numerical Simulation with Experimental Results.............................126

5.9 Temperature Increase Investigation ....................................................................144

5.10 Modified Crystallization Speed by Temperature Proxy ...........................................150

Chapter 6: Conclusions and Recommendations ..................................................................159

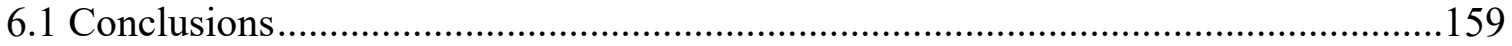

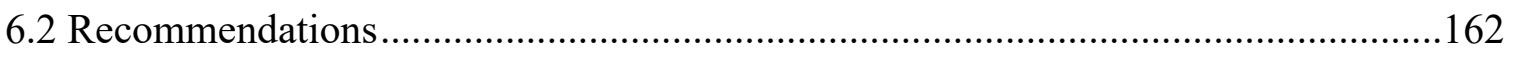

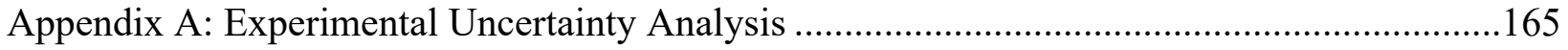

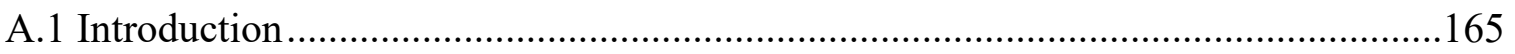

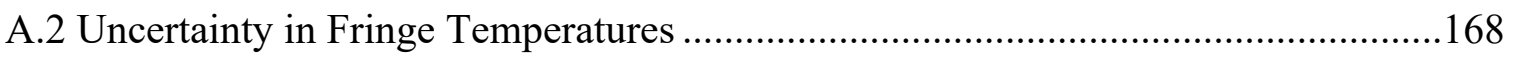

A.3 Uncertainty in Wall Surface Temperature ............................................................170

A.4 Uncertainty in Air Properties .........................................................................170

A.5 Uncertainty in Convective Heat Transfer ................................................................173

A.6 Uncertainty in Measured Convective Heat Transfer Coefficient...............................175

A.7 Uncertainty in Rayleigh number and Prandtl Number..............................................176 
A.8 Uncertainty in Correlated Convective Heat Transfer Coefficient

Appendix B: Tabulated Data

References 


\section{LIST OF FIGURES}

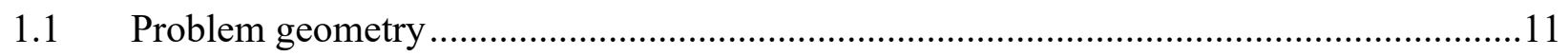

2.1 Overhead view of the Mach-Zehnder Interferometer (MZI) ...........................................13

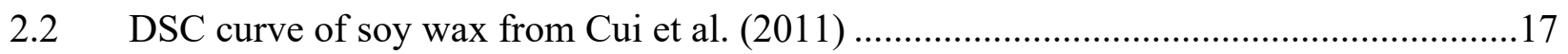

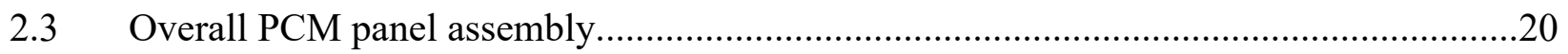

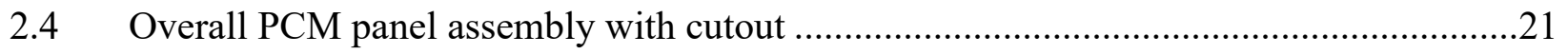

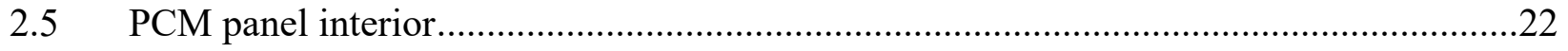

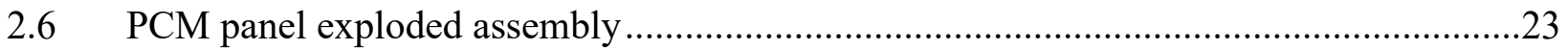

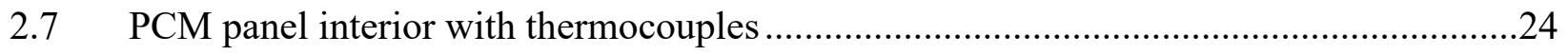

2.8 Overall assembly with PCM panel and support base......................................................27

3.1 High temperature gradient case of finite fringe interferogram .........................................31

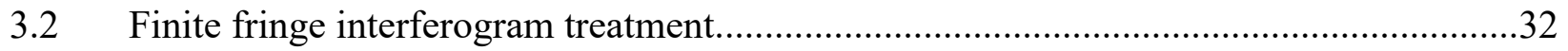

3.3 Illustration of the method used to calculate the surface temperature gradient ...................33

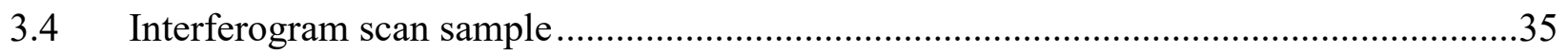

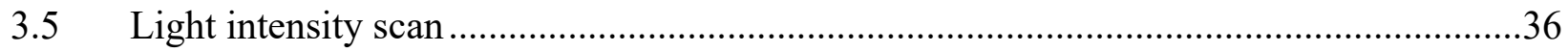

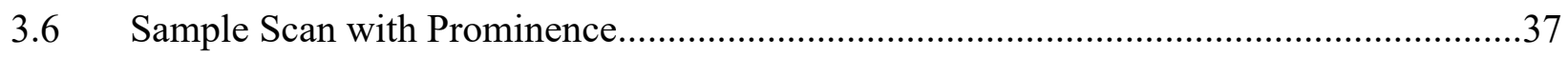

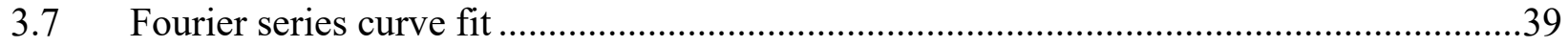

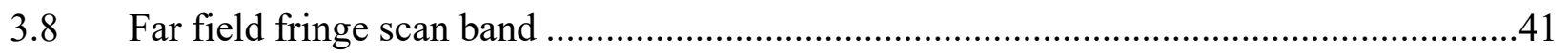

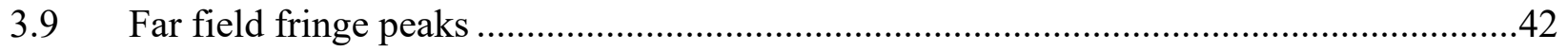

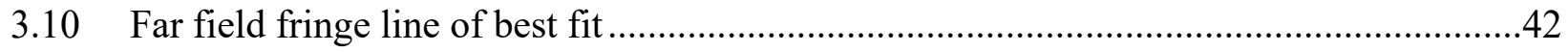

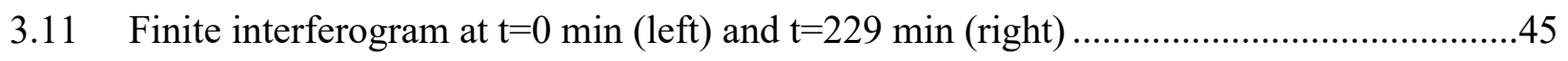

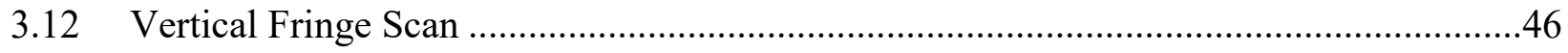

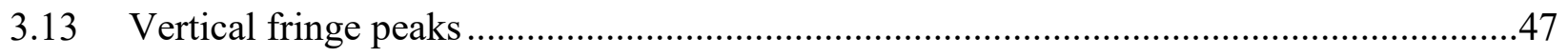




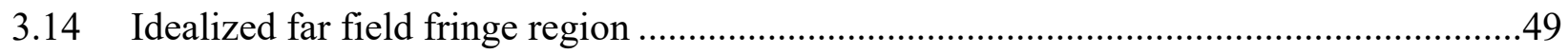

3.15 Interferogram with scan paths at sensor locations .....................................................51

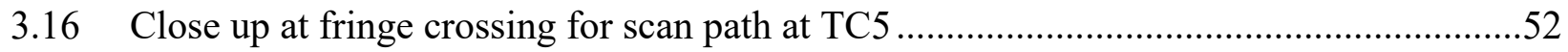

3.17 Fringe crossing location versus far field fringe slope ....................................................53

3.18 Fringe crossing location versus far field fringe slope ……...........................................5

3.19 Illustrated far field fringes with horizontal and vertical fringe crossings .........................56

3.20 Relationship between horizontal and vertical fringe crossings........................................57

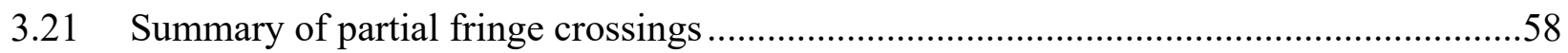

3.22 Wall Profile

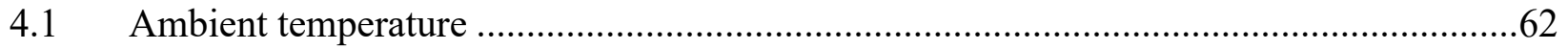

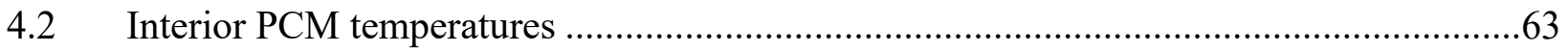

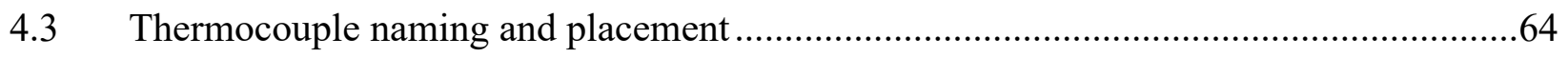

4.4 Optically measured outside surface temperatures........................................................66

4.5 Averaged outside surface, inside surface and center line temperatures............................67

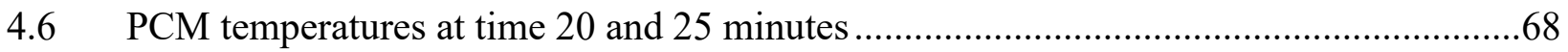

4.7 Surface and PCM temperatures at 20 minutes ..............................................................

$4.8 \quad$ Surface and PCM temperatures at 25 minutes ...............................................................

4.9 Surface and center line temperatures at time 20 and 25 minutes.....................................72

4.10 Center line temperatures from 30 to 35 minutes................................................................74

4.11 Inside surface temperatures from 30 to 35 minutes .......................................................75

4.12 Outside surface temperatures from 30 to 35 minutes ........................................................76

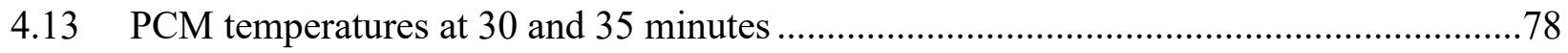

4.14 Surface and center line temperatures at 30 and 35 minutes...........................................79 


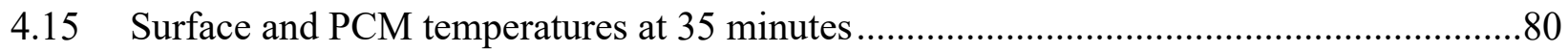

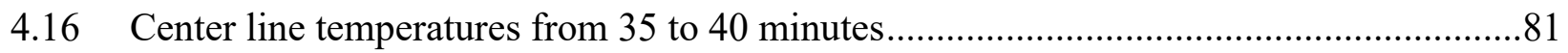

4.17 Inside surface temperatures from 35 to 40 minutes ......................................................

4.18a Raw outside surface temperatures from 35 to 40 minutes................................................84

4.18b Fitted outside surface temperatures from 35 to 40 minutes.............................................85

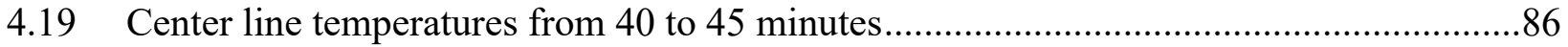

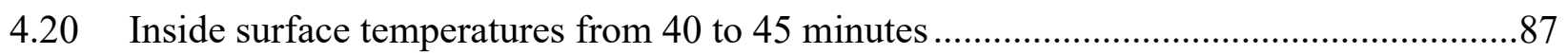

4.21a Raw outside surface temperatures from 40 to 45 minutes...............................................8

4.21b Fitted outside surface temperatures from 40 to 45 minutes.............................................90

4.22 Center line temperatures from 45 to 50 minutes...........................................................91

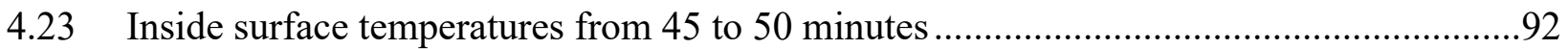

4.24 Outside surface temperatures from 45 to 50 minutes ....................................................93

4.25 Center line temperatures from 50 to 55 minutes...........................................................94

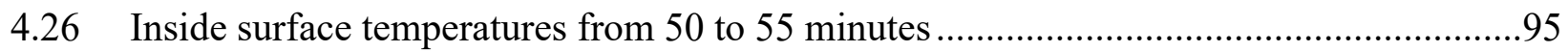

4.27 Outside surface temperatures from 50 to 55 minutes ………............................................96

4.28 Center line temperatures from 55 to 65 minutes............................................................97

4.29 Inside surface temperatures from 55 to 65 minutes .......................................................98

4.30 Outside surface temperatures from 55 to 65 minutes .....................................................99

4.31 PCM temperatures at time 70, 75 and 80 minutes .....................................................101

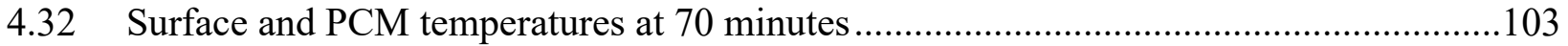

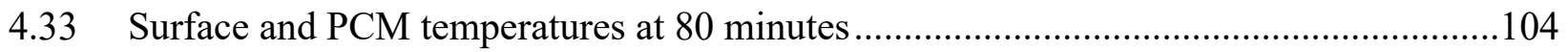

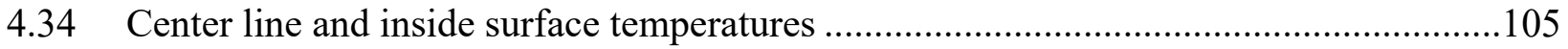

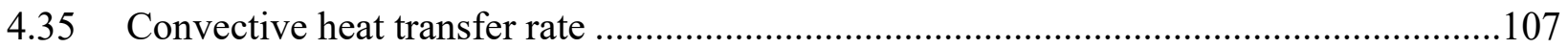




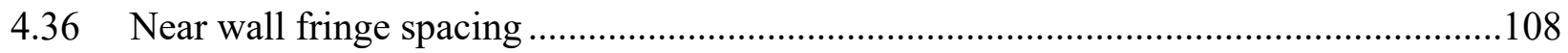

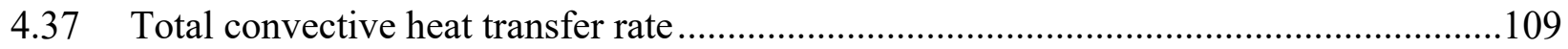

4.38 Average convective heat transfer coefficient..........................................................111

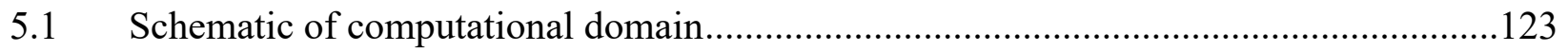

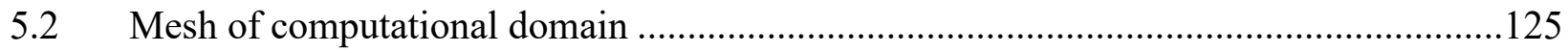

5.3 Average experimental vs simulation temperatures ....................................................128

5.4 Experimental vs simulation center line temperatures at TC2 …..................................129

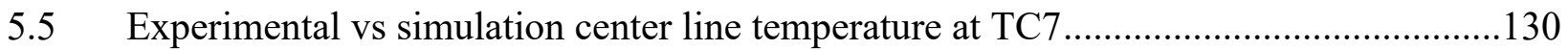

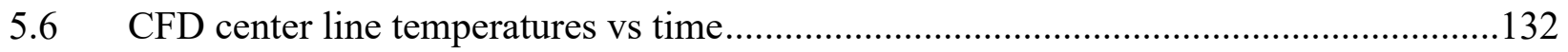

$5.7 \quad$ Experimental center line temperatures vs time .........................................................133

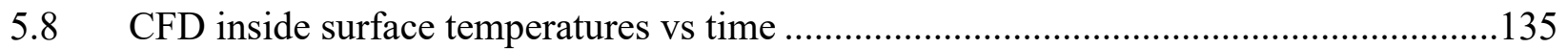

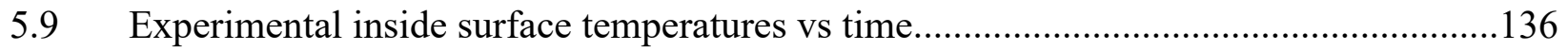

5.10 CFD outside surface temperatures vs time ……........................................................138

5.11 Experimental outside surface temperatures vs time...............................................139

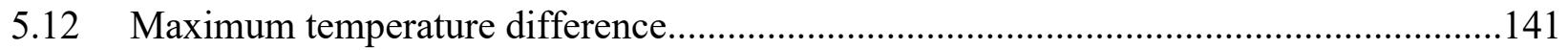

5.13 Temperatures for TC2

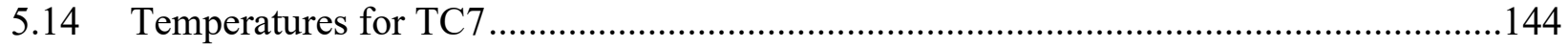

5.15 Experimental apparatus for melting (left) and freezing (right) of sodium acetate trihydrate (Gunther et al. 2007)

5.16 Experimental and numerical results for melting of sodium acetate trihydrate (Gunther et al. 2007)

5.17 1D Numerical domain used for subcooling algorithm (Gunther et al. 2007) ..................148

5.18 Schematic of logic experiment for modified crystallization speed by temperature proxy method 
5.19 Center line temperatures vs time with speed of crystallization highlights

5.20 CFD center line temperatures vs time with temperature jump …………......................156

5.21 CFD inside surface temperatures vs time with temperature jump ...................................157

5.22 CFD outside surface temperatures vs time with temperature jump...............................158 


\section{LIST OF APPENDICES}

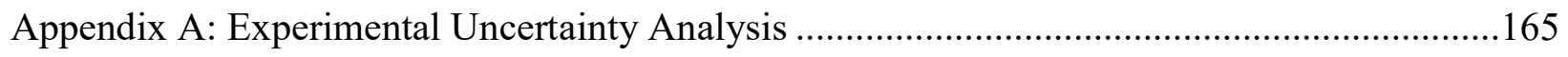

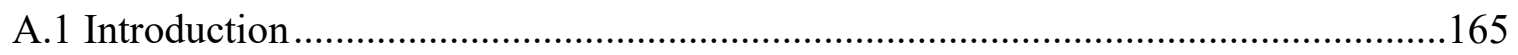

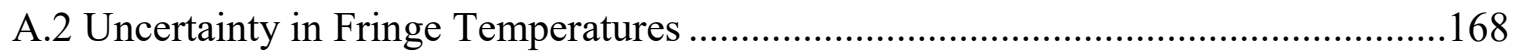

A.3 Uncertainty in Wall Surface Temperature ....................................................... 170

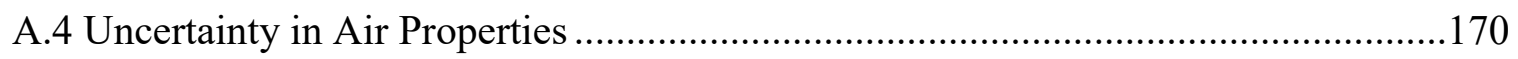

A.5 Uncertainty in Convective Heat Transfer ............................................................173

A.6 Uncertainty in Measured Convective Heat Transfer Coefficient............................175

A.7 Uncertainty in Rayleigh number and Prandtl Number..........................................176

A.8 Uncertainty in Correlated Convective Heat Transfer Coefficient ...........................178

A.9 Summary of Experimental Uncertainty ....................................................... 180

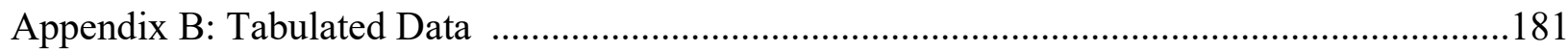

B.1 General Format of Data Tables ..................................................................... 181 


\section{Chapter 1: Introduction}

\subsection{Introduction}

Due to an increased awareness of climate change and other environmental issues, methods to reduce the energy consumption of buildings has become of great importance. One of the ways to improve the efficiency of a building is to use thermal storage material. A recent thermal storage strategy is to use phase change material (PCM) which allows for the storage and release of thermal energy. One of the main advantages of using PCM over traditional thermal storage (like concrete) is that PCM can achieve the same level of thermal storage as concrete while using less material (Sarlos and Dauriat 2003). Using PCM can also reduce and delay peak load, improve thermal comfort, and reduce overall energy consumption of a building (Sharma et al. 2009). A recent trend in energy efficient buildings is to develop net-zero energy buildings that produce as much energy as they consume (in combination of passive/active solar, wind, geothermal, etc.). Net-zero energy buildings usually have large window areas and low infiltration. This can cause more solar energy being absorbed into the PCM than is needed during the day, making it difficult to release all its energy at night. This problem can lead to reduced effectiveness of the PCM and gradual overheating of a building, and affect the thermal comfort of occupants in the building. One of the key parameters that govern the performance and/or effectiveness of a PCM wall is how well it can charge or discharge energy into the built environment can by natural convection. Established correlation that are typically used for building walls may not be applicable for walls with PCM. The proposed work uses experimental measurements and numerical simulations of a phase change panel undergoing solidification to better understand the convective heat transfer rate into the built environment. 
The work includes both experiments and numerical simulation. Experiments are performed to measure the convective heat transfer between a PCM wall and indoor room air. Experiments are performed on a simple vertical flat plate that mimics a building wall. These experiments are used to compare with computer models and established correlations.

\subsection{Literature Review}

Computational Fluid Dynamics (CFD) is used for simulating interior building flows and exterior flows. The focus of this literature review is on indoor building flows. Also, many of the CFD in building research is done on ventilation, where buoyancy is not a great concern. In general, current CFD models can predict air flows well but not heat transfer.

The most common method for turbulence modelling is to use RANS (Reynolds Averaging Navier-Stokes) based models. While more advance turbulence models, such as LES (Large Eddy Simulation), are computationally expensive. Jiang and Chen (2003) studied natural ventilation using RANS and LES and compared with experiments. The LES model took 10 days to compute while the RANS model took 2 days. While the LES was more accurate, the RANS model was still considered reasonable.

The use of CFD can give the engineer or architect a complete view of the air flow patterns and temperature field. This insight can be used for analysis or the investigation of building features. Posner et al. (2003) found that obstructions in a room can greatly affect the air flow and contaminant transport in a room. They performed experiments on models and compared them to a k- $\varepsilon$ model, RNG (Renormalization Group) k- $\varepsilon$ model, and laminar model. This work shows that CFD can be used as an investigative tool to look at how specific details in room air flow. In most studies they only look at idealized rooms (no obstructions) which may not tell the whole story. 
Experiments involving air flow patterns, air velocity and temperature distributions was performed by Koskela et al. (2010) on a typical office building. These experiments were compared with a CFD simulation. In general, the CFD over predicted the air velocities but did obtain the overall air flow pattern. Norton et al. (2010) performed a CFD simulation to study the ventilation effectiveness of a livestock building with different cladding. It was found that the optimum choice of cladding depends on whether there is wind-driven ventilation, or buoyancy influenced ventilation. A historical building in Italy was studied by Balocco and Grazzini (2008). Measurement equipment was placed inside the building and it was found that the CFD simulation was in agreement with the measurements. With minimal intrusion into the building operation to validate the simulation, they now have complete air flow and temperature distribution for inspection. Omri and Galanis (2010) performed a CFD analysis of an indoor ice rink in order to investigate the effect of radiant heat sources.

CFD can be used in the design process for buildings. It can give detailed air flow and temperature distributions within a room. It can be used when traditional methods may be difficult to obtain results. Domingo et al. (2011) used CFD to design a public transit hub in Madrid, Spain. Different ventilation strategies and air conditioning systems were investigated in order to maintain pollutant concentrations from buses at acceptable levels. Traditional methods would not be able to track the pollutant dispersion. An office workspace next to a smelting plant in Quebec was designed by Plikas et al. (2010) using CFD to insure adequate cooling was supplied by the ventilation system, which presented unusual cooling loads. Ascione and Minichiello (2010) studied different diffuser designs for a ventilation system for a museum in Italy. While current building design practices can be used for ventilation design, the unique air quality requirements posed by the museum required a more robust tool to be used. A room-sized experimental facility 
was being designed by Van et al. (2010) in order to study moisture transfer in buildings. In order to design this facility, a HAM (heat, air and moisture) model was coupled to a CFD model. Lien and Ahmed (2010) modelled a rooftop turbine ventilator with CFD using a special rotating mesh generation technique, in order to determine the feasibility of using CFD to model such equipment. They found that the numerical model agreed with experiments.

While CFD can be used to aid in the design of buildings to supplement traditional design practices, it can also be used to investigate new HVAC technologies or techniques. Without the use of CFD it would be difficult and expensive to study new techniques since it would be required to build full scale experiments. Morey and Kershaqw (2010) proposed a novel thermal storage mechanism incorporated into a suspended ceiling. They studied the effect of implementing an air gap in the ceiling so that the room would have thermal access to the cement flooring of the unit above. They found that this new technique can reduce peak room temperatures. A baseboard heater with an integrated air supply was investigated by Ploskic and Holmber (2010). The baseboard heater would preheat the supply air before entering the room. They found that this system can meet thermal comfort conditions and save energy in cold climates. Lo and Novoselac (2010) proposed a method to partition a large office space into multiple thermal zones without using walls. This was done by careful and strategic placement of the ventilation system. They were able to explore many different designs using CFD.

Most of the applications discussed above deal with air velocities and air flow patterns. In general there is good agreement between air flow and CFD simulations. However, there is sometimes a discrepancy in heat transfer rates between experiments and CFD simulations. In a study by Dafraeye et al. (2010), a RANS CFD model was performed of a cube immersed in turbulent flow and compared it to experiments. They found that the convective heat transfer 
coefficient was over predicted when using the standard wall functions. Posner et al. (2003) investigated the effect of obstacles in a room on ventilation with a variety of turbulence models and experiments. They found that while the turbulence models may not have been accurate they did accurately capture the 'features' or trends of the flow. Also, CFD is a powerful and complex tool that required care in order to obtain an accurate solution. Malalasekera (1995) states that CFD is nothing more than the extrapolation of boundary conditions into the domain space. Therefore, the boundary conditions are probably the most important part.

The main reason for this discrepancy is near wall behaviour and thus the choice of wall functions become important. The standard wall function used for the $k-\varepsilon$ model of Launder and Spalding (1974) was derived assuming universal law of the wall behaviour which is true of simple flows like forced convection over a flat plate and pipe flows. Awbi (1998) performed a numerical and experimental study of a test chamber to mimic the typical flow conditions in a room. It was found that the standard ' $\log$ ' wall functions in the standard $k-\varepsilon$ model cannot accurately predict the convective heat transfer coefficient on a heated surface, such as buoyancy driven flows, when compared with experiments. Ferziger (1990) compared a variety of RANS, LES, and zonal models for turbulent flows over obstacles. He found that while different RANS models may work better for different types of flows, one of the main problems is that RANS wall functions are not based on 'sound' theories. For these reasons, Yuan et al. (1993) developed new wall functions appropriate for building flows. However, these wall functions are optimized for vertical flat plates and may not work for different orientations.

Another important near wall effect is grid spacing. Zhang et al. (2010) developed a grid generation tool for use in buildings to ensure that there is an adequate number of grid points within the boundary layer depending on the wall function and turbulence model used. The tool was 
designed to accommodate complex objects inside a room. Ferziger (1990) studied turbulent flows over obstacles and found that the convective heat transfer coefficient was sensitive to grid spacing.

Choice of wall functions and grid spacing may have the most effect on accurately predicting the convective heat transfer coefficients, but the choice of turbulence model is also important. Yimaz and Fraser (2007) performed both numerical and experimental study of vertical parallel-plates with heating. Out of the various turbulence models tried, the low-Reynolds k- $\varepsilon$ predicted the convective heat transfer rates the closest. A numerical study of a double-glazed façade was studied by Coussirat et al. (2008) in order to determine the effects of various numerical models (turbulence models, wall functions, etc.). The results were compared with experiments and it was found that choice of turbulence model did give different results and the RNG k- $\varepsilon$ model performed best for predicting heat transfer rates. Gao et al. (2008) also found that the RNG k- $\varepsilon$ model better predicted the convective heat transfer coefficients when studying thermally stratified flows. However, there was a discrepancy when natural convection was dominant.

One way to improve the accuracy of CFD for building applications is to integrate CFD with building energy simulation software. Negrao (1998) integrated CFD into building simulation software. This paper represents the first work done to integrate CFD in ESP-r (a building simulation software). There are deficiencies in building simulation methods and CFD methods when they are applied to estimating building performance. The rationale is that the two combined (and combined in an intelligent way) can overcome each other's deficiencies. But in order to combine them, there needs to be some procedure to perform the 'hand shaking'. The results of the building simulation are used as the boundary conditions for the CFD. He found that the combined approach performed better than the ESP-r only simulation. For further information about ESP-r's CFD capabilities, please refer to Bartak et al. (2002). Zhai et al. (2002) investigated different 
approaches to integrate building energy simulation with CFD by using EnergyPlus and an in-house CFD code. They performed a case study using a typical office in Boston and found that the accuracy of the CFD prediction was improved by this coupling.

However, the integration of CFD with building simulation can be difficult and a new set of problems arise. Zhai and Chen (2003) coupled building energy simulation with CFD to determine potential numerical issues since each numerical domain contains its own stability criteria. They found that with careful coupling a converged solution between CFD and building energy simulation is possible. As mentioned earlier, the inaccuracies of near wall turbulence might propagate error throughout the combined simulation. Thus, Awbi (1998) recommended the use of empirical correlations along with CFD. As an extension of Negrao (1998) work, BeausoleilMorrison (2002) incorporated a method to determine the correct heat transfer correlation to integrate with the CFD module in ESP-r. They developed an algorithm that manages the 'hand shaking' between the CFD domain and the building simulation domain. The algorithm controls things like applying the appropriate boundary conditions at a given surface, and determines which turbulence model and/or wall functions to be used. These decisions are done based on the nature of the flow.

Obtaining accurate convective heat transfer coefficients are important for accurate modelling of buildings. There are many established convective heat transfer correlations for free edge flows and enclosures, there currently is none available when PCM is present. In the work of Lui and Awbi (2009), an experimental test chamber was built to mimic the conditions of an interior room. The chamber was designed to measure the convective heat transfer coefficient from PCM walls. They found that established correlations under predicted the convective heat transfer coefficient from the PCM wall by a factor of 2. In another PCM study, Zhou et al. (2011) simulated 
a PCM wallboard with sinusoidal heat flux boundary conditions. They found that the natural convective formulas from literature predicted much lower heat transfer rates then what was measured. In a review paper of Kuznik et al. (2011) the authors reviewed the existing literature on the use of PCM applied to building walls. They found that there is a lack of research in understanding the interaction between PCM and convective heat transfer.

In summary, building simulation is a useful tool to help reducing the energy consumption in buildings. While building simulation techniques have evolved over the years, there are still some areas that need improvement. The convective heat transfer coefficient is the 'hand off' of energy between the walls and the air within a room. Building simulations are sensitive to the convective heat transfer coefficient. A full CFD simulation of buildings may not be feasible so a combination of traditional building energy simulation and prescribed convective heat transfer coefficient or correlation CFD is the recommended approach. However, the convective heat transfer coefficient in the presence of PCM is potentially an unknown quantity. For PCM application, the convective heat transfer is important since it affects the performance of the PCM. PCM can be thought of a passive heating mechanism where the convective heat transfer coefficient is the primary driver of the energy charge/discharge. Thus, the proposed research work is to study the effects of convective heat transfer on the thermal performance of PCM with the ultimate goal of optimizing the use of PCM in buildings. 


\subsection{Scope of Research and Motivation}

The focus for the current work is to study the thermal decay behaviour of a vertical heated panel undergoing cooling from natural convection while experiencing phase change from liquid to solid. The primary motivation for this comes from the work of Lui and Awbi (2009), Zhou et al. (2011) and Kuznik et al. (2011) where the thermal behaviour of PCM building walls was studied. The three research groups above found that the measured convective heat transfer rates from a PCM building wall did not match the predicted convective heat transfer rates from established correlation found in ASHRAE and others when the PCM wall was undergoing phase change. The recommended correlation from ASHRAE for a vertical building wall comes from Churchill and Chu (1975). The Churchill and Chu (1975) correlation assumes that the vertical wall surface temperature is either uniform or varies linearly from the bottom of the wall to the top. Scenarios where a deviation from the predicted heat transfer rates of Churchill and Chu (1975) occurs would indicate that this 'uniformity' assumption is not valid. To study these scenarios where deviation from correlations occurs, a PCM panel was devised as a proxy for a PCM building wall.

The investigation includes experiments using Mach-Zehnder Interferometry (MZI) and a numerical study using CFD with Ansys Fluent. An illustration of the PCM panel is shown in Figure 1.1. The PCM panel consists of a vertical flat plate filled with PCM (soy wax) undergoing transient convective heat transfer by natural convection. Soy wax was chosen due to a higher melting/solidification point than typical PCMs used in building applications. The high phase transition temperature was required due to the limitations of the MZI equipment which needs a larger enough of temperature difference between ambient air and the PCM panel. A typical phase transition temperature for building applications is around the room set point temperature (for example $23^{\circ} \mathrm{C}$ ), but if the PCM panel temperature is close to the ambient air temperature then it 
would be difficult to make convective heat transfer measurements using the MZI. The MZI analysis and importance of fringe crossings is explained in Chapter 3.

MZI was used for experiments due to its ability to make non-obstructive near wall heat transfer measurements. Determining convective heat transfer rates directly would require measurements of near wall temperature gradients and surface temperatures. Directly measuring the surface temperature or heat flux with sensors or probes could potentially influence the boundary layer fluid flow and introduce errors. Ansys FLUENT was chosen primary for its ability to simulation both fluid flow and the phase change process simultaneously and for its robust turbulence modelling. A detailed description of the numerical model is in Chapter 5.

For both the experiments and numerical simulation, the PCM panel starts warm and cools off over time. In other words, the PCM wall starts in a liquid state and cools off and solidifies and eventually reaches ambient room temperature. The experiment has thermocouples placed along the inside surface of the shell that measure the inside surface temperatures and another set of thermocouples to measure the mid-plane temperature. The surface temperature and convective heat transfer rates are measured using laser interferometry. The ambient temperature is measured throughout the experiment with a thermocouple near the experimental apparatus. 


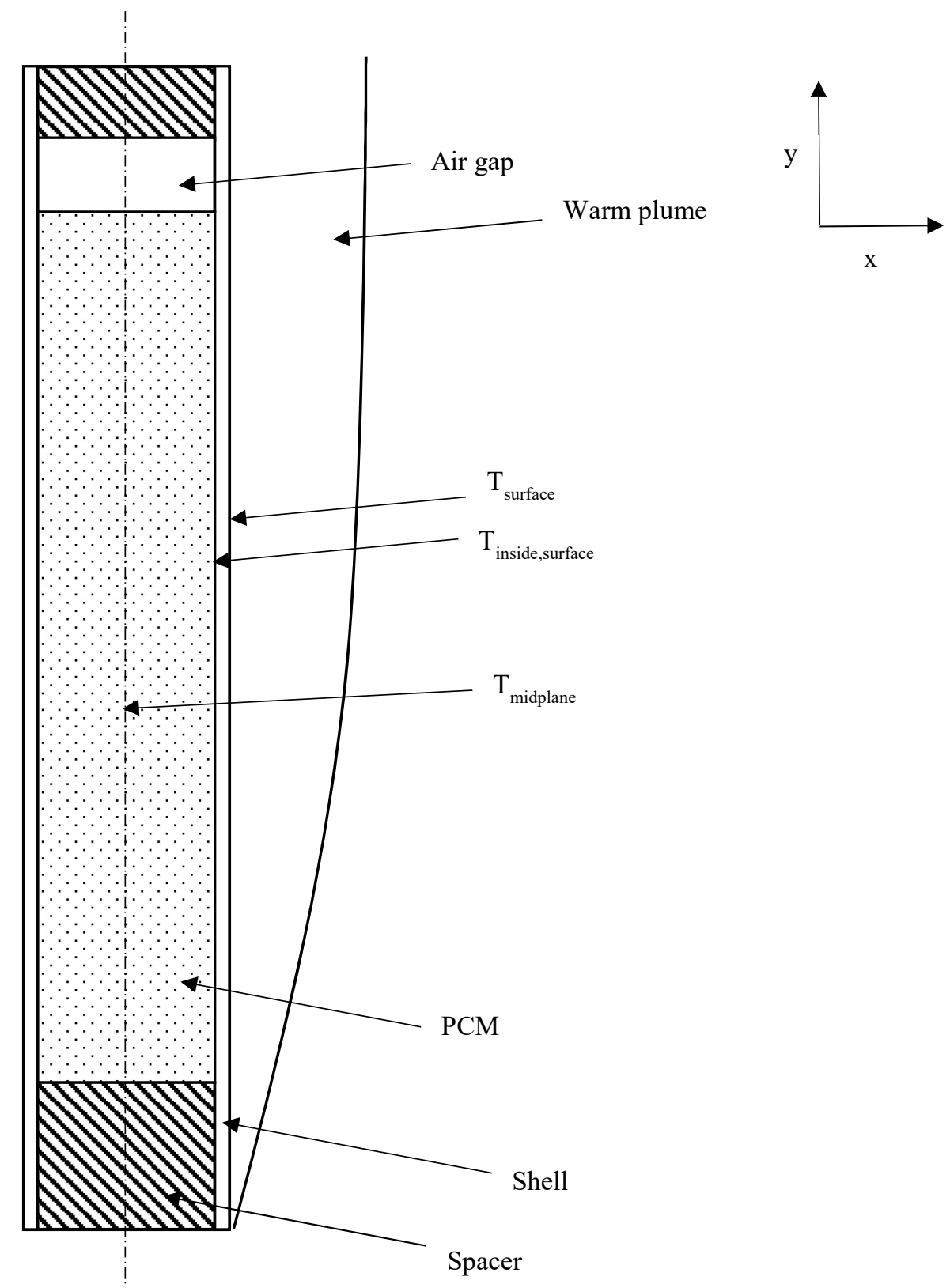

Figure 1.1: Problem Definition 


\section{Chapter 2: Experimental Apparatus and Setup}

\subsection{Introduction}

Experimental measurements were made using a Mach-Zehnder interferometer (MZI). An MZI is an optical instrument for measuring convective heat transfer rates. The MZI provides a full temperature field representation of the experimental domain, which can be used to determine local and average heat transfer rates.

The MZI was used to make measurements on a vertical PCM panel undergoing transient thermal decay. The PCM panel starts warm with the PCM (soy wax) in liquid phase and is allowed to cool off through natural convection. As the PCM panel cools off, the soy wax undergoes phase change and becomes solid. A description of the various components of the Mach-Zehnder interferometer and the experimental apparatus will be discussed next.

\subsection{Interferometer}

The current Mach-Zehnder interferometer used in the laboratory was built by Von Bistram. A more detailed description is found in Von Bistram's undergraduate thesis (1995). That design was based on the interferometer developed by Tarasuk (1968) at the University of Saskatchewan and the interferometer used at University of Western Ontario.

A visual representation of the Mach-Zehnder Interferometer is shown in Figure 2.1. The MZI works by measuring the relative change of the index of refraction of the test fluid. In the current study, the test fluid is air. Assuming an ideal gas, the change in index of refraction can be related to temperature change to give the full temperature field of the problem domain. Using an array of optics, a collimated laser beam (a $15 \mathrm{~mW}$ Helium-Neon laser of wavelength $\lambda=6.328$ 
$\times 10^{-7} \mathrm{~m}$ ) is split into two separate beams. The two beams are initially in phase due to the fact that they come from the same source. One beam passes through ambient air while the other beam passes through the experimental apparatus. The heating of the experimental domain changes the air density, causing a change in index of refraction. The change of index of refraction relative to the ambient beam creates a phase shift. This phase shift appears as constructive and destruction fringes when the two beams recombine. For the MZI shown in Figure 2.1, the final recombined beam is focused in order to be photographed. The output (also known as an interferogram) was captured on a Phase One P45 digital back with a resolution of 39 megapixels.

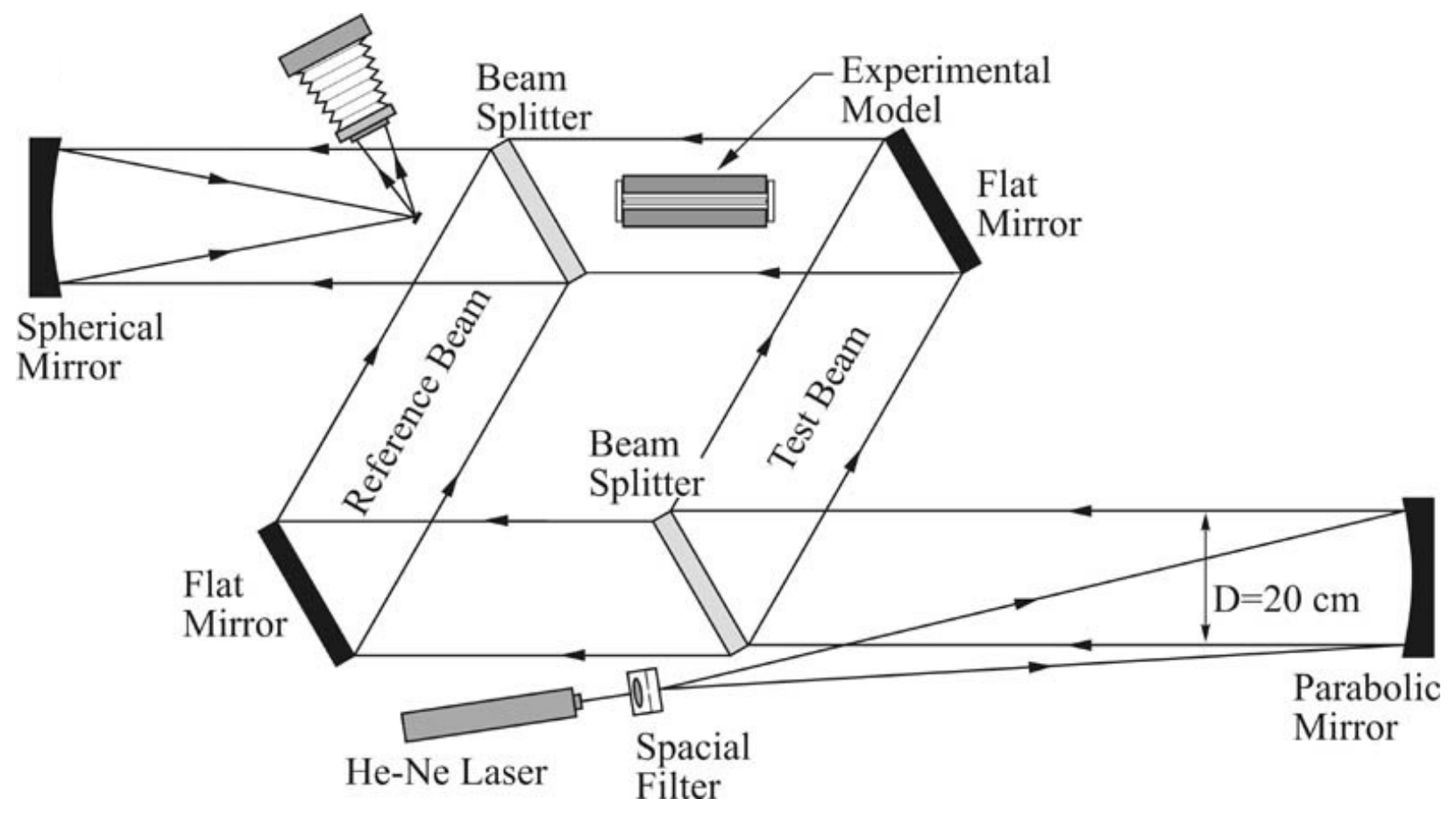

Figure 2.1: Overhead view of the Mach-Zehnder Interferometer (MZI)

The MZI technique is the preferred method of heat transfer measurement for this experiment for many reasons. The MZI is a non-intrusive technique and thus, measurements can be made without affecting the flow pattern or temperature. Also, the MZI is not affected by thermal radiation. So, the convective heat transfer rate is measured directly and the results do not have to be corrected 
for radiation. There is no thermal inertia, giving real time flow measurement. This feature makes it possible to capture time dependent phenomena.

However, there are some disadvantages associated with using the MZI. The MZI obtains the temperature field for a two dimensional slice of the experimental domain by spatially averaging the temperature field along the path of the laser. This averaging makes it difficult to capture turbulence. Also, this characteristic makes the accuracy of two dimensional results susceptible to three dimensional effects. The optical equipment used in the MZI is expensive, fragile, and any imperfections in the optics (i.e., dust, scratches... etc.) can affect the results. Also, the results are sensitive to vibration, which makes obtaining accurate results more difficult. In a transient heat transfer experiment that may take a few hours or longer to perform like in the current research, drifting is another problem. Slowly over time, the alignment of the laser and optics may 'drift' and affects the interferometer output by introducing an artificial fringe gradient.

To minimize the effect of vibrations the entire MZI apparatus was placed on top of a $364 \mathrm{~kg}$ bench of size $1.205 \mathrm{~m} \times 3.03 \mathrm{~m}$ which was elevated on a series of air filled rubber tubes. The surface of the bench has a 'peg-board' like construction with $1 / 4-20 \mathrm{NC}$ tapped holes equally spaced at 25.4 $\mathrm{mm}$ apart. The various optics and laser are mounted to these holes.

\subsection{Differential Scanning Calorimetry}

Thermal capacitance is the ability of a material to store heat and is generally determined by the specific heat capacity of a material. PCM is able to achieve thermal capacitance from the latent heat involved during the solidification or melting process. In general, latent heat is usually much larger than the specific heat capacity for a given temperature range and therefore can achieve more thermal capacitance with less physical space. For example, Microtek Laboratories Inc. (a 
manufacturer of PCM) make a commercial gypsum wallboard with microencapsulated PCM. This PCM wallboard has a thickness of $1.5 \mathrm{~cm}$ and has the same thermal capacitance as $12 \mathrm{~cm}$ brick wall or $9 \mathrm{~cm}$ concrete wall (Microtek Laboratories, Inc.). The latent heat and other thermophysical properties of a material can be found from Differential Scanning Calorimetry (DSC). DSC is a technique where a known heat flux is applied to a small sample of a material and the resultant temperature is measured. The heat flux is applied such that the temperature of the sample increase at a constant rate called the scan rate (for example $5{ }^{\circ} \mathrm{C} / \mathrm{min}$ ). This process of an applied heating rate is applied twice; once for an endothermic process where the sample is heated at a constant rate; and once for an exothermic process where the sample is cooled at a constant rate. The output from this process is called a DSC curve. From this DSC curve the transition temperatures can be found such as the melting point, solidification point, and depending on the type of material used the glass transition point. With the phase transition points known the latent heat can be found as well as the specific heat capacity of the sample at different temperatures.

The DSC curve of soy wax is shown in Figure 2.2 from Cui et al. (2011). Cui et al. studied the thermal behaviour of soy wax and paraffin wax mixed with carbon nanofiber and carbon nanotubes. As part of their study DSC was performed on pure soy wax and pure paraffin wax. The DSC was performed using a TA Instrument, model DSC Q1000 in a nitrogen atmosphere. The soy wax sample had a mass of $5-10 \mathrm{mg}$, the measurement was performed with a scan rate of $5^{\circ} \mathrm{C} / \mathrm{min}$ over a temperature range of $0^{\circ} \mathrm{C}$ to $75^{\circ} \mathrm{C}$. The thermal conductivity of soy wax was measured with a KD2 PRO, Decagon Devices, Inc. The thermal conductivity was measured at $25^{\circ} \mathrm{C}$ and was found to be $0.324 \mathrm{~W} / \mathrm{m} \cdot \mathrm{K}$. The DSC curve in Figure 2.2 contains two sets of data, the solid line represents the endothermic process where the soy wax sample is heated from $0^{\circ} \mathrm{C}$ to $75^{\circ} \mathrm{C}$, and the dashed line represents the exothermic process where the soy wax sample is cooled from $75^{\circ} \mathrm{C}$ to 
$0^{\circ} \mathrm{C}$. The $\mathrm{x}$-axis is the temperature of the sample in ' $\mathrm{K}$ ' and the y-axis represents the applied heat flow in ' $\mathrm{W} / \mathrm{g}$ '. The peaks in each data set represents phase transition points. Phase transition does not occur at discrete temperatures but rather occurs over a range of temperature. For example, looking at the endothermic data, the largest peak represents the melting temperature and occurs at $323.1 \mathrm{~K}\left(50^{\circ} \mathrm{C}\right)$. However, the onset of melting occurs when the applied heat flow increases at 305.4 K. The flat line in heat flow before and after the endothermic peaks represents sensible heating. For the current experiment, the PCM started hot (as a liquid) and cooled off over time by natural convection and allowed to solidify. Looking at the exothermic process the onset of solidification occurs approximately at $311.2 \mathrm{~K}$. For the purposes of the numerical simulation the ending of the solidification process was taken to be right before the next exothermic peak at 296.7 $\mathrm{K}$. With the phase transition temperature range chosen, the latent heat can be found as the area under the curve between the chosen temperatures. The latent heat due to phase change can vary with a different choice of phase temperatures. The specific heat capacity is determined from the flat line portions of the DSC curve before or after a phase transition peak. The specific heat is found by a simple variable conversion of the y-axis by using the experiments scan rate. Dividing the heat flow $(\mathrm{W} / \mathrm{g})$ by the scan rate $\left({ }^{\circ} \mathrm{C} / \mathrm{min}\right)$ and using some conversions gives the specific heat capacity $(\mathrm{J} / \mathrm{g} \cdot \mathrm{K})$.

For the numerical analysis the specific heat was chosen from the endothermic portion of Figure 2.2 at the liquid phase and was found to be $1.613 \mathrm{~J} / \mathrm{g} \cdot \mathrm{K}$. The latent heat due to solidification was chosen from the exothermic portion between $296.7 \mathrm{~K}$ and $311.2 \mathrm{~K}$ and was found to be $56.8 \mathrm{~J} / \mathrm{g}$. 


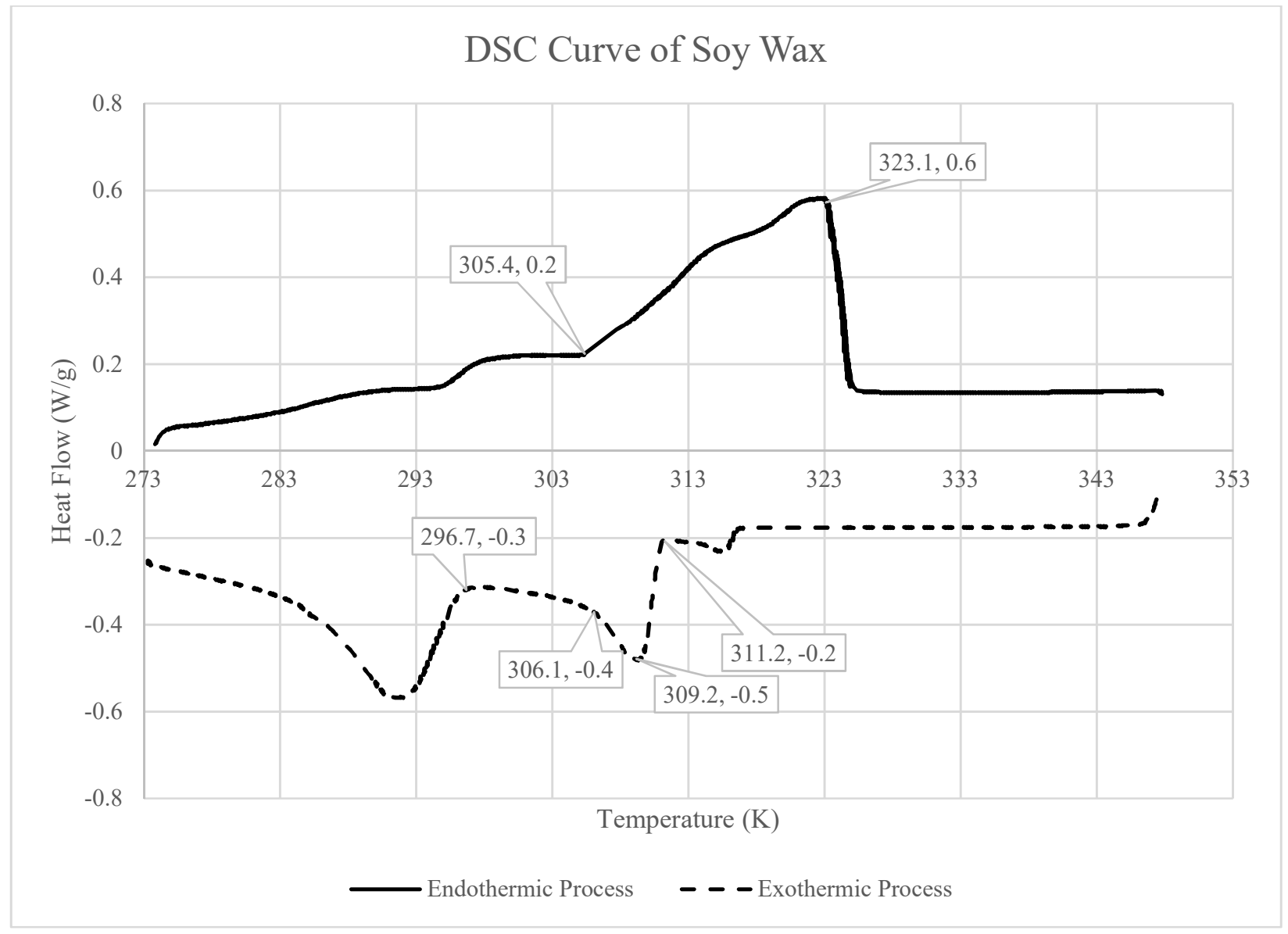

Figure 2.2: DSC curve of soy wax from Cui et al. (2011)

Soy wax was chosen as the PCM primarily due to limitations related to Mach Zehnder Interferometry. The primary output of MZI are images called interferograms. An interferogram image is made up of a series of dark and light fringes that are used to obtain air temperatures. A detailed description of MZI and interferograms is explained in Chapter 3. To obtain optical measurements there needs to be a large enough of a fringe gradient in the region of interest, which in the current work is the near wall heat transfer rates. A larger fringe gradient results in more fringe crossings than a lower fringe gradient. Larger fringe gradients are achieved with increasing 
temperature gradients. Therefore, to obtain measurements with interferometry, there needs to be a large enough of a temperature difference between the PCM panel surface and the ambient air. The soy wax used in the current work has a melting temperature of $38^{\circ} \mathrm{C}$ which assuming an ambient air temperature of $20^{\circ} \mathrm{C}$ results in a temperature difference of $18^{\circ} \mathrm{C}$. This temperature difference along with other parameters such as PCM panel width and laser properties results in a large enough of a fringe gradient that temperature measurements can be made. A larger temperature difference between the PCM panel and ambient air also allows for the buoyancy driven flow to reach turbulence. Due to the short height of the PCM panel (11"), a larger temperature difference was needed to invoke turbulent flow. A detailed description of the PCM panel is explained below in Section 2.4. In addition, soy wax is affordable in large quantities and is easy to obtain.

However, with the reasons for using soy wax as the phase change material explained above, it is important to mention limitations and differences from typical commercial products. The primary difference with typical PCM usage is the choice of melting temperature. For the application of PCM wallboards, the melting temperature should be near the set point temperature of the room where the wallboards are used. This is done so that the passive thermal storage capacity of the PCM can be used to reduce the heating or cooling load of the room. Most commercial PCM wallboards use paraffin wax as the phase change material. An advantage of paraffin wax is the similar exothermic and endothermic behaviour that can be seen on DSC curves with small differences between the melting temperature and solidification temperature. This difference when the solidification temperature is lower than the melting temperature is called subcooling. A symmetric DSC curve and small subcooling allows the paraffin wax to give similar thermal performance for melting and solidification processes. However, for soy wax, the DSC curve in Figure 2.2 demonstrates that the endothermic and exothermic curves are not symmetric and there 
is a large subcooling effect of approximately $13.9^{\circ} \mathrm{C}$. Therefore, due to the hysteresis of soy wax, the thermal performance is different for heating or cooling (charging or discharging of latent heat). The high melting temperature, large subcooling, and asymmetrical DSC curves imply that the soy wax used in the current work is not the ideal PCM to use for PCM wallboards. However, soy wax can still be used to study and highlight the effect of the phase change process on the convective heat transfer rates into the built environment. Additionally, soy wax can be used to study the applicability of established correlations for PCM wallboards such as the ASHRAE recommended correlation for building walls from Churchill and Chu (1975) among others.

\subsection{PCM Panel}

A schematic diagram of the PCM panel is shown in Figure 2.3. The overall dimensions of the PCM panel is $279.4 \mathrm{~mm} \times 355.6 \mathrm{~mm} \times 19.05 \mathrm{~mm}$. Figure 2.4 contains a layered schematic showing the various elements of the PCM panel. The panel consists of two acrylic glass sheets with a height of $279.4 \mathrm{~mm}$ (11"), a width of $355.6 \mathrm{~mm}$ (14") and a thickness of $3.2 \mathrm{~mm}(1 / 8 ")$. The acrylic glass sheets were coated with a white paint to give an emissivity of $\varepsilon_{P}=0.89$. The emissivity was measured using an infrared reflectometer at the University of Waterloo, using a Gier-Dunkle Reflectometer. The corners of the PCM panel where looped with yarn to create a suspension system allowing the PCM panel to hang freely during the experiment with minimal physical contact with other parts so that the heat transfer and air flow around the panel are not interrupted. At the top the PCM panel there is a threaded cap to allow for pouring of the PCM into the cavity. Also shown in Figure 2.3 is a spring mechanism used for thermocouple alignment which will be explained in more detail later.

The dimensions of the PCM panel were determined to optimize the performance of the interferometer. Ideally, the width of the panel should be small in order to give enough visible 
fringes. If the PCM panel width is too large, then refraction errors can be significant. However, if the PCM panel width is too small, then end effects will create three dimensional flows that will skew the results. The chosen dimensions give 9 fringes (or 0.5 fringes per ${ }^{\circ} \mathrm{C}$ ) across the optical window, for a wall-to-air temperature difference of $\Delta \mathrm{T}=18^{\circ} \mathrm{C}$.
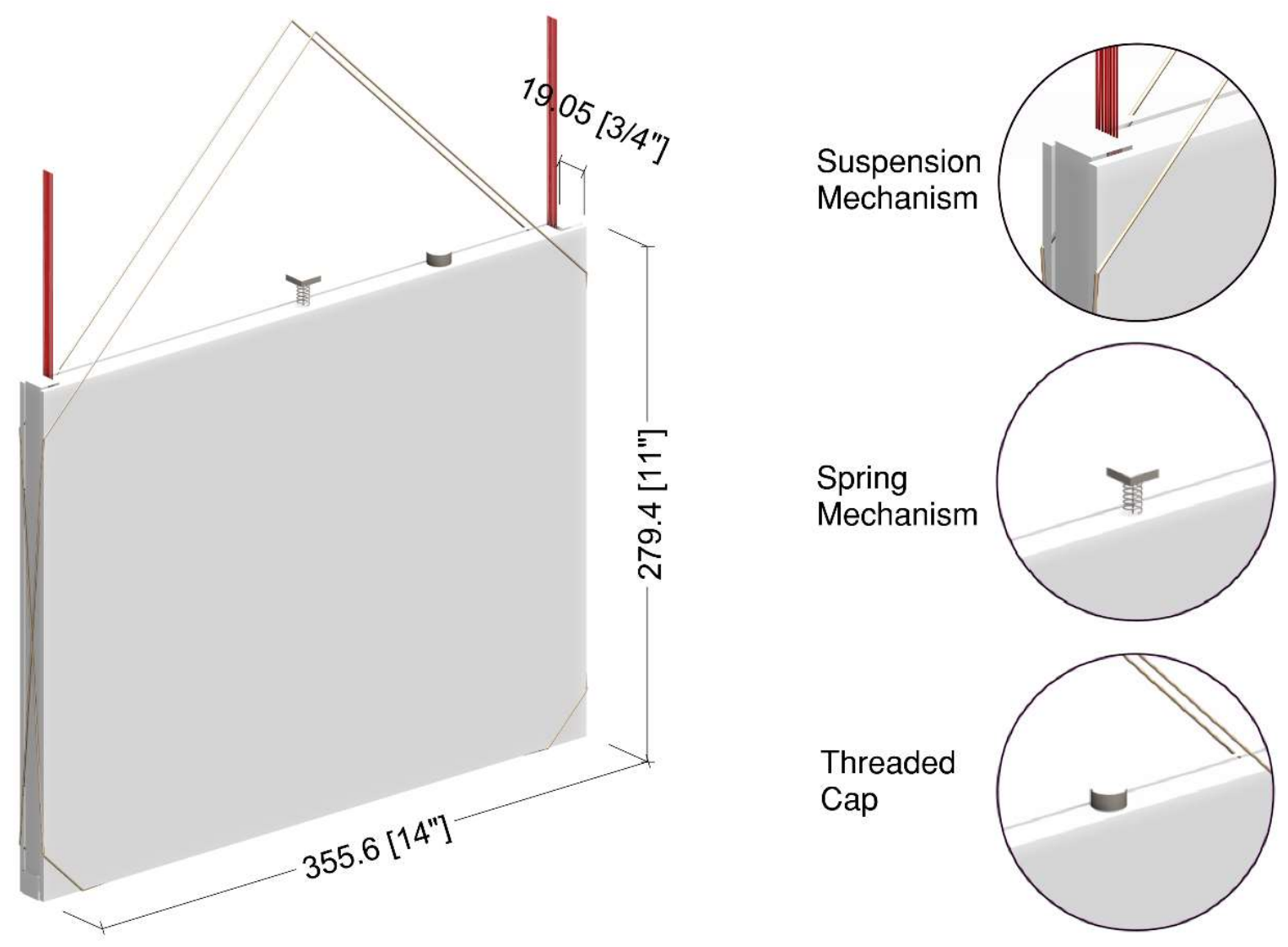

Figure 2.3: Overall PCM panel assembly 


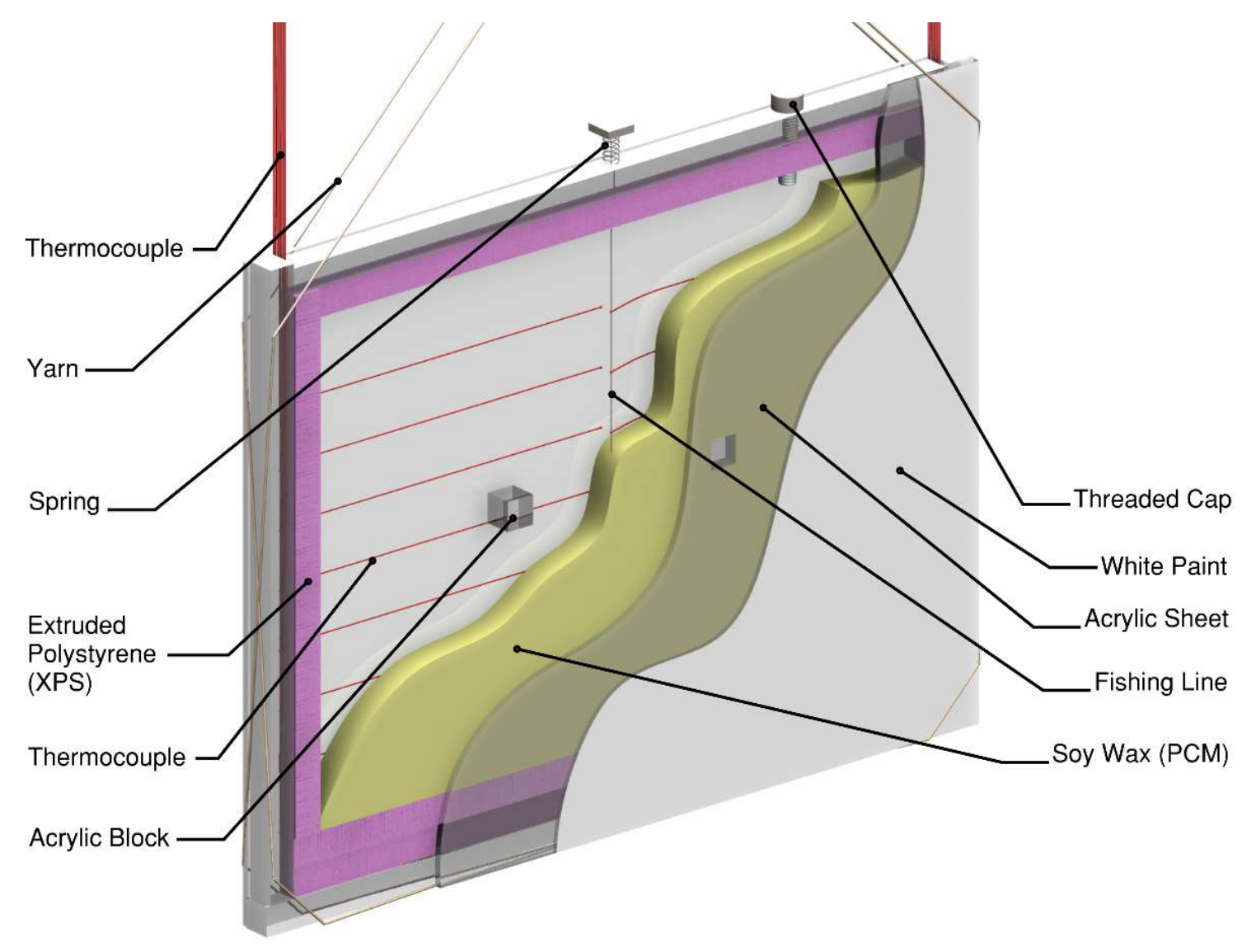

Figure 2.4: Overall PCM panel assembly with cutout

A cavity to hold the soy wax was created by using acrylic glass spacers that where $12.7 \mathrm{~mm}$ by $12.7 \mathrm{~mm}$ in cross section as shown in the cutout shown in Figure 2.5. Four pieces of the acrylic glass spacers where placed around the perimeter of the sheets to create a sealed cavity. The seal was created by using acetone on the acrylic glass sheets and spacers. When used on acrylic, acetone is a solvent-based glue that softens the acrylic creating a kind of weld. This allows the acrylic sheets and spacer to be chemically bonded making a continuous material. Due to how thin the acrylic sheets are, there is a possibility of bowing as a result of the hydrostatic forces of the PCM during the liquid phase. To minimize this effect, two $12.7 \mathrm{~mm} \times 12.7 \mathrm{~mm} \times 12.7 \mathrm{~mm}$ acrylic blocks where placed inside the cavity to hold the acrylic sheets together. The acrylic blocks were chemically bonded to the acrylic sheets with acetone. The interior edges of the panel cavity where 
lined with extruded polystyrene (XPS) to insulate the PCM from the edges. The acrylic sheets, spacer, and blocks were held together under pressure with a series of clamps around the perimeter of the sheets and allowed to cure for 24 hours. Figure 2.6 contains an exploded assembly view of the apparatus which shows the various strips of extruded polystyrene and acrylic spacers. The layers of XPS strips were used to minimize edge affects and to force the heat transfer through the acrylic sheets. The soy wax was heated until it became a liquid and poured through a threaded hole in the top acrylic spacer up to a height of $203.2 \mathrm{~mm}\left(8^{\prime \prime}\right)$. The cavity has an available height of $215.9 \mathrm{~mm}(8.5 ")$. An air gap of $12.7 \mathrm{~mm}(0.5$ ") was left at the top of the cavity to allow the soy wax to expand and contract during the experiment.
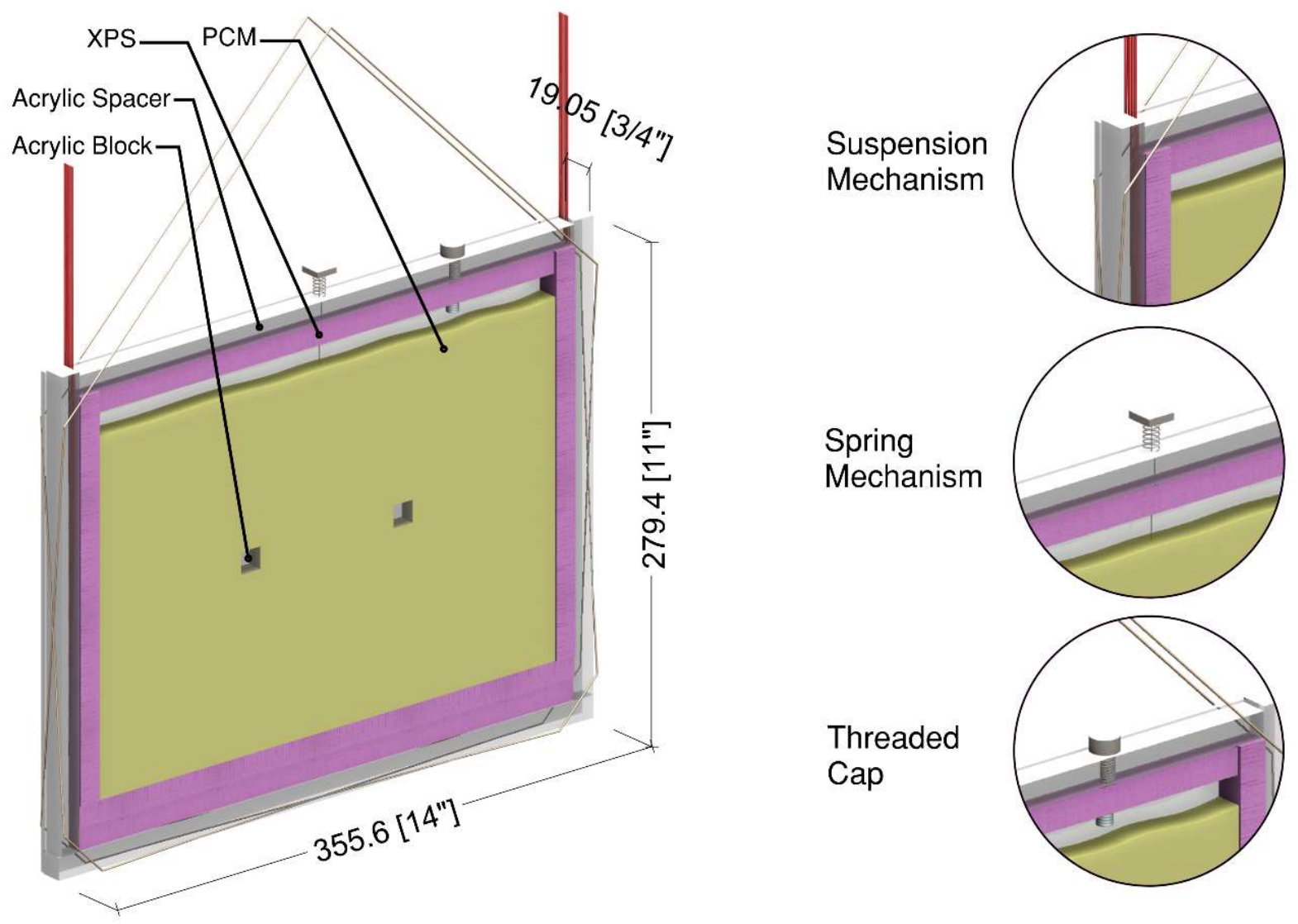

Figure 2.5: PCM panel interior 


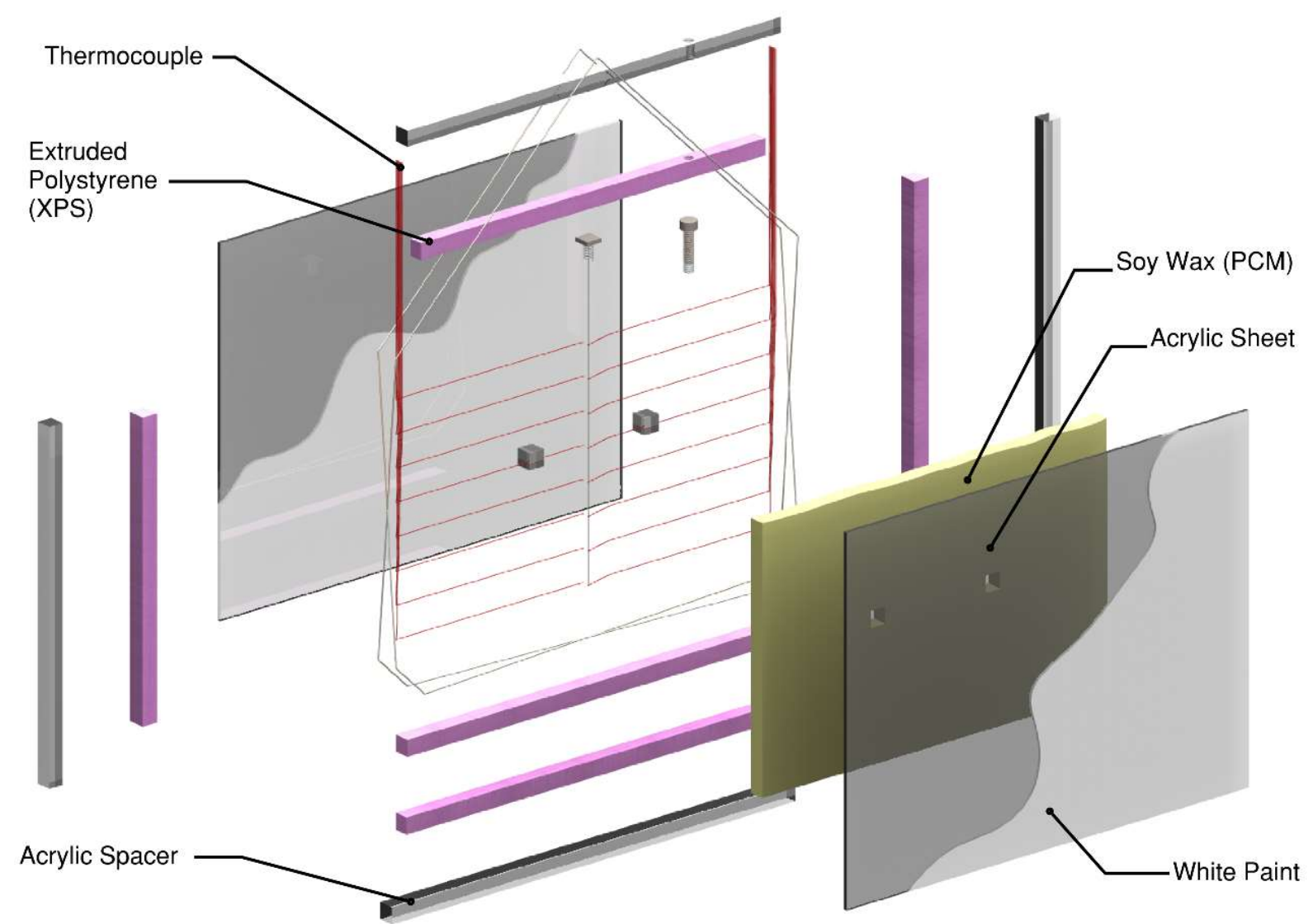

Figure 2.6: PCM panel exploded assembly

Figure 2.7 shows the interior of the panel without PCM exposing the thermocouples. The experimental apparatus was wired with 20 type T thermocouples. All the thermocouple used 40 gauge wire to minimize the impact the thermocouples may have by conducting heat away from the PCM. There are 9 thermocouples placed along the inside surface of one of the acrylic sheets. The plate side sensors were placed vertically at the midpoint of the acrylic sheet. The thermocouples were spaced $25.4 \mathrm{~mm}$ (1") apart from the base of the PCM at the intersection of the PCM and extruded polystyrene spacer up to the air gap above the level of the soy wax. Another set of 9 thermocouples where placed along the center line of the soy wax matching the placement of the plate side thermocouples. To hold the center line thermocouples in place a fishing line was used that runs the height of the PCM panel from the bottom acrylic spacer, through two strips of 
the extruded polystyrene spacer, soy wax, top extruded polystyrene spacer and finally out the top acrylic spacer. The exiting fishing line through the top acrylic spacer was then attached to a spring mechanism in order to keep the fishing line taut. The center line thermocouples were then glued to the fishing line. These 9 center line thermocouples are $6.35 \mathrm{~mm}$ from the acrylic sheets and are spaced $25.4 \mathrm{~mm}$ apart vertically. With these two sets of thermocouples there are 18 sensors in total measuring the temperatures in the cavity. A $19^{\text {th }}$ thermocouple was placed off center on an arbitrary spot on the acrylic sheet to give an indication of the uniformity of the acrylic sheet temperature. Finally, a $20^{\text {th }}$ thermocouple was used to measure the ambient temperature and was not attached to the apparatus. The thermocouples were calibrated with a constant temperature bath using a high precision glass thermometer.

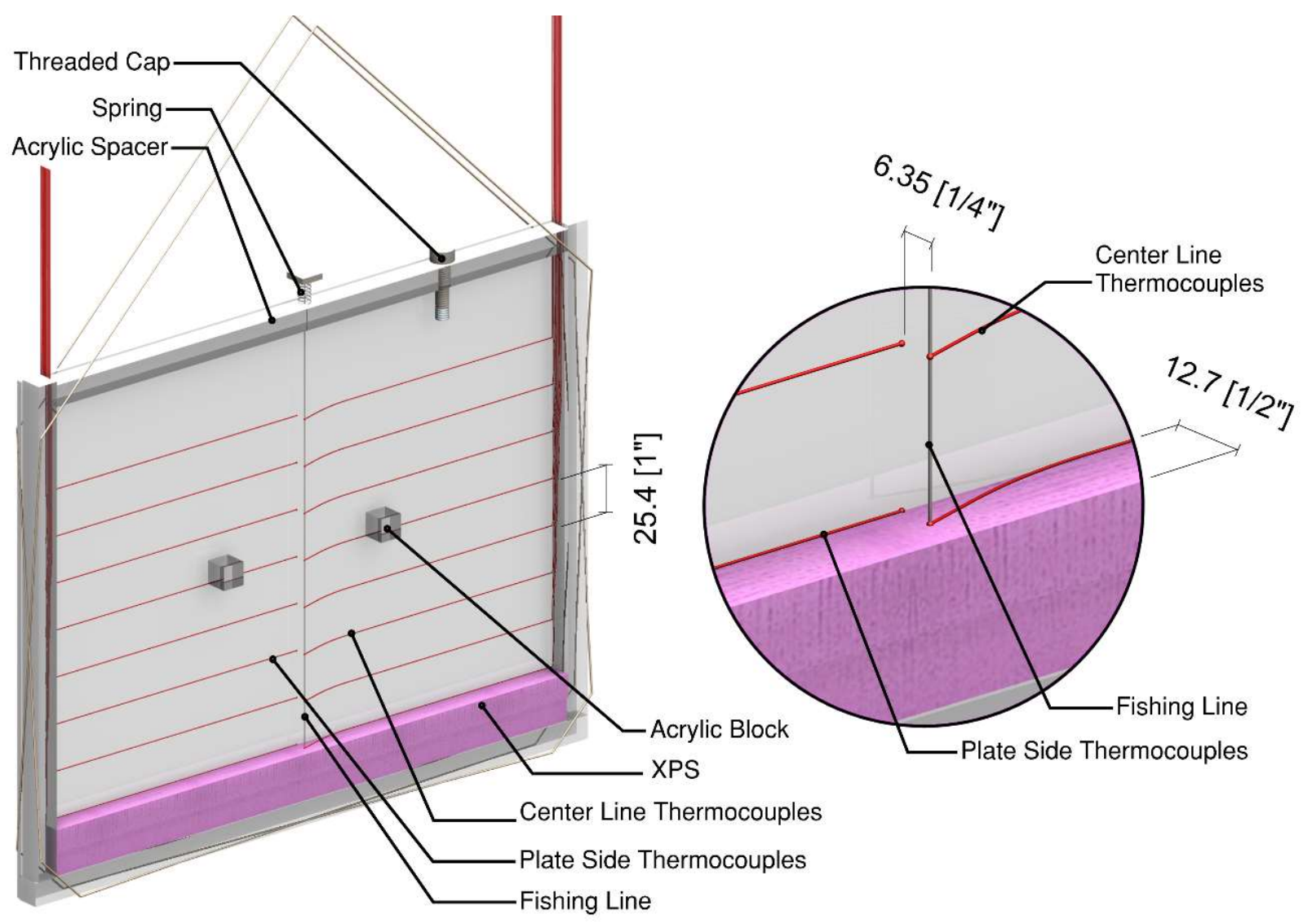

Figure 2.7: PCM panel interior with thermocouples 
In addition to using 40 gauge wire, the impact of the sensors on the PCM heat transfer was minimized by running the thermocouple wiring horizontally along the acrylic sheet and into the vertical strips of extruded polystyrene. By doing this, if the thermocouple wires do conduct heat away from the PCM then the conduction is forced to go laterally instead of vertically reducing the wires influence on the surface temperature profile. It is assumed that the temperature gradient in a vertical flat plate is much greater vertically than it is horizontally. As shown in Figure 2.7 each set of thermocouples runs horizontally to one side or the other. The plate side thermocouples run along the surface of the acrylic sheet to the left vertical spacer while the center line thermocouples run along the sheet surface to the right. The wires then run vertically between the vertical acrylic spacer and vertical extruded polystyrene spacer. The thermocouple wires then come out the top of the PCM panel and are bundled and draped away from the apparatus.

The PCM panel was designed to work within the size limitations and temperature gradient limitations of the interferometer. This results in a few limitations of the PCM panel that do not directly relate to typical commercial PCM wallboard products. The primary difference is the method of soy wax containment in the PCM panel. Typical PCM wallboards use either microencapsulated PCM, where the PCM is contained in microscopic beads, and/or is mixed with typical wallboard constructions (for example, gypsum in drywalls). This is done to modify the thermal behaviour of the PCM wallboard and to reduce 'leaking' in the liquid phase. Microencapsulation or mixed PCM alters the bulk material properties of the PCM and thus alters the thermal performance of the PCM wallboard. Doing this alters the effective thermal conductivity, specific heat, and density among other parameters and typically reduces subcooling and latent heat storage (encapsulation or mixing reduces the amount of PCM used for a given 
volume of wallboard). For the current study, the 'pure' soy wax is contained in a continuous vertical cavity and is not mixed with any other materials. Using a 'pure' PCM in a void cavity (microencapsulation), highlights the effect of subcooling and may allow for some fluid motion to occur when the PCM is in the liquid phase. However, as explained above in Section 2.3 for the reasons to use soy wax as the PCM, the current PCM panel can still be used to determine the applicability of established correlations from ASHRAE and others and to study the effect of the phase change process on free convective heat transfer rates.

\subsection{Overall Assembly}

The overall assembly is shown in Figure 2.8. The PCM panel was designed to be hung without interfering with the optical measurements of the experiment. The chosen suspension mechanism involves running a piece of yarn around the corners of the PCM panel and loop overtop similar in fashion to hanging a picture frame with a wire. The edges of the acrylic sheets have small cut outs that the yarn sits in and was glued in place. The PCM panel hangs from a cantilevered beam that was clamped to a vertical metal pole. The PCM panel was oriented so that the side with the acrylic sheet with embedded thermocouples face outwards away from the support base. Optical measurements with Mach-Zehnder interferometry is sensitive to the alignment of the apparatus in the experiment. The volume of air that is occupied by the path of the laser through the experiment has the effect of averaging the heat transfer results within that volume. Ideally, the vertical surface of the PCM panel should be parallel with the path of the laser otherwise the apparatus may introduce errors to reflections or skewed averaging.

To address this a three point alignment mechanism was created. Three long nylon screws are used to align the PCM panel in the yaw direction and roll direction as indicated in Figure 2.8. The nylon 
screws are threaded and are held in place with a nut to vertical metal poles. Twisting the nylon screws causes the screws to protrude out or retract. Yaw is controlled by two nylon screws that are in contact with the top corners of the PCM panel. Roll is controlled by a single nylon screw that is in contact with the bottom edge of the PCM panel. Pitch is controlled manually by adjusting how the yarn hangs from the cantilevered beam (similar to adjusting a picture frame on a wall).

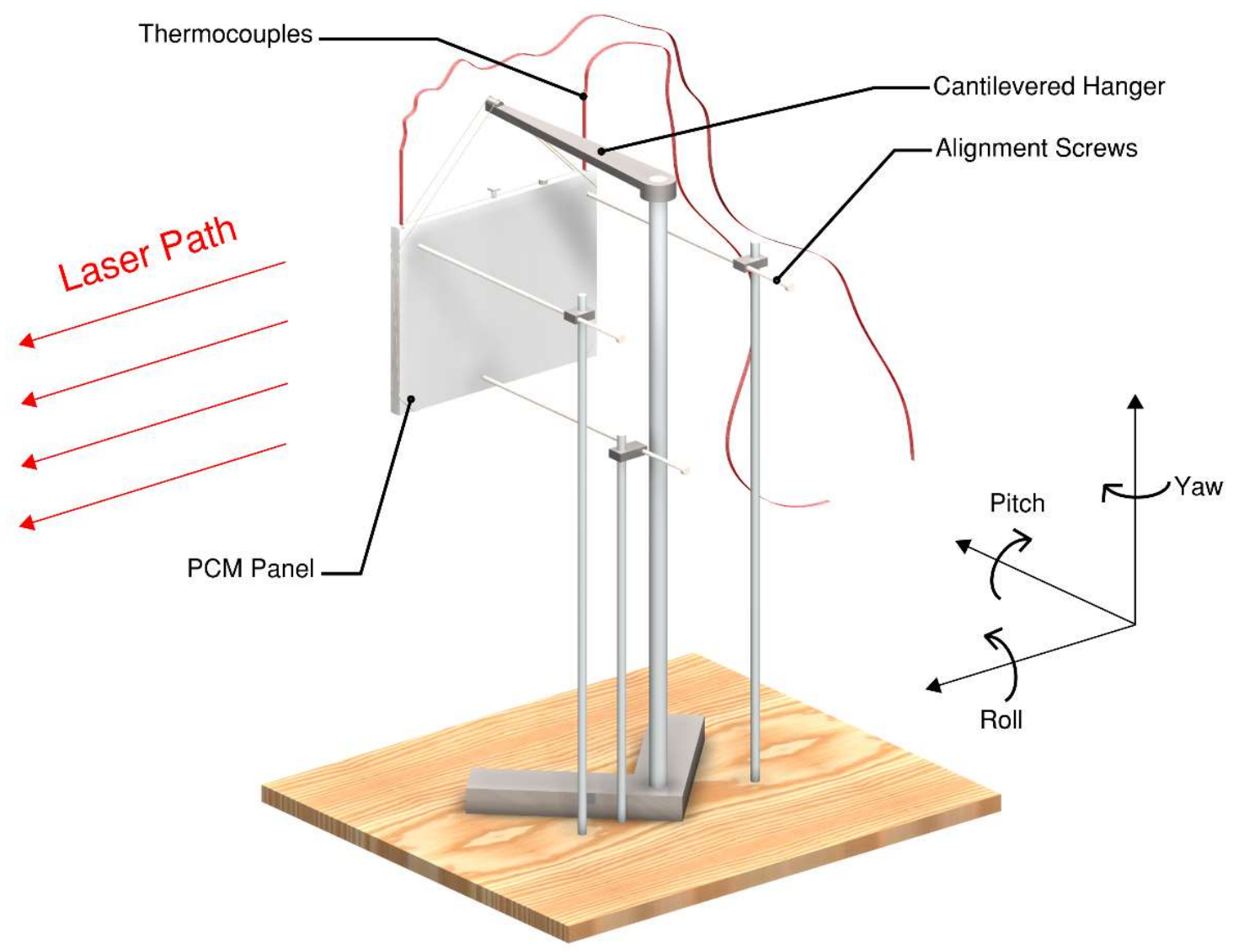

Figure 2.8: Overall assembly with PCM panel and support base

The PCM panel was heated up to a temperature of $70^{\circ} \mathrm{C}$ using a custom built heating bed. The heating bed consisted of a single portable stove heating element with an aluminum panel lying flat on top of the heating element. The heating element and aluminum panel were inclined to approximately $30^{\circ}$. The PCM panel would be placed on top of the aluminum panel and covered in a sheet of extruded polystyrene and inclined such that the threaded cap is elevated. The heating 
bed would be used to heat up the soy wax to $70^{\circ} \mathrm{C}$. During the heating process the thermocouples are connected to a custom built data acquisition system. The temperature readings are monitored until all the embedded thermocouples reach a temperature of $70^{\circ} \mathrm{C}$.

The experimental apparatus as shown in Figure 2.8 was placed on the same large bench that the laser and optics are attached too. The MZI and entire experimental apparatus was located in an enclosure to create a quiescent environment to reduce natural convective errors. The enclosure was custom made using a wood frame and a tarp covering the structure. The enclosure $(3.66 \mathrm{~m} \times$ $6.10 \mathrm{~m} \times 2.44 \mathrm{~m}$ ) is large enough to contain the entire MZI and apparatus. The enclosure was meant to: 1) reduce externally produced convection and 2) create a 'dark' room that is suitable for taking the interferogram images. 


\section{Chapter 3: Experimental Analysis}

\subsection{Interferometric Analysis}

Experimental data was taken using Mach-Zehnder Interferometry (MZI). MZI is a non-intrusive method of measuring the change in index of refraction of air. The index of refraction of air can be related to air temperature and by extension the heat transfer rates. The experiment investigated the thermal decay of a vertical flat plate filled with phase change material (soy wax). The apparatus was heated so that the soy wax was melted and then it was let to cool off in ambient air. The laser interferometry was used to measure the convective heat transfer rates as the PCM solidified. The output of MZI is an image called an interferogram which is made up of dark and light fringes called destructive and constructive fringes respectively. The fringes from MZI can be set up in infinite fringe mode or finite fringe mode. In infinite fringe mode the fringes represent lines of constant temperature which can aid in the visualization of the temperature field. In finite fringe mode the fringes can aid in the visualization of the temperature gradients. For the present study finite fringe mode was used as it is more accurate and easier to obtain measurements. The analysis procedure is the same for finite or infinite fringe modes. Due to the large range of temperatures that the apparatus undergoes, the analysis is done only on the interferograms with a large enough of a temperature difference to give at least three complete fringe crossings.

In previous experiments that used the MZI, the surface temperature of the object of interest was known and is used as the reference temperature to then determine the temperature of all other fringes and to determine the convective heat transfer. However in the current experiments the surface is not known and the reference temperature is taken to be the ambient temperature. This is then used to incrementally determine the fringe temperatures towards the wall surface. The 
actual surface temperature is linearly extrapolated from the two nearest fringe temperatures. A more detailed explanation is shown below.

\subsection{Fringe Temperatures}

Figure 3.1 below is a typical finite fringe interferogram showing a heated vertical flat plate in ambient air. The plate surface is on the left side of the image and the ambient region is on the right side. For a given horizontal slice, each fringe crossing corresponds to a temperature increase of approximately $2 \sim 3 \mathrm{~K}$. Thus, the relative spacing of the fringes can be used as a way to visualize temperature gradients. It can be seen that on the left side (near the wall) the fringe spacing is small and thus indicates an area of high temperature gradient. As the fringe crossing move away from the wall the fringe spacing is increasing indicating a decrease in the temperature gradient. The right half of the Figure 3.1 is the ambient region as shown by the nearly horizontal destructive fringe. Ideally regions with no temperature gradient should have horizontal fringes, however in the current experiment there exists a fringe shift error which effectively adds an artificial temperature gradient to the entire image. Fringe shift errors can be caused by 'drifting' of the MZI optics and the change in ambient temperature from when the optics where first aligned. This error can be seen by the non-horizontal fringe in the ambient region. 


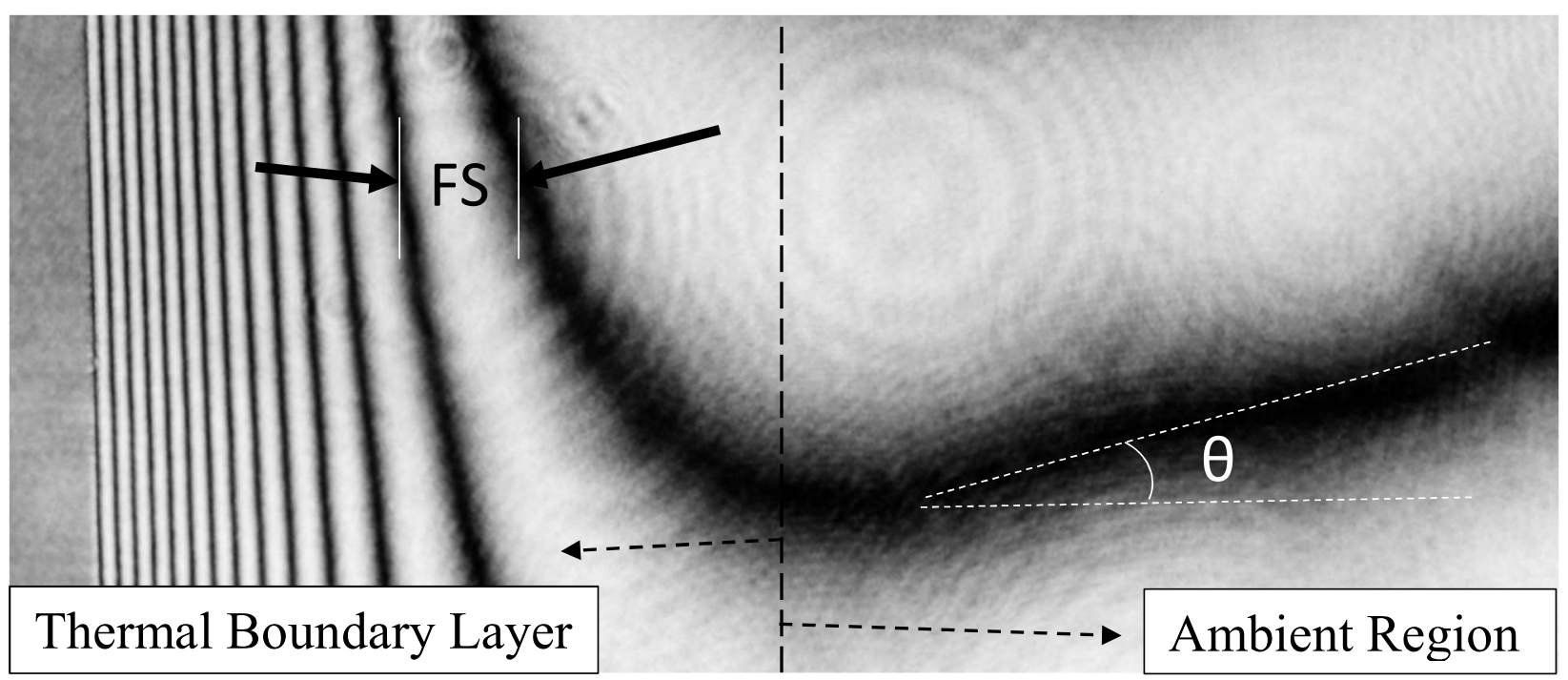

Figure 3.1: High temperature gradient case of finite fringe interferogram

Figure 3.2 illustrates the destructive fringe crossings for a given horizontal scan where 'FS' is fringe spacing and ' $\theta$ ' is the far field fringe angle. Due to the fringe shift error, there needs to be a fringe crossing within the ambient region. Doing this allows using the ambient temperature as a known reference temperature. With this reference temperature known, the subsequent fringes temperatures can be determined towards the wall. In the sample fringe crossings image (Figure 3.2) there are 15 fringe crossings. 


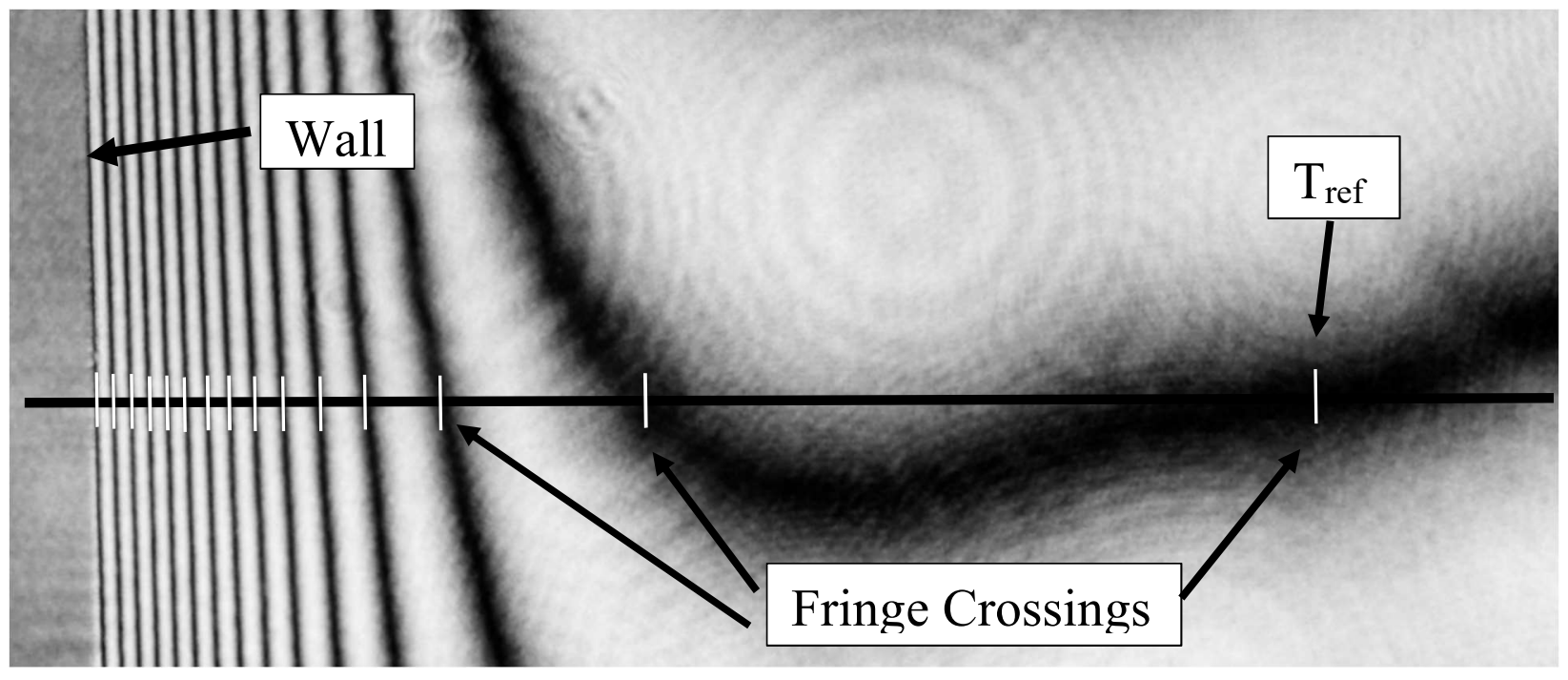

Figure 3.2: Finite fringe interferogram treatment

With the reference temperature known, the 'next' fringe temperature can be found using Equation 3.1.

$$
T_{i+1}=\frac{T_{i}}{1 \pm \frac{R \lambda T_{i}}{W P G}}
$$

where $T_{i}$ is the reference temperature $\left(T_{1}\right.$ is the ambient temperature), $R$ is the universal gas constant $(287 \mathrm{~J} / \mathrm{kg} \cdot \mathrm{K}), \mathrm{G}$ is the Gladstone-Dale constant $\left(\mathrm{G}=0.226 \times 10^{-3} \mathrm{~m}^{3} / \mathrm{kg}\right)$ which is a parameter that describes the relationship between chemical composition, density and refractive index, $\mathrm{P}$ is the absolute ambient pressure (measured from a mercury barometer), $\mathrm{W}$ is the length of the laser path through the experimental model $(\mathrm{W}=355 \mathrm{~mm}), \lambda$ is the vacuum wavelength of the light source $\left(\lambda=6.328 \times 10^{-7} \mathrm{~m}\right)$. Once all the fringe temperatures are known for a given 
horizontal scan, the wall temperature is determined by linear interpolation from the near wall temperature gradient.

\subsection{Local and Average Convective Heat Transfer Rates}

Using Equation 3.1 for a finite fringe interferogram, the temperature gradient normal to the plate surface can be determined. By determining the temperatures of the first two consecutive fringes and assuming a linear interpolation, the temperature gradient at the plate surface can be determined. An illustration of the temperature gradient is shown in Figure 3.3.

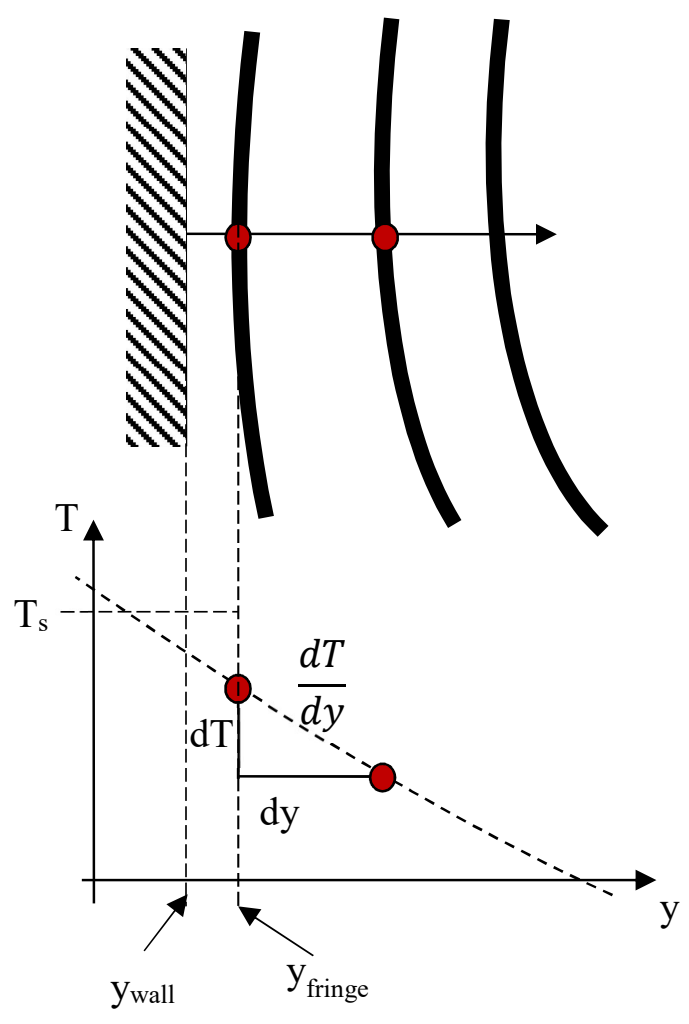

Figure 3.3: Illustration of the method used to calculate the surface temperature gradient 
With the surface temperature gradient known, the convective heat transfer rate can be equated to the conductive heat transfer rate as shown in Equation 3.2:

$$
\begin{aligned}
& q^{\prime \prime}=-\left.k_{S} \frac{d T}{d y}\right|_{y=0}=h\left(T_{S}-T_{\text {ref }}\right) \\
& \left.\frac{d T}{d y}\right|_{y=0}=\left.\frac{R \lambda T_{w}^{2}}{W P G} \frac{\partial \epsilon}{\partial y}\right|_{y=0}=\frac{R \lambda T_{w}^{2}}{W P G}\left(\frac{1}{F S}\right)
\end{aligned}
$$

where ' $\mathrm{k}_{\mathrm{s}}$ ' is the thermal conductivity of the air evaluated at the wall surface temperature, 'dT/dy' is the temperature gradient at the wall surface, ' $h$ ' is the convective heat transfer coefficient, and $\mathrm{q}^{\prime \prime}$ is the local convective heat transfer rate per unit area (heat flux).

The local convective heat flux was calculated at six evenly spaced intervals on the PCM wall panel, over a distance of $127 \mathrm{~mm}$. The entire process was automated using image processing software developed by the author. The average convective heat flux values were calculated by numerically integrating the local convective heat transfer rates using the trapezoidal rule.

\subsection{Image Analysis}

In order to determine the fringe gradient and by extension the temperature gradient, the horizontal location of the fringe crossing need to be known. A custom MATLAB code was written to perform image processing that extracts out these fringe locations. The image analysis involved performing curve fits to the image intensity data. The process is explained in more detail below.

In a gray-scale image (which the interferograms are converted to), each pixel is assigned a value between 0 (white) and 255 (black), this is the light intensity of the pixel. Treating the interferogram 
image as a grid of light intensity, a horizontal scan is simply just a single row from this grid. Figure 3.4 shows an interferogram with the scanned portion highlighted and the corresponding light intensity plot in Figure 3.5. A locating pin is shown as well in Figure 3.4 which is used to measure the length scale and thermocouple locations for each image. The average length scale of all the interferograms used for the analysis is approximately 32 pixels $/ \mathrm{mm}$. The plot in Figure 3.5 was taken from an edited version of Figure 3.4 with an inverted colour spectrum. Thus, in the light intensity plot, the 'highs' correspond to destructive fringes (dark fringes), and the 'lows' corresponds to constructed fringes (light fringes). Each peak was isolated and a curve fit was performed to find the exact location of the fringe peak. A detailed image analysis is explained next.

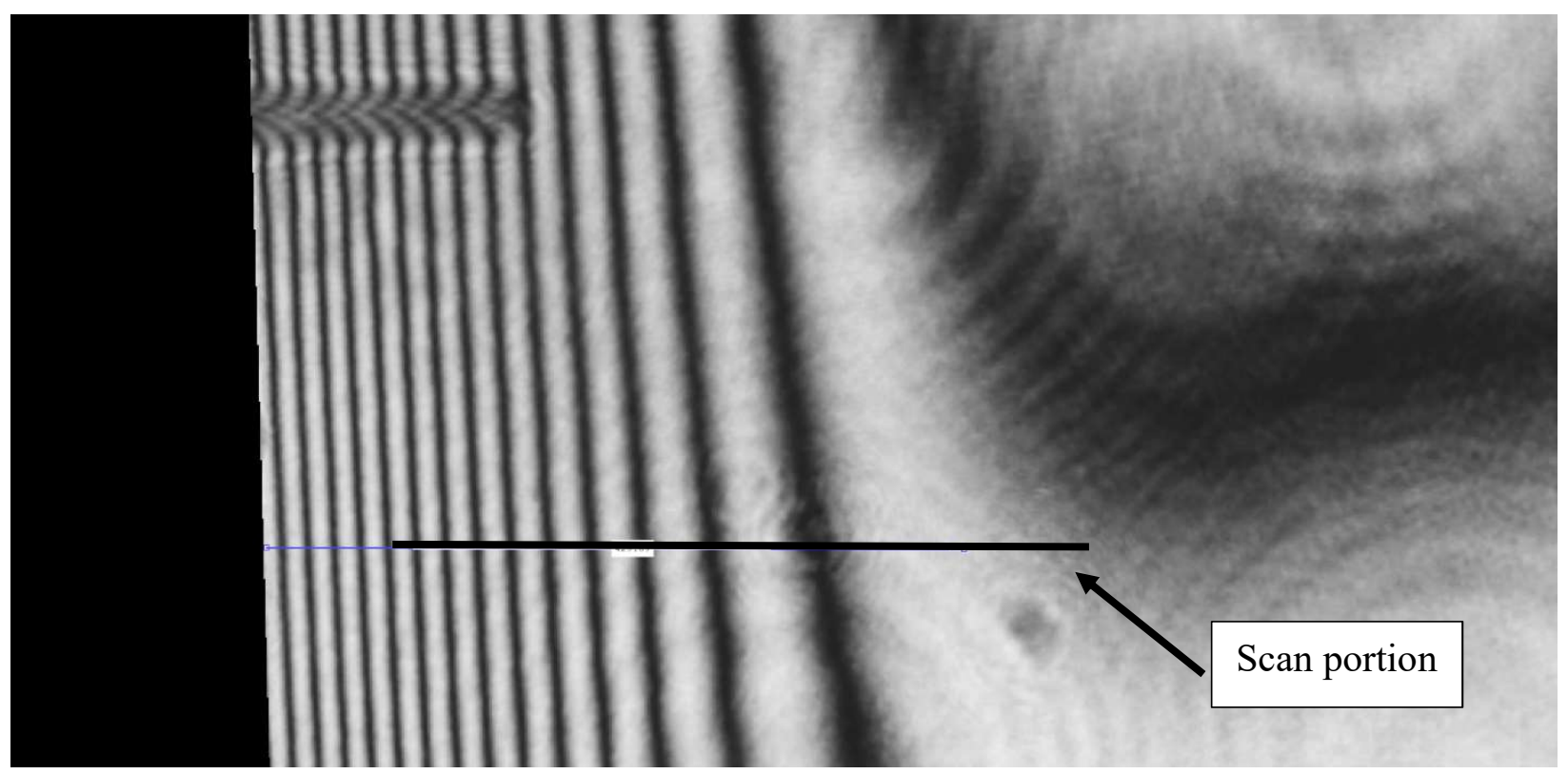

Figure 3.4: Interferogram scan sample

A light intensity curve is shown in Figure 3.5. The light intensity curve does not represent a full horizontal scan, rather it represents the near wall light intensity. The ambient region light intensity treatment is explained in a later section. Each peak in Figure 3.5 is first isolated using a 
MATLAB function called 'findpeaks' which determines the peaks (highs) in a set of data based on the prominence of the curve. The curve prominence is essentially the relative peak height relative to neighbouring peaks. For finding these peaks a minimum prominence of 60 was used to isolate the peaks of interest and ignore miscellaneous peaks due to the noise in the data. A peaks plot with a minimum prominence of 60 is shown in Figure 3.6.

\section{Light Intensity}

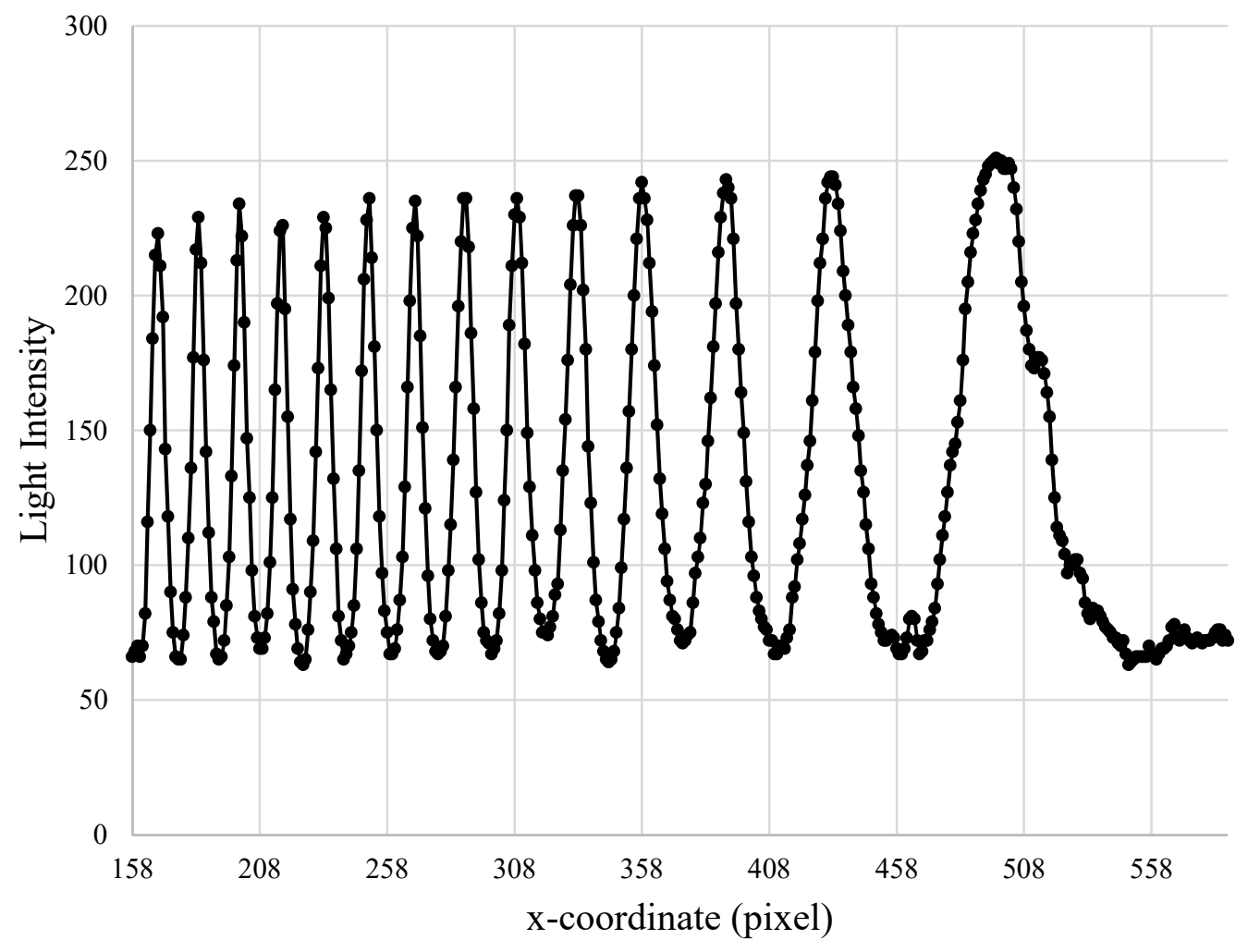

Figure 3.5: Light intensity scan 


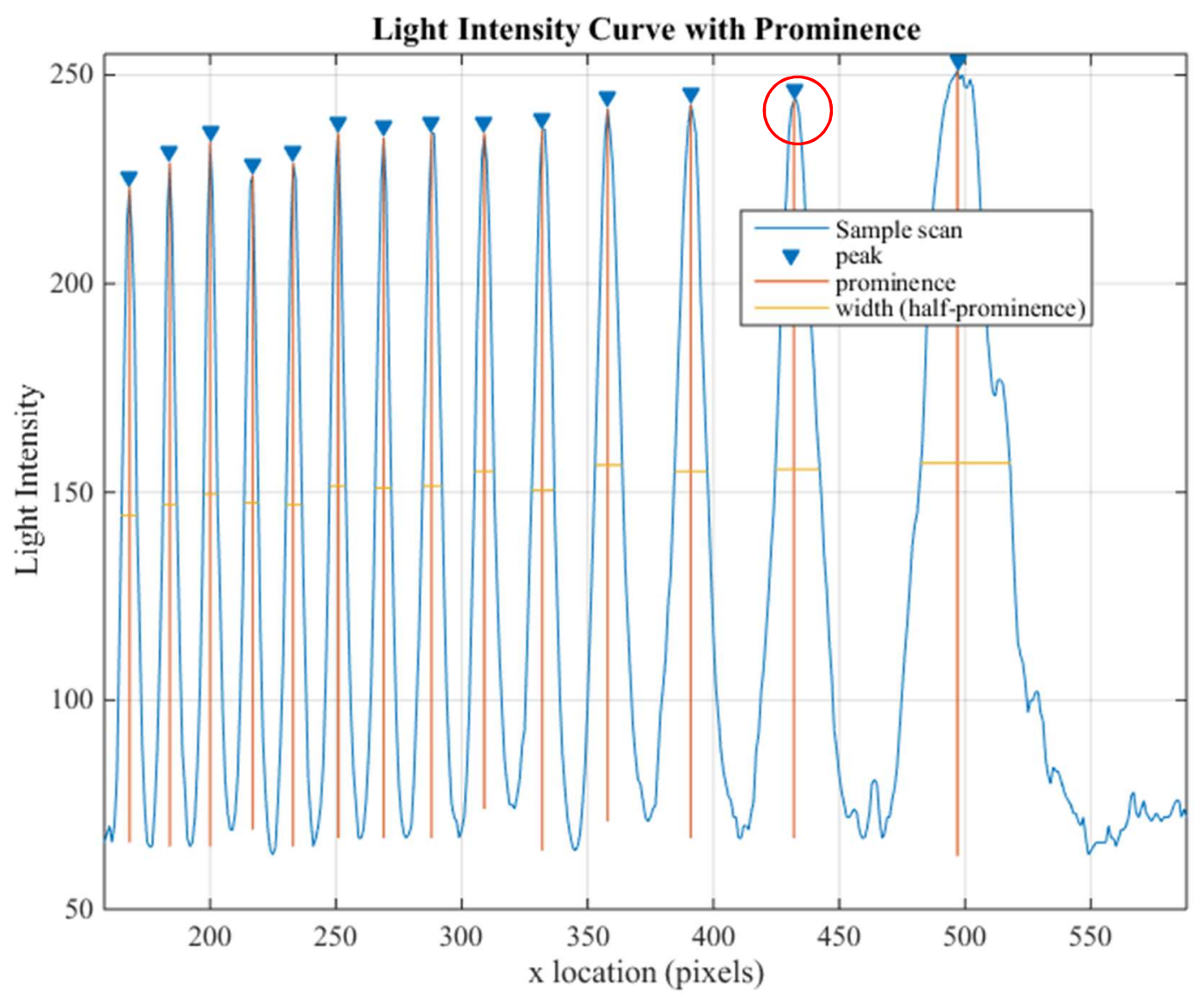

Figure 3.6: Sample Scan with Prominence

The peak locations from Figure 3.6 do not represent the actual location of the light intensity peaks (the fringe crossings). The peak locations correspond to a specific data point which in this case is the integer pixel number. To find the actual peak a single term Fourier series was used to fit a curve on each isolated peak. A nonlinear regression technique was developed by Poulad et al. (2011) to determine the fringe gradient of an interferogram scan by fitting a sinusoidal function to the light intensity curve. For a linear temperature profile, the light intensity follows approximately a sinusoidal pattern. However, for the current study it was found that convergence was difficult to achieve due to the larger number of fringe crossings to find for each scan. The original regression 
technique was used to find the near wall fringe gradient using only the nearest 2 or 3 fringes. However, in this study, the fringe crossings for all the fringes within view of the interferogram output are needed to be obtained. It was found that convergence was greatly improved by using a single term Fourier series which still maintains the 'sinusoidal' shape of the light intensity curve. Each peak was isolated by defining a band that is approximately the quarter peak-to-peak distance on either side of the peak of interest. The Fourier series function used is shown in Equation 3.3:

$$
I(x)=a_{0}+a_{1} \cos (x w)+b_{1} \sin (x w)
$$

where $I(x)$ is the light intensity curve as a function of $x$, and $a_{0}, a_{1}, b_{1}$ are the curve fitting parameters. From Equation 3.3 above the peak fringe location is found by finding the roots of the derivative which becomes Equations 3.4a and 3.4b:

$$
\begin{gathered}
\frac{\partial I(x)}{\partial x}=-a_{1} w \sin (x w)+\mathrm{b}_{1} w \cos (x w)=0 \\
x_{\text {peak }}=\frac{n \pi+\tan ^{-1}\left(\frac{b_{1}}{a_{1}}\right)}{w}
\end{gathered}
$$

where a multiple ' $n$ ' of ' $\pi$ ' is added due to the periodic nature of the tangent function since light intensity curve does not start at zero. The parameters $\mathrm{a}_{1}, \mathrm{~b}_{1}$ and $\mathrm{w}$ are the outputs from the curve fitting process. The integer ' $n$ ' is found by using the original peak location from the 'findpeaks' function as an estimate and rounding to the nearest integer. Thus ' $n$ ' is found from Equation 3.5:

$$
n=\left\|\frac{x_{\text {peak,approx }} w-\tan ^{-1}\left(\frac{b_{1}}{a_{1}}\right)}{\pi}\right\|
$$

A sample output of the curve fit is shown in Figure 3.7. The light intensity data is taken from the $12^{\text {th }}$ peak from Figure 3.6 which is highlighted with a circle. The procedure explained above using Equations 3.4 and 3.5 was applied for each peak along every scan. From this analysis, the fringe 
gradient is obtained from every scan. This procedure only applies to the thermal boundary layer region as shown in Figure 3.1. The additional fringe crossing corresponding due to the ambient fringe is explained next.

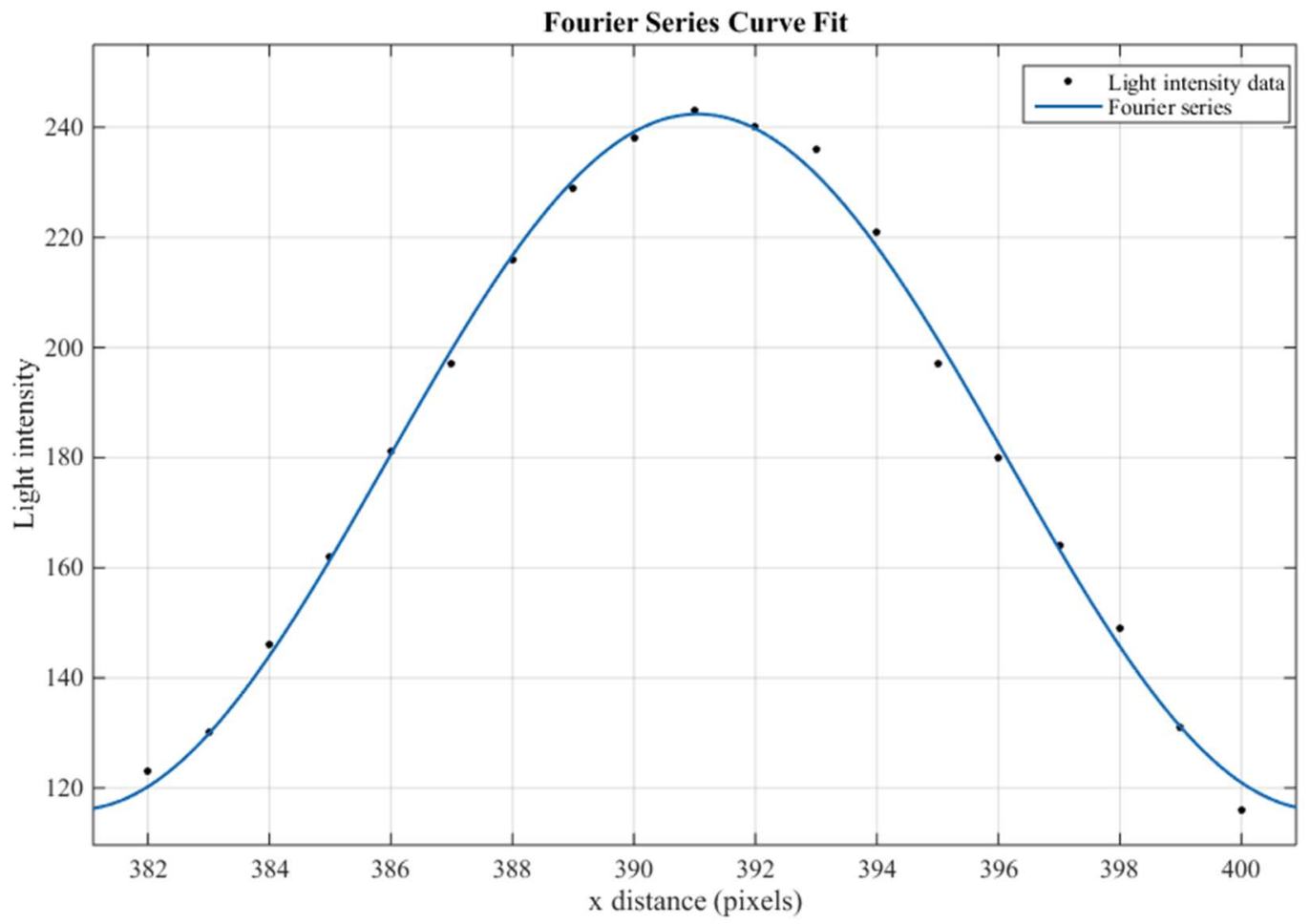

Figure 3.7: Fourier series curve fit

\subsection{Ambient Fringe Scans}

Due to a fringe shift error the ambient fringes are not horizontal as shown in Figure 3.1. Thus for each horizontal scan the fringe crossing of these ambient fringes need to be known as shown in Figure 3.2. For some regions, the interferogram output is not wide enough to either, capture a complete ambient fringe crossing, or obtain a Fourier series curve fit to define a fringe peak. Therefore a different approach is needed than the one explained in Section 3.4. To aid in the 
determination of the far field fringe crossing, a method was developed to measure the location and slope of individual far field fringes. In the ambient region the temperature is assumed to be constant and by extension the temperature gradient is supposed to be zero. With these assumptions the ambient fringes can be expected to be linear (a straight line). The approach to analyze the ambient fringes is to find an equation of a line that represents each fringe. With this line, further analysis can be done to determine the fringe crossing. To do this a vertical scan is performed (as opposed to a horizontal scan used for the thermal boundary layer region) over the thickness of the fringe band. For each vertical scan (or slice) the fringe destructive peak is found using the same procedure as the horizontal scans using a Fourier series curve fit as explained above.

Figure 3.8 illustrates the scanning region of a sample far field fringe. The starting point of each scan is defined by the guide line (blue line defined by P1 and P2) and is parallel to the fringe of interest. The end points of each scan is determined by a scan 'height' (fringe thickness) and is an input into the algorithm and is labeled. A single scan is performed from the vertical slice that begins at the guide line and ends at the scan length (scan band in Figure 3.8). For that vertical slice, the fringe peak is found. This process is repeated for every vertical slice along the scan path (incremented by 1 pixel horizontally). The resulting fringe peaks are shown as a single dot for each scan in Figure 3.9 and is made up of approximately 1200 scans. Finally an equation of a line is fitted to the data from Figure 3.9 and this line can be used to determine the far field fringe crossings or perform additional analysis. A line of best fit for the sample far field fringe is shown in Figure 3.10. If the ambient fringe is sloped large enough (due to a fringe shift error), then the far field fringe crossing is the intersection point of the line of best fit and the horizontal scan line from the Section 3.2. The fringe crossings from Section 3.2 and 3.4 makes a complete horizontal scan with all the fringe crossings from the ambient to the PCM wall. Equation 3.1 can be used to determine 
temperatures of all the fringe crossings where the first temperature (far field fringe crossing) is set as the ambient temperature.

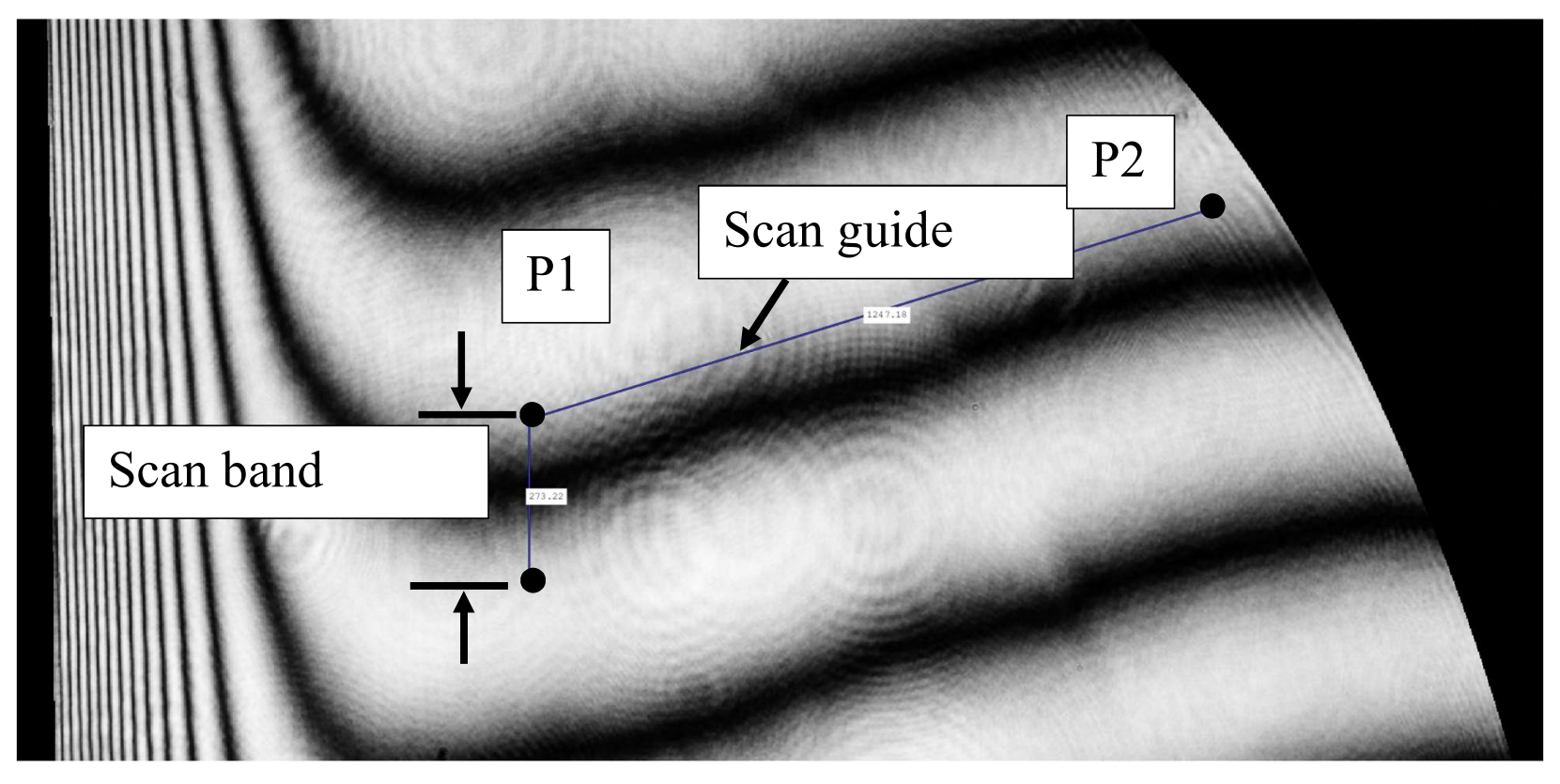

Figure 3.8: Far field fringe scan band 


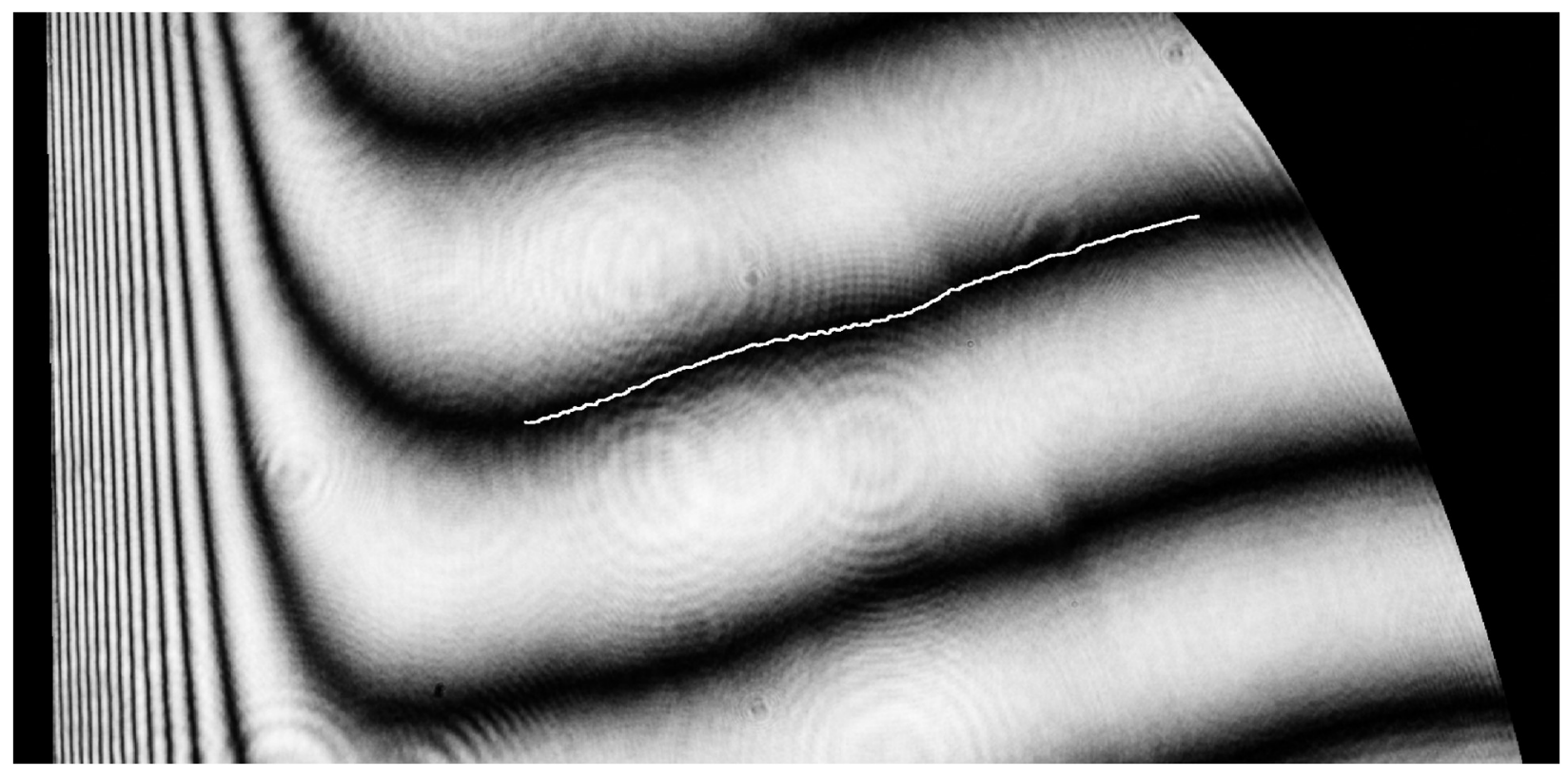

Figure 3.9: Far field fringe peaks

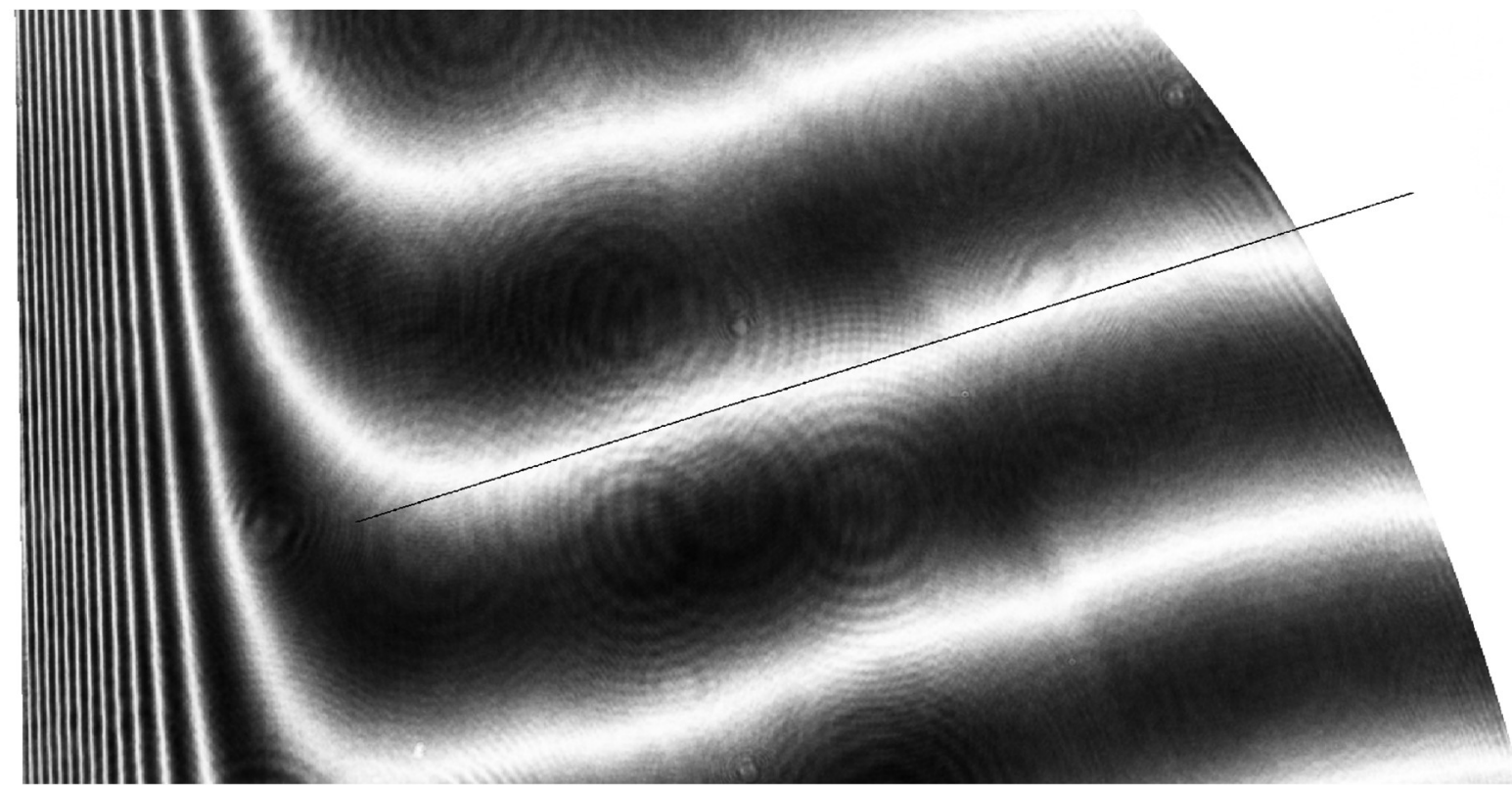

Figure 3.10: Far field fringe line of best fit 


\subsection{Fringe Shift Error}

In a finite fringe interferogram, a zero temperature gradient is evident by a horizontal fringe (and thus no fringe crossings). However, if the optics are not perfectly aligned (or if the optics alignment drifts with time), there will be a non-horizontal fringe. This 'sloped' fringe has the effect of adding an artificial temperature gradient for the entire interferogram. To account for this error, this artificial temperature gradient can be determined and then added/subtracted to the measured temperature gradient. Due to the length of time that the experiments where performed, there is a noticeable drift in the MZI alignment. Figure 3.11 illustrates the change in the far field fringe slope from the beginning of the experiments $(t=0 \mathrm{~min})$ to the end of the experiment $(\mathrm{t}=229 \mathrm{~min})$. At the beginning of the experiment, the downward slope towards the wall of the ambient fringe indicates a reduction of the temperature gradient. Thus this artificial temperature gradient needs to be added to the measured temperature gradients. However, by the end of the experiments the far field fringe slopes upwards towards the wall which has the effect of increasing the temperature gradient. In this case the artificial temperature gradients needs to be subtracted from the measured data. Due to this 'drift' the fringe shift error needs to be determined for each interferogram. This procedure is explained next.

The general procedure involves finding the slope of as many far field fringes as possible and the spacing between them. With this information, the fringe gradient can be determined and by extensions the temperature gradient. The procedure is similar to finding the far field fringe crossing which involves performing a vertical scan across multiple fringe crossing and finding the corresponding fringe peaks. The fringe crossings are found using the same procedure as explained above using Equation 3.4 and Equation 3.5. Consider a single vertical scan and its corresponding light intensity curve shown in Figure 3.12. The blue line in Figure 3.12 represents the location of 
the first vertical scan. The light intensity output is also shown but with inverted intensity levels (black is intensity level 255 and white is intensity level 0). The y-coordinate location of each peak is found for each vertical scan (there are nine peaks in the Figure 3.12). From this the average peak-to-peak distance is obtained from Equation 3.6:

$$
F S y_{j}=\frac{1}{N-1} \sum_{i=1}^{N-1}\left(Y P_{i+1}-Y P_{i}\right)
$$

where $\mathrm{FSy}_{\mathrm{j}}$ is the average peak-to-peak spacing for a given scan ' $\mathrm{j}$ ', $\mathrm{N}$ is the number of peaks in a given scan, and YP is the y-coordinate of a given fringe peak. This vertical scan is repeated along the span of the ambient fringe similarly to scanning a far field fringe as explained above. At every scan the fringe peaks $\left(\mathrm{YP}_{\mathrm{i}}\right)$ and average peak-to-peak spacing $\left(\mathrm{FSy}_{\mathrm{j}}\right)$ are found. For the sample interferogram in Figure 3.12, the resulting fringe peaks are shown as dots superimposed on the interferogram in Figure 3.13 and is made up of 727 vertical scans. 


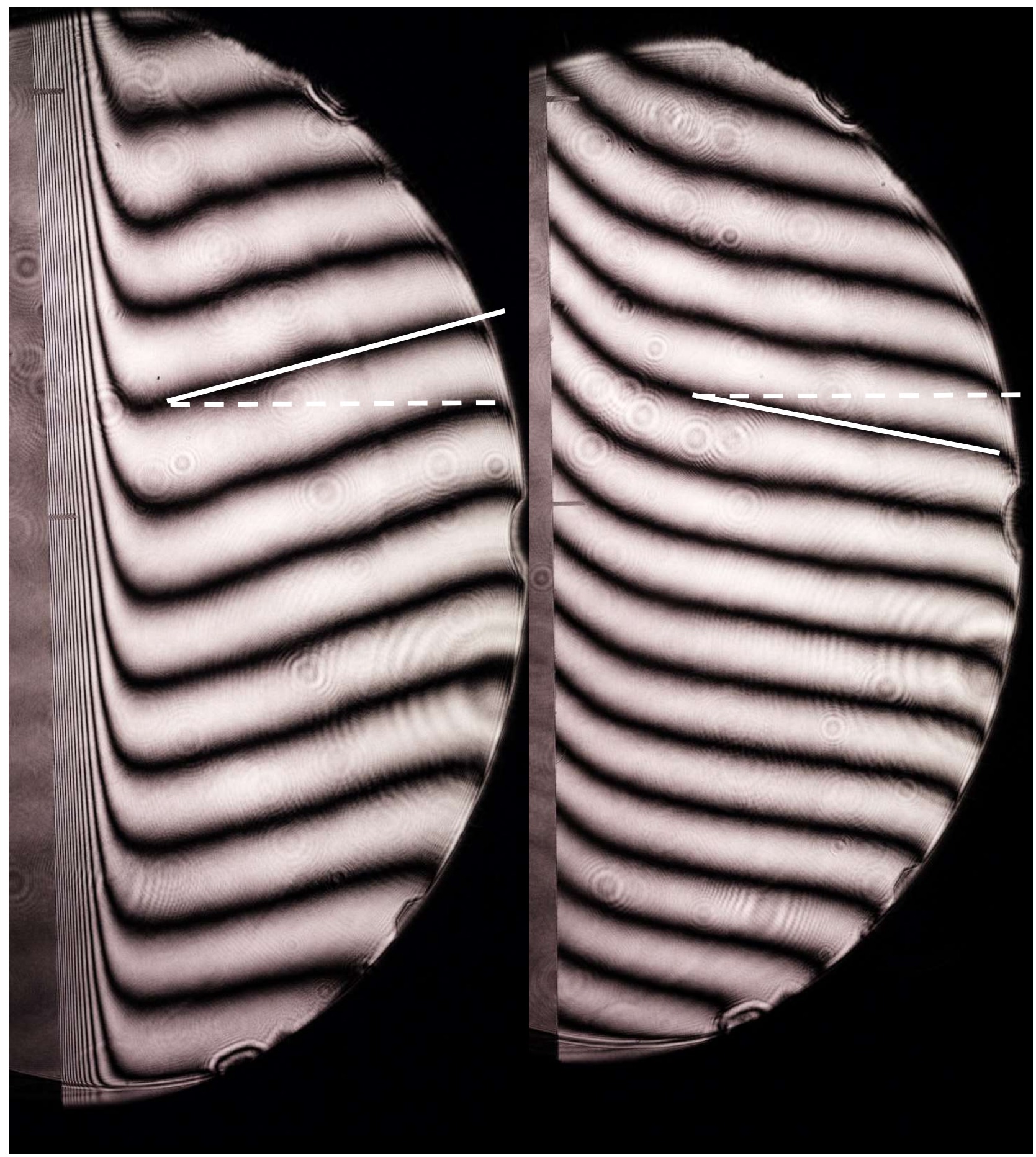

Figure 3.11: Finite interferogram at $t=0$ min (left) and $t=229$ min (right) 

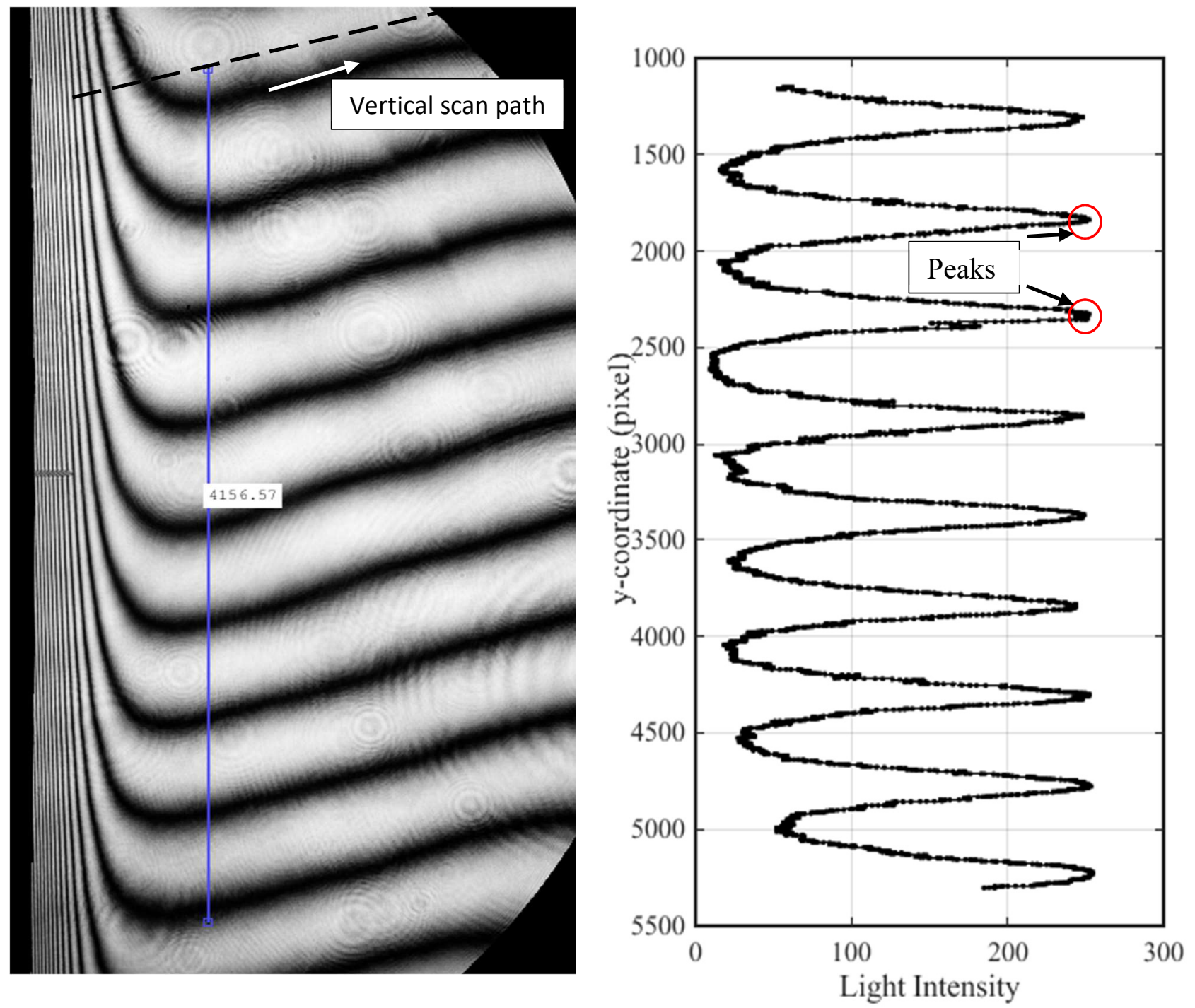

Figure 3.12: Vertical Fringe Scan 


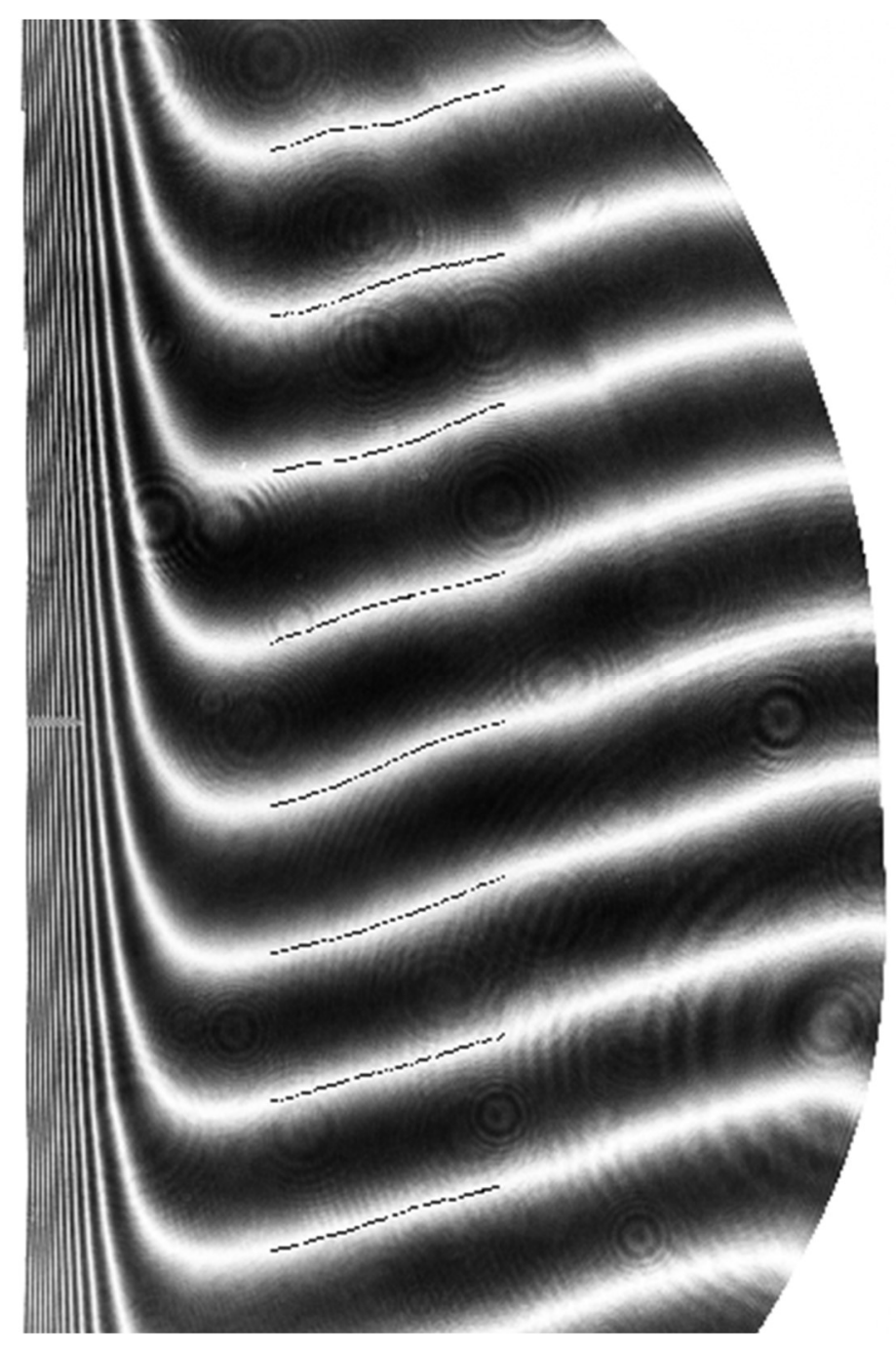

Figure 3.13: Vertical fringe peaks

Once all the vertical scans are obtained the average vertical fringe spacing is found from Equation 3.7.

$$
F S y=\frac{1}{M} \sum_{j=1}^{M} F S y_{j}
$$

A line of best fit is applied to each of the far field fringes and the slope of the fringe is saved and averaged for all the scanned fringes. Together with the averaged slope and averaged vertical fringe 
spacing, an idealized far field fringe pattern is created. This 'idealized' fringe pattern is illustrated in Figure 3.14. In this idealized far field region every fringe has the same slope and are equally spacing by the vertical fringe spacing found above. From Figure 3.14, it can be shown that the horizontal fringe gradient can be found from Equation 3.8.

$$
F S x=\frac{F S y}{\tan \theta}=\frac{F S y}{\bar{m}_{\text {slope }}}
$$

where FSx is the horizontal fringe spacing, $\theta$ is the angle of the far field fringe, and $\bar{m}$ is the averaged slope of the far field fringes.

The horizontal fringe gradient can be determined by considering 2 consecutive horizontal fringe crossings from Figure 3.14. The fringe order, $\varepsilon$, is an integer counter of the number of fringes relative to a reference fringe. Thus the change in fringe order of 2 consecutive fringes is just $\Delta \varepsilon=1$. From Figure 3.14 it can be seen that the length of one complete fringe order is the horizontal fringe spacing FSx. Therefore the fringe gradient from the idealized fringe region can be expressed in Equation 3.9 as:

$$
\frac{\partial \epsilon}{\partial x}=\frac{\Delta \epsilon}{\Delta x}=\frac{1}{F S x}
$$

Finally, the artificial temperature gradient due to the fringe shift error can be found from the fringe gradient using Equation 3.10.

$$
\left(\frac{\partial T}{\partial x}\right)_{\text {error }}=\frac{R \lambda_{o} T_{a m b}^{2}}{W P G} \frac{\partial \epsilon}{\partial x}=\frac{R \lambda_{o} T_{a m b}^{2}}{W P G}\left(\frac{1}{F S x}\right)
$$

The temperature gradient correction from Equation 3.10 is applied to the temperatures found from Equation 3.1. 


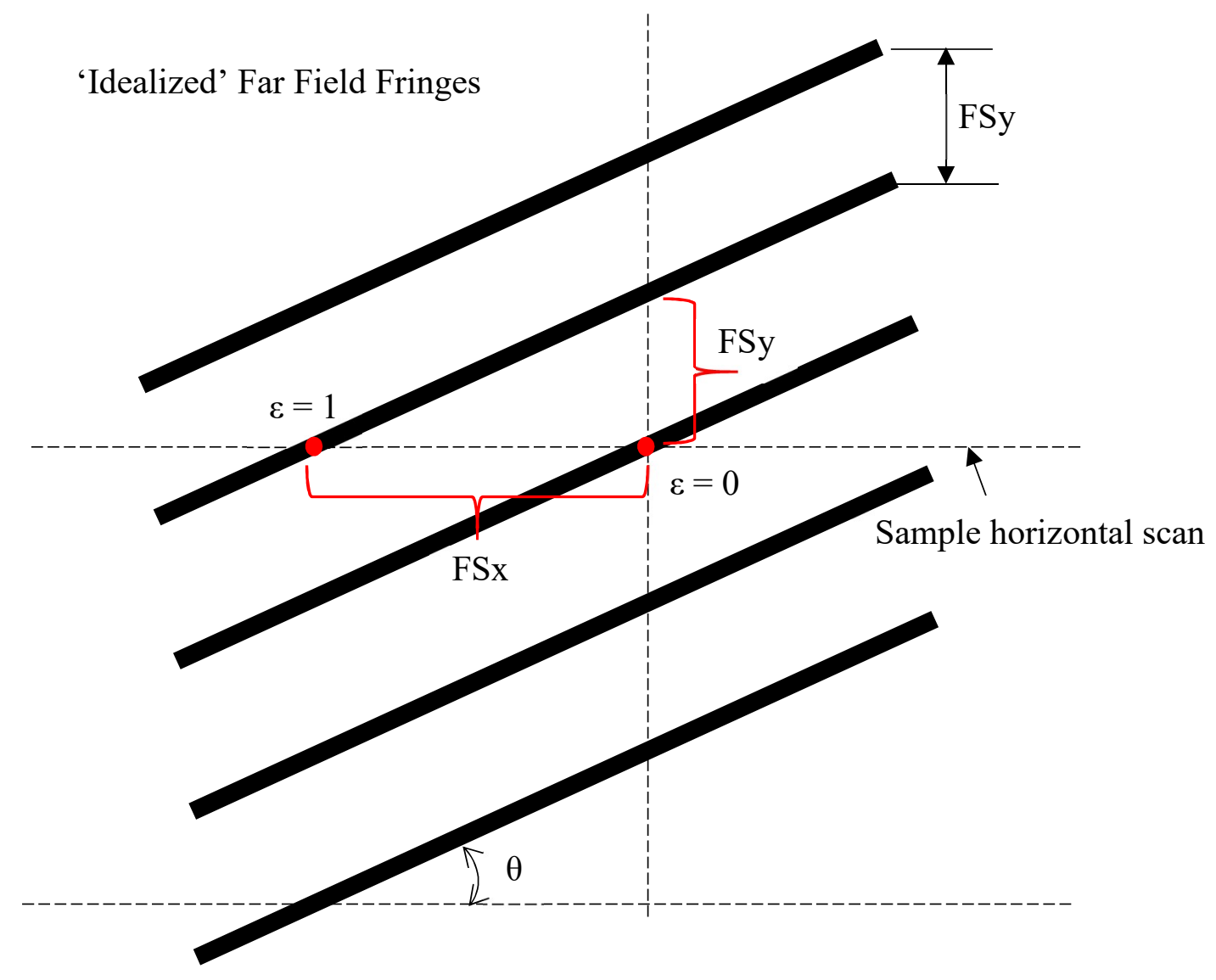

Figure 3.14: Idealized far field fringe region

\subsection{Partial Fringe Crossing}

As explained above, in some regions the interferogram output is not wide enough to define a reference ambient fringe for a given horizontal scan. Knowing the slope of an ambient fringe from the methods above can help this problem in some regions. The slope of a far field fringe can be used to extrapolate a fringe crossing as the intersection between that slope and a horizontal scan path. However, there are 'dead spots' where a given horizontal scan falls between two far field fringes and does not intersect with the horizontal scan within the interferogram window. Figure 3.15 contains a sample interferogram output at time $t=11$ minutes. The figure shows the scan path 
locations (which are in line with the sensor locations) overlaid on the interferogram as horizontal lines labeled TC1 through TC8. Using the methods described above, a line of best fit was found for eight of the far field fringes and is overlaid onto Figure 3.15. With both the scan path and ambient fringe slopes drawn on the same interferogram, potential fringe crossing can be observed. However, some of the scan locations do not cross an ambient fringe. A fringe crossing could be extrapolated from the slope of a far field fringe but that crossing could happen far outside the image domain. For example, the scan path for TC4 does clearly cross an ambient fringe (marked with a red tick) which in turn can be used as the reference temperature fringe to then determine all the other temperatures for each fringe crossings of that scan path. The scan paths for TC5 and TC6 do not contain an ambient fringe crossing within the optical window but a crossing can be found through extrapolating the nearest ambient fringe. However, extrapolating a fringe crossing outside the optical window is not reliable. As the slope of the ambient fringe decreases (flattens), the fringe crossing can occur far outside the optical window. 


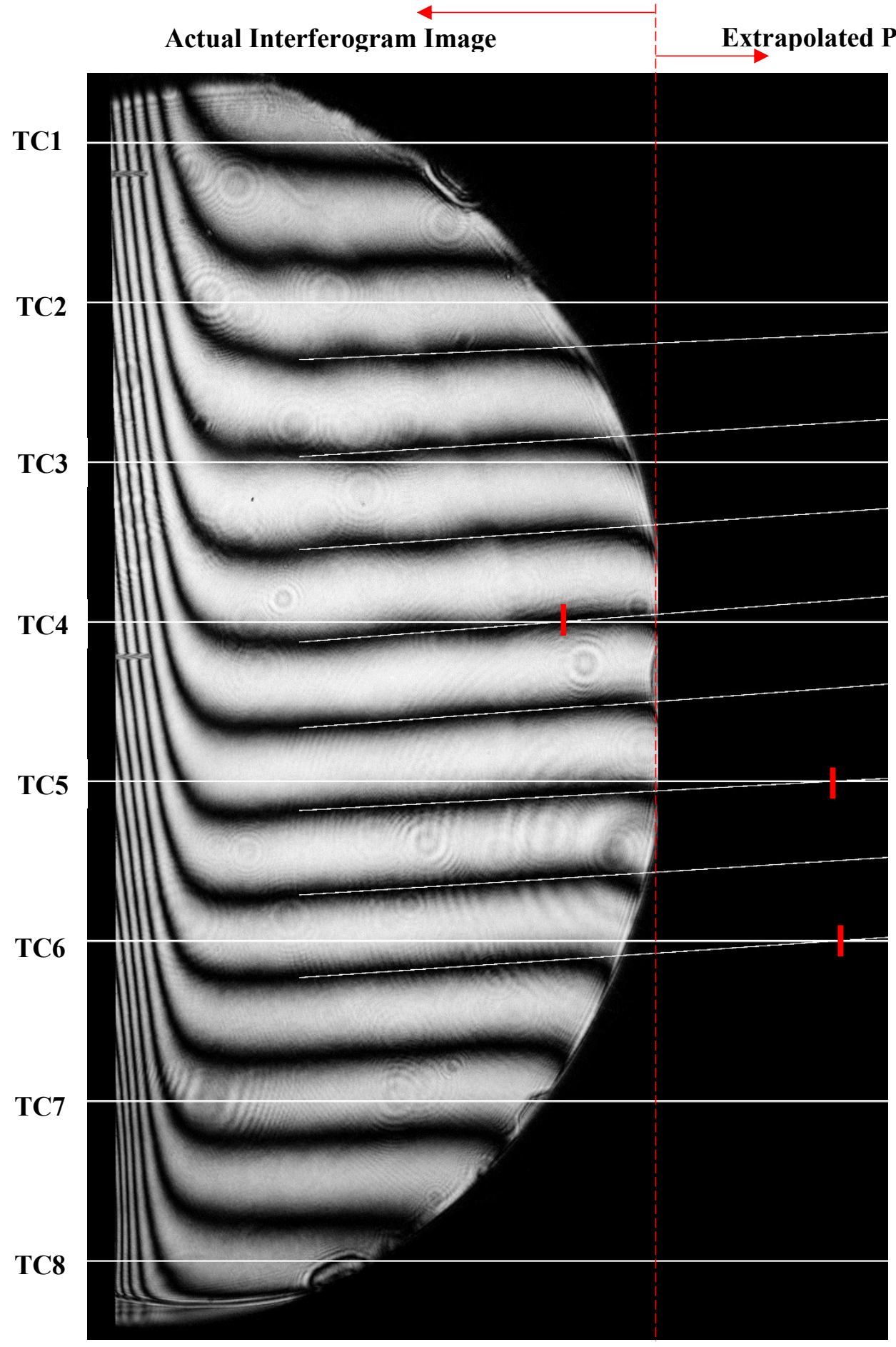

Figure 3.15: Interferogram with scan paths at sensor locations 
A close up of the scan path labelled TC5 is shown in Figure 3.16 with overlaid Cartesian coordinates. The TC5 scan path only crosses an ambient fringe when the slope of the ambient fringe is extrapolated outside the optical window. For an ambient fringe defined by, $y=m x+b$, and a given scan path at location, $y_{T C 5}$, the corresponding fringe crossing occurs at, $x_{T C}=$ $(y-b) / m$. Therefore, the fringe crossing, $x_{T C 5}$, is proportional to, $1 / m$. As the far field fringe slope becomes horizontal, the fringe crossing occurs further and further away from the interferogram optical window. Using equation 3.7, the far field fringe crossing can be shown to be proportional to, $x_{T C} \propto 1 / m=1 / \tan \theta$, where, $\theta$ is the far field fringe slope measured horizontally. Applying the uncertainty analysis method from Kline and McClintock (1953), it can be shown that the error in the far field fringe crossing is given by Equation 3.11:

$$
w_{x}=\frac{\partial x}{\partial \theta} \delta w_{\theta}=\frac{\sec ^{2} \theta}{\tan ^{2} \theta} \delta w_{\theta}
$$

where $w_{x}$ is the error in fringe crossing, and $\delta w_{\theta}$ is the error in far field fringe slope. A complete derivation of Equation 3.11 can be found in Appendix A.

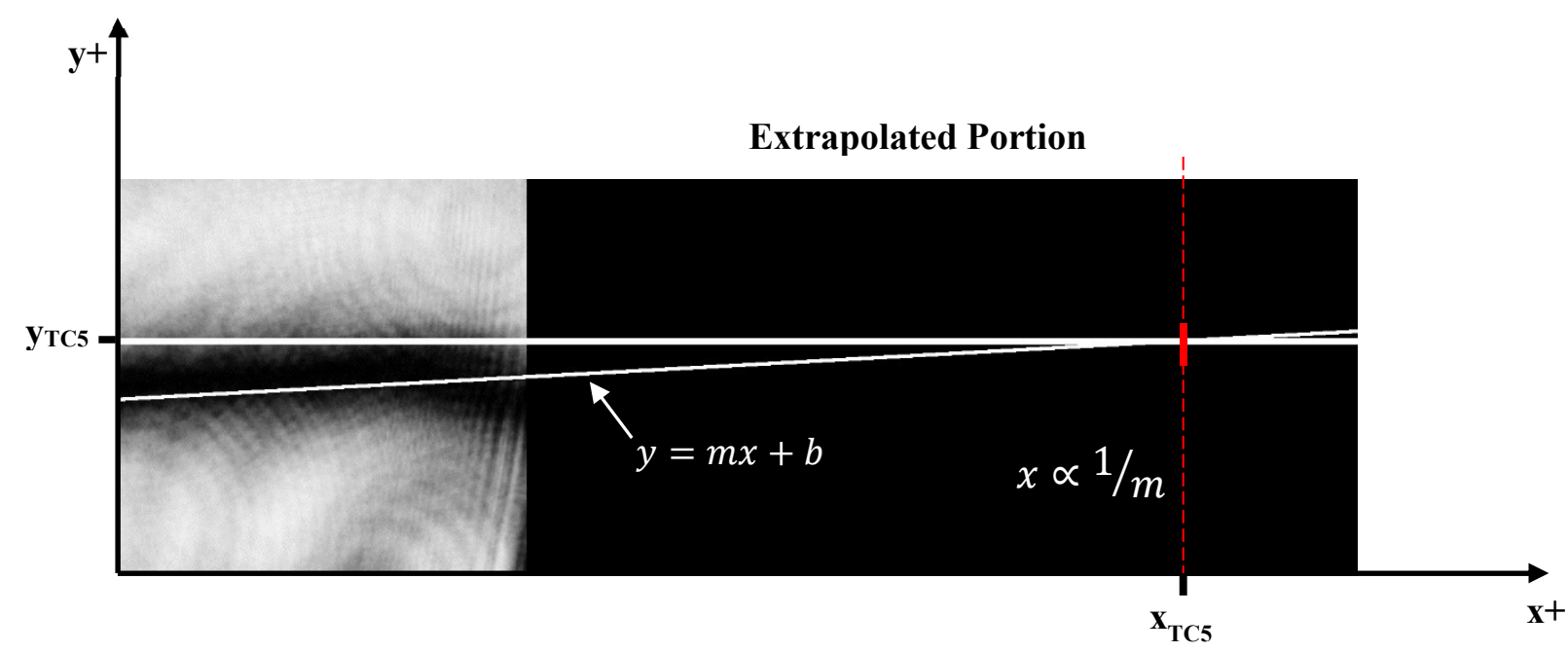

Figure 3.16: Close up at fringe crossing for scan path at TC5 
As an example, consider Figure 3.17 which illustrates a plot of fringe crossing error versus far field fringe slope (Equation 3.11). It can be seen from Figure 3.17 that as the far field fringe slope approaches zero (horizontal), the fringe crossing error goes to infinity. This has the effect of magnifying the error in the fringe crossing. Another approach is to take advantage of the vertical fringe crossing.

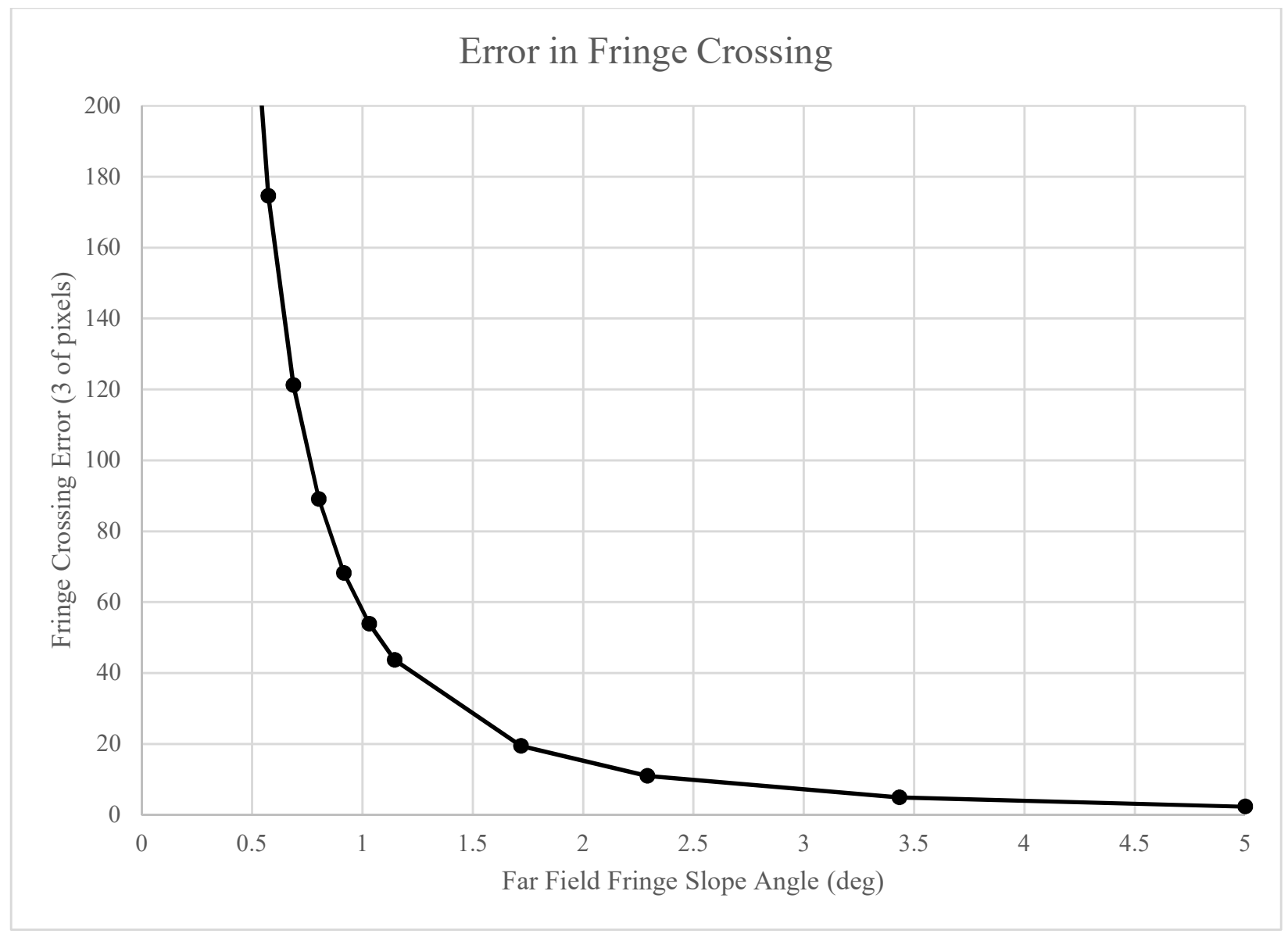

Figure 3.17: Fringe crossing location versus far field fringe slope

The vertical fringe crossing in the ambient region can aid in finding a reference fringe by developing a relationship between the horizontal fringe crossing, FSx, and vertical fringe crossing, FSy. Recall that in the ambient region, the temperature gradient in any direction should be constant (zero if the model is perfectly aligned) and thus the ambient fringes in finite fringe mode should 
be perfectly parallel (horizontal if perfectly aligned). If the interferometer truly was perfectly aligned, then the exact location of the ambient 'fringe crossing' is not needed and the subsequent fringe temperatures can easily be found using only the reference temperature. However, if there is an artificial fringe gradient and the ambient fringes are not horizontal, then the location of that first reference fringe is needed. If there is no fringe crossing, then a 'partial fringe crossing' can be found by taking advantage of the constant artificial fringe gradient and using the horizontal and vertical fringe spacing. The interferogram in Figure 3.18 has a highlighted section of the ambient fringe region showing two consecutive fringe crossings. The yellow line represents a scan path originating from the right side of the image with the reference fringe crossing marked as, $\varepsilon=0$, and the next consecutive fringe crossing on the left side of the image marked as, $\varepsilon=1$. The distance between these two fringe crossings is marked as, FSx. If the temperature at the fringe crossing corresponding to $\varepsilon=0$ is known, then the temperature at the second fringe crossing can easily be found. Due to a constant artificial fringe gradient in this highlighted region, the temperature gradient from the reference fringe to the next fringe crossing is linear and the change in the fringe order from $\varepsilon=0$ to $\varepsilon=1$ is linear also. This linear behaviour is also true for the fringe order along the vertical scan path (shown as a solid red line in Figure 3.18) but not for the temperature gradient. Following along the vertical scan path, the next consecutive fringe crossing crosses the same fringe as the horizontal scan (the fringe corresponding to $\varepsilon=1$ ) giving a fringe spacing marked as, FSy. 


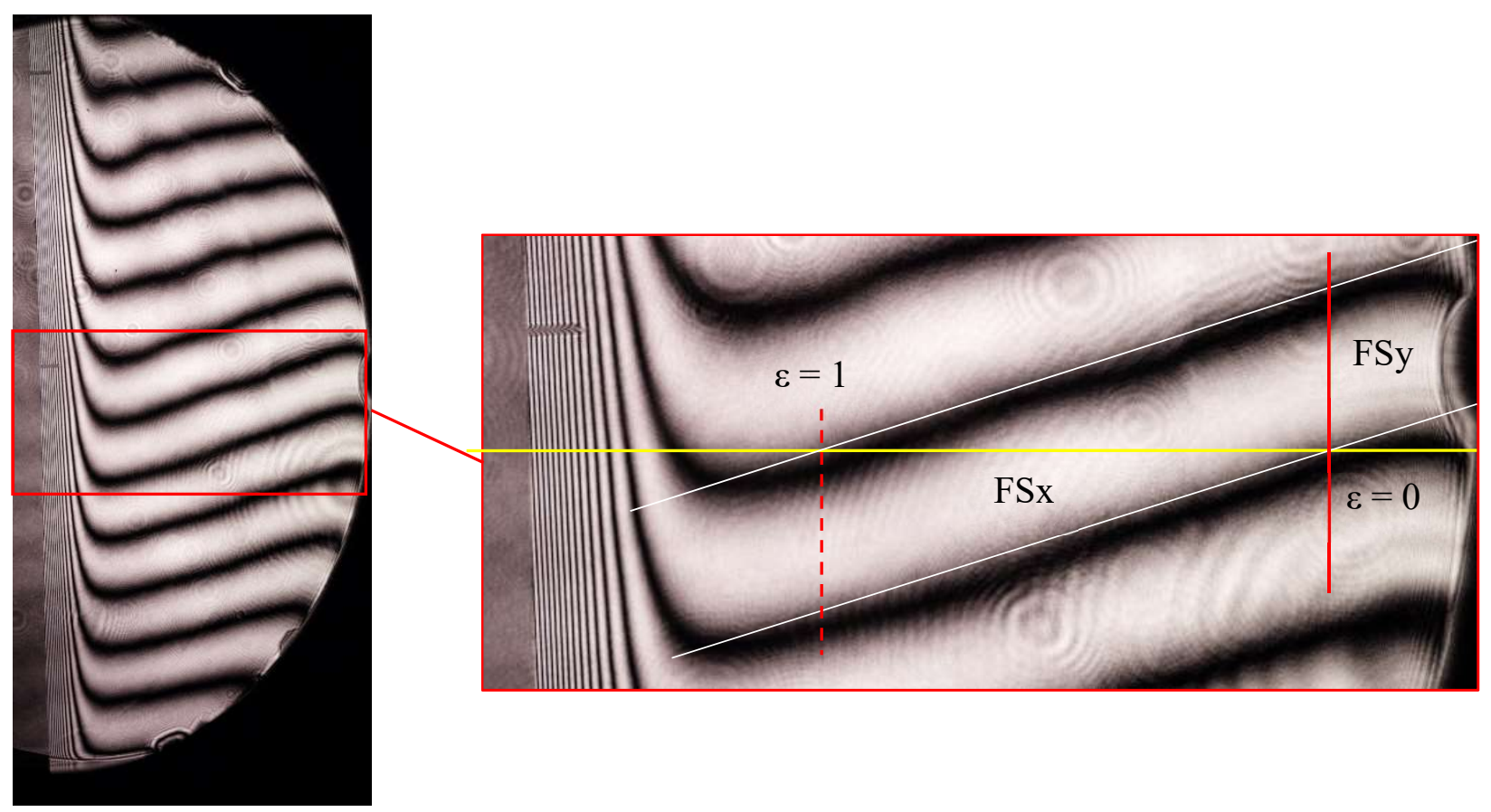

Figure 3.18: Fringe crossing location versus far field fringe slope

An illustrated version of the interferogram from Figure 3.18 is shown in Figure 3.19. Again, a full horizontal fringe order is denoted as FSx and a full vertical fringe order is denoted as FSy. The illustration shows an artificial fringe in grey (labeled ' $\varepsilon=\delta \varepsilon$ ') situated between two fringes in black (labeled as ' $\varepsilon=0$ ' and ' $\varepsilon=1$ '). This illustration is meant to show that a horizontal partial fringe spacing is proportional to a vertical partial fringe spacing. If the partial fringe order is known (some value between 0 and 1), then this partial fringe can be used as the reference fringe crossing to set the reference temperature. The horizontal and vertical distance between fringes ' $\varepsilon=0$ ' and ' $\varepsilon=1$ ' is FSx and FSy respectively. The horizontal and vertical distance between ' $\varepsilon=\delta \varepsilon$ ' and ' $\varepsilon=$ 1 ' is $\Delta F S x_{\varepsilon_{1}-\delta}$ and $\Delta F S y_{\varepsilon_{1}-\delta \varepsilon}$ respectively. Finally, the horizontal and vertical distance between ' $\varepsilon$ $=\delta \varepsilon$ ' and ' $\varepsilon=0$ ' is $\Delta F S x_{\delta \varepsilon-\varepsilon_{0}}$ and $\Delta F S y_{\delta \varepsilon-\varepsilon_{0}}$ respectively. 


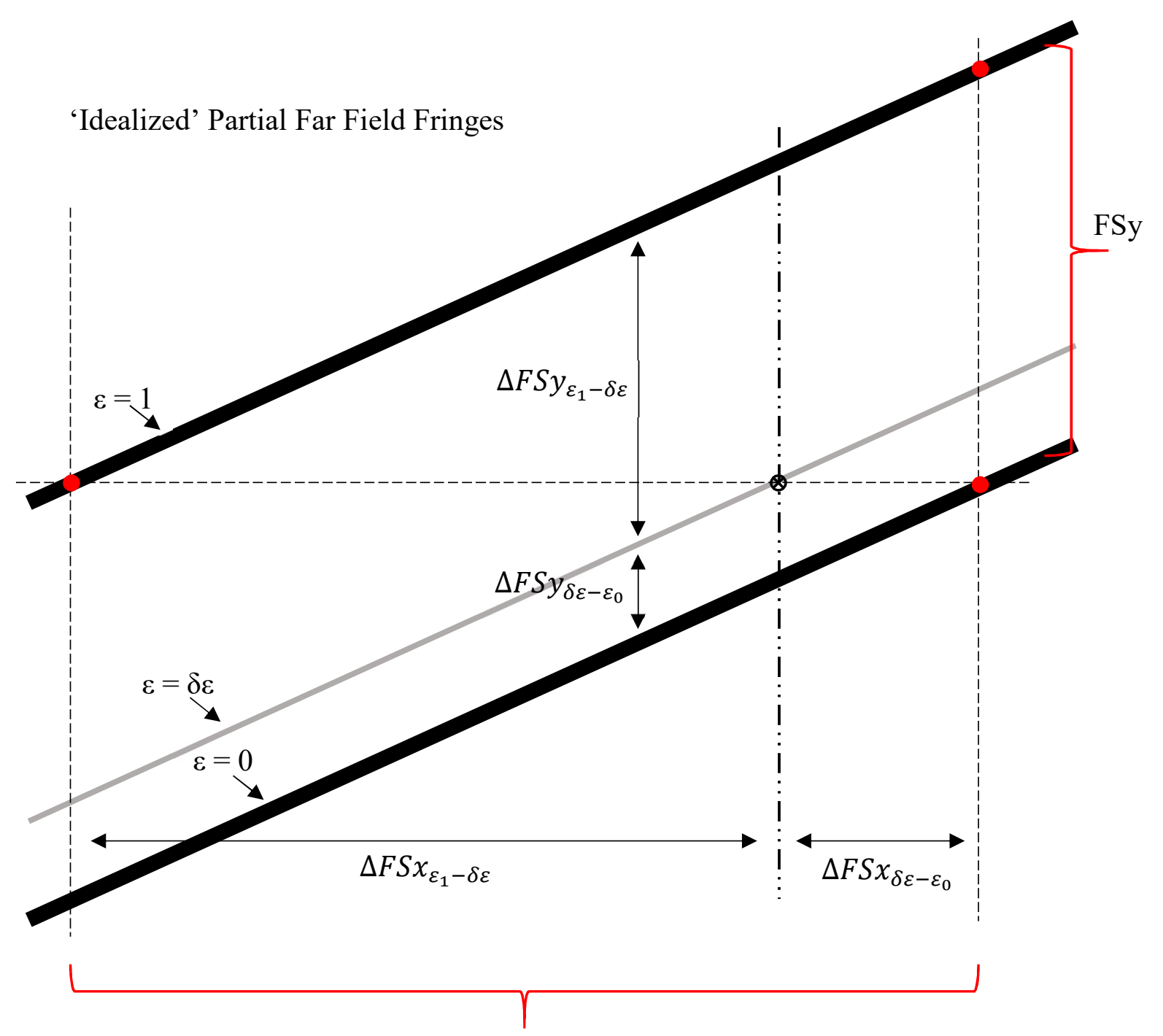

FSx

Figure 3.19: Illustrated far field fringes with horizontal and vertical fringe crossings

With these distances defined, the illustration in Figure 3.20 shows the relationships derived from Figure 3.19 and the distances defined above. It can be seen from Figure 3.20 that if the vertical fringe spacings are known (full and partial spacings), then the full and partial horizontal fringe spacing can be found from the relationships in Equation 3.12 and Equation 3.13. 


$$
\begin{gathered}
\varepsilon_{\varepsilon_{1}-\delta \varepsilon}=\frac{\Delta F S x_{\varepsilon_{1}-\delta \varepsilon}}{\Delta F S x}=\frac{\Delta F S y_{\varepsilon_{1}-\delta \varepsilon}}{\Delta F S y} \\
\varepsilon_{\delta \varepsilon-\varepsilon_{0}}=\frac{\Delta F S x_{\delta \varepsilon-\varepsilon_{0}}}{\Delta F S x}=\frac{\Delta F S y_{\delta \varepsilon-\varepsilon_{0}}}{\Delta F S y}
\end{gathered}
$$

where, $\varepsilon_{\varepsilon_{1}-\delta \varepsilon}$, is the fringe order from the artificial fringe ' $\varepsilon=\delta \varepsilon$ ' to the full fringe ' $\varepsilon=1$ ', and, $\varepsilon_{\delta \varepsilon-\varepsilon_{0}}$, is the fringe order from the full fringe ' $\varepsilon=\delta \varepsilon$ ' to the artificial fringe ' $\varepsilon=\delta \varepsilon$ '.

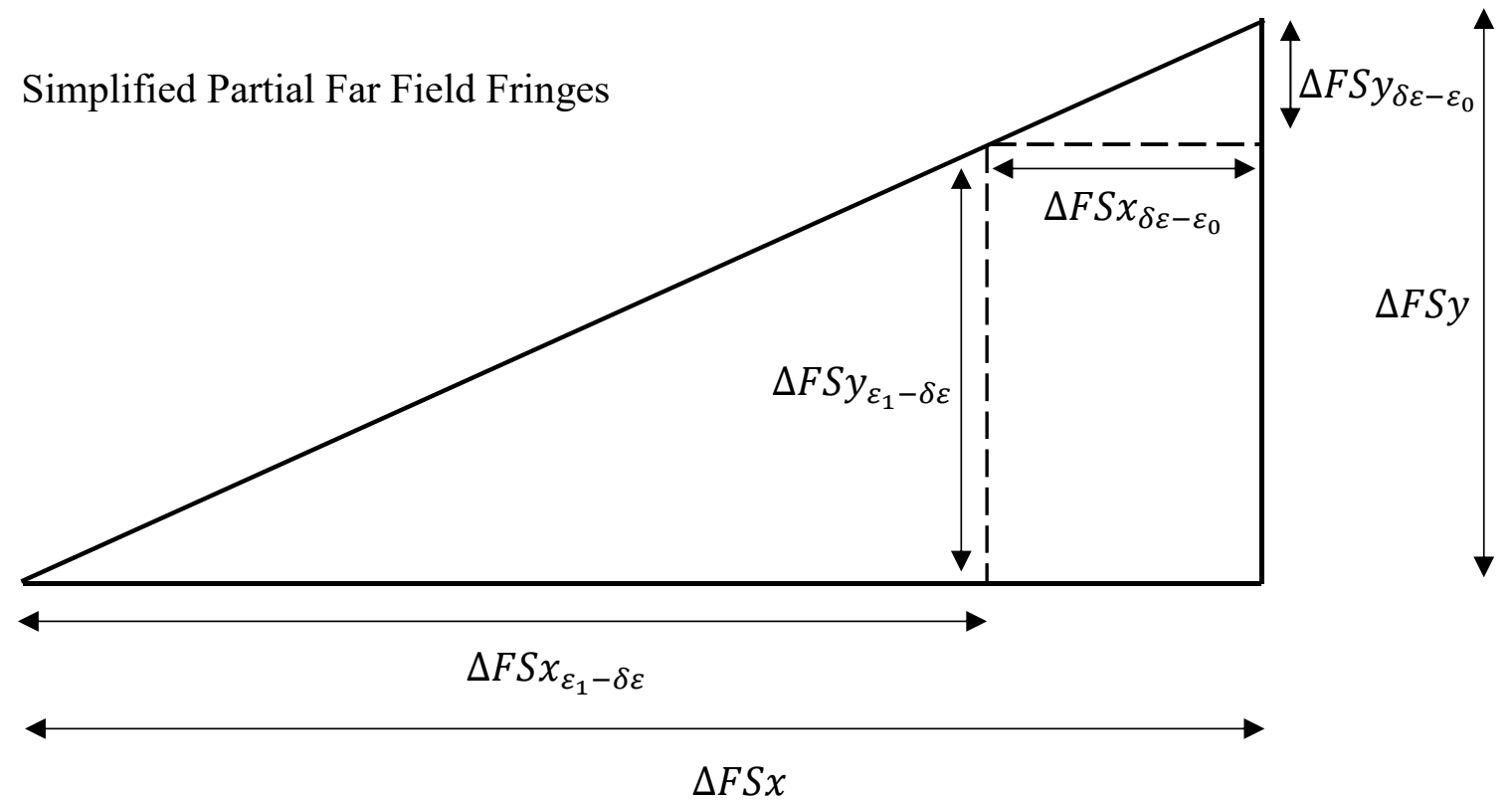

Figure 3.20: Relationship between horizontal and vertical fringe crossings

Equations 3.12 and 3.13 allows for using any arbitrary location in the interferogram as the reference temperature without needing a complete fringe crossing. However, the above derivation of the artificial fringe only applies for the ambient region where there is a zero temperature gradient. A brief summary of the procedure is displayed in Figure 3.21. First the partial and full vertical fringe spacings are determined using the procedures explained in Section 3.4 and 3.5 to 
find the partial fringe order, $\varepsilon_{\varepsilon_{1}-\delta \varepsilon}$. With the partial fringe order determined for that specific point in the interferogram, then the first horizontal fringe crossing and its temperature can be found. The partial fringe order corresponds to the intersection point between the vertical and horizontal scan paths.

Determine the full and partial vertical fringe spacing to get the partial fringe order, $\varepsilon_{\varepsilon_{1}-\delta \varepsilon}$

Use the partial fringe order, $\varepsilon_{\varepsilon_{1}-\delta \varepsilon}$, to determine the first fringe crossing
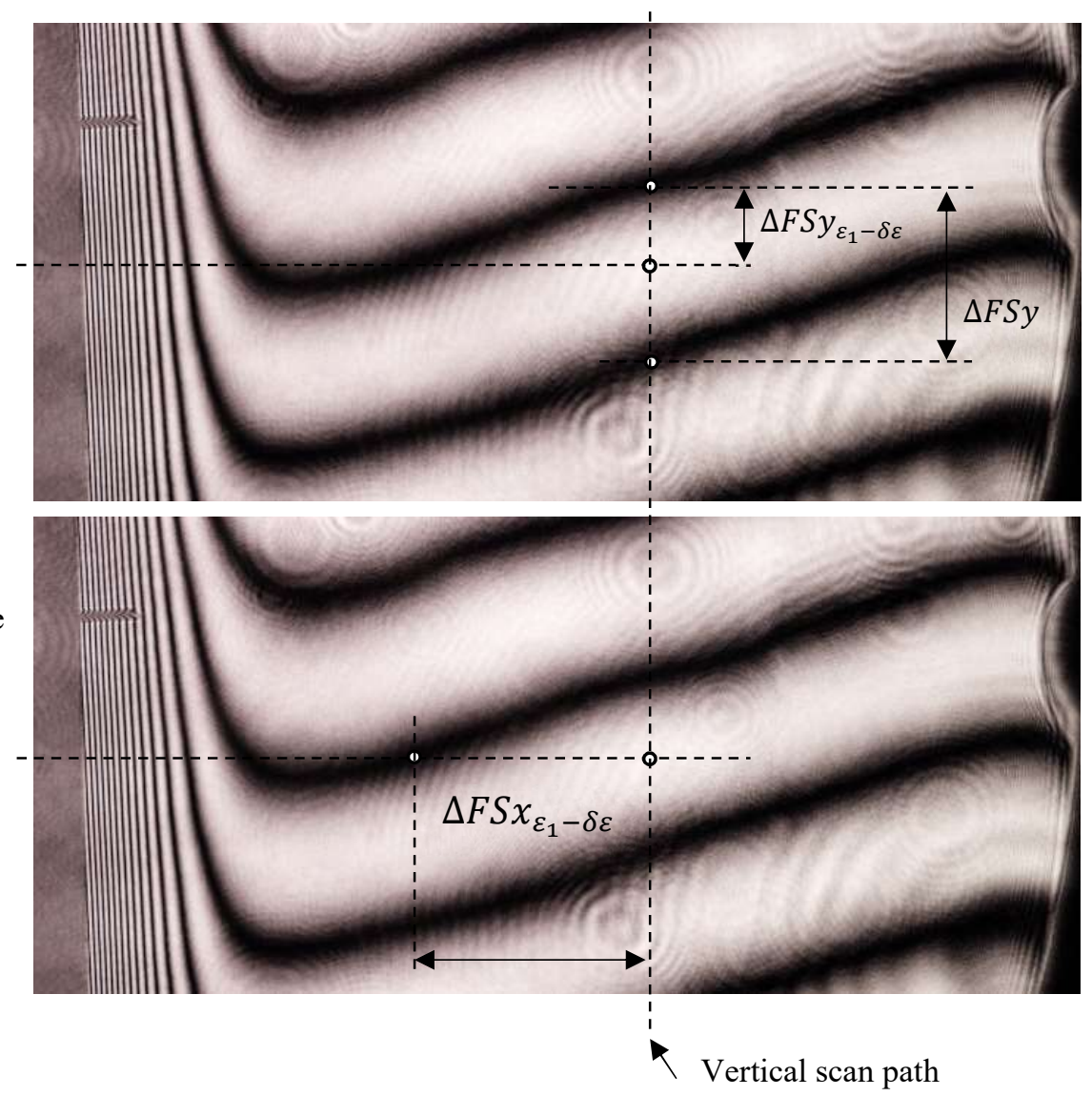

Figure 3.21: Summary of partial fringe crossings 


\subsection{Wall Profile Shift}

In order to determine the local and average convective heat transfer from the PCM wall panel, the near wall temperature gradient and surface temperature is needed. The temperature gradient is determined just from the fringe temperatures and their fringe spacing which are determined using the methodology explained above using Equation 3.1 and Equation 3.2. However, if the surface temperature is required, then the temperature gradient and the wall location is needed. The PCM wall panel has a PCM cavity of $203.2 \mathrm{~mm}$ in height and $304.8 \mathrm{~mm}$ in width. The PCM cavity is sandwiched between two sheets of acrylic glass that is $3.2 \mathrm{~mm}$ thick. When the PCM is in the liquid phase there is a hydrostatic force that is pushing out against the acrylic glass causing the PCM wall panel to bow outwards. This bowing will have an effect in determining the near wall temperature. The surface temperature can be determined from linear extrapolation of the near wall temperature gradient.

To mitigate this issue, for each interferogram, the wall location was defined by a $2^{\text {nd }}$ order polynomial that is unique to that image. With that polynomial, the distance between the nearest fringe crossing and wall is determined and is used to extrapolate the surface temperature from the near wall temperature gradient. The $2^{\text {nd }}$ order polynomial was determined by curve fitting to approximately 12 data points. The wall data points were determined by investigating areas of high contrast on the interferogram along the PCM wall panel. In finite interferogram mode, the light and dark fringes are almost parallel with the wall with portions that intersect the wall. By zooming in on these intersections, the contrast between the wall silhouette and fringes can be seen. The left hand side of Figure 3.22 contains a sample interferogram with the selected wall points circled. With this $2^{\text {nd }}$ order polynomial determined, the interferogram image was modified with custom programming code to 'black out' the wall portion of the image. The right hand side of Figure 3.22 
contains a close of the wall portion before and after being 'blacked out'. With this 'blacked out' image, the wall surface temperature is determined by linear extrapolation from the nearest fringe crossing temperature to the edge of the blackened wall.
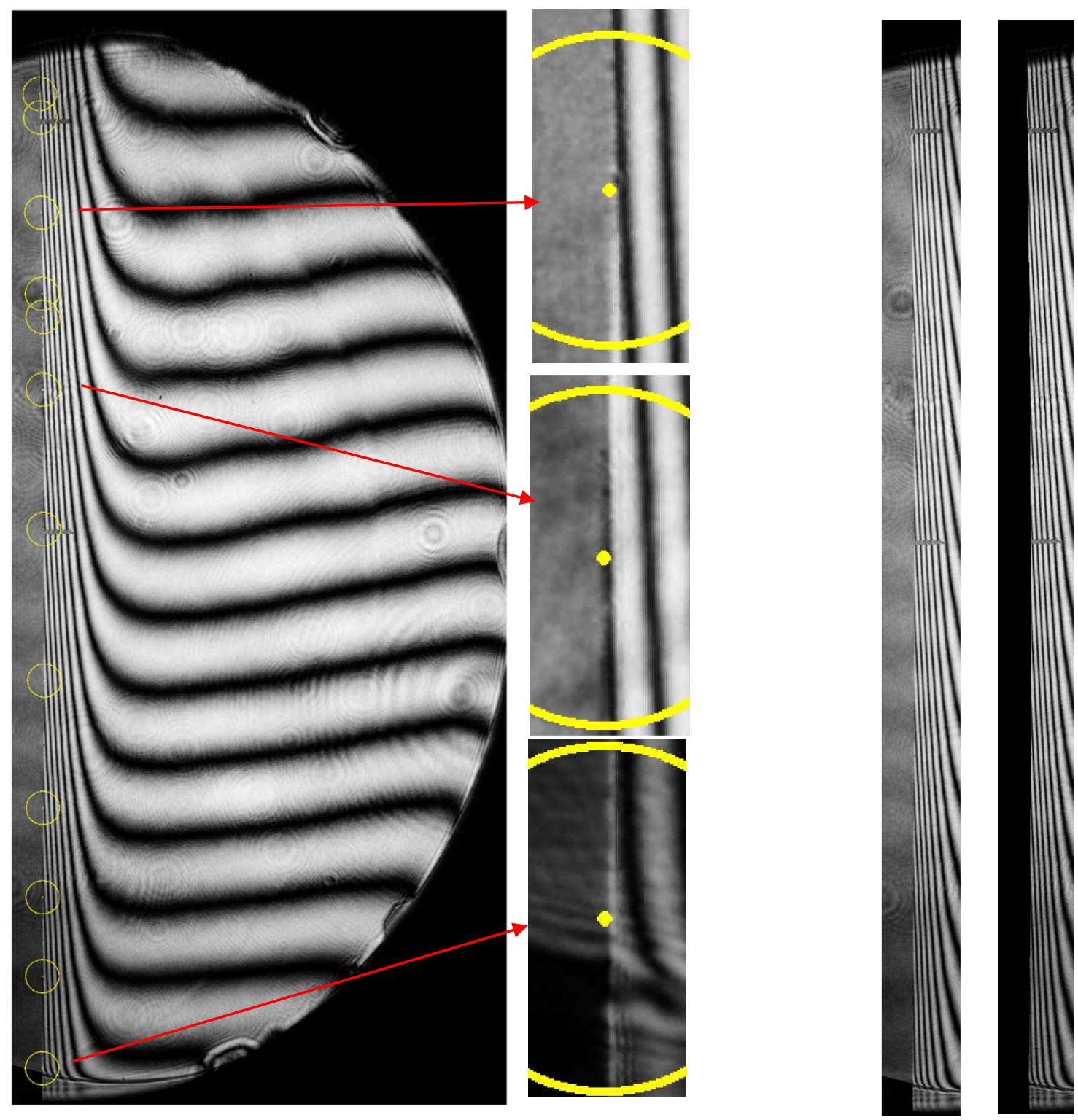

Figure 3.22: Wall Profile 


\section{Chapter 4: Results and Discussions}

\subsection{Overall Experiment}

Due to the length of time, the ambient temperature varies for the duration of the experiments.

Figure 4.1 illustrates the variation of the ambient temperature with time during the length of the experiment. It can be seen that there is a gradual decrease in the ambient temperature from approximately $24.8^{\circ} \mathrm{C}$ to $23.4{ }^{\circ} \mathrm{C}$. The beginning of the experiment ( $\mathrm{t}=0$ seconds in Figure 4.1) corresponds to $3: 59 \mathrm{pm}$ and ends at $8: 45 \mathrm{pm}$ on February $2^{\text {nd }}, 2015$. The drift in ambient temperature is due to the lack of dedicated HVAC controls for the experimental room. The drift could be a result of thermostat placement, a change in HVAC setpoint schedules, and varying internal building loads. The ambient temperature was updated for all interferograms and heat transfer calculations. For all the measured and derived parameters in this section an uncertainty analysis is shown in Appendix A. 


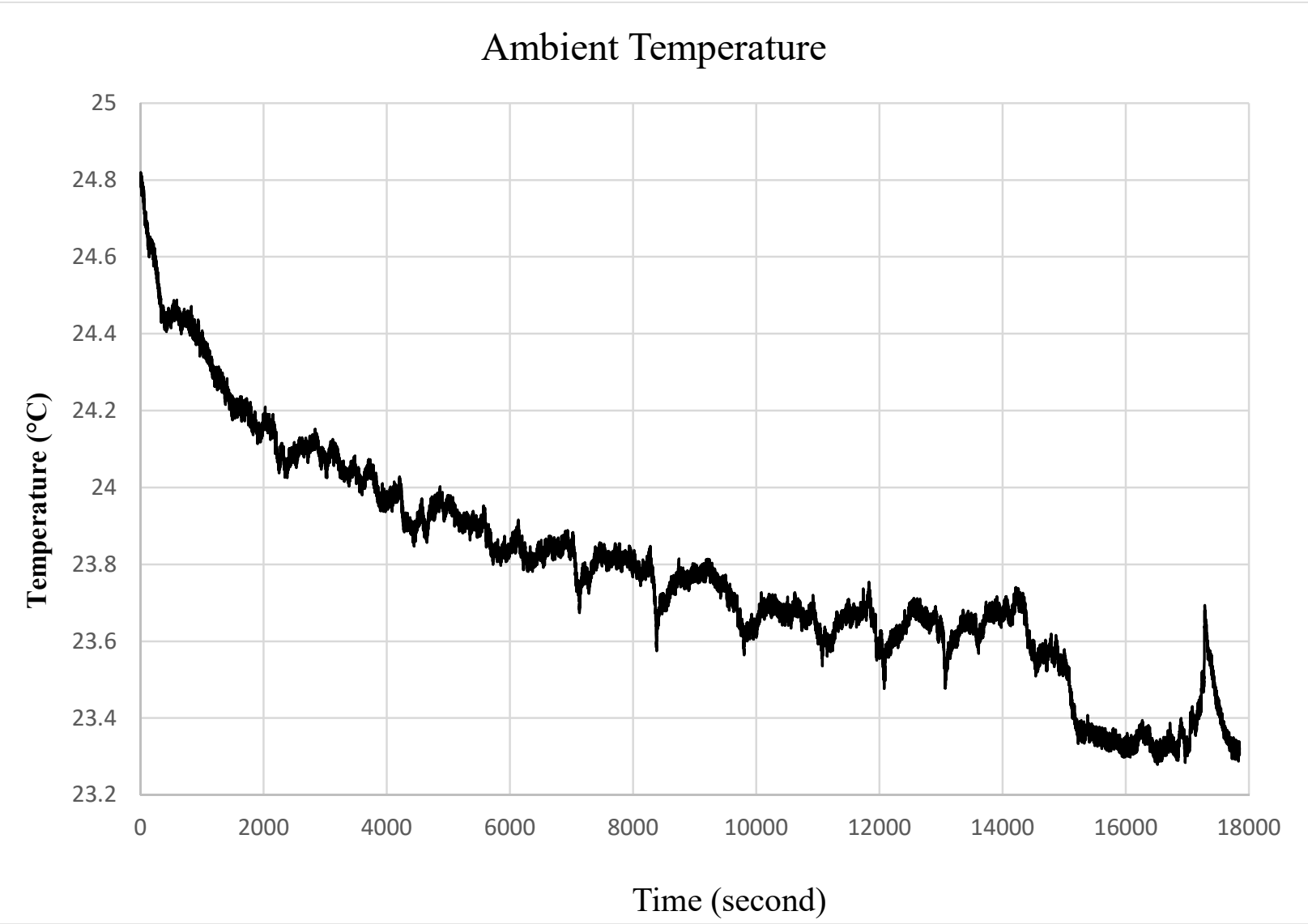

Figure 4.1: Ambient temperature

The experiment can be thought of being split up into three distinct portions for the purposes of the current discussion. There is the high-temperature liquid thermal decay portion, phase change portion, and the low-temperature solid thermal decay portion. Figure 4.2 illustrates the interior PCM temperature for the duration of the entire experiment. The plot includes two thermocouples labelled 'TCM 2' and 'TCM 8'. Both of these thermocouples represent the mid-plane slice of the PCM. TCM 2 is located one inch from the bottom of the PCM cavity and TCM 8 is located $1 / 2$ an inch from the top of the PCM cavity. The placement of the thermocouples is illustrated in Figure 4.3 where TCM indicates the midline placement and TCP indicates inside plate surface placement. The temperatures are measured at 8 locations spaced 1" $(25.4 \mathrm{~mm})$ apart. The first sensor is located at $38.1 \mathrm{~mm}$ from the bottom of the plate and the $8^{\text {th }}$ sensor is located at $215.9 \mathrm{~mm}$. It can be seen 
from Figure 4.2 that the phase change point occurs approximately between time $1700 \mathrm{~s}$ and 4000 s. From $0 \mathrm{~s}$ to $1700 \mathrm{~s}$, it is the high-temperature liquid thermal decay portion and from $4000 \mathrm{~s}$ and on is the low-temperature solid thermal decay portion. From the slopes of the plot it can be seen that in the high-temperature liquid phase, the temperature drops off much faster than the lowtemperature solid phase. This difference in slope is still apparent immediately on either side of the phase change portion. From Figure 4.2 it can be seen that the congealing point $\left(\mathrm{T}_{\text {cong }}\right)$ of soy wax is about $37.5^{\circ} \mathrm{C}(310.5 \mathrm{~K})$. All thermocouple temperature readings have an uncertainty of $\pm 0.5 \mathrm{~K}$.

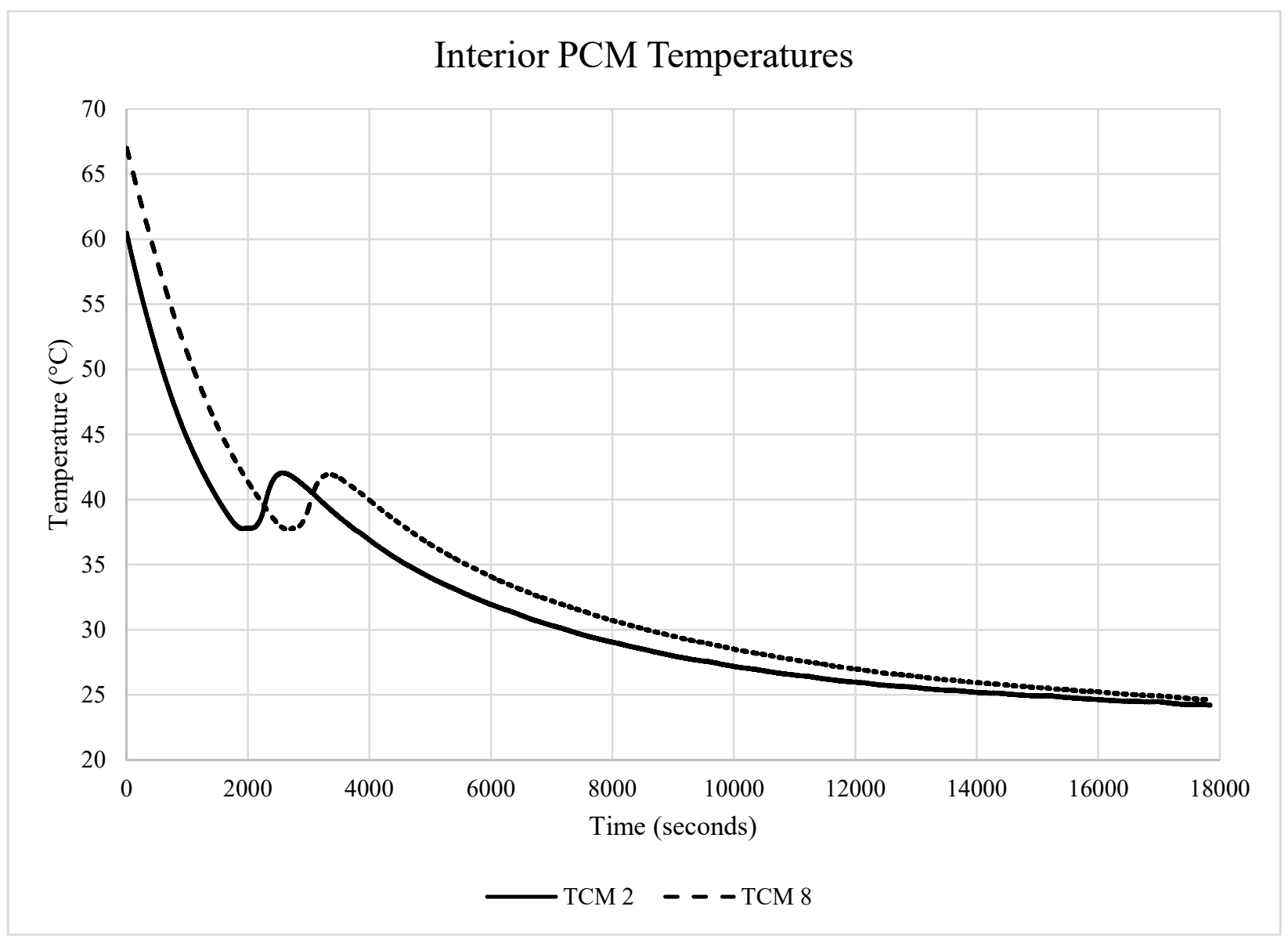

Figure 4.2: Interior PCM temperatures 


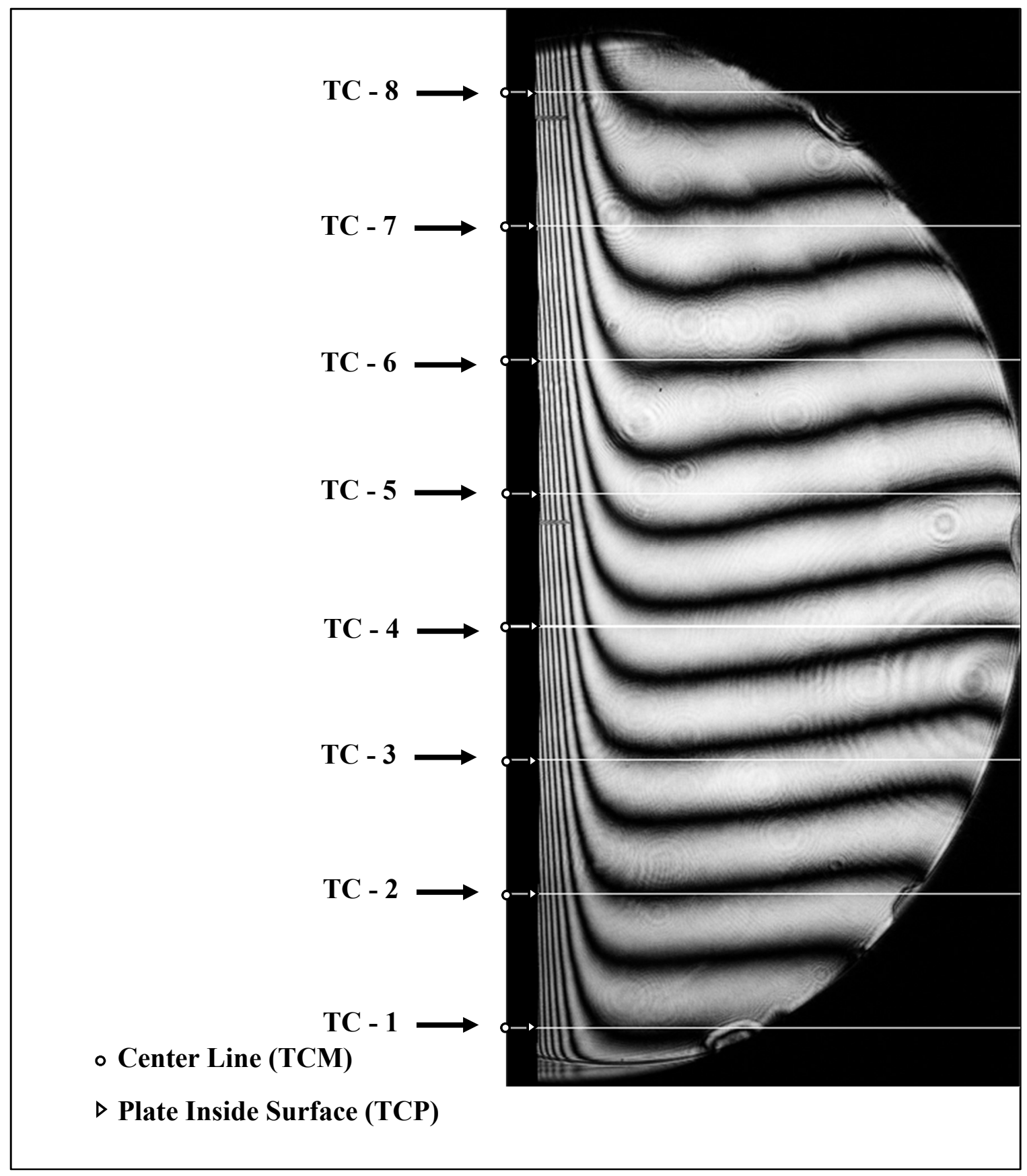

Figure 4.3: Thermocouple naming and placement

Due to optical distortions near the edges of the optical window and a lack of far field fringes, the optically measured surfaces temperatures on the outside surface of the PCM plate are only shown 
for the locations TC2 through TC7. Figure 4.4 contains the optically measured surface temperatures from 20 minutes into the experiment to 80 minutes. From 30 minutes to 70 minutes, the image analysis was performed for intervals of one minutes, while from 20 to 30 minutes and 70 to 80 minutes was analyzed for intervals of five minutes. For the purposes of the current discussion, 30 to 70 minutes was chosen to be the time period where the PCM plate is undergoing phase change and therefore was analyzed at a higher frequency. Before and after this time interval the PCM plate is undergoing sensible heat loss where the shape of the temperature profile does not change with time. Due to the general noise from the MZI output (optical distortion, vibrations, etc.) and the transient nature of the experiment, the optically measured surface temperature data also has noise. To aid further analysis a $5^{\text {th }}$ order Fourier curve fit was performed for each set of surface temperatures from TC2 through TC7 in order to 'smooth' the data. The curve fit is also displayed in Figure 4.4 along with the measured surface temperatures. Similar to the thermocouple data in Figure 4.3, the effect of phase change can be seen in the outside surface temperatures in Figure 4.4 as a temperature jump. As expected at 20 and 25 minutes, the outside surface temperatures follow a somewhat linear temperature profile along the PCM plate with TC2 being the coldest and TC7 the warmest. The onset of phase change can be seen at 30 minutes with the outside surface temperature at TC2 starting to increase while the remaining surface temperatures continue to drop. After 30 minutes, the outside surface temperatures at the remaining locations also increase in a staggered manner giving a temperature profile that varies with time. At approximately 60 minutes the outside surface temperatures return to an expected linear temperature profile. The optically measured wall surface temperatures have a relative uncertainty of $\pm 0.32 \%$ (Appendix A). 


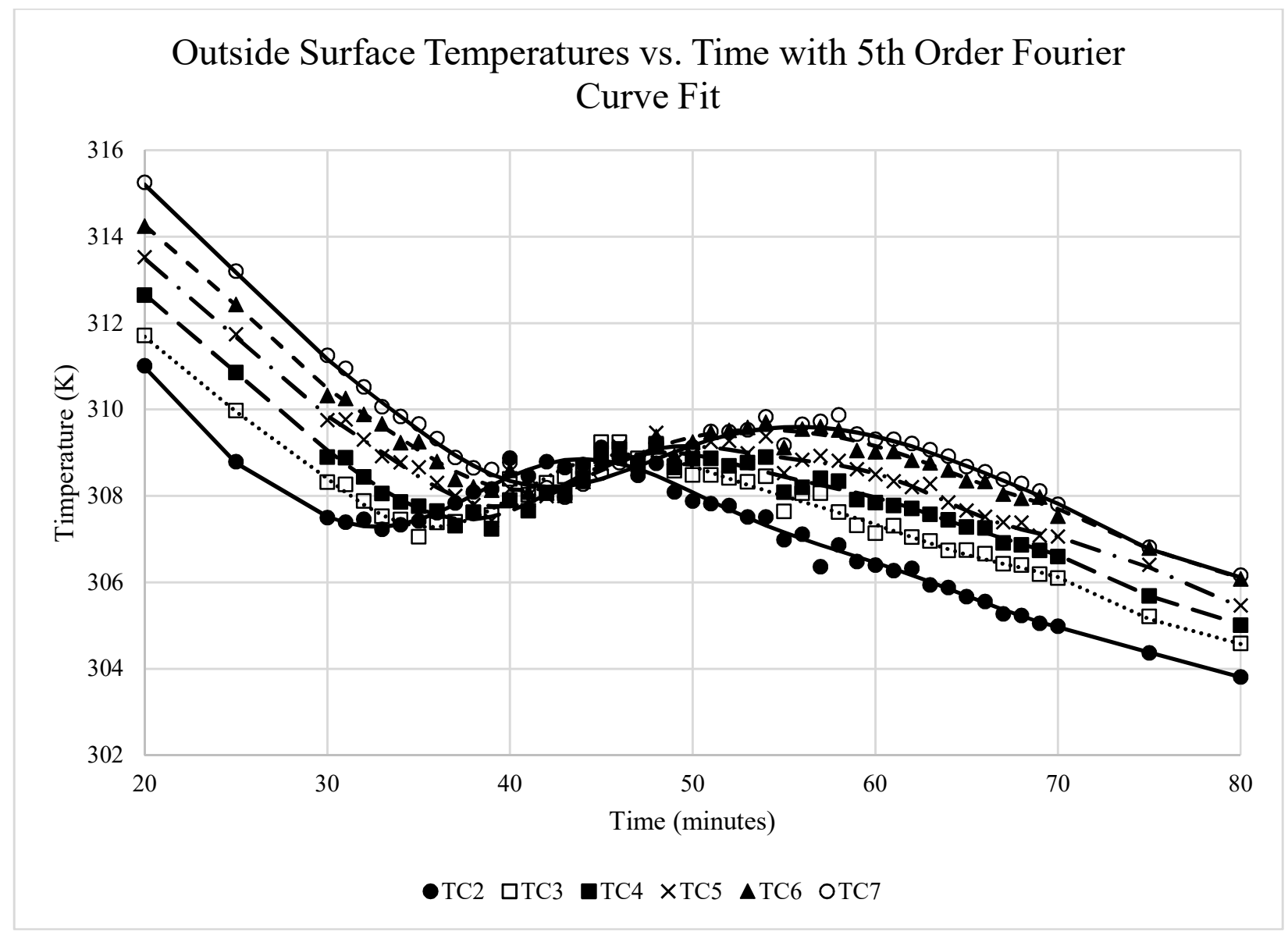

Figure 4.4: Optically measured outside surface temperatures

The averaged outside surface, inside surface and center line temperatures are shown in Figure 4.5 for 20 minutes through 80 minutes. The average outside surface temperatures are from the $5^{\text {th }}$ order Fourier curve fit as explained above. All three averaged temperatures behave in a similar fashion showing three distinct phases; 1) temperature drop during the liquid phase, 2) temperature jump during phase change, and 3) temperature drop during the solid phase. In general, the temperature gradient across the PCM plate is larger between the inside surface and outside surface temperatures than the center line and inside surface temperatures. 


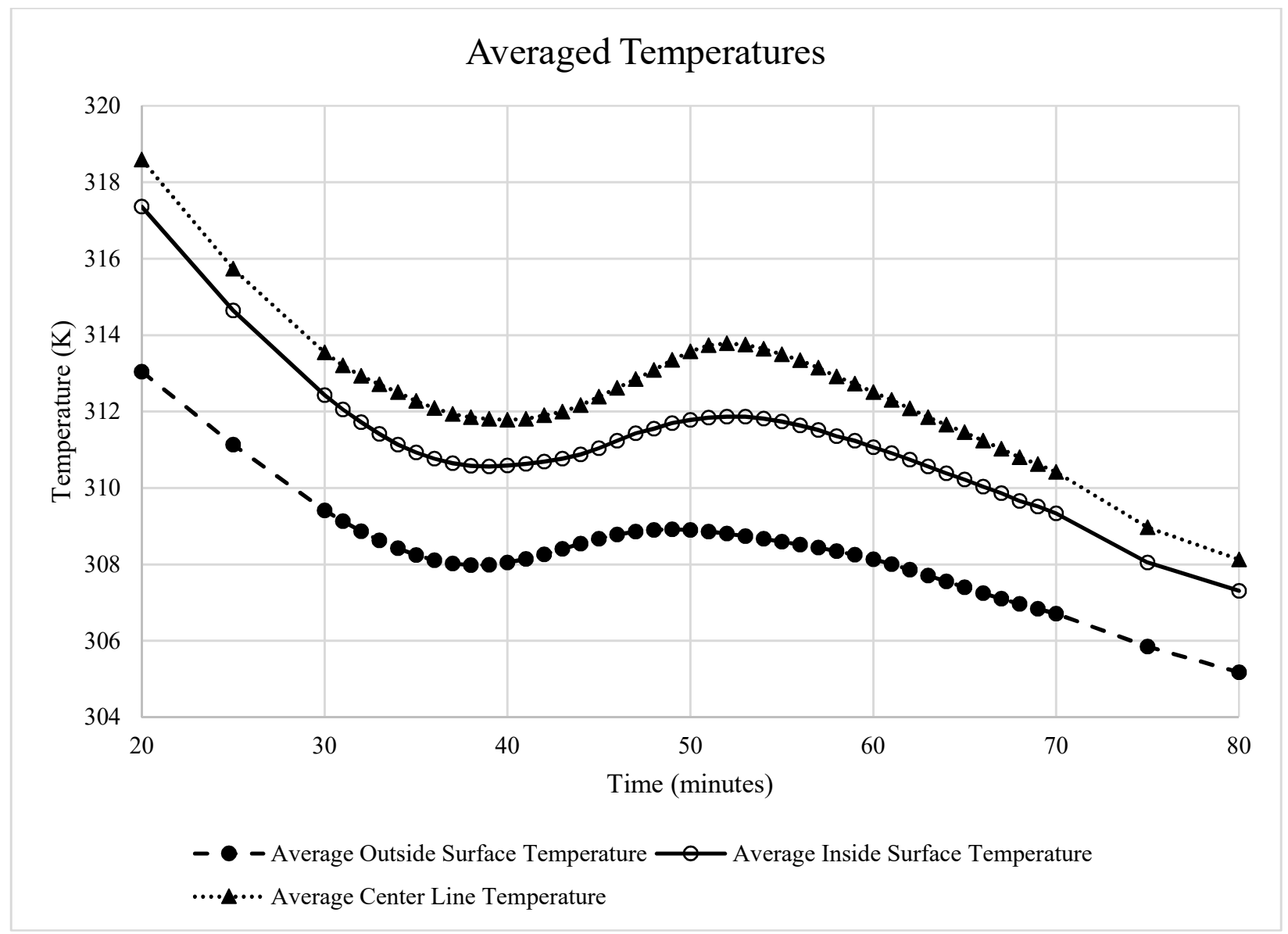

Figure 4.5: Averaged outside surface, inside surface and center line temperatures

\subsection{High Temperature Liquid Phase}

The temperature profiles at time 20 minutes and 25 minutes into the experiment is shown in Figure 4.6. The temperatures shown are from the thermocouple readings during the high-temperature liquid phase of the PCM. The two solid lines represent the the center line temperature (at 20 and 30 minutes) and the two dotted lines represent the inside surface temperature (at 20 and 30 minutes). For a given snap shot in time, the line at the higher temperature represents the thermocouple readings along the center line of the PCM plate. While the lower line represents the thermocouple readings along the inside plate surface. The centerline temperatures are measured at 
8 locations (sensor placements are shown in Figure 4.3). Due to malfunctioning thermocouples, the inside plate surface temperature is missing the $1^{\text {st }}$ and $5^{\text {th }}$ readings. It can be seen from Figure 4.6 that the bottom of the plate is colder than the top of the plate and that the temperature profile is approximately linear along the PCM plate height. At this point in the experiment the PCM plate is completely liquid and is undergoing free-convective cooling. At 20 minutes into the experiment the average inside surface temperature is approximately $1.8 \mathrm{~K}$ lower than the average mid-plane temperature. The mid-plane temperatures vary from $313.9 \mathrm{~K}$ to $322.0 \mathrm{~K}$ over a distance of 177.8 $\mathrm{mm}$. At 25 minutes into the experiment the mid-plane temperatures vary from $311.4 \mathrm{~K}$ to $318.7 \mathrm{~K}$ and the average inside surface temperature is $1.6 \mathrm{~K}$ lower than the mid-plane temperature.

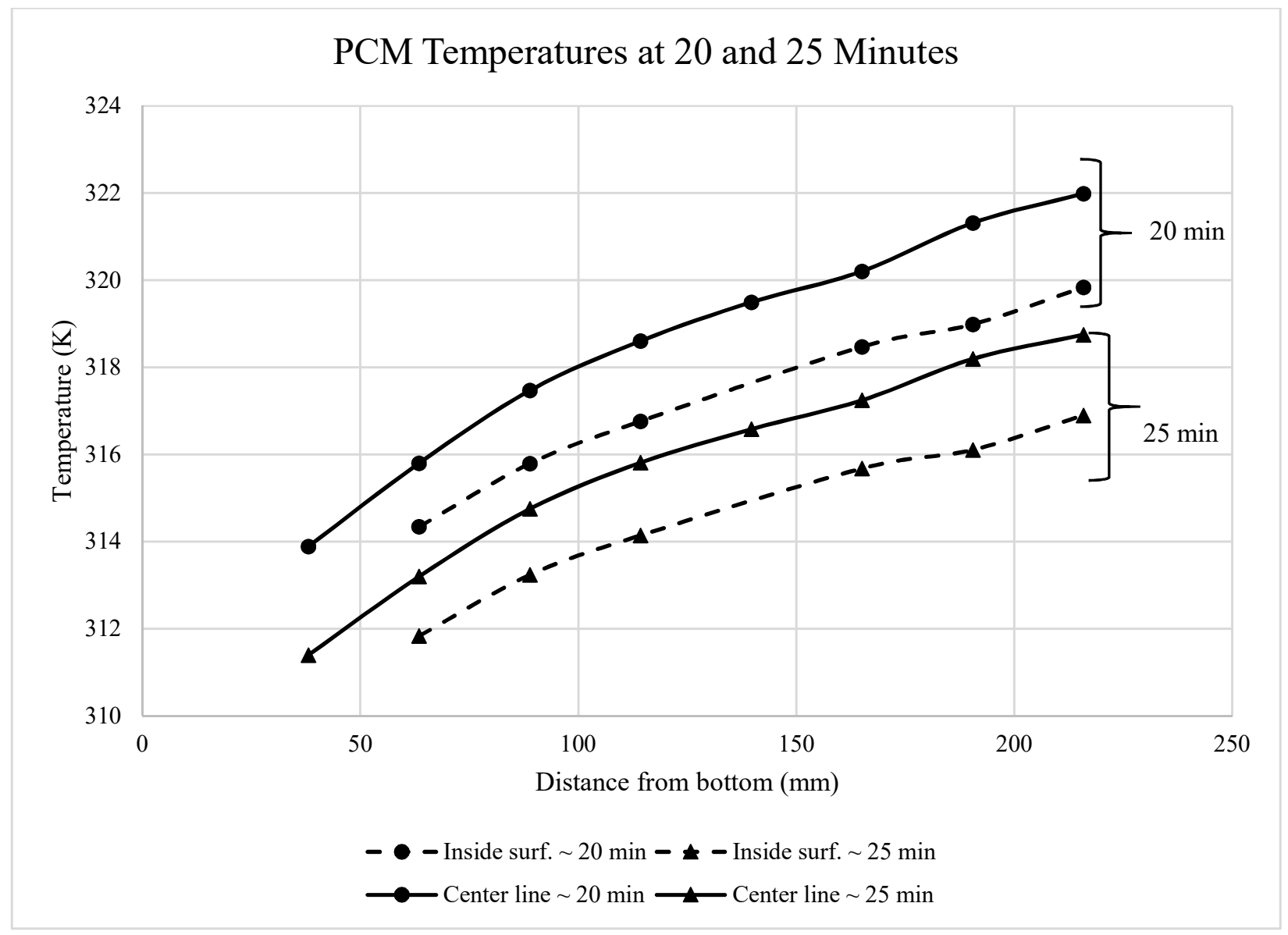

Figure 4.6: PCM temperatures at time 20 and 25 minutes 
The outside surface temperature was determined optically using Mach-Zehnder Interferometry using the process explained in Chapter 4. In summary, the surface temperatures were determined by using the ambient temperature as a reference point to determine the temperatures of all the destructive fringes normal to the wall (sample destructive fringes are shown in Figure 4.3). The wall surface temperature was determined by linear extrapolation of the two nearest fringes temperatures from the wall. The outside surface temperature of the experiment at 20 minutes is shown in Figure 4.7 and at 25 minutes in Figure 4.8. For comparison the inside surface and midplane temperatures are also shown. Due to the size of the optical window and the requirement to use the ambient temperature as a reference point, some of the temperature measurements could not be made. Specifically, the temperature readings near the edge of the optical window (TC1 and TC8) could not be determined. Thus, Figure 4.7 shows the outside surface temperatures from location TC2 to TC7. The outside surface temperatures vary from $311 \mathrm{~K}$ to $315.3 \mathrm{~K}$. Compared to the mid-plane temperature the outside surface temperature is approximately $5.8 \mathrm{~K}$ lower than the mid-plane in Figure 4.7 and $4.8 \mathrm{~K}$ lower in Figure 4.8. The temperature drop is larger in the acrylic sheet (inside and outside sheet surface) than it is within the PCM cavity itself (mid-plane and inside sheet surface). This makes sense since the sheet is exposed to ambient air and the PCM (soy wax) has a low thermal conductivity $(0.324 \mathrm{~W} / \mathrm{m} \cdot \mathrm{K})$. Also, the temperature difference between the outside surface and mid-plane decreases as the plate cools off over the 5 minutes as seen when comparing Figure 4.7 and Figure 4.8. 


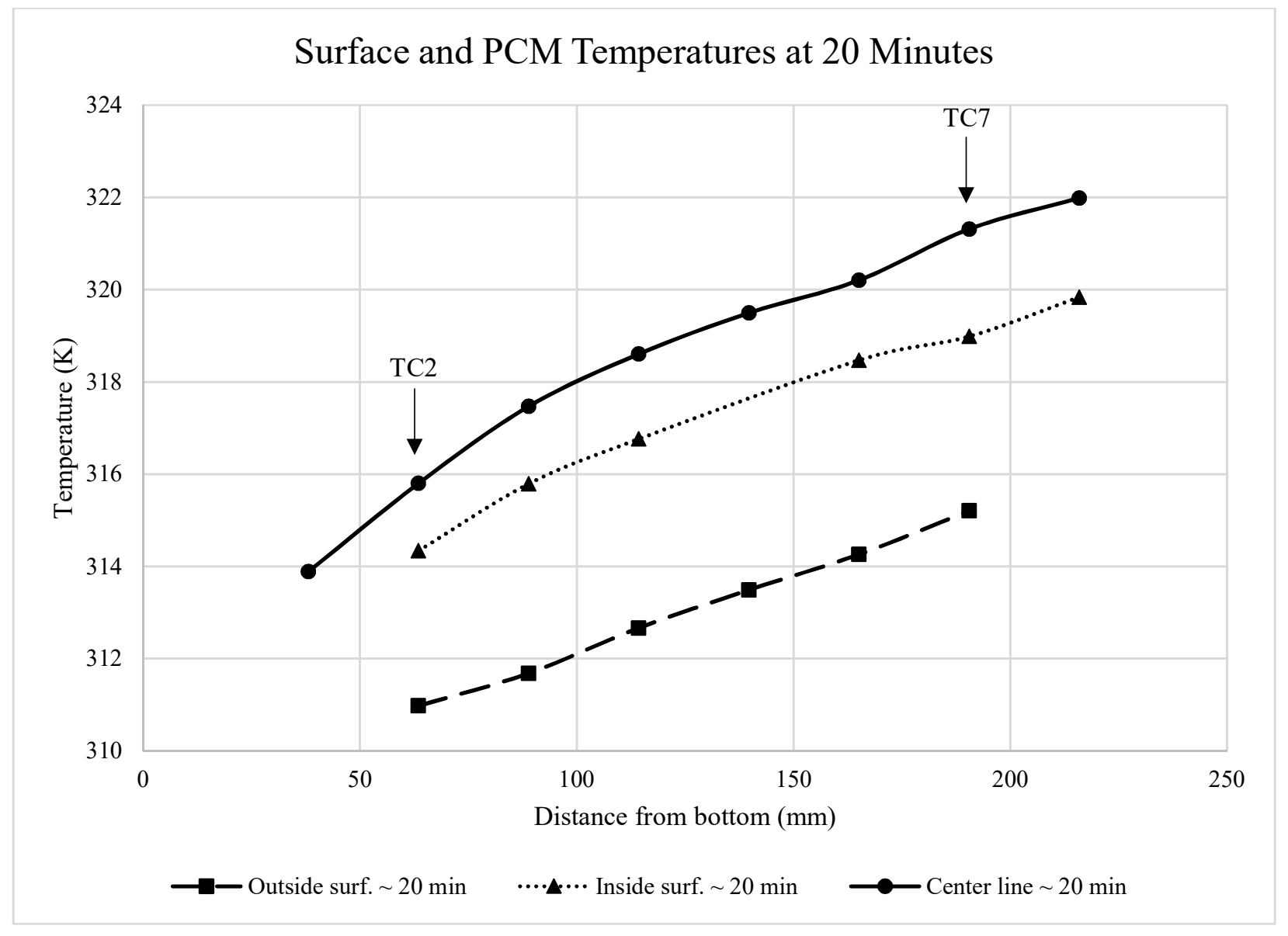

Figure 4.7: Surface and PCM temperatures at 20 minutes 


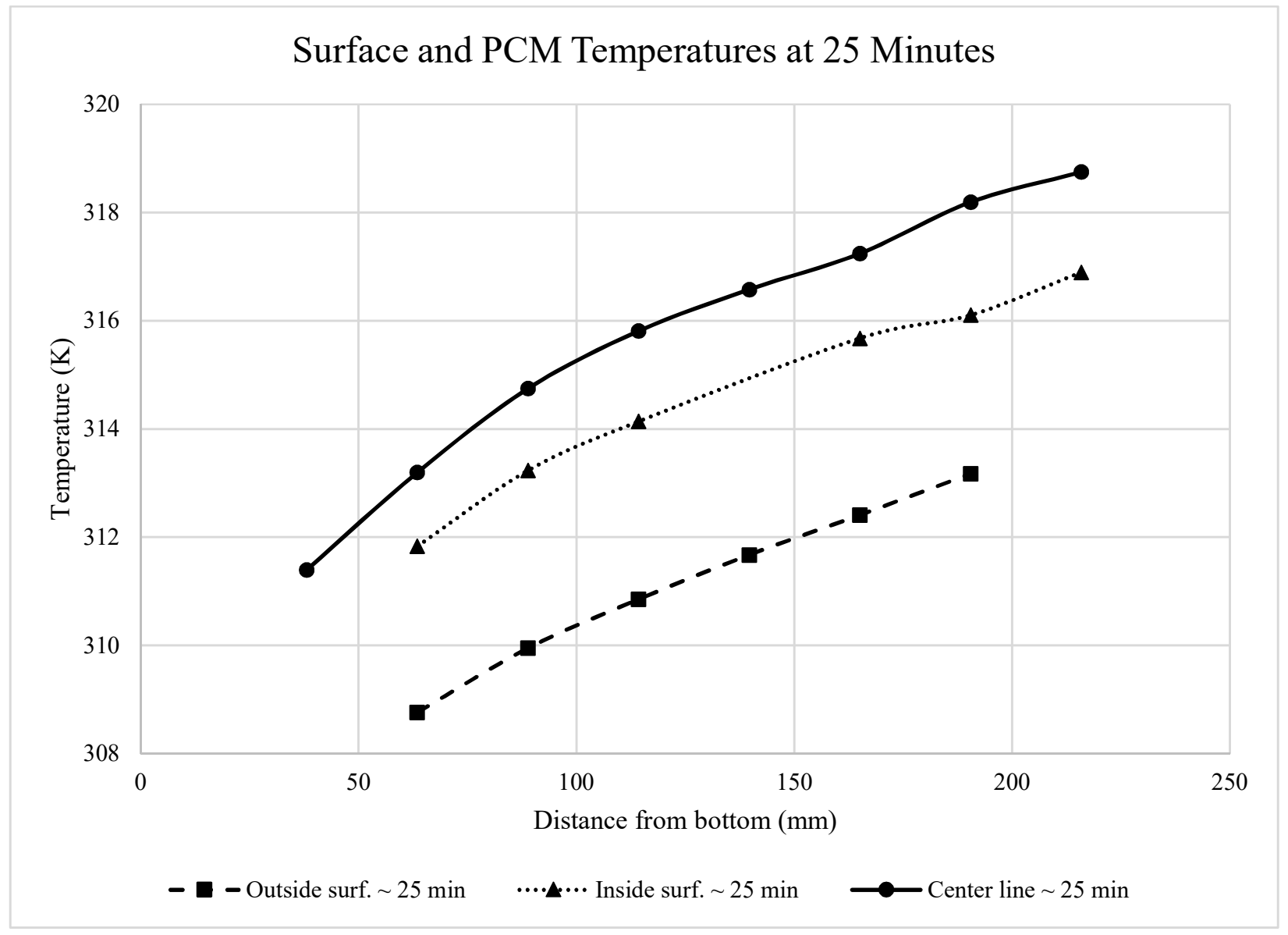

Figure 4.8: Surface and PCM temperatures at 25 minutes

The outside surface temperatures at time 20 and 25 minutes are shown in Figure 4.9. For comparison the center line temperatures at the same times are also shown. In Figure 4.9 the outside surface temperatures where determined optically (through interferometry) and are not as accurate as the thermocouple measurements. Thus the outside surface temperatures will have some 'noise' relative to the center line and inside surface temperatures. However, the outside surface 
temperatures profiles conforms to the thermocouple measurements. Also, the overall temperature trend with time conforms to the center line and inside surface temperatures. An example of this noise can be seen by looking at the TC 2 temperatures. The temperature profile curve at 25 minutes between the center line and outside surface temperature is a better match than the profile curves at 20 minutes. The slope of the temperature profile between TC2 and TC3 is flatter at 20 minutes than at 25 minutes. The 'noise' in the outside surface temperature is more evident during the phase change range of the PCM plate.

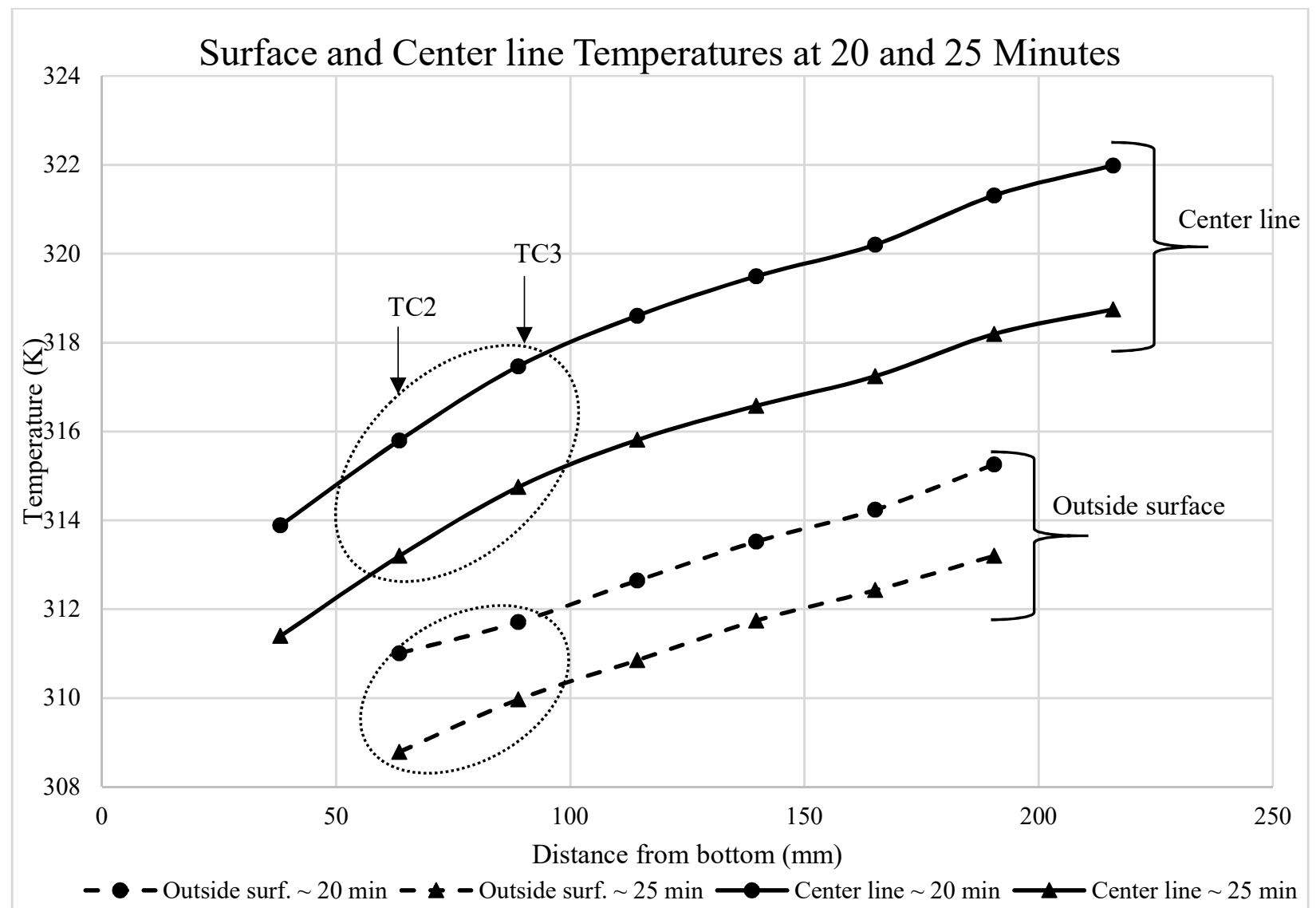

Figure 4.9: Surface and center line temperatures at time 20 and 25 minutes 


\subsection{Phase Change Range}

From the PCM temperature decay plot in Figure 4.2 the phase change process starts at time 1700 $\mathrm{s}(\sim 28 \mathrm{~min})$ and ends at $4000 \mathrm{~s}(\sim 66 \mathrm{~min})$. For the current discussion the phase change time range will be taken to be between 30 and 70 minutes. During this time period the interferograms were analyzed at every minute giving a total of 40 data sets. For visualization purposes, the following discussion on the temperature profiles is broken down into 5 minutes chunks.

\section{Time Period 30 to 35 Minutes}

The center line temperatures are shown from time 30 to 35 minutes in Figure 4.10. There are a total of 6 temperature profiles. From TC3 to TC8 the temperature profile is approximately linear through the 5-minute period. The temperature decay of the liquid soy wax drops steadily at approximately $0.4 \mathrm{~K}$ per minute. The onset of solidification of the soy wax can be seen at $\mathrm{TC} 1$ and TC2. As the soy wax solidifies there is a jump in the temperature at TC1 (bottom of the plate) from $310.7 \mathrm{~K}$ at 30 minutes to $312.8 \mathrm{~K}$ at 35 minutes. However, at TC8 (top of plate) the temperature drops from $316 \mathrm{~K}$ at 30 minutes to $313.8 \mathrm{~K}$ at 35 minutes. There is a smaller change in temperature at TC2 starting at $311.2 \mathrm{~K}$ at 30 minutes and dropping to $310.0 \mathrm{~K}$ at 35 minutes. For comparison the inside surface temperatures from time 30 to 35 minutes is shown in Figure 4.11. The inside surface temperature is missing data for TC1 and TC5 due to broken sensors. The temperature profiles of the inside surface temperature match the behaviour of the center line temperatures. This can be seen by looking at TC2 where the temperature profiles 'converge' to a point. Also the slope of the temperature profile between TC6 and TC7 increases slightly relative to the rest of the temperature profiles. 


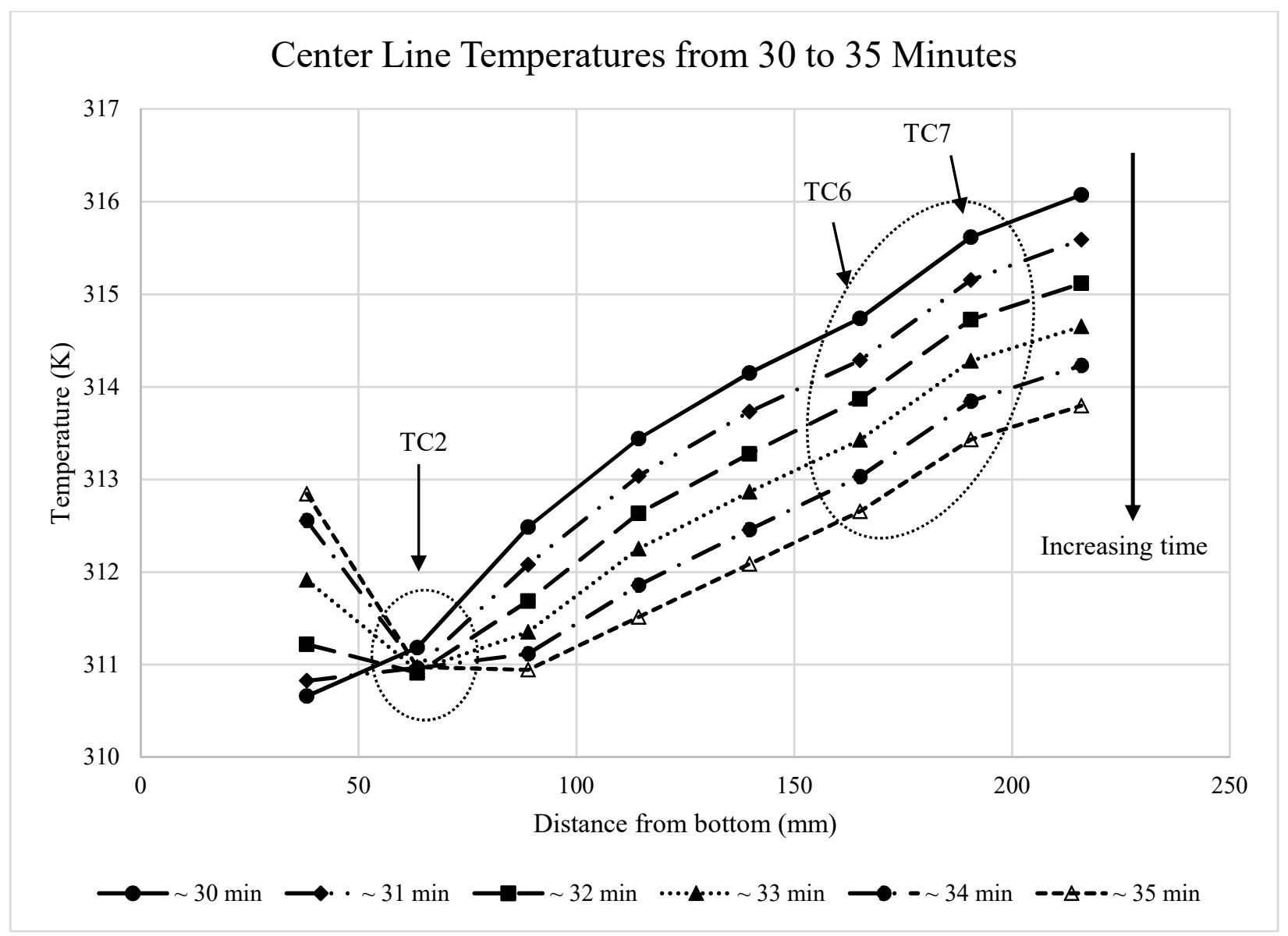

Figure 4.10: Center line temperatures from 30 to 35 minutes. 


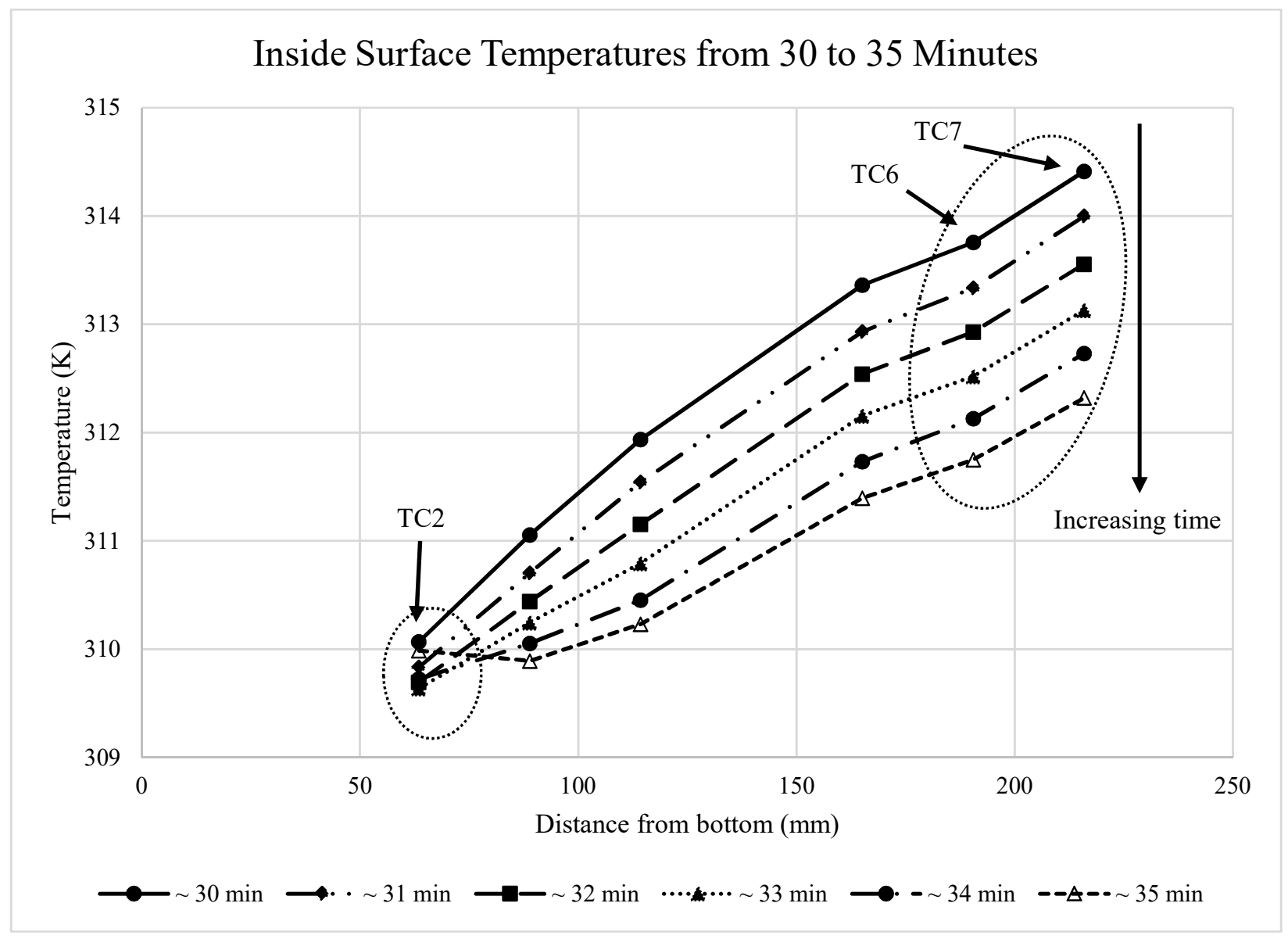

Figure 4.11: Inside surface temperatures from 30 to 35 minutes

The outside surface temperature profiles from time 30 to 35 minutes are shown in Figure 4.12. Due to the size of the optical windows of the interferometer, TC1 and TC8 was not measured since there is not enough fringe crossings in those regions to 'capture' the ambient air. Therefore, the temperatures shown are from TC2 to TC7. In can be seen from Figure 4.12 that there is noise in the optically measured temperatures. The temperature profiles do not fall in a uniform manner which was seen in Figure 4.10 and Figure 4.11. For example, the temperature profiles for time 30 minutes and 31 minutes is almost identical (average temperature drop of approximately $0.08 \mathrm{~K}$ ) while the temperature profiles between 31 minutes and 32 minutes have an observable temperature 
drop of approximately $0.4 \mathrm{~K}$. Similarly, the temperature profiles at time 34 minutes and 35 minutes almost identical. However, the overall trend in the temperature profiles in Figure 4.12 conforms to the behaviour that was seen in Figure 4.10 and Figure 4.11. Another observable trend in Figure 4.12 that conforms to the thermocouple data can be seen at time 35 minutes. There is a temperature jump at TC2 at 35 minutes due to the onset of solidification which was also observed in Figures 4.10 and Figures 4.11.

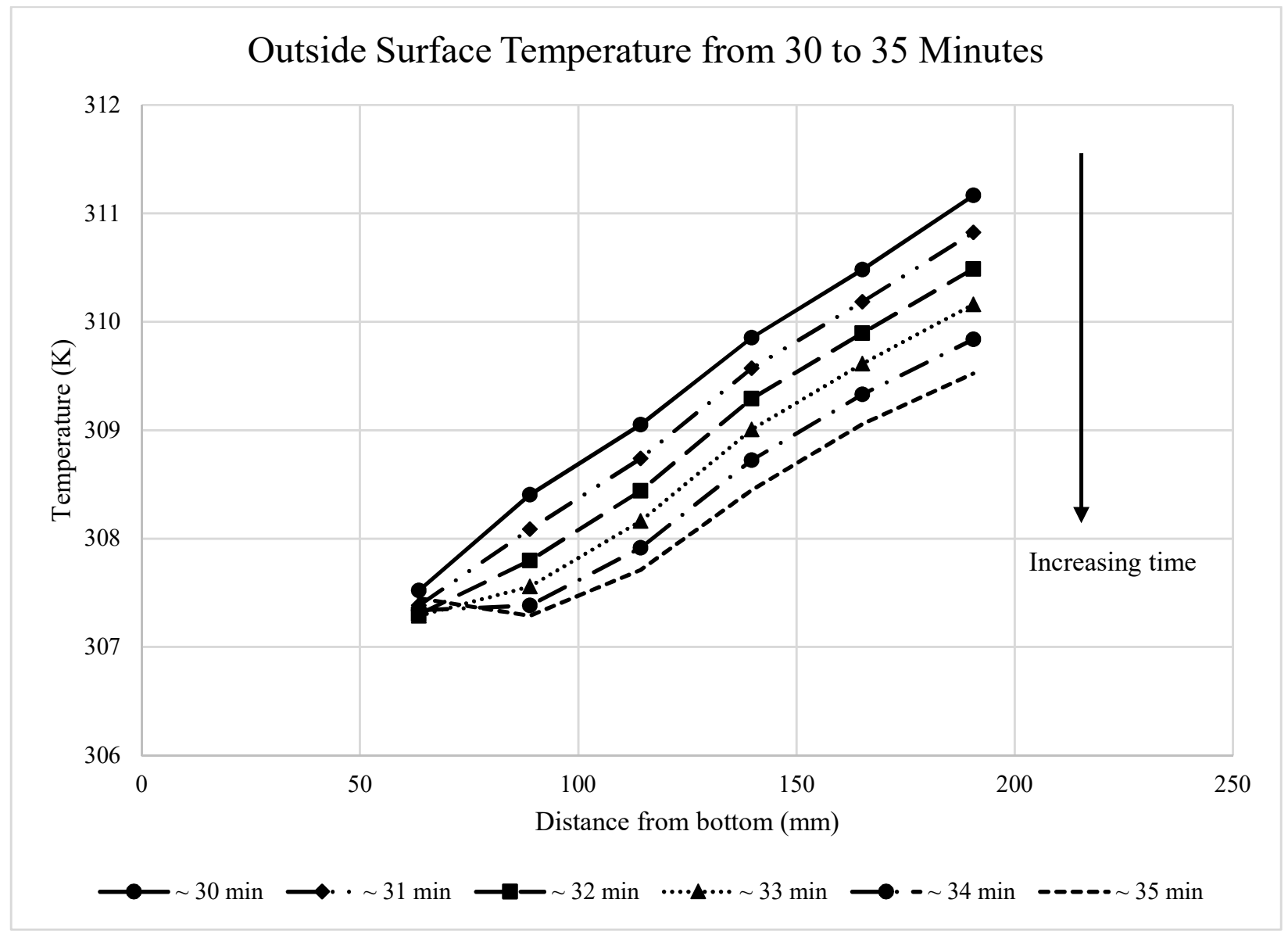

Figure 4.12: Outside surface temperatures from 30 to 35 minutes

For a clearer comparison consider the temperature profiles at 30 minutes and 35 minutes only. The center line temperature and inside surface temperatures are shown in Figure 4.13 and the center 
line and outside surface temperatures are shown in Figure 4.14. In both Figure 4.13 and Figure 4.14 the center line temperature profile at 35 minutes shows a jump in temperature at $\mathrm{TC} 1$ that is $2.18 \mathrm{~K}$ higher than the temperature at 30 minutes. In order to compare the temperature profiles between 30 and 35 minutes, consider the slope between TC2 and TC3 (circled region in Figure 4.13). The slope of the center line between $\mathrm{TC} 2$ and $\mathrm{TC} 3$ at 30 minutes is approximately parallel with the slope of the inside surface temperatures. Similarly, the slope of the center line at 35 minutes is approximately parallel with the inside surface temperatures. The slope comparison between the center line and outside surface temperature profiles in Figure 4.14 are not as parallel as the slopes in Figure 4.13. For example, at 35 minutes, the temperature slope between TC2 and TC3 is steeper than the center line slope. This is due to the noise that occurs when using MZI to obtain outside surface temperatures. 


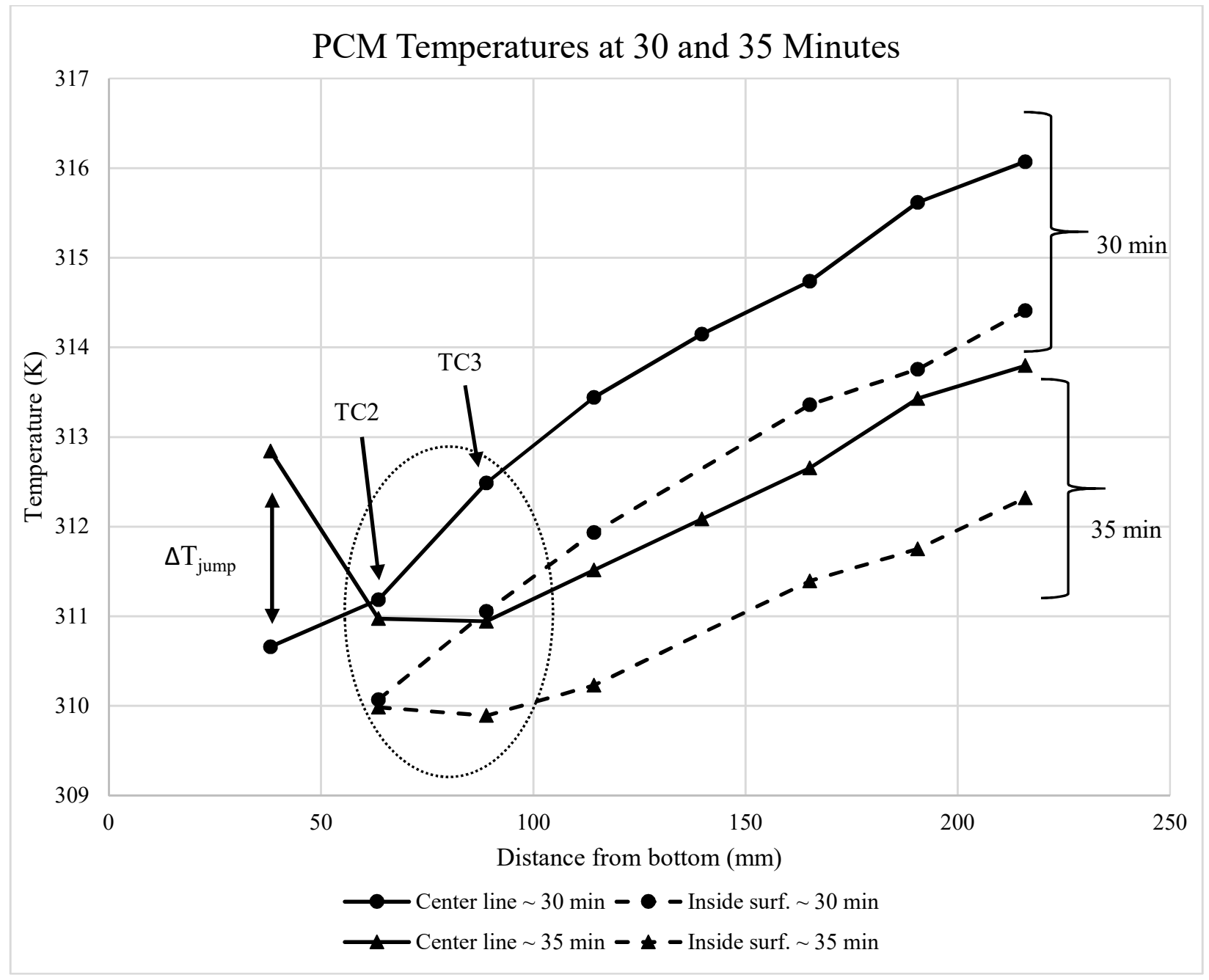

Figure 4.13: PCM temperatures at 30 and 35 minutes 


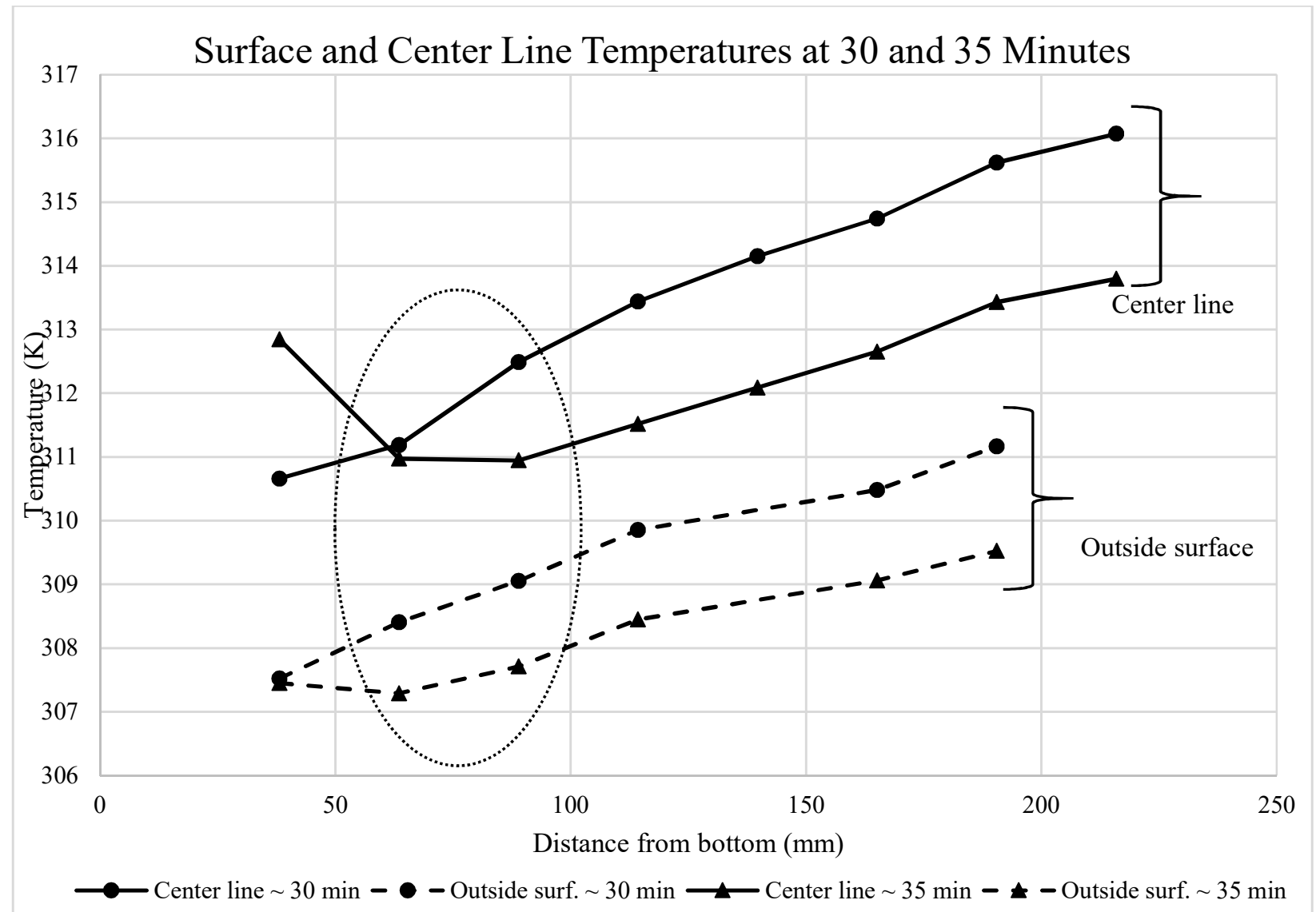

Figure 4.14: Surface and center line temperatures at 30 and 35 minutes

The center line, inside surface, and outside temperature profiles are shown together in Figure 4.15. As discussed above, the slope of the temperature profile between TC2 and TC3 is approximately parallel between the center line and inside surface temperatures while the slope of the outside surface temperature is steeper. The temperature drop between the inside and outside temperature profiles is larger than the temperature drop between the inside and center line temperature. The average inside surface temperature is $1.35 \mathrm{~K}$ lower than the average center line temperature. The average outside surface temperature is $2.63 \mathrm{~K}$ lower than the average inside surface temperature. This is due to the acrylic sheet that contains the PCM which provides an insulating effect (thermal 
conductivity of $0.324 \mathrm{~W} / \mathrm{m} \cdot \mathrm{K}$ ) giving a temperature drop that is smaller within the PCM than the temperature drop within the acrylic sheet.

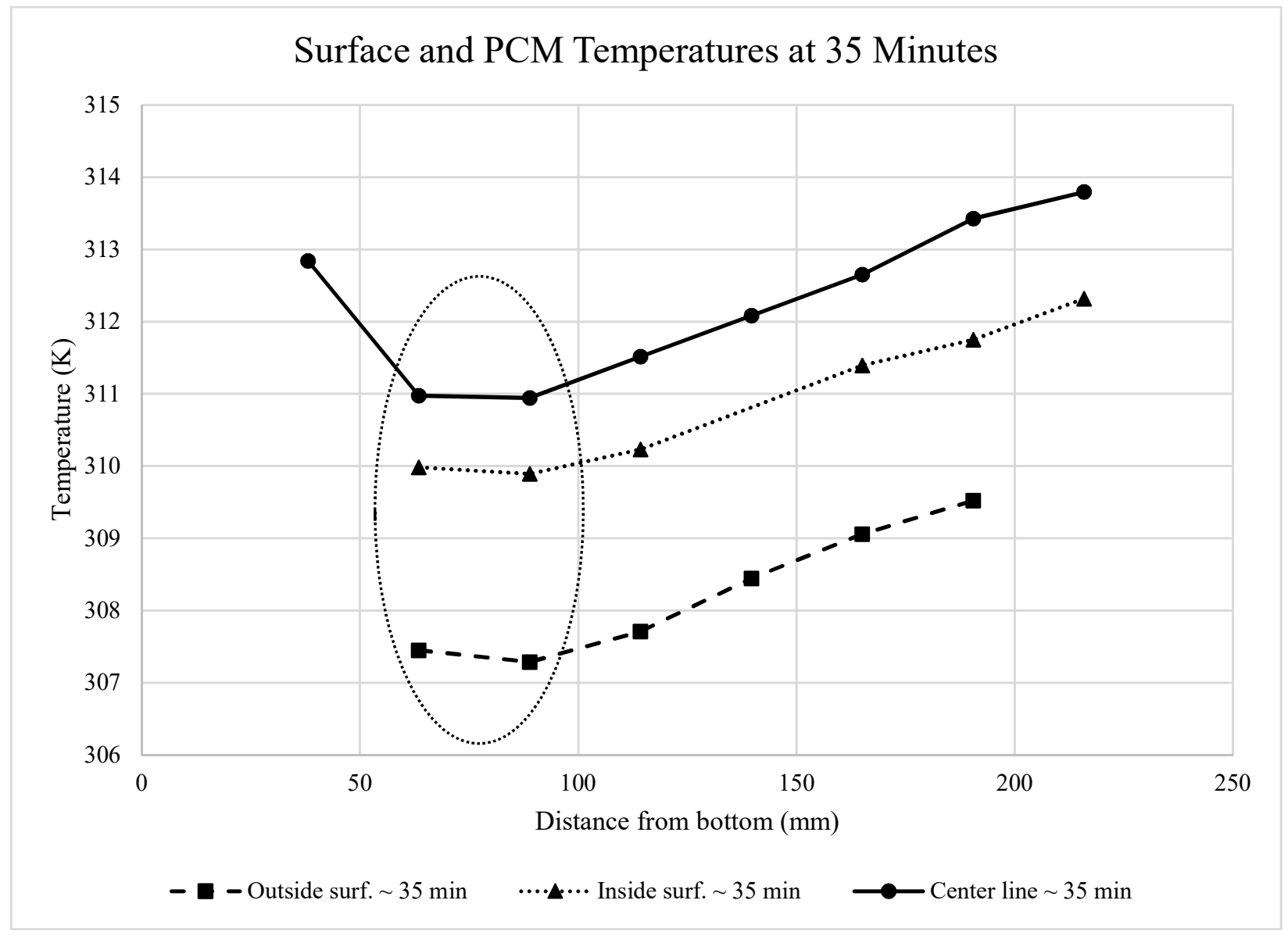

Figure 4.15: Surface and PCM temperatures at 35 minutes

\section{Time Period 35 and 40 Minutes}

The center line temperature profiles from 35 minutes to 40 minutes are shown in Figure 4.16. The largest temperature jump occurs at location TC2. This is in contrast to Figure 4.10 which has the largest temperature jump at TC1. During this 5-minute time period, the highest temperature occurs at time 40 minutes at location TC2. In other words, the temperature at TC2 actually increases with time. Treating the temperature axis as a unit of length, the 'linear' portion of the temperature 
profiles (TC6 through TC8) drops in temperature by $2.01 \mathrm{~K}$ during the 5 -minute period. However, at TC2 the temperature increases by $3.56 \mathrm{~K}$ during the same time period. The bottom of the plate increases in temperature faster than the top of the plate. By visual inspection of Figure 4.16, it can be seen that the TC temperatures flat lines at approximately $311 \mathrm{~K}$ which is approximately the congealing point $\left(\mathrm{T}_{\text {cong }}\right)$ of soy wax.

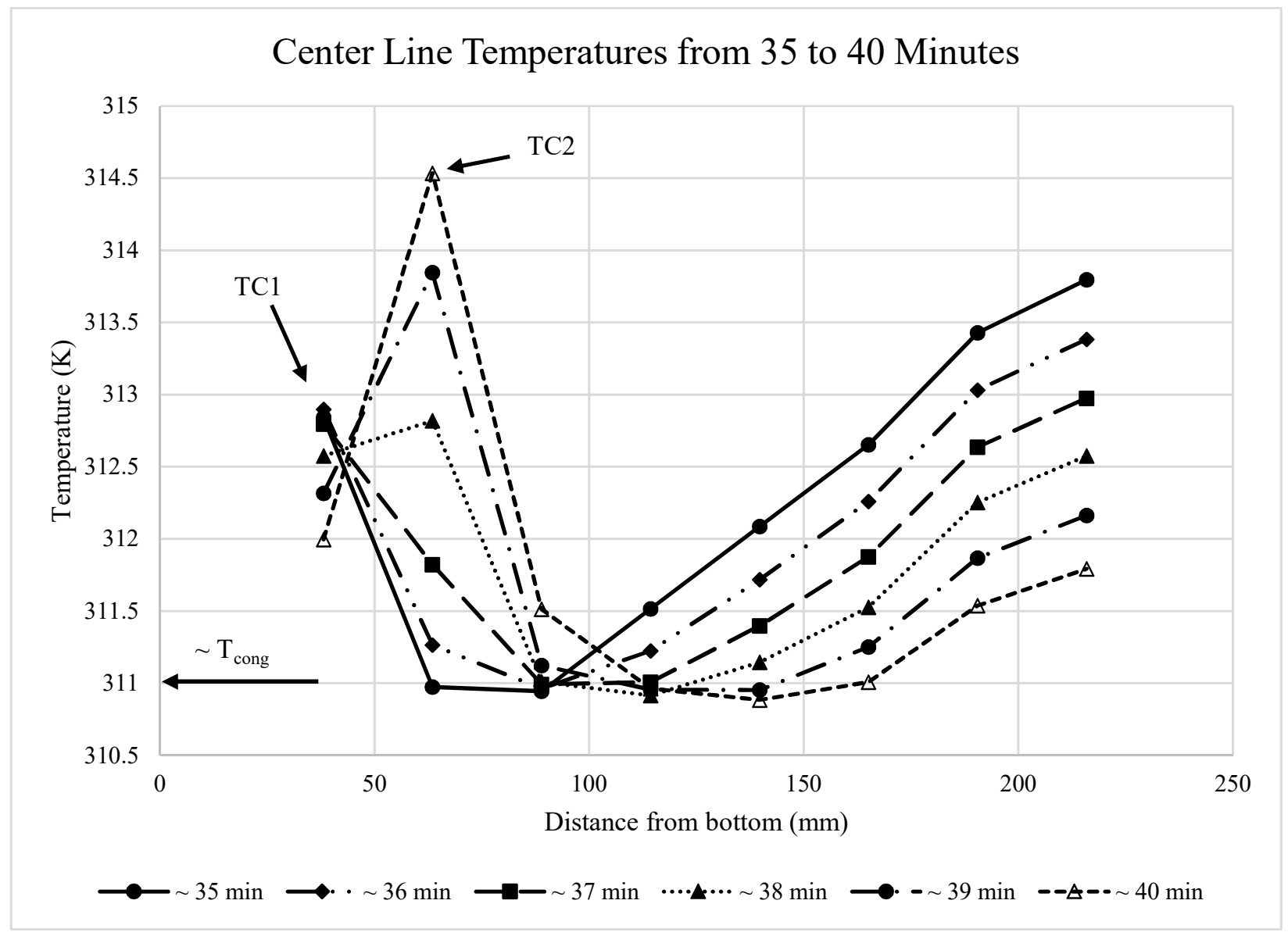

Figure 4.16: Center line temperatures from 35 to 40 minutes

For comparison consider the inside surface temperature profiles from 35 minutes to 40 minutes shown in Figure 4.17. In Figure 4.17 temperatures measurements at TC1 and TC5 are missing. 
The temperature drop of the linear portion of the temperature profiles drops by $1.74 \mathrm{~K}$ during this 5-minute period. However, at location TC2, the temperature jumps by $1.75 \mathrm{~K}$ during the same time period. In contrast to the center line temperatures in Figure 4.16, the temperature jumps and drops are smaller in Figure 4.17. Also, in Figure 4.17, the temperature flat line is not as pronounced and is at a lower temperature than Figure 4.16. This is expected since the inside surface thermocouples are in contact with both the PCM and acrylic sheet which is in turn exposed to the ambient.

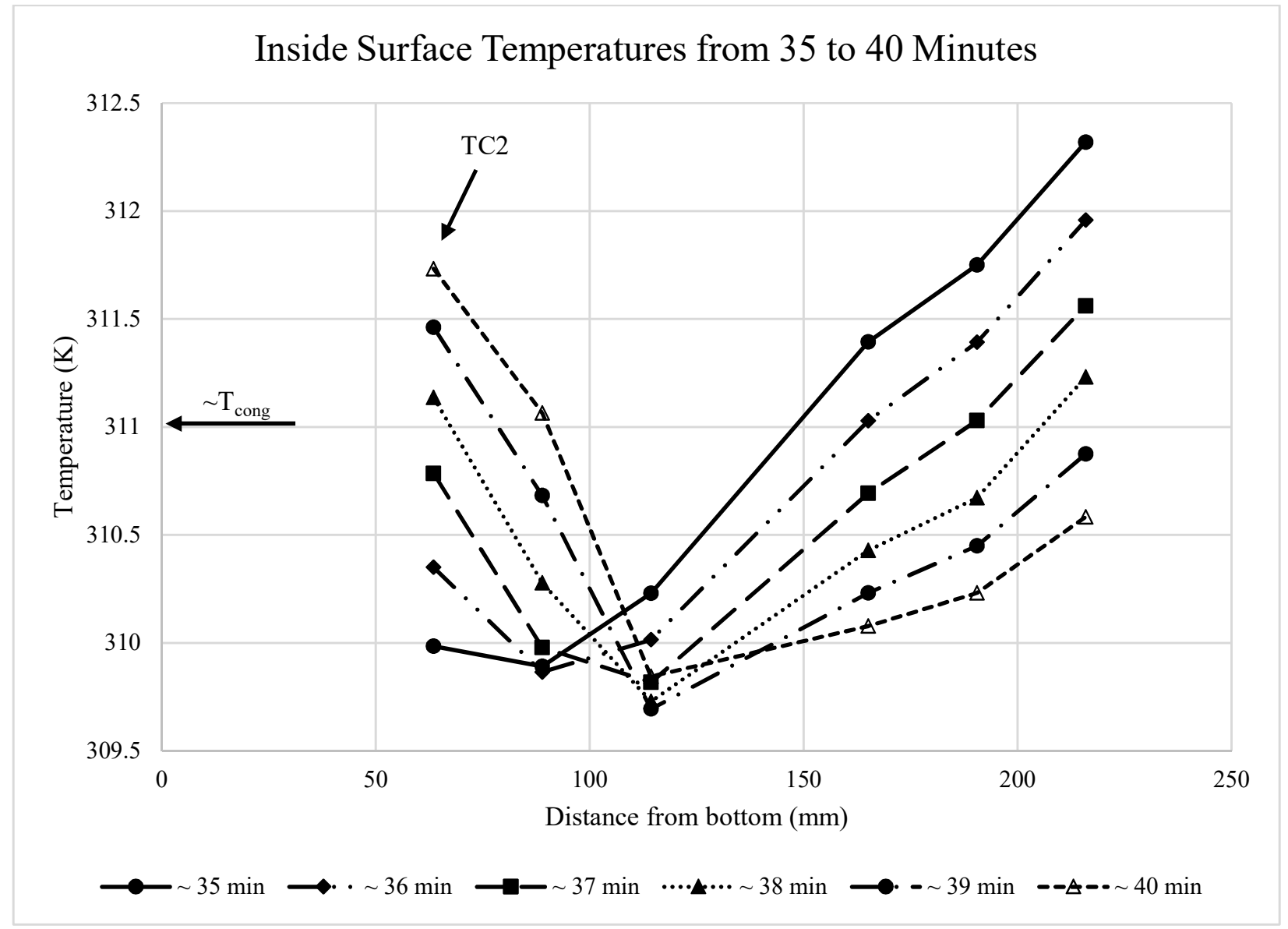

Figure 4.17: Inside surface temperatures from 35 to 40 minutes

The outside surface temperatures from 35 minutes to 40 minutes is shown in Figure 4.18a and Figure $4.18 \mathrm{~b}$ for unfitted and curve fitted surface temperatures respectively. Due to the analysis 
procedure of obtaining the temperatures using interferometry, TC1 and TC8 are not available. As explained before, obtaining outside surface temperatures using interferometry introduces noise to the data. This noise is visible in the temperature profiles in Figure 4.18a. For example consider the minute by minute temperature drop at location TC7. The expectation would be for the TC7 temperature to steadily drop with time, which was seen in Figure 4.16 and Figure 4.17. However, in Figure 4.18a, the temperature profiles at time 39 minutes and 40 minutes does not follow this trend. The TC7 temperatures drops steadily at 35, 36, 37 and 38 minutes, but flat lines at 39 minutes, and actually increases at 40 minutes. For a given point in time, the overall trend in the temperature profile does match the profiles seen in Figure 4.16 and Figure 4.17. For a given temperature profile, the highest temperature is at TC7, the lowest temperature is at TC4, and there is a temperature jump at TC2 and TC3 (same pattern as the center line and inside surface temperatures above). In Figure 4.18b the minute by minute temperature at location TC7 behaves as expected by having a consistent temperature drop throughout this block of time. 


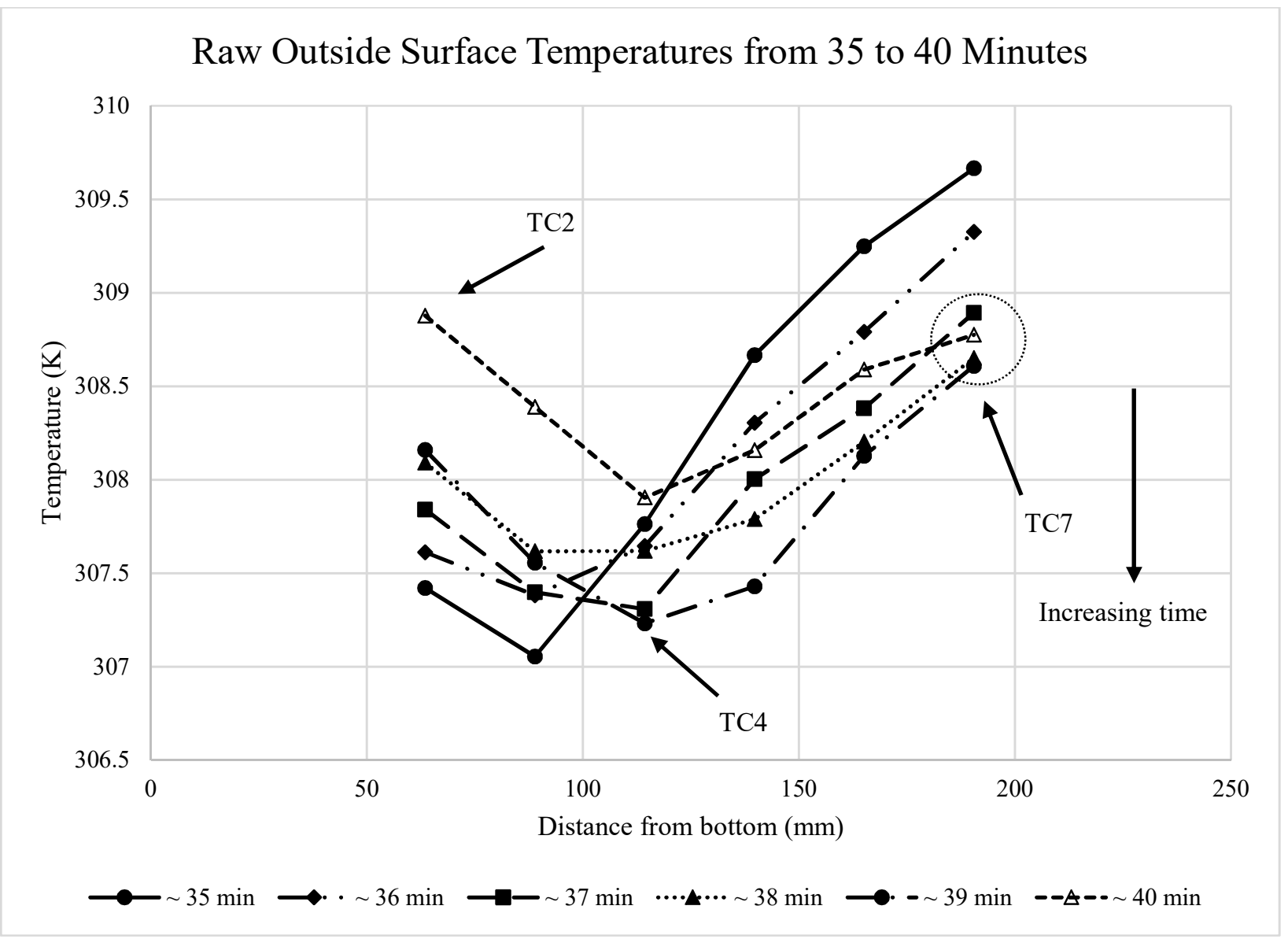

Figure 4.18a: Raw outside surface temperatures from 35 to 40 minutes 


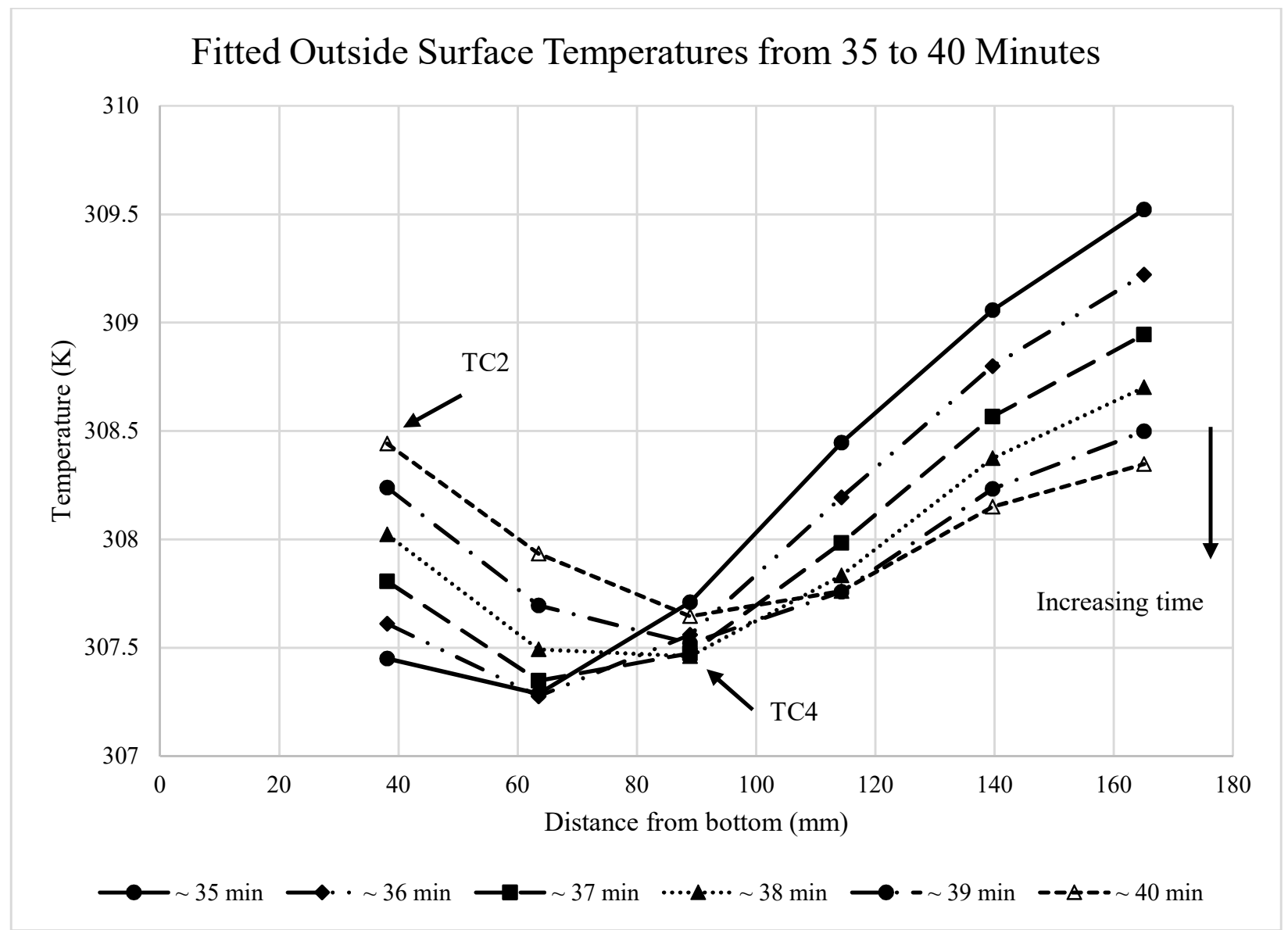

Figure 4.18b: Fitted outside surface temperatures from 35 to 40 minutes

\section{Time Period 40 and 45 Minutes}

The next 5-minute block progression of the center line temperature profiles from 40 minutes to 45 minutes of the experiment is shown in Figure 4.19. The previous 5-minute center line temperature profile progression is in Figure 4.16. In Figure 4.19 one can see the continuation of the temperature jump which is now progressing upward along the PCM panel. From the previous progression in Figure 4.16 location TC2 experienced the largest jump in temperature during that 5-minute period. Now in Figure 4.19 the temperature at location TC2 continues to rise by an additional $0.8 \mathrm{~K}$ but it is TC3 that has the largest jump in temperature going from $311.5 \mathrm{~K}$ to $315 \mathrm{~K}$. The temperatures at 
locations TC4, TC5, and TC6 are also experiencing a temperature jump but by a smaller amount than TC3. In the previous progression in Figure 4.16 only two of the thermocouples (TC2 and TC3) measured a temperature jump; however, in the current progression there are five thermocouples, encompassing the region between TC2 through TC6. Also, the temperature at the top of the PCM panel at TC8 dropped by $1 \mathrm{~K}$ while in the previous 5-minute block that same location dropped by $2 \mathrm{~K}$.

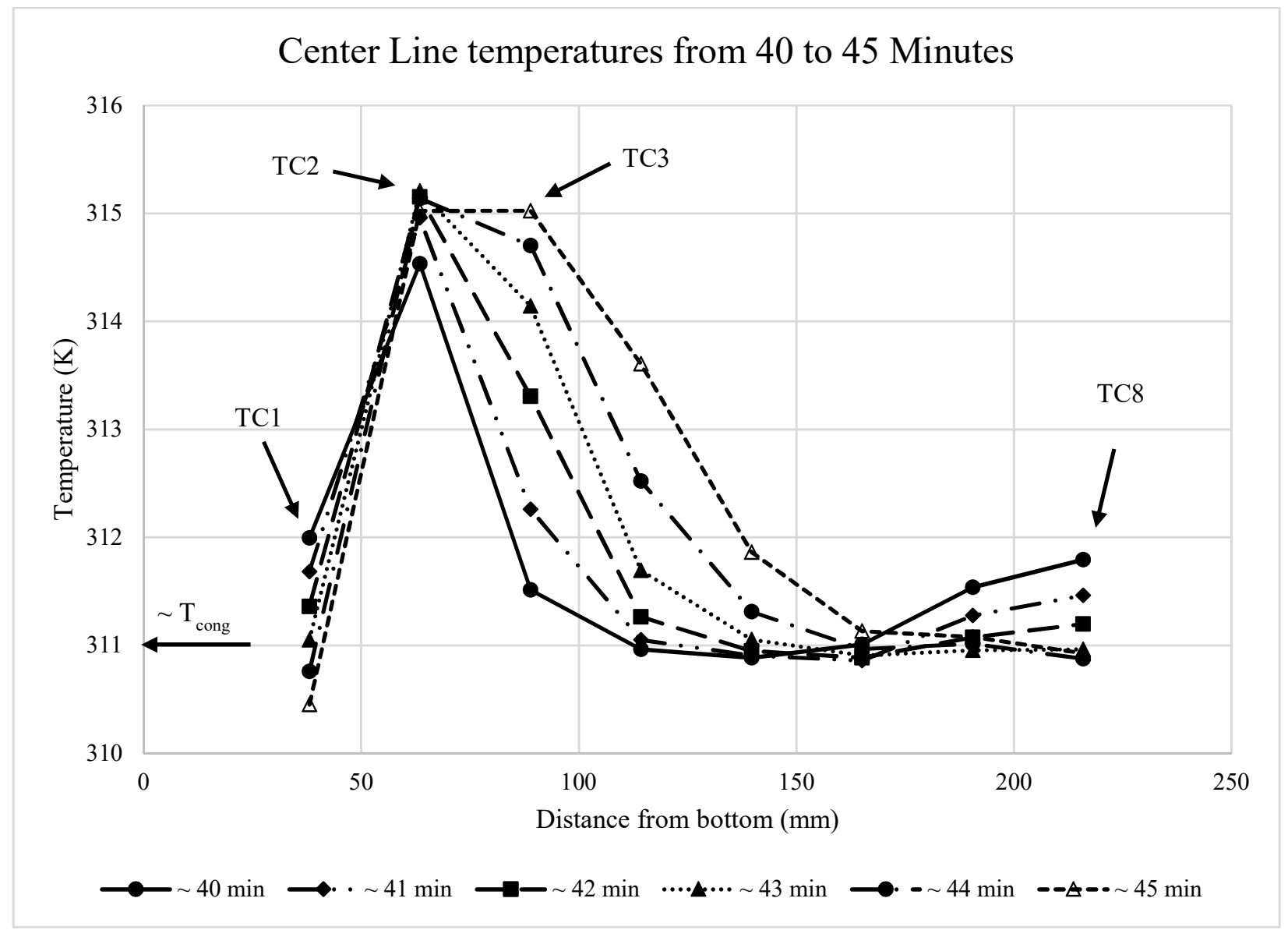

Figure 4.19: Center line temperatures from 40 to 45 minutes

The inside surface temperature profiles from 40 to 45 minutes is shown Figure 4.20. The previous time period for the inside surface temperature profiles are shown in Figure 4.17. The temperature jump that started at the bottom of the PCM wall is continuing to rise and move upward along the 
plate. The locations TC2, TC3 and TC4 are all experiencing temperature jumps. At locations TC6, TC7 and TC 8 the temperatures are continuing to drop from the previous time period, however, the temperature drops are smaller and eventually are overtaken by the temperature jump that is propagating along the wall. At location TC2 the temperature rise is not as pronounced as in Figure 4.17 and at time 45 minutes the temperature is even starting to drop again.

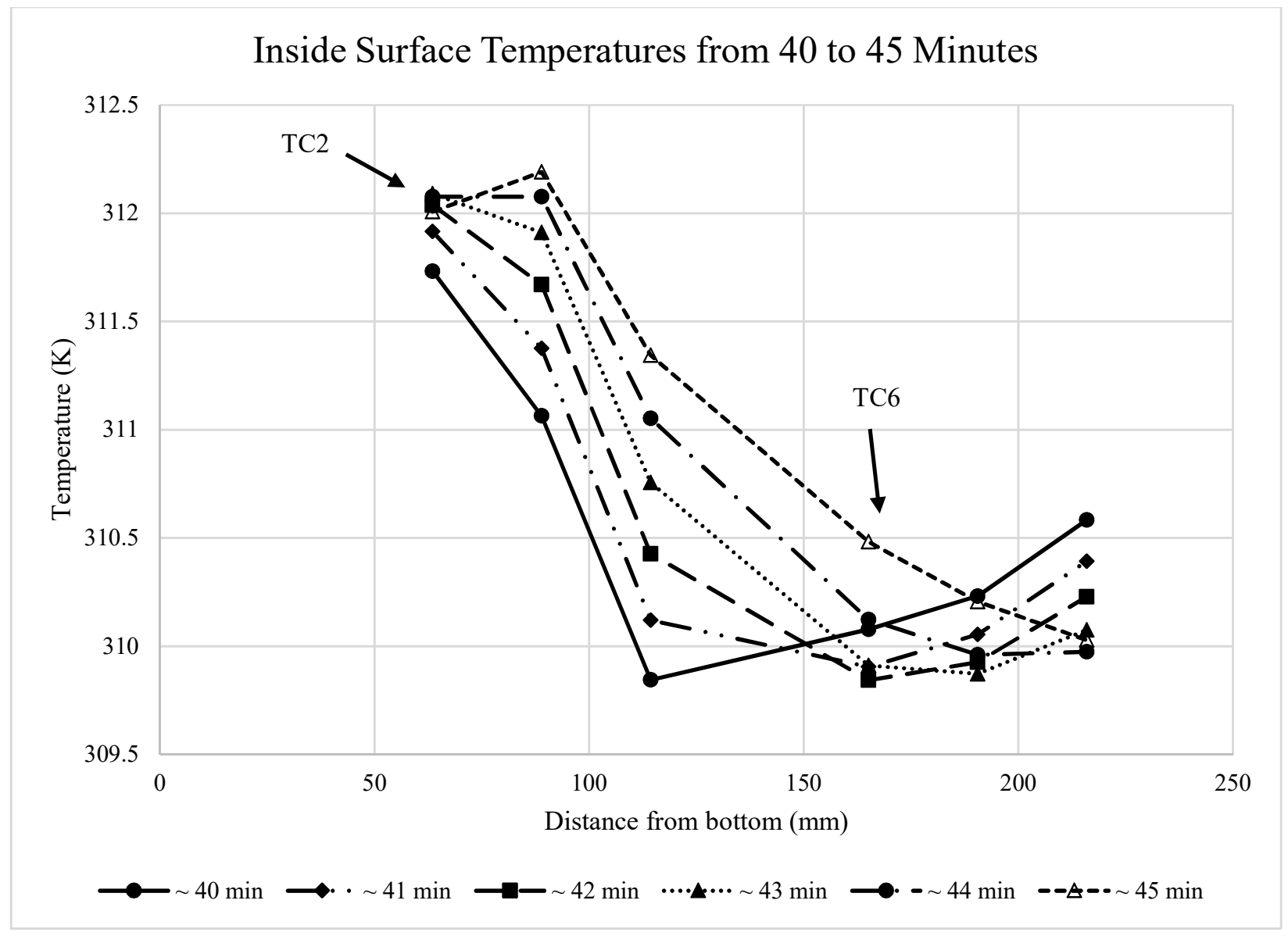

Figure 4.20: Inside surface temperatures from 40 to 45 minutes

The outside surface temperature profiles for time period 40 to 45 minutes are shown in Figure 4.21a and Figure $4.21 \mathrm{~b}$ for the unfitted and curve fitted surface temperatures respectively. These outside surface temperatures are a continuation from Figure 4.18a and 4.18b. As mentioned in the discussion for Figure 4.18a, the results gathered by MZI have additional 'noise' in the data which 
is apparent here in Figure 4.21a. For example, the progression from 40 minutes to 41 minutes does not conform to the pattern seen from the thermocouple data or from the MZI results in Figure 4.18a. The expectation would be that the temperature profile at 41 minutes should be higher than the temperature profile at 40 minutes. However, here in Figure 4.21a the temperature profile drops from 40 to 41 minutes. However, if the temperature profile at 40 minutes is ignored, the other temperature profile progressions from 41 to 45 minutes do behave as expected. Also the shapes of the temperature profiles behave as expected for the entire range of 40 to 45 minutes. The 'smoothed' outside surface temperature profiles in Figure $4.21 \mathrm{~b}$ do behave as expected relative to the general behaviour in Figure 4.20 by seeing a consistent jump in temperature over time for most of the TC locations. For the remainder of the discussion the fitted outside surface temperatures will just be referenced as the outside surface temperatures. 


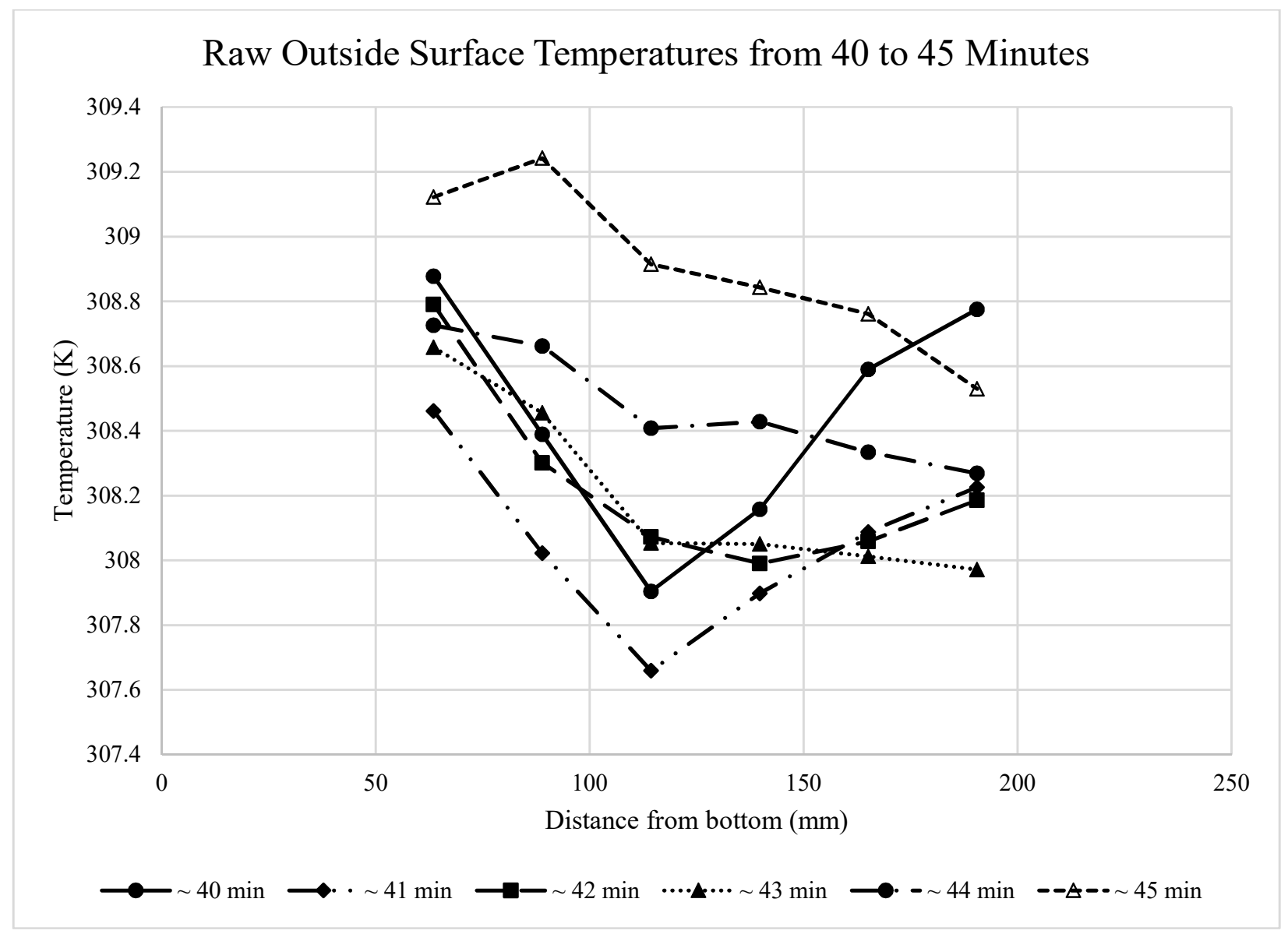

Figure 4.21a: Raw outside surface temperatures from 40 to 45 minutes 


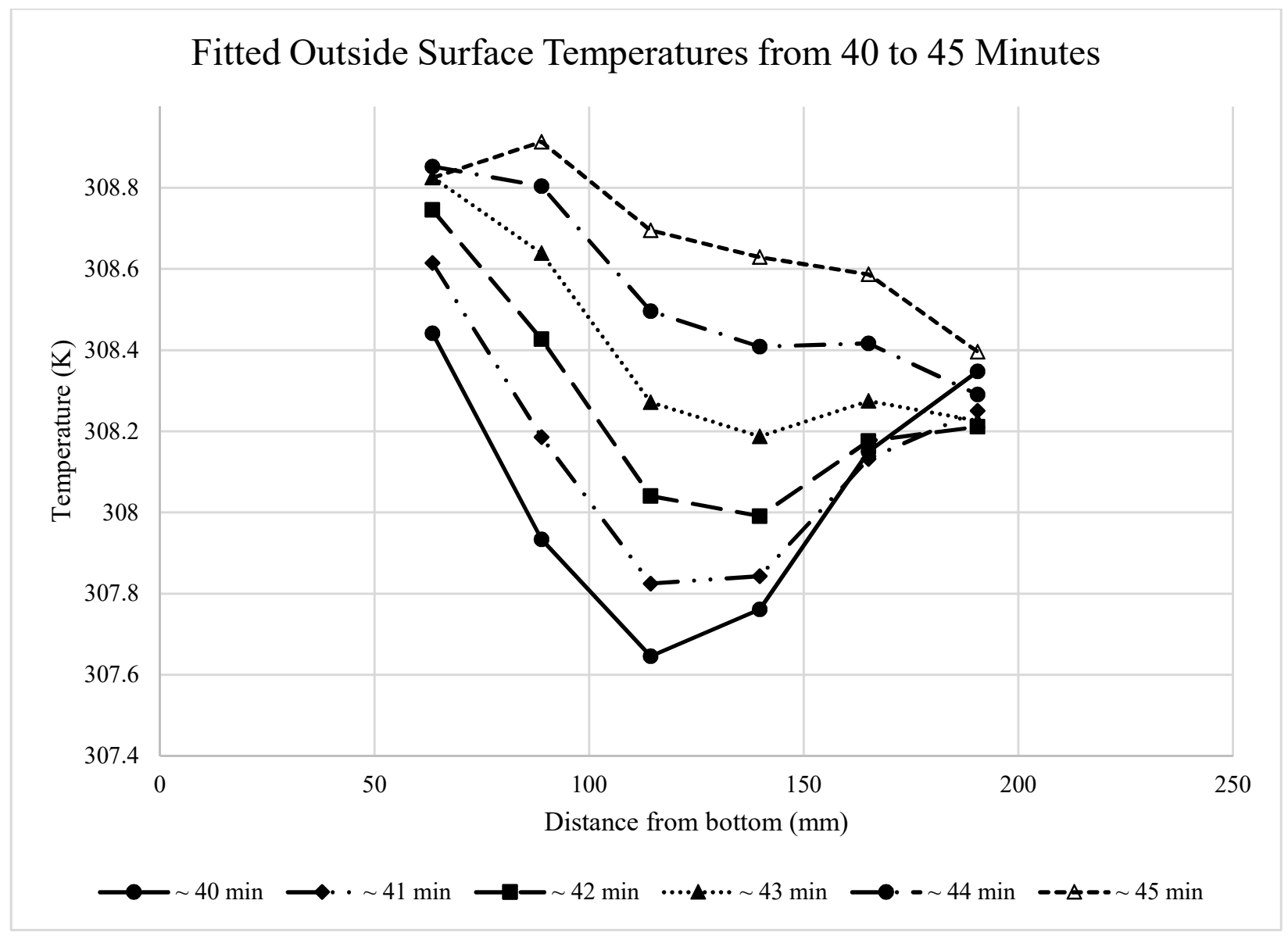

Figure 4.21b: Fitted outside surface temperatures from 40 to 45 minutes

\section{Time Period 45 and 50 Minutes}

The center line temperature profiles from 45 to 50 minutes are shown in Figure 4.22 and is a continuation of Figure 4.19. The temperatures at TC1 and TC2 are continuing to drop from the previous block and both appear to fall at the same rate. The temperature at TC3 rises slightly from 45 to 47 minutes then starts to drop. The temperatures at the remaining locations from TC4 through 
TC8 are all rising with TC6 having the largest jump in temperature during this 5-minute block at approximately $3.4 \mathrm{~K}$.

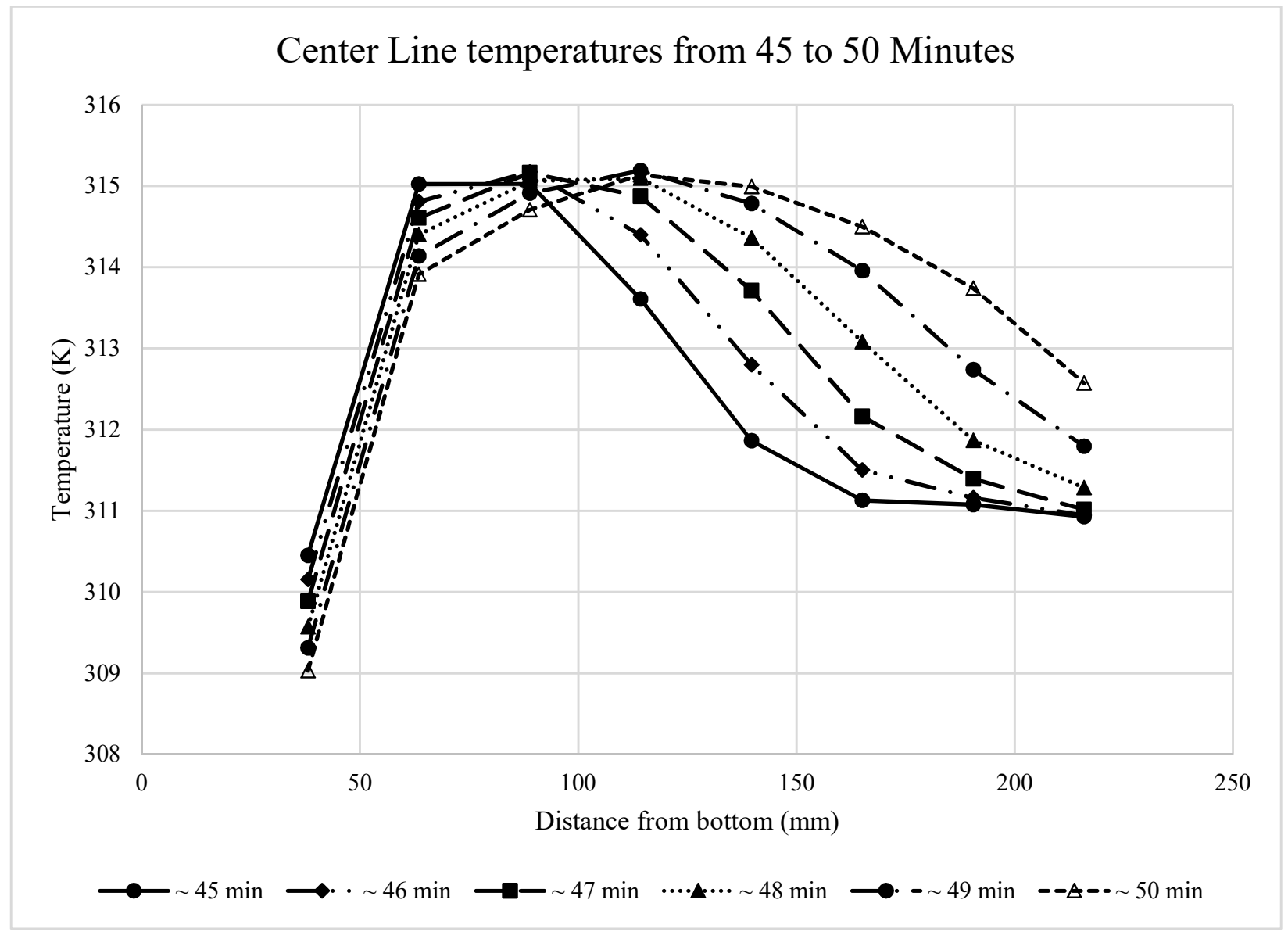

Figure 4.22: Center line temperatures from 45 to 50 minutes

The inside surface temperature profiles for the time period 45 to 50 minutes are shown in Figure 4.23 and is a continuation of Figure 4.20. The inside surface temperatures are following a similar trend as the center line temperatures. The temperature at TC2 drops throughout this time block while TC3 rises slightly for one minute then slowly drops. The remaining temperatures at TC4, TC6, TC7 and TC8 all increase in temperature with TC8 having the largest jump at $1.61 \mathrm{~K}$ during this 5-minute period. From the shape of the temperature profiles one can see that the temperature jump propagates up the plate. The largest temperature drop is at the bottom of the plate at TC2 
followed by TC3 with the second largest drop. For the remaining thermocouples the temperature jumps get larger as the higher up the thermocouples are on the PCM panel.

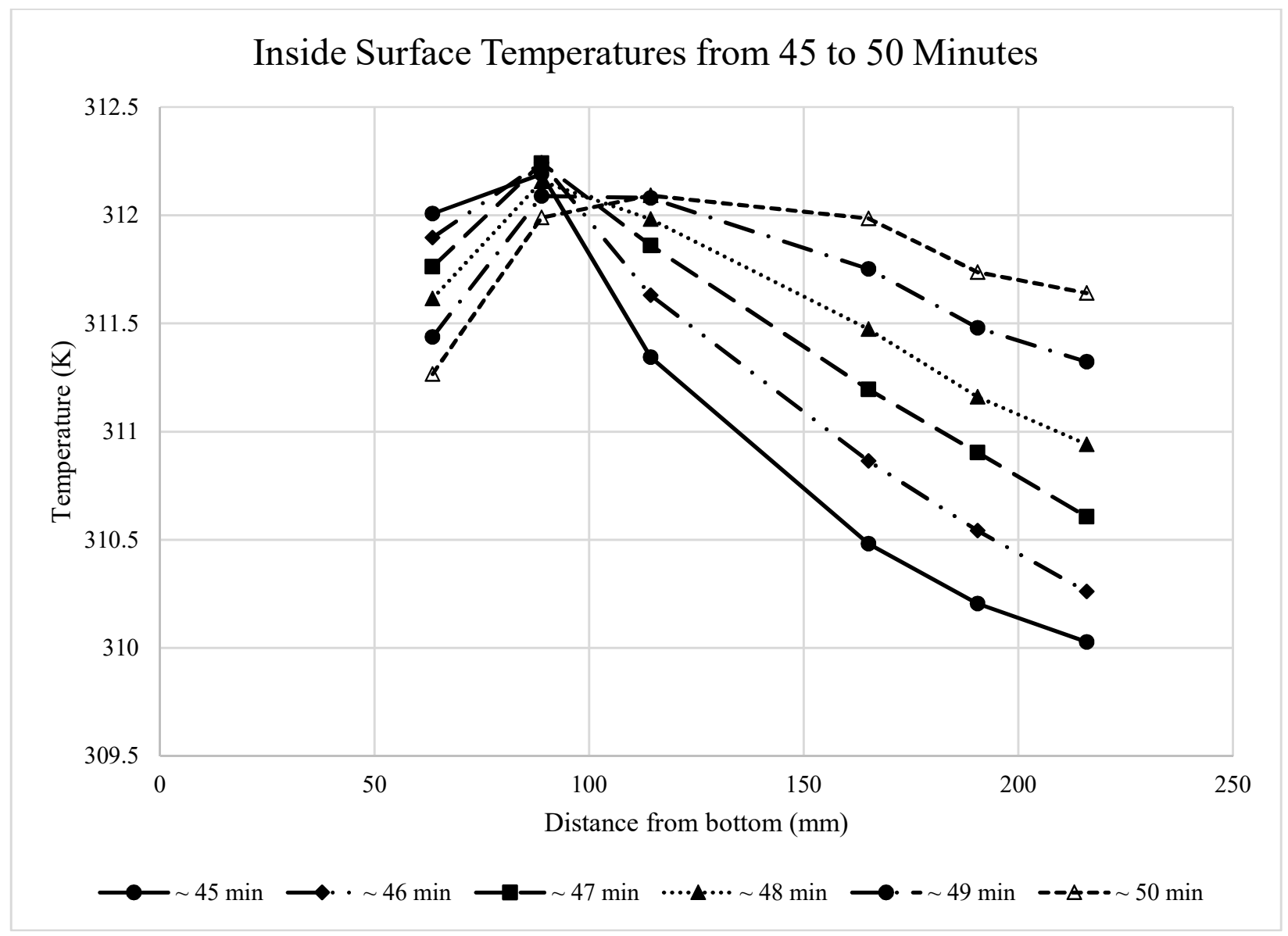

Figure 4.23: Inside surface temperatures from 45 to 50 minutes

The outside surface temperature profiles for the time period 45 to 50 minutes are shown in Figure 4.24 and is a continuation of Figure 4.19. Similar to the center line and inside surface temperature profiles for the same block of time, some locations on the plate drop in temperature and some jump in temperature. However, the temperature drops and jumps are not as pronounced as the center line and inside surface temperatures. The location at TC2 is showing a continuous temperature drop from $308.8 \mathrm{~K}$ to $308.1 \mathrm{~K}$. The locations at TC3 and TC4 initially rise in temperature then 
drops with TC3 having a larger drop than TC4. The remaining locations at TC5, TC6, and TC7 are all experiencing a temperature jump.

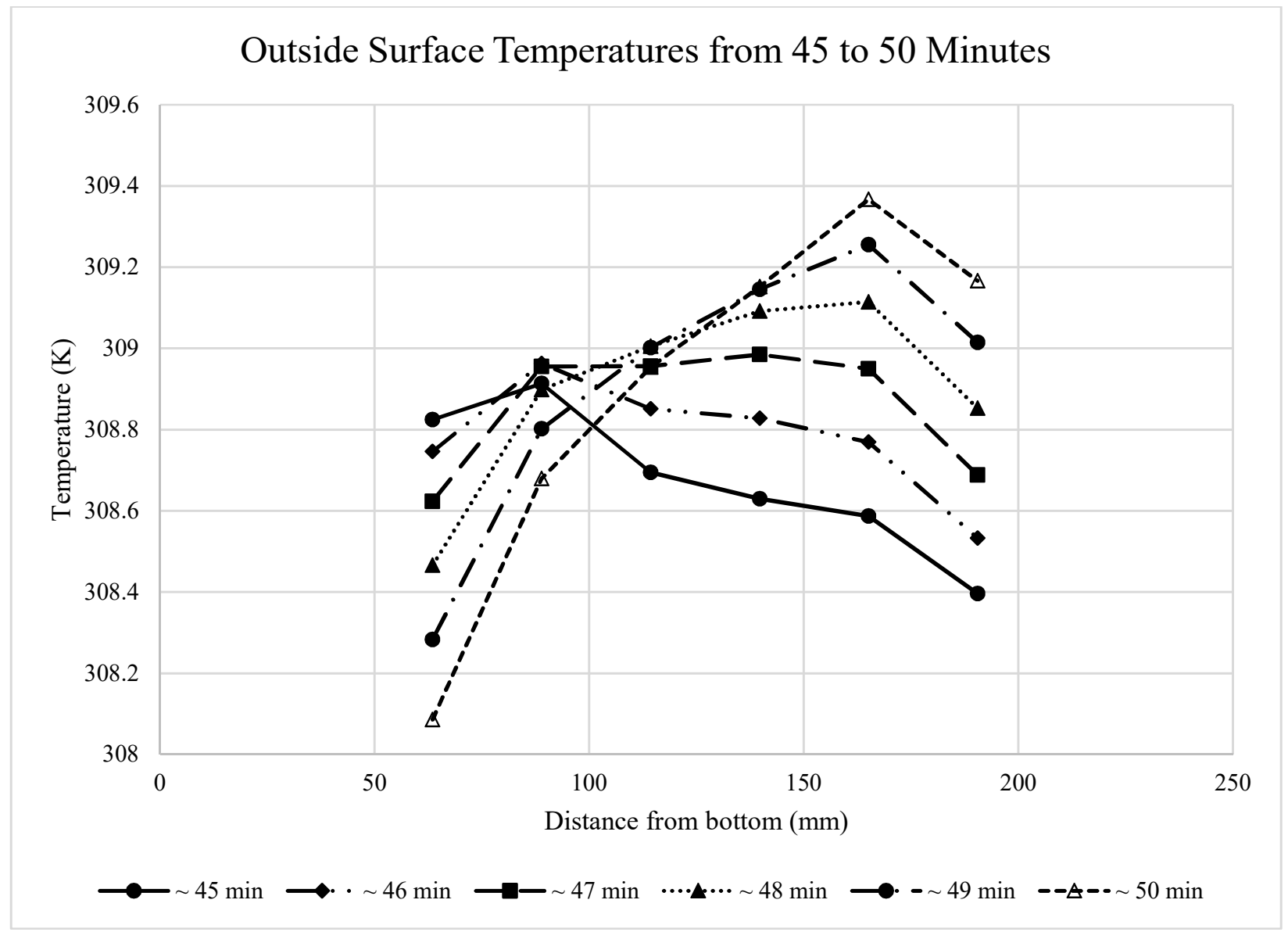

Figure 4.24: Outside surface temperatures from 45 to 50 minutes

\section{Time Period 50 and 55 Minutes}

The center line temperature profiles for the time period 50 to 55 minutes are shown in Figure 4.25.

With the temperature jump propagating up the plate, the lower portion of the PCM panel is taking on the expected free convective temperature drop that was seen before in the hot liquid phase. Starting with the previous time black one can start to see from the temperature profiles the PCM starting to solidify. The temperatures at TC1 and TC2 continue the steady drop in temperature as seen from Figure 4.22 indicating the PCM has solidified at these locations. During this time block 
the locations TC3 and TC4 also take on a steady drop in temperature indicating a solidification phase front is propagating up the plate. The top portion of the plate is still experiencing a temperature jump as seen at locations TC5 through TC8 with the largest temperature jump at TC8 with a jump of $2.5 \mathrm{~K}$. The locations TC5, TC6 and TC7 initially have a temperature rise but eventually drops.

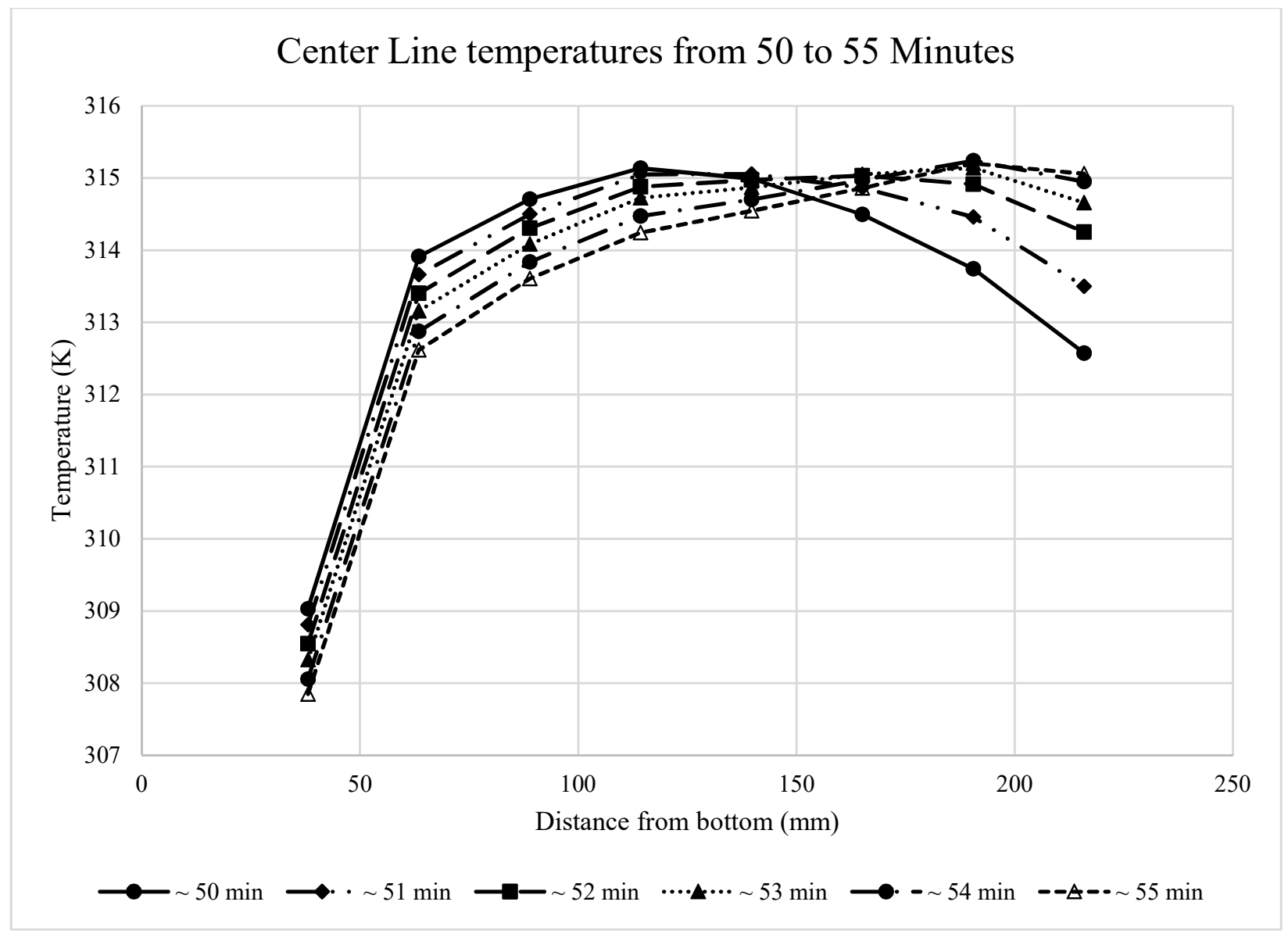

Figure 4.25: Center line temperatures from 50 to 55 minutes

The inside surface temperature profiles are shown in Figure 4.26 for the time period 50 to 55 minutes. As expected the temperatures at TC2 and TC3 are continuing the steady drop as seen from Figure 4.23 and it matches the trend in the center line temperatures from Figure 4.25. In the 
previous time block the temperature at TC4 was experiencing a jump and in the current time block of 50 to 55 minutes the temperature at TC4 initially drops slightly then conforms to the steady temperature drop trend as seen in TC2 and TC3. The remaining locations at TC6, TC7 and TC8 are all experiencing a temperature jump with the largest jump at TC 8 by $1 \mathrm{~K}$.

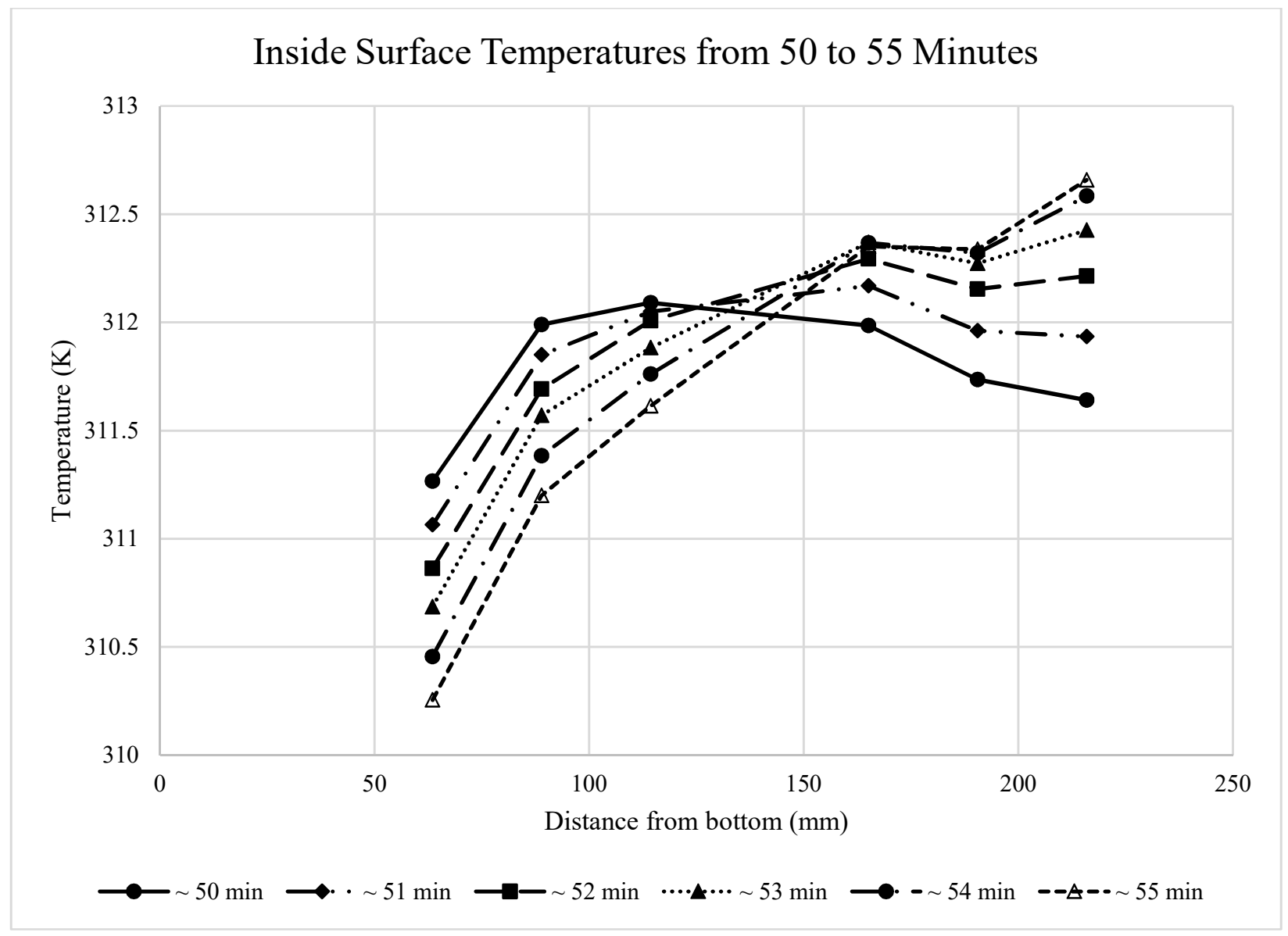

Figure 4.26: Inside surface temperatures from 50 to 55 minutes

The outside surface temperature profiles for the time period 50 to 55 minutes are shown in Figure 4.27. Similar to the inside surface and center line temperatures, the temperatures at location TC2 and TC 3 continue to drop steadily as seen from the previous time block of 45 to 50 minutes. In the previous time block the temperatures at TC4 and TC5 experienced a jump then a drop and in the current time block the temperatures at TC4 and TC5 are dropping steadily. The temperatures at 
the remaining locations TC6 and TC7 are continuing to increase but by a smaller amount than the previous time block.

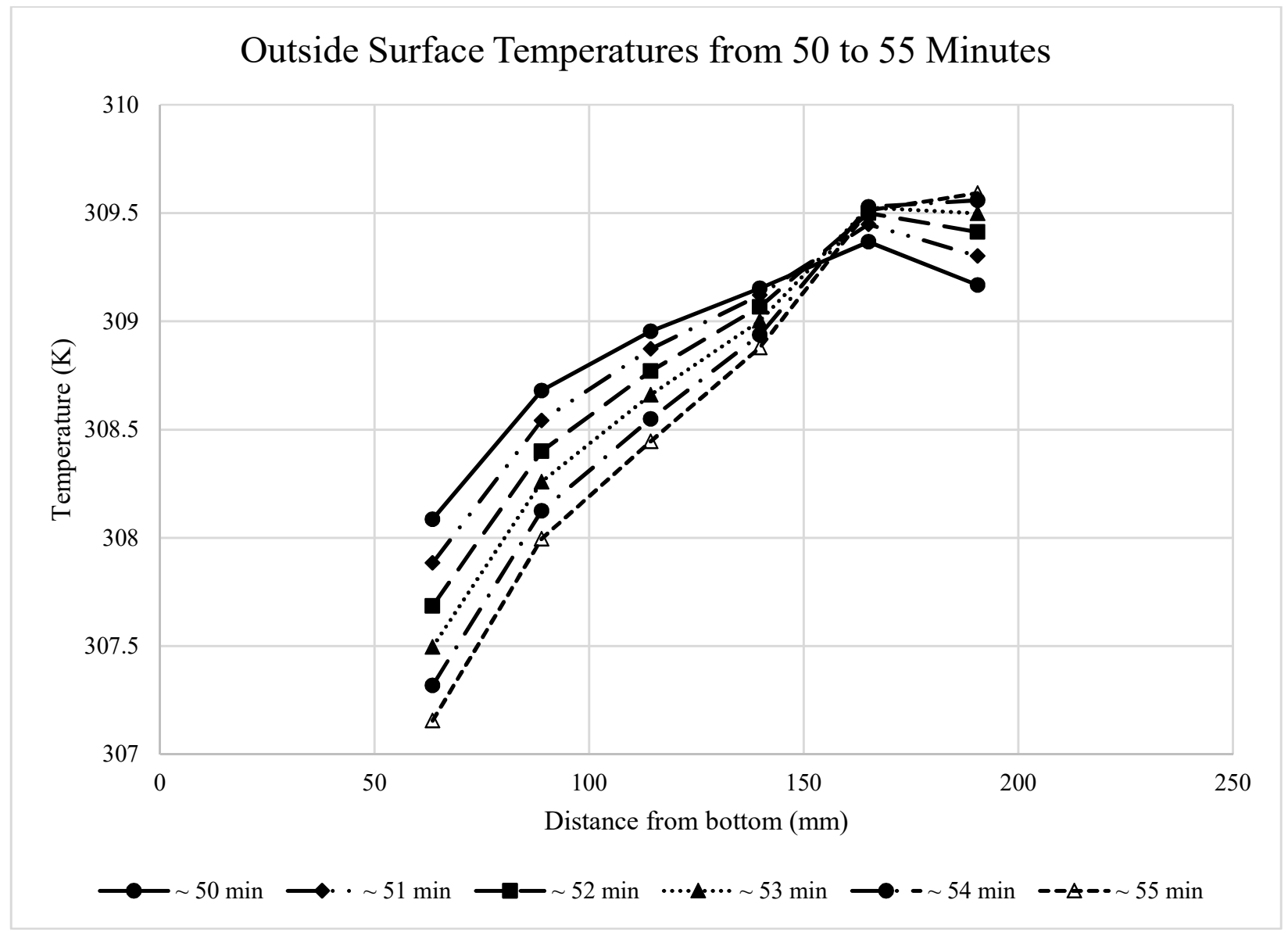

Figure 4.27: Outside surface temperatures from 50 to 55 minutes

\section{Time Period 55 and 65 Minutes}

The center line temperature profiles for the time period 55 to 65 minutes are shown in Figure 4.28. The temperature profiles are shown for every other minute as opposed to every minute as has been the case for the previous results above. The only temperature jump is at location TC 8 from 55 to 57 minutes then conforms to a steady drop from 57 to 65 minutes. All the remaining locations 
from $\mathrm{TC} 1$ to $\mathrm{TC} 7$ for the entire time block are all undergoing steady temperature drop while maintaining a consistent temperature profile 'shape'. From TC2 to TC8 the temperature profile is mostly linear starting at TC2 and linearly gets warmer as one moves upward along the PCM panel to TC8. However, the temperature at TC1 is not consistent with the uniformity of the rest of the temperature profile. The temperature at location TC1 is significantly colder than the rest of the PCM panel. The thermocouple at TC1 is placed at the very bottom of the PCM cavity adjacent to the polystyrene spacer.

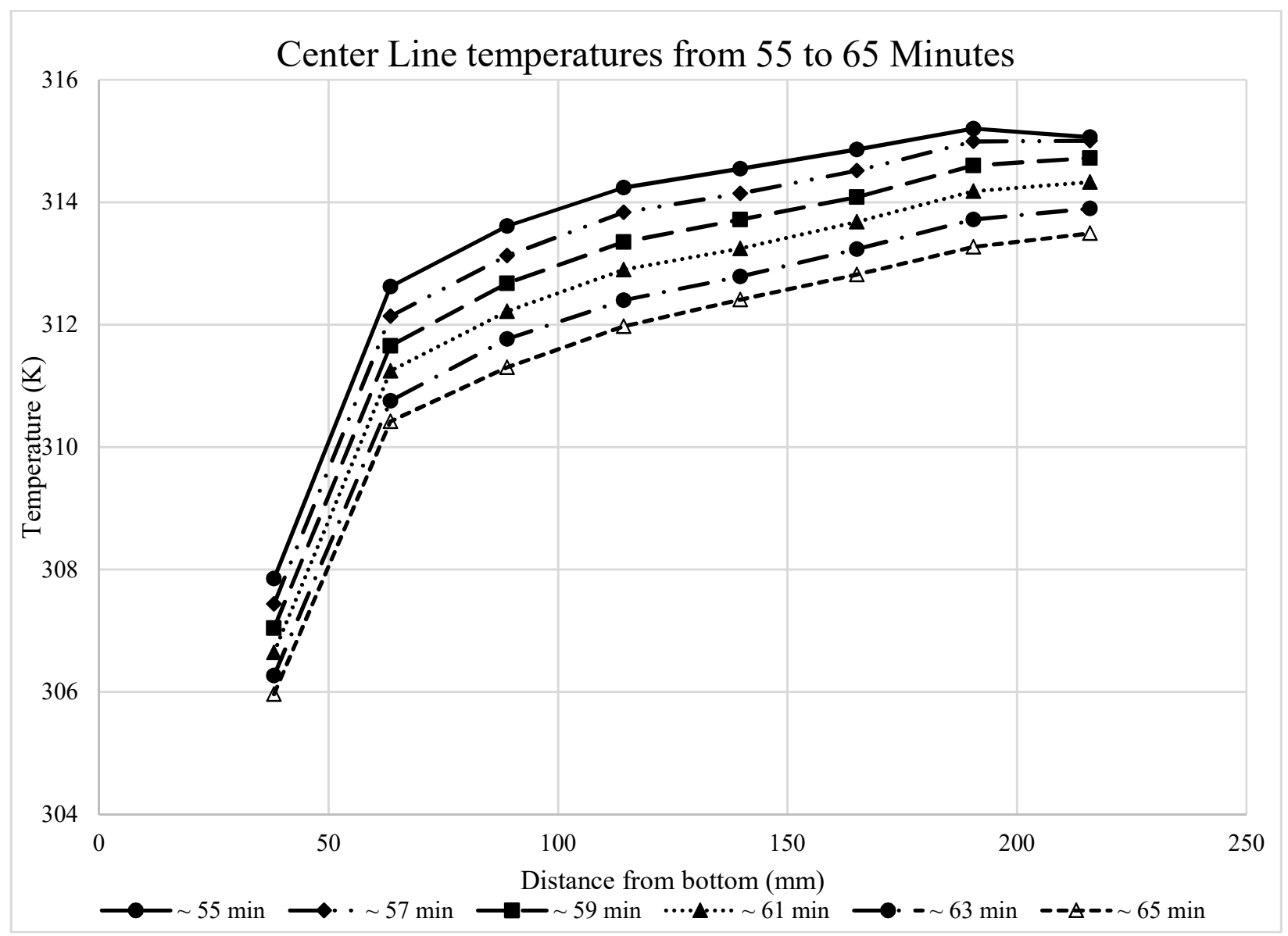

Figure 4.28: Center line temperatures from 55 to 65 minutes

The inside surface temperatures for the time period 55 to 65 minutes are shown in Figure 4.29. Similar to the center line temperatures from Figure 4.28, the only temperature jump is at location 
TC 8 between 55 and 57 minutes. With that one exception, for all locations from TC2 through TC7 for the entire time period of 55 to 65 minutes, the temperatures are undergoing a steady temperature drop. The 'shape' of the temperature profile while not as linear as the center line temperatures, do steadily increase from the bottom of the PCM panel at TC2 to the top at TC8. From a visual inspection it appears that the temperatures at TC2 and TC7 deviate the most from a linear temperature profile.

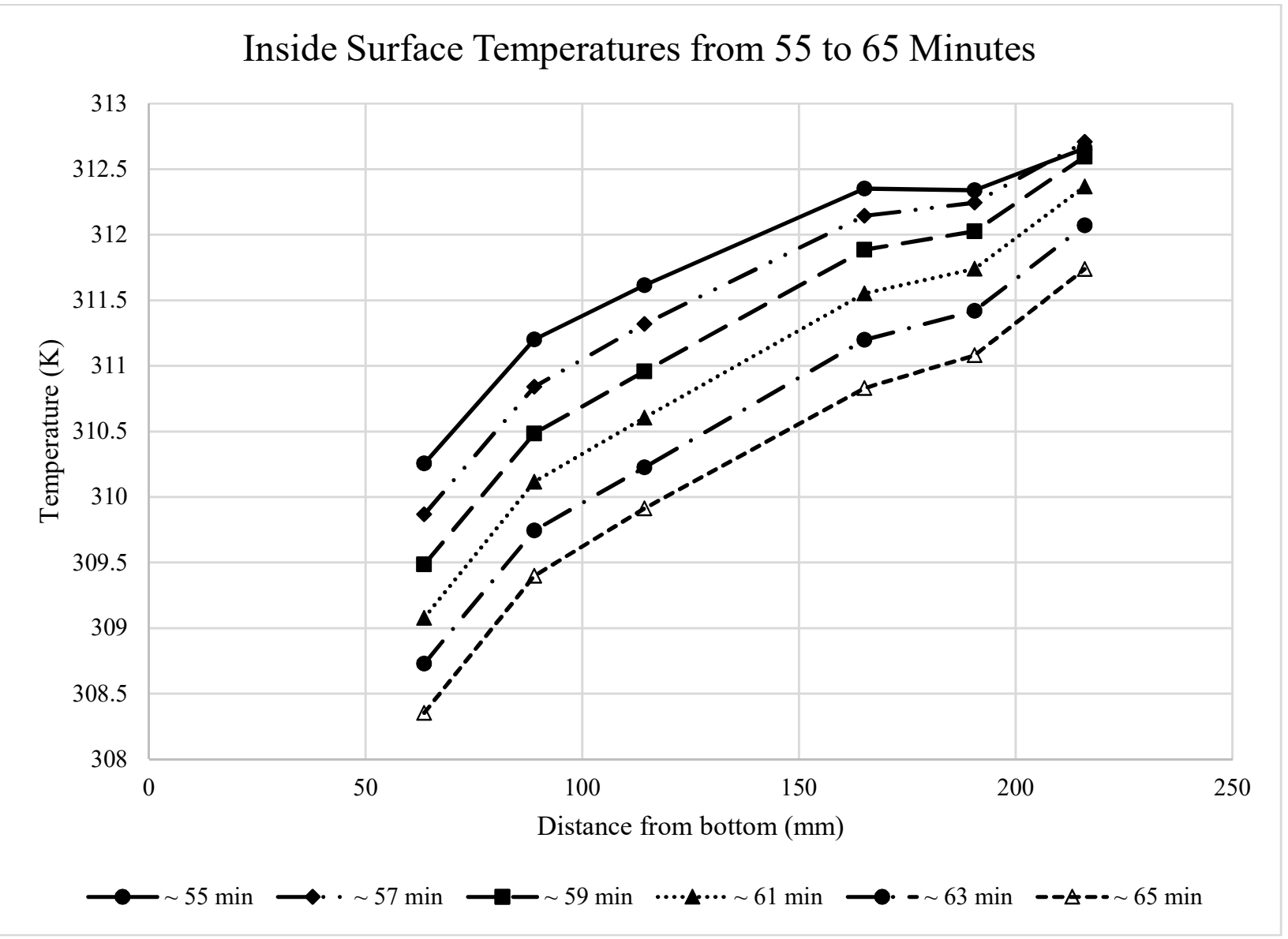

Figure 4.29: Inside surface temperatures from 55 to 65 minutes

The outside surface temperature profiles for the time period 55 to 65 minutes are shown in Figure 4.30. For the outside surface temperatures, the location TC 8 was not measured and therefore cannot directly be compared. However, similar to the inside surface temperatures in Figure 4.29 
and the center line temperatures in Figure 4.28 there is a very slight temperature drop at TC7 from 55 to 57 minutes then drops more from 57 to 65 minutes. For the remaining locations there is a steady temperature drop from 55 to 65 minutes.

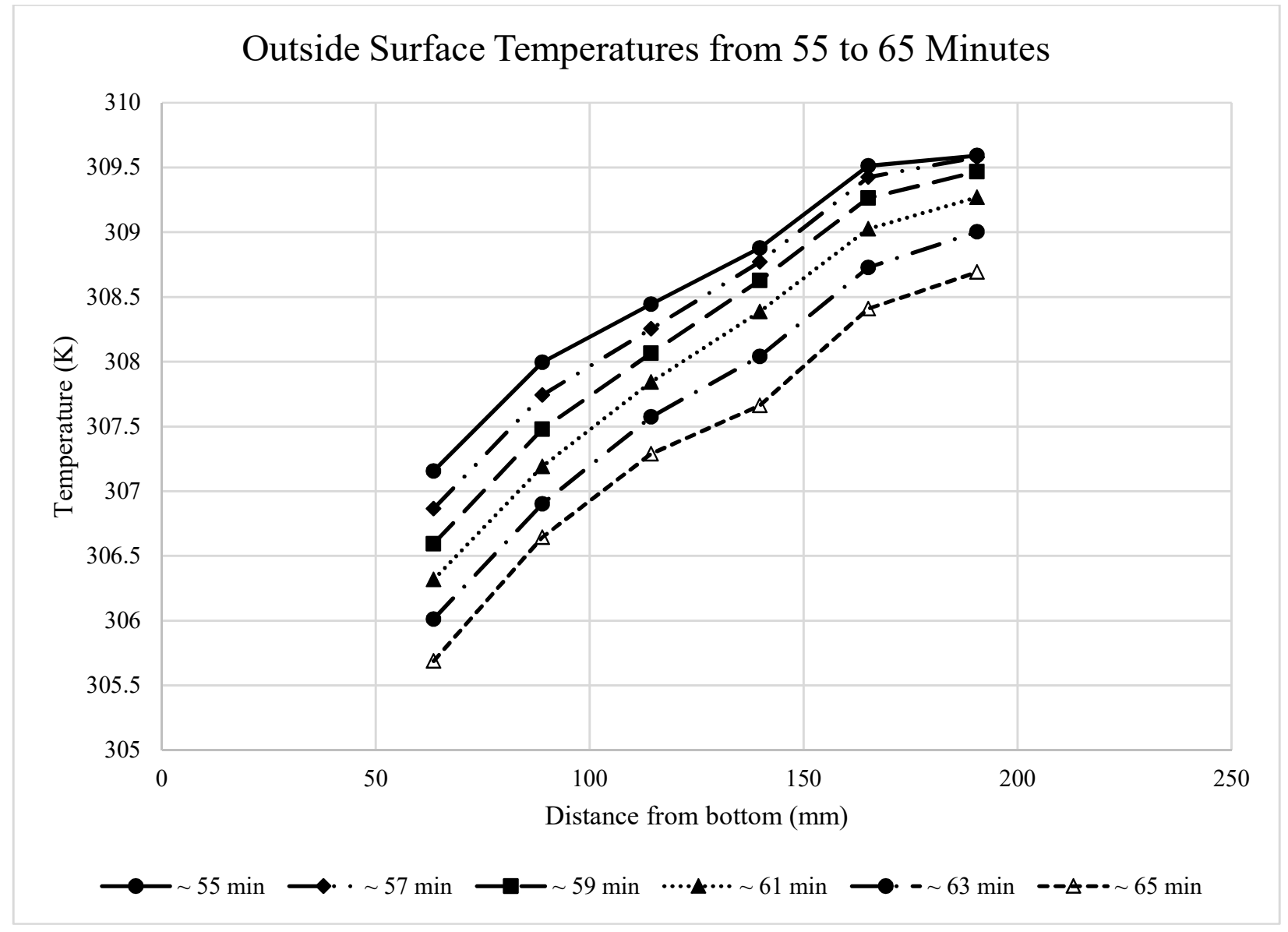

Figure 4.30: Outside surface temperatures from 55 to 65 minutes

\subsection{Low Temperature Solid Phase}

The PCM temperature profiles at time 70, 75, and 80 minutes is shown in Figure 4.31. The PCM temperatures shown correspond to the center line and inside surface temperatures. The temperature profiles are shown in three pairs of two with each pair corresponding to the three time stamps at 70,75 and 80 minutes. For a given pair of temperature profiles the results are separated between 
center line temperatures and inside surface temperatures. For the current time block between 70 and 80 minutes, the PCM has solidified and is undergoing free-convective cooling. This is evident by the linear temperature profile between TC2 and TC 8 indicating that the PCM is behaving like a solid. For a given time period pair, the center line temperatures are higher than the inside surface for each thermocouple location. On average, the center line temperature is $1.8 \mathrm{~K}$ higher than the inside surface temperature at 70 minutes, $1.6 \mathrm{~K}$ higher at 75 minutes, and $1.5 \mathrm{~K}$ higher at 80 minutes. The temperature difference across the PCM between the center line and inside surface is getting smaller as time goes on. This is expected since the PCM is in free cooling and will eventually reach equilibrium with the ambient air with uniform temperature within the PCM cavity. The average center line temperature is $310.4 \mathrm{~K}$ at 70 minutes, $309.0 \mathrm{~K}$ at 75 minutes and $308.1 \mathrm{~K}$ at 80 minutes giving a temperature drop of $1.4 \mathrm{~K}$ and $0.9 \mathrm{~K}$ at 75 and 80 minutes respectively. The drop in the average center line temperature gets smaller as time goes on. For comparison the average inside surface temperature is $309.3 \mathrm{~K}$ at 70 minutes, $308.1 \mathrm{~K}$ at 75 minutes, and $307.3 \mathrm{~K}$ at 80 minutes giving an average temperature drop of $1.2 \mathrm{~K}$ and $0.8 \mathrm{~K}$ at 75 and 80 minutes respectively. 


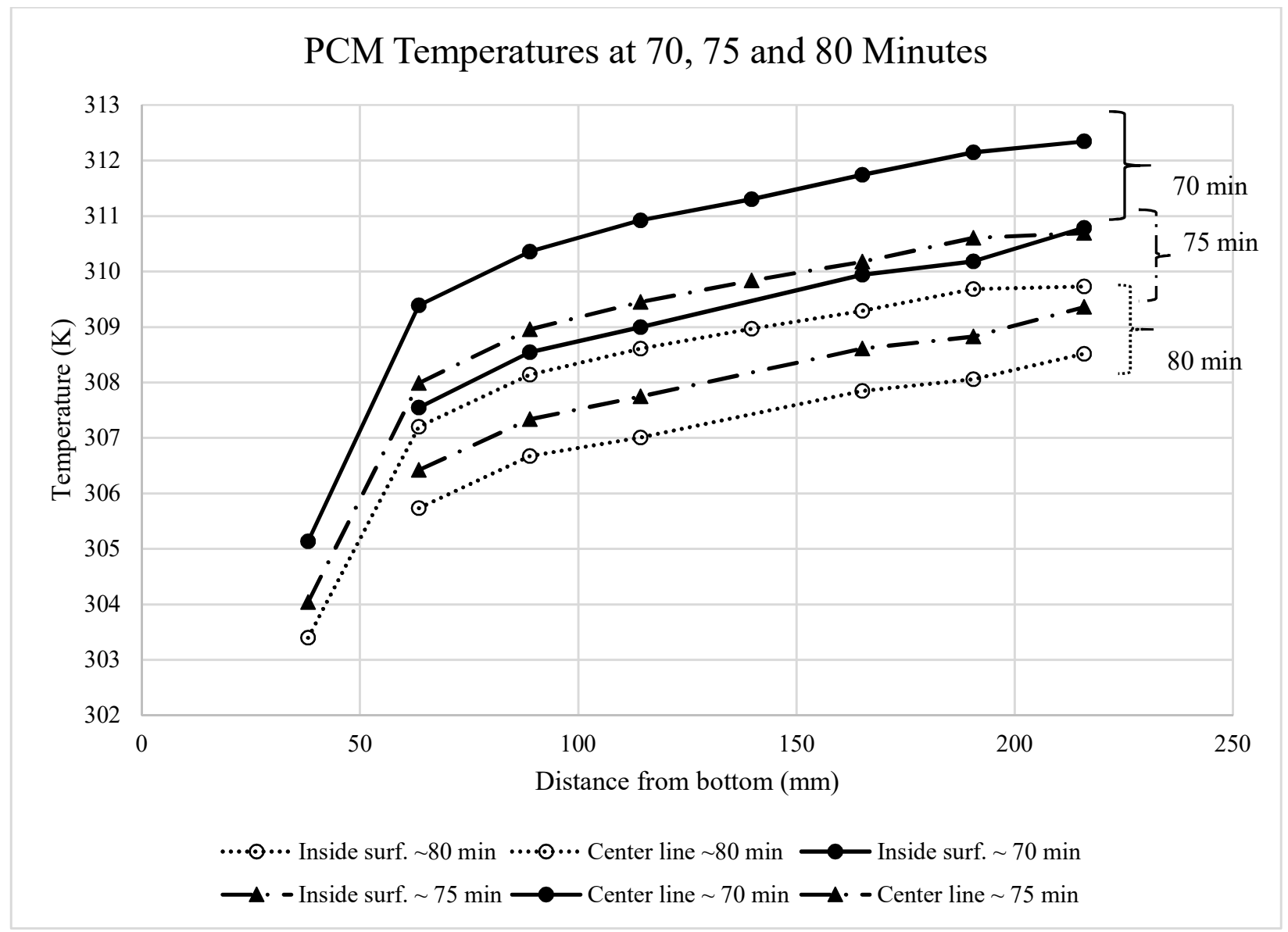

Figure 4.31: PCM temperatures at time 70, 75 and 80 minutes

The center line temperatures, inside surface temperatures, and outside surface temperatures at 70 minutes are shown in Figure 4.32 and at 80 minutes in Figure 4.33. At 70 minutes, the outside surface temperature varies from $305 \mathrm{~K}$ to $307.8 \mathrm{~K}$ giving an increase in temperature of $2.8 \mathrm{~K}$ along the plate. This is in contrast to the $4.3 \mathrm{~K}$ temperature increase seen at 20 minutes from Figure 4.7. At 80 minutes the outside surface temperature increases by $2.3 \mathrm{~K}$ from $303.8 \mathrm{~K}$ to $306.1 \mathrm{~K}$. The temperature increase along the plate is getting smaller as time goes on giving a 'flatter' temperature profile. A major difference between the high temperature liquid phase and the low temperature solid phase is the behaviour of the temperature at the TC1 location. The center line temperature at 20 minutes, as seen in Figure 4.7, has TC1 conforming to an approximately linear temperature 
profile from TC2 through TC8. However, in Figure 4.32 and Figure 4.33 the temperature at TC1 drops off from the linear temperature profile indicated by the other TC locations. Another point of comparison is the temperature gradient between the center line temperatures, inside surface temperatures, and the outside surface temperatures. For this comparison the average temperature will be taken to be between the temperatures at TC2 through TC7. The center line and inside surface are $6.35 \mathrm{~mm}$ apart while the inside surface and outside surface are spaced $3.175 \mathrm{~mm}$ apart. In Figure 4.7 at 20 minutes, the average center line temperature is $1.9 \mathrm{~K}$ higher than the inside surface temperature and in Figure 4.8 at 25 minutes the average center line temperature is $1.8 \mathrm{~K}$ higher. For comparison, in the low temperature solid phase shown in Figure 4.32 at 70 minutes the center line temperature is $1.9 \mathrm{~K}$ higher than the inside surface temperature and at 80 minutes as shown in Figure 4.33 the center line temperature is $1.7 \mathrm{~K}$ higher. The temperature difference between the inside surface and outside surface is larger than the center line to inside surface for the same time period despite the distance between the inside surface and outside surface is smaller than the center line to inside surface. At 20 minutes the average inside surface temperature is 3.8 $\mathrm{K}$ higher than the outside surface temperature and at 25 minutes it is $3.1 \mathrm{~K}$ higher. At 70 minutes the temperature difference is $1.9 \mathrm{~K}$ and at 80 minutes it is also $1.9 \mathrm{~K}$. 


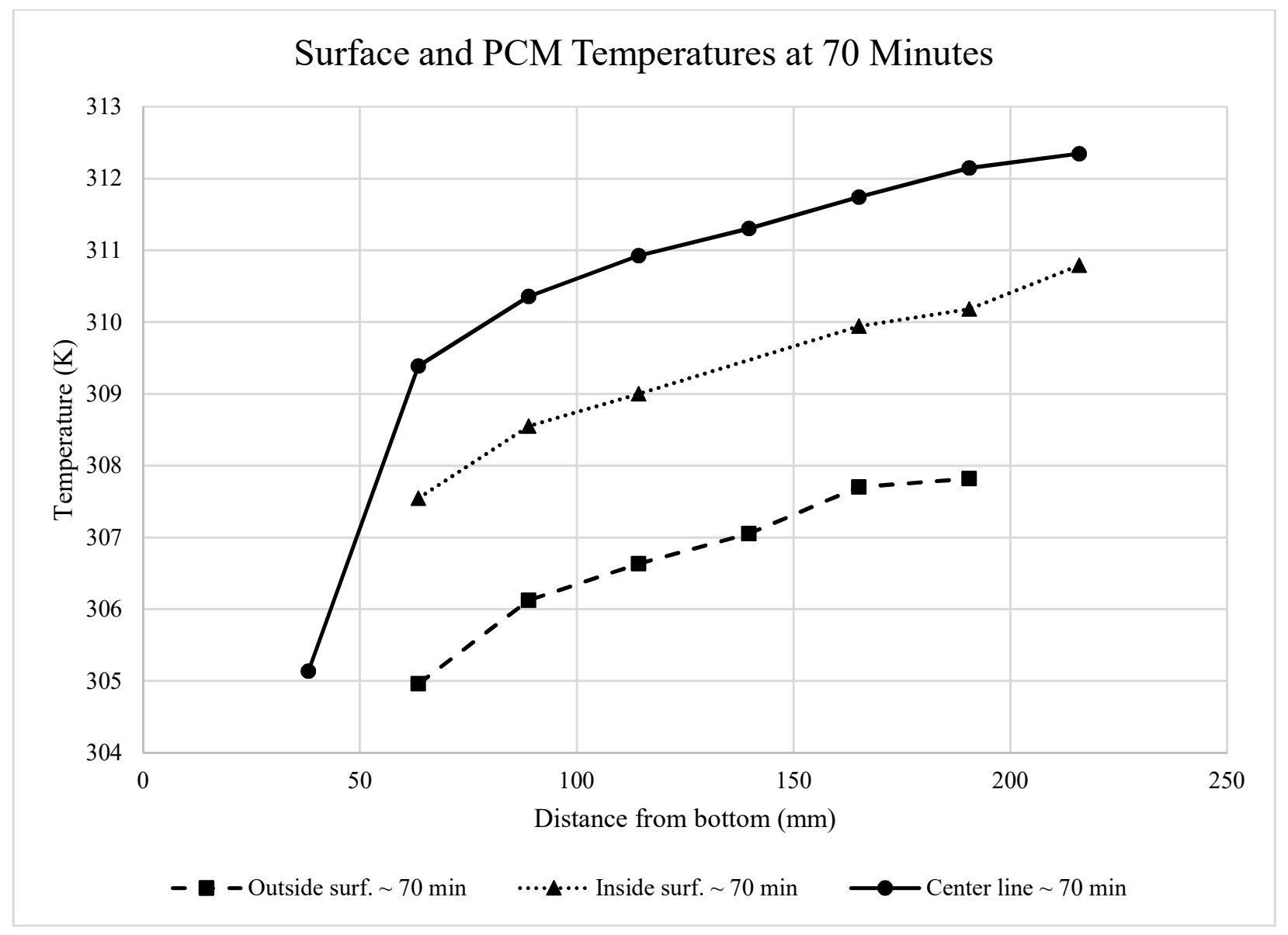

Figure 4.32: Surface and PCM temperatures at 70 minutes 


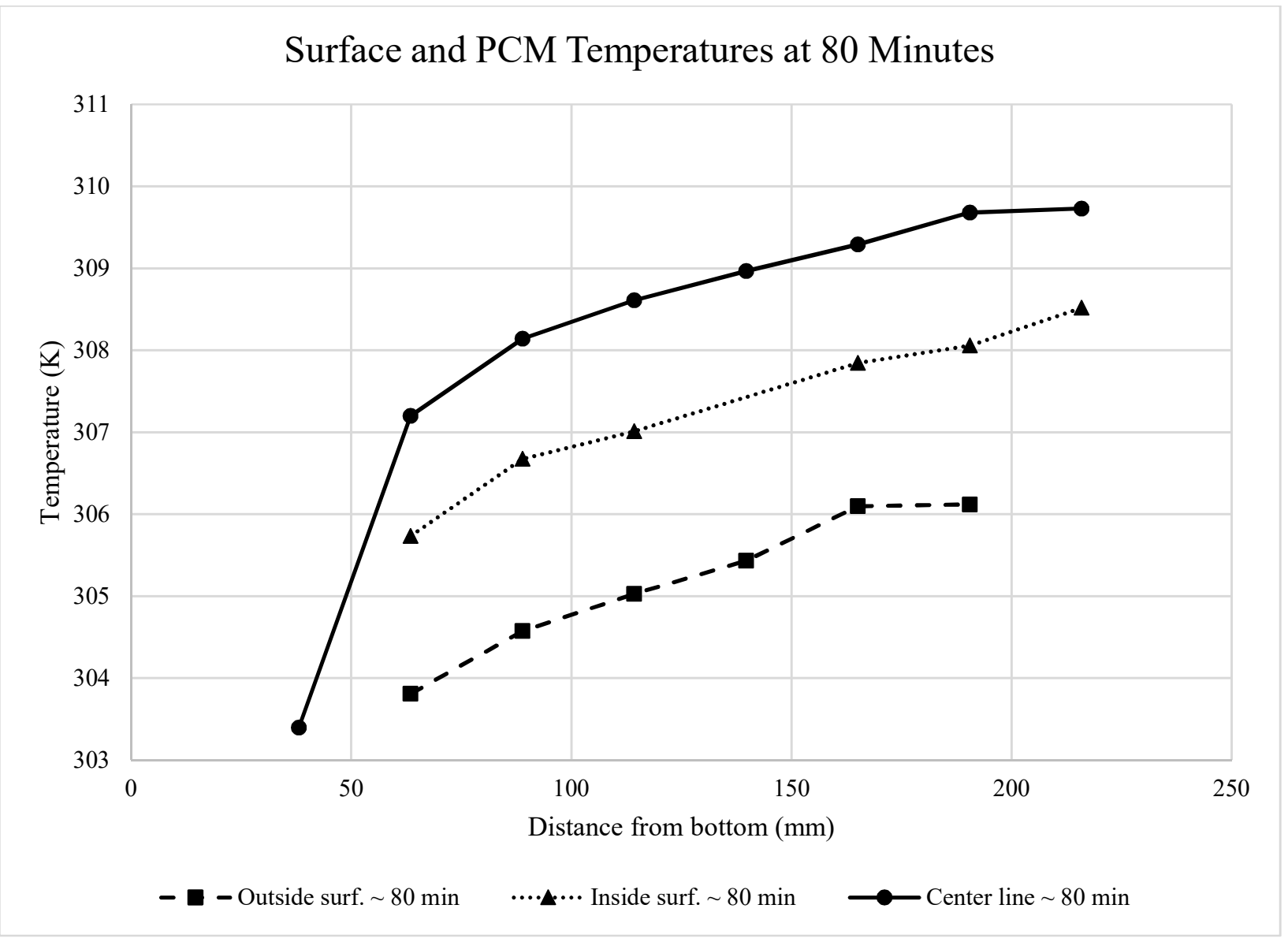

Figure 4.33: Surface and PCM temperatures at 80 minutes

In general, the trend is for this temperature difference to decrease over time as seen from 20 to 25 minutes and 70 to 80 minutes. However, due to the phase change process the temperature difference increased. This can be seen in the thermocouple data in Figure 4.34 which displays the average center line temperature and the average inside surface temperature versus time. The phase change portion can be seen as the 'bump' occurring between approximately 35 and 60 minutes. Before and after this phase change 'bump' the temperature gradient across the PCM (center line and inside surface temperatures) gets smaller over time and during the phase change the temperature gradient increases. This results in having the same temperature difference at 20 minutes and at 70 minutes. 


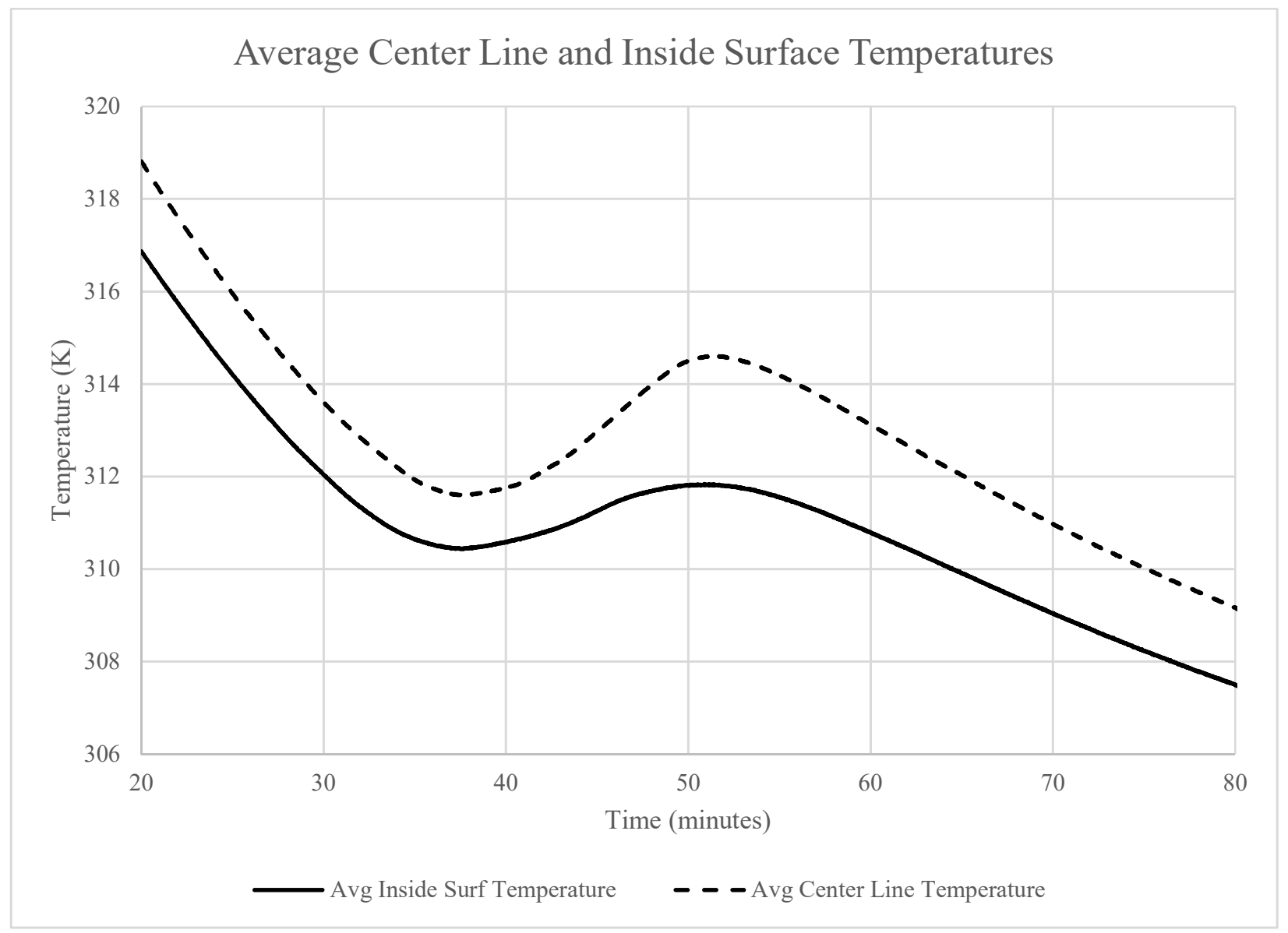

Figure 4.34: enter line and inside surface temperatures

\subsection{Convective Heat Transfer}

As explained in section 3.3, the temperature gradient, and by extensions the heat transfer rate, can be found from the surface temperature and near wall fringe gradient using Equation 3.2. The convective heat transfer rate from 20 minutes to 80 minutes for the locations at TC2 through TC7 is shown in Figure 4.35. The image analysis was done in one-minute intervals from 30 minutes to 70 minutes (during phase change) but in 5-minute interval at 20-30 minutes and 70-80 minutes (during sensible heat transfer). The analysis of heat transfer rate was done using the curve fitted surface temperatures. However, it can be seen in Figure 4.35 that despite using the 'smoothed' 
surface temperatures there is still noise in the heat transfer results. This is due to the impact of the fringe gradient on Equation 3.2 where a small variation in fringe spacing results in a larger fringe gradient. For comparison, the near wall fringe spacing that was used to calculate the convective heat transfer rate is shown in Figure 4.36 at each thermocouple location. In both Figure 4.35 and Figure 4.36 the effect of phase change can be seen between 30 minutes and 60 minutes. In Figure 4.35 the variation in the convective heat transfer rate along the PCM wall is small during the hightemperature liquid phase and low-temperature solid phase. However, the variation in convective heat transfer rate increases during the phase change range matching the variation in surface temperature as shown in Figure 4.5. The same behaviour exists in the fringe spacing in Figure 4.36 where the variation of fringe spacing along the PCM plate is small before and after the phase change range but the variation increases during the phase change. Another trend that matches the behaviour of the outside surface temperatures is the staggered delay of the temperature jump that was seen previously. The convective heat transfer rate at the TC2 location initially drops from 20 to 30 minutes then increases. As the experiment continues the convective heat transfer rate at TC 3 increases at approximately 38 minutes followed by TC4 at 40 minutes, TC5 at 42 minutes, TC6 at 43 minutes, and TC7 at 45 minutes. In terms of the near wall fringe spacing in Figure 4.36 the temperature jump is expressed as a drop in fringe spacing. The fringe spacing initially increases with time then drops during the phase change while the surface temperature initially decreases with time then jumps during the phase change. The drop in fringe spacing for each thermocouple location is staggered with the drop occurring at the same time as the convective heat transfer rate from Figure 4.35. The convective heat transfer rates have a relative uncertainty of $\pm 2.67 \%$. 


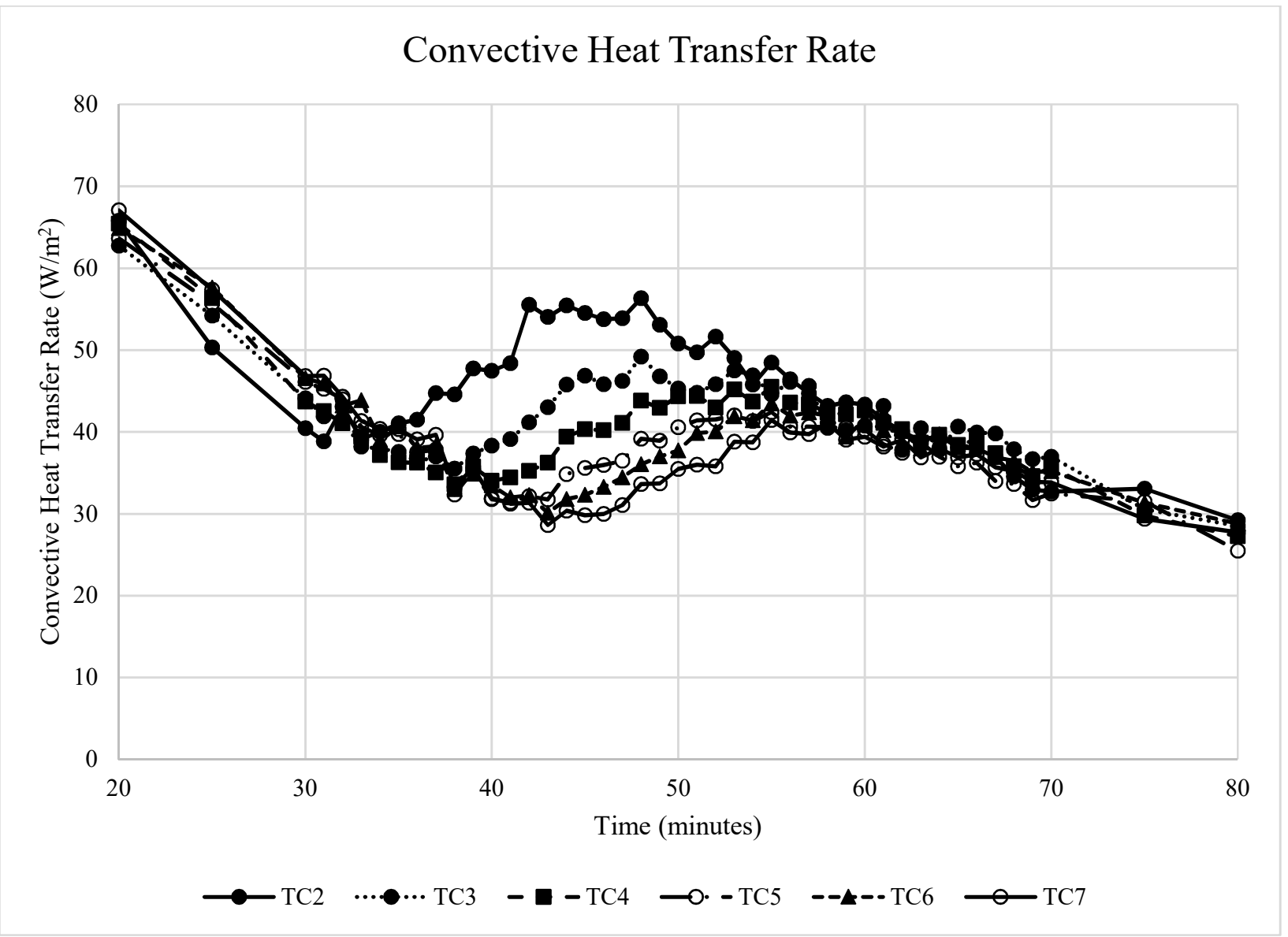

Figure 4.35: Convective heat transfer rate 


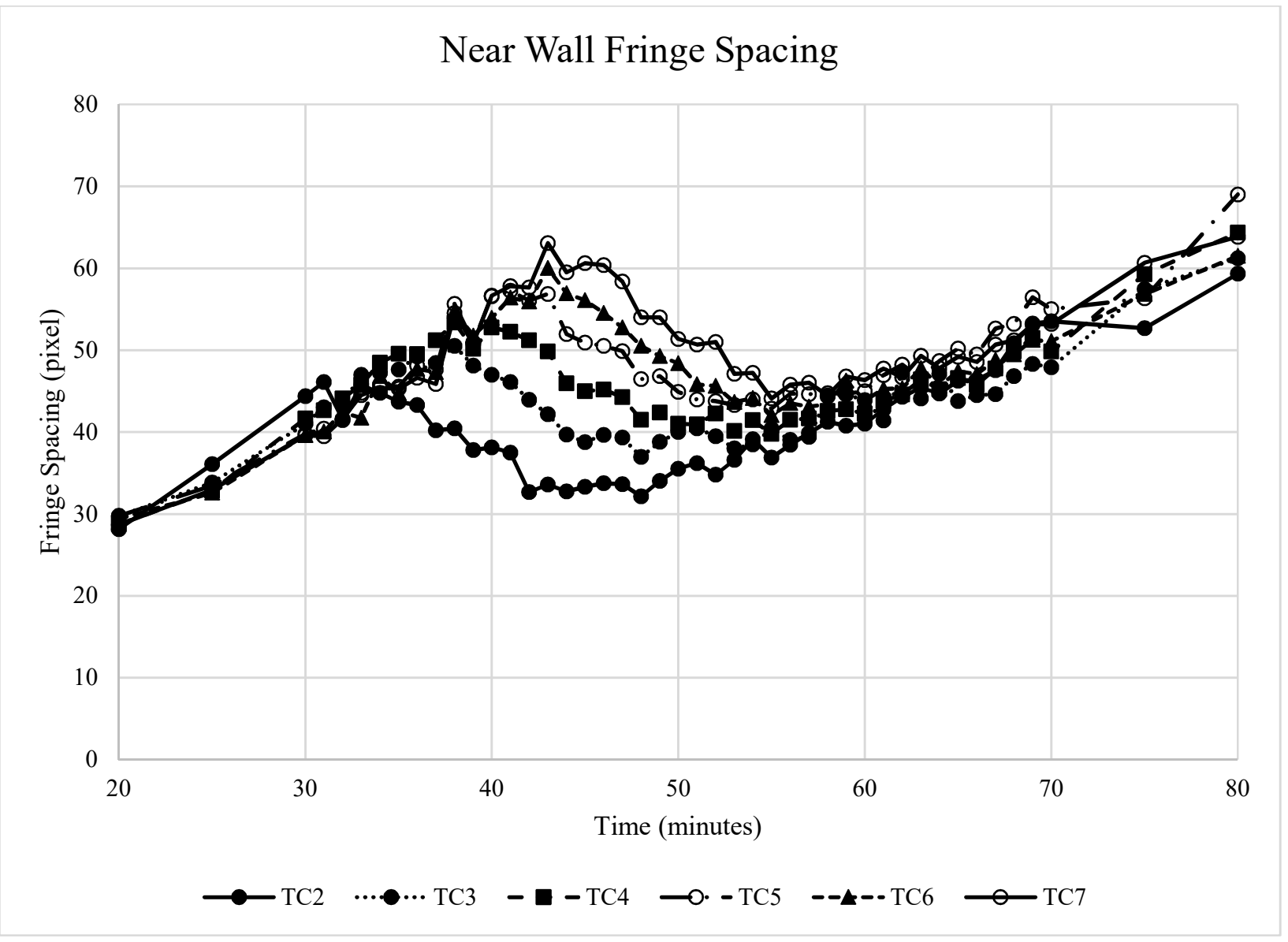

Figure 4.36: Near wall fringe spacing

The total convective heat transfer rate from the PCM plate was determined by numerical integration of the locally measured convective heat transfer rate at each thermocouple location using the trapezoid rule. The total convective heat transfer is shown in Figure 4.37 for the entire duration of the experiment. The total convective heat transfer rate corresponds to a portion of the PCM plate that spans a height of $127.1 \mathrm{~mm}$ (5 in.) starting $63.5 \mathrm{~mm}(2.5 \mathrm{in}$.$) above the bottom$ edge of the plate. At 20 minutes into the experiment the total convective heat transfer rate is 64.6 $\mathrm{W} / \mathrm{m}^{2}$ and linearly drops down to $34.7 \mathrm{~W} / \mathrm{m}^{2}$ at 38 minutes. At this point the effect of phase change can be seen as the total convective heat transfer rate curves upwards and increases to a peak of $44.2 \mathrm{~W} / \mathrm{m}^{2}$ at 55 minutes. After the peak the total convective heat transfer rate curves downward 
and at approximately 60 minutes' drops linearly down to $27.7 \mathrm{~W} / \mathrm{m}^{2}$ at 80 minutes. For a given time period, the total convective heat transfer rate decreases faster in the high-temperature liquid phase than the low-temperature solid phase. In the liquid phase the total convective heat transfer rate drops $1.98 \mathrm{~W} / \mathrm{m}^{2}$ per minute while in the solid phase the total convective heat transfer rate drops $0.70 \mathrm{~W} / \mathrm{m}^{2}$ per minute.

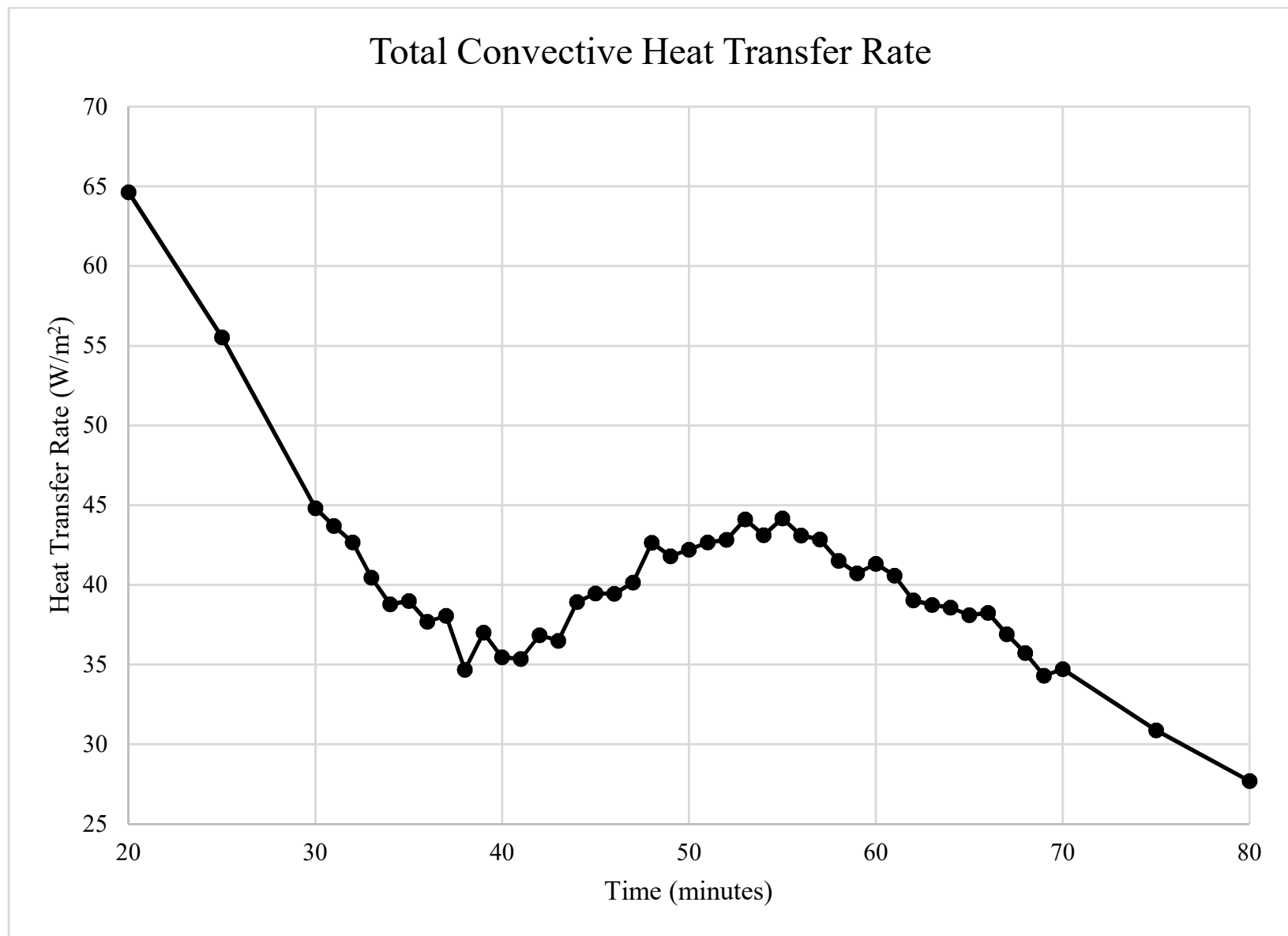

Figure 4.37: Total convective heat transfer rate

With the total convective heat transfer and surface temperature known, the convective heat transfer coefficient can be determined using Equation 3.2. Figure 4.38 contains the measured averaged 
convective heat transfer coefficient from 20 minutes to 80 minutes. Figure 4.38 also contains the predicted heat transfer coefficient from the correlation of Churchill and Chu (1975) for a vertical heated plate which is valid for any Rayleigh number. Similar to the total convective heat transfer results in Figure 4.37, the phase change range can be seen from the variation (drop or jump) of the convective heat transfer coefficient. During the high-temperature liquid phase at 20 and 25 minutes, the measured convective heat transfer coefficient is in good agreement with the correlation with the measured result being within $1 \%$ of the correlation. There is also good agreement with the correlation from 53 minutes to 80 minutes which includes the ending portion of the phase change range and the low-temperature solid range with the measured convective heat transfer coefficient being within $3 \%$ of the predicted coefficient. The measured convective heat transfer coefficient deviates from the predicted correlated value during most of the phase change range from 25 minutes to 53 minutes. The deviation starts small at 25 minutes with the measured value being within $4 \%$ of predicted and continues to increase with the largest deviation being $14 \%$ occurring at 39 minutes and 41 minutes. Comparing to Figure 4.37 and Figure 4.5 , the time interval of 39 to 41 minutes corresponds to when the average surface temperature plateaus and starts to increase. The deviation decreases after 41 minutes until the measured convective heat transfer coefficient is within agreement of the correlation at 53 minutes. Comparing to Figure 4.37 and Figure 4.5, 53 minutes corresponds to when the increase of the average surface temperature plateaus and starts to decrease. The convective heat transfer coefficients have an uncertainty of $\pm 0.35 \mathrm{~W} / \mathrm{m}^{2} \cdot \mathrm{K}$. 


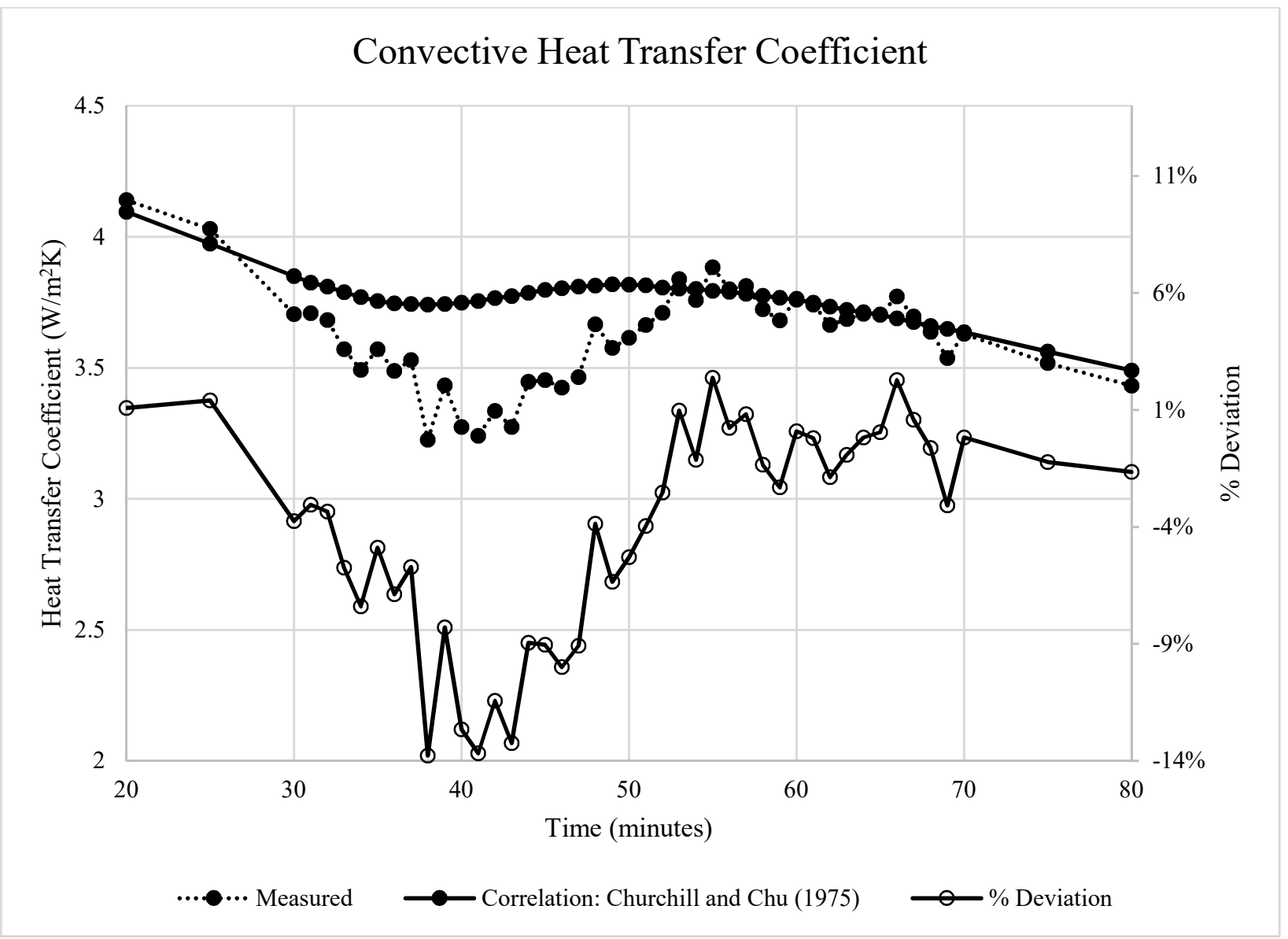

Figure 4.38: Average convective heat transfer coefficient 


\section{Chapter 5: Numerical Design and Results}

\subsection{Governing Equations}

The governing equation for fluid flows consists of a set of partial differential equations that describe the conservations of mass and momentum. Collectively these equations are referred to as the Navier-Stokes Equations. In the present study conservation of energy is also involved. For the current simulation, the explanation of the governing equations will be limited to unsteady two dimensional flow, assuming that the width of the PCM panel is wide enough to render negligible end effects.

The conservation of mass, also known as the continuity equation is shown in Equation 5.1 for two dimensional unsteady flow. For incompressible flow the density is constant and therefore the steady and unsteady versions of the continuity equation is the same.

$$
\frac{\partial u}{\partial x}+\frac{\partial v}{\partial y}=0
$$

The left hand side of Equation 5.1 relates to the mass flux passing through the surface of the control volume in the ' $\mathrm{x}$ ' and ' $\mathrm{y}$ ' direction, respectively. The sum of these two terms are zero and thus the mass is conserved. This equation gives a scalar field in the fluid domain.

The conservation of momentum can be described as the application of a transport equation on momentum. The conservation of momentum is shown in Equation 5.2 and Equation 5.3 for the ' $\mathrm{x}$ ' and ' $y$ ' directions respectively for incompressible unsteady flow. Equation 5.2 and Equation 5.3 
effectively represents a sum of forces with each term representing some component of force acting on an infinitesimal small fluid volume.

$$
\begin{gathered}
\rho\left(\frac{\partial u}{\partial t}+u \frac{\partial u}{\partial x}+v \frac{\partial u}{\partial y}\right)=-\frac{\partial p}{\partial x}+\mu\left(\frac{\partial^{2} u}{\partial x^{2}}+\frac{\partial^{2} u}{\partial y^{2}}\right) \\
\rho\left(\frac{\partial v}{\partial t}+u \frac{\partial v}{\partial x}+v \frac{\partial v}{\partial y}\right)=-\frac{\partial p}{\partial y}+\mu\left(\frac{\partial^{2} v}{\partial x^{2}}+\frac{\partial^{2} v}{\partial y^{2}}\right)+\rho_{\infty} g \beta\left(T-T_{\infty}\right)
\end{gathered}
$$

The first term in the left hand side of Equations 5.2 and Equation 5.3 represents the time rate of change of momentum in some fluid volume. The second and third terms from the left hand side represents the convective transport of momentum through the surface of a fluid volume. In other words, the kinetic motion of some quantity (in this case momentum) entering and exiting a fluid element volume. The first term on the right hand side represents the force due to pressure acting in the ' $\mathrm{x}$ ' or ' $\mathrm{y}$ ' direction. The last term in the y-momentum equation in Equation 5.3 is the buoyancy term based on the Boussinesq approximation. The buoyancy force is treated as a body force acting on the fluid. In the Boussinesq approximation that buoyancy body force is assumed to be proportional to the temperature difference between the buoyant fluid temperature and some reference temperature (usually ambient temperature). The $2^{\text {nd }}$ order velocity gradients on the right hand side of the equations represents the diffusive transport of momentum through the surface of a fluid volume. In this case diffusive transport is the motion of some quantity due to the viscous shear stresses acting on the fluid volume surface. In alternate forms of the conservation of 
momentum equations, the $2^{\text {nd }}$ order velocity terms can be expressed in terms of shear stresses as shown in Equation 5.4, Equation 5.5 and Equation 5.6.

$$
\begin{gathered}
\tau_{x x}=-2 \mu \frac{\partial u}{\partial x}+\frac{2}{3} \mu\left(\frac{\partial u}{\partial x}+\frac{\partial v}{\partial y}\right) \\
\tau_{y y}=-2 \mu \frac{\partial v}{\partial y}+\frac{2}{3} \mu\left(\frac{\partial u}{\partial x}+\frac{\partial v}{\partial y}\right) \\
\tau_{x y}=-\mu\left(\frac{\partial u}{\partial y}+\frac{\partial v}{\partial x}\right)=\tau_{y x}
\end{gathered}
$$

A transport equation can be applied to the fluid temperature or total specific energy to describe the conservation of energy and is an application of the $1^{\text {st }}$ Law of Thermodynamics. The form of the conservation of energy equation applied to temperature is shown in Equation 5.7

$$
\rho c_{p}\left(\frac{\partial T}{\partial t}+u \frac{\partial T}{\partial x}+v \frac{\partial T}{\partial y}\right)-u \frac{\partial p}{\partial x}-v \frac{\partial p}{\partial y}=k\left(\frac{\partial^{2} T}{\partial x^{2}}+\frac{\partial^{2} T}{\partial y^{2}}\right)
$$

The above equations are considered exact, and the complete natural convection flow can be solved. Turbulence can be solved as well but it would require significant computational cost due to small grid sizes. Solving the Navier-Stokes equations directly is called DNS (Direct Numerical Simulation) and it requires small grid sizes according to Kolomogrov theory (1941). This is difficult and not practical for engineering applications. An alternative approach to model turbulence is to apply Reynolds averaging which results in the Reynolds Averaged Navier-Stokes 
(RANS) equations. Simulating turbulence with RANS modeling can be considered a category of turbulence modeling with many applications. In this approach obtaining the chaotic and time varying nature of turbulence is ignored and only the average flow properties are of concern. In Reynolds averaging flow properties are decomposed into an average and fluctuating component. For example, the $\mathrm{x}$ component of velocity can be decomposed to an average and fluctuating component as shown in Equation 5.8 and then averaged as shown in Equation 5.9.

$$
\begin{gathered}
u(x, t)=\bar{U}(x)+u^{\prime}(x, t) \\
\bar{u}=\overline{\bar{U}(x)+u^{\prime}(x, t)}=U+\overline{u^{\prime}}=U
\end{gathered}
$$

Here, the flow velocity, which is a function of space and time, is decomposed into a mean component and the turbulent fluctuating component. In Equation 5.9 the fluctuating component is averaged as well and is no longer varying in time. A property of Reynolds averaging is that the average of a single fluctuating component is zero (for example: $\bar{u}^{\prime}=0$ ) while the average of a multiple of fluctuating components is not (for example: $\overline{u^{\prime} u^{\prime}} \neq \overline{v^{\prime} v^{\prime}} \neq \overline{u^{\prime} v^{\prime}} \neq 0$ ). It is important to recognize that in Reynolds averaging there is a separation of time scales. In other words, the averaged quantities, such as mean flow velocity and averaged fluctuations, can vary 'slowly' in time for a transient analysis while the 'fast' fluctuations due to turbulence can be averaged. In this case it is assumed that the transient analysis has a time scale that is larger than the turbulent time scale. This distinction in time scales allows a RANS simulation to be performed on transient phenomena. This decomposition can be applied to the flow velocity, temperature, and pressure and then substituted into the Navier-Stokes and energy equations. The continuity equation from 
Equation 5.1 now transforms to Equation 5.10 after the substituting the Reynolds decomposition. Similarly the conservation of momentum equations in Equation 5.2 and Equation 5.3 now transforms to Equation 5.11 and Equation 5.12 respectively, and the conservation of energy equation from Equation 5.7 now transforms into Equation 5.13

$$
\begin{aligned}
& \frac{\partial U}{\partial x}+\frac{\partial V}{\partial y}=0 \\
& \rho\left(\frac{\partial U}{\partial t}+U \frac{\partial U}{\partial x}+V \frac{\partial U}{\partial y}\right)=-\frac{\partial P}{\partial x}+\mu\left(\frac{\partial^{2} U}{\partial x^{2}}+\frac{\partial^{2} U}{\partial y^{2}}\right)-\rho\left(\frac{\partial \overline{u^{\prime} u^{\prime}}}{\partial x}+\frac{\partial \overline{u^{\prime} v^{\prime}}}{\partial y}\right) \\
& \rho\left(\frac{\partial V}{\partial t}+U \frac{\partial V}{\partial x}+V \frac{\partial V}{\partial y}\right) \\
& =-\frac{\partial P}{\partial y}+\mu\left(\frac{\partial^{2} V}{\partial x^{2}}+\frac{\partial^{2} V}{\partial y^{2}}\right)-\rho\left(\frac{\partial \overline{u^{\prime} v^{\prime}}}{\partial x}+\frac{\partial \overline{v^{\prime} v^{\prime}}}{\partial y}\right) \\
& +\rho_{\infty} g \beta\left(\bar{T}-T_{\infty}\right) \\
& \rho c_{p}\left(\frac{\partial \bar{T}}{\partial t}+U \frac{\partial \bar{T}}{\partial x}+V \frac{\partial \bar{T}}{\partial y}\right)-U \frac{\partial P}{\partial x}-V \frac{\partial P}{\partial y} \\
& =k\left(\frac{\partial^{2} \bar{T}}{\partial x^{2}}+\frac{\partial^{2} \bar{T}}{\partial y^{2}}\right)-\rho c_{p}\left(\frac{\partial \overline{u^{\prime} T^{\prime}}}{\partial x}+\frac{\partial \overline{v^{\prime} T^{\prime}}}{\partial y}\right)
\end{aligned}
$$

The new Reynolds averaged Navier-Stokes and energy equations are almost identical to the original, except for the addition of new terms. These new terms are the average of two point turbulence correlations and create an open set of differential equations that require additional 
equations for closure. In the case of Equation 5.11 and Equation 5.12 the averaged two point turbulence correlations are called the Reynolds stresses. The fundamental problem of RANS based turbulence modelling is in defining these terms to close the set of equations from Equation 5.10 through 5.13 .

\subsection{Turbulence Modelling}

One way of understanding the $\mathrm{k}-\varepsilon$ model can best be described by explaining the history of turbulence modelling. One of the approaches to RANS turbulence modeling is to create additional transport equations to solve for the Reynolds stresses. These additional transport equations are based on the Boussinesq approximation for turbulence. The Boussinesq approximation for turbulence is explained next.

Turbulence can be conceptualized as a cascade of turbulent eddy motion or swirls that break off into smaller eddies until the length scale is small enough that the viscosity of the fluid dissipates that eddy motion. In the Boussinesq approximation for turbulence, turbulent eddy motion is treated as analogues to molecular motion. From this, turbulent motion can be thought of as molecular diffusion. It was postulated that the Reynolds stress tensor can take the form as shown in Equation 5.14, Equation 5.15 and Equation 5.16.

$$
\begin{gathered}
-\overline{u^{\prime} u^{\prime}}=2 \mu_{T} \frac{\partial U}{\partial x}-\frac{1}{3} k \\
-\overline{u^{\prime} v^{\prime}}=\mu_{T}\left(\frac{\partial U}{\partial y}+\frac{\partial V}{\partial x}\right)=-\overline{v^{\prime} u^{\prime}}
\end{gathered}
$$




$$
-\overline{v^{\prime} v^{\prime}}=2 \mu_{T} \frac{\partial V}{\partial y}-\frac{1}{3} k
$$

In the above equation a new term, $\mu_{T}$ is introduced and is known as the eddy viscosity. The last term in Equation 5.14 and Equation 5.16 include the variable ' $\mathrm{k}$ ' which is the turbulent kinetic energy that is due to turbulent fluctuations and is shown in Equation 5.17.

$$
k=\frac{1}{2}\left(\overline{u^{\prime} u^{\prime}}+\overline{v^{\prime} v^{\prime}}\right)
$$

\section{3 k-E Turbulence Model}

One common turbulence model is the k- $\varepsilon$ model. In this model two additional transport equations are devised to solve for the turbulent eddy viscosity in Equation 5.14 through Equation 5.16. There is one transport equation for the turbulent kinetic energy and another transport equation for the turbulent dissipation rate. The turbulent kinetic energy is defined above, the turbulent dissipation rate is the rate at which viscosity dissipates turbulent energy. In other words, ' $k$ ' can be thought of as the production of turbulent energy and ' $\varepsilon$ ' is the destruction of turbulent energy. In the k- $\varepsilon$ turbulence model the turbulent eddy viscosity is defined in Equation 5.18 and relates to the turbulent kinetic energy and turbulent dissipation rate.

$$
\mu_{T}=\rho C_{\mu} \frac{k^{2}}{\epsilon}
$$


where $\mathrm{C}_{\mu}$ is a constant. The transport equation for the turbulent kinetic energy is shown in Equation 5.19 and the transport equation for the turbulent dissipation rate is shown in Equation 5.20 in tensor notation for compactness.

$$
\begin{gathered}
\rho \frac{\partial k}{\partial t}+\rho U_{i} \frac{\partial k}{\partial x_{i}}=\frac{\partial}{\partial x_{j}}\left[\left(\mu+\frac{\mu_{t}}{\sigma_{k}}\right) \frac{\partial k}{\partial x_{j}}\right]+G_{k}+G_{b}-\rho \epsilon \\
\rho \frac{\partial \epsilon}{\partial t}+\rho U_{i} \frac{\partial \epsilon}{\partial x_{i}}=\frac{\partial}{\partial x_{j}}\left[\left(\mu+\frac{\mu_{t}}{\sigma_{\epsilon}}\right) \frac{\partial \epsilon}{\partial x_{j}}\right]+C_{1 \epsilon} \frac{\epsilon}{k}\left(G_{k}+C_{3 \epsilon} G_{b}\right)-C_{2 \epsilon} \frac{\epsilon^{2}}{k}
\end{gathered}
$$

where

$$
\begin{gathered}
G_{k}=-\rho \overline{u_{i}^{\prime} u_{j}^{\prime}} \frac{\partial u_{j}}{\partial x_{i}} \\
G_{b}=\beta g_{i} \frac{\mu_{t}}{\operatorname{Pr}_{t}} \frac{\partial T}{\partial x_{i}}
\end{gathered}
$$

where $G_{k}$ is the production of turbulent kinetic energy due to shearing and $G_{b}$ is the production of turbulent kinetic energy due to buoyancy. $\mathrm{C}_{1 \varepsilon}, \mathrm{C}_{2 \varepsilon}, \mathrm{C}_{3 \varepsilon}, \mathrm{Pr}_{\mathrm{t}}, \sigma_{\mathrm{k}}$, and $\sigma_{\varepsilon}$ are constants. For the current simulation the following parameters were used: $\mathrm{C}_{1 \varepsilon}=1.44, \mathrm{C}_{2 \varepsilon}=1.92, \mathrm{C}_{3 \varepsilon}=0, \operatorname{Pr}_{\mathrm{t}}=0.85, \sigma_{\mathrm{k}}=1, \sigma_{\varepsilon}=$ 1.3.

\subsection{Wall Functions}

The $\mathrm{k}-\varepsilon$ model poses some numerical problems for near wall turbulence. This is due to the complex nature of boundary layer flows. A boundary layer that is formed near a wall can be split up into 
three overlapping regions. The outer most region is called the defect layer and it interfaces with the upstream flow. The middle layer is usually called the Log layer since it follows a logarithmic profile. The inner most region is called the viscous sublayer, where due to the no slip condition at the wall surface, the flow is moving slowly enough to be considered laminar and thus the viscous effects of the fluid completely dampen the turbulent fluctuations. It is mainly in the two regions nearest the wall, log layer and viscous sublayer, that the $\mathrm{k}-\varepsilon$ model is unable to be resolved. A common approach to resolve this issue is to bypass the turbulence model completely and just apply an algebraic expression that are usually called wall functions.

The Log-law is perhaps the most common wall function used with the $\mathrm{k}-\varepsilon$ model. The derivation of this wall function is based on the physical nature of near wall turbulence of forced flow over a flat plate. Since the velocity profile in the boundary layer follow a known shape, it is used as the foundation for the Log-law function. The wall functions are used as the boundary conditions that are then fed into the k- $\varepsilon$ turbulence model. In essence, the wall shear stress and wall convection are replaced with the Log-law functions. For near wall heat transfer phenomena a better approach is to use the enhanced wall functions. Walsh and Leong (2004) determined that using the enhanced wall functions were more accurate for heated vertical flat plate. The enhanced wall functions were used for the current simulation.

\subsection{Solidification and Melting}

For the current simulation, soy wax was modelled as the phase change material. In order to match with the experiments the soy wax starts warm as a liquid and cools off and solidifies. Simulating 
phase change requires the capability to model the material as a liquid and a solid. In addition the latent heat in the material needs to be taken into account for either absorbing energy as latent heat when the material is melting or discharging the latent heat when the material solidifies. To simulate the presence of phase change in the model, the enthalpy-porosity method was used and is described in the ANSYS FLUENT User's Guide (2010). The enthalpy-porosity method was developed by Voller and Prakash (1987). In the enthalpy-porosity method every finite volume element is assigned a liquid fraction between 0 and 1 which is used to describe the phase state of the material where ' 0 ' signifies a solid state and ' 1 ' signifies a liquid state. A phase change material is allowed to fall between 0 and 1 by defining a mushy zone. In the mushy zone the phase change material is treated as a porous medium with the porosity assigned between 1 and 0 . A porosity of ' 1 ' indicates fully free flowing liquid, while a ' 0 ' indicates no fluid motion and therefore behaves as a solid.

To use the enthalpy-porosity method, the conservation of energy as shown in Equation 5.13 is manipulated to describe the energy in terms of total enthalpy ' $\mathrm{H}$ ' and is defined in Equation 5.23.

$$
H=h+\Delta H=h+\beta L
$$

where ' $h$ ' is the sensible enthalpy, $\Delta H$ is the latent heat, $\beta$ is the liquid fraction, and $L$ is the latent heat of the material.

For the conservation of momentum, the presence of phase change is treated as a momentum sink term that is added to Equation 5.11 and Equation 5.12 for the $\mathrm{x}$-momentum and $\mathrm{y}$-momentum equations. The momentum sink term is defined in Equation 5.24. 


$$
S=\frac{(1-\beta)^{2}}{\left(\beta^{3}+\epsilon\right)} A_{m u s h}\left(\vec{v}-\overrightarrow{v_{p}}\right)
$$

where $\varepsilon, \mathrm{A}_{\text {mush }}$ are constants, $\vec{v}$ is the flow velocity and $\overrightarrow{v_{p}}$ is the pull velocity (velocity of the solid phase). For the current simulation the constants are left at defaults as $\mathrm{A}_{\text {mush }}=100,000$, and $\varepsilon=$ 0.001 and the pull velocity is also set at default as $\overrightarrow{v_{p}}=0$.

Similarly, for k- $\varepsilon$ turbulence model, the effect of phase change is treated as a sink term and is added to both transport equations for the turbulent kinetic energy and turbulent dissipation rate in Equation 5.19 and Equation 5.20 respectively. The turbulent kinetic energy sink term and turbulent dissipation sink term is defined in Equation 5.25 and Equation 5.26 respectively.

$$
\begin{aligned}
& S=\frac{(1-\beta)^{2}}{\left(\beta^{3}+\epsilon\right)} A_{m u s h} k \\
& S=\frac{(1-\beta)^{2}}{\left(\beta^{3}+\epsilon\right)} A_{m u s h} \epsilon
\end{aligned}
$$

\subsection{Problem Domain}

A schematic of the computational domain is shown in Figure 5.1 and includes an overall domain and a close up of the PCM panel. These images are outputs of ANSYS FLUENT with shaded hatch marks overlaid on top to better view the various components. The simulated geometry was matched as closely as possible to the physical geometry of the experiment and matches closely with the schematic shown in Figure 1.1. The overall modeled domain is $950 \mathrm{~mm}$ in height and 400 $\mathrm{mm}$ in width. The PCM panel is elevated $200 \mathrm{~mm}$ from the bottom of the domain and is $279.4 \mathrm{~mm}$ 
tall. Due to the symmetry of the PCM panel geometry, only half of it was modeled. In the experimental apparatus the PCM cavity is $12.7 \mathrm{~mm}$ wide while in the modeled geometry the PCM cavity is $6.35 \mathrm{~mm}$. The acrylic sheets that contain the soy wax are $3.175 \mathrm{~mm}$ thick and span the entire height of the PCM panel. The bottom of the PCM panel is enclosed by $12.7 \mathrm{~mm}$ thick acrylic glass and $25.4 \mathrm{~mm}$ thick extruded polystyrene. The top of the PCM panel is enclosed by a 12.7 mm thick air gap, a $12.7 \mathrm{~mm}$ thick extruded polystyrene and $12.7 \mathrm{~mm}$ thick of acrylic glass.

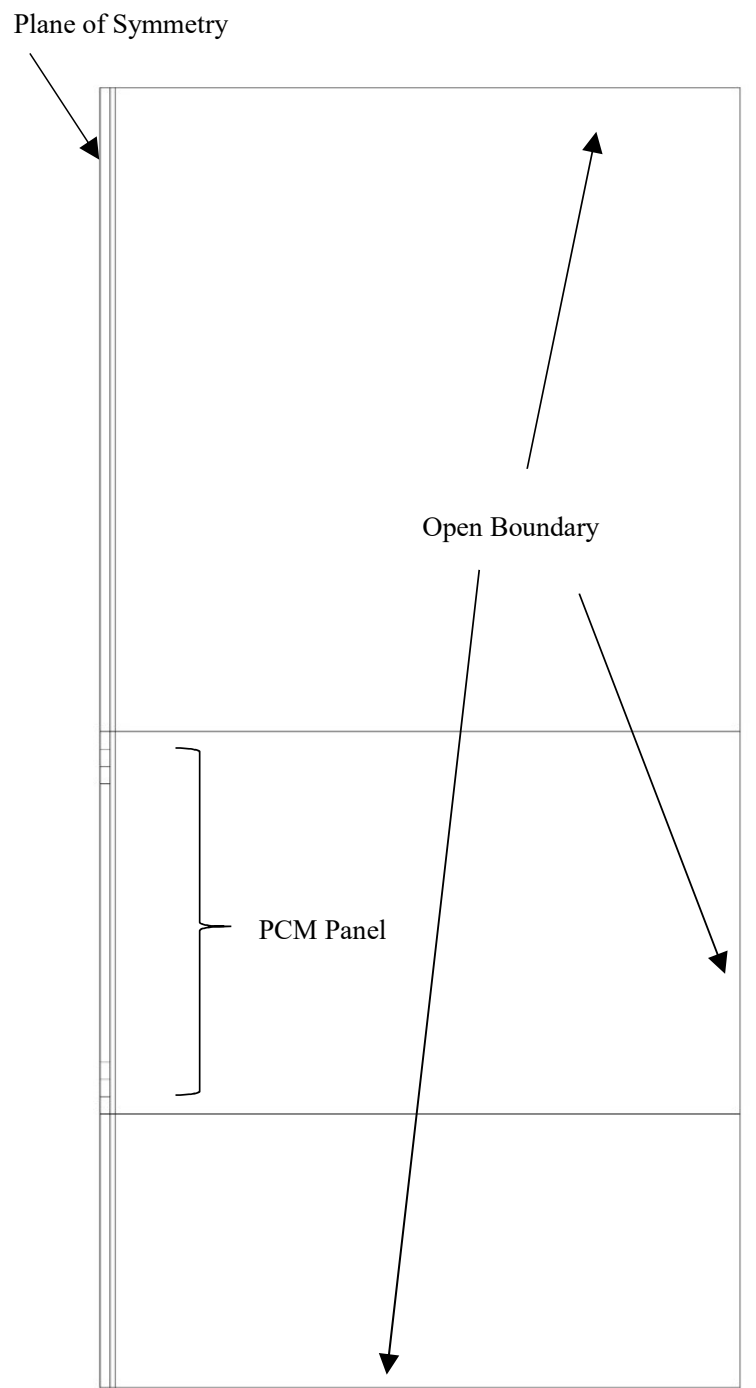

Overall Computational Domain

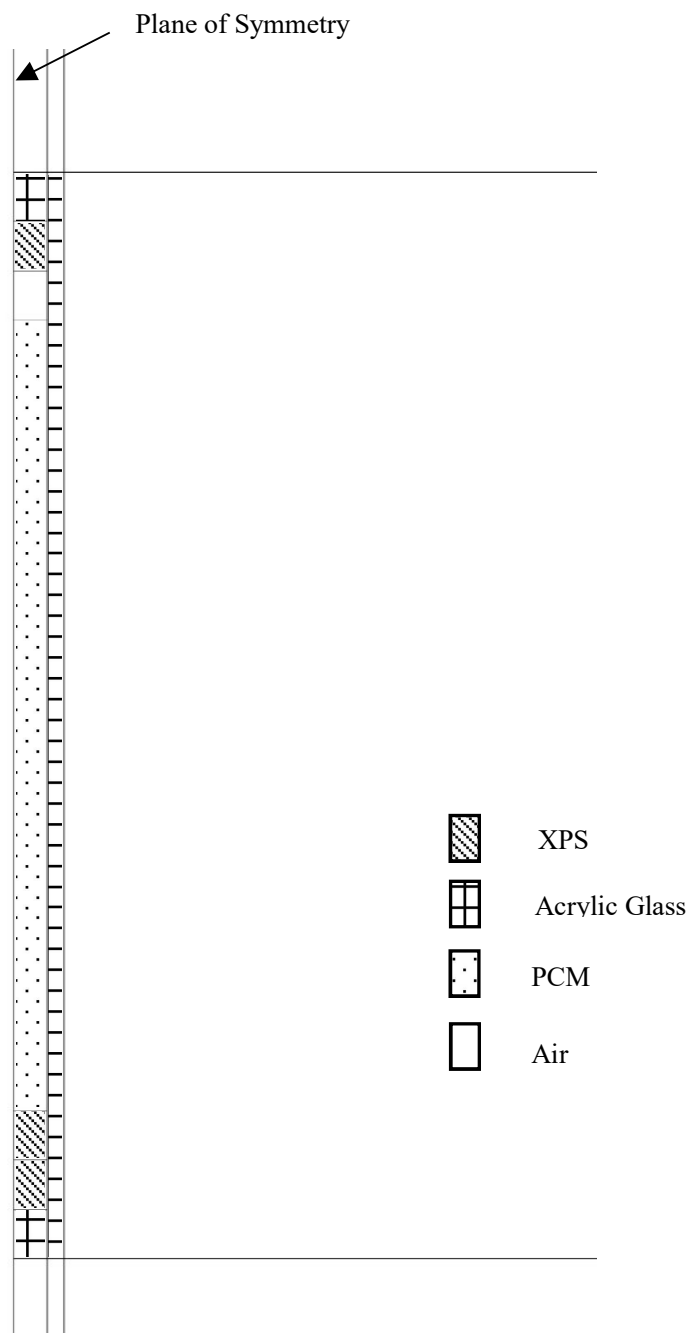

Zoomed on PCM Panel

Figure 5.1: Schematic of computational domain 
Figure 5.2 contains the mesh used for the current simulation is shown for the overall computational domain and a zoomed in portion of the PCM panel. The outline of the various components of the PCM panel is also highlighted. As can be seen the fluid mesh is finer along the plane of symmetry and along the surface of the PCM panel. This is due to the increase in velocity and thermal gradients near a wall due to boundary layers and thus requires more discretization to probably capture the physics. The mesh gradually becomes less dense towards the open boundary away from the PCM panel in order to reduce computational cost. The air and soy wax were set up as a fluid. The remaining materials, acrylic glass and extruded polystyrene, were set up as a solid. For the acrylic glass, the assumed material properties were density of $\rho=1170 \mathrm{~kg} / \mathrm{m}^{3}$, specific heat of $\mathrm{c}_{\mathrm{p}}=1466 \mathrm{~J} / \mathrm{kg} \cdot \mathrm{K}$, and thermal conductivity of $\mathrm{k}=0.17 \mathrm{~W} / \mathrm{m} \cdot \mathrm{K}$. For the extruded polystyrene, the assumed material properties were density of $\rho=28 \mathrm{~kg} / \mathrm{m}^{3}$, specific heat of $\mathrm{c}_{\mathrm{p}}=1220 \mathrm{~J} / \mathrm{kg} \cdot \mathrm{K}$, and thermal conductivity of $\mathrm{k}=0.029 \mathrm{~W} / \mathrm{m} \cdot \mathrm{K}$. For the soy wax the density was assumed to be governed by the Boussinesq approximation for buoyancy with an assumed reference density of $\rho=890$ $\mathrm{kg} / \mathrm{m}^{3}$, and viscosity was assumed to be $0.009 \mathrm{~kg} / \mathrm{m} \cdot \mathrm{s}$. The remaining soy wax material properties were determined from differential scanning calorimetry (DSC) as explained in Section 2.3 and from the work of Cui et al. (2011). The specific heat was found to be $c_{p}=2118 \mathrm{~J} / \mathrm{kg} \cdot \mathrm{K}$; the measured thermal conductivity was $\mathrm{k}=0.324 \mathrm{~W} / \mathrm{m} \cdot \mathrm{K}$. The solidification temperature was found from the peak of the DSC curve in Figure 2.2 and was found to be $309.3 \mathrm{~K}$. The latent of the solidification process was determined by taking the phase change range to be $296.7 \mathrm{~K}$ to $311.2 \mathrm{~K}$ which gives a latent heat of $\mathrm{L}=31429 \mathrm{~J} / \mathrm{kg}$. 


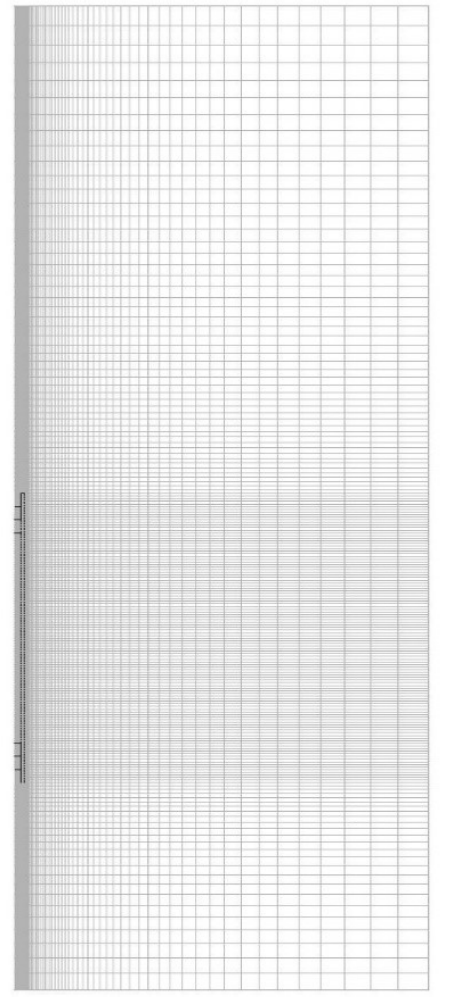

Overall Computational Domain

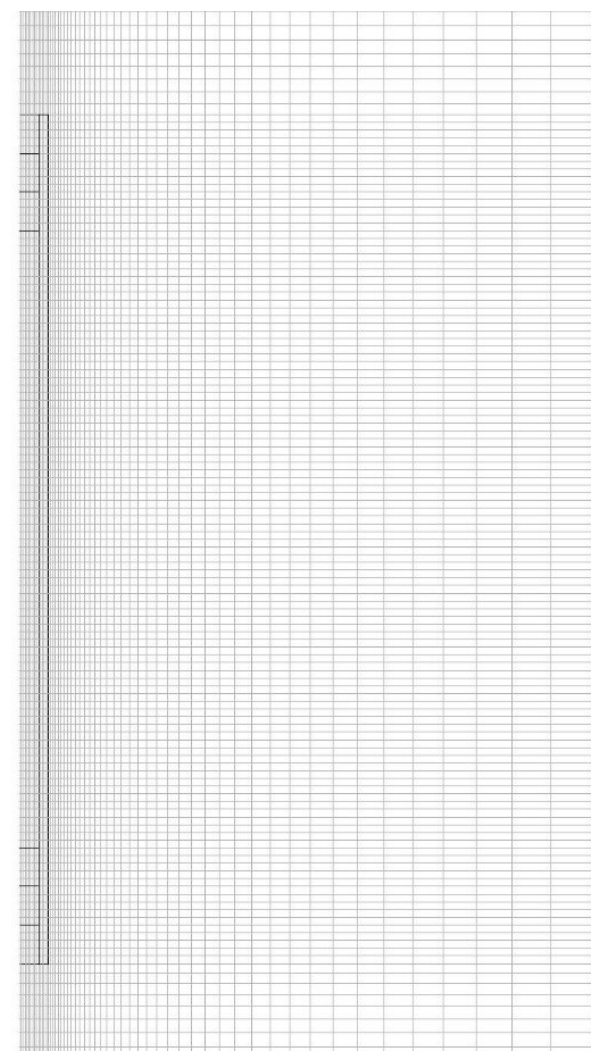

Zoomed on PCM Panel

Figure 5.2: Mesh of computational domain

\subsection{Boundary Conditions and Initial Conditions}

As mentioned above, a plane of symmetry was used to reduce the model size and a symmetry boundary condition was applied along the left edge of the domain in Figures 5.1 and 5.2. Along the bottom edge, right edge, and top edge of the domain is treated as an open boundary where air can flow freely across the boundary. This was done by setting those boundaries as a pressure outlet boundary condition with any 'new' air flowing in at ambient temperature. Any fluid to solid interface was set as a wall boundary condition with the no slip condition where the flow velocity 
is set to zero on the surface. Air density was allowed to vary according to the Boussinesq approximation for buoyancy with the reference density set to $1.17 \mathrm{~kg} / \mathrm{m}^{3}$. To account for thermal radiation the Surface to Surface (S2S) radiation model was chosen which computes the surface to surface view factors for all participating elements. All exposed solid surface were given an emissivity of 0.89 which is the measured emissivity of the white spray paint that was applied to the experimental apparatus. The pressure outlet boundary conditions was assumed to behave as a black body with an emissivity of 1 and participates in the view factor calculation. The components of the PCM panel were initialized at a temperature of $343 \mathrm{~K}$. The air was initialized to ambient temperature and the turbulent kinetic energy and turbulent dissipation rate was initialized to zero.

The pressure-velocity coupling was set to use the SIMPLEC method (Van Doormaal and Raithby 1984). In terms of the spatial discretization schemes, pressure was discretized with the PRESTO! Scheme (Patankar 1980), the momentum equations with Second Order Upwind scheme, and both the turbulent kinetic energy and turbulent dissipation rate was discretized with First Order Upwind scheme. For the transient formulation, time was discretized with First Order Implicit scheme. To aid in convergence the following under relaxation factors were used; 0.3 for pressure, 1 for density and body forces, 0.7 for momentum, and 0.8 for turbulent kinetic energy. The simulation was run with a time step size of 1 second for a total time of 5300 seconds.

\subsection{Comparison of Numerical Simulation with Experimental Results}

A computational fluid dynamics (CFD) simulation was performed on the same geometry as the experimental apparatus using ANSYS FLUENT, based on the models and parameters described in the previous sections. A comparison of the averaged center line temperatures, inside surface temperature and outside surface temperatures between the experiment and numerical simulation is 
shown in Figure 5.3. For the averaged numerical results to be consistent with the measures results, only the temperatures from location TC2 through TC7 was included in the averaging. The numerical results are displayed for the entire range of the simulation from 0 minutes to 90 minutes. For the experimental results the thermocouple data for the center line temperatures and inside surface temperatures are shown for 5 minutes through 85 minutes. For the optically measured outside surface temperatures only the range of approximately 20 minutes to 80 minutes is shown since that was the range that interferogram analysis was performed. All the experimental results displayed in Figure 5.3 was collectively shifted in order to be synced with the numerical results. In general the sensible heat transfer portions of the numerical results and experimental results match closely for the center line temperatures as shown from 0 minutes to 40 minutes in Figure 5.3. Comparing the inside surface temperatures, the experimental results are consistently at a lower temperature than the numerical results. However the thermal decay of the experimental inside surface temperatures decreases at the same rate as the numerical temperatures. One explanation for this is due to the large temperature gradient within the acrylic sheet. The thermocouples on the experiment were embedded slightly into the acrylic sheet. This was done to secure the thermocouples in place but has the unintended consequence of not truly measuring the inside surface temperatures. The numerical results deviate from the measured temperatures during the phase change range. In the experiments a temperature jump was observed, however in the numerical simulation the temperatures flat line. For the sensible heat transfer in the solid phase the numerical results and measured results match in terms of being almost parallel with each other. However the experimental results are consistently higher than the numerical results. This might be due to the temperature jump that the experimental results experience. 


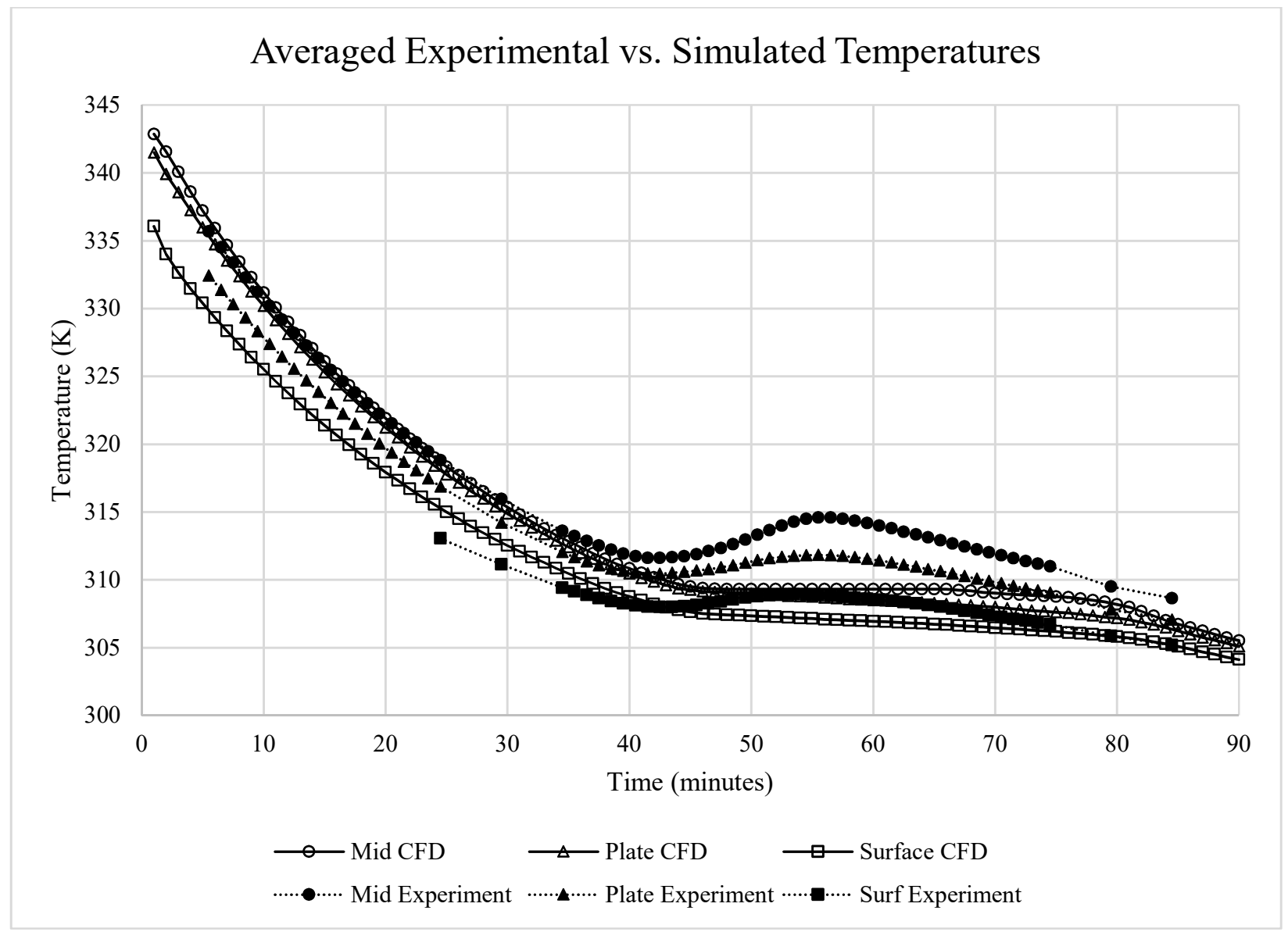

Figure 5.3: Average experimental vs. simulation temperatures

Figure 5.4 contains the center line temperatures for the location at TC2 from the numerical simulation and measured values. Similarly Figure 5.5 contains the center line temperatures for the location at TC7 from the numerical simulation and measured values. In general the sensible heat transfer portions for the liquid and phase and solid phase show agreement. During the phase change portion in both Figure 5.4 and Figure 5.5 it appears that the length of time that the soy wax is undergoing phase change is the same in the experimental results and numerical simulation. At approximately 38 minutes both the experimental and numerical simulation show the onset of phase change in Figure 5.4. At this point the experimental results show the temperature jump that has 
been explained before while the simulation temperatures flat line. After this flat line portion at approximately 66 minutes the simulated results finish the phase change process and is in sensible thermal decay. This thermal decay of the simulated results parallels the thermal decay of the experiments. A similar trend is seen at location TC7 in Figure 5.5 except the onset of phase change occurs at approximately 46 minutes and finishes at approximately 82 minutes.

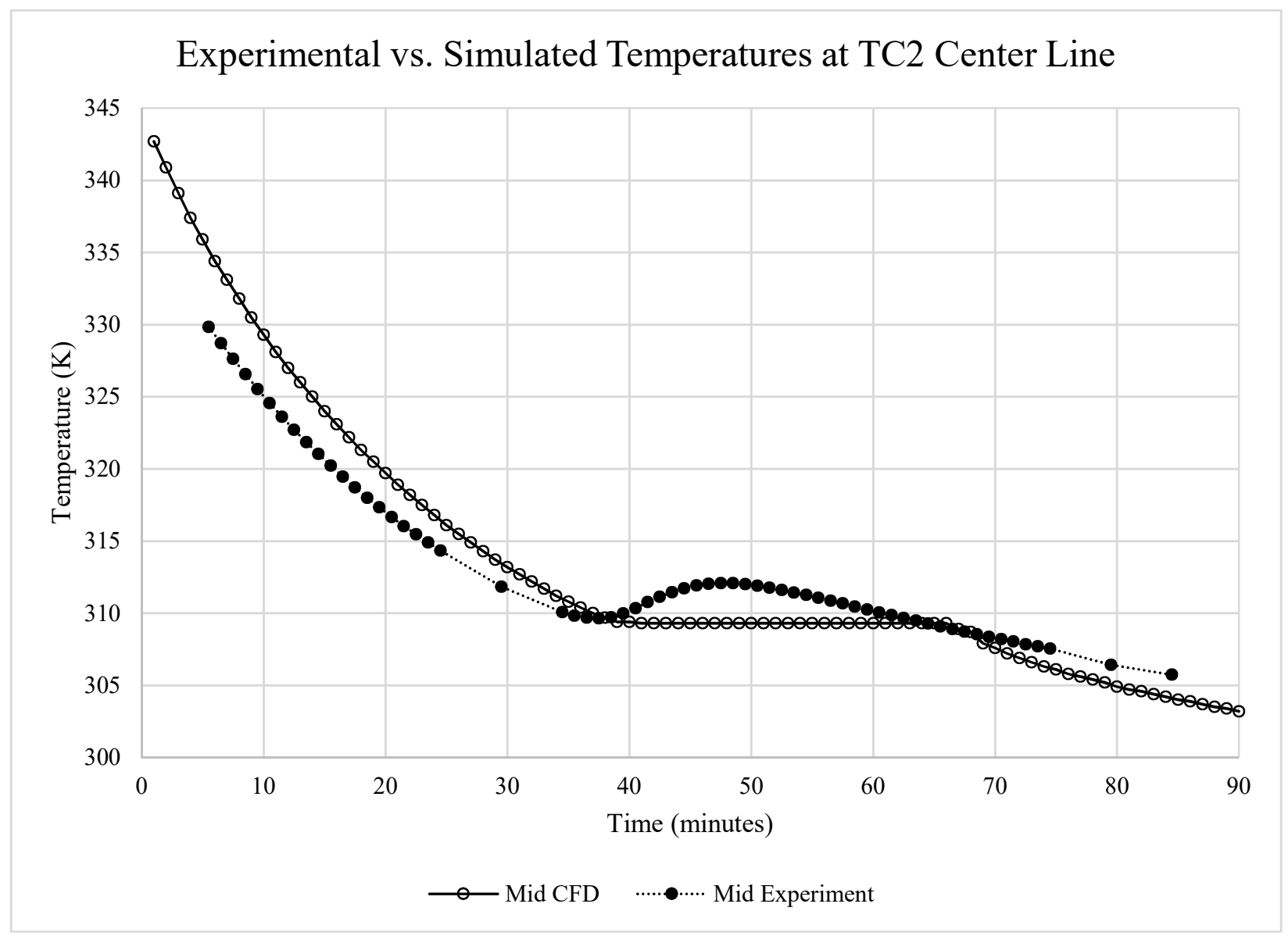

Figure 5.4: Experimental vs. simulation center line temperatures at TC2 


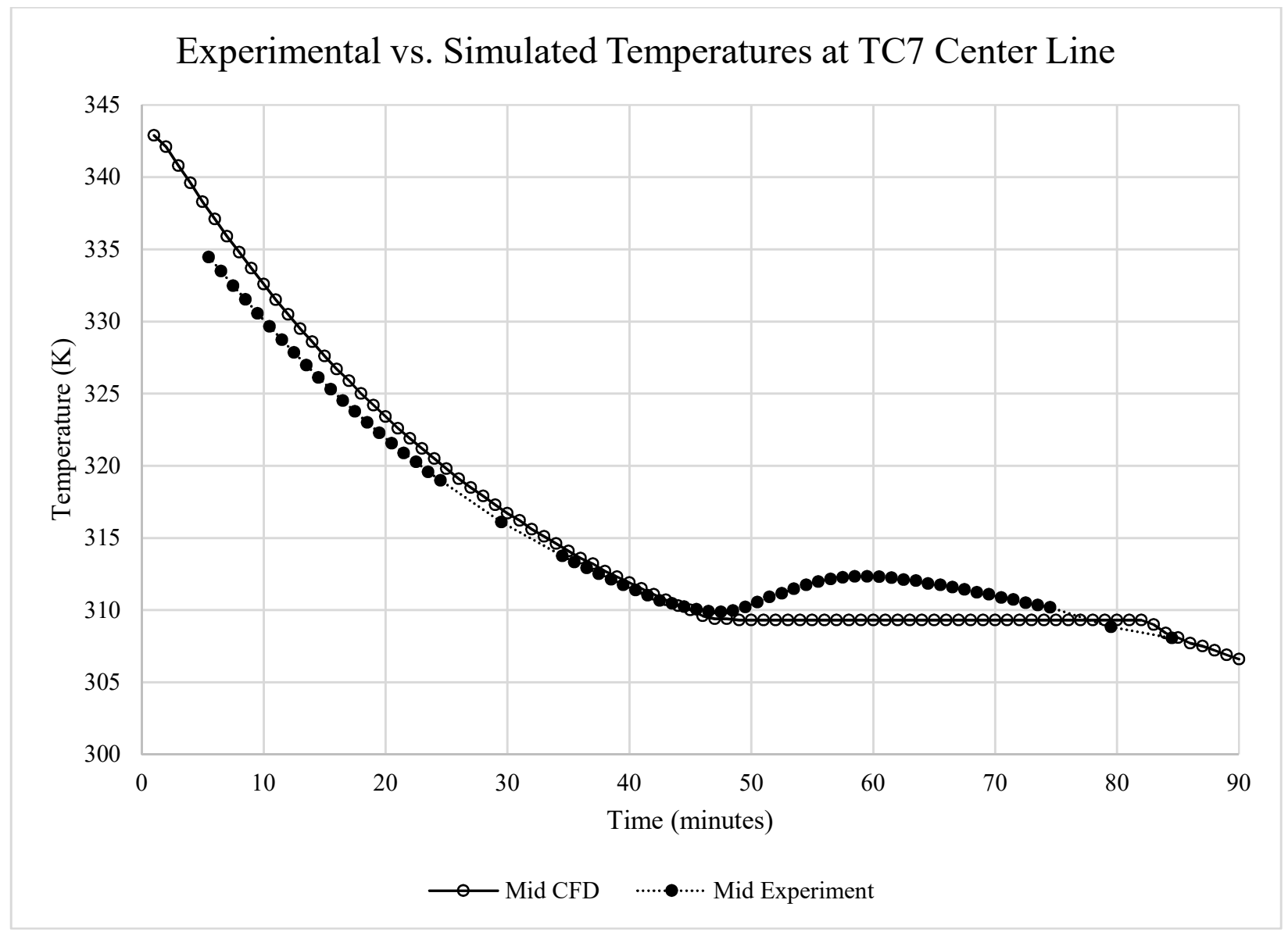

Figure 5.5: Experimental vs. simulation center line temperatures at TC7

The center line temperatures from thermocouple locations TC2 through TC7 are shown in Figure 5.6 for an 80 minute time interval. For comparison the equivalent graph for the experimental center line temperatures for thermocouple locations TC2 through TC7 are shown in Figure 5.7. The time scale between these two graphics are not equivalent due to the chosen initial conditions in the numerical simulation. The contrast between Figure 5.6 and Figure 5.7 highlight the temperature jump effect. In the numerical simulation in Figure 5.6 the presence of phase change has the effect of flattening the temperature decay with time. In contrast, the experimental results in Figure 5.6, the presence of phase change creates a temperature jump. Prior to phase change occurring, the 
temperatures at all thermocouple locations decay linearly with time in both the experimental results and CFD simulation. The onset of phase change occurs in a staggered fashion with time with thermocouple location TC2 experiencing phase change first, then followed by TC3, TC4, etc. This staggering is seen in both sets of data from Figure 5.6 and Figure 5.7. This is due to the 'linear' temperature profile from the bottom of the PCM panel to the top during the sensible heat transfer portion when the soy wax is in a liquid state. Another point of similarity is the return to sensible heat transfer when the soy wax is in a solid state. In the numerical results in Figure 5.6 the return to sensible heat transfer more pronounced since the phase change portion is flat making the change to sensible heat transfer more noticeable. Affectively, the latent heat storage potential can be observed by visual inspection as the length of time of the 'flat line' phase change portion between the liquid states and solid states. Once the latent heat has discharged into the air by natural convection, the temperatures drop again with the temperature at thermocouple location TC2 dropping first then followed by TC3, then TC4, etc. The return to a linear drop in temperature for sensible heat transfer is also observed in Figure 5.6 after the temperature jump during phase change. Similar to the numerical results in Figure 5.6, the temperature drop occurs in a staggered fashion with time with the temperature at thermocouple location TC2 dropping first, followed by TC3, etc. 


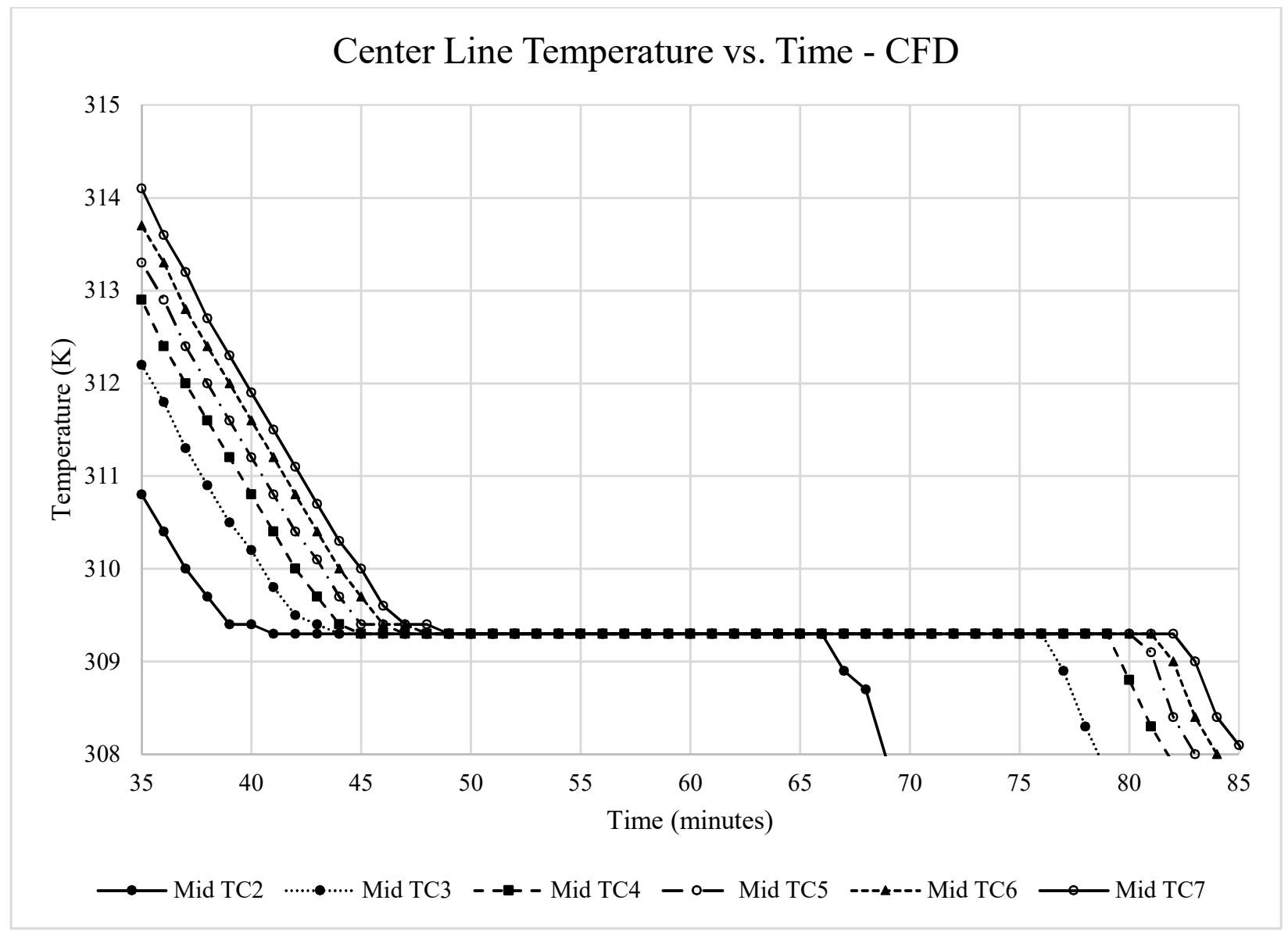

Figure 5.6: CFD center line temperatures vs. time 


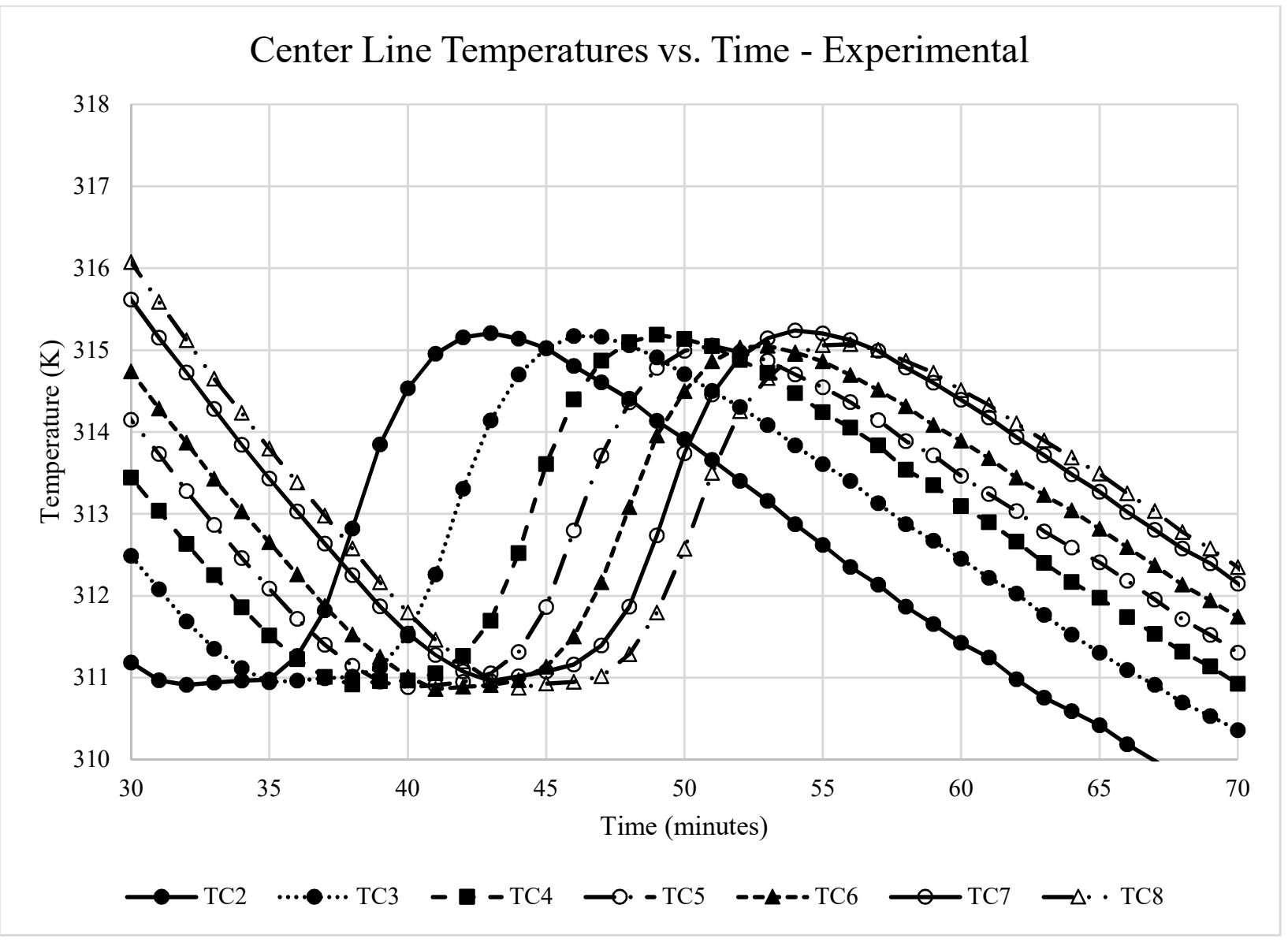

Figure 5.7: Experimental center line temperatures vs. time

The inside surface temperatures vs. time is shown in Figure 5.8 for the numerical simulation and Figure 5.9 for the experimental results. Similar to the center line temperatures shown above in Figure 5.6 and Figure 5.7 there is a distinctive temperature jump in the experimental results that is not seen in the numerical simulation. The temperature jump occurs during the phase change range of the soy wax in the experiments in Figure 5.9. However, in the numerical simulation in Figure 5.8 the effect of the phase change is to 'flat line' the temperature drop. In both Figure 5.8 and Figure 5.9 the temperatures at all thermocouple locations from TC2 through TC7 are experiencing a temperature drop during the sensible heat transfer portion while the soy wax is in liquid phase. In Figure 5.8 for the numerical simulation, the onset of phase change occurs at approximately 39 
minutes. In Figure 5.7 for the experimental simulation, the onset of phase change occurs at approximately 33 minutes. In Figure 5.8 and Figure 5.9, same as in Figure 5.6 and Figure 5.7, the time periods do not match due to the initial conditions chosen for the numerical simulation. A key difference between the numerical inside surface temperatures from Figure 5.8 and the numerical center line temperatures in Figure 5.6 is the behaviour of the temperatures during the phase change range. In Figure 5.6, the center line temperatures were completely flat for the duration of the phase change and all thermocouple readings from TC2 through TC7 were at the same temperature. In contrast, the numerical simulation for the inside surface temperatures in Figure 5.8 do not show a complete 'flat line' during the phase change, and each thermocouple reading from TC2 through TC7 are showing different temperatures. During the phase change range in Figure 5.8 the temperatures at all the thermocouple locations drop linearly with time. However, that linear drop is less steep than during the sensible heat transfer range with liquid soy wax. After the phase change range the temperatures on the inside surface of the acrylic sheet drop at a steeper rate than during the phase change. Similar to the center line temperatures described above in Figure 5.6 and Figure 5.7, after the phase change range the temperatures at the thermocouple locations from TC2 through TC7 drop in a staggered fashion. The experimental results in Figure 5.9 show a smaller temperature jump on the inside surface temperatures than the center line temperatures shown in Figure 5.7. The presence of the acrylic sheet has the effect of dampening the temperature jump since it can conduct heat away from the soy wax into the surrounding air. This dampening is also seen in the difference between the numerical simulations in Figure 5.6 and Figure 5.8 by the variation of temperature with time during the phase change range. The center line temperatures in Figure 5.6 are flat with time during phase change while in Figure 5.8 the inside surface temperatures drop slowing with time during the phase change range. 


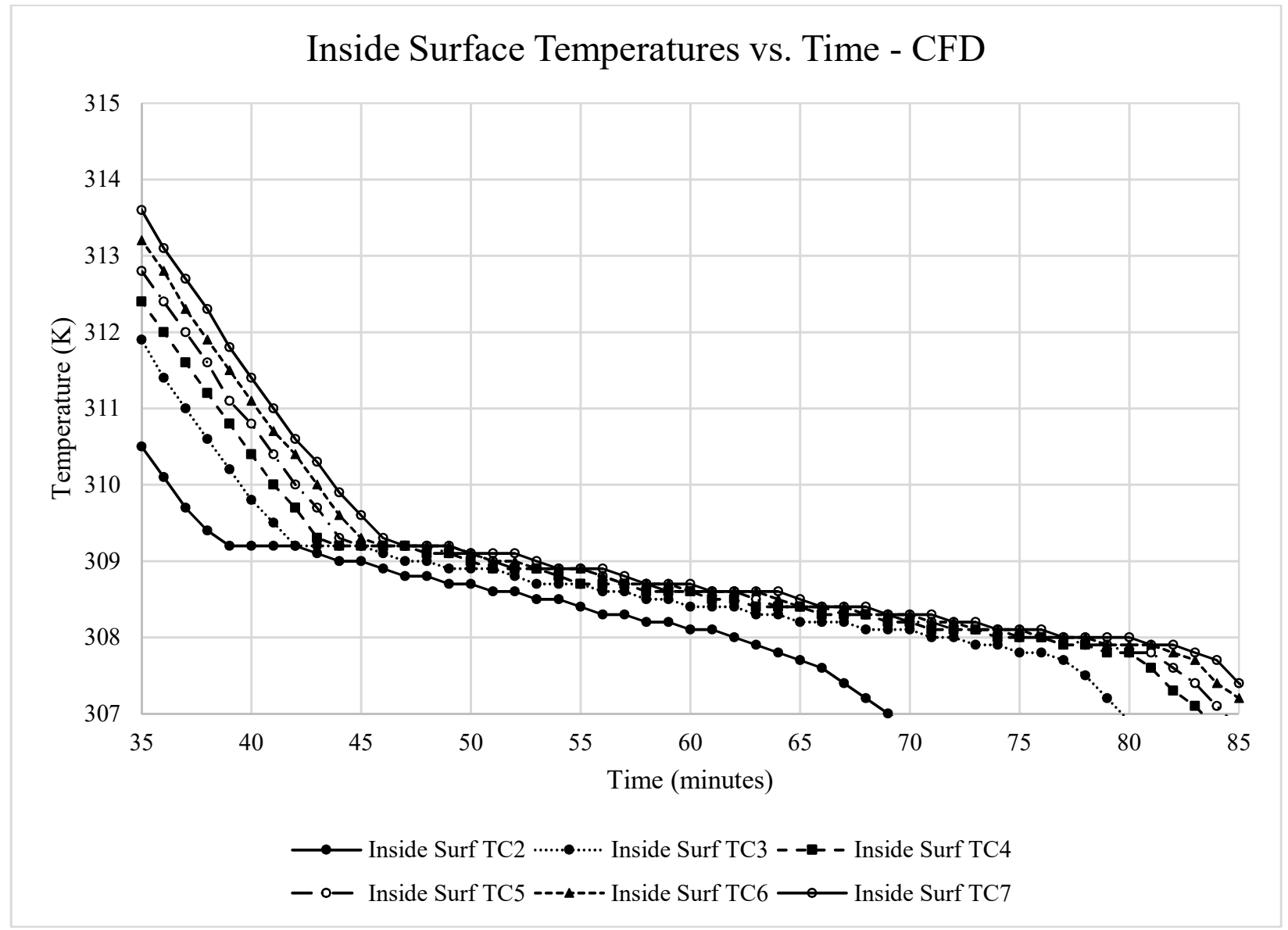

Figure 5.8: CFD inside surface temperatures vs. time 


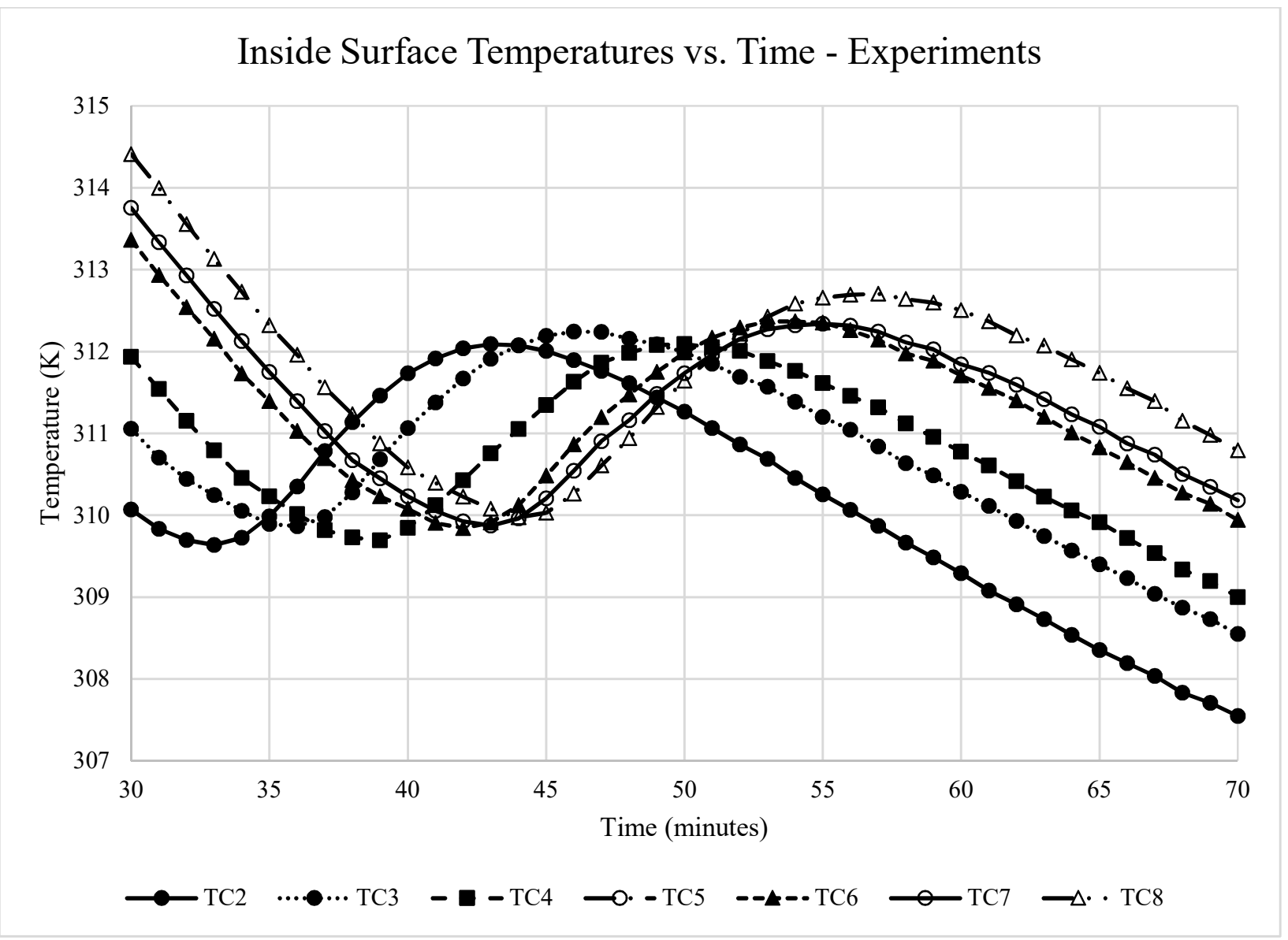

Figure 5.9: Experimental inside surface temperatures vs. time

The outside surface temperatures are shown in Figure 5.10 for the numerical simulation and in Figure 5.11 for the experimental results. The experimental results shown in Figure 5.11 is for 'smoothed' case with a $5^{\text {th }}$ order Fourier curve fit, the original experimental data can be seen in Figure 4.4. Similar to the center line temperatures in Figure 5.6 and Figure 5.7, and to the inside surface temperatures in Figure 5.8 and Figure 5.9, there is a distinct temperature jump observed in the experiments during the phase change range that is not seen in the numerical results. However, the general trends seen for the inside surface temperatures in Figure 5.8 and Figure 5.9 continue here for the numerical simulation in Figure 5.10 and experimental results in Figure 5.11. In the 
numerical results in Figure 5.10 all temperatures at thermocouple locations TC2 through TC7 are experiencing a linear temperature drop with time during the sensible heat transfer portion when the soy wax is in a liquid state. This is followed by the phase change portion where each thermocouple location hits phase change at different times in a staggered fashion where the onset of phase change seen in TC2 first at approximately 40 minutes into the simulation, then TC 3 at approximately 42 minutes and so on. During the phase change portion, the temperature profile at all thermocouple locations with time is not flat as was seen in the center line temperature in Figure 5.6 but drops slowly in a linear fashion as seen on the inside surface temperatures in Figure 5.8. However, this linear temperature drop during the phase change portion is steeper than the temperature drop during phase change from the inside surface temperatures in Figure 5.8. In Figure 5.8 the linear temperature drop is approximately $1 \mathrm{~K}$ during the phase change portion. In Figure 5.10 the linear temperature drop during phase change at each thermocouple location is approximately $1.5 \mathrm{~K}$. 


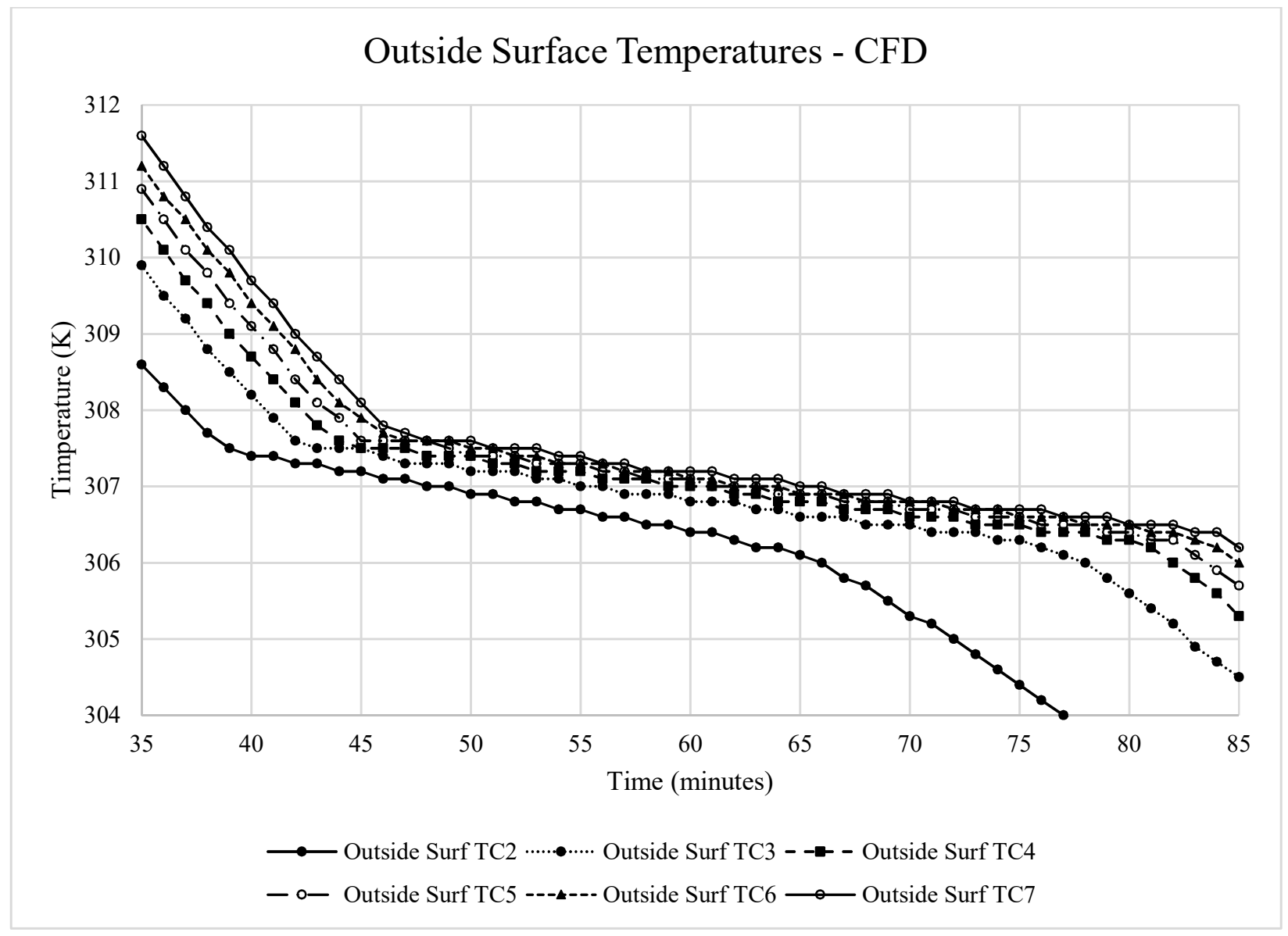

Figure 5.10: CFD outside surface temperatures vs. time 


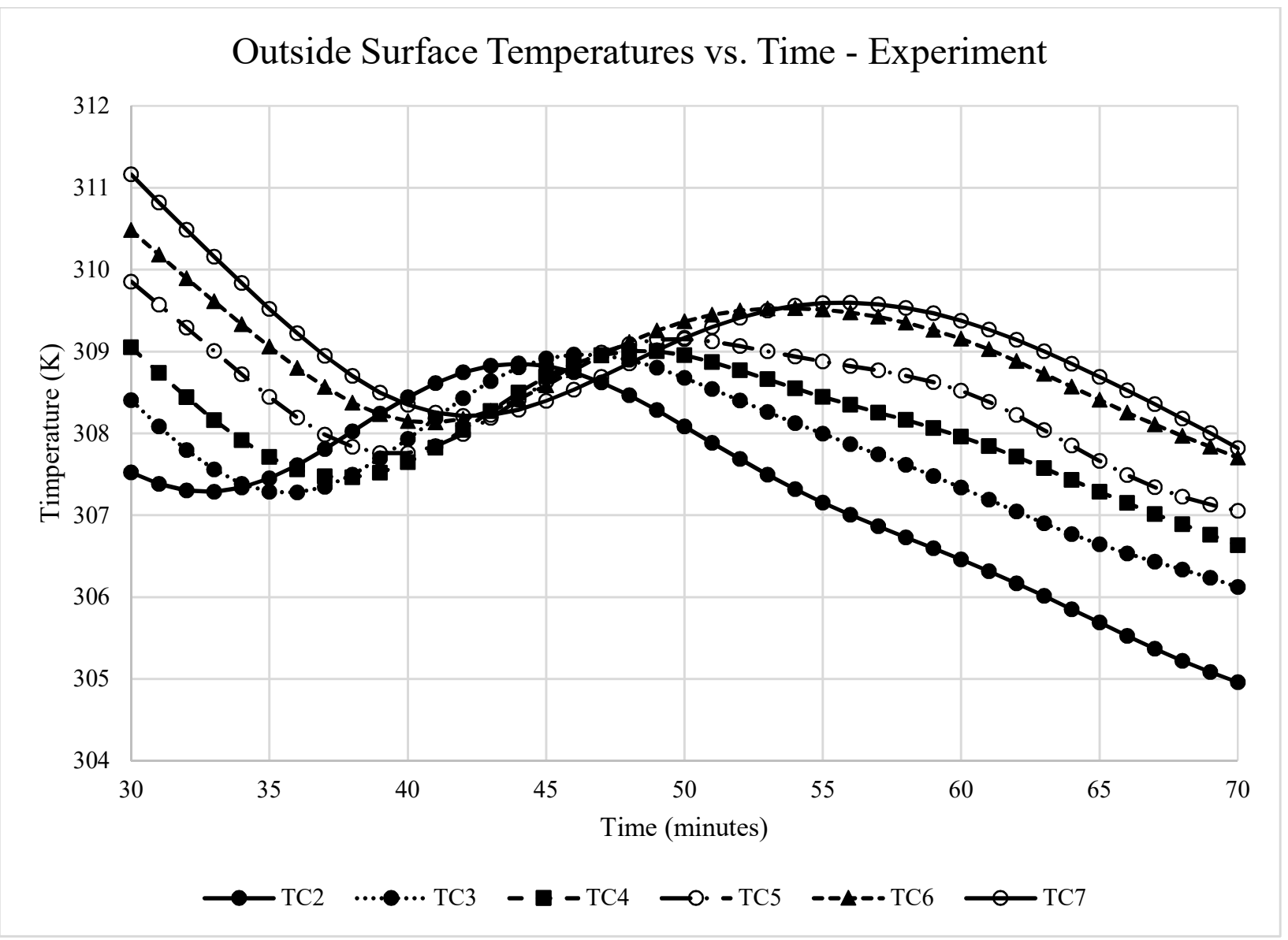

Figure 5.11: Experimental outside surface temperatures vs. time

Another difference between the simulated inside surface temperatures and the outside surface temperatures is the level of 'spread' between the thermocouple temperatures during the phase change range. For example, the temperatures at thermocouple location TC2 through TC7 at the 50 minute mark is shown in Figure 5.8 and in Figure 5.10. The inside surface temperatures at 50 minutes covers a band of approximately $0.4 \mathrm{~K}$ between thermocouple locations TC7 and TC2. However, the outside surface temperatures at 50 minutes between TC7 and TC2 covers a band of approximately $0.7 \mathrm{~K}$. While the temperature differences are small relative to the absolute temperatures at a given thermocouple location, this results in a temperature spread during the phase change portion that is $75 \%$ larger on the outside surface than the inside surface. In other words, 
the outside side surface temperatures are less uniform than the inside surface temperatures. The 'band' of temperatures for a given slice of time gives an indication to the temperature uniformity for a given plane on the PCM wall panel. For comparison the temperature 'band' is shown in Figure 5.6 for the numerically simulated center line temperatures. During the phase change portion the temperature 'band' is $0 \mathrm{~K}$ and does not vary with time. This indicates that there is no variation in temperature along the center line plane from thermocouple locations TC2 through TC7. Temperature uniformity measured as the maximum temperature difference is shown in Figure 5.12 for the center line, inside surface, and outside surface temperatures. During the sensible heat transfer portion when the soy wax is in a liquid state, the maximum temperature difference (or temperature uniformity) is similar for the center line, inside surface, and outside surface temperatures up to approximately 45 minutes but with the center line temperatures showing the largest temperature difference. However, during the phase change portion there is a distinct difference across the three different planes of measurements with the center line temperatures showing no variation in temperature while the outside surface temperatures are showing the largest temperature difference. After the phase change portion when the soy wax is in a solid stated, the maximum temperature differences flip with the center line temperatures showing the largest temperature difference and the outside surface temperatures showing the smallest temperature difference. This effect shows a kind of 'delay' of the influence of the phase change process on the outside surface temperatures. 


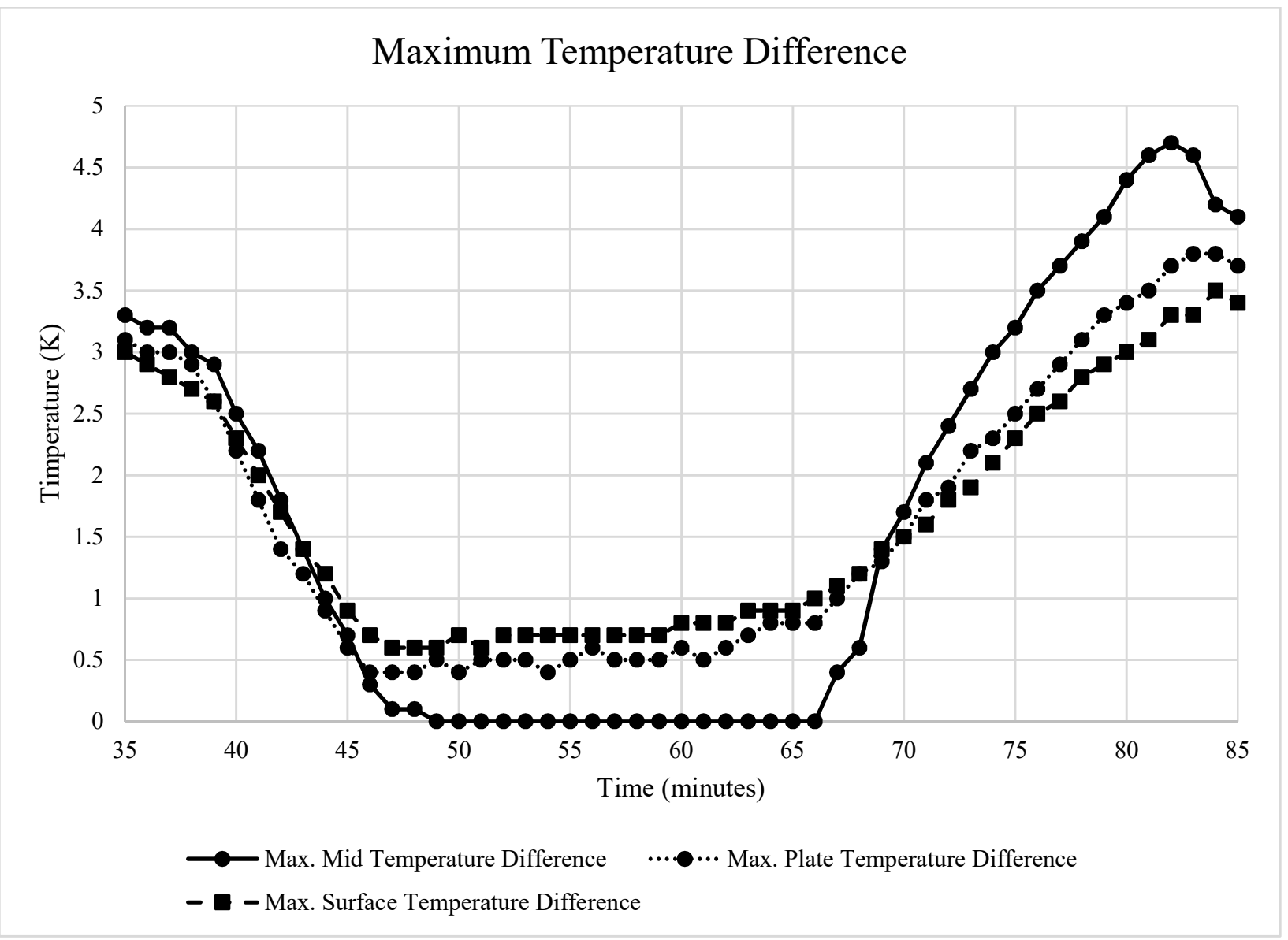

Figure 5.12: Maximum temperature difference

The 'delay' effect of the phase change process on the outside surface temperatures is due to the dampening effect of the acrylic sheets. Figure 5.13 contains the temperatures for thermocouple location TC2 for the center line, inside surface, and outside surface temperatures. For comparison, Figure 5.14 contains the temperatures for thermocouple location TC7 for the center line, inside surface, and outside surface temperatures. The behaviour of the temperature decay over time is similar at both locations at TC2 and TC7. To observe the 'dampening' influence of the acrylic sheet, consider the slope of the temperature profiles for each plane of temperature measurements within the PCM wall panel. In Figure 5.13, the center line temperatures initially are at a negative 
slope during the sensible heat transfer phase when the soy wax is in liquid phase, then the slope 'flattens' during phase change, and finally, the temperatures return to a negative slope during the sensible heat transfer phase when the soy wax is in solid phase. The changing of the temperature slopes is different for the inside surface and outside surface temperatures. For example, looking at the slope of the inside surface temperatures in Figure 5.13 or 5.14, the slope is initially negative, same as the center line temperatures, then the slope decreases during the phase change range but is still negative. Finally the temperature slope increases during the sensible heat transfer portion when the soy wax is in solid phase and matches the slope seen in the center line temperatures. The presence of the acrylic sheet dampens the sharp 'flattening' of the center line temperatures and the sharp return to a negative temperature slope on the outside surface temperatures. This dampening is due the specific heat capacity of the acrylic sheets. The same pattern is seen in Figure 5.14 for thermocouple location TC7 with the only difference being the length of time that TC7 is experiencing phase change. 


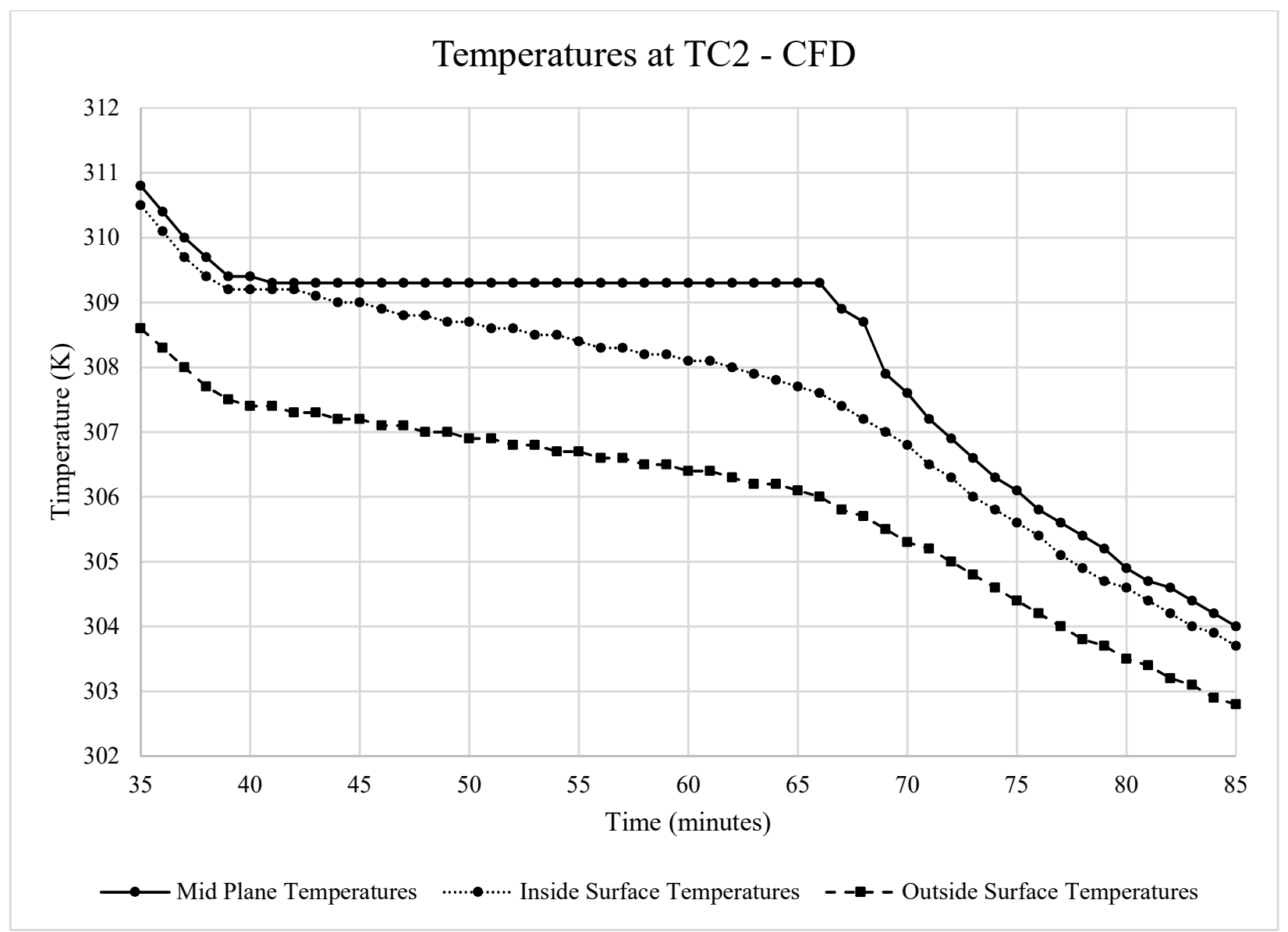

Figure 5.13: Temperatures for TC2 


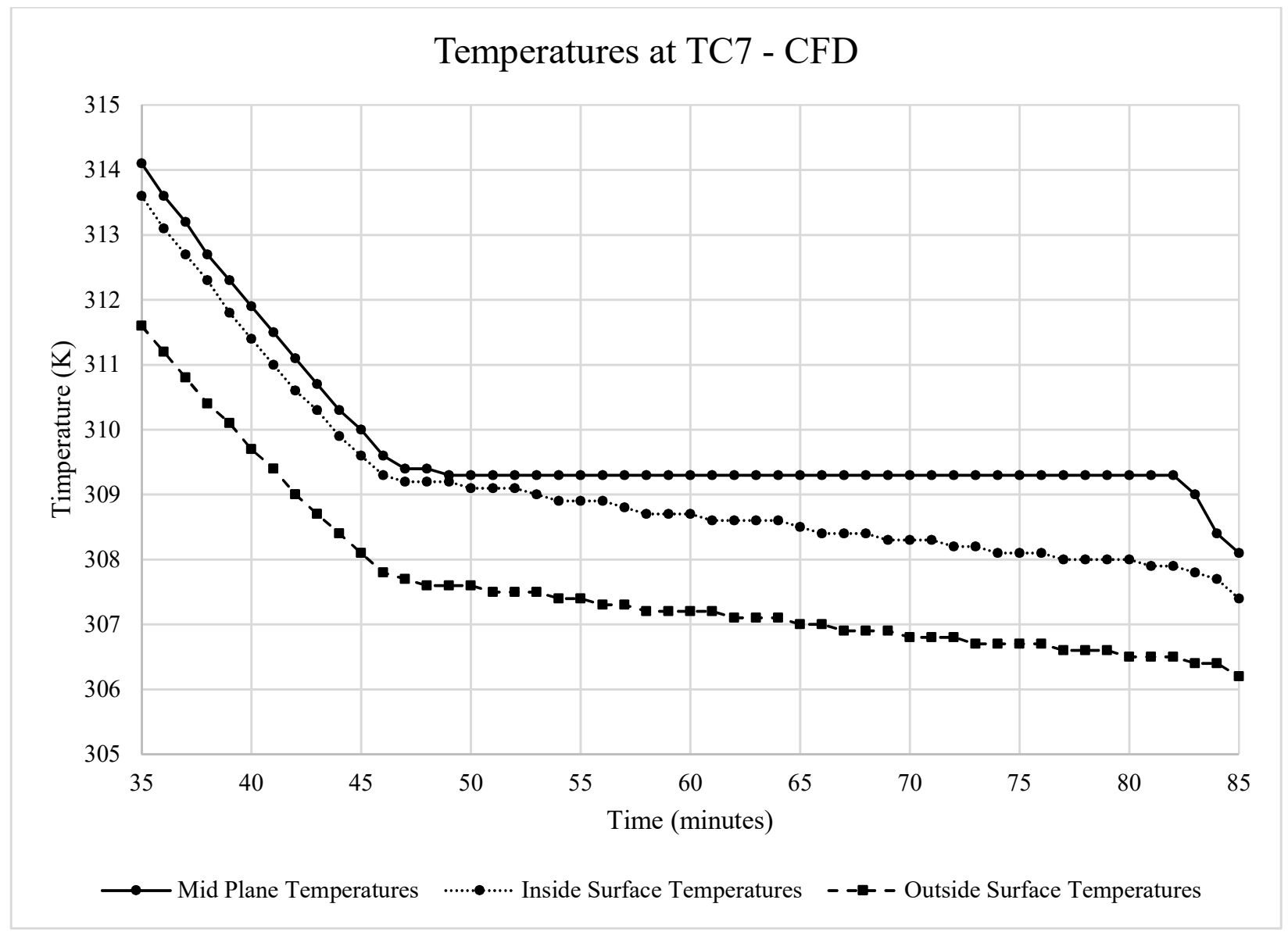

Figure 5.14: Temperatures for TC7

\subsection{Temperature Increase Investigation}

An interesting observed behaviour that occurs in the experiments but not in the numerical simulation is the temperature jump during phase change. This is fundamentally a limit of the enthalpy-porosity method used in ANSYS Fluent for modeling of solidification and freezing processes. The temperature jump is a consequence of a phase change phenomenon called 'subcooling'. Subcooling occurs when the solidification temperature is lower than the melting temperature. The DSC curve of soy wax is shown in Figure 2.2. On the endothermic curve the soy wax is heated and indicates a melting temperature of $323.1 \mathrm{~K}$. However, on the exothermic curve where the soy wax is cooled, the solidification temperature is $309.2 \mathrm{~K}$. In an 'ideal' phase change 
material, the two curves (endothermic and exothermic) would be mirror images of each other giving the same temperature for both the melting temperature and solidification temperature. For the soy wax in Figure 2.2, there is a shift of approximately $13.9 \mathrm{~K}$. This $13.9 \mathrm{~K}$ band is considered the subcooling range. This subcooling range indicates that the soy wax 'could' be triggered to solidify anywhere between $309.2 \mathrm{~K}$ and $323.1 \mathrm{~K}$. The onset of solidification is governing by when nucleation occurs in the soy wax.

The subcooling phenomenon can be a difficult problem to analysis numerically as it needs to model crystallization growth within the PCM and requires the knowledge of specific material properties like enthalpy curves for both endothermic and exothermic directions and crystallization speed which are difficult to measure (Mehling and Cabeza 2008). Crystallization speed can be thought of as the speed of the phase front while the PCM is undergoing melting. If the crystallization speed is fast enough, only a small subcooling effect would be seen. However, there have been some numerical treatment of subcooling. Gunther et al. 2007 have developed a numerical model which was validated with experiments. A brief description of their model and experiments will be discussed next as it explains the subcooling process.

An example of the subcooling process can be seen in experiments using sodium acetate trihydrate, $\mathrm{NaOAc} \cdot 3 \mathrm{H}_{2} \mathrm{O}$, (sometimes referred to as 'hot ice'). Figure 5.15 and Figure 5.16 contains the work of Gunther et al. 2007 showing a comparison between experiments and simulation of the melting of sodium acetate trihydrate. Figure 5.15 contains the experimental apparatus used to for their measurement with thermocouples embedded at various depths into the PCM. The authors chose sodium acetate trihydrate due to significant subcooling. It is interesting to in Figure 5.7 there is a distinct temperature jump observed. The temperature jump is even more pronounced than the current experiments with soy wax. For the experiments of Gunther et al. 2007, the temperature 
jumps and plateaus at the melting temperature and eventually cools off again. The temperature jump occurs because when a given molecule of PCM solidifies, it releases latent heat causing the surrounding material to increase in temperature up to the melting temperature. If the thermal conductivity of the material is low enough, the phase front moves slowly and is unable to conduct away its latent heat and therefore the phase front increases in temperature. However, if the phase front moves fast enough then the temperature jump is less pronounced. A key observation of Gunther et al. 2007 is that once a region has crystallized (nucleation), the surrounding material will also crystallize without have to hit the subcooled solidification temperature. In other words, the presence of crystallization has the effect of 'triggering' nearby molecules to crystallize as long as they are somewhere within the subcooled range between the solidification temperature and melting temperature. Affectively the solidification temperature can vary with time starting at the lower subcooled solidification temperature then gradually increasing with time up to a maximum which is the melting temperature.

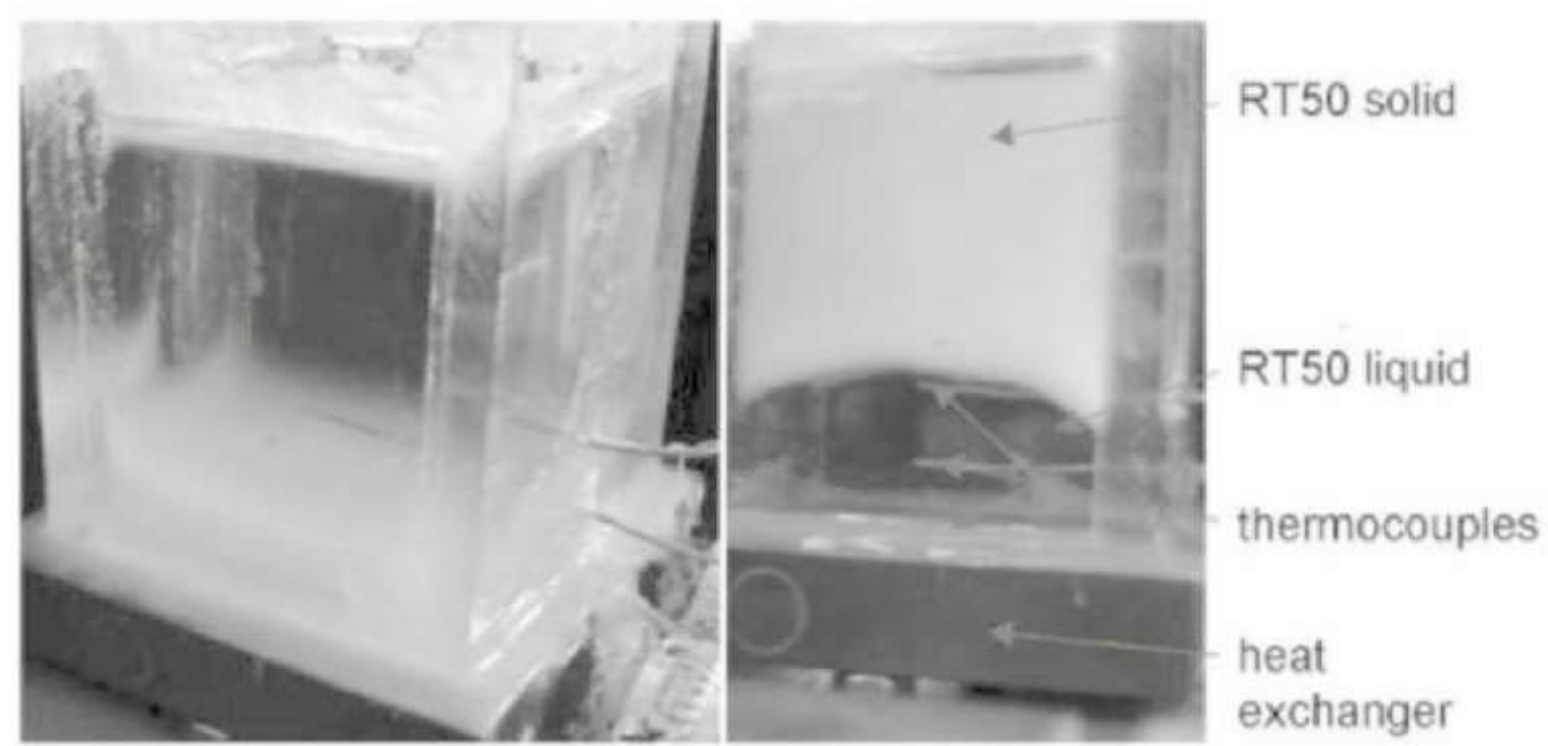

Figure 5.15: Experimental apparatus for melting (left) and freezing (right) of sodium acetate trihydrate (Gunther et al. 2007) 


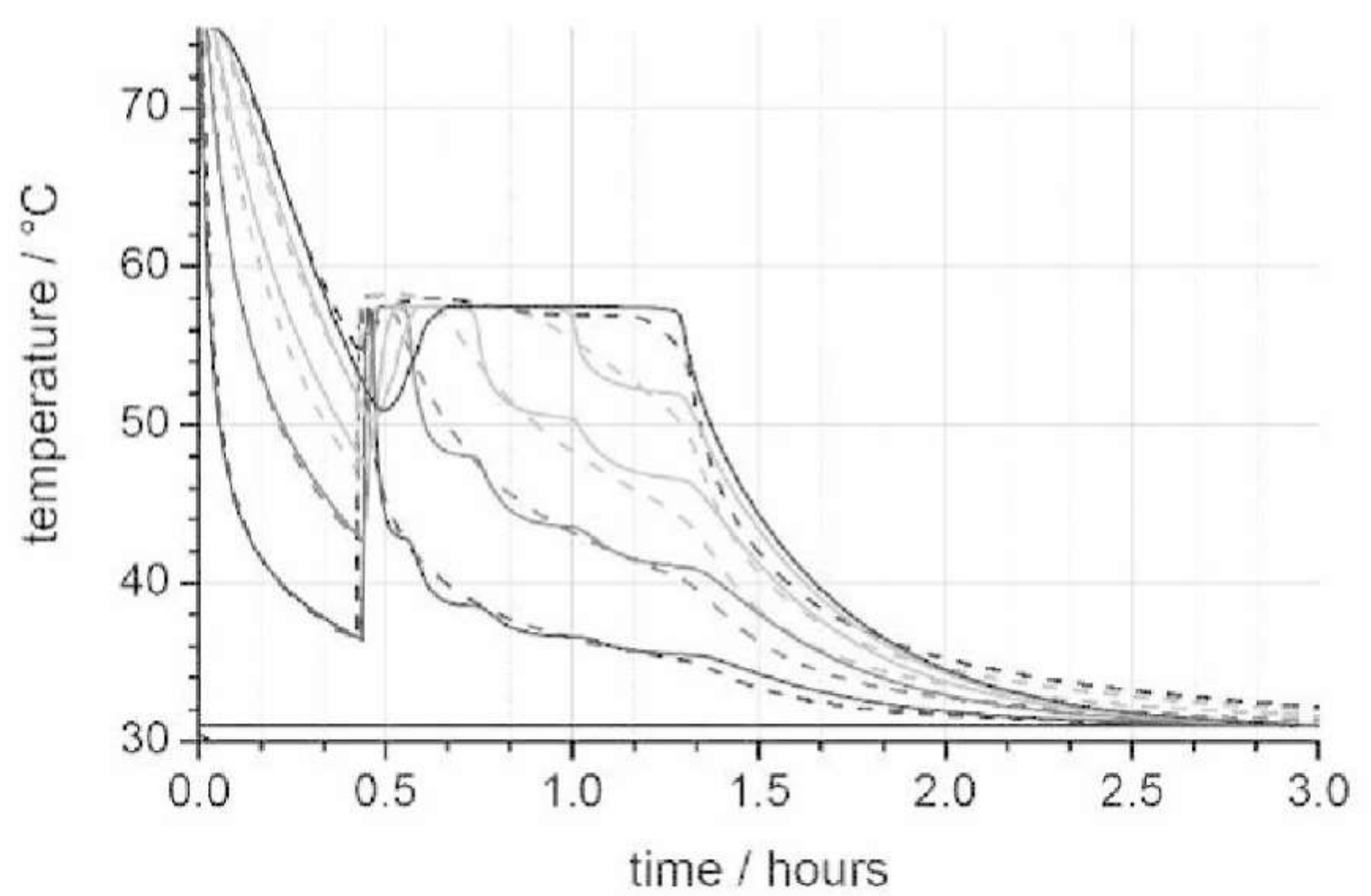

Figure 5.16: Experimental and numerical results for melting of sodium acetate trihydrate (Gunther et al. 2007)

To account for subcooling, Gunther et al. (2007) developed a numerical model where two sets of material properties are defined for the PCM at a given temperature. Their numerical model was applied to a 1D conduction problem with PCM. An example of their numerical domain is shown in Figure 5.17. One set of material properties are used when the PCM is undergoing cooling in liquid phase. In the numerical model the PCM is allowed to cool below the melting temperature until the nucleation temperature (for example, solidification temperature on the exothermic side of a DSC curve). Once nucleation begins, the other set of material properties are used. In addition, once nucleation occurs, the surrounding nodes can be 'triggered' to solidify even though they may be at a higher temperature than the subcooled solidification temperature. Therefore there are two 
conditions for when a given node is allowed to 'switch' to the second set of material properties. The node would have to be within the subcooled range and have a neighbouring node that has already been triggered. This triggering allows the numerical model to capture the speed of crystallization as the phase front propagates through the PCM domain.

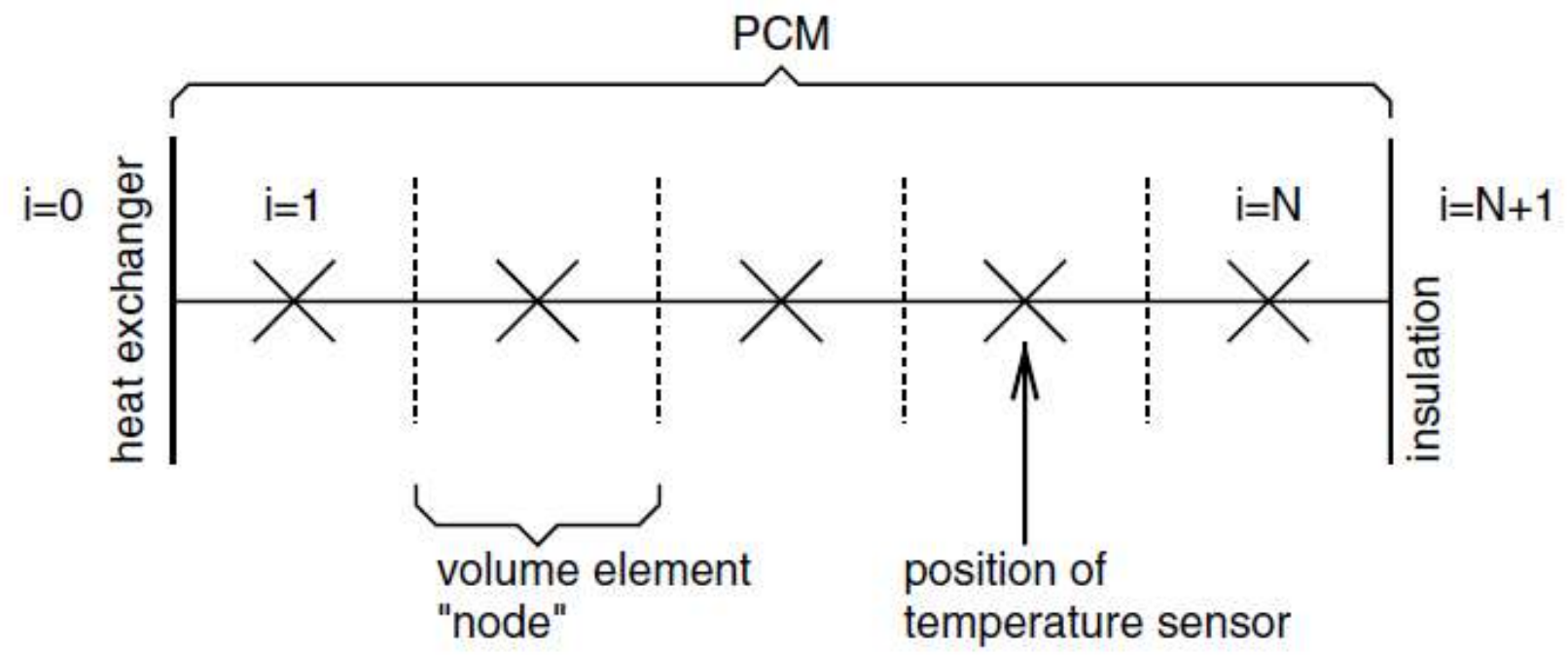

Figure 5.17: 1D Numerical domain used for subcooling algorithm (Gunther et al. 2007)

To 'properly' account for the effect of subcooling using the method described by Gunther et al. 2007, additional material properties are needed for the PCM of interest which have to be determined experimentally. Specifically, the method of Gunther et al. 2007 requires knowledge of the speed of crystallization growth. For the specific example of sodium acetate trihydrate, $\mathrm{NaOAc} \cdot 3 \mathrm{H}_{2} \mathrm{O}$ and the parametric simulation performed on it, Gunther et al. 2007 devised the following expression as an approximation for speed of crystallization as a function of temperature as shown in Equation 5.6.

$$
v(T)=v_{0} \cdot\left(a_{0}+a_{1} T+a_{2} T^{2}\right)
$$


Currently no commercial software incorporates the method of Gunther et al. 2007 nor can any commercial software account for the effect subcooling (PCM text book reference). For the current work, the numerical simulation was performed with ANSYS Fluent which incorporates the enthalpy-porosity method to model solidification and freezing which is explained in Section 5.5. The current implementation of the enthalpy-porosity method in ANSYS Fluent does not account for the effect of subcooling when modeling phase change. However, an ad-hoc method to approximate the subcooling effect based on Gunther et al. 2007 method was devised for the current work and is described next.

\subsection{Modified Crystallization Speed by Temperature Proxy}

To explain the new modified method to model subcooling it is usefully to consider a logic experiment. The current experimental apparatus consists of a vertical cavity filled with a heated phase change material in liquid phase undergoing convective cooling by natural convection. As explained in Chapter 4, while undergoing convective cooling in the liquid phase, the coldest temperature is at the bottom of the PCM cavity and the warmest temperature is at the top of the PCM cavity. For simplicity, assume an arbitrary PCM which has a melting temperature of $320 \mathrm{~K}$ and a solidification temperature of $310 \mathrm{~K}$. Since the solidification temperature is lower than the melting temperature, the subcooling effect exists and the degree of subcooling will be labelled as $\Delta \mathrm{T}_{\mathrm{sub}}=10 \mathrm{~K}$ which is simply the temperature difference between the solidification temperature and melting temperature. For the case of subcooling, the solidification temperature of $310 \mathrm{~K}$ can also be called the nucleation temperature since that is the temperature at which crystallization occurs. As explained in the work of Gunther et al. 2007, the nucleation temperature can vary within a material. A schematic of this 'idealized' PCM logic experiment is shown in Figure 5.18 at different points in time. Similar to the experiments explained in Chapter 4, this PCM logic experiment starts 
in liquid phase and is allowed to solidify. Initially this 'idealized' PCM cavity has a linear temperature profile from $340 \mathrm{~K}$ at the bottom of the cavity to $360 \mathrm{~K}$ at the top of the cavity at time, $\mathrm{t}=0$ (for simplicity, time will be treated as a unitless integer) and is allowed to cool off by natural convection such that all the temperatures drop in lock step. At time, $t=1$, the bottom of the PCM cavity now hits the melting temperature but remains as a liquid due to subcooling. Then at time, $t$ $=2$, the bottom of the PCM cavity is $310 \mathrm{~K}$ which is the solidification temperature and nucleation begins with the black arrow indicating the subcooled region. Under the schematic for time, $\mathrm{t}=2$, the nucleation temperature is listed as, $\mathrm{T}_{\mathrm{n}}=310 \mathrm{~K}$. At time, $\mathrm{t}=3$, the material immediately adjacent to the now crystallized PCM will get 'triggered' into nucleation because it is currently within the subcooled region and is next to an already crystallized material. For this simplified geometry where the phase front, between solid and liquid, is marching upwards and the pragmatic effect is that the nucleation temperature is now higher than the solidification temperature. Due to the aspect ratio of the PCM cavity there is a large temperature gradient vertically during sensible heat transfer. A given arbitrary portion of material will be at a higher temperature than the material below it. Therefore, as solidification continues, the nucleation temperature will continue to increase since the adjacent material above it is warmer. At time, $t=4$, the nucleation temperature increases again and will continue to increase up to a maximum. The maximum nucleation temperature, if reached, would be the melting point which in this logic experiment was $\mathrm{T}_{\text {melt }}=320 \mathrm{~K}$ and is shown at time, $\mathrm{t}=7$. 

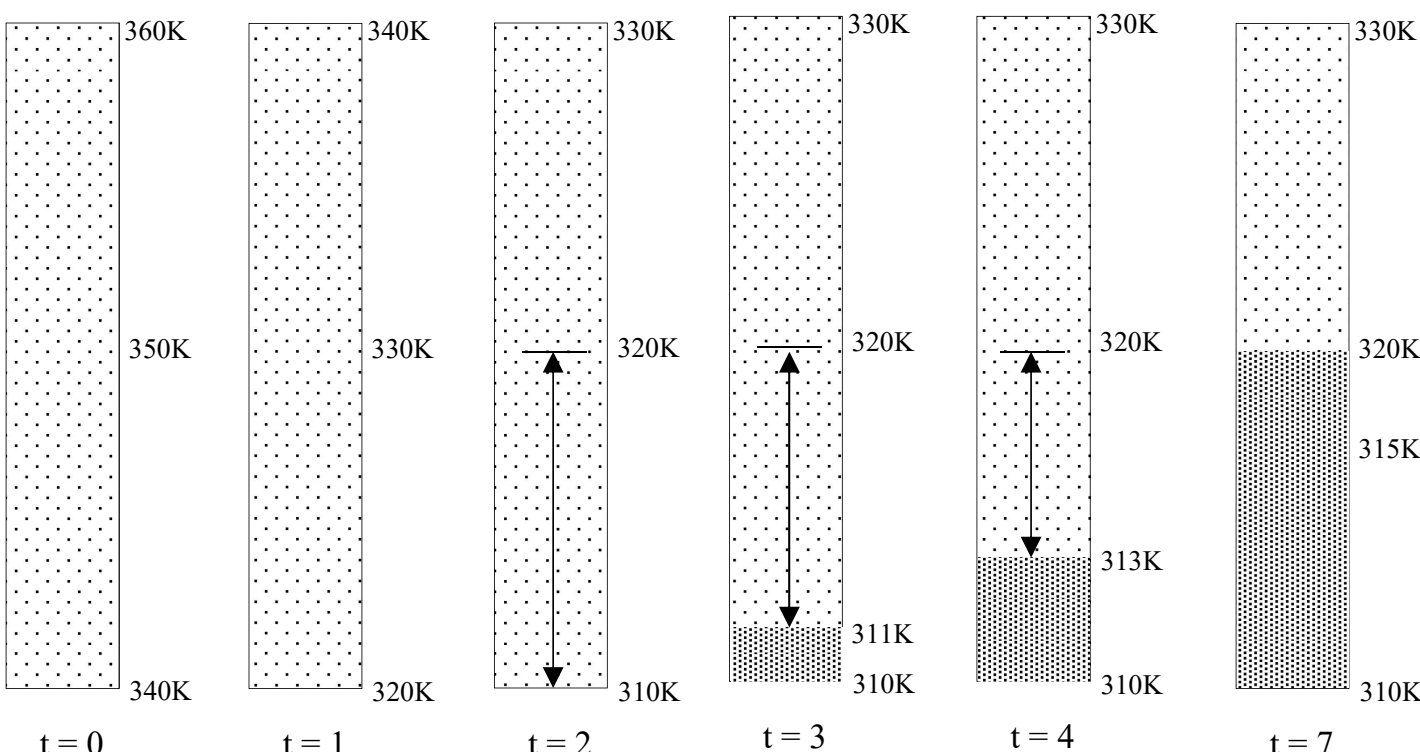

$$
\mathrm{t}=2
$$

$\mathrm{t}=3$

$\mathrm{t}=7$

$\mathrm{T}_{\mathrm{n}}=310 \mathrm{~K} \quad \mathrm{~T}_{\mathrm{n}}=311 \mathrm{~K}$

$\mathrm{T}_{\mathrm{n}}=313 \mathrm{~K}$

$\mathrm{T}_{\mathrm{n}}=\mathrm{T}_{\mathrm{melt}}=320 \mathrm{~K}$

Liquid

Solid

Double arrow indicates subcooled region

Figure 5.18: Schematic of logic experiment for modified crystallization speed by temperature proxy method

From the schematic in Figure 5.18 and the discussion above, the incremental change of the nucleation temperature as the phase front between liquid and solid moves upward along PCM cavity can be thought of as a crystallization velocity. Gunther et al. (2007) have found that an important parameter for numerically predicting subcooling is the "speed of crystallization". For their work on sodium acetate trihydrate, $\mathrm{NaOAc} \cdot 3 \mathrm{H}_{2} \mathrm{O}$ they used an equation in the form of Equation 5.26. However, the speed of crystallization is something that would need to be measured experimentally. 
For the specific geometry of the current experimental apparatus explained in Chapter 4 and the numerical domain explained previously, the expected behavior for nucleation is similar to what was discussed in the logic experiment which is shown in Figure 5.18. The nucleation temperature would increase along with the phase front. In terms of the enthalpy-porosity method in ANSYS Fluent, the practical implication is that the solidification temperature would increase with time. However, the manner of increase and the magnitude of increase would be related to the speed of crystallization.

To implement the effect of subcooling into ANSYS Fluent, the proposed method is to estimate the speed of crystallization from the experimental results by looking at the propagation of the temperature jump along the center line temperatures. From this speed of crystallization, the increase in nucleation temperature with time can be estimated. For example, in the logic experiment in Figure 5.18, the nucleation temperature increases by $1 \mathrm{~K}$ from $\mathrm{t}=2 \mathrm{to} t=3$, then increases by $2 \mathrm{~K}$ from $\mathrm{t}=3$ to $\mathrm{t}=4$. Then the solidification temperature in the enthalpy-porosity method is changed to match the increase in nucleation temperature. Therefore, setting the solidification temperature to match the increase in nucleation temperature becomes the mechanism by which temperature can be used as a proxy to recreate the speed of crystallization. The estimation of crystallization velocity and nucleation temperature time rate is explained next.

Crystallization velocity was estimated from the measured temperature jump along the center line temperatures. The center line temperatures was chosen since it is a plane of symmetry where there is no lateral heat flux across the soy wax. Therefore the center line temperatures represents a phase front between liquid and solid phases which only moves vertically from the bottom of the PCM cavity to the top. The center line temperatures for thermocouple locations TC2 through TC8 is shown in Figure 5.19. The speed of crystallization was determined by inspecting the peak 
temperature jump at each thermocouple location and tracking the time at which that peak occurs. These peak temperatures used are highlighted in Figure 5.19 with a red circle. For thermocouple TC2 which is located at $63.5 \mathrm{~mm}$ from the bottom edge of the PCM panel the peak temperature jump occurs at 43 minutes. For thermocouple TC 8 which is located at $215.9 \mathrm{~mm}$ from the bottom edge has a peak temperature jump occurring at 56 minutes. That is a time period of 13 minutes over a distance of $152.4 \mathrm{~mm}$ resulting in a speed of crystallization of $0.195 \mathrm{~mm} / \mathrm{s}$. For comparison, the speed of crystallization in the work of Gunther et al. 2007 for sodium acetate trihydrate, $\mathrm{NaOAc} \cdot 3 \mathrm{H}_{2} \mathrm{O}$, was $0.382 \mathrm{~mm} / \mathrm{s}$. For the modified method to be implement in ANSYS Fluent, the speed of crystallization is not used directly. Rather, a characteristic temperature rise is found that is associated with the speed of crystallization. The temperature rise was found by taking into account the time it took for the onset of nucleation to travel from TC2 to TC8, then looking at the maximum temperature difference between these times. The temperature and time scales used is highlighted in Figure 5.19 in red rectangles. The period of time chosen was between 35 minutes which is the start of nucleation at thermocouple location TC2 and time 46 minutes which is the start of nucleation at thermocouple location TC8. Within this 11 minute period the maximum temperature difference is $4.23 \mathrm{~K}$ which results in a temperature rise $0.3845 \mathrm{~K} / \mathrm{min}$. 


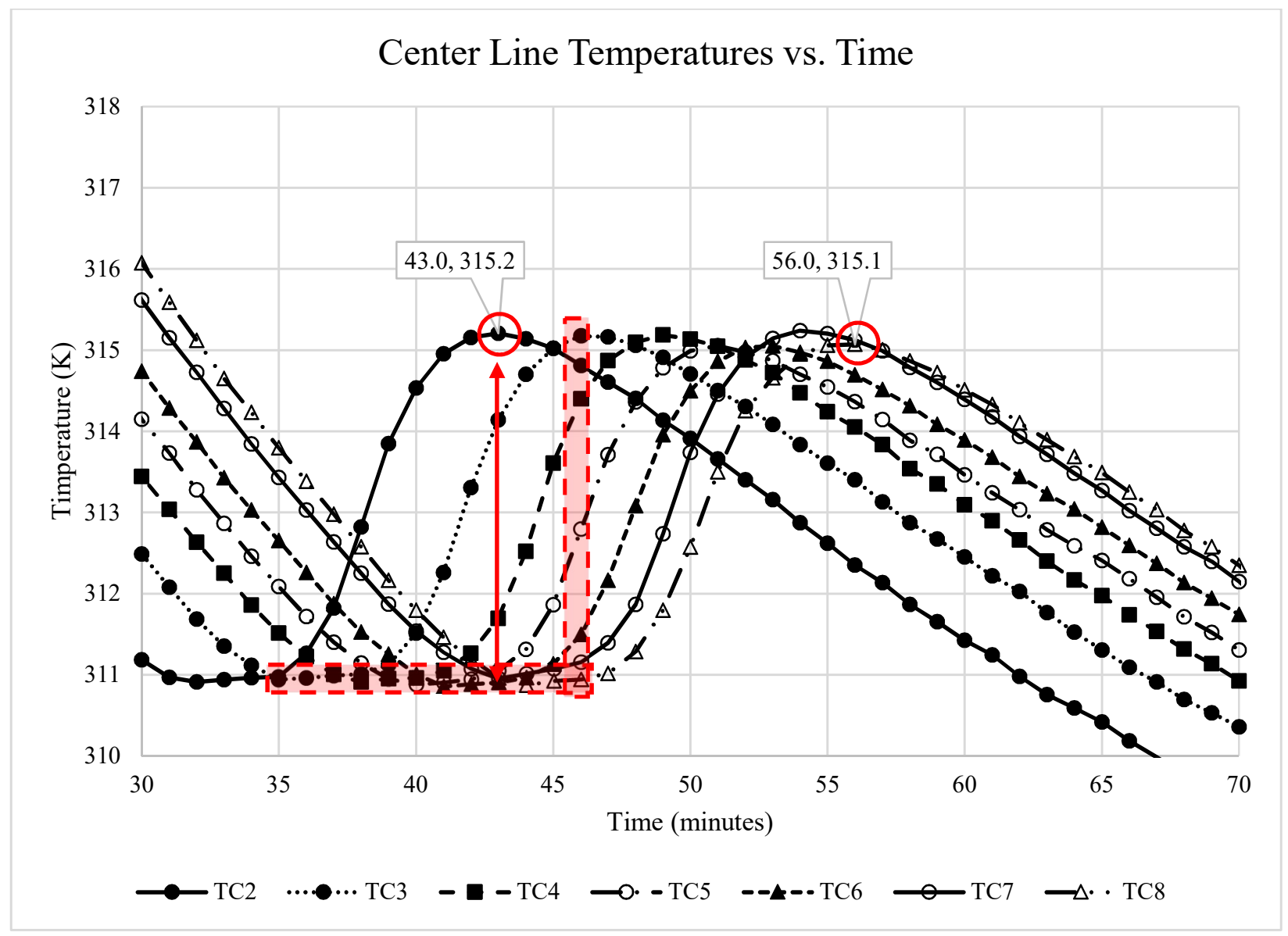

Figure 5.19: Center line temperatures vs. time with speed of crystallization highlights

The numerical simulation performed above was re-run to apply a varying solidification temperature with time. The simulation was run at the same initial conditions and boundary conditions. The temperatures within the PCM cavity was monitored to track for the minimum temperatures. Once the onset of phase change occurred at the model of the PCM cavity, then the modified nucleation temperatures were applied. The solidification temperature was changed by increasing it to match the estimated nucleation temperature using the parameters discussed above. Specifically, the solidification temperature was increased by $0.3845 \mathrm{~K}$ every minute for a total of nine minutes. The results of the simulation are discussed next. 
Figure 5.20 contains the simulated center line temperatures using the modified solidification temperature procedure explained above. The equivalent figure for comparison is Figure 5.6 which contains the original CFD simulation for the center line temperatures vs. time. In Figure 5.20 there is now a distinct temperature jump that was not seen before. The simulated inside surface temperatures are shown in Figure 5.21 with the modified solidification temperature which is related to the original CFD results from Figure 5.8. And finally, the simulated outside surface temperatures are shown in Figure 5.22 using the modified solidification temperature and its equivalent figure from the original CFD simulation is in Figure 5.10. In all planes of measurement there is now a temperature jump during the phase change range which then returns to sensible heat transfer when the soy wax is in solid phase. It is important to repeat that the enthalpy-porosity method in ANSYS Fluent is not able to directly account for subcooling effect. The temperature jump presented below is an ad-hoc approximation to account for this behaviour. Also, to accurately simulate subcooling would require the knowledge of additional material properties of the PCM of interest. However, with an understanding of the nucleation process it is possible to simulate the subcooling effect. 


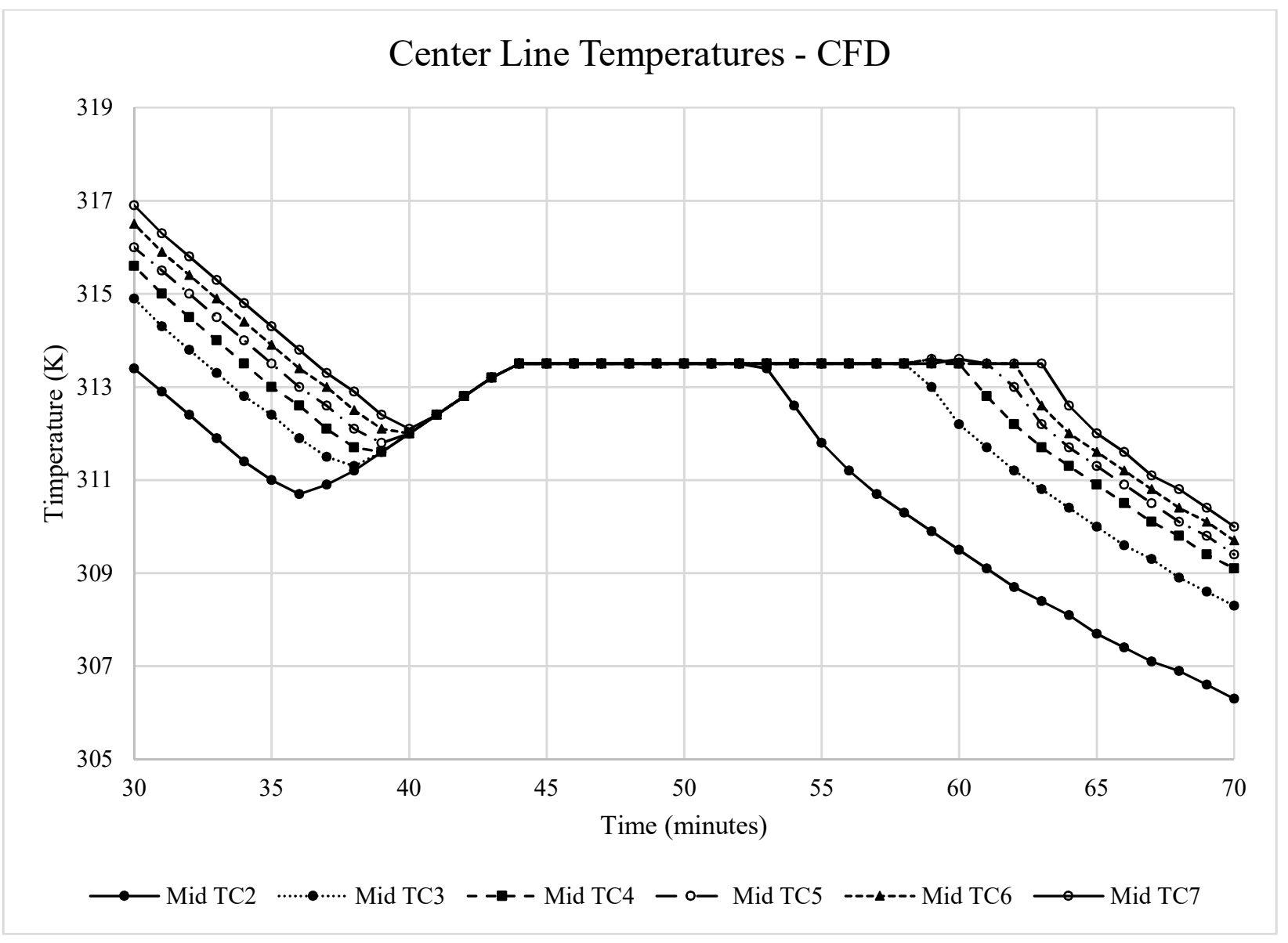

Figure 5.20: CFD center line temperatures vs. time with temperature jump 


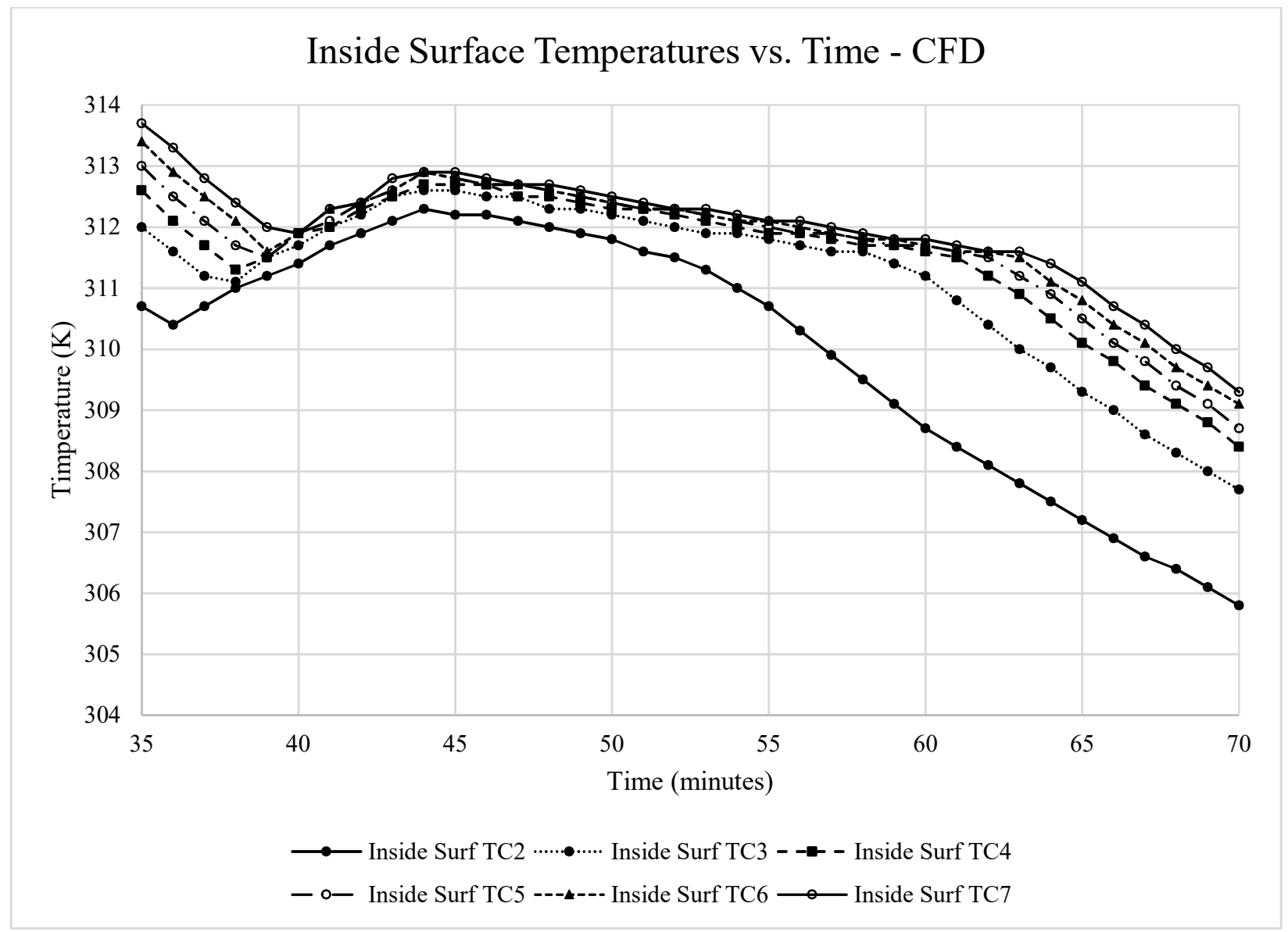

Figure 5.21: CFD inside surface temperatures vs. time with temperature jump 


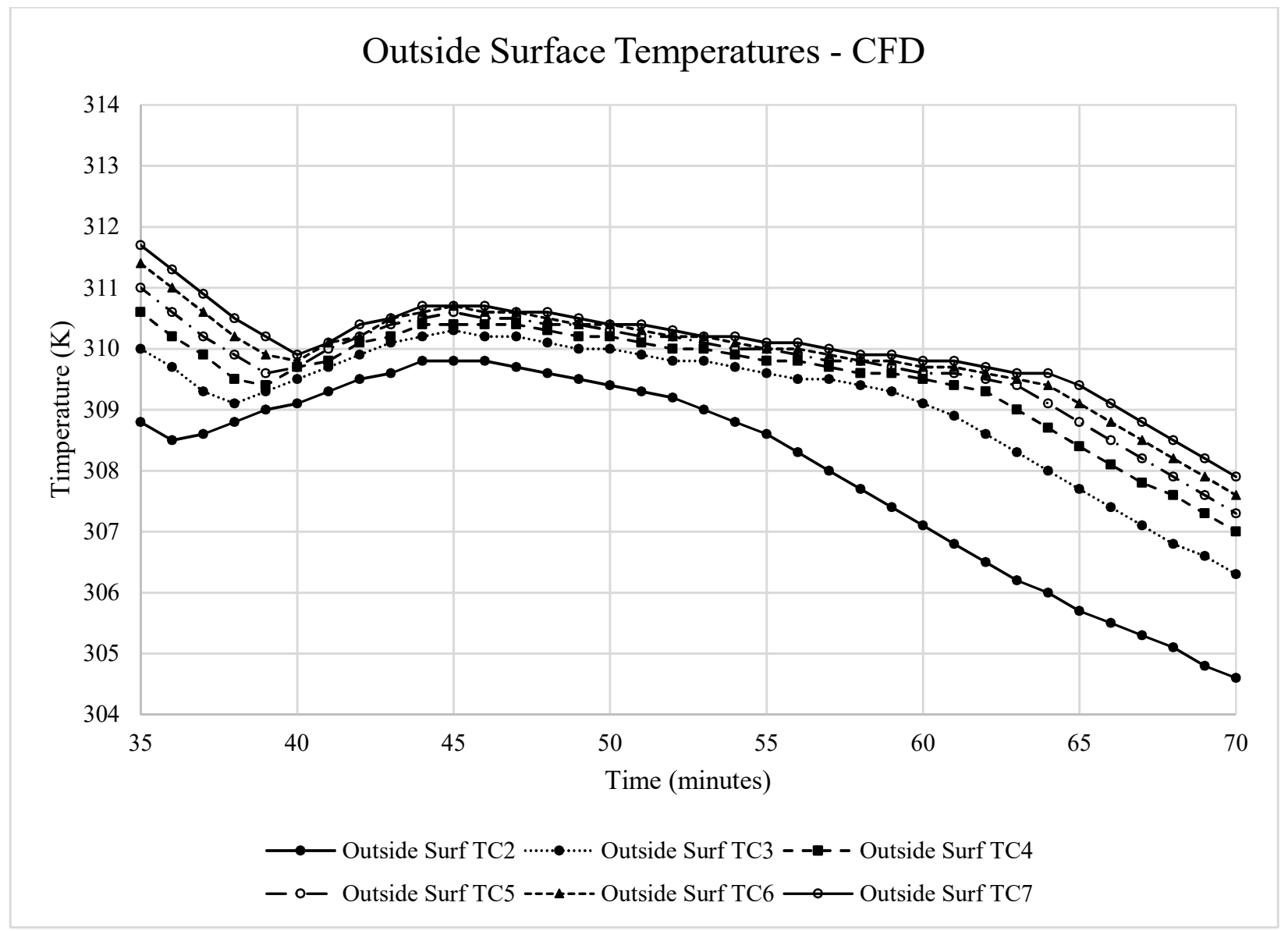

Figure 5.22: CFD outside surface temperatures vs. time with temperature jump 


\section{Chapter 6: Conclusions and Recommendations}

\subsection{Conclusion}

An experimental and numerical study was performed to measure the convective heat transfer and thermal decay of a heated vertical flat panel with soy wax as a phase change material by natural convection. Temperature field visualization and convective heat transfer measurements were obtained using laser interferometry and compared with numerical CFD simulations and established correlations. The primary focus of the current work was to investigate the deviation of convective heat transfer coefficients between the experimentally measured coefficients and the predicted coefficients from established correlations when a PCM panel is undergoing phase change. Finite fringe interferograms were used to obtain surface temperatures and used for heat transfer analysis. For the interferogram analysis, a novel partial fringe methodology was developed that uses ambient air as the reference temperature instead of using a known surface temperature. The optically measured convective heat transfer was used to determine convective heat transfer coefficients and was compared to a Nusselt number correlation. A numerical simulation was performed and compared with the experimental results. To capture the significant subcooling effect of the soy wax an ad-hoc method was implemented in the CFD simulation that estimates the speed of crystallization by a temperature proxy from the experimental data.

The results reproduce and explain the deviation of the measured convective heat transfer coefficients from established correlations that was seen in literature for PCM walls. The deviation of the measured convective heat transfer coefficient is due to the 'non-uniformity' of the PCM panel surface temperature that occurs during phase change. The implementation of crystallization speed in the numerical simulation also reproduced this 'non-uniform' surface temperature and 
reproduced the temperature jump effect due to subcooling that was seen in the experiments and in literature. The current work highlights the inapplicability of the recommended correlations for vertical flat plates undergoing natural convection during the phase change process.

The main findings are summarized as follows:

1) Finite fringe interferograms can be used to determine the surface temperatures and convective heat transfer rates of a heated vertical flat plate.

2) A new method of interferogram analysis was developed based on vertical fringe crossings in the ambient air region and can be used to derive a partial fringe crossing for use on horizontal fringe crossings to determine surface temperatures and convective heat transfer rates.

3) In general, the transient thermal decay of the PCM panel is linear with time when the soy wax is undergoing sensible heat transfer during the liquid only phase and solid only phase.

4) There is a distinct linear temperature variation vertically along the PCM panel at each plane of measurements at the center line, inside plate surface, and outside plate surface temperatures during sensible heat transfer of the soy wax in the liquid only and solid only phases.

5) There is a distinct jump in temperature across all planes of measurement when the soy wax is undergoing phase change. The temperature jump occurs sequentially from the bottom of 
the PCM panel first and propagates upwards. After the phase change is complete, the temperature measurement return to sensible heat transfer behaviour.

6) Convective heat transfer coefficients were determined from optically measured surface temperatures and near-wall temperature gradients using Mach-Zehnder interferometry. There are good agreements (within $\pm 4 \%$ difference) between the optically measured convective heat transfer coefficients and the convective heat transfer coefficients from a Nusselt number correlation, which is for isothermal flat plates experiencing steady free convection, during the sensible heat transfer portion of the soy wax in the liquid only and solid only phases.

7) Related to the above point, there are deviations (up to $14 \%$ lower) between the optically measured convective heat transfer coefficient and the convective heat transfer coefficients from the above-mentioned Nusselt number correlation while the soy wax is undergoing phase change. This is partially a consequence of the variation of the surface temperature and the transient process during phase change. Thus using the above-mentioned Nusselt number correlation can over-estimate convective heat transfer from a PCM wall.

8) The largest deviation in the convective heat transfer coefficient occurs at approximately 40 minutes into the transient decay of the PCM panel. This time period coincides with the initial plateau of the averaged temperatures at each plane of measurement before the temperature jump. 
9) A numerical simulation was compared with experiments and displayed some agreement with the results. The overall trends in the simulation matched with the experiments. Specifically, the sensible heat transfer portions of the experiment and the duration of phase change.

10) However, the original numerical simulation was not able to recreate the temperature jump phenomena that was observed in the experiments. At some thermocouple locations this resulted in a temperature 'shift' of the solid sensible heat transfer portion between the simulation and experiments.

11) An ad-hoc solution to account for this temperature jump was developed based on the speed of crystallization and the subcooling effect seen in PCM.

\subsection{Recommendations}

The following recommendations are made for future studies:

1) Smoke flow visualization is needed to determine, more accurately, the flow behaviour during the transient thermal decay process. In addition it would be useful to see if the flow pattern changes during phase change.

2) Larger scale experiments with a taller PCM panel would be beneficial. One factor in the deviation of the measured convective heat transfer coefficient with a correlation is the variation of the vertical temperature gradient along the panel surface. A taller PCM panel 
might show an even larger variation in the temperature gradient and therefore deviate even more from the correlation.

3) Perform experiments on different phase change materials with different melting/solidification points. The current experiment uses soy wax with a solidification temperature of $38^{\circ} \mathrm{C}$. For the application of PCM in interior building walls, a solidification temperature of $38^{\circ} \mathrm{C}$ is too high. A more practical melting/solidification temperature should be around the set-point temperature of a room (for example $24^{\circ} \mathrm{C}$ ).

4) Perform experiments where the PCM is undergoing melting instead of just solidification. This could be done by using a PCM that has a melting temperature below room temperature. The experiment could be done with the PCM panel in a frozen state and allowed to melt under natural convection.

5) Another reason to do larger scale experiments is to enforce fully turbulent flow. The current experimental apparatus is short enough that despite a large temperature difference between the PCM panel and ambient air, the flow may not be turbulent. Based on the Grashof number criteria the current experimental apparatus might be turbulent during the hot liquid phase and in transition during the phase change range, and laminar during the cold solid phase. Also the choice of the PCM panel characteristic length and choice of surface temperature effects the Grashof number criteria. Therefore it would be useful to perform experiments on a taller PCM panel that enforces turbulence.

6) Perform multiple experiments with the interferometer optics 'tuned' for certain temperature ranges. For the current experiments, the interferometer is used during a large 
range of temperatures with the PCM panel starting at $70^{\circ} \mathrm{C}$ and allowed to cool to room temperature. It is difficult to obtain optical measurements if there are too many fringes in a small space or too few fringes. One way of solving this problem is to repeat the experiments with the interferometer 'tuned' for different temperature ranges. For example, perform one experimental run with the interferometer tuned for 50 to $70^{\circ} \mathrm{C}$, then another experimental run tuned for 30 to $50^{\circ} \mathrm{C}$, etc.

7) Additional numerical simulation is needed with special attention to the modeling of a nonNewtonian fluids. The soy wax in the liquid phase is a non-Newtonian fluid and care must be taken to describe the viscosity.

8) Related to the above point, in order to bring the simulation even closer to the experimental results, the material properties of the components used in the experiment needs to be independently measured instead of using published material properties. Since the properties of extruded polystyrene and acrylic glass can vary from product to product.

9) Additional DSC measurements would be needed to capture the material properties of soy wax or other phase change materials including to determine the speed of crystallization. Also, DSC measurements at varying scanning rates are needed to better mimic the temporal temperature change in typical PCM wallboard applications.

10) Further investigation is need to simulate the subcooling effect of PCM and a full implementation of the crystallization speed method needs to be implemented. 


\section{Appendix A: Experimental Uncertainty Analysis}

\section{A.1 Introduction}

The results from the Mach-Zehnder Interferometer (MZI) have a degree of uncertainty from a variety of sources. One of the earliest works involving a MZI to make convective heat transfer measurements was the research of Machin (1997). A detailed description of probable sources of error can be found in Machin's thesis (1997).

The current uncertainty analysis was performed on the results from the finite fringe interferogram image \#470 which corresponds to 40 minutes into the experiment. Interferogram image \#470 is shown in Figure A.1. This case was chosen because it represents typical experimental parameters and offers a range of variables to investigate the errors of the measurements.
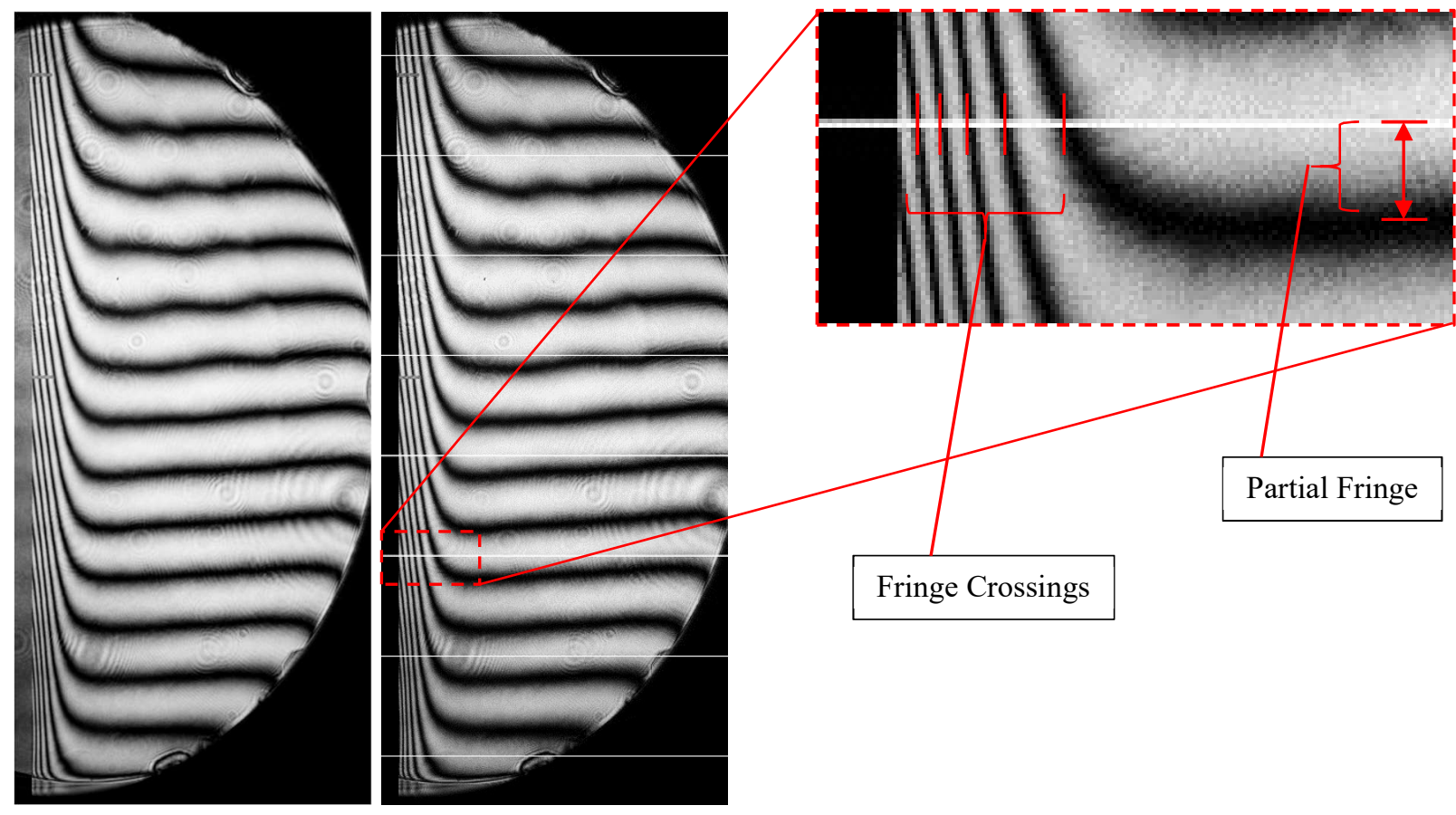

Figure A.1: Interferogram image \#470: Raw image (left); processed image with TC locations (middle); Zoomed on TC3 (right) 
Each interferogram image was analyzed at six thermocouple locations (TC2 through TC7) and each thermocouple location was made up of 17 scans which covers a band of approximately $0.53 \mathrm{~mm}$. The wall temperatures and fringe spacing were determined for each of the 17 scans using the procedures explained in Chapter 3. The wall temperature and fringe spacing at a specific thermocouple location was determined as the average of the 17 scans. The uncertainty analysis was performed for the first scan at TC3. Where appropriate the average of the 17 scans was used to determine the uncertainty in derived parameters such as convective heat transfer, Rayleigh number etc.

In the finite fringe mode, the fringes are set in a horizontal position while the model is unheated. When the model is heated, these fringes bend up in areas of increasing temperature and bend down in areas of decreasing temperature. The accuracy of these fringe bends can be affected by any misalignment of the optics. This misalignment would create an artificial fringe gradient resulting in a fringe shift error.

The digital image processing techniques contain new kinds of errors not found in previous analog image analysis procedures. The main measurement made by the image processing software is the number of pixels between two consecutive fringe peaks. Each fringe peak could be off by one pixel giving a maximum error of two pixels. One pixel equals $0.0142 \mathrm{~mm}$ nominally giving the error in the peak to peak distance as $0.028 \mathrm{~mm}$. By observation, due to the 'noise' in the data, there is an additional pixel worth of potential error. Thus, the total fringe peak to peak error is $0.042 \mathrm{~mm}$.

To quantify the fringe shift error $(\delta \varepsilon)$, the following expression was derived from the geometry of the problem: 


$$
\delta \varepsilon= \pm \frac{\Delta x}{d} \tan \Psi
$$

where $\Delta \mathrm{x}$ is the fringe distance between two consecutive fringes, $\mathrm{d}$ is the fringe spacing, and $\Psi$ is the fringe misalignment relative to the horizontal. Before every experiment, the finite fringe interferograms were set at a spacing of $d=1 \mathrm{~mm}$ while the model was unheated. By inspection, it was determined that the maximum the fringe alignment could be off by is approximately $\varphi=1^{\circ}$. With all of these parameters known, the fringe shift error was calculated to be \pm 0.014 . A summary of the uncertainties and the propagated errors are shown in Table A.1.

Table A.1 Uncertainty in measured quantities

\begin{tabular}{|l|l|}
\hline Quantity & Uncertainty \\
\hline Ambient pressure $\delta \mathrm{P}$ & $\pm 66.64 \mathrm{~Pa}$ \\
\hline Ambient Temp $\delta \mathrm{T}_{\infty}$ & $\pm 0.5 \mathrm{~K}$ \\
\hline Optical length of plate $\delta \mathrm{W}$ & $\pm 0.005 \mathrm{~m}$ \\
\hline Height of Plate $\delta \mathrm{H}$ & $\pm 0.0005 \mathrm{~m}$ \\
\hline Fringe distance $\delta \Delta \mathrm{x}$ & $\pm 0.000028 \mathrm{~m}$ \\
\hline Fringe shift $\delta \varepsilon$ & \pm 0.014 \\
\hline
\end{tabular}

The experimental uncertainty was determined by using the methods of Kline and McClintock (1953). This method assumes that all the uncertainties have the same odds of occurring. Given the result $\mathrm{R}$ is a function of $\mathrm{n}$ independent variables, $\mathrm{x}_{1}, \mathrm{x}_{2}, \mathrm{x}_{3}, \ldots, \mathrm{x}_{\mathrm{n}}$ then;

$$
R=R\left(x_{1}, x_{2}, x_{3}, \ldots, x_{n}\right)
$$

And letting $\mathrm{w}_{\mathrm{R}}$ be the uncertainty of $\mathrm{R}$ and $\mathrm{w}_{1}, \mathrm{w}_{2}, \mathrm{w}_{3}, \ldots, \mathrm{w}_{\mathrm{n}}$ be the uncertainty of the independent variables, then the uncertainty of $\mathrm{R}$ can be expressed as follows:

$$
w_{R}=\left[\left(\frac{\partial R}{\partial x_{1}} w_{1}\right)^{2}+\left(\frac{\partial R}{\partial x_{2}} w_{2}\right)^{2}+\cdots+\left(\frac{\partial R}{\partial x_{n}} w_{n}\right)^{2}\right]^{1 / 2}
$$




\section{A.2 Uncertainty in Fringe Temperatures}

From Chapter 3, the formulation to determine the fringe temperature is given by Equation A.3:

$$
T=\frac{T_{R E F}}{1 \pm \frac{\varepsilon R \lambda T_{R E F}}{G P W}}
$$

To determine the surface temperature, Equation A.2 is applied at each fringe crossing from the partial fringe in the ambient region all the way to the last fringe near the wall. Errors in the first fringe crossing will propagate through the remaining fringe crossings. Due to this propagation the error in the wall surface temperature needs to account for the error in all the fringe crossing temperatures. The first fringe crossing labeled ' $\mathrm{T}_{1}$ ' is shown in Equation A.4.

$$
T_{1}=\frac{T_{\infty}}{1-\frac{\delta \varepsilon R \lambda T_{\infty}}{G P W}}
$$

where $T_{\infty}$, is the ambient temperature and $\delta \varepsilon$, is the partial fringe order. Applying Equation A.1 to Equation A.4 results in the following expression for the error in the first fringe crossing temperature shown in Equation A.5.

$$
w_{T_{1}}=\left[\left(\frac{\partial T_{1}}{\partial P} w_{P}\right)^{2}+\left(\frac{\partial T_{1}}{\partial W} w_{W}\right)^{2}+\left(\frac{\partial T_{1}}{\partial T_{\infty}} w_{T_{\infty}}\right)^{2}+\left(\frac{\partial T_{1}}{\partial \delta \varepsilon} w_{\delta \varepsilon}\right)^{2}\right]^{1 / 2}
$$

To solve Equation A.5 the four derivatives need to be known. The expression for each derivative is shown below from Equation A.6 through Equation A.9.

$$
\begin{gathered}
\frac{\partial T_{1}}{\partial P}=\frac{R T_{\infty}^{2} \delta \epsilon \lambda}{G P^{2} W\left(\frac{R T_{\infty} \delta \epsilon \lambda}{G P W}-1\right)^{2}}=-8.4546 \times 10^{-6} \\
\frac{\partial T_{1}}{\partial W}=\frac{R T_{\infty}^{2} \delta \epsilon \lambda}{G P W^{2}\left(\frac{R T_{\infty} \delta \epsilon \lambda}{G P W}-1\right)^{2}}=-2.4091
\end{gathered}
$$




$$
\begin{gathered}
\frac{\partial T_{1}}{\partial T_{\infty}}=\frac{R T_{\infty} \delta \epsilon \lambda}{G P W\left(\frac{R T_{\infty} \delta \epsilon \lambda}{G P W}-1\right)^{2}}-\frac{1}{\frac{R T_{\infty} \delta \epsilon \lambda}{G P W}-1}=1.0057 \\
\frac{\partial T_{1}}{\partial \delta \varepsilon}=\frac{R T_{\infty}^{2} \lambda}{G P W\left(\frac{R T_{\infty} \delta \epsilon \lambda}{G P W}-1\right)^{2}}=1.9873
\end{gathered}
$$

Subbing in the results from Equation A.6 through Equation A.9 into Equation A.5, the uncertainty of the first fringe crossing temperature is $\pm 0.5038 \mathrm{~K}$.

The process explained above to find the uncertainty in the first fringe crossing temperature can be repeated for the remaining fringe crossings. Equation A.4 through Equation A.9 can be reused as is for each 'next' fringe crossing temperature. For the first fringe crossing solved above, the partial fringe order was used since the reference temperature was from the ambient environment. For the remaining fringe crossing, the fringe order is equal to $\varepsilon=1$ since the reference temperature comes from a known fringe crossing. For the specific case of interferogram image $\# 470$, there are a total of 5 fringe crossings which is shown on the right hand side in Figure A.1. Each calculated fringe temperature uncertainty becomes the uncertainty for the reference temperature for the next fringe crossing temperature. A summary of the fringe crossings, along with their corresponding temperatures and uncertainty is shown in Table A.2.

Table A.2 Fringe Crossing Temperature Uncertainty

\begin{tabular}{|c|c|c|c|c|}
\hline $\begin{array}{c}\text { Fringe } \\
\text { Crossing } \\
\#\end{array}$ & $\begin{array}{c}\text { Reference } \\
\text { Temperature } \\
(\mathrm{K})\end{array}$ & $\begin{array}{c}\text { Uncertainty in } \\
\text { Reference Fringe } \\
\text { Temperature (K) }\end{array}$ & $\begin{array}{c}\text { Fringe } \\
\text { Temperature } \\
(\mathrm{K})\end{array}$ & $\begin{array}{c}\text { Uncertainty in } \\
\text { Fringe } \\
\text { Temperature (K) }\end{array}$ \\
\hline Ambient & N/A & N/A & 297.65 & \pm 0.5 \\
\hline 1 & 297.65 & \pm 0.5 & 298.70 & \pm 0.5038 \\
\hline 2 & 298.70 & \pm 0.5038 & 300.74 & \pm 0.5121 \\
\hline 3 & 300.74 & \pm 0.5121 & 302.79 & \pm 0.5206 \\
\hline 4 & 302.79 & \pm 0.5206 & 304.85 & \pm 0.5293 \\
\hline 5 & 304.85 & \pm 0.5293 & 306.96 & \pm 0.5382 \\
\hline
\end{tabular}




\section{A.3 Uncertainty in Wall Surface Temperature}

The wall surface temperature is determined from linear interpolation from the two nearest fringe crossings and temperatures from the wall. The expression for the wall surface temperature is shown in Equation A.10.

$$
T_{w}=\left(\frac{x_{w}-F X_{5}}{F X_{4}-F X_{5}}\right)\left(T_{4}-T_{5}\right)+T_{5}
$$

Applying Equation A.2 results in the following expressions for the uncertainty for wall surface temperature.

$$
\begin{gathered}
w_{T_{w}}=\left[\left(\frac{\partial T_{w}}{\partial F X_{5}} w_{F X_{5}}\right)^{2}+\left(\frac{\partial T_{w}}{\partial F X_{4}} w_{F X_{4}}\right)^{2}+\left(\frac{\partial T_{w}}{\partial x_{w}} w_{x_{w}}\right)^{2}+\left(\frac{\partial T_{w}}{\partial T_{4}} w_{T_{4}}\right)^{2}+\left(\frac{\partial T_{w}}{\partial T_{5}} \delta w_{T_{5}}\right)^{2}\right]^{1 / 2} \\
\frac{\partial T_{w}}{\partial F X_{5}}=-\frac{T_{4}-T_{5}}{F X_{4}-F X_{5}}-\frac{\left(T_{4}-T_{5}\right)\left(F X_{5}-x_{w}\right)}{\left(F X_{4}-F X_{5}\right)^{2}}=2.4270 e+03 \\
\frac{\partial T_{w}}{\partial F X_{4}}=\frac{\left(T_{4}-T_{5}\right)\left(F X_{5}-x_{w}\right)}{\left(F X_{4}-F X_{5}\right)^{2}}=-983.5232 \\
\frac{\partial T_{w}}{\partial x_{w}}=\frac{T_{4}-T_{5}}{F X_{4}-F X_{5}}=-1.4435 e+03 \\
\frac{\partial T_{w}}{\partial T_{4}}=-\frac{F X_{5}-x_{w}}{F X_{4}-F X_{5}}=-0.6813 \\
\frac{\partial T_{w}}{\partial T_{5}}=\frac{F X_{5}-x_{w}}{F X_{4}-F X_{5}}+1=1.6813
\end{gathered}
$$

Combining the results from Equation A.12 through Equation A.16 and submitting into Equation A.11 results in an uncertainty of the wall surface temperature of $\pm 0.9777 \mathrm{~K}$.

\section{A.4 Uncertainty in Air Properties}

To describe the uncertainty in the air material properties it is useful to discuss total uncertainty and the components that make up total uncertainty. Total uncertainty can be thought of to be made up of bias uncertainty and random uncertainty which can be referred to as systematic 
and precision uncertainty respectively. With those two components of total uncertainty defined an expression for total uncertainty can be developed and is shown in Equation A.17.

$$
\delta R_{\text {total }}=\left[\left(\delta R_{\text {bias }}\right)^{2}+\left(\delta R_{\text {random }}\right)^{2}\right]^{\frac{1}{2}}
$$

In the current experimental results the air material properties are used to determine the convective heat transfer rate from the PCM wall panel and are used to evaluate convective heat transfer correlations. For the convective heat transfer rates shown in Equation 3.2 the thermal conductivity of air is needed and is evaluated at the wall surface temperature. The convective heat transfer correlations of Churchill and Chu (1975) are evaluated at the air film temperature. An expression for film temperature is shown in Equation A.18 along with the uncertainty shown in Equation A.19 and Equation A.20.

$$
\begin{gathered}
T_{f}=\frac{T_{w}+T_{\infty}}{2} \\
w_{T_{f}}=\left[\left(\frac{\partial T_{f}}{\partial T_{w}} w_{T_{w}}\right)^{2}+\left(\frac{\partial T_{f}}{\partial T_{\infty}} w_{T_{\infty}}\right)^{2}\right]^{1 / 2} \\
\frac{\partial T_{f}}{\partial T_{w}}=\frac{\partial T_{f}}{\partial T_{\infty}}=\frac{1}{2}
\end{gathered}
$$

The uncertainty in wall temperature and ambient temperature was already determined above with $w_{T_{w}} \pm 0.9777 \mathrm{~K}$ and $w_{T_{\infty}} \pm 0.5 \mathrm{~K}$. Subbing these uncertainties into Equation A.19 the uncertainty in film temperature is $\pm 0.5491 \mathrm{~K}$. Note that the conductivity of air in Equation 3.2 is evaluated at the surface temperature $\mathrm{T}_{\mathrm{w}}$.

For the current experimental analysis all air properties were evaluated using the temperature dependent regression correlations from Touloukian and Makita (1975) for dynamic viscosity, thermal conductivity and specific heat. An expression for dynamic viscosity, thermal 
conductivity and specific heat are shown in Equation A.21a, Equation A.21b, and Equation A.21c respectively and coefficients are shown in Table A.3. Equation A.21 were developed by applying regression techniques on measured experimental data. The uncertainty in Equations A.21 can be obtained from scatter charts from Touloukian et al. (1975) for dynamic viscosity, Touloukian et al. (1970) for thermal conductivity, and Touloukian and Makita (1970) for specific heat. The uncertainty from scatter charts are shown along with their respective equations in Equations A.21.

$$
\begin{gathered}
\mu=D_{\mu}\left[b_{0}^{\mu}+b_{1}^{\mu} T+b_{2}^{\mu} T^{2}\right] \quad ; \delta \mu_{\text {scatter }}= \pm 0.005 \\
k=D_{k}\left[b_{0}^{k}+b_{1}^{k} T+b_{2}^{k} T^{2}\right] ; \delta k_{\text {scatter }}= \pm 0.01 \\
c_{p}=4184\left[b_{0}^{c_{p}}+b_{1}^{c_{p}} T+b_{2}^{c_{p}} T^{2}+b_{3}^{c_{p}} T^{3}\right] \quad ; \delta c_{p_{\text {scatter }}}= \pm 0.0025
\end{gathered}
$$

Table A.3 Regression Coefficients

\begin{tabular}{|c|c|c|c|c|c|}
\hline \multicolumn{2}{|c|}{ Dynamic Viscosity } & \multicolumn{2}{c|}{ Thermal Conductivity } & \multicolumn{2}{c|}{ Specific Heat } \\
\hline$b_{0}^{\mu}$ & 1.439285714 & $b_{0}^{k}$ & -0.028742857 & $b_{0}^{c_{p}}$ & 0.249679 \\
\hline$b_{1}^{\mu}$ & 0.066607143 & $b_{1}^{k}$ & 0.001188333 & $b_{1}^{c_{p}}$ & $-7.55179 \times 10^{-5}$ \\
\hline$b_{2}^{\mu}$ & $-3.21429 \times 10^{-5}$ & $b_{2}^{k}$ & $-7.38095 \times 10^{-7}$ & $b_{2}^{c_{p}}$ & $1.69194 \times 10^{-7}$ \\
\hline$D_{\mu}$ & 0.00001 & $D_{k}$ & 0.1 & $b_{3}^{C_{p}}$ & $\begin{array}{c}-6.46128 \times 10^{-} \\
11\end{array}$ \\
\hline
\end{tabular}

Combining Equation A.21 and Equation A.2 the uncertainty in the air material properties can be expressed as shown in Equation A.22, Equation A.23, and Equation A.24 evaluated at the film temperature.

$$
\begin{gathered}
\frac{\delta \mu}{\mu}=\left[\left(\delta \mu_{\text {scatter }}\right)^{2}+\left(\frac{\partial \mu}{\partial T_{f}} \frac{w_{T_{f}}}{\mu}\right)^{2}\right]^{1 / 2} \\
\frac{\partial \mu}{\partial T_{f}}=D_{\mu}\left(b_{1}^{\mu}+2 T_{f} b_{2}^{\mu}\right)\left(b_{2}^{\mu} T_{f}^{2}+b_{1}^{\mu} T_{f}+b_{0}^{\mu}\right)^{D-1}=4.6782 e-07
\end{gathered}
$$




$$
\begin{gathered}
\frac{\delta k}{k}=\left[\left(\delta k_{\text {scatter }}\right)^{2}+\left(\frac{\partial k}{\partial T_{f}} \frac{w_{T_{f}}}{k}\right)^{2}\right]^{1 / 2} \\
\frac{\partial k}{\partial T_{f}}=D_{k}\left(b_{1}^{k}+2 T_{f} b_{2}^{k}\right)=7.4159 e-05 \\
\frac{\delta c_{p}}{c_{p}}=\left[\left(\delta c_{p_{\text {scatter }}}\right)^{2}+\left(\frac{\partial c_{p}}{\partial T_{f}} \frac{w_{T_{f}}}{c_{p}}\right)^{2}\right]^{1 / 2} \\
\frac{\partial c_{p}}{\partial T_{f}}=12552 b_{3}^{c_{p}} T_{f}^{2}+8368 b_{2}^{c_{p}} T+4184 b_{1}^{c_{p}}=0.0382
\end{gathered}
$$

Combining Equations A.22, Equations A.23, and Equations A.24 with Equations A.21 and recognizing that the quantity $\delta R /{ }_{R}$ is a percentage or 'relative uncertainty' the uncertainty in dynamic viscosity is $\pm 9.6791 \times 10^{-8} \mathrm{~kg} / \mathrm{m} \cdot \mathrm{s}$, the uncertainty in thermal conductivity is $\pm 2.6642 \times 10^{-}$

${ }^{4} \mathrm{~W} / \mathrm{m} \cdot \mathrm{K}$ and the uncertainty in specific heat is $\pm 2.5160 \mathrm{~J} / \mathrm{kg} \cdot \mathrm{K}$. When evaluated at the wall temperature, the uncertainty in thermal conductivity is $\pm 2.7697 \times 10^{-4} \mathrm{~W} / \mathrm{m} \cdot \mathrm{K}$.

\section{A.5 Uncertainty in Convective Heat Transfer}

From Equation $3.2 \mathrm{~b}$ the expression for the convective heat transfer rate at the wall is shown below in Equation A.25 as a function of measured variables.

$$
q_{w}=\left.k_{s} \frac{d T}{d x}\right|_{w a l l}=k_{s} \frac{R \lambda T_{w}^{2}}{W P G}\left(\frac{\partial \epsilon}{\partial x}\right)=k_{s} \frac{R \lambda T_{w}^{2}}{W P G}\left(\frac{1}{F_{4}-F X_{5}}\right)
$$


In the above equation the fringe gradient, $\partial \epsilon \backslash \partial x$, substituted directly by the near wall measured fringe crossings. Applying Equation A.2 to Equation A.25 results in the following expression for the uncertainty of the convective heat transfer rate shown in Equation A.26.

$$
\begin{gathered}
w_{q_{w}}=\left[\left(\frac{\partial q_{w}}{\partial T_{w}} w_{T_{w}}\right)^{2}+\left(\frac{\partial q_{w}}{\partial P} w_{P}\right)^{2}+\left(\frac{\partial q_{w}}{\partial W} w_{w}\right)^{2}+\left(\frac{\partial q_{w}}{\partial k_{s}} w_{k_{s}}\right)^{2}+\left(\frac{\partial q_{w}}{\partial F X_{4}} w_{F X_{4}}\right)^{2}\right. \\
\left.+\left(\frac{\partial q_{w}}{\partial F X_{5}} w_{F X_{5}}\right)^{2}\right]^{1 / 2}
\end{gathered}
$$

The expressions for each partial derivative is shown below from Equation A.27 through Equation A.32 along with its evaluation.

$$
\begin{gathered}
\frac{\partial q_{w}}{\partial T_{w}}=\frac{2 R T_{w} k_{s} \lambda}{G P W\left(F X_{4}-F X_{5}\right)}=0.2518 \\
\frac{\partial q_{w}}{\partial P}=-\frac{R T_{w}^{2} k_{s} \lambda}{G P^{2} W\left(F X_{4}-F X_{5}\right)}=-3.8320 e-04 \\
\frac{\partial q_{w}}{\partial W}=-\frac{R T_{w}^{2} k_{s} \lambda}{G P W^{2}\left(F X_{4}-F X_{5}\right)}=-109.1884 \\
\frac{\partial q_{w}}{\partial k_{s}}=\frac{R T_{w}^{2} \lambda}{G P W\left(F X_{4}-F X_{5}\right)}=1.4511 e+03 \\
\frac{\partial q_{w}}{\partial F X_{4}}=-\frac{R T_{w}^{2} k_{s} \lambda}{G P W\left(F X_{4}-F X_{5}\right)^{2}}=-2.6563 e+04 \\
\frac{\partial q_{w}}{\partial F X_{5}}=\frac{R T_{w}^{2} k_{s} \lambda}{G P W\left(F X_{4}-F X_{5}\right)^{2}}=2.6563 e+04
\end{gathered}
$$

Submitting Equation A.27 through Equation A.32 into Equation A.26 and using the uncertainties in Table A.1 and the uncertainties calculated above, the uncertainty in the convective heat transfer rate is $\pm 1.0364 \mathrm{~W} / \mathrm{m}^{2}$. 


\section{A.6 Uncertainty in Measured Convective Heat Transfer Coefficient}

With the convective heat transfer rate known, Equation 3.2a can be used to determine the convective heat transfer coefficient as shown in Equation A.33. Here the subscript 'MZI' is used to indicate that the convective heat transfer coefficient is optically measured.

$$
h_{M Z I}=\frac{q_{w}}{T_{w}-T_{\infty}}
$$

Applying Equation A.2 to Equation A.33 gives the expression for the uncertainty in the convective heat transfer coefficient as shown in Equation A.34.

$$
w_{h_{M Z I}}=\left[\left(\frac{\partial h_{M Z I}}{\partial q_{w}} w_{q_{w}}\right)^{2}+\left(\frac{\partial h_{M Z I}}{\partial T_{w}} w_{T_{w}}\right)^{2}+\left(\frac{\partial h_{M Z I}}{\partial T_{\infty}} w_{T_{\infty}}\right)^{2}\right]^{\frac{1}{2}}
$$

The three partial derivatives in Equation A.34 are shown expanded in Equation A.35 and evaluated at the appropriate conditions.

$$
\begin{gathered}
\frac{\partial h_{M Z I}}{\partial q_{w}}=-\frac{1}{T_{\infty}-T_{w}}=0.0923 \\
\frac{\partial h_{M Z I}}{\partial T_{w}}=-\frac{q_{w}}{\left(T_{\infty}-T_{w}\right)^{2}}=-0.3023 \\
\frac{\partial h_{M Z I}}{\partial T_{\infty}}=\frac{q_{w}}{\left(T_{\infty}-T_{w}\right)^{2}}=0.3023
\end{gathered}
$$

Subbing the results of Equation A.35 into Equation A.34 results in an uncertainty in the measured convective heat transfer coefficient of $\pm 0.0 .3455 \mathrm{~W} / \mathrm{m}^{2} \cdot \mathrm{K}$. Recalling that the current uncertainty analysis was performed on the interferogram image \#470 (at time 40 minutes), the measured convective heat transfer coefficient at this time was $3.2743 \mathrm{~W} / \mathrm{m}^{2} \cdot \mathrm{K}$. The uncertainty on the measured convective heat transfer coefficient can be expressed as $\pm 10.55 \%$. 


\section{A.7 Uncertainty in Rayleigh number and Prandtl Number}

Many natural convection corrections are expressed in terms of Nusselt number and is a function of the Rayleigh number 'Ra' and the Prandtl number 'Pr'. The Nusselt number correlation is evaluated using measured parameters and therefore will have an uncertainty associated with it. The Rayleigh number and Prandtl number are defined below in Equation A.36 and Equation A.37 respectively.

$$
\begin{gathered}
\operatorname{Pr}=\frac{c_{p} \mu}{k} \\
R a=\frac{g \rho^{2} \beta\left(T_{w}-T_{\infty}\right) H^{3}}{\mu^{2}} \operatorname{Pr}=\frac{g\left(\frac{P}{R T_{f}}\right)^{2}\left(1 / T_{\infty}\right)\left(T_{w}-T_{\infty}\right) H^{3}}{\mu^{2}} \operatorname{Pr}
\end{gathered}
$$

Note that in Equation A.37, the density of air can be assumed to be an ideal gas and therefore can

be substituted with $\rho=P / R T_{f}$ and the thermal expansion coefficient can be approximated with $\beta=1 / T_{\infty}$. In terms of using Equation A.37 for Nusselt number correlations, the characteristic plate height was chosen to be from the bottom leading edge of the plate to the height of the PCM cavity since it is mainly this region that is involved in convective heat transfer with the environment. Using Equation A.2 an expression for the uncertainty in Prandtl number can be derived and is shown in Equation A.38.

$$
\begin{aligned}
w_{P r} & =\left[\left(\frac{\partial P r}{\partial c_{p}} w_{c_{p}}\right)^{2}+\left(\frac{\partial P r}{\partial \mu} w_{\mu}\right)^{2}+\left(\frac{\partial P r}{\partial k} w_{k}\right)^{2}\right]^{1 / 2} \\
& =\left[\left(\frac{\mu}{k} w_{c_{p}}\right)^{2}+\left(\frac{c_{p}}{k} w_{\mu}\right)^{2}+\left(\frac{-c_{p} \mu}{k^{2}} w_{k}\right)^{2}\right]^{1 / 2}
\end{aligned}
$$


The uncertainty in the air material properties were already derived above and can be submitted into Equation A.38 to get an uncertainty in Prandtl number of \pm 0.0081 .

Similarly, an expression for the uncertainty of Rayleigh number can be derived using Equation A.2 and is shown in Equation A.39.

$$
\begin{gathered}
w_{R a}=\left[\left(\frac{\partial R a}{\partial P} w_{P}\right)^{2}+\left(\frac{\partial R a}{\partial T_{f}} w_{T_{f}}\right)^{2}+\left(\frac{\partial R a}{\partial T_{w}} w_{T_{w}}\right)^{2}+\left(\frac{\partial R a}{\partial T_{\infty}} w_{T_{\infty}}\right)^{2}+\left(\frac{\partial R a}{\partial H} w_{H}\right)^{2}\right. \\
\left.+\left(\frac{\partial R a}{\partial \mu} w_{\mu}\right)^{2}+\left(\frac{\partial R a}{\partial P r} w_{P r}\right)^{2}\right]^{1 / 2}
\end{gathered}
$$

The seven partial derivatives in Equation A.39 are shown below and evaluation in Equation A.40.

$$
\begin{gathered}
\frac{\partial R a}{\partial P}=-\frac{2 H^{3} P \operatorname{Pr} g\left(T_{\infty}-T_{w}\right)}{R^{2} T_{f}^{2} T_{\infty} \mu^{2}}=278.1381 \\
\frac{\partial R a}{\partial T_{f}}=\frac{2 H^{3} P^{2} \operatorname{Pr} g\left(T_{\infty}-T_{w}\right)}{R^{2} T_{f}^{3} T_{\infty} \mu^{2}}=-9.3680 e+04 \\
\frac{\partial R a}{\partial T_{w}}=\frac{H^{3} P^{2} \operatorname{Pr} g}{R^{2} T_{f}^{2} T_{\infty} \mu^{2}}=1.3088 e+06 \\
\frac{\partial R a}{\partial T_{\infty}}=\frac{H^{3} P^{2} \operatorname{Pr} g\left(T_{\infty}-T_{w}\right)}{R^{2} T_{f}^{2} T_{\infty} \mu^{2}}-\frac{H^{3} P^{2} \operatorname{Pr} g}{R^{2} T_{f}^{2} T_{\infty} \mu^{2}}=-1.3565 e+06 \\
\frac{\partial R a}{\partial H}=-\frac{3 H^{2} P^{2} \operatorname{Pr} g\left(T_{\infty}-T_{w}\right)}{R^{2} T_{f}^{2} T_{\infty} \mu^{2}}=1.7624 e+08 \\
\frac{\partial R a}{\partial \mu}=\frac{2 H^{3} P^{2} \operatorname{Pr} g\left(T_{\infty}-T_{w}\right)}{R^{2} T_{f}^{2} T_{\infty} \mu^{3}}=-1.5199 e+12 \\
\frac{\partial R a}{\partial P r}=-\frac{H^{3} P^{2} g\left(T_{\infty}-T_{w}\right)}{R^{2} T_{f}^{2} T_{\infty} \mu^{2}}=1.9882 e+07
\end{gathered}
$$

Substituting in the results from Equation A.40 into Equation A.39 and using the uncertainties from Table A.1 and the uncertainties derived above, the uncertainty of the Rayleigh number is 
$\pm 1.4682 \times 10^{6}$. For the case of interferogram image $\# 470$ (40 minutes into the experiment) the Rayleigh number using Equation A.37 was found to be $1.4175 \times 10^{7}$ which results in a relative uncertainty of $\pm 10.36 \%$. Note that the uncertainties of the constant ' $\mathrm{g}$ ' and ' $\mathrm{R}$ ' was not included in the current analysis.

\section{A.8 Uncertainty in Correlated Convective Heat Transfer Coefficient}

The measured results were compared to the correlation of Churchill and Chu (1975) and shown in Equation A.36 in terms of Nusselt number. The correlation of Churchill and Chu (1975) is for natural convection of a vertical heated flat plate and is a function of air properties and plate geometry. While Equation A.41 is a correlation, some of the parameters needed to evaluate the Prandtl number and Rayleigh number come from experimental measurements and therefore will have an uncertainty associated with it.

$$
N u=\frac{h_{\text {corr } H}}{k}=\left\{0.825+\frac{0.387 R a^{1 / 6}}{\left(1+\left(\frac{0.492}{P r}\right)^{9 / 16}\right)^{8 / 27}}\right\}^{2}
$$

The subscript 'corr' is used to indicate that the heat transfer coefficient corresponds to the Churchill and Chu (1975) correlation. Rearranging Equation A.41 for the convective heat transfer coefficient gives Equation A.42 shown below.

$$
h_{\text {corr }}=\frac{k}{H}\left\{0.825+\frac{0.387 R a^{1 / 6}}{\left(1+\left(\frac{0.492}{P r}\right)^{9 / 16}\right)^{8 / 27}}\right\}^{2}
$$


Applying Equation A.2 on Equation A.42 results in the following expression for the uncertainty in the correlated convective heat transfer coefficient shown in Equation A.43.

$$
w_{h_{c o r r}}=\left[\left(\frac{\partial h_{\text {corr }}}{\partial k} w_{k}\right)^{2}+\left(\frac{\partial h_{\text {corr }}}{\partial H} w_{H}\right)^{2}+\left(\frac{\partial h_{\text {corr }}}{\partial R a} w_{R a}\right)^{2}+\left(\frac{\partial h_{\text {corr }}}{\partial P r} w_{P r}\right)^{2}\right]^{1 / 2}
$$

The four partial derivatives in Equation A.43 are shown below and evaluated in Equation A.44.

$$
\begin{aligned}
& \frac{\partial h_{\text {corr }}}{\partial k}=\frac{1}{H}\left\{0.825+\frac{0.387 R a^{1 / 6}}{\left(1+\left(\frac{0.492}{P r}\right)^{9 / 16}\right)^{8 / 27}}\right\}^{2}=142.9696 \\
& \frac{\partial h_{\text {corr }}}{\partial H}=-\frac{k}{H^{2}}\left\{0.825+\frac{0.387 R a^{1 / 6}}{\left(1+\left(\frac{0.492}{P r}\right)^{9 / 16}\right)^{8 / 27}}\right\}^{2}=-15.5996 \\
& \frac{\partial h_{\text {corr }}}{\partial R a}=\left(\frac{0.129 k}{H R a^{5 / 6}\left(1+\left(\frac{0.492}{P r}\right)^{9 / 16}\right)^{8 / 27}}\right)\left\{0.825+\frac{0.387 R a^{1 / 6}}{\left(1+\left(\frac{0.492}{P r}\right)^{9 / 16}\right)^{8 / 27}}\right\} \\
& =7.6082 e-8 \\
& \frac{\partial h_{\text {corr }}}{\partial P r}=\left(\frac{\left(\frac{15867}{250000}\right) R a^{1 / 6 k}}{\operatorname{HPr}^{2}\left(\frac{0.492}{P r}\right)^{7 / 16}\left(1+\left(\frac{0.492}{P r}\right)^{9 / 16}\right)^{35 / 27}}\right)\left\{0.825+\frac{0.387 R a^{1 / 6}}{\left(1+\left(\frac{0.492}{P r}\right)^{9 / 16}\right)^{8 / 27}}\right\} \\
& =0.6777
\end{aligned}
$$

Substituting the results from Equation A.44 into Equation A.43 results in an uncertainty of the correlation heat transfer coefficient of $\pm 0.1184 \mathrm{~W} / \mathrm{m}^{2} \cdot \mathrm{K}$. At 40 minutes into the experiment the 
calculated heat transfer coefficient using Equation A.42 was found to be $3.7642 \mathrm{~W} / \mathrm{m}^{2} \cdot \mathrm{K}$ which results in a relative uncertainty of the correlated heat transfer coefficient of $\pm 3.1455 \%$.

\section{A.9 Summary of Experimental Uncertainty}

The previous sections of this appendix describes the experimental uncertainty for various measured and derived parameters. The uncertainty analysis was done using the methods of Kline and McClinkton (1953). For convenience a summary of the uncertainty analysis is shown below in Table A.4. The uncertainty analysis was performed using measured quantities from interferogram image \#470 which corresponds to 40 minutes into the experiment. Relative uncertainty was determined from the uncertainty determined in this appendix and the absolute value of the quantity being measured or calculated.

Table A.4 Uncertainty and relative uncertainty in measured and derived quantities

\begin{tabular}{|l|l|c|}
\hline Quantity & Uncertainty & $\begin{array}{c}\text { Relative } \\
\text { Uncertainty }\end{array}$ \\
\hline Ambient pressure $\delta \mathrm{P}$ & $\pm 66.64 \mathrm{~Pa}$ & $\pm 0.07 \%$ \\
\hline Ambient Temp $\delta \mathrm{T}_{\infty}$ & $\pm 0.5 \mathrm{~K}$ & $\pm 0.17 \%$ \\
\hline Optical length of plate $\delta \mathrm{W}$ & $\pm 0.005 \mathrm{~m}$ & $\pm 0.14 \%$ \\
\hline Height of Plate $\delta \mathrm{H}$ & $\pm 0.0005 \mathrm{~m}$ & $\pm 0.18 \%$ \\
\hline Fringe distance $\delta \Delta \mathrm{x}$ & $\pm 0.000028 \mathrm{~m}$ & $\pm 1.77 \%$ \\
\hline Fringe shift $\delta \varepsilon$ & \pm 0.014 & $\pm 2.99 \%$ \\
\hline First fringe crossing temperature & $\pm 0.5038 \mathrm{~K}$ & $\pm 0.17 \%$ \\
\hline Surface temperature & $\pm 0.9777 \mathrm{~K}$ & $\pm 0.32 \%$ \\
\hline Dynamic viscosity & $\pm 9.6791 \times 10^{-8} \mathrm{~kg} / \mathrm{m} \cdot \mathrm{s}$ & $\pm 0.52 \%$ \\
\hline Thermal conductivity @ $\mathrm{T}_{\text {film }}$ & $\pm 2.6642 \times 10^{-4} \mathrm{~W} / \mathrm{m} \cdot \mathrm{K}$ & $\pm 1.01 \%$ \\
\hline Specific heat & $\pm 2.5160 \mathrm{~J} / \mathrm{kg} \cdot \mathrm{K}$ & $\pm 0.25 \%$ \\
\hline Thermal conductivity @ $\mathrm{T}_{\text {wall }}$ & $\pm 1.03 \%$ \\
\hline Convective heat transfer & $\pm 2.7697 \times 10^{-4} \mathrm{~W} / \mathrm{m} \cdot \mathrm{K}$ & $\pm 2.67 \%$ \\
\hline Convective heat transfer coefficient & $\pm 1.0364 \mathrm{~W} / \mathrm{m}^{2}$ & $\pm 10.55 \%$ \\
\hline Prandtl number & $\pm 0.3455 \mathrm{~W} / \mathrm{m}^{2} \cdot \mathrm{K}$ & $\pm 1.14 \%$ \\
\hline Rayleigh number & \pm 0.0081 & $\pm 10.36 \%$ \\
\hline Correlation convective heat transfer coefficient & $\pm 0.1184 \mathrm{~W} / \mathrm{m}^{2} \cdot \mathrm{K}$ & $\pm 3.15 \%$ \\
\hline
\end{tabular}




\section{Appendix B: Tabulated Data}

\section{B. 1 General Format of Data Tables}

The optically measured surface temperatures were determined using the procedure described in Chapter 3. The analysis was performed on a set of images called interferograms. A sample interferogram with sensor locations and grid is shown in Figure B.1. The interferograms are Tagged Image File Format (TIFF) files which treats images as a 2D grid where each cell represents a single pixel. The data described in the tables below references the coordinate system of these TIFF files. In TIFF files, the coordinate system origin is located at top left corner of the image as shown in Figure B.1. The xy-coordinates referenced in the tables below assumes the x-coordinate is in the horizontal direction and the y-coordinate is in the vertical direction.
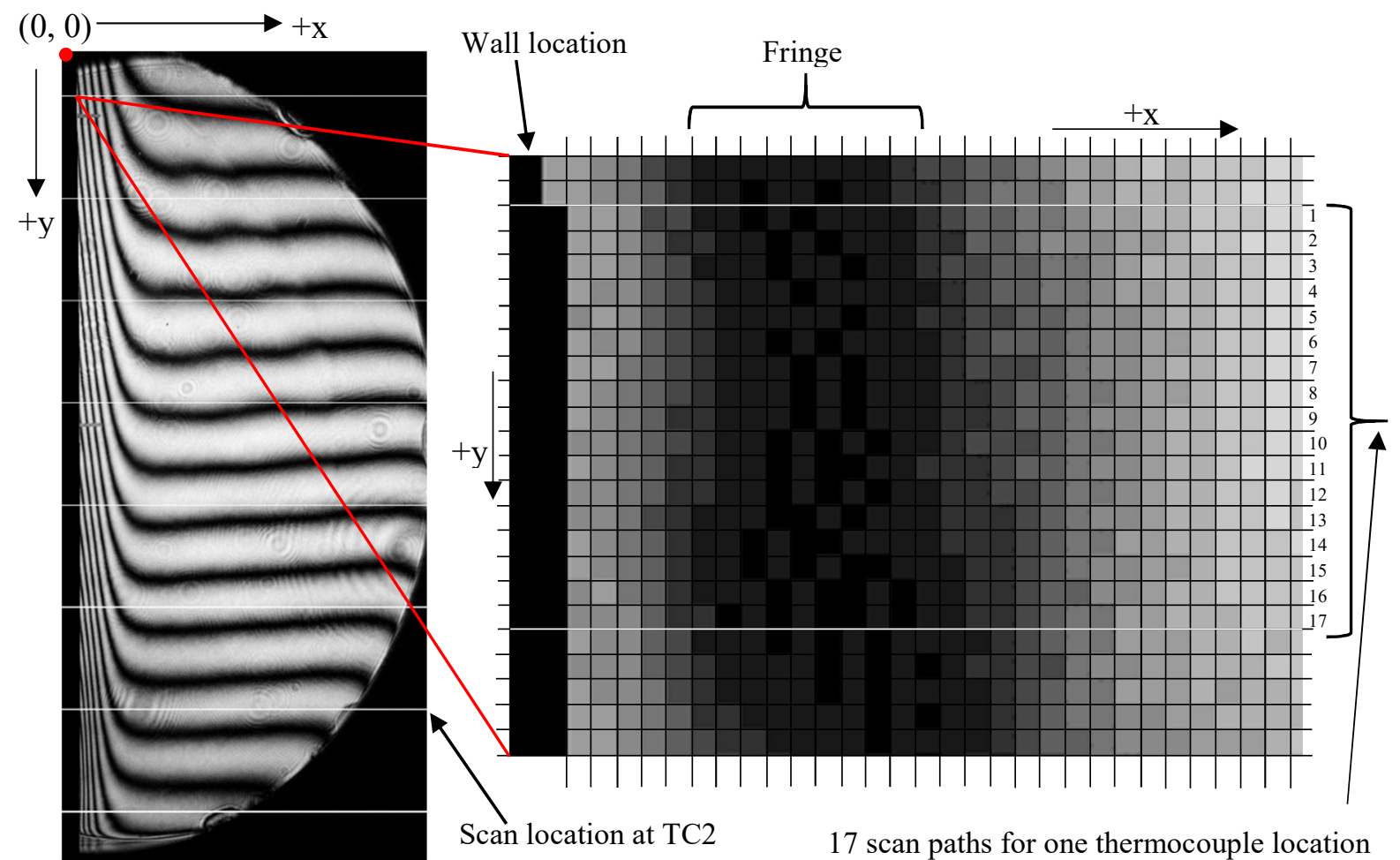

Figure B.1: Sample interferogram with sensor locations and grid 
A sample data table is shown in Table B.1. Each table contains the fringe crossing and fringe temperatures for each sensor location for each image. Optical measurements were performed at locations TC2 through TC7 resulting in 102 measurements per interferogram image. A single table contains data for 17 scans location for one thermocouple location for one image. Note that a single thermocouple location is made up of 17 scans that covers a band of approximately $0.53 \mathrm{~mm}$. Each interferogram image is referenced in terms of image number instead of time period. Table B.2 contains a legend correlating the image number with time. The top row of each table contains a summary of the measurement parameters and results for each scan location. The first entries along the top row contains the interferogram image number and the thermocouple location. The next two entries along the top row contains the measured parameters used to determine the partial fringe crossing that was explained in Chapter 3. The next two entries in the top row contains the averaged near wall fringe spacing and the averaged surface temperature. The last entry along the top row contains the temperature gradient error due to fringe shift error that was explained in Chapter 3.

\section{Table B.1: Sample format of tabulated data}

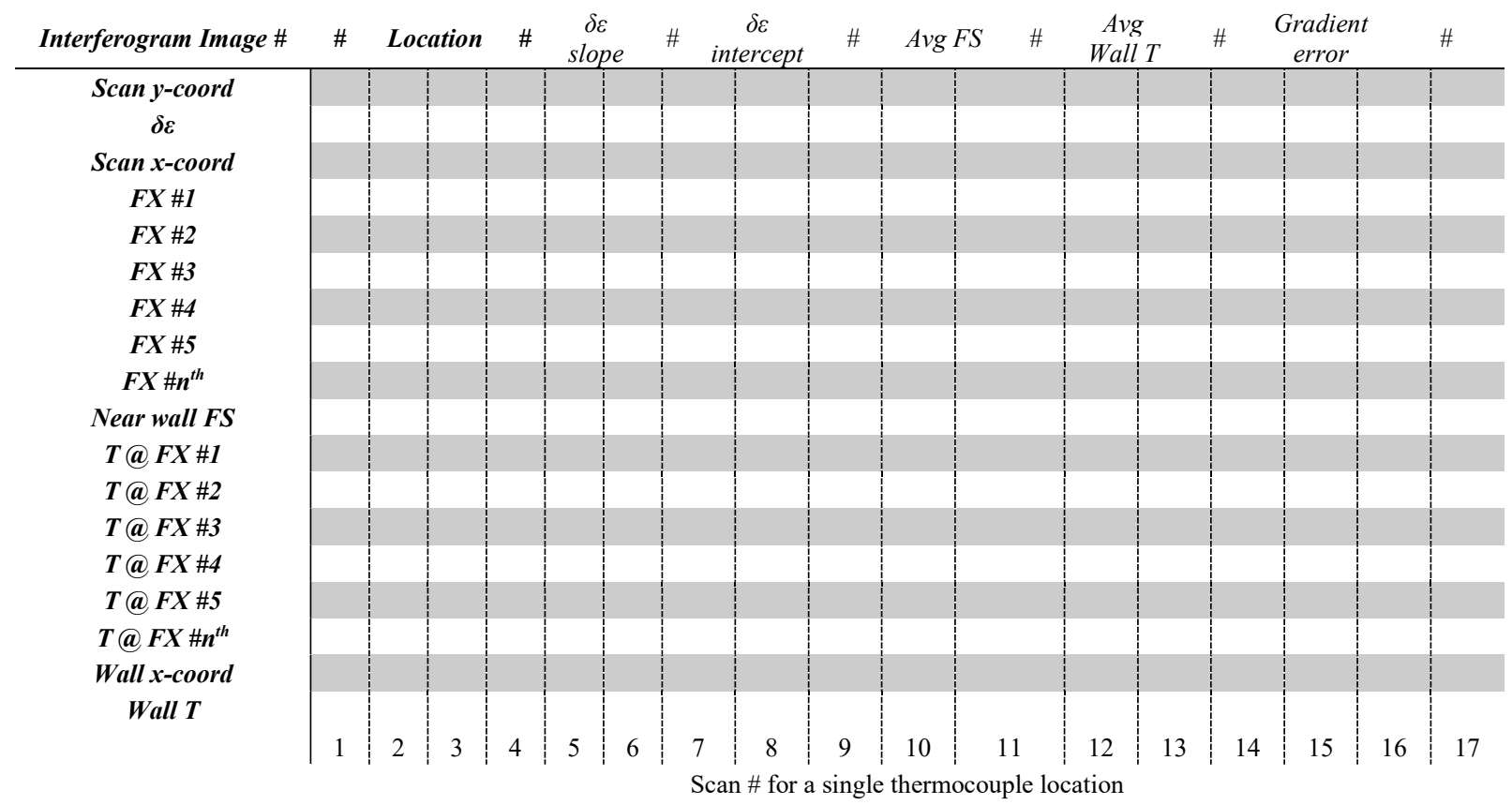


Table B.2: Interferogram image number and time

\begin{tabular}{|cc|cc|cc|}
\hline Time (min) & Image \# & Time (min) & Image \# & Time (min) & Image \# \\
\hline 20 & 450 & 43 & 473 & 58 & 488 \\
25 & 455 & 44 & 474 & 59 & 489 \\
30 & 460 & 45 & 475 & 60 & 490 \\
31 & 461 & 46 & 476 & 61 & 491 \\
32 & 462 & 47 & 477 & 62 & 492 \\
33 & 463 & 48 & 478 & 63 & 493 \\
34 & 464 & 49 & 479 & 64 & 494 \\
35 & 465 & 50 & 480 & 65 & 495 \\
36 & 466 & 51 & 481 & 66 & 496 \\
37 & 467 & 52 & 482 & 67 & 497 \\
38 & 468 & 53 & 483 & 68 & 498 \\
39 & 469 & 54 & 484 & 69 & 499 \\
40 & 470 & 55 & 485 & 70 & 500 \\
41 & 471 & 56 & 486 & 75 & 505 \\
42 & 472 & 57 & 487 & 80 & 510 \\
\hline
\end{tabular}

The rest of the table contains the tabulated data for the 17 scans that make up one thermocouple location. For each scan the y-coordinate is listed in units of pixel relative to the coordinate system shown in Figure B.1. Next the $\delta \varepsilon$, which is a number between 0 and 1 is listed and is determined from the equation of a line using the $\delta \varepsilon$ slope and $\delta \varepsilon$ intercept that is listed along the top row of the table. The $\delta \varepsilon$ corresponds to a specific $\mathrm{x}$-coordinate that is the starting point along the horizontal scan path. The entries in the table next to label "FX \#_" denotes the x-coordinate for each fringe crossing along the horizontal scan path. The last two fringe crossings are used to determine the near wall fringe spacing which is denoted as "Near wall FS". With all the fringe crossings and $\delta \varepsilon$ known, the temperatures can be determined from Equation 3.1 and is listed in the tables and labelled as “T @ FX\#_". Note that there is a temperature for each corresponding fringe crossing. The $\delta \varepsilon$ is used to determine the temperature at the first fringe crossing. Next the $\mathrm{x}-$ coordinate of the wall is listed for each horizontal scan path and is denoted as "Wall x-coord". The 
location of the wall was determined by curve fitting and is explained in Chapter 3. The wall location can be seen in Figure B.1 as the solid black cells on the left hand side of the gridded close up. Using Equation 3.1 along with the near wall fringe spacing and fringe temperatures, the wall temperature is determined by linear interpolation from the $\mathrm{x}$-coordinate of the nearest fringe to the $\mathrm{x}$-coordinate of the wall and is labelled as "Wall T". The parameters explained above are determined for 17 separate scans and is then averaged to give the wall temperature and fringe spacing for that specific thermocouple location and is summarized along the top row of the table.

The remainder of this appendix contains the tabulated data for all measurements made on the chosen interferograms. There is a total of 45 interferograms that was used for the current analysis with six thermocouple locations measured for each image and 17 scans performed for each thermocouple location resulting in 4,590 scans. A summary of the data table labels explained above is shown in Table B.3.

Table B.3: Summary of tabulated data labels

Label

Description

\begin{tabular}{|c|c|}
\hline Interferogram Image \# & Interferogram image number from $450(20 \mathrm{~min})$ to $510(80 \mathrm{~min})$ \\
\hline Location & Thermocouple location from TC2 to TC7 \\
\hline 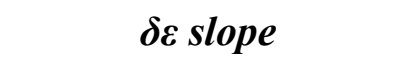 & Parameter used to determine $\delta \varepsilon$ \\
\hline$\delta \varepsilon$ intercept & Parameter used to determine $\delta \varepsilon$ \\
\hline Gradient error & Temperature gradient error due to fringe shift error \\
\hline Scan y-coord & Vertical location of a single scan \\
\hline$\delta \varepsilon$ & Partial fringe order between 0 and 1 \\
\hline Scan $x$-coord & Horizontal location of the start of a single scan \\
\hline$F X \# n^{\text {th }}$ & Horizontal location of the $\mathrm{n}^{\text {th }}$ fringe crossing \\
\hline Near wall FS & Near wall fringe spacing between the two nearest fringe crossings \\
\hline$T @ F X \# n^{t h}$ & Fringe temperature that corresponds to the $\mathrm{n}^{\text {th }}$ fringe crossing \\
\hline Wall $x$-coord & Horizontal location of wall \\
\hline Wall T & Wall temperature corresponding to a single scan \\
\hline Avg FS & $\begin{array}{c}\text { Averaged fringe spacing for a given thermocouple location } \\
\text { determined from } 17 \text { scans }\end{array}$ \\
\hline Avg Wall T & $\begin{array}{l}\text { Averaged wall temperature for a given thermocouple location } \\
\text { determined from } 17 \text { scans }\end{array}$ \\
\hline
\end{tabular}




\section{B.2 Tabulated Data for Interferogram 450}

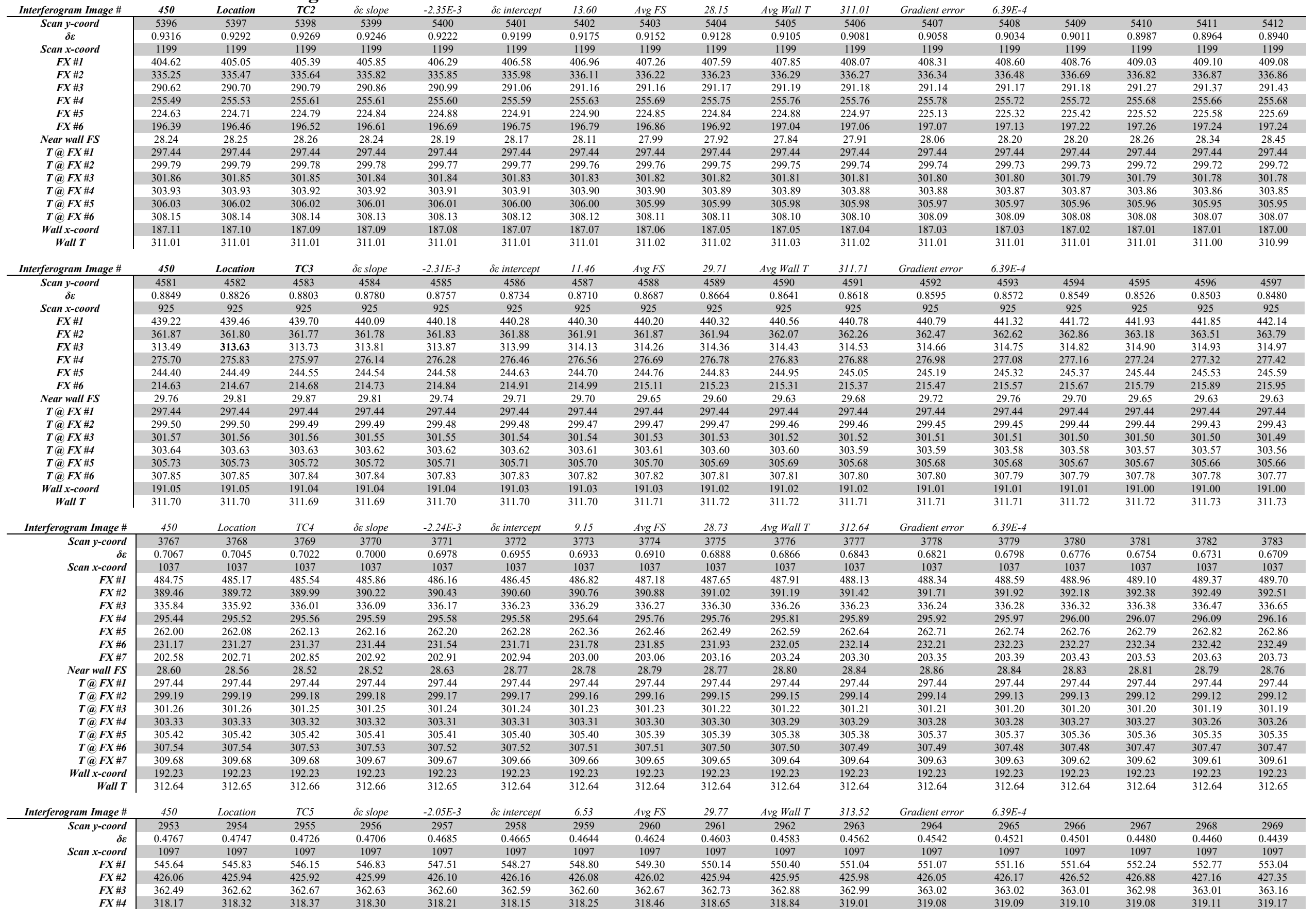




\begin{tabular}{|c|c|c|c|c|c|c|c|c|c|c|c|c|c|c|c|c|c|}
\hline$F X \# 5$ & 281.63 & 281.68 & 281.80 & 281.98 & 282.20 & 282.30 & 282.37 & 282.39 & 282.39 & 282.42 & 282.54 & 282.70 & 282.85 & 283.03 & 283.04 & 283.10 & 283.04 \\
\hline$F X \# 6$ & 249.53 & 249.52 & 249.56 & 249.66 & 249.87 & 250.06 & 250.25 & 250.37 & 250.46 & 250.48 & 250.46 & 250.48 & 250.55 & 250.67 & 250.82 & 250.97 & 250.98 \\
\hline$F X \# 7$ & 219.84 & 220.01 & 220.09 & 220.14 & 220.20 & 220.42 & 220.65 & 220.80 & 220.89 & 220.89 & 220.81 & 220.65 & 220.57 & 220.56 & 220.61 & 220.72 & 220.71 \\
\hline Near wall FS & 29.68 & 29.51 & 29.47 & 29.52 & 29.67 & 29.64 & 29.60 & 29.57 & 29.56 & 29.60 & 29.65 & 29.82 & 29.98 & 30.10 & 30.22 & 30.25 & 30.27 \\
\hline$T @ F X \# 1$ & 297.44 & 297.44 & 297.44 & 297.44 & 297.44 & 297.44 & 297.44 & 297.44 & 297.44 & 297.44 & 297.44 & 297.44 & 297.44 & 297.44 & 297.44 & 297.44 & 297.44 \\
\hline$T @ F X \# 2$ & 298.73 & 298.73 & 298.72 & 298.72 & 298.71 & 298.71 & 298.71 & 298.70 & 298.70 & 298.69 & 298.69 & 298.68 & 298.68 & 298.68 & 298.67 & 298.67 & 298.66 \\
\hline$T$ (a) $F X \# 3$ & 300.81 & 300.81 & 300.80 & 300.80 & 300.80 & 300.79 & 300.79 & 300.78 & 300.78 & 300.78 & 300.77 & 300.77 & 300.76 & 300.76 & 300.75 & 300.75 & 300.75 \\
\hline$T @ F X \# 4$ & 302.88 & 302.88 & 302.88 & 302.87 & 302.87 & 302.86 & 302.86 & 302.86 & 302.85 & 302.85 & 302.84 & 302.84 & 302.83 & 302.83 & 302.83 & 302.82 & 302.82 \\
\hline$T(F X \# 5$ & 304.97 & 304.97 & 304.96 & 304.96 & 304.96 & 304.95 & 304.95 & 304.94 & 304.94 & 304.93 & 304.93 & 304.93 & 304.92 & 304.92 & 304.91 & 304.91 & 304.90 \\
\hline$T @ F X \# 6$ & 307.08 & 307.08 & 307.08 & 307.07 & 307.07 & 307.06 & 307.06 & 307.05 & 307.05 & 307.05 & 307.04 & 307.04 & 307.03 & 307.03 & 307.02 & 307.02 & 307.02 \\
\hline$T @ \boldsymbol{F X} \# 7$ & 309.22 & 309.22 & 309.21 & 309.21 & 309.21 & 309.20 & 309.20 & 309.19 & 309.19 & 309.18 & 309.18 & 309.17 & 309.17 & 309.17 & 309.16 & 309.16 & 309.15 \\
\hline Wall $x$-coord & 190.65 & 190.66 & 190.66 & 190.66 & 190.67 & 190.67 & 190.67 & 190.68 & 190.68 & 190.68 & 190.69 & 190.69 & 190.70 & 190.70 & 190.70 & 190.71 & 190.71 \\
\hline Wall $T$ & 313.52 & 313.54 & 313.54 & 313.54 & 313.53 & 313.54 & 313.56 & 313.56 & 313.57 & 313.56 & 313.54 & 313.52 & 313.49 & 313.48 & 313.47 & 313.47 & 313.46 \\
\hline Interferogram Image \# & 450 & Location & $T C 6$ & $\delta$ s slope & $-2.13 E-3$ & $\delta \varepsilon$ intercept & 4.66 & $\operatorname{Avg} F S$ & 29.41 & Avg Wall T & 314.24 & Gradient error & $6.39 E-4$ & & & & \\
\hline Scan y-coord & 2138 & 2139 & 2140 & 2141 & 2142 & 2143 & 2144 & 2145 & 2146 & 2147 & 2148 & 2149 & 2150 & 2151 & 2152 & 2153 & 2154 \\
\hline & 0.1027 & 0.1006 & 0.0985 & 0.0963 & 0.0942 & 0.0921 & 0.0899 & 0.0878 & 0.0857 & 0.0836 & 0.0814 & 0.0793 & 0.0772 & 0.0750 & 0.0729 & 0.0708 & 0.0686 \\
\hline Scan $x$-coord & 1117 & 1117 & 1117 & 1117 & 1117 & 1117 & 1117 & 1117 & 1117 & 1117 & 1117 & 1117 & 1117 & 1117 & 1117 & 1117 & 1117 \\
\hline$F X \# 1$ & 691.41 & 691.69 & 694.84 & 695.61 & 697.16 & 698.75 & 677.49 & 680.89 & 679.99 & 680.30 & 680.67 & 680.77 & 682.18 & 740.70 & 676.28 & 715.51 & 717.16 \\
\hline$F X \# 2$ & 462.69 & 462.75 & 462.90 & 463.08 & 463.20 & 463.33 & 463.51 & 463.66 & 463.89 & 464.08 & 464.28 & 464.57 & 464.81 & 465.03 & 465.13 & 464.90 & 464.95 \\
\hline$F X \# 3$ & 386.35 & 386.53 & 386.64 & 386.76 & 386.90 & 387.13 & 387.32 & 387.48 & 387.71 & 387.94 & 388.08 & 388.20 & 388.30 & 388.45 & 388.64 & 388.76 & 388.86 \\
\hline$F X \# 4$ & 337.19 & 337.24 & 337.25 & 337.29 & 337.39 & 337.53 & 337.69 & 337.89 & 338.11 & 338.37 & 338.51 & 338.62 & 338.71 & 338.79 & 338.85 & 338.89 & 338.92 \\
\hline$F X \# 5$ & 298.63 & 298.67 & 298.72 & 298.81 & 298.93 & 299.04 & 299.08 & 299.19 & 299.25 & 299.35 & 299.44 & 299.52 & 299.61 & 299.75 & 299.93 & 300.07 & 300.22 \\
\hline$F X \# 6$ & 265.39 & 265.47 & 265.49 & 265.48 & 265.46 & 265.47 & 265.49 & 265.54 & 265.58 & 265.64 & 265.79 & 265.95 & 266.08 & 266.20 & 266.44 & 266.62 & 266.74 \\
\hline$F X \# 7$ & 235.56 & 235.66 & 235.73 & 235.79 & 235.86 & 235.93 & 235.96 & 235.95 & 235.94 & 235.99 & 236.03 & 236.09 & 236.20 & 236.35 & 236.38 & 236.42 & 236.50 \\
\hline$F X \# 8$ & 206.17 & 206.27 & 206.33 & 206.36 & 206.38 & 206.38 & 206.40 & 206.43 & 206.47 & 206.48 & 206.52 & 206.63 & 206.75 & 206.96 & 207.11 & 207.29 & 207.44 \\
\hline Near wall FS & 29.40 & 29.39 & 29.40 & 29.43 & 29.48 & 29.55 & 29.56 & 29.52 & 29.47 & 29.51 & 29.51 & 29.46 & 29.45 & 29.38 & 29.27 & 29.13 & 29.06 \\
\hline$T @ F X \# 1$ & 297.44 & 297.44 & 297.44 & 297.44 & 297.44 & 297.44 & 297.44 & 297.44 & 297.44 & 297.44 & 297.44 & 297.44 & 297.44 & 297.44 & 297.44 & 297.44 & 297.44 \\
\hline$T @ F X \# 2$ & 297.91 & 297.91 & 297.90 & 297.90 & 297.89 & 297.89 & 297.89 & 297.89 & 297.88 & 297.88 & 297.88 & 297.87 & 297.87 & 297.82 & 297.86 & 297.83 & 297.83 \\
\hline$T @ F X \# 3$ & 300.05 & 300.05 & 300.04 & 300.04 & 300.03 & 300.03 & 300.02 & 300.02 & 300.01 & 300.01 & 300.01 & 300.00 & 300.00 & 299.99 & 299.99 & 299.98 & 299.98 \\
\hline$T @ F X \# 4$ & 302.12 & 302.12 & 302.11 & 302.11 & 302.10 & 302.10 & $\begin{array}{l}3002.02 \\
302\end{array}$ & $\begin{array}{l}3000.02 \\
302.09\end{array}$ & 302.08 & 302.08 & 302.08 & 302.07 & 302.07 & 302.06 & 302.06 & 302.05 & 302.05 \\
\hline$T(F X \# 5$ & 304.20 & 304.20 & 304.19 & 304.19 & 304.18 & 304.18 & 304.17 & 304.17 & 304.17 & 304.16 & 304.16 & 304.15 & 304.15 & 304.14 & 304.14 & 304.13 & 304.13 \\
\hline$T @ F X \# 6$ & 306.30 & 306.30 & 306.29 & 306.29 & 306.29 & 306.28 & 306.28 & 306.27 & 306.27 & 306.26 & 306.26 & 306.25 & 306.25 & 306.24 & 306.24 & 306.24 & 306.23 \\
\hline$T$ @ $F X \# 7$ & 308.43 & 308.43 & 308.42 & 308.42 & 308.41 & 308.41 & 308.40 & 308.40 & 308.40 & 308.39 & 308.39 & 308.38 & 308.38 & 308.37 & 308.37 & 308.36 & 308.36 \\
\hline$T @ F X \# 8$ & 310.59 & 310.58 & 310.58 & 310.57 & 310.57 & 310.56 & 310.56 & 310.55 & 310.55 & 310.55 & 310.54 & 310.54 & 310.53 & 310.53 & 310.52 & 310.52 & 310.51 \\
\hline Wall $x$-coord & 186.31 & 186.32 & 186.32 & 186.33 & 186.34 & 186.35 & 186.35 & 186.36 & 186.37 & 186.37 & 186.38 & 186.39 & 186.39 & 186.40 & 186.41 & 186.42 & 186.42 \\
\hline Wall $T$ & 314.25 & 314.25 & 314.25 & 314.25 & 314.24 & 314.23 & 314.23 & 314.23 & 314.23 & 314.22 & 314.22 & 314.22 & 314.23 & 314.24 & 314.25 & 314.27 & 314.28 \\
\hline Interferogram Image \# & 450 & Location & $T C 7$ & $\delta \varepsilon$ slope & $-2.07 E-3$ & $\delta \varepsilon$ intercept & 3.45 & $A v g F S$ & 28.69 & Avg Wall $T$ & 315.26 & Gradient error & $6.39 E-4$ & & & & \\
\hline Scan y-coord & 1324 & 1325 & 1326 & 1327 & 1328 & 1329 & 1330 & 1331 & 1332 & 1333 & 1334 & 1335 & 1336 & 1337 & 1338 & 1339 & 1340 \\
\hline & 0.7067 & 0.7046 & 0.7025 & 0.7005 & 0.6984 & 0.6963 & 0.6942 & 0.6922 & 0.6901 & 0.6880 & 0.6860 & 0.6839 & 0.6818 & 0.6797 & 0.6777 & 0.6756 & 0.6735 \\
\hline Scan $x$-coord & 1332 & 1332 & 1332 & 1332 & 1332 & 1332 & 1332 & 1332 & 1332 & 1332 & 1332 & 1332 & 1332 & 1332 & 1332 & 1332 & 1332 \\
\hline$F X \# 1$ & 526.26 & 526.36 & 526.77 & 526.98 & 527.30 & 527.63 & 528.16 & 528.89 & 529.50 & 529.98 & 530.48 & 531.11 & 531.89 & 532.53 & 532.82 & 532.75 & $\begin{array}{l}1532 \\
532.42\end{array}$ \\
\hline$F X \# 2$ & 422.81 & 422.96 & 423.09 & 423.28 & 423.32 & 423.49 & 423.75 & 424.02 & 424.18 & 424.30 & 424.39 & 424.51 & 424.61 & 424.77 & 424.85 & 424.95 & 425.15 \\
\hline$F X \# 3$ & 363.22 & 363.29 & 363.30 & 363.35 & 363.30 & 363.34 & 363.41 & 363.54 & 363.62 & 363.64 & 363.72 & 363.88 & 363.96 & 364.07 & 364.20 & 364.32 & 364.51 \\
\hline$F X \# 4$ & 319.43 & 319.52 & 319.57 & 319.60 & 319.69 & 319.84 & 319.92 & 319.92 & 319.91 & 319.93 & 320.06 & 320.13 & 320.13 & 320.22 & 320.32 & 320.53 & 320.69 \\
\hline$F X \# 5$ & 282.89 & 282.99 & 283.07 & 283.17 & 283.23 & 283.24 & 283.29 & 283.36 & 283.42 & 283.50 & 283.51 & 283.54 & 283.59 & 283.72 & 283.86 & 283.98 & 284.07 \\
\hline$F X \# 6$ & 250.87 & 251.03 & 251.13 & 251.22 & 251.31 & 251.38 & 251.48 & 251.59 & 251.67 & 251.78 & 251.84 & 251.90 & 251.98 & 252.03 & 252.18 & 252.22 & 252.25 \\
\hline$F X \# 7$ & 220.97 & 221.13 & 221.23 & 221.34 & 221.41 & 221.44 & 221.49 & 221.57 & 221.64 & 221.64 & 221.66 & 221.68 & 221.74 & 221.79 & 221.84 & 221.83 & 221.87 \\
\hline$F X \# 8$ & 192.29 & 192.39 & 192.50 & 192.60 & 192.71 & 192.77 & 192.83 & 192.94 & 192.98 & 193.01 & 193.03 & 193.04 & 193.07 & 193.10 & 193.08 & 193.09 & 193.12 \\
\hline Near wall FS & 28.69 & 28.74 & 28.73 & 28.74 & 28.71 & 28.67 & 28.66 & 28.64 & 28.65 & 28.64 & 28.63 & 28.63 & 28.67 & 28.69 & 28.76 & 28.74 & 28.75 \\
\hline$T @ F X \# 1$ & 297.44 & 297.44 & 297.44 & 297.44 & 297.44 & 297.44 & 297.44 & 297.44 & 297.44 & 297.44 & 297.44 & 297.44 & 297.44 & 297.44 & 297.44 & 297.44 & 297.44 \\
\hline$T(F X \# 2$ & 299.35 & 299.35 & 299.34 & 299.34 & 299.33 & 299.33 & 299.33 & 299.32 & 299.32 & 299.31 & 299.31 & 299.30 & 299.30 & 299.29 & 299.29 & 299.29 & 299.28 \\
\hline$T @ F X \# 3$ & 301.43 & 301.43 & 301.42 & 301.42 & 301.41 & 301.41 & 301.40 & 301.40 & 301.40 & 301.39 & 301.39 & 301.38 & 301.38 & 301.37 & 301.37 & 301.37 & 301.36 \\
\hline$T @ F X \# 4$ & 303.51 & 303.50 & 303.50 & 303.50 & 303.49 & 303.49 & 303.48 & 303.48 & 303.47 & 303.47 & 303.47 & 303.46 & 303.46 & 303.45 & 303.45 & 303.44 & 303.44 \\
\hline$T @ F X \# 5$ & 305.60 & 305.60 & 305.60 & 305.59 & 305.59 & 305.58 & 305.58 & 305.57 & 305.57 & 305.57 & 305.56 & 305.56 & 305.55 & 305.55 & 305.54 & 305.54 & 305.54 \\
\hline$T @ F X \# 6$ & 307.73 & 307.72 & 307.72 & 307.71 & 307.71 & 307.70 & 307.70 & 307.69 & 307.69 & 307.69 & 307.68 & 307.68 & 307.67 & 307.67 & 307.66 & 307.66 & 307.65 \\
\hline$T @ F X \# 7$ & 309.87 & 309.87 & 309.86 & 309.86 & 309.85 & 309.85 & 309.85 & 309.84 & 309.84 & 309.83 & 309.83 & 309.82 & 309.82 & 309.81 & 309.81 & 309.81 & 309.80 \\
\hline$T(\boldsymbol{F} X \# 8$ & 312.05 & 312.04 & 312.04 & 312.03 & 312.03 & 312.03 & 312.02 & 312.02 & 312.01 & 312.01 & 312.00 & 312.00 & 311.99 & 311.99 & 311.98 & 311.98 & 311.98 \\
\hline Wall $x$-coord & 179.22 & 179.23 & 179.24 & 179.25 & 179.26 & 179.27 & 179.28 & 179.29 & 179.30 & 179.31 & 179.32 & 179.33 & 179.34 & 179.35 & 179.36 & 179.37 & 179.38 \\
\hline Wall T & 315.26 & 315.26 & 315.26 & 315.26 & 315.27 & 315.27 & 315.27 & 315.27 & 315.27 & 315.27 & 315.26 & 315.26 & 315.25 & 315.25 & 315.24 & 315.24 & 315.23 \\
\hline \multicolumn{18}{|c|}{ B.3 Tabulated Data for Interferogram 455} \\
\hline Interferogram Image \# & 455 & Location & $T C 2$ & $\delta \varepsilon$ slope & $-2.41 E-3$ & $\delta \varepsilon$ intercept & 13.28 & $\operatorname{Avg} F S$ & 36.07 & Avg Wall T & 308.79 & Gradient error & $5.01 E-4$ & & & & \\
\hline Scan y-coord & 5322 & 5323 & 5324 & 5325 & 5326 & 5327 & 5328 & 5329 & 5330 & 5331 & 5332 & 5333 & 5334 & 5335 & 5336 & 5337 & 5338 \\
\hline$\delta \varepsilon$ & 0.4678 & 0.4654 & 0.4629 & 0.4605 & 0.4581 & 0.4557 & 0.4533 & 0.4509 & 0.4485 & 0.4461 & 0.4437 & 0.4413 & 0.4389 & 0.4365 & 0.4340 & 0.4316 & 0.4292 \\
\hline Scan $x$-coord & 783 & 783 & 783 & 783 & 783 & 783 & 783 & 783 & 783 & 783 & 783 & 783 & 783 & 783 & 783 & 783 & 783 \\
\hline$F X \# 1$ & 402.03 & 402.65 & 402.32 & 401.76 & 402.80 & 403.27 & 404.64 & 405.38 & 404.65 & 405.93 & 408.06 & 408.46 & 408.58 & 408.95 & 409.45 & 411.09 & 411.40 \\
\hline$F X \# 2$ & 297.86 & 297.86 & 297.76 & 297.75 & 297.86 & 298.02 & 298.20 & 298.28 & 298.33 & 298.51 & 298.77 & 298.98 & 299.25 & 299.64 & 300.01 & 300.36 & 300.58 \\
\hline$F X \# 3$ & 240.18 & 240.43 & 240.69 & 240.92 & 241.14 & 241.43 & 241.66 & 241.85 & 241.94 & 242.03 & 242.12 & 242.26 & 242.42 & 242.56 & 242.64 & 242.70 & 242.74 \\
\hline$F X \# 4$ & 198.55 & 198.58 & 198.65 & 198.65 & 198.73 & 198.80 & 198.85 & 198.86 & 198.88 & 198.88 & 198.89 & 198.97 & 199.03 & 199.06 & 199.07 & 199.13 & 199.20 \\
\hline & 162.33 & 162.43 & 162.54 & 162.63 & 162.69 & 162.72 & 162.73 & 162.80 & 162.92 & 162.96 & 163.03 & 163.04 & 163.01 & 162.92 & 162.90 & 162.92 & 162.99 \\
\hline \multirow{2}{*}{ Near wall FS } & 36.22 & 36.15 & 36.10 & 36.02 & 36.04 & 36.07 & 36.12 & 36.06 & 35.95 & 35.91 & 35.86 & 35.93 & 36.02 & 36.14 & 36.17 & 36.21 & 36.21 \\
\hline & & & & & & & & 186 & & & & & & & & & \\
\hline
\end{tabular}




\begin{tabular}{|c|c|c|c|c|c|c|c|c|c|c|c|c|c|c|c|c|c|}
\hline$T @ F X \# 1$ & 297.36 & 297.36 & 297.36 & 297.36 & 297.36 & 297.36 & 297.36 & 297.36 & 297.36 & 297.36 & 297.36 & 297.36 & 297.36 & 297.36 & 297.36 & 297.36 & 297.36 \\
\hline$T$ @ $F X \# 2$ & 298.47 & 298.47 & 298.47 & 298.46 & 298.46 & 298.45 & 298.44 & 298.44 & 298.44 & 298.43 & 298.42 & 298.42 & 298.41 & 298.41 & 298.40 & 298.40 & 298.39 \\
\hline$T @ F X \# 3$ & 300.53 & 300.52 & 300.52 & 300.51 & 300.51 & 300.50 & 300.50 & 300.49 & 300.49 & 300.48 & 300.48 & 300.47 & 300.47 & 300.46 & 300.46 & 300.45 & 300.45 \\
\hline$T @ F X \# 4$ & 302.58 & 302.58 & 302.57 & 302.57 & 302.56 & 302.56 & 302.55 & 302.55 & 302.54 & 302.54 & 302.53 & 302.53 & 302.52 & 302.52 & 302.51 & 302.51 & 302.50 \\
\hline$T @ F X \# 5$ & 304.66 & 304.66 & 304.65 & 304.65 & 304.64 & 304.64 & 304.63 & 304.63 & 304.62 & 304.62 & 304.61 & 304.61 & 304.60 & 304.60 & 304.59 & 304.59 & 304.58 \\
\hline Wall $x$-coord & 127.38 & 127.37 & 127.37 & 127.37 & 127.36 & 127.36 & 127.35 & 127.35 & 127.34 & 127.34 & 127.34 & 127.33 & 127.33 & 127.32 & 127.32 & 127.32 & 127.31 \\
\hline Wall T & 308.79 & 308.80 & 308.80 & 308.81 & 308.80 & 308.80 & 308.79 & 308.79 & 308.80 & 308.80 & 308.80 & 308.80 & 308.78 & 308.77 & 308.76 & 308.75 & 308.75 \\
\hline Interferogram Image \# & 455 & Location & $T C 3$ & de slope & $-2.38 E-3$ & $\delta \varepsilon$ intercept & 11.16 & $\operatorname{Avg} F S$ & 33.85 & Avg Wall T & 309.97 & Gradient error & $5.01 E-4$ & & & & \\
\hline Scan y-coord & 4507 & 4508 & 4509 & 4510 & 4511 & 4512 & 4513 & 4514 & 4515 & 4516 & 4517 & 4518 & 4519 & 4520 & 4521 & 4522 & 4523 \\
\hline & 0.4137 & 0.4113 & 0.4089 & 0.4065 & 0.4041 & 0.4017 & 0.3994 & 0.3970 & 0.3946 & 0.3922 & 0.3898 & 0.3874 & 0.3850 & 0.3827 & 0.3803 & 0.3779 & 0.3755 \\
\hline Scan $x$-coord & 845 & 845 & 845 & 845 & 845 & 845 & 845 & 845 & 845 & 845 & 845 & 845 & 845 & 845 & 845 & 845 & 845 \\
\hline$F X \# I$ & 438.03 & 438.62 & 439.26 & 439.84 & 440.57 & 440.88 & 440.82 & 442.26 & 442.12 & 442.29 & 442.45 & 442.92 & 442.68 & 443.33 & 443.71 & 444.48 & 444.18 \\
\hline$F X \# 2$ & 323.77 & 323.79 & 323.70 & 323.64 & 323.49 & 323.41 & 323.31 & 323.23 & 323.14 & 323.10 & 323.19 & 323.32 & 323.50 & 323.74 & 324.07 & 324.47 & 324.90 \\
\hline$F X \# 4$ & 219.50 & 219.58 & 219.61 & 219.67 & 219.64 & 219.67 & 219.77 & 219.81 & 219.85 & 219.98 & 220.13 & 220.33 & 220.47 & 220.57 & 220.65 & 220.71 & 220.75 \\
\hline$F X \# 5$ & 182.14 & 182.19 & 182.18 & 182.19 & 182.31 & 182.36 & 182.44 & 182.50 & 182.55 & 182.60 & 182.70 & 182.82 & 182.88 & 182.97 & 183.06 & 183.15 & 183.22 \\
\hline$F X \# 6$ & 148.02 & 148.16 & 148.21 & 148.27 & 148.37 & 148.50 & 148.60 & 148.76 & 148.91 & 148.99 & 149.05 & 149.11 & 149.09 & 149.11 & 149.17 & 149.21 & 149.30 \\
\hline Near wall FS & 34.13 & 34.04 & 33.97 & 33.92 & 33.94 & 33.87 & 33.84 & 33.74 & 33.64 & 33.61 & 33.66 & 33.71 & 33.79 & 33.86 & 33.90 & 33.94 & 33.92 \\
\hline$T @ F X \# 1$ & 297.36 & 297.36 & 297.36 & 297.36 & 297.36 & 297.36 & 297.36 & 297.36 & $\begin{array}{l}297.04 \\
297\end{array}$ & $\begin{array}{l}297.31 \\
296\end{array}$ & 297.36 & 297.36 & 297.36 & $\begin{array}{r}297.00 \\
296\end{array}$ & 297.36 & 297.36 & 297.36 \\
\hline$T @ F X \# 2$ & 298.38 & 298.38 & 298.37 & 298.37 & 298.36 & 298.36 & 298.35 & 298.35 & 298.34 & 298.34 & 298.33 & 298.33 & 298.32 & 298.32 & 298.31 & 298.31 & 298.30 \\
\hline$T$ (a) $F X \# 3$ & 300.44 & 300.43 & 300.43 & 300.42 & 300.42 & 300.41 & 300.41 & 300.40 & 300.40 & 300.39 & 300.39 & 300.38 & 300.38 & 300.37 & 300.37 & 300.36 & 300.36 \\
\hline$T @ F X \# 4$ & 302.49 & 302.49 & 302.48 & 302.48 & 302.47 & 302.47 & 302.46 & 302.46 & 302.45 & 302.45 & 302.44 & 302.44 & 302.43 & 302.43 & 302.43 & 302.42 & 302.42 \\
\hline$T @ F X \# 5$ & 304.57 & 304.57 & 304.56 & 304.56 & 304.55 & 304.55 & 304.54 & 304.54 & 304.53 & 304.53 & 304.52 & 304.52 & 304.51 & 304.51 & 304.50 & 304.50 & 304.49 \\
\hline$T \cong F X \# 6$ & 306.67 & 306.67 & 306.66 & 306.66 & 306.65 & 306.65 & 306.64 & 306.64 & 306.63 & 306.63 & 306.62 & 306.62 & 306.61 & 306.61 & 306.60 & 306.60 & 306.59 \\
\hline Wall $x$-coord & 129.53 & 129.53 & 129.53 & 129.53 & $\begin{array}{l}300.05 \\
129.53\end{array}$ & 129.53 & 129.53 & 129.52 & $\begin{array}{l}300.03 \\
129.52\end{array}$ & 129.52 & $\begin{array}{l}300.02 \\
129.52\end{array}$ & $\begin{array}{l}300.02 \\
129.52\end{array}$ & 129.52 & 129.52 & 129.52 & 129.51 & 129.51 \\
\hline Wall T & 309.95 & 309.96 & 309.96 & 309.96 & 309.96 & 309.97 & 309.97 & 309.98 & 309.99 & 309.99 & 309.98 & 309.98 & 309.97 & 309.97 & 309.96 & 309.96 & 309.96 \\
\hline Interferogram Image \# & 455 & Location & $T C 4$ & $\delta \varepsilon$ slope & $-2.28 E-3$ & $\delta \varepsilon$ intercept & 8.68 & $\operatorname{Avg} F S$ & 32.84 & Avg Wall T & 310.85 & Gradient error & $5.01 E-4$ & & & & \\
\hline Scan y-coord & 3692 & 3693 & 3694 & 3695 & 3696 & 3697 & 3698 & 3699 & 3700 & 3701 & 3702 & 3703 & 3704 & 3705 & 3706 & 3707 & 3708 \\
\hline & 0.2482 & 0.2460 & 0.2437 & 0.2414 & 0.2391 & 0.2368 & 0.2345 & 0.2323 & 0.2300 & 0.2277 & 0.2254 & 0.2231 & 0.2208 & 0.2185 & 0.2163 & 0.2140 & 0.2117 \\
\hline Scan $x$-coord & 983 & 983 & 983 & 983 & 983 & 983 & 983 & 983 & 983 & 983 & 983 & 983 & 983 & 983 & 983 & 983 & 983 \\
\hline$F X \# 1$ & 497.77 & 498.25 & 498.87 & 499.46 & 499.98 & 502.25 & 502.88 & 501.08 & 501.46 & 501.74 & 502.55 & 503.94 & 506.75 & 507.32 & 507.71 & 507.13 & 507.56 \\
\hline$F X \# 2$ & 351.71 & 351.91 & 352.12 & 352.38 & 352.76 & 353.18 & 353.49 & 353.69 & 353.90 & 354.04 & 354.17 & 354.46 & 354.79 & 355.01 & 355.13 & 355.17 & 355.27 \\
\hline$F X \# 3$ & 285.66 & 285.80 & 285.93 & 286.10 & 286.30 & 286.43 & 286.60 & 286.64 & 286.69 & 286.70 & 286.75 & 286.81 & 286.88 & 286.96 & 287.08 & 287.21 & 287.37 \\
\hline$F X \# 4$ & 238.52 & 238.62 & 238.68 & 238.77 & 238.81 & 238.88 & 238.96 & 239.04 & 239.08 & 239.13 & 239.20 & 239.33 & 239.41 & 239.49 & 239.60 & 239.78 & 239.84 \\
\hline$F X \# 5$ & 199.93 & 199.98 & 200.01 & 200.01 & 200.05 & 200.12 & 200.24 & 200.39 & 200.52 & 200.59 & 200.67 & 200.72 & 200.75 & 200.81 & 200.91 & 201.09 & 201.23 \\
\hline$F X \# 6$ & 165.02 & 165.03 & 165.05 & 165.12 & 165.27 & 165.40 & 165.55 & 165.78 & 165.92 & 165.98 & 166.05 & 166.10 & 166.10 & 166.09 & 166.10 & 166.14 & 166.21 \\
\hline$F X \# 7$ & 133.07 & 133.03 & 132.90 & 132.86 & 132.68 & 132.58 & 132.67 & 132.47 & 132.39 & 132.56 & 132.70 & 132.84 & 132.95 & 133.10 & 133.23 & 133.33 & 133.30 \\
\hline Near wall FS & 31.96 & 32.00 & 32.14 & 32.26 & 32.59 & 32.82 & 32.89 & 33.31 & 33.53 & 33.42 & 33.34 & 33.27 & 33.15 & 32.99 & 32.88 & 32.81 & 32.91 \\
\hline$T @ F X \# 1$ & 297.36 & 297.36 & 297.36 & 297.36 & 297.36 & 297.36 & 297.36 & 297.36 & 297.36 & 297.36 & 297.36 & 297.36 & 297.36 & 297.36 & 297.36 & 297.36 & 297.36 \\
\hline$T$ (a) $F X \# 2$ & 298.09 & 298.09 & 298.08 & 298.08 & 298.07 & 298.07 & 298.06 & 298.06 & 298.05 & 298.05 & 298.04 & 298.04 & 298.03 & 298.03 & 298.02 & 298.02 & 298.01 \\
\hline$T @ F X \# 3$ & 300.16 & 300.16 & 300.15 & 300.15 & 300.14 & 300.14 & 300.13 & 300.13 & 300.12 & 300.12 & 300.11 & 300.11 & 300.10 & 300.10 & 300.09 & 300.09 & 300.09 \\
\hline$T @ F X \# 4$ & 302.22 & 302.21 & 302.21 & 302.20 & 302.20 & 302.19 & 302.19 & 302.18 & 302.18 & 302.17 & 302.17 & 302.16 & 302.16 & 302.16 & 302.15 & 302.15 & 302.14 \\
\hline$T @ F X \# 5$ & 304.29 & 304.29 & 304.28 & 304.28 & 304.27 & 304.27 & 304.26 & 304.26 & 304.25 & 304.25 & 304.24 & 304.24 & 304.23 & 304.23 & 304.22 & 304.22 & 304.21 \\
\hline$T @ F X \# 6$ & 306.39 & 306.38 & 306.38 & 306.37 & 306.37 & 306.37 & 306.36 & 306.36 & 306.35 & 306.35 & 306.34 & 306.34 & 306.33 & 306.33 & 306.32 & 306.32 & 306.31 \\
\hline$T$ @ $\boldsymbol{F} \boldsymbol{F} \# \mathbf{7}$ & 308.51 & 308.51 & 308.51 & 308.50 & 308.50 & 308.49 & 308.49 & 308.48 & 308.48 & 308.47 & 308.47 & 308.46 & 308.46 & 308.45 & 308.45 & 308.44 & 308.44 \\
\hline Wall $x$-coord & 129.46 & 129.46 & 129.46 & 129.47 & 129.47 & 129.47 & 129.47 & 129.47 & 129.47 & $\begin{array}{l}300.47 \\
129.47\end{array}$ & 129.48 & $\begin{array}{l}300.40 \\
129.48\end{array}$ & $\begin{array}{l}700.40 \\
129.48\end{array}$ & $\begin{array}{l}300.45 \\
129.48\end{array}$ & 129.48 & 129.48 & 129.48 \\
\hline Wall T & 310.91 & 310.90 & $\begin{array}{r}3127.40 \\
310.89\end{array}$ & 310.88 & 310.86 & 310.85 & 310.85 & 310.83 & 310.82 & 310.82 & 310.83 & 310.83 & 310.84 & 310.84 & 310.85 & 310.85 & 310.84 \\
\hline Interferogram Image \# & 455 & Location & $T C 5$ & $\delta \varepsilon$ slope & $-2.03 E-3$ & $\delta \varepsilon$ intercept & 5.93 & $\operatorname{Avg} F S$ & 33.48 & Avg Wall T & 311.74 & Gradient error & $5.01 E-4$ & & & & \\
\hline Scan y-coord & 2877 & 2878 & 2879 & 2880 & 2881 & 2882 & 2883 & 2884 & 2885 & 2886 & 2887 & 2888 & 2889 & 2890 & 2891 & 2892 & 2893 \\
\hline$\delta \varepsilon$ & 0.0857 & 0.0836 & 0.0816 & 0.0796 & 0.0776 & 0.0755 & 0.0735 & 0.0715 & 0.0694 & 0.0674 & 0.0654 & 0.0633 & 0.0613 & 0.0593 & 0.0573 & 0.0552 & 0.0532 \\
\hline Scan $x$-coord & 952 & 952 & 952 & 952 & 952 & 952 & 952 & 952 & 952 & 952 & 952 & 952 & 952 & 952 & 952 & 952 & 952 \\
\hline$F X \# 1$ & 618.79 & 620.41 & 621.72 & 607.90 & 619.89 & 635.75 & 636.10 & 731.93 & 732.33 & 638.03 & 638.91 & 638.74 & 639.12 & 657.36 & 648.26 & 651.75 & 654.46 \\
\hline$F X \# 2$ & 386.31 & 386.77 & 387.27 & 387.74 & 388.10 & 388.21 & 388.44 & 388.52 & 388.55 & 388.74 & 388.81 & 389.40 & 389.74 & 389.56 & 390.23 & 389.89 & 389.87 \\
\hline$F X \# 3$ & 310.82 & 310.73 & 310.69 & 310.76 & 310.86 & 310.96 & 311.05 & 311.12 & 311.16 & 311.17 & 311.20 & 311.30 & 311.57 & 311.87 & 312.05 & 312.15 & 312.10 \\
\hline$F X \# 4$ & 260.25 & 260.37 & 260.44 & 260.49 & 260.57 & 260.53 & 260.62 & 260.72 & 260.76 & 260.86 & 260.95 & 261.04 & 261.03 & 261.05 & 261.08 & 261.22 & 261.31 \\
\hline$F X \# 5$ & 219.31 & 219.39 & 219.47 & 219.50 & 219.50 & 219.51 & 219.55 & 219.60 & 219.65 & 219.82 & 219.94 & 220.02 & 220.05 & 220.06 & 220.11 & 220.21 & 220.32 \\
\hline$F X \# 6$ & $\begin{array}{l}219.31 \\
183.46\end{array}$ & 183.49 & $\begin{array}{l}219.47 \\
183.55\end{array}$ & 183.71 & $\begin{array}{l}219.50 \\
183.77\end{array}$ & $\begin{array}{l}219.51 \\
183.76\end{array}$ & $\begin{array}{l}219.53 \\
183.73\end{array}$ & $\begin{array}{l}219.00 \\
183.67\end{array}$ & 183.63 & $\begin{array}{l}193.82 \\
183.71\end{array}$ & $\begin{array}{l}219.94 \\
183.81\end{array}$ & $\begin{array}{l}220.02 \\
183.93\end{array}$ & 184.07 & 184.11 & 184.06 & 184.06 & 184.09 \\
\hline$F \boldsymbol{F} \# \mathbf{7}$ & 149.83 & 149.94 & 149.99 & 150.01 & 150.08 & 150.28 & 150.34 & 150.41 & 150.38 & 150.46 & 150.55 & 150.59 & 150.62 & 150.60 & 150.52 & 150.45 & 150.41 \\
\hline Near wall FS & 33.62 & 33.55 & 33.56 & 33.69 & 33.70 & 33.48 & 33.39 & 33.26 & 33.25 & 33.25 & 33.25 & 33.33 & 33.45 & 33.51 & 33.54 & 33.61 & 33.68 \\
\hline$T @ F X \# 1$ & 297.36 & 297.36 & 297.36 & 297.36 & 297.36 & 297.36 & 297.36 & 297.36 & 297.36 & 297.36 & 297.36 & 297.36 & 297.36 & 297.36 & 297.36 & 297.36 & 297.36 \\
\hline$T @ F X \# 2$ & 297.69 & 297.69 & 297.69 & 297.69 & 297.68 & 297.67 & 297.66 & 297.61 & 297.61 & 297.65 & 297.64 & 297.64 & 297.64 & 297.62 & 297.62 & 297.62 & 297.61 \\
\hline$T(F X \# 3$ & 299.80 & 299.80 & 299.79 & 299.79 & 299.78 & 299.78 & 299.78 & 299.77 & 299.77 & 299.76 & 299.76 & 299.75 & 299.75 & 299.75 & 299.74 & 299.74 & 299.73 \\
\hline$T(\mathrm{a} X \# \#$ & 301.86 & 301.85 & 301.85 & 301.84 & 301.84 & 301.84 & 301.83 & 301.83 & 301.82 & 301.82 & 301.82 & 301.81 & 301.81 & 301.80 & 301.80 & 301.79 & 301.79 \\
\hline$T @ F X \# 5$ & 303.93 & 303.92 & 303.92 & 303.92 & 303.91 & 303.91 & 303.90 & 303.90 & 303.89 & 303.89 & 303.89 & 303.88 & 303.88 & 303.87 & 303.87 & 303.86 & 303.86 \\
\hline$T @ F X \# 6$ & 306.02 & 306.02 & 306.01 & 306.01 & 306.01 & 306.00 & 306.00 & 305.99 & 305.99 & 305.98 & 305.98 & 305.98 & 305.97 & 305.97 & 305.96 & 305.96 & 305.95 \\
\hline$T @ F X \# 7$ & 308.14 & 308.14 & 308.14 & 308.13 & 308.13 & 308.12 & 308.12 & 308.11 & 308.11 & 308.10 & 308.10 & 308.10 & 308.09 & 308.09 & 308.08 & 308.08 & 308.07 \\
\hline Wall $x$-coord & 127.16 & 127.16 & 127.17 & 127.17 & 127.18 & 127.18 & 127.18 & $\begin{array}{l}500.11 \\
127.19\end{array}$ & $\begin{array}{l}300.11 \\
127.19\end{array}$ & 127.20 & 127.20 & 127.21 & $\begin{array}{l}30.09 \\
127.21\end{array}$ & 127.21 & 127.22 & $\begin{array}{l}30.08 \\
127.22\end{array}$ & 127.23 \\
\hline Wall T & 311.74 & 311.75 & 311.75 & 311.74 & 311.74 & 311.75 & 311.76 & 311.76 & 311.76 & 311.76 & 311.76 & 311.75 & 311.74 & 311.74 & 311.72 & 311.71 & 311.70 \\
\hline
\end{tabular}




\begin{tabular}{|c|c|c|c|c|c|c|c|c|c|c|c|c|c|c|c|c|c|}
\hline Interferogram Image \# & 455 & Location & TC6 & $\delta \varepsilon$ slope & $-1.99 E-3$ & $\delta \varepsilon$ intercept & 4.80 & $\operatorname{Avg} F S$ & 32.58 & Avg Wall $T$ & 312.43 & Gradient error & $5.01 E-4$ & & & & \\
\hline Scan y-coord & 2062 & 2063 & 2064 & 2065 & 2066 & 2067 & 2068 & 2069 & 2070 & 2071 & 2072 & 2073 & 2074 & 2075 & 2076 & 2077 & 2078 \\
\hline & 0.7014 & 0.6994 & 0.6975 & 0.6955 & 0.6935 & 0.6915 & 0.6895 & 0.6875 & 0.6855 & 0.6836 & 0.6816 & 0.6796 & 0.6776 & 0.6756 & 0.6736 & 0.6716 & 0.6696 \\
\hline Scan $x$-coord & 1025 & 1025 & 1025 & 1025 & 1025 & 1025 & 1025 & 1025 & 1025 & 1025 & 1025 & 1025 & 1025 & 1025 & 1025 & 1025 & 1025 \\
\hline$F X \# 1$ & 429.74 & 430.01 & 430.36 & 430.75 & 431.16 & 431.51 & 431.77 & 431.83 & 431.68 & 431.88 & 432.21 & 432.47 & 432.79 & 433.29 & 433.21 & 433.33 & 434.01 \\
\hline$F X \# 2$ & 340.15 & 340.33 & 340.48 & 340.57 & 340.65 & 340.74 & 340.88 & $\begin{array}{r}341.03 \\
341.01\end{array}$ & 341.19 & $\begin{array}{r}341.30 \\
341.38\end{array}$ & 341.56 & $\begin{array}{r}352.47 \\
341.63\end{array}$ & 341.68 & 341.67 & $\begin{array}{l}35.21 \\
341.69\end{array}$ & $\begin{array}{l}35.05 \\
341.69\end{array}$ & 341.82 \\
\hline$F X \# 3$ & 282.09 & 282.24 & 282.33 & 282.35 & 282.30 & 282.20 & 282.07 & 281.96 & 281.91 & 281.94 & 282.01 & 282.20 & 282.36 & 282.54 & 282.68 & 282.76 & 282.80 \\
\hline$F X \# 5$ & 199.82 & 199.88 & 199.92 & 199.94 & 199.94 & 199.90 & 199.93 & 199.93 & 199.98 & 200.10 & 200.28 & 200.46 & 200.65 & 200.83 & 201.01 & 201.18 & 201.42 \\
\hline$F X \# 6$ & 166.43 & 166.50 & 166.59 & 166.72 & 166.79 & 166.86 & 167.00 & 167.12 & 167.24 & 167.35 & 167.51 & 167.60 & 167.69 & 167.75 & 167.80 & 167.84 & 167.88 \\
\hline$F \boldsymbol{F} \# \mathbf{7}$ & 133.78 & 134.06 & 134.39 & 134.36 & 134.52 & 134.41 & 134.31 & 134.24 & 134.24 & 134.38 & 134.60 & 134.79 & 134.93 & 135.01 & 135.20 & 135.65 & 135.97 \\
\hline Near wall FS & 32.65 & 32.44 & 32.20 & 32.36 & 32.27 & 32.45 & 32.68 & 32.87 & 33.00 & 32.97 & 32.91 & 32.81 & 32.76 & 32.74 & 32.60 & 32.19 & 31.92 \\
\hline$T @ F X \# 1$ & 297.36 & 297.36 & 297.36 & 297.36 & 297.36 & 297.36 & 297.36 & 297.36 & 297.36 & 297.36 & 297.36 & 297.36 & 297.36 & 297.36 & 297.36 & 297.36 & 297.36 \\
\hline$T @ F X \# 2$ & 299.05 & 299.04 & 299.04 & 299.03 & 299.03 & 299.03 & 299.02 & 299.02 & 299.01 & 299.01 & 299.01 & 299.00 & 299.00 & 298.99 & 298.99 & 298.99 & 298.98 \\
\hline$T @ F X \# 4$ & 303.16 & 303.16 & 303.16 & 303.15 & 303.15 & 303.14 & 303.14 & 303.14 & 303.13 & 303.13 & 303.12 & 303.12 & 303.12 & 303.11 & 303.11 & 303.10 & 303.10 \\
\hline$T @ F X \# 5$ & 305.25 & 305.25 & 305.24 & 305.24 & 305.23 & 305.23 & 305.22 & 305.22 & 305.22 & 305.21 & 305.21 & 305.20 & 305.20 & 305.20 & 305.19 & 305.19 & 305.18 \\
\hline$T @ F X \# 6$ & 307.36 & 307.36 & 307.35 & 307.35 & 307.34 & 307.34 & 307.34 & 307.33 & 307.33 & 307.32 & 307.32 & 307.32 & 307.31 & 307.31 & 307.30 & 307.30 & 307.29 \\
\hline$T @ F X \# 7$ & 309.50 & 309.50 & 309.49 & 309.49 & 309.48 & 309.48 & 309.47 & 309.47 & 309.47 & 309.46 & 309.46 & 309.45 & 309.45 & 309.44 & 309.44 & 309.44 & 309.43 \\
\hline Wall $x$-coord & 122.63 & 122.64 & 122.64 & 122.65 & 122.66 & 122.66 & 122.67 & 122.68 & 122.68 & 122.69 & 122.70 & 122.70 & 122.71 & 122.72 & 122.73 & 122.73 & 122.74 \\
\hline Wall T & 312.41 & 312.43 & 312.45 & 312.44 & 312.45 & 312.43 & 312.41 & 312.40 & 312.39 & 312.40 & 312.41 & 312.42 & 312.42 & 312.42 & 312.44 & 312.47 & 312.50 \\
\hline
\end{tabular}

Interferogram Image

\begin{tabular}{|c|c|c|c|c|c|c|c|c|c|c|c|c|c|c|c|c|c|}
\hline ggram Image \# & 455 & Location & $T C 7$ & $\delta \varepsilon$ slope & $-1.84 E-3$ & $\delta \varepsilon$ intercept & 2.67 & $\operatorname{Avg} F S$ & 32.94 & Avg Wall T & 313.20 & Gradient error & $5.01 E-4$ & & & & \\
\hline Scan y-coord & 1247 & 1248 & 1249 & 1250 & 1251 & 1252 & 1253 & 1254 & 1255 & 1256 & 1257 & 1258 & 1259 & 1260 & 1261 & 1262 & 1263 \\
\hline & 0.3767 & 0.3749 & 0.3731 & 0.3712 & 0.3694 & 0.3676 & 0.3657 & 0.3639 & 0.3620 & 0.3602 & 0.3584 & 0.3565 & 0.3547 & 0.3529 & 0.3510 & 0.3492 & 0.3474 \\
\hline Scan $x$-coord & 1014 & 1014 & 1014 & 1014 & 1014 & 1014 & 1014 & 1014 & 1014 & 1014 & 1014 & 1014 & 1014 & 1014 & 1014 & 1014 & 1014 \\
\hline$F X \# 1$ & 527.78 & 528.35 & 529.14 & 529.96 & 530.50 & 531.14 & 532.03 & 532.90 & 533.66 & 534.21 & 534.42 & 534.63 & 534.98 & 535.67 & 537.32 & 538.19 & 537.38 \\
\hline$F X \# 2$ & 382.37 & 382.44 & 382.68 & 383.04 & 383.39 & 383.67 & 383.94 & 384.13 & 384.34 & 384.49 & 384.62 & 384.73 & 384.89 & 385.07 & 385.26 & 385.61 & 385.89 \\
\hline$F X \# 3$ & $\begin{array}{l}302.51 \\
310.99\end{array}$ & 311.05 & 311.12 & $\begin{array}{l}30.04 \\
311.24\end{array}$ & 311.23 & $\begin{array}{l}30.01 \\
311.23\end{array}$ & 311.28 & 311.38 & $\begin{array}{l}304.04 \\
311.54\end{array}$ & 311.72 & $\begin{array}{l}304.02 \\
311.84\end{array}$ & 311.98 & $\begin{array}{l}30.67 \\
312.12\end{array}$ & 312.23 & 312.33 & $\begin{array}{l}30.01 \\
312.46\end{array}$ & 312.58 \\
\hline$F X \# 4$ & 260.50 & 260.56 & 260.58 & 260.67 & 260.78 & 260.89 & 260.96 & 261.04 & 261.09 & 261.10 & 261.15 & 261.17 & 261.26 & 261.33 & 261.41 & 261.57 & 261.79 \\
\hline$F X \# 5$ & 219.54 & 219.61 & 219.66 & 219.69 & 219.71 & 219.74 & 219.80 & 219.82 & 219.86 & 219.89 & 219.94 & 220.01 & 220.07 & 220.26 & 220.43 & 220.53 & 220.57 \\
\hline$F X \# 6$ & 183.31 & 183.34 & 183.40 & 183.48 & 183.57 & 183.68 & 183.81 & 183.86 & 183.93 & 184.02 & 184.10 & 184.16 & 184.15 & 184.19 & 184.15 & 184.13 & 184.13 \\
\hline$F X \# 7$ & 149.83 & 149.99 & 150.08 & 150.22 & 150.31 & 150.40 & 150.41 & 150.39 & 150.39 & 150.44 & 150.45 & 150.41 & 150.41 & 150.41 & 150.45 & 150.50 & 150.56 \\
\hline$F X \# 8$ & 117.33 & 116.80 & 116.49 & 116.67 & 116.80 & 117.13 & 117.18 & 117.18 & 117.29 & 117.39 & 117.49 & 117.70 & 117.91 & 118.08 & 118.10 & 118.11 & 117.97 \\
\hline Near wall FS & 32.49 & 33.19 & 33.59 & 33.55 & 33.51 & 33.27 & 33.23 & 33.21 & 33.10 & 33.04 & 32.96 & 32.72 & 32.50 & 32.33 & 32.35 & 32.39 & 32.59 \\
\hline$T @ F X \# 1$ & 297.36 & 297.36 & 297.36 & 297.36 & 297.36 & 297.36 & 297.36 & 297.36 & 297.36 & 297.36 & 297.36 & 297.36 & 297.36 & 297.36 & 297.36 & 297.36 & 297.36 \\
\hline$T @ F X \# 2$ & 298.35 & 298.34 & 298.34 & 298.34 & 298.33 & 298.33 & 298.32 & 298.32 & 298.31 & 298.31 & 298.31 & 298.30 & 298.30 & 298.30 & 298.29 & 298.29 & 298.28 \\
\hline$T$ (a) $F X \# 3$ & 300.42 & 300.41 & 300.41 & 300.41 & 300.40 & 300.40 & 300.40 & 300.39 & 300.39 & 300.38 & 300.38 & 300.38 & 300.37 & 300.37 & 300.37 & 300.36 & 300.36 \\
\hline$T(F X \# 4$ & 302.48 & 302.48 & 302.47 & 302.47 & 302.47 & 302.46 & 302.46 & 302.45 & 302.45 & 302.45 & 302.44 & 302.44 & 302.44 & 302.43 & 302.43 & 302.42 & 302.42 \\
\hline$T @ F X \# 5$ & 304.56 & 304.56 & 304.55 & 304.55 & 304.54 & 304.54 & 304.54 & 304.53 & 304.53 & 304.53 & 304.52 & 304.52 & 304.51 & 304.51 & 304.51 & 304.50 & 304.50 \\
\hline$T$ @ $F X \# 6$ & 306.66 & 306.66 & 306.66 & 306.65 & 306.65 & 306.64 & 306.64 & 306.64 & 306.63 & 306.63 & 306.63 & 306.62 & 306.62 & 306.61 & 306.61 & 306.61 & 306.60 \\
\hline$T(F X \# 7$ & 308.79 & 308.79 & 308.79 & 308.78 & 308.78 & 308.77 & 308.77 & 308.77 & 308.76 & 308.76 & 308.75 & 308.75 & 308.75 & 308.74 & 308.74 & 308.73 & 308.73 \\
\hline$T @ F X \# 8$ & 310.95 & 310.95 & 310.94 & 310.94 & 310.94 & 310.93 & 310.93 & 310.92 & 310.92 & 310.92 & 310.91 & 310.91 & 310.90 & 310.90 & 310.90 & 310.89 & 310.89 \\
\hline Wall $x$-coord & 115.87 & 115.88 & 115.89 & 115.90 & 115.91 & 115.92 & 115.93 & 115.94 & 115.95 & 115.96 & 115.97 & 115.98 & 115.98 & 115.99 & 116.00 & 116.01 & 116.02 \\
\hline Wall T & 313.24 & 313.20 & 313.17 & 313.18 & 313.18 & 313.20 & 313.20 & 313.19 & 313.20 & 313.20 & 313.20 & 313.21 & 313.22 & 313.23 & 313.22 & 313.22 & 313.21 \\
\hline
\end{tabular}

\section{B.4 Tabulated Data for Interferogram 460}

\begin{tabular}{|c|c|c|c|c|c|c|c|c|c|c|c|c|c|c|c|c|c|}
\hline Interferogram Image \# & 460 & Location & $T C 2$ & $\delta \varepsilon$ slope & $-2.50 E-3$ & $\delta \varepsilon$ intercept & 14.00 & $\operatorname{Avg} F S$ & 44.37 & Avg Wall $T$ & 307.50 & Gradient error & $4.88 E-4$ & & & & \\
\hline Scan y-coord & 5267 & 5268 & 5269 & 5270 & 5271 & 5272 & 5273 & 5274 & 5275 & 5276 & 5277 & 5278 & 5279 & 5280 & 5281 & 5282 & 5283 \\
\hline$\delta \varepsilon$ & 0.8499 & 0.8474 & 0.8449 & 0.8424 & 0.8399 & 0.8374 & 0.8349 & 0.8324 & 0.8299 & 0.8274 & 0.8249 & 0.8224 & 0.8199 & 0.8174 & 0.8150 & 0.8125 & 0.8100 \\
\hline Scan $x$-coord & 872 & 872 & 872 & 872 & 872 & 872 & 872 & 872 & 872 & 872 & 872 & 872 & 872 & 872 & 872 & 872 & 872 \\
\hline$F X \# 1$ & 327.46 & 328.04 & 328.67 & 328.29 & 328.93 & 329.58 & 333.68 & 334.04 & 334.91 & 335.29 & 334.72 & 335.20 & 335.86 & 336.95 & 337.38 & 332.89 & 332.55 \\
\hline$F X \# 2$ & 247.56 & 247.70 & 247.80 & 248.02 & 248.29 & 248.53 & 248.70 & 248.88 & 248.95 & 249.03 & 249.18 & 249.28 & 249.35 & 249.42 & 249.46 & 249.67 & 249.87 \\
\hline$F X \# 3$ & 194.24 & 194.35 & 194.42 & 194.54 & 194.68 & 194.77 & 194.91 & 195.05 & 195.02 & 195.08 & 195.20 & 195.29 & 195.44 & 195.62 & 195.70 & 195.82 & 195.92 \\
\hline$F X \# 4$ & 149.87 & 149.93 & 149.97 & 150.10 & 150.21 & 150.34 & 150.50 & 150.66 & 150.80 & 150.86 & 150.89 & 150.92 & 151.02 & 151.16 & 151.28 & 151.49 & 151.68 \\
\hline Near wall FS & 44.37 & 44.42 & 44.45 & 44.44 & 44.46 & 44.43 & 44.41 & 44.39 & 44.22 & 44.22 & 44.31 & 44.36 & 44.42 & 44.46 & 44.41 & 44.33 & 44.24 \\
\hline$T @ F X \# 1$ & 297.32 & 297.32 & 297.32 & 297.32 & 297.32 & 297.32 & 297.32 & 297.32 & 297.32 & 297.32 & 297.32 & 297.32 & 297.32 & 297.32 & 297.32 & 297.32 & 297.32 \\
\hline$T(F X \# 2$ & 299.27 & 299.26 & 299.26 & 299.25 & 299.25 & 299.24 & 299.24 & 299.23 & 299.23 & 299.22 & 299.22 & 299.21 & 299.21 & 299.20 & 299.19 & 299.19 & 299.19 \\
\hline$T(a) F X \#$ & 301.32 & 301.31 & 301.31 & 301.30 & 301.30 & 301.29 & 301.29 & 301.28 & 301.28 & 301.27 & 301.27 & 301.26 & 301.26 & 301.25 & 301.25 & 301.24 & 301.24 \\
\hline$T$ (a) $F X \# 4$ & 303.38 & 303.38 & 303.37 & 303.37 & 303.36 & 303.36 & 303.35 & 303.35 & 303.34 & 303.34 & 303.33 & 303.33 & 303.32 & 303.32 & 303.31 & 303.31 & 303.30 \\
\hline Wall $x$-coord & 106.80 & 106.79 & 106.79 & 106.78 & 106.78 & 106.77 & 106.77 & 106.77 & 106.76 & 106.76 & 106.75 & 106.75 & 106.74 & 106.74 & 106.73 & 106.73 & 106.72 \\
\hline
\end{tabular}

\begin{tabular}{|c|c|c|c|c|c|c|c|c|c|c|c|c|c|c|c|c|c|}
\hline Interferogram Image \# & 460 & Location & $T C 3$ & $\delta \varepsilon$ slope & $-2.32 E-3$ & $\delta \varepsilon$ intercept & 11.09 & $\operatorname{Avg} F S$ & 41.01 & Avg Wall $T$ & 308.32 & Gradient error & $4.88 E-4$ & & & & \\
\hline Scan y-coord & 4453 & 4454 & 4455 & 4456 & 4457 & 4458 & 4459 & 4460 & 4461 & 4462 & 4463 & 4464 & 4465 & 4466 & 4467 & 4468 & 4469 \\
\hline & 0.7729 & 0.7705 & 0.7682 & 0.7659 & 0.7636 & 0.7613 & 0.7589 & 0.7566 & 0.7543 & 0.7520 & 0.7497 & 0.7474 & 0.7450 & 0.7427 & 0.7404 & 0.7381 & 0.7358 \\
\hline Scan $x$-coord & 878 & 878 & 878 & 878 & 878 & 878 & 878 & 878 & 878 & 878 & 878 & 878 & 878 & 878 & 878 & 878 & 878 \\
\hline$F X \# 1$ & 362.09 & 360.55 & 359.78 & 360.13 & 360.48 & 360.86 & 361.22 & 366.23 & 366.58 & 367.00 & 367.00 & 367.28 & 367.62 & 367.88 & 368.11 & 368.12 & 368.21 \\
\hline$F X \# 2$ & 270.47 & 270.54 & 270.54 & 270.54 & 270.62 & 270.66 & 270.80 & 270.91 & 271.03 & 271.12 & 271.27 & 271.41 & 271.59 & 271.83 & 271.99 & 272.20 & 272.38 \\
\hline$F X \# 3$ & 213.03 & 213.13 & 213.21 & 213.33 & 213.52 & 213.65 & 213.79 & 213.89 & 214.02 & 214.17 & 214.31 & 214.46 & 214.64 & 214.78 & 214.89 & 214.99 & 215.08 \\
\hline$F X \# 4$ & 167.76 & 167.86 & 167.96 & 168.08 & 168.18 & 168.33 & 168.40 & 168.47 & 168.55 & 168.66 & 168.75 & 168.82 & 168.96 & 169.06 & 169.17 & 169.23 & 169.33 \\
\hline$F X \# 5$ & 126.57 & 126.73 & 126.94 & 127.10 & 127.26 & 127.40 & 127.48 & 127.46 & 127.54 & 127.64 & 127.79 & 127.95 & 128.03 & 128.06 & 128.09 & 128.12 & 128.21 \\
\hline Near wall FS & 41.19 & 41.12 & 41.02 & 40.98 & 40.91 & 40.93 & 40.92 & 41.02 & 41.01 & 41.02 & 40.95 & 40.87 & 40.92 & 41.00 & 41.08 & 41.11 & 41.12 \\
\hline
\end{tabular}




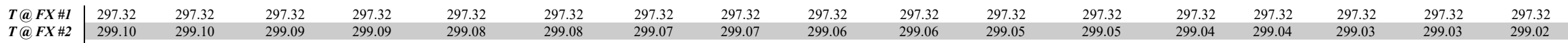

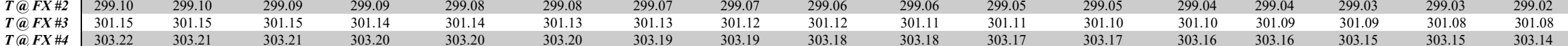

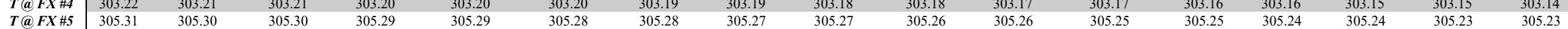

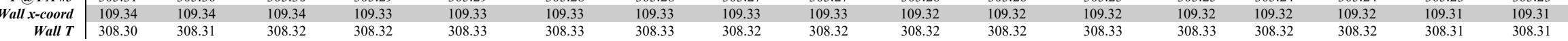

\begin{tabular}{|c|c|c|c|c|c|c|c|c|c|c|c|c|c|c|c|c|c|}
\hline Interferogram Image \# & 460 & Location & $T C 4$ & $\delta$ slope & $-2.29 E-3$ & $\delta \varepsilon$ intercept & 8.92 & $\operatorname{Avg} F S$ & 41.65 & Avg Wall $T$ & 308.90 & Gradient error & $4.88 E-4$ & & & & \\
\hline Scan y-coord & 3640 & 3641 & 3642 & 3643 & 3644 & 3645 & 3646 & 3647 & 3648 & 3649 & 3650 & 3651 & 3652 & 3653 & 3654 & 3655 & 3656 \\
\hline & 0.5792 & 0.5769 & 0.5746 & 0.5723 & 0.5700 & 0.5677 & 0.5654 & 0.5631 & 0.5609 & 0.5586 & 0.5563 & 0.5540 & 0.5517 & 0.5494 & 0.5471 & 0.5448 & 0.5425 \\
\hline Scan $x$-coord & 1045 & 1045 & 1045 & 1045 & 1045 & 1045 & 1045 & 1045 & 1045 & 1045 & 1045 & 1045 & 1045 & 1045 & 1045 & 1045 & 1045 \\
\hline$F X \# 1$ & 402.62 & 403.07 & 403.56 & 404.13 & 404.63 & 405.02 & 405.28 & 405.44 & 405.62 & 405.85 & 406.21 & 406.58 & 406.95 & 407.42 & 408.08 & 408.28 & 408.72 \\
\hline$F X \# 2$ & 296.77 & 296.89 & 296.99 & 297.15 & 297.42 & 297.66 & 297.87 & 298.05 & 298.19 & 298.34 & 298.50 & 298.73 & 298.94 & 299.15 & 299.30 & 299.38 & 299.57 \\
\hline$F X \# 3$ & 234.16 & 234.23 & 234.39 & 234.60 & 234.80 & 234.86 & 234.96 & 235.04 & 235.09 & 235.09 & 235.11 & 235.20 & 235.27 & 235.40 & 235.56 & 235.71 & 235.84 \\
\hline$F X \# 4$ & 186.94 & 187.04 & 187.11 & 187.10 & 187.13 & 187.18 & 187.33 & 187.44 & 187.56 & 187.67 & 187.80 & 187.90 & 187.93 & 187.94 & 187.95 & 187.99 & 188.02 \\
\hline$F X \# 5$ & 145.13 & 145.28 & 145.33 & 145.41 & 145.48 & 145.57 & 145.68 & 145.85 & 145.96 & 146.05 & 146.09 & 146.12 & 146.25 & 146.36 & 146.45 & 146.51 & 146.55 \\
\hline Near wall FS & 41.81 & 41.77 & 41.78 & 41.70 & 41.65 & 41.61 & 41.65 & 41.59 & 41.60 & 41.62 & 41.71 & 41.78 & 41.69 & 41.58 & 41.49 & 41.48 & 41.47 \\
\hline$T @ F X \# 1$ & 297.32 & 297.32 & 297.32 & 297.32 & 297.32 & 297.32 & 297.32 & 297.32 & 297.32 & 297.32 & 297.32 & 297.32 & 297.32 & 297.32 & 297.32 & 297.32 & 297.32 \\
\hline$T(F X \# 2$ & 298.78 & 298.77 & 298.77 & 298.76 & 298.76 & 298.75 & 298.75 & 298.74 & 298.74 & 298.74 & 298.73 & 298.73 & 298.72 & 298.72 & 298.71 & 298.71 & 298.70 \\
\hline$T$ (a) $F X \# 3$ & 300.83 & 300.83 & 300.82 & 300.82 & 300.81 & 300.81 & 300.81 & 300.80 & 300.80 & 300.79 & 300.79 & 300.78 & 300.78 & 300.77 & 300.77 & 300.76 & 300.76 \\
\hline$T$ (a) $F X \# 4$ & 302.90 & 302.89 & 302.89 & 302.88 & 302.88 & 302.87 & 302.87 & 302.86 & 302.86 & 302.85 & 302.85 & 302.84 & 302.84 & 302.83 & 302.83 & 302.83 & 302.82 \\
\hline$T$ @ $F X \# 5$ & 304.98 & 304.97 & 304.97 & 304.97 & 304.96 & 304.96 & 304.95 & 304.95 & 304.94 & 304.94 & 304.93 & 304.93 & 304.92 & 304.92 & 304.91 & 304.91 & 304.90 \\
\hline Wall $x$-coord & 109.38 & 109.39 & 109.39 & 109.39 & 109.39 & 109.39 & 109.39 & 109.39 & 109.40 & 109.40 & 109.40 & 109.40 & 109.40 & 109.40 & 109.40 & 109.41 & 109.41 \\
\hline Wall T & 308.89 & 308.90 & 308.89 & 308.90 & 308.90 & 308.90 & 308.90 & 308.90 & 308.90 & 308.90 & 308.89 & 308.89 & 308.89 & 308.90 & 308.90 & 308.90 & 308.90 \\
\hline
\end{tabular}

\begin{tabular}{|c|c|c|c|c|c|c|c|c|c|c|c|c|c|c|c|c|c|}
\hline Interferogram Image \# & 460 & Location & $T C 5$ & $\delta \varepsilon$ slope & $-2.16 E-3$ & $\delta \varepsilon$ intercept & 6.53 & $A v g F S$ & 39.76 & Avg Wall T & 309.75 & Gradient error & 4.88E-4 & & & & \\
\hline Scan y-coord & 2826 & 2827 & 2828 & 2829 & 2830 & 2831 & 2832 & 2833 & 2834 & 2835 & 2836 & 2837 & 2838 & 2839 & 2840 & 2841 & 2842 \\
\hline & 0.4112 & 0.4091 & 0.4069 & 0.4048 & 0.4026 & 0.4004 & 0.3983 & 0.3961 & 0.3939 & 0.3918 & 0.3896 & 0.3874 & 0.3853 & 0.3831 & 0.3809 & 0.3788 & 0.3766 \\
\hline Scan $x$-coord & 950 & $\begin{array}{l}950 \\
950\end{array}$ & 950 & $\begin{array}{l}9.40+6 \\
950\end{array}$ & $\begin{array}{l}950 \\
950\end{array}$ & 950 & 950 & 950 & 950 & 950 & 950 & 950 & 950 & 950 & 950 & 950 & 950 \\
\hline$F X \# 1$ & 458.88 & 459.36 & 459.88 & 460.35 & 461.46 & 461.88 & 462.21 & 463.45 & 463.95 & 463.55 & 465.25 & 465.85 & 466.23 & 466.80 & 467.31 & 467.66 & 467.89 \\
\hline$F X \# 2$ & 327.31 & 327.55 & 327.77 & 328.02 & 328.36 & 328.54 & 328.75 & 328.92 & 329.13 & 329.29 & 329.61 & 329.75 & 329.85 & 330.00 & 330.07 & 330.05 & 330.16 \\
\hline$F X \# 3$ & 258.54 & 258.51 & 258.52 & 258.62 & 258.82 & 259.00 & 259.21 & 259.37 & 259.49 & 259.48 & 259.51 & 259.58 & 259.71 & 259.91 & 260.11 & 260.28 & 260.37 \\
\hline$F X \# 4$ & 207.47 & 207.50 & 207.55 & 207.67 & 207.83 & 208.04 & 208.33 & 208.49 & 208.64 & 208.72 & 208.85 & 209.02 & 209.17 & 209.32 & 209.51 & 209.66 & 209.71 \\
\hline$F X \# 5$ & 164.35 & 164.44 & 164.58 & 164.67 & 164.80 & 164.88 & 164.96 & 165.04 & 165.07 & 165.16 & 165.25 & 165.29 & 165.31 & 165.36 & 165.46 & 165.51 & 165.56 \\
\hline$F X \# 6$ & 124.82 & 124.86 & 124.93 & 124.86 & 124.80 & 124.74 & 124.84 & 125.01 & 125.20 & 125.45 & 125.57 & 125.66 & 125.77 & 125.79 & 125.79 & 125.82 & 125.93 \\
\hline Near wall FS & 39.53 & 39.59 & 39.65 & 39.81 & 40.00 & 40.14 & 40.13 & 40.03 & 39.87 & 39.71 & 39.68 & 39.63 & 39.53 & 39.58 & 39.67 & 39.69 & 39.62 \\
\hline & 297.32 & 297.32 & 297.32 & 297.32 & 297.32 & 297.32 & 297.32 & 297.32 & 297.32 & 297.32 & 297.32 & 297.32 & 297.32 & 297.32 & 297.32 & 297.32 & 297.32 \\
\hline$T @ F X \# 2$ & 298.37 & 298.37 & 298.36 & 298.36 & 298.35 & 298.35 & 298.34 & 298.34 & 298.33 & 298.33 & $\begin{array}{l}291.52 \\
298.32\end{array}$ & $\begin{array}{l}291.32 \\
298.32\end{array}$ & 298.32 & 298.31 & 298.31 & 298.30 & 298.30 \\
\hline$T @ F X \# 3$ & 300.43 & 300.43 & 300.42 & 300.42 & 300.42 & 300.41 & 300.41 & 300.40 & 300.40 & 300.39 & 300.39 & 300.38 & 300.38 & 300.38 & 300.37 & 300.37 & 300.36 \\
\hline$T$ (a) $F X \# 4$ & 302.49 & 302.49 & 302.49 & 302.48 & 302.48 & 302.47 & 302.47 & 302.46 & 302.46 & 302.45 & 302.45 & 302.44 & 302.44 & 302.44 & 302.43 & 302.43 & 302.42 \\
\hline$T @ F X \# 5$ & 304.57 & 304.57 & 304.56 & 304.56 & 304.56 & 304.55 & 304.55 & 304.54 & 304.54 & 304.53 & 304.53 & 304.52 & 304.52 & 304.51 & 304.51 & 304.51 & 304.50 \\
\hline$T @ F X \# 6$ & 306.68 & 306.67 & 306.67 & 306.66 & 306.66 & 306.65 & 306.65 & 306.65 & 306.64 & 306.64 & 306.63 & 306.63 & 306.62 & 306.62 & 306.61 & 306.61 & 306.60 \\
\hline Wall $x$-coord & 106.93 & 106.94 & 106.94 & 106.95 & 106.95 & 106.95 & 106.96 & 106.96 & 106.97 & 106.97 & 106.98 & 106.98 & 106.99 & 106.99 & 106.99 & 107.00 & 107.00 \\
\hline Wall $T$ & 309.77 & 309.77 & 309.77 & 309.75 & 309.74 & 309.73 & 309.73 & 309.74 & 309.75 & 309.76 & 309.76 & 309.76 & 309.77 & 309.76 & 309.75 & 309.75 & 309.75 \\
\hline
\end{tabular}

\begin{tabular}{|c|c|c|c|c|c|c|c|c|c|c|c|c|c|c|c|c|c|}
\hline Interferogram Image \# & 460 & Location & TC6 & SE slope & $-2.24 E-3$ & $\delta \varepsilon$ intercept & 4.56 & $\operatorname{Avg} F S$ & 125.43 & Avg Wall T & 310.32 & Gradient error & $4.88 E-4$ & & & & \\
\hline Scan y-coord & 2012 & 2013 & 2014 & 2015 & 2016 & 2017 & 2018 & 2019 & 2020 & 2021 & 2022 & 2023 & 2024 & 2025 & 2026 & 2027 & 2028 \\
\hline & 0.0658 & 0.0636 & 0.0613 & 0.0591 & 0.0568 & 0.0546 & 0.0524 & 0.0501 & 0.0479 & 0.0457 & 0.0434 & 0.0412 & 0.0390 & 0.0367 & 0.0345 & 0.0323 & 0.0300 \\
\hline Scan $x$-coord & 1023 & 1023 & 1023 & 1023 & 1023 & 1023 & 1023 & 1023 & 1023 & 1023 & 1023 & 1023 & 1023 & 1023 & 1023 & 1023 & 1023 \\
\hline 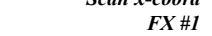 & 664.00 & 664.00 & 664.00 & 664.00 & 664.00 & 664.00 & 664.00 & 664.00 & 664.00 & 664.00 & 664.00 & 664.00 & 664.00 & 664.00 & 664.00 & 664.00 & 664.00 \\
\hline$F X \# 2$ & 365.40 & 365.93 & 366.19 & 366.46 & 366.77 & 364.95 & 365.15 & 365.33 & 365.49 & 365.59 & 365.63 & 365.77 & 365.87 & 366.06 & 366.15 & 366.29 & 366.43 \\
\hline$F X \# 3$ & 284.33 & 284.54 & 284.80 & 285.12 & 285.44 & 285.74 & 286.01 & 286.23 & 286.32 & 286.27 & 286.11 & 285.94 & 285.90 & 285.92 & 286.06 & 286.27 & 286.50 \\
\hline$F X \# 4$ & 229.64 & 229.68 & 229.69 & 229.71 & 229.79 & 229.91 & 230.07 & 230.24 & 230.35 & 230.37 & 230.36 & 230.43 & 230.56 & 230.65 & 230.76 & 230.81 & 230.85 \\
\hline$F X \# 5$ & 182.99 & 183.02 & 183.04 & 183.09 & 183.20 & 183.30 & 183.41 & 183.59 & 183.77 & 183.92 & 184.10 & 184.28 & 184.42 & 184.56 & 184.73 & 184.98 & 185.31 \\
\hline$F X \# 6$ & 143.11 & 143.25 & 143.39 & 143.46 & 143.58 & 143.68 & 143.71 & 143.76 & 143.84 & 143.97 & 144.10 & 144.31 & 144.50 & 144.61 & 144.69 & 144.71 & 144.78 \\
\hline$F \boldsymbol{X} \# 7$ & 105.16 & 105.09 & 104.93 & 0.00 & 0.00 & 0.00 & 0.00 & 0.00 & 0.00 & 0.00 & 0.00 & 0.00 & 0.00 & 0.00 & 0.00 & 0.00 & 0.00 \\
\hline Near wall FS & 37.95 & 38.16 & 38.46 & 143.46 & 143.58 & 143.68 & 143.71 & 143.76 & 143.84 & 143.97 & 144.10 & 144.31 & 144.50 & 144.61 & 144.69 & 144.71 & 144.78 \\
\hline$T @ F X \# 1$ & 297.32 & 297.32 & 297.32 & 297.32 & 297.32 & 297.32 & 297.32 & 297.32 & 297.32 & 297.32 & 297.32 & 297.32 & 297.32 & 297.32 & 297.32 & 297.32 & 297.32 \\
\hline$T @ F X \# 2$ & 297.62 & 297.62 & 297.61 & 297.61 & 297.61 & 297.60 & 297.60 & 297.59 & 297.59 & 297.58 & 297.58 & 297.57 & 297.57 & 297.57 & 297.56 & 297.56 & 297.55 \\
\hline$T$ (a) $F X \# 4$ & 301.81 & 301.81 & 301.81 & 301.80 & 301.80 & 301.79 & 301.79 & 301.78 & 301.78 & 301.77 & 301.77 & 301.76 & 301.76 & 301.75 & 301.75 & 301.75 & 301.74 \\
\hline$T$ (a) $F X \# 5$ & 303.89 & 303.88 & 303.88 & 303.87 & 303.87 & 303.86 & 303.86 & 303.85 & 303.85 & 303.84 & 303.84 & 303.84 & 303.83 & 303.83 & 303.82 & 303.82 & 303.81 \\
\hline$T(a) F X \# 6$ & 305.98 & 305.98 & 305.97 & 305.97 & 305.96 & 305.96 & 305.95 & 305.95 & 305.95 & 305.94 & 305.94 & 305.93 & 305.93 & 305.92 & 305.92 & 305.91 & 305.91 \\
\hline$T @ F X \# 7$ & 308.10 & 308.10 & 308.10 & 308.09 & 308.09 & 308.08 & 308.08 & 308.07 & 308.07 & 308.06 & 308.06 & 308.05 & 308.05 & 308.04 & 308.04 & 308.03 & 308.03 \\
\hline Wall $x$-coord & 101.98 & 101.99 & 102.00 & 102.00 & 102.01 & 102.02 & 102.03 & 102.03 & 102.04 & 102.05 & 102.06 & 102.07 & 102.07 & 102.08 & 102.09 & 102.10 & 102.10 \\
\hline Wall T & 310.44 & 310.43 & 310.41 & 310.31 & 310.31 & 310.31 & 310.30 & 310.29 & 310.29 & 310.29 & 310.29 & 310.29 & 310.30 & 310.30 & 310.30 & 310.28 & 310.26 \\
\hline Interferogram Image \# & 460 & Location & $T C 7$ & $\delta \varepsilon$ slope & $-2.17 E-3$ & $\delta \varepsilon$ intercept & 3.37 & $\operatorname{Avg} F S$ & 39.62 & Avg Wall T & 311.25 & Gradient error & $4.88 E-4$ & & & & \\
\hline Scan y-coord & 1199 & 1200 & 1201 & 1202 & 1203 & 1204 & 1205 & 1206 & 1207 & 1208 & 1209 & 1210 & 1211 & 1212 & 1213 & 1214 & 1215 \\
\hline$\delta \varepsilon$ & 0.7656 & 0.7634 & 0.7612 & 0.7591 & 0.7569 & 0.7547 & 0.7525 & 0.7504 & 0.7482 & 0.7460 & 0.7439 & 0.7417 & 0.7395 & 0.7374 & 0.7352 & 0.7330 & 0.7309 \\
\hline Scan $x$-coord & 1321 & 1321 & 1321 & 1321 & 1321 & 1321 & 1321 & 1321 & 1321 & 1321 & 1321 & 1321 & 1321 & 1321 & 1321 & 1321 & 1321 \\
\hline
\end{tabular}




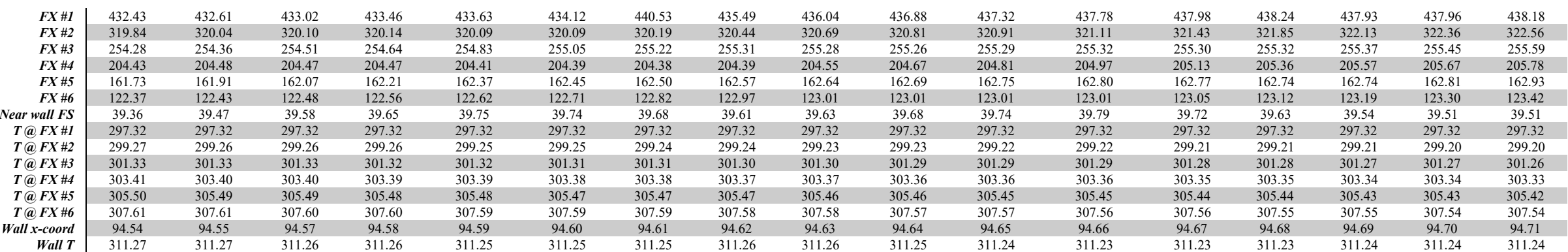

\section{B.5 Tabulated Data for Interferogram 46}

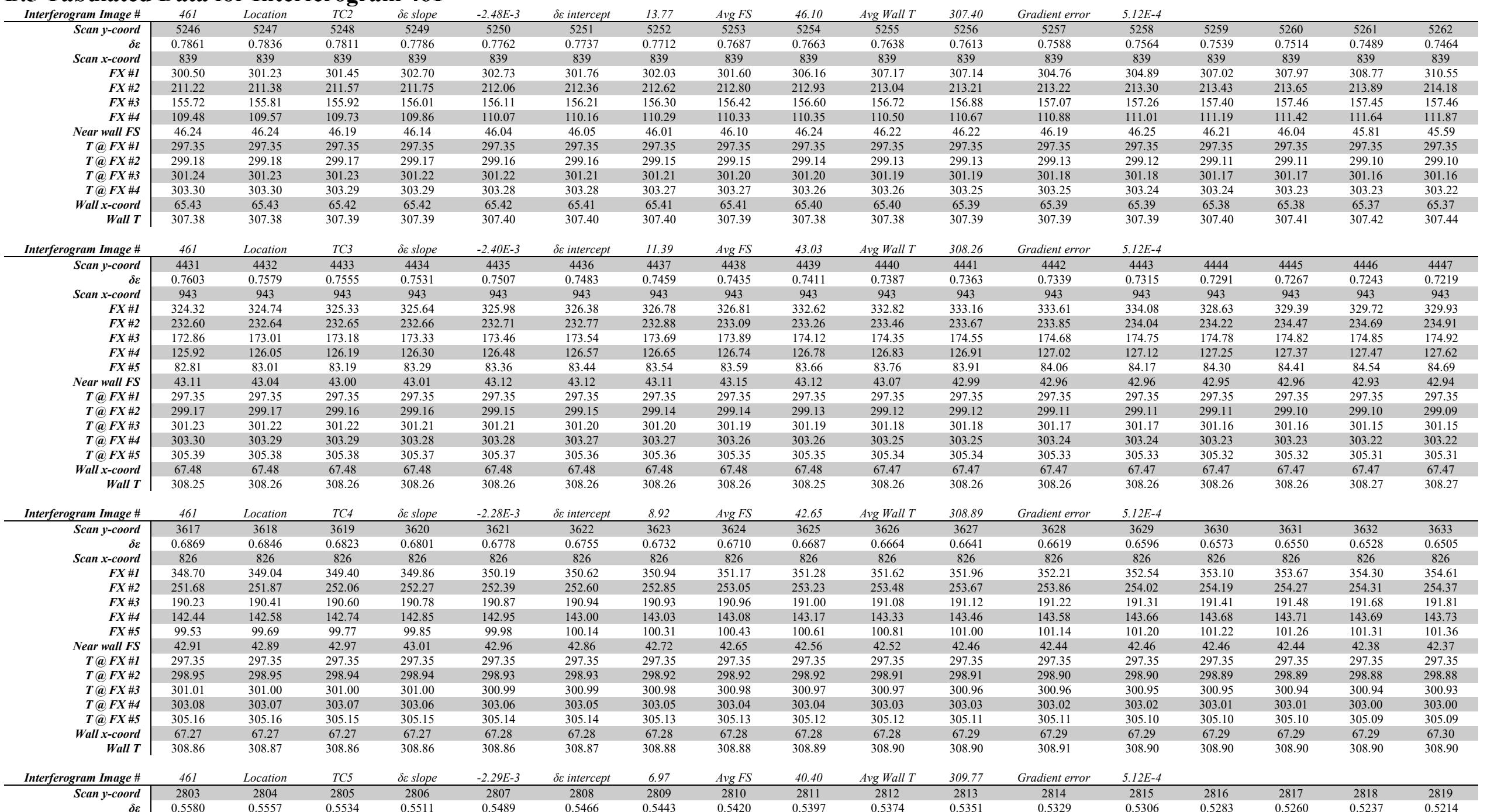




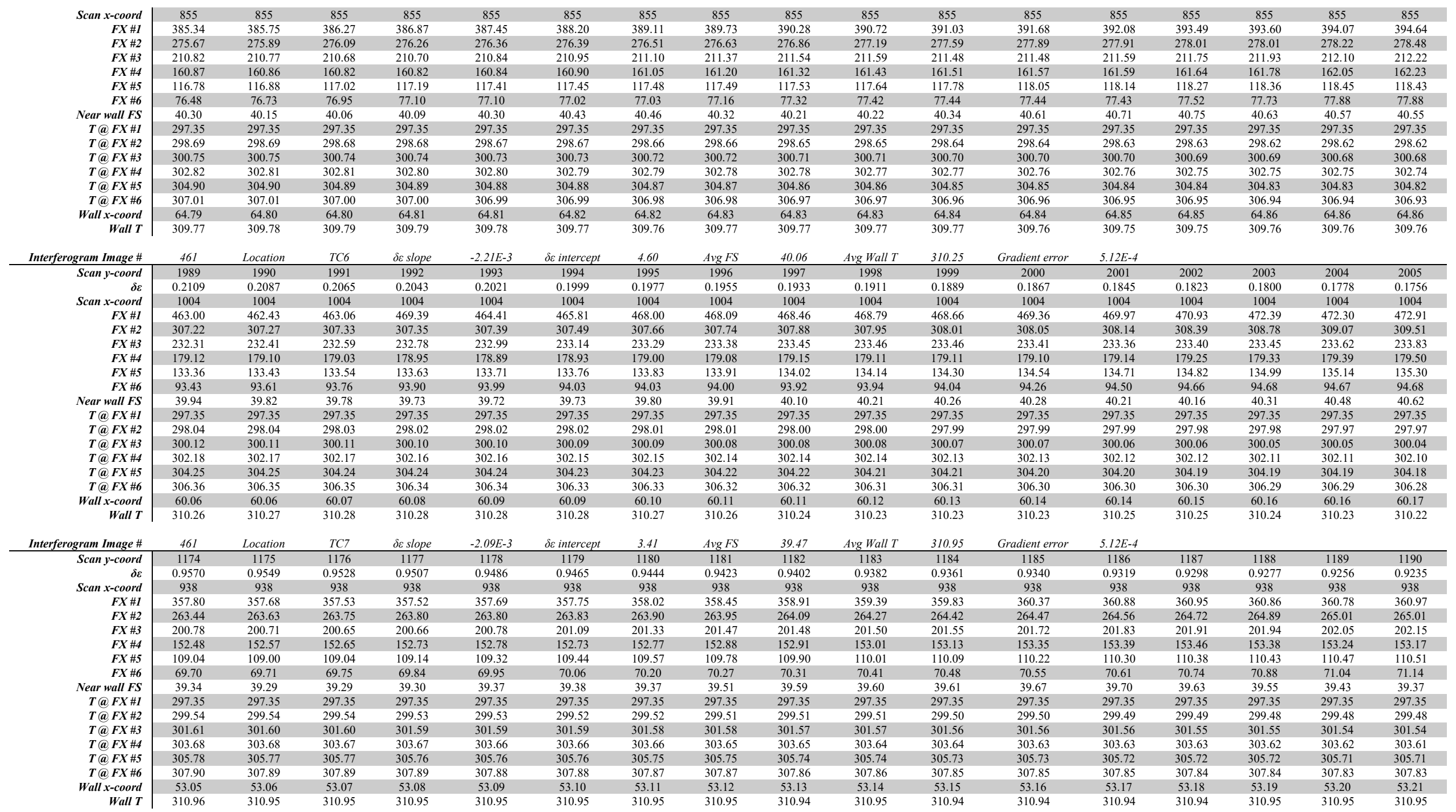

\section{B.6 Tabulated Data for Interferogram 462}

\begin{tabular}{|c|c|c|c|c|c|c|c|c|c|c|c|c|c|c|c|c|c|}
\hline Interferogram Image \# & 462 & Location & $T C 2$ & $\delta \varepsilon$ slope & $-2.52 E-3$ & $\delta \varepsilon$ intercept & 13.76 & $\operatorname{Avg} F S$ & 41.53 & Avg Wall $T$ & 307.46 & Gradient error & 4.93E-4 & & & & \\
\hline Scan $y$-coord & 5202 & 5203 & 5204 & 5205 & 5206 & 5207 & 5208 & 5209 & 5210 & 5211 & 5212 & 5213 & 5214 & 5215 & 5216 & 5217 & 5218 \\
\hline & 0.6658 & 0.6632 & 0.6607 & 0.6582 & 0.6557 & 0.6532 & 0.6507 & 0.6481 & 0.6456 & 0.6431 & 0.6406 & 0.6381 & 0.6355 & 0.6330 & 0.6305 & 0.6280 & 0.6255 \\
\hline Scan $x$-coord & 730 & 730 & 730 & 730 & 730 & 730 & 730 & 730 & 730 & 730 & 730 & 730 & 730 & 730 & 730 & 730 & 730 \\
\hline$F X \# 1$ & 309.14 & 309.87 & 310.12 & 310.52 & 310.70 & 310.13 & 305.68 & 306.47 & 307.50 & 308.44 & 307.94 & 309.97 & 310.91 & 311.30 & 311.85 & 312.14 & 312.31 \\
\hline$F X \# 2$ & 213.58 & 213.70 & 213.98 & 214.25 & 214.61 & 214.98 & 215.26 & 215.43 & 215.58 & 215.61 & 215.70 & 215.71 & 215.80 & 215.94 & 216.10 & 216.38 & 216.78 \\
\hline$F X \# 3$ & 159.84 & 159.92 & 160.00 & 160.12 & 160.23 & 160.38 & 160.51 & 160.52 & 160.51 & 160.53 & 160.53 & 160.56 & 160.56 & 160.61 & 160.63 & 160.63 & 160.66 \\
\hline$F X \# 4$ & 114.44 & 114.53 & 114.63 & 114.71 & 114.79 & 114.95 & 115.01 & 115.12 & 115.21 & 115.28 & 115.29 & 115.29 & 115.31 & 115.38 & 115.55 & 115.70 & 115.80 \\
\hline$F X \# 5$ & 72.64 & 72.70 & 72.79 & 72.94 & 73.21 & 73.43 & 73.66 & 73.70 & 73.67 & 73.63 & 73.84 & 74.00 & 74.10 & 74.13 & 74.14 & 74.18 & 74.17 \\
\hline Near wall FS & 41.80 & 41.83 & 41.84 & 41.77 & 41.58 & 41.53 & 41.34 & 41.42 & 41.54 & 41.66 & 41.45 & 41.29 & 41.21 & 41.25 & 41.41 & 41.52 & 41.64 \\
\hline$T @ F X \# 1$ & 297.28 & 297.28 & 297.28 & 297.28 & 297.28 & 297.28 & 297.28 & 297.28 & 297.28 & 297.28 & 297.28 & 297.28 & 297.28 & 297.28 & 297.28 & 297.28 & 297.28 \\
\hline$T$ (a) $F X \# 2$ & 298.81 & 298.80 & 298.80 & 298.79 & 298.79 & 298.78 & 298.78 & 298.78 & 298.77 & 298.77 & 298.76 & 298.75 & 298.75 & 298.74 & 298.74 & 298.73 & 298.73 \\
\hline$T(a) F X$ & 300.86 & 300.86 & 300.85 & 300.85 & 300.84 & 300.84 & 300.83 & 300.83 & 300.82 & 300.82 & 300.81 & 300.80 & 300.80 & 300.79 & 300.79 & 300.78 & 300.78 \\
\hline$T @ F X \# 4$ & 302.92 & 302.92 & 302.91 & 302.91 & 302.90 & 302.89 & 302.89 & 302.88 & 302.88 & 302.87 & 302.87 & 302.86 & 302.86 & 302.85 & 302.85 & 302.84 & 302.84 \\
\hline$T$ (a) FX\#5 & 305.00 & 305.00 & 304.99 & 304.99 & 304.98 & 304.98 & 304.97 & 304.97 & 304.96 & 304.96 & 304.95 & 304.95 & 304.94 & 304.94 & 304.93 & 304.92 & 304.92 \\
\hline
\end{tabular}




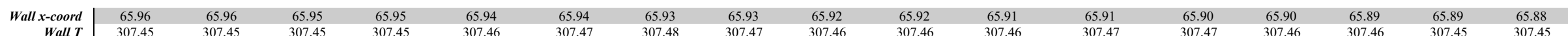

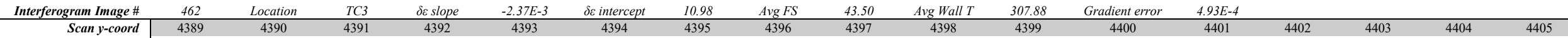

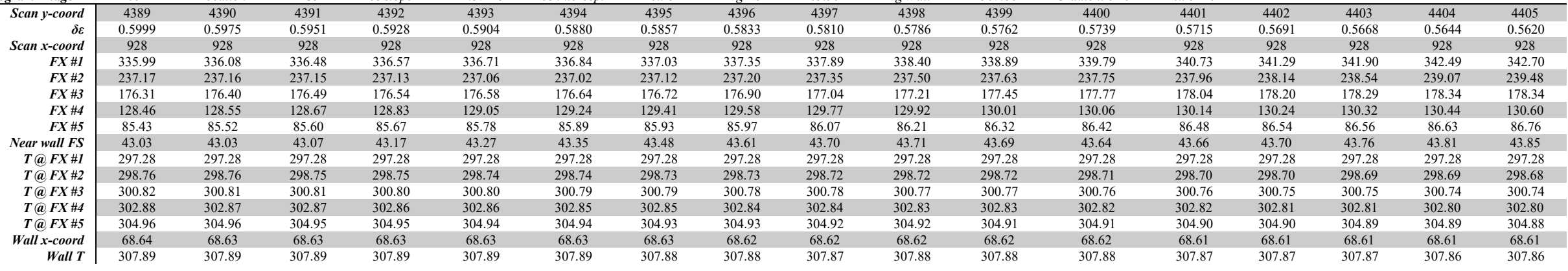

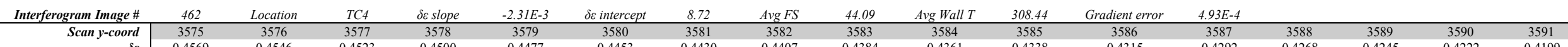

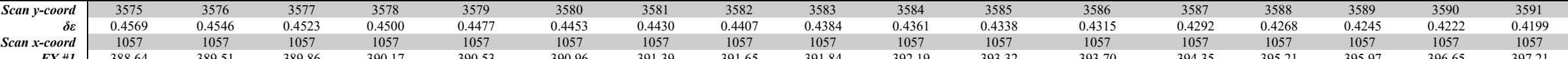

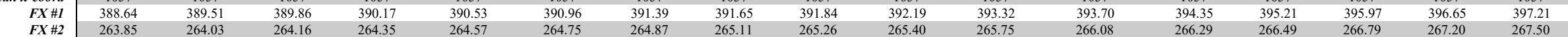

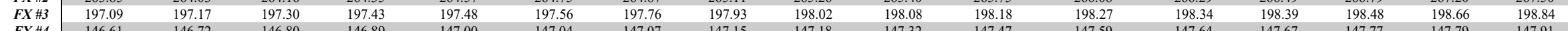

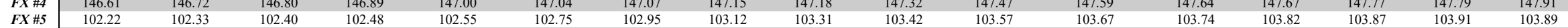

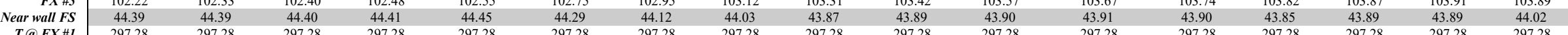

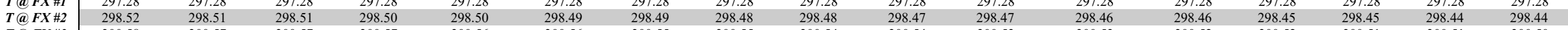

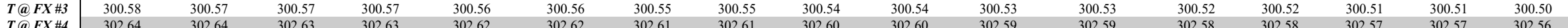

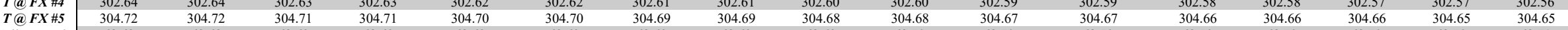

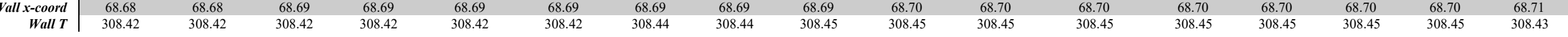

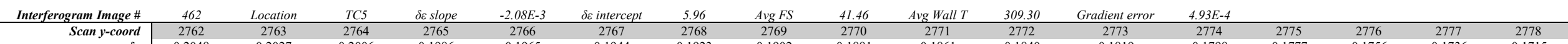

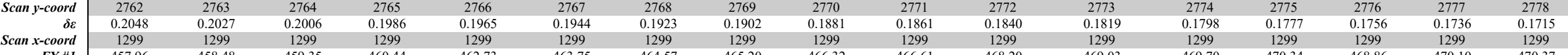

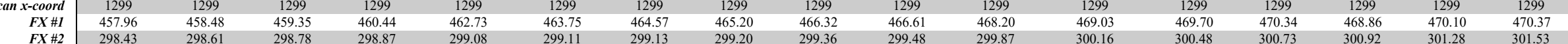

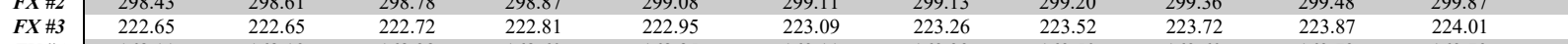

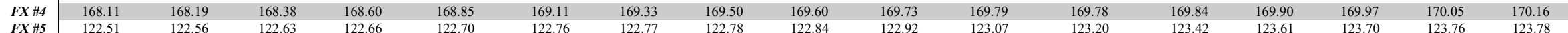

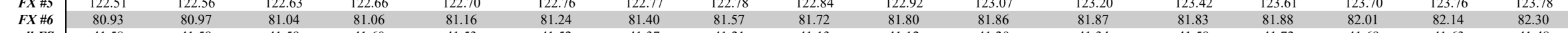

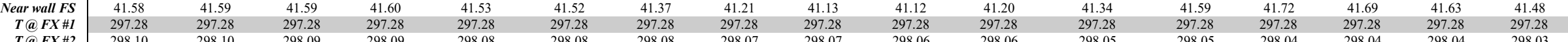

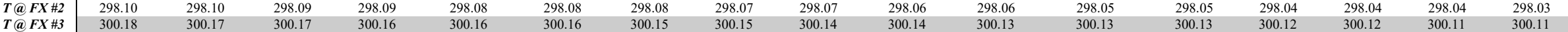

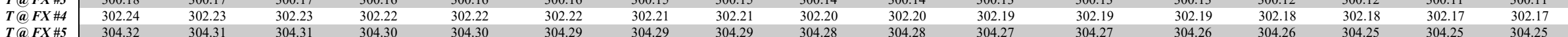

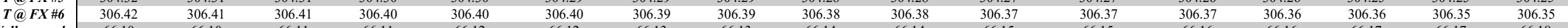

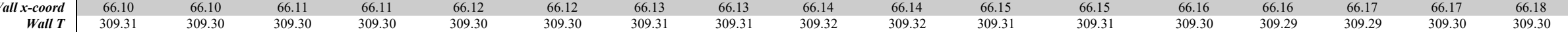

\begin{tabular}{|c|c|c|c|c|c|c|c|c|c|c|c|c|c|c|c|c|c|}
\hline Interferogram Image \# & 462 & Location & TC6 & $\delta \varepsilon$ slope & $-2.21 E-3$ & $\delta \varepsilon$ intercept & 5.25 & $\operatorname{Avg} F S$ & 42.43 & Avg Wall T & 309.88 & Gradient error & 4.93E-4 & & & & \\
\hline Scan y-coord & 1948 & 1949 & 1950 & 1951 & 1952 & 1953 & 1954 & 1955 & 1956 & 1957 & 1958 & 1959 & 1960 & 1961 & 1962 & 1963 & 1964 \\
\hline & 0.9472 & 0.9450 & 0.9428 & 0.9406 & 0.9384 & 0.9362 & 0.9340 & 0.9318 & 0.9296 & 0.9274 & 0.9252 & 0.9230 & 0.9208 & 0.9186 & 0.9164 & 0.9141 & 0.9119 \\
\hline Scan $x$-coord & 1215 & 1215 & 1215 & 1215 & 1215 & 1215 & 1215 & 1215 & 1215 & 1215 & 1215 & 1215 & 1215 & 1215 & 1215 & 1215 & 1215 \\
\hline$F X \# 1$ & 336.81 & 336.50 & 335.70 & 335.53 & 335.97 & 335.80 & 335.70 & 335.72 & 336.38 & 337.24 & 338.08 & 339.44 & 340.07 & 340.42 & 340.69 & 339.68 & 339.71 \\
\hline$F X \# 2$ & 248.72 & 249.05 & 249.37 & 249.62 & 249.75 & 249.81 & 249.77 & 249.73 & 249.80 & 249.92 & 250.18 & 250.42 & 250.68 & 250.88 & 251.08 & 251.25 & 251.44 \\
\hline$F X \# 3$ & 190.07 & 190.26 & 190.40 & 190.53 & 190.79 & 191.09 & 191.24 & 191.33 & 191.32 & 191.29 & 191.29 & 191.35 & 191.42 & 191.48 & 191.58 & 191.80 & 192.09 \\
\hline$F X \# 4$ & 142.10 & 142.12 & 142.15 & 142.24 & 142.28 & 142.45 & 142.54 & 142.64 & 142.75 & 142.87 & 143.13 & 143.34 & 143.44 & 143.47 & 143.62 & 143.69 & 143.74 \\
\hline$F X \# 5$ & 99.87 & 99.86 & 99.95 & 100.02 & 100.07 & 100.15 & 100.27 & 100.42 & 100.59 & 100.67 & 100.66 & 100.70 & 100.73 & 100.76 & 100.78 & 100.86 & 100.96 \\
\hline Near wall FS & 42.23 & 42.27 & 42.20 & 42.22 & 42.21 & 42.29 & 42.26 & 42.22 & 42.16 & 42.20 & 42.47 & 42.64 & 42.71 & 42.71 & 42.84 & 42.84 & 42.78 \\
\hline$T @ F X \# 1$ & 297.28 & 297.28 & 297.28 & 297.28 & 297.28 & 297.28 & 297.28 & 297.28 & 297.28 & 297.28 & 297.28 & 297.28 & 297.28 & 297.28 & 297.28 & 297.28 & 297.28 \\
\hline$T$ @ $F X \# 2$ & 299.60 & 299.59 & 299.59 & 299.58 & 299.58 & 299.57 & 299.57 & 299.57 & 299.56 & 299.56 & 299.55 & 299.55 & 299.54 & 299.54 & 299.53 & 299.53 & 299.52 \\
\hline$T @ F X \# 3$ & 301.66 & 301.65 & 301.65 & 301.64 & 301.64 & 301.63 & 301.63 & 301.62 & 301.62 & 301.61 & 301.61 & 301.61 & 301.60 & 301.60 & 301.59 & 301.59 & 301.58 \\
\hline
\end{tabular}




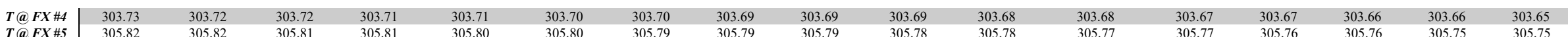

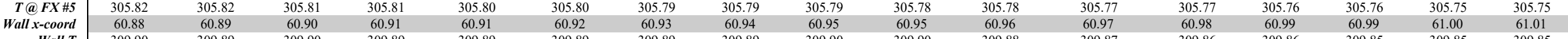

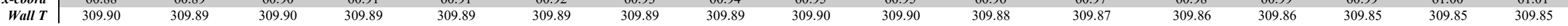

\begin{tabular}{|c|c|c|c|c|c|c|c|c|c|c|c|c|c|c|c|c|c|}
\hline Interferogram Image \# & & & & & & & & & & & & alent error & & & & & \\
\hline Scan y-coord & 1135 & 1136 & 1137 & 1138 & 1139 & 1140 & $\begin{array}{l}1141 \\
05812\end{array}$ & $\begin{array}{l}1142 \\
05793\end{array}$ & $\begin{array}{l}1143 \\
05775\end{array}$ & 1144 & $\begin{array}{l}1145 \\
05738\end{array}$ & $\begin{array}{l}1146 \\
05719\end{array}$ & $\begin{array}{l}1147 \\
05700\end{array}$ & $\begin{array}{l}1148 \\
05682\end{array}$ & 1149 & $\begin{array}{l}1150 \\
05644\end{array}$ & 1151 \\
\hline & $\begin{array}{c}0.5924 \\
979\end{array}$ & $\begin{array}{c}0.5905 \\
979\end{array}$ & $\begin{array}{c}0.5887 \\
979\end{array}$ & $\begin{array}{c}0.5868 \\
979\end{array}$ & $\begin{array}{c}0.5849 \\
979\end{array}$ & $\begin{array}{c}0.5831 \\
979\end{array}$ & $\begin{array}{c}0.5812 \\
979\end{array}$ & $\begin{array}{c}0.5793 \\
979\end{array}$ & $\begin{array}{c}0.5775 \\
979\end{array}$ & $\begin{array}{c}0.5756 \\
979\end{array}$ & $\begin{array}{c}0.5738 \\
979\end{array}$ & $\begin{array}{c}0.5719 \\
979\end{array}$ & $\begin{array}{c}0.5700 \\
979\end{array}$ & $\begin{array}{c}0.5682 \\
979\end{array}$ & $\begin{array}{c}0.5663 \\
979\end{array}$ & $\begin{array}{c}0.5644 \\
979\end{array}$ & $\begin{array}{l}0.5626 \\
979\end{array}$ \\
\hline $\begin{array}{r}\text { Scan } x \text {-coord } \\
F X \# 1\end{array}$ & $\begin{array}{c}979 \\
410.09\end{array}$ & $\begin{array}{c}979 \\
410.59\end{array}$ & $\begin{array}{c}979 \\
410.95\end{array}$ & $\begin{array}{c}979 \\
411.23\end{array}$ & $\begin{array}{c}979 \\
411.92\end{array}$ & $\begin{array}{c}979 \\
414.10\end{array}$ & $\begin{array}{c}979 \\
414.94\end{array}$ & $\begin{array}{c}979 \\
415.61\end{array}$ & $\begin{array}{c}979 \\
415.66\end{array}$ & $\begin{array}{c}979 \\
415.91\end{array}$ & $\begin{array}{c}979 \\
416.63\end{array}$ & $\begin{array}{c}979 \\
416.89\end{array}$ & $\begin{array}{c}979 \\
416.93\end{array}$ & $\begin{array}{c}979 \\
417.24\end{array}$ & $\begin{array}{c}979 \\
417.22\end{array}$ & 417.42 & 417.67 \\
\hline$F X \# 2$ & 288.47 & 288.49 & 288.44 & 288.45 & 288.65 & 289.00 & 289.26 & 289.57 & 289.77 & 289.87 & 290.16 & 290.31 & 290.42 & 290.56 & 290.61 & 290.71 & 290.82 \\
\hline$F X \# 3$ & 218.16 & 218.08 & 217.90 & 217.87 & 217.92 & 217.99 & 218.03 & 218.04 & 218.12 & 218.29 & 218.34 & 218.33 & 218.26 & 218.13 & 218.06 & 218.11 & 218.20 \\
\hline$F X \# 4$ & 165.53 & 165.65 & 165.90 & 165.99 & 165.97 & 165.88 & 165.85 & 165.90 & 165.98 & 166.04 & 166.12 & 166.24 & 166.20 & 166.20 & 166.22 & 166.36 & 166.41 \\
\hline$F X \# 5$ & 120.33 & 120.41 & 120.48 & 120.55 & 120.65 & 120.74 & 120.86 & 121.03 & 121.31 & 121.44 & 121.52 & 121.62 & 121.65 & 121.73 & 121.75 & 12195 & 122.07 \\
\hline$F X \# 6$ & 79.14 & 79.14 & 79.08 & 79.08 & 79.14 & 79.23 & 79.32 & 79.43 & 79.62 & 79.82 & 79.91 & 79.96 & 79.98 & 79.97 & 79.92 & 79.87 & 79.80 \\
\hline Near wall FS & 41.19 & 41.27 & 41.39 & 41.47 & 41.51 & 41.51 & 41.54 & 41.60 & 41.69 & 41.62 & 41.61 & 41.67 & 41.67 & 41.75 & 41.83 & 42.08 & 42.26 \\
\hline$T @ F X \# 1$ & 297.28 & 297.28 & 297.28 & 297.28 & 297.28 & 297.28 & 297.28 & 297.28 & 297.28 & 297.28 & 297.28 & 297.28 & 297.28 & 297.28 & 297.28 & 297.28 & 297.28 \\
\hline$T$ (a) $F X \# 2$ & 298.74 & 298.73 & 298.73 & 298.73 & 298.72 & 298.72 & 298.71 & 298.71 & 298.70 & 298.70 & 298.70 & 298.69 & 298.69 & 298.69 & 298.68 & 298.68 & 298.67 \\
\hline$T(\mathbb{a} F X \# 3$ & 300.80 & 300.80 & 300.79 & 300.79 & 300.79 & 300.78 & 300.78 & 300.77 & 300.77 & 300.77 & 300.76 & 300.76 & 300.75 & 300.75 & 300.75 & 300.74 & 300.74 \\
\hline$T(a) F X \# 4$ & 302.87 & 302.86 & 302.86 & 302.86 & 302.85 & 302.85 & 302.84 & 302.84 & 302.84 & 302.83 & 302.83 & 302.83 & 302.82 & 302.82 & 302.81 & 302.81 & 302.81 \\
\hline$T @ F X \# 6$ & 307.06 & 307.06 & 307.06 & 307.05 & 307.05 & 307.04 & 307.04 & 307.04 & 307.03 & 307.03 & 307.02 & 307.02 & 307.02 & 307.01 & 307.01 & 307.00 & 307.00 \\
\hline Wall $x$-coord & 53.04 & 53.05 & 53.07 & 53.08 & 53.09 & 53.10 & 53.11 & 53.12 & 53.13 & 53.14 & 53.16 & 53.17 & 53.18 & 53.19 & 53.20 & 53.21 & 53.22 \\
\hline Wall T & 310.56 & 310.55 & 310.54 & 310.53 & 310.53 & 310.53 & 310.53 & 310.52 & 310.53 & 310.53 & 310.54 & 310.53 & $\begin{array}{l}310.10 \\
310.53\end{array}$ & 310.52 & 310.51 & 310.50 & 310.48 \\
\hline
\end{tabular}

\section{B.7 Tabulated Data for Interferogram 463}

\begin{tabular}{|c|c|c|c|c|c|c|c|c|c|c|c|c|c|c|c|c|c|}
\hline Interferogram Image \# & 463 & Location & $T C 2$ & $\delta \varepsilon$ slope & $-2.37 E-3$ & $\delta \varepsilon$ intercept & 12.88 & $\operatorname{Avg} F S$ & 45.74 & Avg Wall T & 307.23 & Gradient error & 4.17E-4 & & & & \\
\hline Scan y-coord & 5277 & 5278 & 5279 & 5280 & 5281 & 5282 & 5283 & 5284 & 5285 & 5286 & 5287 & 5288 & 5289 & 5290 & 5291 & 5292 & 5293 \\
\hline & 0.3751 & 0.3728 & 0.3704 & 0.3680 & 0.3657 & 0.3633 & 0.3609 & 0.3586 & 0.3562 & 0.3538 & 0.3514 & 0.3491 & 0.3467 & 0.3443 & 0.3420 & 0.3396 & 0.3372 \\
\hline Scan $x$-coord & 810 & 810 & 810 & 810 & 810 & 810 & 810 & 810 & 810 & 810 & 810 & 810 & 810 & 810 & 810 & 810 & 810 \\
\hline$F X \# 1$ & 384.02 & 384.73 & 380.93 & 384.95 & 386.04 & 385.75 & 386.03 & 387.03 & 387.25 & 386.81 & 387.42 & 388.74 & 389.06 & 388.46 & 389.89 & 390.58 & 440.88 \\
\hline$F X \# 2$ & 256.69 & 256.92 & 257.20 & 258.12 & 258.54 & 258.81 & 259.31 & 259.73 & 260.14 & 260.46 & 260.88 & 261.26 & 261.77 & 262.33 & 262.96 & 263.54 & 264.01 \\
\hline$F X \# 3$ & 197.98 & 197.98 & 197.99 & 198.04 & 198.24 & $\begin{array}{l}198.01 \\
198.39\end{array}$ & 198.63 & 198.79 & 198.90 & 198.91 & 198.87 & 198.85 & 198.89 & 198.95 & 199.05 & 199.15 & 199.23 \\
\hline$F X \# 5$ & 101.18 & 101.27 & 101.32 & 101.46 & 101.72 & 102.05 & 102.36 & 102.63 & 102.77 & 102.77 & 102.76 & 102.85 & 103.00 & 103.11 & 103.14 & 103.27 & 103.40 \\
\hline Near wall FS & 46.28 & 46.27 & 46.36 & 46.32 & 46.22 & 45.95 & 45.76 & 45.57 & 45.50 & 45.58 & 45.60 & 45.49 & 45.37 & 45.29 & 45.31 & 45.28 & 45.40 \\
\hline$T @ F X \# 1$ & 297.30 & 297.30 & 297.30 & 297.30 & 297.30 & 297.30 & 297.30 & 297.30 & 297.30 & 297.30 & 297.30 & 297.30 & 297.30 & 297.30 & 297.30 & 297.30 & 297.30 \\
\hline$T @ F X \# 2$ & 298.22 & 298.22 & 298.21 & 298.21 & 298.20 & 298.20 & 298.19 & 298.19 & 298.18 & 298.18 & 298.17 & 298.17 & 298.16 & 298.16 & 298.15 & 298.15 & 298.12 \\
\hline$T @ F X \# 3$ & 300.27 & 300.27 & 300.26 & 300.26 & 300.25 & 300.25 & 300.24 & 300.24 & 300.23 & 300.23 & 300.22 & 300.22 & 300.21 & 300.21 & 300.20 & 300.20 & 300.19 \\
\hline$T @ F X \# 4$ & 302.32 & 302.31 & 302.31 & 302.30 & 302.30 & 302.29 & 302.29 & 302.28 & 302.28 & 302.28 & 302.27 & 302.27 & 302.26 & 302.26 & 302.25 & 302.25 & 302.24 \\
\hline Wall $x$-coord & 85.54 & 85.54 & 85.53 & 85.53 & 85.53 & 85.52 & 85.52 & 85.51 & 85.51 & 85.51 & 85.50 & 85.50 & 85.49 & 85.49 & 85.49 & 85.48 & 85.48 \\
\hline Wall T & 307.20 & 307.20 & 307.20 & 307.20 & 307.21 & 307.22 & 307.23 & $\begin{array}{l}8.51 \\
307.25\end{array}$ & $\begin{array}{l}30.51 \\
307.25\end{array}$ & $\begin{array}{l}8.51 \\
307.24\end{array}$ & $\begin{array}{l}8.50 \\
307.24\end{array}$ & $\begin{array}{l}8.50 \\
307.24\end{array}$ & $\begin{array}{l}8.49 \\
307.24\end{array}$ & $\begin{array}{l}8.49 \\
307.24\end{array}$ & $\begin{array}{l}8.49 \\
307.24\end{array}$ & $\begin{array}{r}8.48 \\
307.24\end{array}$ & $\begin{array}{r}307.24 \\
3072\end{array}$ \\
\hline Interferogram Image \# & 463 & Location & $T C 3$ & $\delta \varepsilon$ slope & $-2.28 E-3$ & $\delta \varepsilon$ intercept & 10.47 & $\operatorname{Avg} F S$ & 46.99 & Avg Wall T & 307.53 & Gradient error & 4. $17 E-4$ & & & & \\
\hline Scan y-coord & 4463 & 4464 & 4465 & 4466 & $\frac{4.20 L-1}{4467}$ & 4468 & 4469 & 4470 & 4471 & 4472 & 4473 & 4474 & $\frac{7.176-7}{445}$ & 4476 & 4477 & 4478 & 4479 \\
\hline & 0.2802 & 0.2779 & 0.2756 & 0.2733 & 0.2710 & 0.2687 & 0.2665 & 0.2642 & 0.2619 & 0.2596 & 0.2573 & 0.2551 & 0.2528 & 0.2505 & 0.2482 & 0.2459 & 0.2436 \\
\hline Scan $x$-coord & 837 & 837 & 837 & 837 & 837 & 837 & 837 & 837 & 837 & 837 & 837 & 837 & 837 & 837 & 837 & 837 & 837 \\
\hline$F X \# 1$ & 442.97 & 443.66 & 443.45 & 444.58 & 445.04 & 445.52 & 445.77 & 445.41 & 460.94 & 446.65 & 449.40 & 449.87 & 452.02 & 452.32 & 451.16 & 451.91 & 452.58 \\
\hline$F X \# 2$ & 289.98 & 290.11 & 290.23 & 290.56 & 290.77 & 291.01 & 291.03 & $\begin{array}{l}291.09 \\
291.09\end{array}$ & 291.19 & 291.24 & 291.49 & 291.65 & $\begin{array}{l}432.02 \\
291.82\end{array}$ & $\begin{array}{l}52.32 \\
291.88\end{array}$ & $\begin{array}{l}\begin{array}{l}51.16 \\
291.88\end{array} \\
\text {. }\end{array}$ & $\begin{array}{l}\begin{array}{l}51.91 \\
291.94\end{array} \\
\end{array}$ & $\begin{array}{l}452.58 \\
292.02\end{array}$ \\
\hline$F X \# 3$ & 217.15 & 217.19 & 217.23 & 217.29 & 217.37 & 217.49 & 217.53 & 217.60 & 217.70 & 217.83 & 218.01 & 218.18 & 218.38 & 218.59 & 218.69 & 218.76 & 218.81 \\
\hline$F X \# 4$ & 161.68 & 161.73 & 161.89 & 162.09 & 162.26 & 162.42 & 162.62 & 162.81 & 163.00 & 163.28 & 163.43 & 163.59 & 163.72 & 163.82 & 163.99 & 164.14 & 164.26 \\
\hline$F X \# 5$ & 114.48 & 114.70 & 114.94 & 115.20 & 115.43 & 115.58 & 115.70 & 115.90 & 116.06 & 116.26 & 116.45 & 116.60 & 116.71 & 116.83 & 116.92 & 117.03 & 117.15 \\
\hline Near wall FS & 47.20 & 47.04 & 46.94 & 46.89 & 46.83 & 46.84 & 46.92 & 46.91 & 46.95 & 47.02 & 46.98 & 46.99 & 47.01 & 46.99 & 47.07 & 47.11 & 47.11 \\
\hline$T @ F X \# 3$ & 300.08 & 300.07 & 300.07 & 300.06 & 300.06 & 300.05 & 300.05 & 300.04 & 300.04 & 300.03 & 300.03 & 300.03 & 300.02 & 300.02 & 300.01 & 300.01 & 300.00 \\
\hline$T @ F X \# 4$ & 302.13 & 302.12 & 302.12 & 302.11 & 302.11 & 302.11 & 302.10 & 302.10 & 302.09 & 302.09 & 302.08 & 302.08 & 302.07 & 302.07 & 302.06 & 302.06 & 302.05 \\
\hline$T @ F X \# 5$ & 304.20 & 304.20 & 304.19 & 304.19 & 304.18 & 304.18 & 304.17 & 304.17 & 304.16 & 304.16 & 304.15 & 304.15 & 304.14 & 304.14 & 304.13 & 304.13 & 304.13 \\
\hline Wall $x$-coord & 87.64 & 87.64 & 87.64 & 87.64 & 87.64 & 87.64 & 87.64 & 87.64 & 87.63 & 87.63 & 87.63 & 87.63 & 87.63 & 87.63 & 87.63 & 87.63 & 87.63 \\
\hline Wall T & 307.49 & 307.50 & 307.51 & 307.52 & 307.52 & 307.53 & 307.52 & 307.53 & 307.53 & 307.53 & 307.54 & 307.54 & 307.54 & 307.54 & 307.54 & 307.54 & 307.54 \\
\hline Interferogram Image \# & 463 & Location & $T C 4$ & $\delta \varepsilon$ slope & $-2.31 E-3$ & $\delta \varepsilon$ intercept & 8.53 & $\operatorname{Avg} F S$ & 60.47 & Avg Wall $T$ & 308.05 & Gradient error & 4.17E-4 & & & & \\
\hline Scan y-coord & 3649 & 3650 & 3651 & 3652 & 3653 & 3654 & 3655 & 3656 & 3657 & 3658 & 3659 & 3660 & 3661 & 3662 & 3663 & 3664 & 3665 \\
\hline & 0.0987 & 0.0964 & 0.0941 & 0.0918 & 0.0895 & 0.0872 & 0.0849 & 0.0826 & 0.0802 & 0.0779 & 0.0756 & 0.0733 & 0.0710 & 0.0687 & 0.0664 & 0.0641 & 0.0618 \\
\hline Scan $x$-coord & 977 & 977 & 977 & 977 & 977 & 977 & 977 & 977 & 977 & 977 & 977 & 977 & 977 & 977 & 977 & 977 & 977 \\
\hline$F X \# 1$ & 556.70 & 557.34 & 561.39 & 562.28 & 561.06 & 564.33 & 565.30 & 568.71 & 570.95 & 573.03 & 575.32 & 574.41 & 575.16 & 574.99 & 588.01 & 589.94 & 592.02 \\
\hline$F X \# 2$ & 321.21 & 321.55 & 321.76 & $\begin{array}{l}5022.28 \\
322.03\end{array}$ & $\begin{array}{l}501.00 \\
322.33\end{array}$ & $\begin{array}{l}504.35 \\
322.54\end{array}$ & $\begin{array}{l}305.50 \\
322.95\end{array}$ & 323.21 & 323.49 & 323.62 & 323.76 & $\begin{array}{l}3 / 4.41 \\
323.93\end{array}$ & 324.11 & 324.34 & $\begin{array}{l}588.01 \\
324.54\end{array}$ & $\begin{array}{l}589.94 \\
324.80\end{array}$ & 324.96 \\
\hline$F X \# 3$ & 239.06 & 239.22 & 239.36 & 239.50 & 239.68 & 239.89 & 240.00 & 240.13 & 240.21 & 240.34 & 240.42 & 240.48 & 240.56 & 240.68 & 240.77 & 240.88 & 241.10 \\
\hline$F X \# 4$ & 181.13 & 181.18 & 181.28 & 181.39 & 181.42 & 181.41 & 181.58 & 181.65 & 181.68 & 181.78 & 181.89 & 182.02 & 182.17 & 182.33 & 182.47 & 182.57 & 182.64 \\
\hline$F X \# 5$ & 132.62 & 132.28 & $\begin{array}{l}101.20 \\
132.27\end{array}$ & 132.29 & $\begin{array}{l}101.42 \\
132.71\end{array}$ & $\begin{array}{l}101.41 \\
132.75\end{array}$ & 132.87 & 132.96 & $\begin{array}{l}10.00 \\
133.08\end{array}$ & 133.25 & 133.40 & 133.50 & 133.56 & 133.61 & 133.67 & 133.80 & 133.95 \\
\hline$F X \# 6$ & 88.23 & 0.00 & 0.00 & 0.00 & 88.02 & 87.90 & 87.87 & 88.13 & 88.34 & 88.09 & 88.09 & 88.07 & 88.09 & 88.10 & 88.21 & 88.60 & 88.84 \\
\hline
\end{tabular}




\begin{tabular}{|c|c|c|c|c|c|c|c|c|c|c|c|c|c|c|c|c|c|}
\hline Near wall FS & 44.38 & 132.28 & 132.27 & 132.29 & 44.70 & 44.85 & 45.00 & 44.83 & 44.74 & 45.16 & 45.31 & 45.43 & 45.47 & 45.50 & 45.46 & 45.20 & 45.12 \\
\hline$T @ F X \# 1$ & 297.30 & 297.30 & 297.30 & 297.30 & 297.30 & 297.30 & 297.30 & 297.30 & 297.30 & 297.30 & 297.30 & 297.30 & 297.30 & 297.30 & 297.30 & 297.30 & 297.30 \\
\hline$T @ F X \# 2$ & 297.67 & 297.67 & 297.66 & 297.66 & 297.65 & 297.65 & 297.64 & 297.63 & 297.63 & 297.62 & 297.62 & 297.61 & 297.61 & 297.60 & 297.59 & 297.59 & 297.58 \\
\hline$T @ F X \# 3$ & 299.76 & 299.75 & 299.75 & 299.74 & 299.74 & 299.74 & 299.73 & 299.73 & 299.72 & 299.72 & 299.71 & 299.71 & 299.70 & 299.70 & 299.69 & 299.69 & 299.68 \\
\hline$T(F X \# 4$ & 301.81 & 301.81 & 301.80 & 301.80 & 301.79 & 301.79 & 301.78 & 301.78 & 301.77 & 301.77 & 301.76 & 301.76 & 301.75 & 301.75 & 301.74 & 301.74 & 301.73 \\
\hline$T @ F X \# 5$ & 303.88 & 303.88 & 303.87 & 303.87 & 303.86 & 303.86 & 303.85 & 303.85 & 303.84 & 303.84 & 303.83 & 303.83 & 303.82 & 303.82 & 303.81 & 303.81 & 303.80 \\
\hline$T @ F X \# 6$ & 305.97 & 305.97 & 305.96 & 305.96 & 305.95 & 305.95 & 305.94 & 305.94 & 305.93 & 305.93 & 305.93 & 305.92 & 305.92 & 305.91 & 305.91 & 305.90 & 305.90 \\
\hline Wall $x$-coord & 87.35 & 87.35 & 87.35 & 87.35 & 87.35 & 87.35 & 87.36 & 87.36 & 87.36 & 87.36 & 87.36 & 87.37 & 87.37 & 87.37 & 87.37 & 87.37 & 87.37 \\
\hline Wall T & 308.14 & 307.89 & 307.88 & 307.88 & 308.11 & 308.10 & 308.09 & 308.10 & 308.10 & 308.08 & 308.08 & 308.07 & 308.07 & 308.07 & 308.07 & 308.08 & 308.08 \\
\hline
\end{tabular}

\begin{tabular}{|c|c|c|c|c|c|c|c|c|c|c|c|c|c|c|c|c|c|}
\hline Interferogram Image \# & 463 & Location & $T C 5$ & $\delta \varepsilon$ slope & $-2.23 E-3$ & $\delta \varepsilon$ intercept & 7.27 & $\operatorname{Avg} F S$ & 44.80 & Avg Wall $T$ & 308.92 & Gradient error & $4.17 E-4$ & & & & \\
\hline Scan $y$-coord & 2834 & 2835 & 2836 & 2837 & 2838 & 2839 & 2840 & 2841 & 2842 & 2843 & 2844 & 2845 & 2846 & 2847 & 2848 & 2849 & 2850 \\
\hline$\delta \varepsilon$ & 0.9493 & 0.9471 & 0.9449 & 0.9426 & 0.9404 & 0.9382 & 0.9359 & 0.9337 & 0.9315 & 0.9293 & 0.9270 & 0.9248 & 0.9226 & 0.9203 & 0.9181 & 0.9159 & 0.9136 \\
\hline Scan $x$-coord & 987 & 987 & 987 & 987 & 987 & 987 & 987 & 987 & 987 & 987 & 987 & 987 & 987 & 987 & 987 & 987 & 987 \\
\hline$F X \# I$ & 359.28 & 359.72 & 358.62 & 360.48 & 360.89 & 358.66 & 359.03 & 359.17 & 359.11 & 359.20 & 359.22 & 359.52 & 359.87 & 360.12 & 360.56 & 360.85 & 361.32 \\
\hline$F X \# 2$ & 264.67 & 264.83 & 264.97 & 265.05 & 265.11 & 265.24 & 265.28 & 265.33 & 265.40 & 265.54 & 265.70 & 265.90 & 266.02 & 266.11 & 266.30 & 266.42 & 266.57 \\
\hline$F X \# 3$ & 203.57 & 203.71 & 203.86 & 204.05 & 204.18 & 204.33 & 204.51 & 204.73 & 204.93 & 205.10 & 205.28 & 205.47 & 205.52 & 205.49 & 205.42 & 205.36 & 205.37 \\
\hline$F X \# 4$ & 153.87 & 153.89 & 153.94 & 153.93 & 153.92 & 154.07 & 154.23 & 154.47 & 154.65 & 154.79 & 154.93 & 155.07 & 155.20 & 155.42 & 155.52 & 155.56 & 155.57 \\
\hline$F X \# 5$ & 108.79 & 108.97 & 109.18 & 109.38 & 109.49 & 109.65 & 109.77 & 109.89 & 109.97 & 110.00 & 110.14 & 110.22 & 110.32 & 110.41 & 110.44 & 110.42 & 110.41 \\
\hline Near wall FS & 45.08 & 44.92 & 44.76 & 44.55 & 44.43 & 44.42 & 44.46 & 44.58 & 44.68 & 44.78 & 44.79 & 44.85 & 44.88 & 45.00 & 45.08 & 45.13 & 45.16 \\
\hline$T @ F X \# 1$ & 297.30 & 297.30 & 297.30 & 297.30 & 297.30 & 297.30 & 297.30 & 297.30 & 297.30 & 297.30 & 297.30 & 297.30 & 297.30 & 297.30 & 297.30 & 297.30 & 297.30 \\
\hline$T$ (a) $F X \# 2$ & 299.45 & 299.44 & 299.44 & 299.43 & 299.43 & 299.42 & 299.42 & 299.42 & 299.41 & 299.41 & 299.40 & 299.40 & 299.39 & 299.39 & 299.38 & 299.38 & 299.37 \\
\hline$T @ F X \# 3$ & 301.50 & 301.49 & 301.49 & 301.49 & 301.48 & 301.48 & 301.47 & 301.47 & 301.46 & 301.46 & 301.45 & 301.45 & 301.44 & 301.44 & 301.44 & 301.43 & 301.43 \\
\hline$T$ (a) $F X \# 4$ & 303.57 & 303.56 & 303.56 & 303.55 & 303.55 & 303.54 & 303.54 & 303.53 & 303.53 & 303.52 & 303.52 & 303.51 & 303.51 & 303.51 & 303.50 & 303.50 & 303.49 \\
\hline Wall $x$-coord & 84.64 & 84.65 & 84.65 & 84.66 & 84.66 & 84.67 & 84.67 & 84.68 & 84.68 & 84.69 & 84.69 & 84.70 & 84.70 & 84.71 & 84.71 & 84.72 & 84.72 \\
\hline Wall $T$ & 308.91 & 308.91 & 308.92 & 308.93 & 308.94 & 308.94 & 308.94 & 308.94 & 308.93 & 308.93 & 308.93 & 308.92 & 308.92 & 308.92 & 308.91 & 308.91 & 308.90 \\
\hline
\end{tabular}

\begin{tabular}{|c|c|c|c|c|c|c|c|c|c|c|c|c|c|c|c|c|c|}
\hline Interferogram Image \# & 463 & Location & TC6 & $\delta \varepsilon$ slope & $-2.21 E-3$ & $\delta \varepsilon$ intercept & 5.08 & $\operatorname{Avg} F S$ & 41.72 & Avg Wall $T$ & 309.67 & Gradient error & 4.17E-4 & & & & \\
\hline Scan y-coord & 2020 & 2021 & 2022 & 2023 & 2024 & 2025 & 2026 & 2027 & 2028 & 2029 & 2030 & 2031 & 2032 & 2033 & 2034 & 2035 & 2036 \\
\hline & 0.6070 & 0.6048 & 0.6026 & 0.6004 & 0.5982 & 0.5960 & 0.5938 & 0.5915 & 0.5893 & 0.5871 & 0.5849 & 0.5827 & 0.5805 & 0.5783 & 0.5760 & 0.5738 & 0.5716 \\
\hline Scan $x$-coord & 1154 & 1154 & 1154 & 1154 & 1154 & 1154 & 1154 & 1154 & 1154 & 1154 & 1154 & 1154 & 1154 & 1154 & 1154 & 1154 & 1154 \\
\hline$F X \# 1$ & 411.34 & 411.60 & 411.61 & 412.34 & 412.83 & 413.27 & 413.70 & 413.93 & 414.14 & 414.36 & 414.61 & 414.98 & 415.47 & 415.99 & 416.81 & 417.40 & 417.97 \\
\hline $\begin{array}{l}F X+1 \\
F X \# 2\end{array}$ & $\begin{array}{l}411.04 \\
294.52\end{array}$ & $\begin{array}{l}411.00 \\
294.72\end{array}$ & 294.87 & $\begin{array}{l}4125.07 \\
295.07\end{array}$ & $\begin{array}{l}412.03 \\
295.29\end{array}$ & $\begin{array}{l}4155.47 \\
295.47\end{array}$ & 295.70 & $\begin{array}{l}41.95 \\
295.94\end{array}$ & $\begin{array}{l}414.14 \\
296.23\end{array}$ & 296.51 & $\begin{array}{l}414.01 \\
296.72\end{array}$ & 296.81 & $\begin{array}{l}4156.90 \\
2969\end{array}$ & 296.96 & $\begin{array}{l}410.01 \\
29699\end{array}$ & 297.01 & 297.04 \\
\hline$F X \# 3$ & 226.50 & 226.62 & 226.77 & 226.86 & 226.98 & 227.11 & 227.30 & 227.49 & 227.65 & 227.78 & 227.92 & 228.04 & 228.13 & 228.19 & 228.19 & 228.19 & 228.22 \\
\hline$F X \# 5$ & 129.30 & 129.37 & 129.47 & 129.63 & 129.73 & 129.82 & 129.94 & 130.10 & 130.27 & 130.39 & 130.49 & 130.56 & 130.62 & 130.64 & 130.66 & 130.70 & 130.73 \\
\hline$F X \# 6$ & 87.97 & 88.16 & 88.36 & 88.54 & 88.64 & 88.66 & 88.59 & 88.36 & 88.14 & 88.01 & 87.99 & 88.18 & 88.39 & 88.55 & 88.69 & 88.86 & 89.14 \\
\hline Near wall FS & 41.33 & 41.21 & 41.11 & 41.09 & 41.09 & 41.16 & 41.36 & 41.73 & 42.12 & 42.38 & 42.50 & 42.38 & 42.23 & 42.09 & 41.96 & 41.84 & 41.59 \\
\hline$T @ F X \# 1$ & 297.30 & 297.30 & 297.30 & 297.30 & 297.30 & 297.30 & 297.30 & 297.30 & 297.30 & 297.30 & 297.30 & 297.30 & 297.30 & 297.30 & 297.30 & 297.30 & 297.30 \\
\hline$T(F X \# 2$ & 298.81 & 298.81 & 298.80 & 298.80 & 298.79 & 298.79 & 298.78 & 298.78 & 298.78 & 298.77 & 298.77 & 298.76 & 298.76 & 298.75 & 298.75 & 298.74 & 298.74 \\
\hline$T(a) F X \# 3$ & 300.87 & 300.86 & 300.86 & 300.85 & 300.85 & 300.84 & 300.84 & 300.83 & 300.83 & 300.82 & 300.82 & 300.82 & 300.81 & 300.81 & 300.80 & 300.80 & 300.79 \\
\hline$T$ (a) $F X \# 5$ & 305.01 & 305.00 & 305.00 & 304.99 & 304.99 & 304.99 & 304.98 & 304.98 & 304.97 & 304.97 & 304.96 & 304.96 & 304.95 & 304.95 & 304.94 & 304.94 & 304.93 \\
\hline$T @ F X \# 6$ & 307.12 & 307.11 & 307.11 & 307.10 & 307.10 & 307.09 & 307.09 & 307.08 & 307.08 & 307.07 & 307.07 & 307.06 & 307.06 & 307.06 & 307.05 & 307.05 & 307.04 \\
\hline Wall $x$-coord & 79.55 & 79.55 & 79.56 & 79.57 & 79.58 & 79.58 & 79.59 & 79.60 & 79.61 & 79.62 & 79.62 & 79.63 & 79.64 & 79.65 & 79.65 & 79.66 & 79.67 \\
\hline Wall T & 309.69 & 309.69 & 309.70 & 309.70 & 309.70 & 309.70 & 309.69 & 309.67 & 309.65 & 309.63 & 309.63 & 309.63 & 309.64 & 309.64 & 309.65 & 309.65 & 309.66 \\
\hline
\end{tabular}

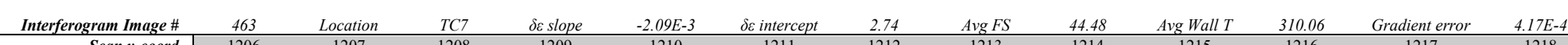

\begin{tabular}{|c|c|c|c|c|c|c|c|c|c|c|c|c|c|c|c|c|c|}
\hline & & & & & & & & & & & & & & & & & \\
\hline Scan $y$-coord & 1206 & 1207 & 1208 & 1209 & 1210 & 1211 & 1212 & 1213 & 1214 & 1215 & 1216 & 1217 & 1218 & 1219 & 1220 & 1221 & 1222 \\
\hline$\delta \varepsilon$ & 0.2199 & 0.2178 & 0.2157 & 0.2137 & 0.2116 & 0.2095 & 0.2074 & 0.2053 & 0.2032 & 0.2011 & 0.1990 & 0.1969 & 0.1948 & 0.1927 & 0.1907 & 0.1886 & 0.1865 \\
\hline Scan $x$-coord & 1144 & 1144 & 1144 & 1144 & 1144 & 1144 & 1144 & 1144 & 1144 & 1144 & 1144 & 1144 & 1144 & 1144 & 1144 & 1144 & 1144 \\
\hline$F X \# 1$ & 550.12 & 550.33 & 550.51 & 550.87 & 552.45 & 552.82 & 553.96 & 553.84 & 559.01 & 560.24 & 561.31 & 561.77 & 562.71 & 563.43 & 563.88 & 564.43 & 564.74 \\
\hline$F X \# 2$ & 348.76 & 348.98 & 349.17 & 349.35 & 349.45 & 349.33 & 349.21 & 349.21 & 349.42 & 349.51 & 349.55 & 349.60 & 349.73 & 349.77 & 349.67 & 349.45 & 349.22 \\
\hline$F X \# 3$ & 257.31 & 257.48 & 257.57 & 257.61 & 257.56 & 257.54 & 257.59 & 257.67 & 257.84 & 258.10 & 258.35 & 258.66 & 258.93 & 259.20 & 259.55 & 259.78 & 259.92 \\
\hline$F X \# 4$ & 198.98 & 199.15 & 199.32 & 199.40 & 199.46 & 199.59 & 199.83 & 200.10 & 200.30 & 200.39 & 200.49 & 200.62 & 200.80 & 200.91 & 200.98 & 200.99 & 201.04 \\
\hline$F X \# 5$ & 150.99 & 151.11 & 151.30 & 151.42 & 151.52 & 151.58 & 151.71 & 151.78 & 151.79 & 151.95 & 152.01 & 152.05 & 152.08 & 152.10 & 152.14 & 152.27 & 152.33 \\
\hline$F X \# 6$ & 106.70 & 106.72 & 106.75 & 106.88 & 106.92 & 106.91 & 106.92 & 107.00 & 107.14 & 107.22 & 107.41 & 107.61 & 107.75 & 107.88 & 107.96 & 108.05 & 108.09 \\
\hline Near wall FS & 44.29 & 44.39 & 44.54 & 44.55 & 44.59 & 44.67 & 44.80 & 44.77 & 44.65 & 44.73 & 44.60 & 44.43 & 44.33 & 44.22 & 44.18 & 44.23 & 44.24 \\
\hline$T @ F X \# 1$ & 297.30 & 297.30 & 297.30 & 297.30 & 297.30 & 297.30 & 297.30 & 297.30 & 297.30 & 297.30 & 297.30 & 297.30 & 297.30 & 297.30 & 297.30 & 297.30 & 297.30 \\
\hline$T(F X \# 2$ & 297.98 & 297.98 & 297.97 & 297.97 & 297.97 & 297.96 & 297.96 & 297.95 & 297.95 & 297.94 & 297.94 & 297.93 & 297.93 & 297.92 & 297.92 & 297.92 & 297.91 \\
\hline$T$ (a) $F X \# 3$ & 300.06 & 300.06 & 300.05 & 300.05 & 300.04 & 300.04 & 300.04 & 300.03 & 300.03 & 300.02 & 300.02 & 300.01 & 300.01 & 300.01 & 300.00 & 300.00 & 299.99 \\
\hline$T @ F X \# 4$ & 302.12 & 302.12 & 302.11 & 302.11 & 302.10 & 302.10 & 302.09 & 302.09 & 302.09 & 302.08 & 302.08 & 302.07 & 302.07 & 302.06 & 302.06 & 302.06 & 302.05 \\
\hline$T @ F X \# 5$ & 304.19 & 304.19 & 304.19 & 304.18 & 304.18 & 304.17 & 304.17 & 304.16 & 304.16 & 304.15 & 304.15 & 304.15 & 304.14 & 304.14 & 304.13 & 304.13 & 304.12 \\
\hline$T @ F X \# 6$ & 306.29 & 306.29 & 306.28 & 306.28 & 306.27 & 306.27 & 306.27 & 306.26 & 306.26 & 306.25 & 306.25 & 306.24 & 306.24 & 306.23 & 306.23 & 306.23 & 306.22 \\
\hline Wall $x$-coord & 72.05 & 72.06 & 72.07 & 72.08 & 72.09 & 72.10 & 72.11 & 72.12 & 72.13 & 72.15 & 72.16 & 72.17 & 72.18 & 72.19 & 72.20 & 72.21 & 72.22 \\
\hline Wall T & 310.08 & 310.07 & 310.06 & 310.06 & 310.06 & 310.05 & 310.04 & 310.04 & 310.05 & 310.04 & 310.05 & 310.06 & 310.07 & 310.07 & 310.07 & 310.07 & 310.07 \\
\hline
\end{tabular}

\section{B.8 Tabulated Data for Interferogram 464}

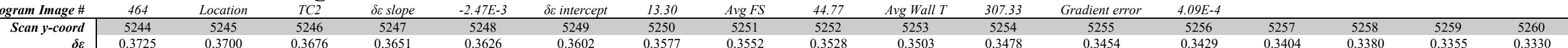




\begin{tabular}{|c|c|c|c|c|c|c|c|c|c|c|c|c|c|c|c|c|c|}
\hline Scan $x$-coord & 879 & 879 & 879 & 879 & 879 & 879 & 879 & 879 & 879 & 879 & 879 & 879 & 879 & 879 & 879 & 879 & 879 \\
\hline$F X \# 1$ & 391.45 & 391.95 & 392.51 & 392.99 & 399.69 & 389.95 & 405.70 & 403.25 & 403.97 & 404.58 & 405.47 & 406.10 & 406.90 & 407.47 & 407.97 & 408.68 & 409.21 \\
\hline$F X \# 2$ & 277.00 & 277.16 & 277.29 & 277.31 & 277.46 & 277.36 & 277.51 & 277.97 & 278.15 & 278.40 & 278.59 & 278.71 & 278.82 & 278.94 & 279.16 & 279.41 & 279.74 \\
\hline$F X \# 3$ & 210.59 & 210.69 & 210.78 & 210.91 & 211.14 & 211.19 & 211.40 & 211.60 & 211.71 & 211.79 & 211.98 & 212.13 & 212.33 & 212.51 & 212.86 & 212.95 & 213.07 \\
\hline$F X \# 4$ & 161.47 & 161.67 & 161.87 & 162.04 & 162.12 & 162.20 & 162.31 & 162.50 & 162.66 & 162.79 & 162.96 & 163.04 & 163.12 & 163.13 & 163.15 & 163.23 & 163.39 \\
\hline$F X \# 5$ & 116.90 & 116.97 & 117.01 & 117.01 & 117.08 & 117.25 & 117.45 & 117.67 & 117.91 & 118.09 & 118.12 & 118.23 & 118.27 & 118.42 & 118.55 & 118.75 & 118.90 \\
\hline Near wall FS & 44.57 & 44.70 & 44.87 & 45.04 & 45.04 & 44.96 & 44.87 & 44.83 & 44.75 & 44.71 & 44.84 & 44.81 & 44.85 & 44.70 & 44.60 & 44.49 & 44.49 \\
\hline$T @ F X \# 1$ & 297.32 & 297.32 & 297.32 & 297.32 & 297.32 & 297.32 & 297.32 & 297.32 & 297.32 & 297.32 & 297.32 & 297.32 & 297.32 & 297.32 & 297.32 & 297.32 & 297.32 \\
\hline$T @ F X \# 2$ & 298.25 & 298.25 & 298.24 & 298.24 & 298.23 & 298.23 & 298.22 & 298.21 & 298.21 & 298.20 & 298.20 & 298.19 & 298.19 & 298.18 & 298.18 & 298.17 & 298.17 \\
\hline$T$ (a) $F X \# 3$ & 300.30 & 300.29 & 300.29 & 300.28 & 300.28 & 300.27 & 300.27 & 300.26 & 300.26 & 300.25 & 300.25 & 300.24 & 300.24 & 300.23 & 300.23 & 300.22 & 300.22 \\
\hline$T(F X \# 4$ & 302.35 & 302.34 & 302.34 & 302.33 & 302.33 & 302.32 & 302.32 & 302.31 & 302.31 & 302.30 & 302.30 & 302.29 & 302.29 & 302.28 & 302.28 & 302.27 & 302.27 \\
\hline$T$ (a) $F X \# 5$ & 304.42 & 304.42 & 304.41 & 304.41 & 304.40 & 304.40 & 304.39 & 304.39 & 304.38 & 304.37 & 304.37 & 304.36 & 304.36 & 304.35 & 304.35 & 304.34 & 304.34 \\
\hline Wall $x$-coord & 99.64 & 99.64 & 99.63 & 99.63 & 99.63 & 99.62 & 99.62 & 99.61 & 99.61 & 99.61 & 99.60 & 99.60 & 99.60 & 99.59 & 99.59 & 99.58 & 99.58 \\
\hline Wall T & 307.33 & 307.33 & 307.32 & 307.31 & 307.31 & 307.32 & 307.32 & 307.33 & 307.34 & 307.34 & 307.33 & 307.34 & 307.33 & 307.34 & 307.34 & 307.35 & 307.35 \\
\hline Interferogram Image \# & 464 & Location & $T C 3$ & $\delta \varepsilon$ slope & $-2.32 E-3$ & $\delta \varepsilon$ intercept & 10.59 & $\operatorname{Avg} F S$ & 47.22 & Avg Wall T & 307.45 & Gradient error & $4.09 E-4$ & & & & \\
\hline Scan y-coord & 4430 & 4431 & 4432 & 4433 & 4434 & 4435 & 4436 & 4437 & 4438 & 4439 & 4440 & 4441 & 4442 & 4443 & 4444 & 4445 & 4446 \\
\hline$\delta \varepsilon$ & 0.3199 & 0.3176 & 0.3152 & 0.3129 & 0.3106 & 0.3083 & 0.3060 & 0.3036 & 0.3013 & 0.2990 & 0.2967 & 0.2944 & 0.2920 & 0.2897 & 0.2874 & 0.2851 & 0.2828 \\
\hline Scan $x$-coord & 979 & 979 & 979 & 979 & 979 & 979 & 979 & 979 & 979 & 979 & 979 & 979 & 979 & 979 & 979 & 979 & 979 \\
\hline$F X \# 1$ & 428.85 & 428.62 & 429.06 & 430.16 & 430.88 & 431.66 & 432.31 & 433.65 & 434.05 & 434.40 & 434.62 & 435.00 & 435.42 & 435.98 & 436.97 & 437.57 & 438.54 \\
\hline$F X \# 2$ & 294.56 & 294.59 & 294.73 & 294.89 & 294.98 & 295.11 & 295.21 & 295.39 & 295.55 & 295.60 & 295.60 & 295.67 & 295.73 & 295.95 & 296.11 & 296.28 & 296.49 \\
\hline$F X \# 3$ & 224.87 & 225.09 & 225.25 & 225.44 & 225.61 & 225.72 & 225.77 & 225.80 & 225.83 & 225.87 & 225.95 & 226.11 & 226.28 & 226.46 & 226.56 & 226.66 & 226.79 \\
\hline$F X \# 4$ & 170.63 & 170.76 & 170.93 & 171.14 & 171.27 & 171.51 & 171.72 & 171.96 & 172.11 & 172.30 & 172.57 & 172.84 & 173.09 & 173.33 & 173.52 & 173.76 & 173.94 \\
\hline$F X \# 5$ & 123.61 & 123.79 & 123.99 & 124.19 & 124.33 & 124.61 & 124.76 & 124.87 & 125.04 & 125.24 & 125.44 & 125.59 & 125.65 & 125.74 & 125.87 & 125.94 & 125.96 \\
\hline Near wall FS & 47.02 & 46.97 & 46.94 & 46.95 & 46.94 & 46.90 & 46.97 & 47.09 & 47.07 & 47.06 & 47.13 & 47.25 & 47.43 & 47.59 & 47.65 & 47.82 & 47.98 \\
\hline$T @ F X \# 1$ & 297.32 & $\begin{array}{l}29.91 .32 \\
297\end{array}$ & $\begin{array}{r}40.94 \\
297.32\end{array}$ & $\begin{array}{l}29.93 \\
292\end{array}$ & $\begin{array}{r}40.94 \\
297.32\end{array}$ & $\begin{array}{r}49.90 \\
297.32\end{array}$ & $\begin{array}{l}40.91 \\
297.32\end{array}$ & 297.32 & 297.32 & 297.32 & 297.32 & $\begin{array}{l}4.29 \\
297.32\end{array}$ & $\begin{array}{l}4.43 \\
297.32\end{array}$ & 297.32 & $\begin{array}{l}497.32 \\
297\end{array}$ & $\begin{array}{l}497.32 \\
292\end{array}$ & 297.32 \\
\hline$T @ F X \# 2$ & 298.17 & 298.17 & 298.16 & 298.16 & 298.15 & 298.15 & 298.14 & 298.14 & 298.14 & 298.13 & 298.13 & 298.12 & 298.12 & 298.11 & 298.11 & 298.10 & 298.10 \\
\hline$T(F X \# 3$ & 300.23 & 300.22 & 300.22 & 300.21 & 300.21 & 300.20 & 300.20 & 300.19 & 300.19 & 300.18 & 300.18 & 300.17 & 300.17 & 300.16 & 300.16 & 300.15 & 300.15 \\
\hline$T$ (a) $F X \# 5$ & 304.35 & 304.35 & 304.34 & 304.34 & 304.33 & 304.33 & 304.32 & 304.32 & 304.31 & 304.31 & 304.30 & 304.30 & 304.29 & 304.29 & 304.28 & 304.28 & 304.27 \\
\hline Wall $x$-coord & 101.58 & 101.58 & 101.58 & 101.58 & 101.58 & 101.58 & 101.58 & 101.57 & 101.57 & 101.57 & 101.57 & 101.57 & 101.57 & 101.57 & 101.57 & 101.57 & 101.57 \\
\hline Wall T & 307.43 & 307.44 & 307.44 & 307.45 & 307.45 & 307.46 & 307.46 & 307.45 & 307.46 & 307.46 & 307.46 & 307.46 & 307.46 & 307.45 & 307.45 & 307.45 & 307.44 \\
\hline Interferogram Image \# & 464 & Location & $T C 4$ & $\delta \varepsilon$ slope & $-2.40 E-3$ & $\delta \varepsilon$ intercept & 8.87 & $\operatorname{Avg} F S$ & 48.47 & Avg Wall $T$ & 307.86 & Gradient error & $4.09 E-4$ & & & & \\
\hline Scan y-coord & 3616 & 3617 & 3618 & 3619 & 3620 & 3621 & 3622 & 3623 & 3624 & 3625 & 3626 & 3627 & 3628 & 3629 & 3630 & 3631 & 3632 \\
\hline & 0.1744 & 0.1720 & 0.1696 & 0.1672 & 0.1648 & 0.1624 & 0.1600 & 0.1576 & 0.1552 & 0.1528 & 0.1504 & 0.1480 & 0.1456 & 0.1432 & 0.1408 & 0.1384 & 0.1360 \\
\hline Scan $x$-coord & 1071 & 1071 & 1071 & 1071 & 1071 & 1071 & 1071 & 1071 & 1071 & 1071 & 1071 & 1071 & 1071 & 1071 & 1071 & 1071 & 1071 \\
\hline 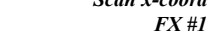 & 536.43 & 534.55 & 535.44 & 544.27 & 545.15 & 546.21 & 547.26 & $\begin{array}{l}1011 \\
548.42\end{array}$ & 549.36 & 550.82 & $\begin{array}{l}1011 \\
552.12\end{array}$ & 552.84 & 554.13 & 555.40 & 556.41 & 557.49 & 558.54 \\
\hline$F X \# 2$ & 325.89 & 326.06 & 326.29 & 326.63 & 326.96 & 327.27 & 327.53 & 327.87 & 328.22 & 328.41 & 328.68 & 328.96 & 329.17 & 329.40 & 329.64 & 329.88 & 330.07 \\
\hline$F X \# 3$ & 245.91 & 246.11 & 246.30 & 246.38 & 246.43 & 246.51 & 246.56 & 246.64 & 246.68 & 246.75 & 246.84 & 246.91 & 247.01 & 247.08 & 247.17 & 247.23 & 247.36 \\
\hline$F X \# 4$ & 188.94 & 189.03 & 189.10 & 189.16 & 189.25 & 189.37 & 189.56 & 189.70 & 189.83 & 189.91 & 189.96 & 189.98 & 190.07 & 190.15 & 190.19 & 190.23 & 190.23 \\
\hline$F X \# 5$ & 140.50 & 140.57 & 140.59 & 140.64 & 140.73 & 140.87 & 140.97 & 141.05 & 141.13 & 141.24 & 141.38 & 141.56 & 141.73 & 141.79 & 141.88 & 141.97 & 142.05 \\
\hline Near wall FS & 48.45 & 48.46 & 48.51 & 48.52 & 48.52 & 48.50 & 48.59 & 48.65 & 48.70 & 48.67 & 48.58 & 48.42 & 48.34 & 48.35 & 48.30 & 48.26 & 48.18 \\
\hline$T @ F X \# 1$ & 297.32 & 297.32 & 297.32 & 297.32 & 297.32 & 297.32 & 297.32 & 297.32 & 297.32 & 297.32 & 297.32 & 297.32 & 297.32 & 297.32 & 297.32 & 297.32 & 297.32 \\
\hline$T @ F X \# 2$ & 297.88 & 297.88 & 297.87 & 297.86 & 297.86 & 297.85 & 297.85 & 297.84 & 297.84 & 297.83 & 297.83 & 297.82 & 297.82 & 297.81 & 297.81 & 297.80 & 297.79 \\
\hline$T(F) F \# 3$ & 299.96 & 299.95 & 299.95 & 299.94 & 299.94 & 299.93 & 299.93 & 299.92 & $\begin{array}{l}291.84 \\
299.92\end{array}$ & 299.91 & 299.91 & 299.90 & $\begin{array}{l}291.82 \\
299.90\end{array}$ & 299.89 & 299.89 & 299.88 & 299.88 \\
\hline$T @ F X \# 4$ & 302.01 & 302.01 & 302.00 & 302.00 & 301.99 & 301.99 & 301.98 & 301.98 & 301.97 & 301.97 & 301.96 & 301.96 & 301.95 & 301.95 & 301.94 & 301.94 & 301.93 \\
\hline$T$ (a) $F X \# 5$ & 304.08 & 304.08 & 304.07 & 304.07 & 304.06 & 304.06 & 304.05 & 304.05 & 304.04 & 304.04 & 304.03 & 304.03 & 304.02 & 304.02 & 304.01 & 304.01 & 304.00 \\
\hline Wall $x$-coord & 101.33 & 101.34 & 101.34 & 101.34 & 101.34 & 101.34 & 101.34 & 101.35 & 101.35 & 101.35 & 101.35 & 101.35 & 101.35 & 101.36 & 101.36 & 101.36 & 101.36 \\
\hline Wall $T$ & 307.87 & 307.87 & 307.86 & 307.86 & 307.86 & 307.86 & 307.86 & 307.85 & 307.85 & 307.85 & 307.85 & 307.86 & 307.87 & 307.86 & 307.87 & 307.87 & 307.87 \\
\hline Interferogram Image \# & 464 & Location & $T C 5$ & $\delta \varepsilon$ slope & $-2.05 E-3$ & $\delta \varepsilon$ intercept & 5.82 & $\operatorname{Avg} F S$ & 45.80 & Avg Wall $T$ & 308.78 & Gradient error & $4.09 E-4$ & & & & \\
\hline Scan y-coord & 2802 & 2803 & 2804 & 2805 & 2806 & 2807 & 2808 & 2809 & 2810 & 2811 & 2812 & 2813 & 2814 & 2815 & 2816 & 2817 & 2818 \\
\hline & 0.0784 & 0.0764 & 0.0743 & 0.0723 & 0.0702 & 0.0682 & 0.0661 & 0.0641 & 0.0620 & 0.0600 & 0.0579 & 0.0559 & 0.0538 & 0.0518 & 0.0497 & 0.0477 & 0.0456 \\
\hline Scan $x$-coord & 1065 & 1065 & 1065 & 1065 & 1065 & 1065 & 1065 & 1065 & 1065 & 1065 & 1065 & 1065 & 1065 & 1065 & 1065 & 1065 & 1065 \\
\hline$F X \# I$ & 687.00 & 687.00 & 687.00 & 687.00 & 687.00 & 687.00 & 687.00 & 687.00 & 687.00 & 687.00 & 687.00 & 687.00 & 687.00 & 687.00 & 687.00 & 687.00 & 687.00 \\
\hline$F X \# 6$ & 113.58 & 113.59 & 113.66 & 113.95 & 114.24 & 114.50 & 114.66 & 114.82 & 114.84 & 114.79 & 114.78 & 114.84 & 114.90 & 115.07 & 115.32 & 115.43 & 115.46 \\
\hline Near wall FS & 46.07 & 46.13 & 46.15 & 46.07 & 46.01 & 45.93 & 45.88 & 45.72 & 45.63 & 45.54 & 45.51 & 45.48 & 45.62 & 45.72 & 45.71 & 45.72 & 45.67 \\
\hline$T @ F X \# 1$ & 297.32 & 297.32 & 297.32 & 297.32 & 297.32 & 297.32 & 297.32 & 297.32 & 297.32 & 297.32 & 297.32 & 297.32 & 297.32 & 297.32 & 297.32 & 297.32 & 297.32 \\
\hline$T(F X \# 2$ & 297.63 & 297.62 & 297.62 & 297.61 & 297.61 & 297.61 & 297.60 & 297.60 & 297.59 & 297.59 & 297.59 & 297.58 & 297.58 & 297.57 & 297.57 & 297.57 & 297.56 \\
\hline$T(F X \# 3$ & 299.75 & 299.74 & 299.74 & 299.74 & 299.73 & 299.73 & 299.72 & 299.72 & 299.71 & 299.71 & 299.71 & 299.70 & 299.70 & 299.69 & 299.69 & 299.69 & 299.68 \\
\hline$T @ F X \# 4$ & 301.80 & 301.80 & 301.79 & 301.79 & 301.79 & 301.78 & 301.78 & 301.77 & 301.77 & 301.76 & 301.76 & 301.76 & 301.75 & 301.75 & 301.74 & 301.74 & 301.74 \\
\hline$T @ F X \# 5$ & 303.87 & 303.87 & 303.86 & 303.86 & 303.86 & 303.85 & 303.85 & 303.84 & 303.84 & 303.83 & 303.83 & 303.83 & 303.82 & 303.82 & 303.81 & 303.81 & 303.80 \\
\hline$T @ F X \# 6$ & 305.97 & 305.96 & 305.96 & 305.95 & 305.95 & 305.95 & 305.94 & 305.94 & 305.93 & 305.93 & 305.92 & 305.92 & 305.92 & 305.91 & 305.91 & 305.90 & 305.90 \\
\hline Wall $x$-coord & 98.90 & 98.90 & 98.91 & 98.91 & 98.92 & 98.92 & 98.93 & 98.93 & 98.93 & 98.94 & 98.94 & 98.95 & 98.95 & 98.96 & 98.96 & 98.96 & 98.97 \\
\hline Wall $T$ & 308.76 & 308.76 & 308.76 & 308.77 & 308.78 & 308.79 & 308.79 & 308.79 & 308.79 & 308.79 & 308.78 & 308.78 & 308.78 & 308.78 & 308.79 & 308.79 & 308.78 \\
\hline Interferogram Image \# & 464 & Location & TC6 & $\delta \varepsilon$ slope & $-2.18 E-3$ & $\delta \varepsilon$ intercept & 5.09 & $\operatorname{Avg} F S$ & 46.81 & Avg Wall T & 309.23 & Gradient error & $4.09 E-4$ & & & & \\
\hline
\end{tabular}




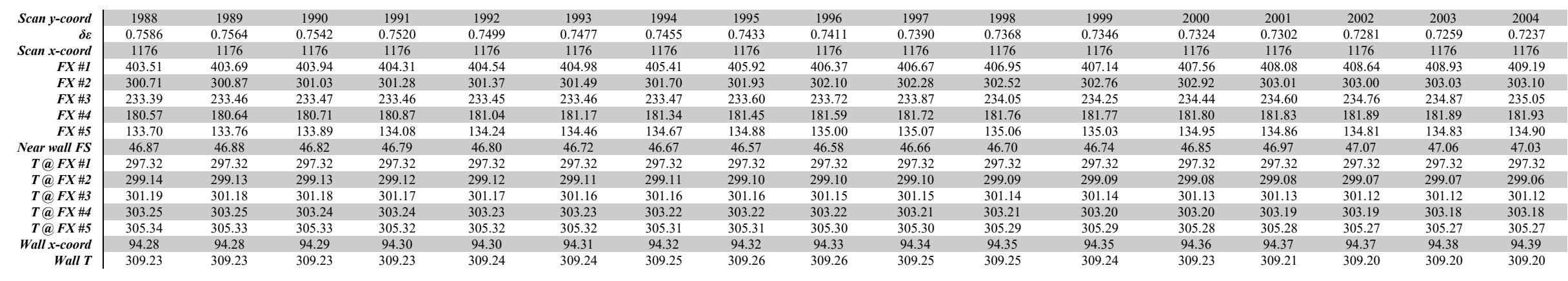

\begin{tabular}{|c|c|c|c|c|c|c|c|c|c|c|c|c|c|c|c|c|c|}
\hline Interferogram Image \# & 464 & Location & $T C 7$ & $\delta \varepsilon$ slope & $-2.09 E-3$ & $\delta \varepsilon$ intercept & 2.81 & $\operatorname{Avg} F S$ & 45.39 & Avg Wall $T$ & 309.84 & Gradient error & 4.09E-4 & & & & \\
\hline Scan y-coord & 1174 & 1175 & 1176 & 1177 & 1178 & 1179 & 1180 & 1181 & 1182 & 1183 & 1184 & 1185 & 1186 & 1187 & 1188 & 1189 & 1190 \\
\hline & 0.3539 & 0.3518 & 0.3497 & 0.3476 & 0.3455 & 0.3434 & 0.3413 & 0.3392 & 0.3371 & 0.3351 & 0.3330 & 0.3309 & 0.3288 & 0.3267 & 0.3246 & 0.3225 & 0.3204 \\
\hline Scan $x$-coord & 1196 & 1196 & 1196 & 1196 & 1196 & 1196 & 1196 & 1196 & 1196 & 1196 & 1196 & 1196 & 1196 & 1196 & 1196 & 1196 & 1196 \\
\hline$F X \# 1$ & 504.81 & 505.76 & 506.64 & 507.60 & 510.06 & 510.89 & 511.61 & 513.01 & 514.40 & 515.23 & 516.83 & 517.93 & 518.90 & 519.74 & 521.00 & 521.91 & 522.48 \\
\hline$F X \# 2$ & 344.65 & 344.92 & 345.22 & 345.46 & 345.70 & 345.83 & 346.01 & 346.29 & 346.67 & 346.95 & 347.30 & 347.63 & 347.94 & 348.35 & 348.52 & 348.67 & 348.80 \\
\hline$F X \# 3$ & 265.03 & 265.15 & 265.30 & 265.49 & 265.56 & 265.53 & 265.46 & 265.47 & 265.55 & 265.69 & 265.82 & 266.00 & 266.16 & 266.40 & 266.65 & 266.96 & 267.19 \\
\hline$F X \# 5$ & 156.99 & 157.07 & 157.10 & 157.08 & 157.09 & 157.00 & 157.01 & 157.07 & 157.12 & 157.22 & 157.38 & 157.52 & 157.64 & 157.83 & 158.00 & 158.12 & 158.23 \\
\hline$F X \# 6$ & 111.51 & 111.56 & 111.68 & 111.76 & 111.85 & 111.89 & 111.92 & 111.94 & 111.98 & 112.02 & 112.08 & 112.11 & 112.16 & 112.24 & 112.31 & 112.38 & 112.39 \\
\hline Near wall FS & 45.48 & 45.51 & 45.42 & 45.32 & 45.24 & 45.11 & 45.08 & 45.13 & 45.15 & 45.20 & 45.29 & 45.41 & 45.48 & 45.59 & 45.69 & 45.74 & 45.83 \\
\hline$T @ F X \# 1$ & 297.32 & 297.32 & 297.32 & 297.32 & 297.32 & 297.32 & 297.32 & 297.32 & 297.32 & 297.32 & 297.32 & 297.32 & 297.32 & 297.32 & 297.32 & 297.32 & 297.32 \\
\hline$T @ F X \# 2$ & 298.30 & 298.29 & 298.29 & 298.29 & 298.28 & 298.28 & 298.27 & 298.27 & 298.26 & 298.26 & 298.25 & 298.25 & 298.24 & 298.24 & 298.23 & 298.23 & 298.23 \\
\hline$T @ F X \# 3$ & 300.36 & 300.36 & 300.35 & 300.35 & 300.35 & 300.34 & 300.34 & 300.33 & 300.33 & 300.32 & 300.32 & 300.31 & 300.31 & 300.31 & 300.30 & 300.30 & 300.29 \\
\hline$T @ F X \# 5$ & 304.50 & 304.49 & 304.49 & 304.49 & 304.48 & 304.48 & 304.47 & 304.47 & 304.46 & 304.46 & 304.45 & 304.45 & 304.45 & 304.44 & 304.44 & 304.43 & 304.43 \\
\hline$T @ F X \# 6$ & 306.60 & 306.60 & 306.59 & 306.59 & 306.58 & 306.58 & 306.57 & 306.57 & 306.57 & 306.56 & 306.56 & 306.55 & 306.55 & 306.54 & 306.54 & 306.53 & 306.53 \\
\hline Wall $x$-coord & 87.46 & 87.47 & 87.48 & 87.49 & 87.50 & 87.51 & 87.52 & 87.53 & 87.54 & 87.55 & 87.56 & 87.57 & 87.58 & 87.59 & 87.60 & 87.61 & 87.62 \\
\hline Wall $T$ & 309.86 & 309.85 & 309.86 & 309.86 & 309.86 & 309.86 & 309.86 & 309.85 & 309.85 & 309.84 & 309.84 & 309.83 & 309.83 & 309.82 & 309.82 & 309.82 & 309.81 \\
\hline
\end{tabular}

\section{B.9 Tabulated Data for Interferogram 465}

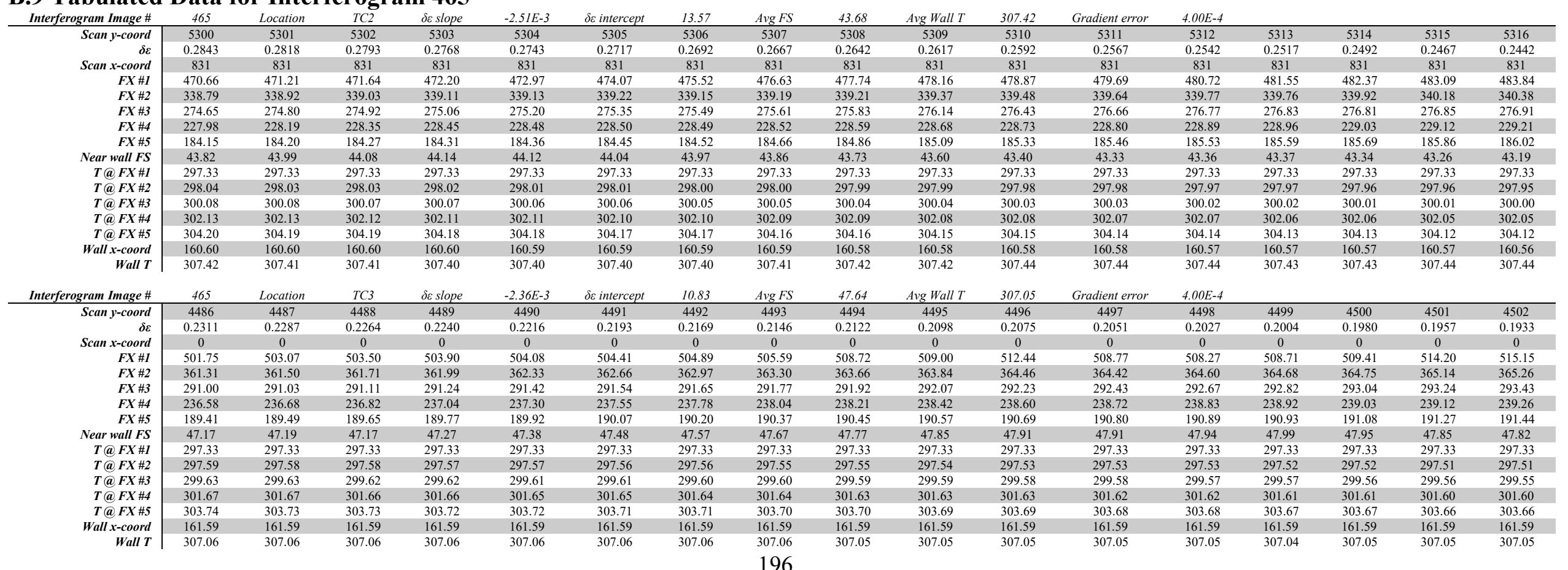




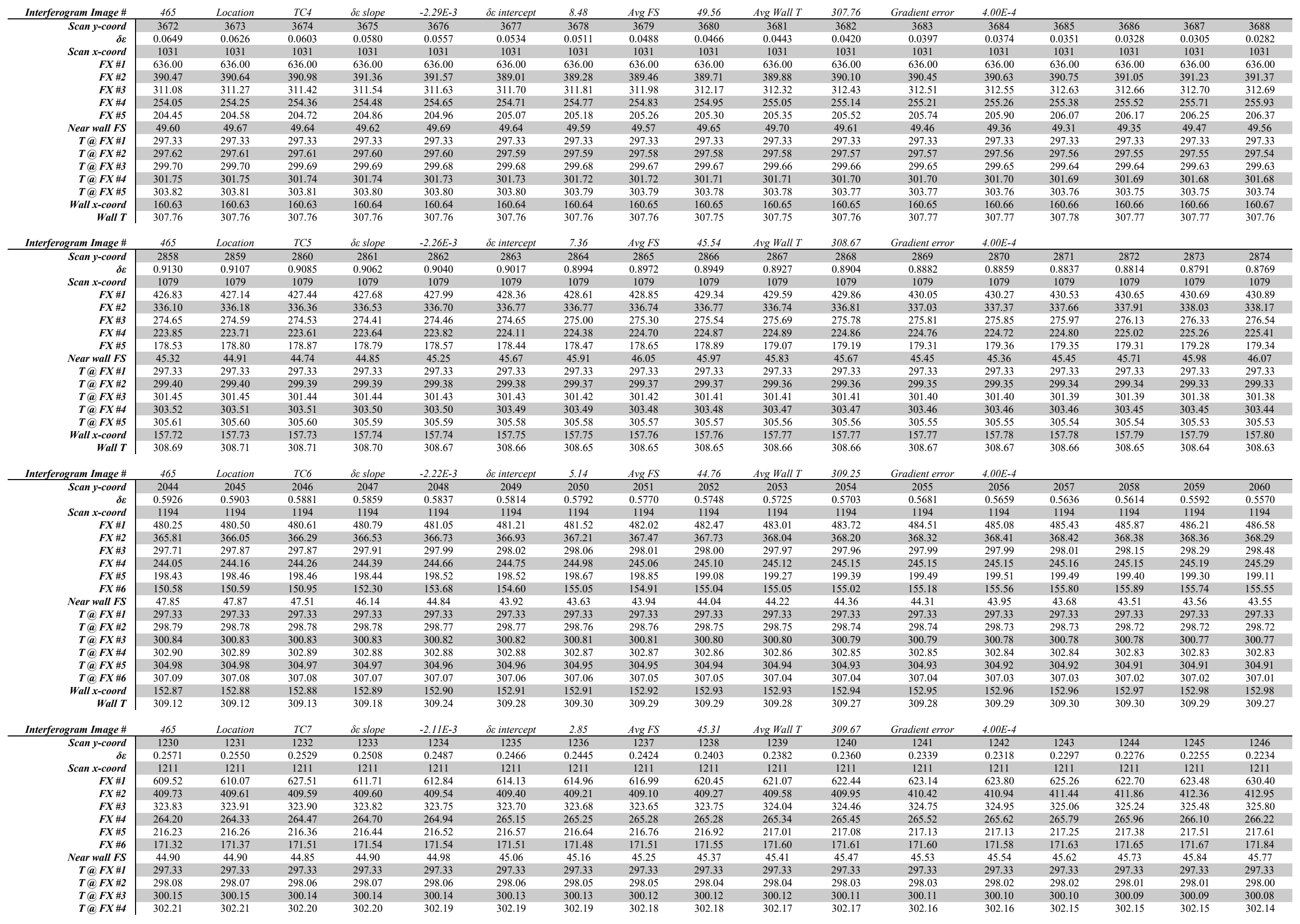




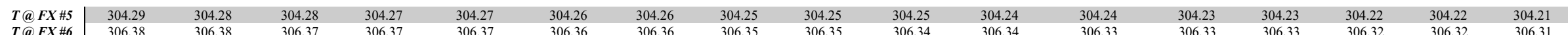

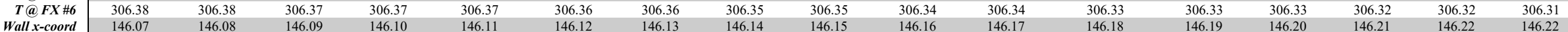

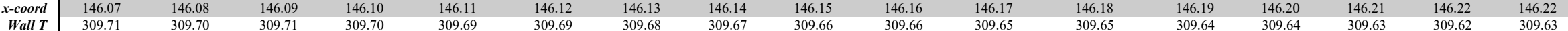

\section{B.10 Tabulated Data for Interferogram 466}

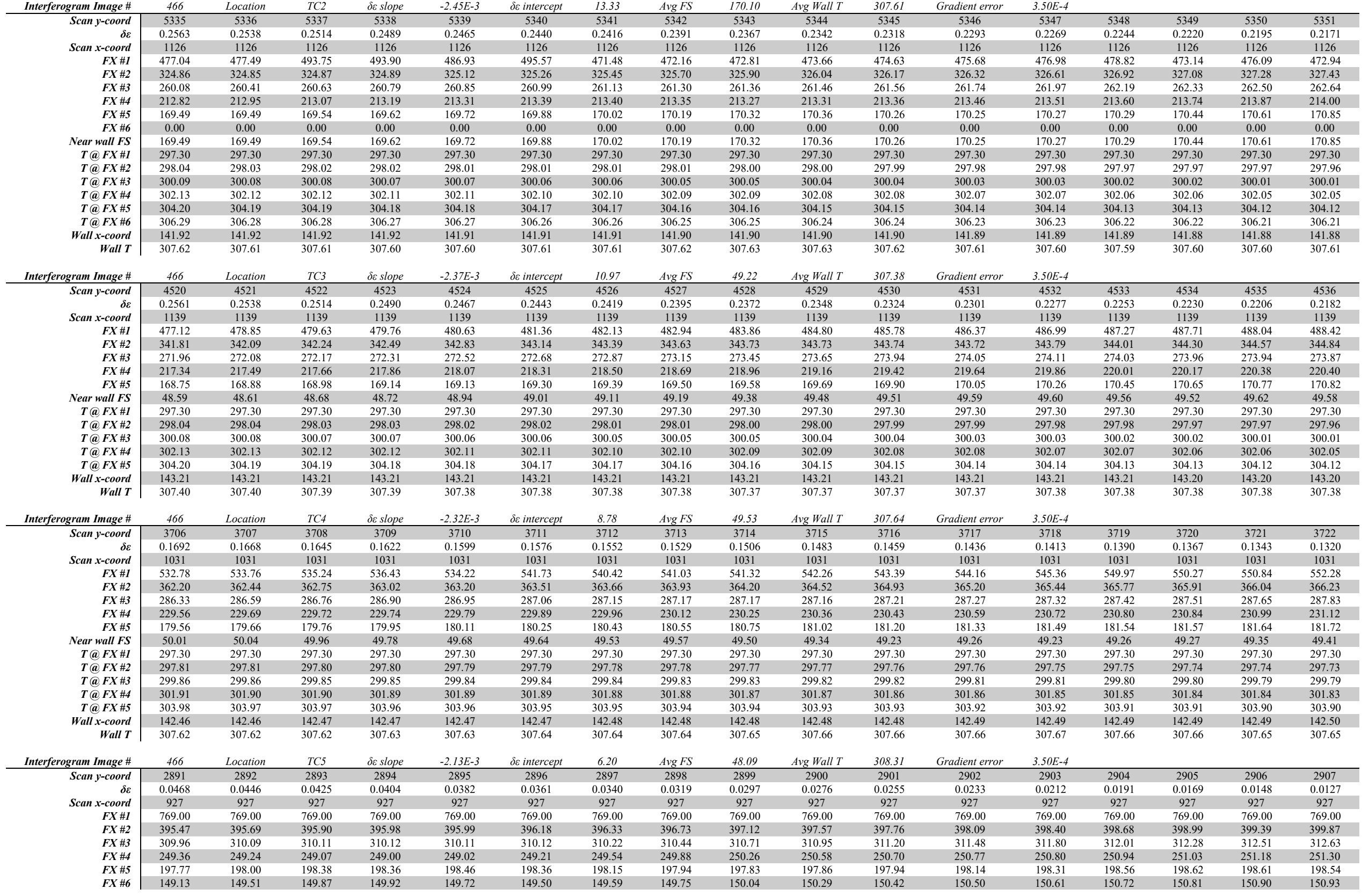




\begin{tabular}{|c|c|c|c|c|c|c|c|c|c|c|c|c|c|c|c|c|c|}
\hline Near wall FS & 48.64 & 48.49 & 48.51 & 48.44 & 48.74 & 48.86 & 48.56 & 48.19 & 47.79 & 47.57 & 47.52 & 47.64 & 47.70 & 47.84 & 47.81 & 47.71 & 47.60 \\
\hline$T @ F X \# I$ & 297.30 & 297.30 & 297.30 & 297.30 & 297.30 & 297.30 & 297.30 & 297.30 & 297.30 & 297.30 & 297.30 & 297.30 & 297.30 & 297.30 & 297.30 & 297.30 & 297.30 \\
\hline$T @ F X \# 2$ & 297.45 & 297.45 & 297.44 & 297.44 & 297.44 & 297.43 & 297.43 & 297.42 & 297.42 & 297.41 & 297.41 & 297.41 & 297.40 & 297.40 & 297.39 & 297.39 & 297.38 \\
\hline$T @ F X \# 3$ & 299.57 & 299.56 & 299.56 & 299.56 & 299.55 & 299.55 & 299.54 & 299.54 & 299.53 & 299.53 & 299.53 & 299.52 & 299.52 & 299.51 & 299.51 & 299.50 & 299.50 \\
\hline$T @ F X \# 4$ & 301.61 & 301.61 & 301.61 & 301.60 & 301.60 & 301.59 & 301.59 & 301.58 & 301.58 & 301.57 & 301.57 & 301.57 & 301.56 & 301.56 & 301.55 & 301.55 & 301.54 \\
\hline$T @ F X \# 5$ & 303.68 & 303.67 & 303.67 & 303.66 & 303.66 & 303.66 & 303.65 & 303.65 & 303.64 & 303.64 & 303.63 & 303.63 & 303.62 & 303.62 & 303.62 & 303.61 & 303.61 \\
\hline$T @ F X \# 6$ & 305.77 & 305.76 & 305.76 & 305.75 & 305.75 & 305.74 & 305.74 & 305.74 & 305.73 & 305.73 & 305.72 & 305.72 & 305.71 & 305.71 & 305.70 & 305.70 & 305.70 \\
\hline Wall $x$-coord & 139.68 & 139.69 & 139.69 & 139.70 & 139.70 & 139.70 & 139.71 & 139.71 & 139.72 & 139.72 & 139.73 & 139.73 & 139.74 & 139.74 & 139.75 & 139.75 & 139.76 \\
\hline Wall T & 308.29 & 308.31 & 308.32 & 308.32 & 308.30 & 308.29 & 308.29 & 308.29 & 308.30 & 308.31 & 308.31 & 308.31 & 308.31 & 308.31 & 308.31 & 308.31 & 308.31 \\
\hline
\end{tabular}

\begin{tabular}{|c|c|c|c|c|c|c|c|c|c|c|c|c|c|c|c|c|c|}
\hline Interferogram Image \# & 466 & Location & $T C 6$ & $\delta \varepsilon$ slope & $-2.26 E-3$ & $\delta \varepsilon$ intercept & 5.41 & $\operatorname{Avg} F S$ & 47.71 & Avg Wall $T$ & 308.79 & Gradient error & $3.50 E-4$ & & & & \\
\hline Scan y-coord & 2076 & 2077 & 2078 & 2079 & 2080 & 2081 & 2082 & 2083 & 2084 & 2085 & 2086 & 2087 & 2088 & 2089 & 2090 & 2091 & 2092 \\
\hline & 0.7176 & 0.7154 & 0.7131 & 0.7108 & 0.7086 & 0.7063 & 0.7041 & 0.7018 & 0.6995 & 0.6973 & 0.6950 & 0.6928 & 0.6905 & 0.6883 & 0.6860 & 0.6837 & 0.6815 \\
\hline Scan $x$-coord & 1310 & 1310 & 1310 & 1310 & 1310 & 1310 & 1310 & 1310 & 1310 & 1310 & 1310 & 1310 & 1310 & 1310 & 1310 & 1310 & 1310 \\
\hline$F X \# 1$ & 434.18 & 434.51 & 434.87 & 435.26 & 435.66 & 435.99 & 436.20 & 436.26 & 436.41 & 436.58 & 436.54 & 436.56 & 436.60 & 436.70 & 436.83 & 437.11 & 437.57 \\
\hline$F X \# 2$ & 334.90 & 334.91 & 334.92 & 334.94 & 335.01 & 335.13 & 335.38 & 335.65 & 335.98 & 336.31 & 336.65 & 336.97 & 337.15 & 337.25 & 337.26 & 337.23 & 337.21 \\
\hline$F X \# 3$ & 269.03 & 269.08 & 269.19 & 269.30 & 269.41 & 269.57 & 269.73 & 269.88 & 270.02 & 270.14 & 270.19 & 270.22 & 270.21 & 270.20 & 270.18 & 270.24 & 270.35 \\
\hline$F X \# 4$ & 216.00 & 216.13 & 216.24 & 216.32 & 216.38 & 216.43 & 216.45 & 216.44 & 216.48 & 216.64 & 216.83 & 217.02 & 217.20 & 217.32 & 217.33 & 217.24 & 217.11 \\
\hline$F X \# 5$ & 167.61 & 167.85 & 168.15 & 168.43 & 168.67 & 168.89 & 169.10 & 169.30 & 169.34 & 169.33 & 169.33 & 169.31 & 169.33 & 169.43 & 169.49 & 169.47 & 169.45 \\
\hline Near wall FS & 48.38 & 48.28 & 48.09 & 47.88 & 47.71 & 47.53 & 47.34 & 47.15 & 47.15 & 47.31 & 47.50 & 47.72 & 47.87 & 47.89 & 47.84 & 47.77 & 47.66 \\
\hline$T @ F X \# 1$ & 297.30 & 297.30 & 297.30 & 297.30 & 297.30 & 297.30 & 297.30 & 297.30 & 297.30 & 297.30 & 297.30 & 297.30 & 297.30 & 297.30 & 297.30 & 297.30 & 297.30 \\
\hline$T @ F X \# 2$ & 299.03 & 299.03 & 299.02 & 299.02 & 299.01 & 299.01 & 299.00 & 299.00 & 299.00 & 298.99 & 298.99 & 298.98 & 298.98 & 298.97 & 298.97 & 298.96 & 298.96 \\
\hline$T @ F X \# 3$ & 301.07 & 301.07 & 301.07 & 301.06 & 301.06 & 301.05 & 301.05 & 301.04 & 301.04 & 301.03 & 301.03 & 301.02 & 301.02 & 301.01 & 301.01 & 301.01 & 301.00 \\
\hline$T(F X \# 4$ & 303.13 & 303.13 & 303.12 & 303.12 & 303.11 & 303.11 & 303.11 & 303.10 & 303.10 & 303.09 & 303.09 & 303.08 & 303.08 & 303.07 & 303.07 & 303.06 & 303.06 \\
\hline Wall $x$-coord & 134.86 & 134.87 & 134.88 & 134.89 & 134.89 & 134.90 & 134.91 & 134.91 & 134.92 & 134.93 & 134.94 & 134.94 & 134.95 & 134.96 & 134.96 & 134.97 & 134.98 \\
\hline Wall T & 308.75 & 308.76 & 308.77 & 308.79 & 308.80 & 308.81 & 308.82 & 308.83 & 308.82 & 308.81 & 308.80 & 308.79 & 308.78 & 308.78 & 308.78 & 308.77 & 308.77 \\
\hline
\end{tabular}

\begin{tabular}{|c|c|c|c|c|c|c|c|c|c|c|c|c|c|c|c|c|c|}
\hline Interferogram Image \# & 466 & Location & $T C 7$ & $\delta \varepsilon$ slope & $-2.20 E-3$ & $\delta \varepsilon$ intercept & 3.16 & $\operatorname{Avg} F S$ & 141.68 & Avg Wall $T$ & 309.33 & Gradient error & $3.50 E-4$ & & & & \\
\hline Scan y-coord & 1261 & 1262 & 1263 & 1264 & 1265 & 1266 & 1267 & 1268 & 1269 & 1270 & 1271 & 1272 & 1273 & 1274 & 1275 & 1276 & 1277 \\
\hline & 0.3907 & 0.3885 & 0.3863 & 0.3841 & 0.3819 & 0.3797 & 0.3775 & 0.3753 & 0.3731 & 0.3710 & 0.3688 & 0.3666 & 0.3644 & 0.3622 & 0.3600 & 0.3578 & 0.3556 \\
\hline Scan $x$-coord & 1270 & 1270 & 1270 & 1270 & 1270 & 1270 & 1270 & 1270 & 1270 & 1270 & 1270 & 1270 & 1270 & 1270 & 1270 & 1270 & 1270 \\
\hline$F X \# 1$ & 535.00 & 535.11 & 535.47 & 536.07 & 536.69 & 538.22 & 534.20 & 540.38 & 543.06 & 545.31 & 545.66 & 546.06 & 546.33 & 546.39 & 544.51 & 544.68 & 545.92 \\
\hline$F X \# 2$ & 374.03 & 373.83 & 373.91 & 374.21 & 374.47 & 374.55 & 374.67 & 374.98 & 375.44 & 375.98 & 376.25 & 376.52 & 376.73 & 376.92 & 377.02 & 377.24 & 377.52 \\
\hline$F X \# 3$ & 294.70 & 294.90 & 295.06 & 295.18 & 295.40 & 295.62 & 295.77 & 295.88 & 295.91 & 295.95 & 295.91 & 295.97 & 296.04 & 296.09 & 296.16 & 296.29 & 296.39 \\
\hline$F X \# 5$ & 187.65 & 187.84 & 187.90 & 187.91 & 187.97 & 188.04 & 188.11 & 187.99 & 188.11 & 188.24 & 188.36 & 188.50 & 188.62 & 188.84 & 189.07 & 189.11 & 189.05 \\
\hline$F X \# 6$ & 141.60 & 141.53 & 141.45 & 141.42 & 141.43 & 141.50 & 141.55 & 141.62 & 141.76 & 141.89 & 141.95 & 141.94 & 141.86 & 141.81 & 141.79 & 141.73 & 141.74 \\
\hline$F X \# 7$ & 0.00 & 0.00 & 0.00 & 0.00 & 0.00 & 0.00 & 0.00 & 0.00 & 0.00 & 0.00 & 0.00 & 0.00 & 0.00 & 0.00 & 0.00 & 0.00 & 0.00 \\
\hline Near wall FS & 141.60 & 141.53 & 141.45 & 141.42 & 141.43 & 141.50 & 141.55 & 141.62 & 141.76 & 141.89 & 141.95 & 141.94 & 141.86 & 141.81 & 141.79 & 141.73 & 141.74 \\
\hline$T @ F X \# 1$ & 297.30 & 297.30 & 297.30 & 297.30 & 297.30 & 297.30 & 297.30 & 297.30 & 297.30 & 297.30 & 297.30 & 297.30 & 297.30 & 297.30 & 297.30 & 297.30 & 297.30 \\
\hline$T @ F X \# 2$ & 298.33 & 298.33 & 298.33 & 298.32 & 298.32 & 298.31 & 298.31 & 298.30 & 298.30 & 298.29 & 298.29 & 298.28 & 298.28 & 298.27 & 298.27 & 298.27 & 298.26 \\
\hline$T @ F X \# 4$ & 302.44 & 302.44 & 302.43 & 302.43 & 302.42 & 302.42 & 302.42 & 302.41 & 302.41 & 302.40 & 302.40 & 302.39 & 302.39 & 302.38 & 302.38 & 302.37 & 302.37 \\
\hline$T @ F X \# 5$ & 304.52 & 304.51 & 304.51 & 304.50 & 304.50 & 304.49 & 304.49 & 304.48 & 304.48 & 304.48 & 304.47 & 304.47 & 304.46 & 304.46 & 304.45 & 304.45 & 304.44 \\
\hline$T @ F X \# 6$ & 306.62 & 306.61 & 306.61 & 306.60 & 306.60 & 306.59 & 306.59 & 306.58 & 306.58 & 306.57 & 306.57 & 306.57 & 306.56 & 306.56 & 306.55 & 306.55 & 306.54 \\
\hline$T @ \boldsymbol{F} \boldsymbol{T} \# \mathbf{7}$ & $\begin{array}{l}300.02 \\
3084\end{array}$ & 308.74 & 308.73 & 308.73 & 308.72 & 308.72 & 308.72 & 308.71 & 308.71 & 308.70 & 308.70 & 308.69 & 308.69 & 308.68 & 308.68 & 308.67 & 308.67 \\
\hline Wall $x$-coord & 128.01 & 128.02 & 128.03 & 128.04 & 128.05 & 128.06 & 128.07 & 128.08 & 128.09 & 128.10 & 128.11 & 128.12 & 128.13 & 128.14 & 128.15 & 128.16 & 128.17 \\
\hline Wall T & 309.37 & 309.36 & 309.35 & 309.34 & 309.34 & 309.33 & 309.33 & 309.33 & 309.33 & 309.33 & 309.33 & 309.32 & 309.31 & 309.30 & 309.29 & 309.28 & 309.28 \\
\hline
\end{tabular}

\section{B.11 Tabulated Data for Interferogram 467}

\begin{tabular}{|c|c|c|c|c|c|c|c|c|c|c|c|c|c|c|c|c|c|}
\hline Interferogram Image \# & 467 & Location & $T C 2$ & $\delta \varepsilon$ slope & $-2.44 E-3$ & $\delta \varepsilon$ intercept & 13.02 & $\operatorname{Avg} F S$ & 40.20 & Avg Wall T & 307.84 & Gradient error & 3.11E-4 & & & & \\
\hline Scan y-coord & 5302 & 5303 & 5304 & 5305 & 5306 & 5307 & 5308 & 5309 & 5310 & 5311 & 5312 & 5313 & 5314 & 5315 & 5316 & 5317 & 5318 \\
\hline & 0.1001 & 0.0977 & 0.0952 & 0.0928 & 0.0903 & 0.0879 & 0.0855 & 0.0830 & 0.0806 & 0.0782 & 0.0757 & 0.0733 & 0.0708 & 0.0684 & 0.0660 & 0.0635 & 0.0611 \\
\hline Scan $x$-coord & 825 & 825 & 825 & 825 & 825 & 825 & 825 & 825 & 825 & 825 & 825 & 825 & 825 & 825 & 825 & 825 & 825 \\
\hline$F X \# I$ & 488.00 & 488.00 & 488.00 & 488.00 & 488.00 & 488.00 & 488.00 & 488.00 & 488.00 & 488.00 & 488.00 & 488.00 & 488.00 & 488.00 & 488.00 & 488.00 & 488.00 \\
\hline$F X \# 2$ & 277.88 & 278.13 & 278.42 & 278.68 & 278.80 & 278.80 & 278.93 & 279.04 & 279.10 & 279.22 & 279.40 & 279.37 & 279.58 & 279.93 & 280.22 & 280.54 & 280.85 \\
\hline$F X \# 3$ & 213.28 & 213.57 & 213.88 & 214.13 & 214.39 & 214.64 & 214.81 & 214.93 & 214.94 & 214.95 & 214.95 & 215.01 & 215.09 & 215.22 & 215.33 & 215.38 & 215.41 \\
\hline$F X \# 5$ & 127.31 & 127.41 & 127.49 & 127.61 & 127.75 & 127.86 & 127.96 & 128.03 & 128.12 & 128.16 & 128.21 & 128.36 & 128.40 & 128.47 & 128.47 & 128.50 & 128.51 \\
\hline Near wall FS & 39.90 & 39.88 & 39.84 & 39.78 & 39.78 & 39.85 & 39.93 & 40.03 & 40.14 & 40.26 & 40.36 & 40.35 & 40.46 & 40.53 & 40.71 & 40.81 & 40.84 \\
\hline$T$ (a) $F X \# 1$ & 297.24 & 297.24 & 297.24 & 297.24 & 297.24 & 297.24 & 297.24 & 297.24 & 297.24 & 297.24 & 297.24 & 297.24 & 297.24 & 297.24 & 297.24 & 297.24 & 297.24 \\
\hline$T @ F X \# 2$ & 297.54 & 297.54 & 297.53 & 297.53 & 297.52 & 297.52 & 297.51 & 297.51 & 297.50 & 297.50 & 297.49 & 297.49 & 297.48 & 297.48 & 297.47 & 297.47 & 297.46 \\
\hline$T @ F X \# 3$ & 299.59 & 299.59 & 299.58 & 299.58 & 299.57 & 299.57 & 299.56 & 299.56 & 299.55 & 299.55 & 299.54 & 299.54 & 299.53 & 299.53 & 299.52 & 299.52 & 299.51 \\
\hline$T @ F X \# 4$ & 301.63 & 301.62 & 301.62 & 301.61 & 301.61 & 301.60 & 301.60 & 301.59 & 301.59 & 301.58 & 301.58 & 301.57 & 301.57 & 301.56 & 301.56 & 301.55 & 301.55 \\
\hline$T @ F X \# 5$ & 303.69 & 303.68 & 303.68 & 303.67 & 303.67 & 303.66 & 303.66 & 303.65 & 303.65 & 303.64 & 303.64 & 303.63 & 303.62 & 303.62 & 303.61 & 303.61 & 303.60 \\
\hline Wall $x$-coord & 87.30 & 87.30 & 87.30 & 87.29 & 87.29 & 87.29 & 87.29 & 87.28 & 87.28 & 87.28 & 87.27 & 87.27 & 87.27 & 87.27 & 87.26 & 87.26 & 87.26 \\
\hline Wall $T$ & 307.86 & 307.86 & 307.86 & 307.86 & 307.87 & 307.86 & 307.86 & 307.85 & 307.85 & 307.84 & 307.83 & 307.83 & 307.82 & 307.82 & 307.81 & 307.80 & 307.79 \\
\hline Interferogram Image \# & 467 & Location & $T C 3$ & $\delta \varepsilon$ slope & $-2.33 E-3$ & $\delta \varepsilon$ intercept & 10.52 & $\operatorname{Avg} F S$ & 48.43 & Avg Wall $T$ & 307.40 & Gradient error & $3.11 E-4$ & & & & \\
\hline Scan y-coord & 4487 & 4488 & 4489 & 4490 & 4491 & 4492 & 4493 & 4494 & 4495 & 4496 & 4497 & 4498 & 4499 & 4500 & 4501 & 4502 & 4503 \\
\hline & 0.0843 & 0.0820 & 0.0797 & 0.0773 & 0.0750 & 0.0727 & 0.0704 & 0.0680 & 0.0657 & 0.0634 & 0.0611 & 0.0587 & 0.0564 & 0.0541 & 0.0518 & 0.0494 & 0.0471 \\
\hline
\end{tabular}




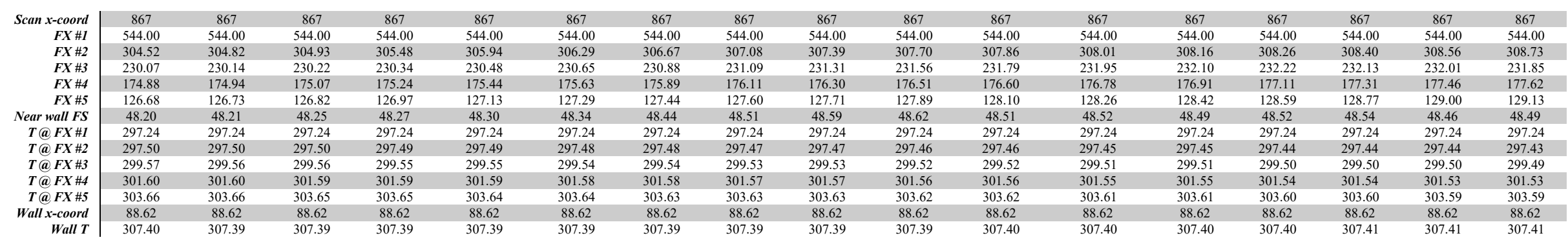

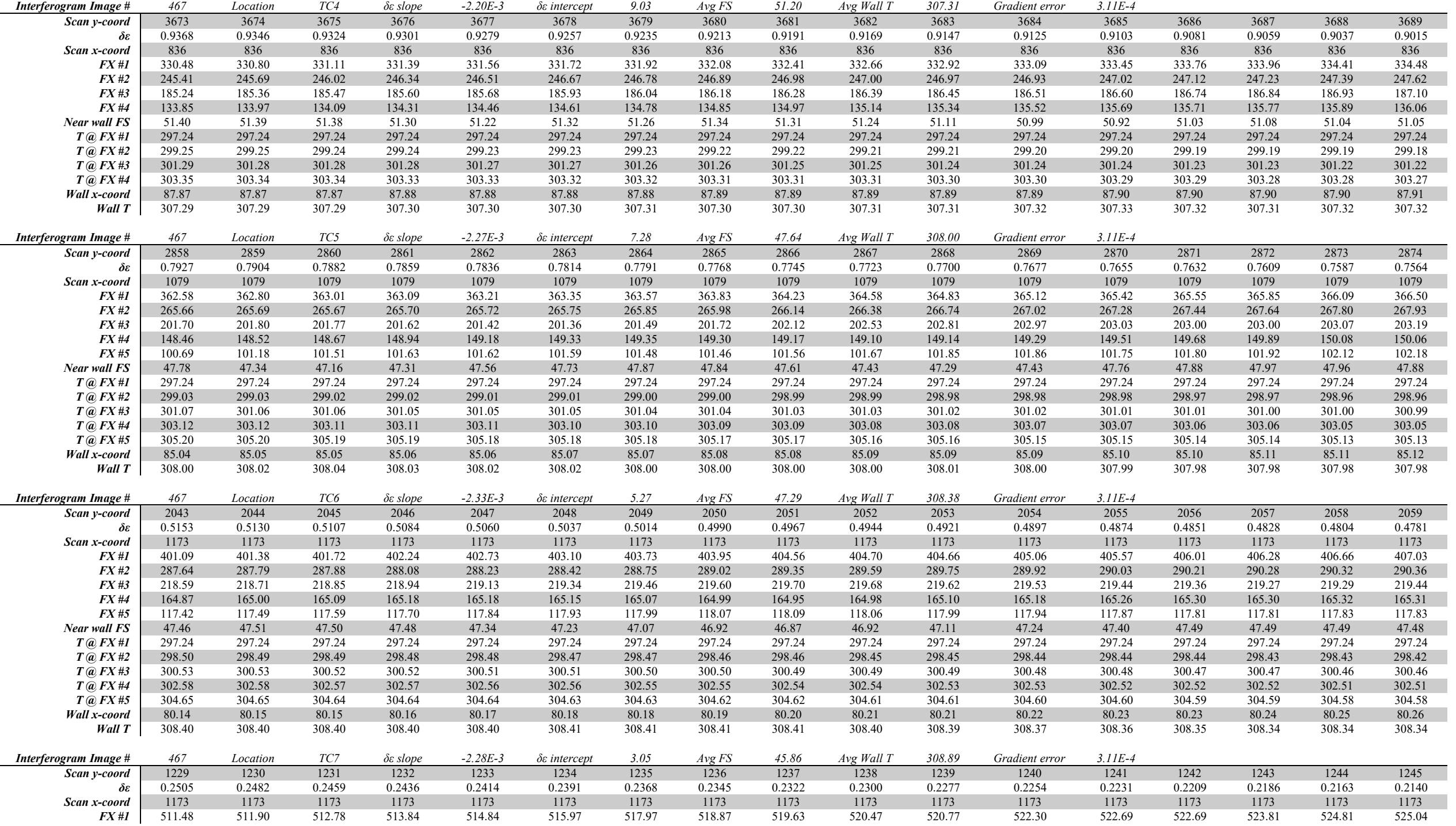




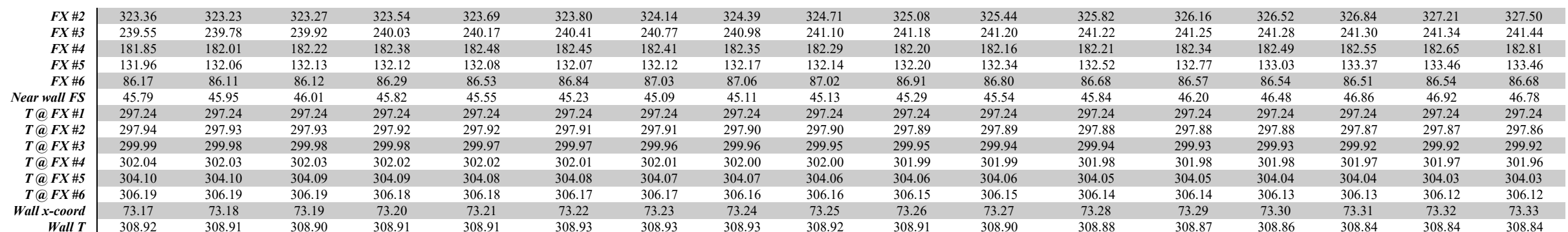

\section{B.12 Tabulated Data for Interferogram 468}

\begin{tabular}{|c|c|c|c|c|c|c|c|c|c|c|c|c|c|c|c|c|c|}
\hline Interferogram Image \# & 468 & Location & $T C 2$ & $\delta \varepsilon$ slope & $-2.48 E-3$ & $\delta$ E intercept & 13.84 & $\operatorname{Avg} F S$ & 40.44 & Avg Wall T & 308.09 & Gradient error & $3.48 E-4$ & & & & \\
\hline Scan y-coord & 5251 & 5252 & 5253 & 5254 & 5255 & 5256 & 5257 & 5258 & 5259 & 5260 & 5261 & 5262 & 5263 & 5264 & 5265 & 5266 & 5267 \\
\hline & 0.8370 & 0.8345 & 0.8320 & 0.8295 & 0.8271 & 0.8246 & 0.8221 & 0.8196 & 0.8172 & 0.8147 & 0.8122 & 0.8097 & 0.8073 & 0.8048 & 0.8023 & 0.7998 & 0.7974 \\
\hline Scan $x$-coord & 903 & 903 & 903 & 903 & 903 & 903 & 903 & 903 & 903 & 903 & 903 & 903 & 903 & 903 & 903 & 903 & 903 \\
\hline$F X \# 1$ & 365.87 & 366.00 & 366.07 & 366.23 & 366.67 & 366.81 & 367.28 & 367.59 & 367.79 & 368.00 & 368.08 & 368.27 & 368.41 & 368.55 & 368.85 & 369.02 & 369.34 \\
\hline$F X \# 2$ & 281.87 & 281.91 & 282.14 & 282.42 & 282.64 & 282.77 & 282.94 & 283.07 & 283.13 & 283.16 & 283.22 & 283.24 & 283.22 & 283.27 & 283.27 & 283.31 & 283.44 \\
\hline$F X \# 3$ & 229.09 & 229.19 & 229.30 & 229.40 & 229.49 & 229.59 & 229.74 & 229.91 & 229.96 & 229.97 & 230.04 & 230.04 & 230.13 & 230.21 & 230.31 & 230.45 & 230.55 \\
\hline$F X \# 5$ & 145.80 & 145.82 & 145.98 & 146.20 & 146.41 & 146.56 & 146.77 & 146.94 & 147.06 & 147.16 & 147.25 & 147.44 & 147.63 & 147.90 & 148.14 & 148.30 & 148.39 \\
\hline Near wall FS & 40.60 & 40.81 & 40.87 & 40.77 & 40.63 & 40.53 & 40.45 & 40.43 & 40.45 & 40.44 & 40.43 & 40.34 & 40.29 & 40.15 & 40.09 & 40.06 & 40.07 \\
\hline$T$ (a) $F X \# 1$ & 297.23 & 297.23 & 297.23 & 297.23 & 297.23 & 297.23 & 297.23 & 297.23 & 297.23 & 297.23 & 297.23 & 297.23 & 297.23 & 297.23 & 297.23 & 297.23 & 297.23 \\
\hline$T$ (a) $F X \# 2$ & 299.08 & 299.07 & 299.07 & 299.06 & 299.06 & 299.05 & 299.05 & 299.04 & 299.04 & 299.03 & 299.03 & 299.02 & 299.02 & 299.01 & 299.01 & 299.00 & 299.00 \\
\hline$T$ (a) $F X \# 3$ & 301.12 & 301.11 & 301.11 & 301.10 & 301.10 & 301.09 & 301.09 & 301.08 & 301.08 & 301.07 & 301.07 & 301.06 & 301.06 & 301.05 & 301.05 & 301.04 & 301.04 \\
\hline$T$ @ $F X \# 4$ & 303.17 & 303.16 & 303.16 & 303.15 & 303.15 & 303.14 & 303.14 & 303.13 & 303.13 & 303.12 & 303.12 & 303.11 & 303.11 & 303.10 & 303.10 & 303.09 & 303.09 \\
\hline Wall $x$-coord & 132.14 & 132.14 & 132.13 & 132.13 & 132.13 & 132.13 & 132.13 & 132.12 & 132.12 & 132.12 & 132.12 & 132.11 & 132.11 & 132.11 & 132.11 & 132.10 & 132.10 \\
\hline Wall T & 308.06 & 308.06 & 308.06 & 308.07 & 308.07 & 308.08 & 308.09 & 308.09 & 308.09 & 308.09 & 308.09 & 308.10 & 308.10 & 308.11 & 308.12 & 308.13 & 308.13 \\
\hline
\end{tabular}

\begin{tabular}{|c|c|c|c|c|c|c|c|c|c|c|c|c|c|c|c|c|c|}
\hline Interferogram Image \# & 468 & Location & $T C 3$ & $\delta \varepsilon$ slope & $-2.37 E-3$ & $\delta \varepsilon$ intercept & 11.29 & $\operatorname{Avg} F S$ & 50.51 & Avg Wall $T$ & 307.62 & Gradient error & $3.48 E-4$ & & & & \\
\hline Scan y-coord & 4437 & 4438 & 4439 & 4440 & 4441 & 4442 & 4443 & 4444 & 4445 & 4446 & 4447 & 4448 & 4449 & 4450 & 4451 & 4452 & 4453 \\
\hline & 0.7867 & 0.7843 & 0.7819 & 0.7796 & 0.7772 & 0.7748 & 0.7725 & 0.7701 & 0.7677 & 0.7654 & 0.7630 & 0.7606 & 0.7583 & 0.7559 & 0.7535 & 0.7512 & 0.7488 \\
\hline Scan $x$-coord & 1077 & 1077 & 1077 & 1077 & 1077 & 1077 & 1077 & 1077 & 1077 & 1077 & 1077 & 1077 & 1077 & 1077 & 1077 & 1077 & 1077 \\
\hline$F X \# 1$ & 396.82 & 397.25 & 397.70 & 398.16 & 398.72 & 399.13 & 399.61 & 400.13 & 400.32 & 400.31 & 401.52 & 401.50 & 401.95 & 402.91 & 403.26 & 403.70 & 404.21 \\
\hline$F X \# 2$ & 304.58 & 304.43 & 304.31 & 304.28 & 304.35 & 304.54 & 304.77 & 305.09 & 305.42 & 305.66 & 305.99 & 306.24 & 306.49 & 306.81 & 307.02 & 307.21 & 307.34 \\
\hline$F X \# 3$ & 242.66 & 242.67 & 242.70 & 242.69 & 242.84 & 242.87 & 242.93 & 243.10 & 243.28 & 243.48 & 243.66 & 243.89 & 244.13 & 244.39 & 244.65 & 244.82 & 244.99 \\
\hline$F X \# 5$ & 140.35 & 140.53 & 140.55 & 140.49 & 140.63 & 140.75 & 140.96 & 141.06 & 141.29 & 141.43 & 141.56 & 141.78 & 141.89 & 141.93 & 142.00 & 142.25 & 142.45 \\
\hline Near wall $F S$ & 50.35 & $\begin{array}{l}140.53 \\
50.27\end{array}$ & 50.37 & $\begin{array}{l}400.54 \\
50.54\end{array}$ & $\begin{array}{l}140.03 \\
50.52\end{array}$ & 50.53 & 50.45 & $\begin{array}{l}141.00 \\
50.50\end{array}$ & $\begin{array}{l}141.29 \\
50.48\end{array}$ & $\begin{array}{l}141.45 \\
50.48\end{array}$ & $\begin{array}{l}50.50 \\
50.50\end{array}$ & 50.42 & $\begin{array}{l}141.09 \\
50.45\end{array}$ & 50.63 & 50.75 & $\begin{array}{l}14.25 \\
50.67\end{array}$ & $\begin{array}{l}12.40 \\
50.71\end{array}$ \\
\hline$T @ F X \# 1$ & 297.23 & 297.23 & 297.23 & 297.23 & 297.23 & 297.23 & 297.23 & 297.23 & 297.23 & 297.23 & 297.23 & 297.23 & 297.23 & 297.23 & 297.23 & 297.23 & 297.23 \\
\hline$T @ F X \# 2$ & 299.03 & 299.02 & 299.02 & 299.01 & 299.01 & 299.00 & 299.00 & 298.99 & 298.99 & 298.98 & 298.98 & 298.97 & 298.97 & 298.96 & 298.96 & 298.95 & 298.95 \\
\hline$T @ F X \# 3$ & 301.07 & 301.06 & 301.06 & 301.05 & 301.05 & 301.04 & 301.04 & 301.03 & 301.03 & 301.02 & 301.02 & 301.01 & 301.01 & 301.00 & 301.00 & 300.99 & 300.99 \\
\hline$T @ F X \# 4$ & 303.12 & 303.12 & 303.11 & 303.11 & 303.10 & 303.10 & 303.09 & 303.09 & 303.09 & 303.08 & 303.08 & 303.07 & 303.07 & 303.06 & 303.06 & 303.05 & 303.05 \\
\hline Wall $x$-coord & 133.09 & 133.09 & 133.09 & 133.09 & 133.09 & 133.09 & 133.09 & 133.09 & 133.09 & 133.09 & 133.09 & 133.09 & 133.09 & 133.09 & 133.09 & 133.09 & 133.09 \\
\hline Wall T & $\begin{array}{l}307.69 \\
307.62\end{array}$ & 307.62 & 307.62 & 307.61 & 307.61 & 307.61 & 307.61 & 307.61 & 307.62 & 307.62 & 307.62 & 307.62 & 307.62 & 307.62 & 307.61 & 307.62 & 307.62 \\
\hline
\end{tabular}

\begin{tabular}{|c|c|c|c|c|c|c|c|c|c|c|c|c|c|c|c|c|c|}
\hline Interferogram Image \# & 468 & Location & $1 C 4$ & $\partial \varepsilon$ slope & & de intercept & 9.11 & $\operatorname{Avg} F S$ & 53.38 & Avg Wall T & 307.62 & Gradient error & $3.48 E-4$ & & & & \\
\hline Scan $y$-coord & 3623 & 3624 & 3625 & 3626 & 3627 & 3628 & 3629 & 3630 & 3631 & 3632 & 3633 & 3634 & 3635 & 3636 & 3637 & 3638 & 3639 \\
\hline & 0.6377 & 0.6354 & 0.6330 & 0.6307 & 0.6284 & 0.6260 & 0.6237 & 0.6213 & 0.6190 & 0.6167 & 0.6143 & 0.6120 & 0.6097 & 0.6073 & 0.6050 & 0.6026 & 0.6003 \\
\hline Scan $x$-coord & 1443 & 1443 & 1443 & 1443 & 1443 & 1443 & 1443 & 1443 & 1443 & 1443 & 1443 & 1443 & 1443 & 1443 & 1443 & 1443 & 1443 \\
\hline$F X \# 1$ & 440.06 & 440.42 & 440.79 & 441.06 & 441.42 & 441.72 & 442.25 & 443.20 & 444.36 & 444.73 & 444.74 & 445.20 & 445.45 & 445.99 & 446.60 & 447.01 & 447.45 \\
\hline$F X \# 2$ & 326.10 & 326.27 & 326.47 & 326.70 & 326.99 & 327.31 & 327.64 & 327.91 & 328.20 & 328.41 & 328.56 & 328.71 & 328.84 & 329.05 & 329.25 & 329.36 & 329.49 \\
\hline$F X \# 3$ & 255.51 & 255.66 & 255.83 & 256.18 & 256.41 & 256.59 & 256.74 & 256.82 & 256.89 & 256.95 & 257.05 & 257.17 & 257.30 & 257.33 & 257.40 & 257.60 & 257.84 \\
\hline$F X \# 4$ & 197.39 & 197.51 & 197.72 & 197.88 & 198.09 & 198.29 & 198.48 & 198.70 & 198.80 & 198.96 & 199.04 & 199.09 & 199.23 & 199.40 & 199.56 & 199.67 & 199.79 \\
\hline$F X \# 5$ & 144.23 & 144.27 & 144.41 & 144.48 & 144.59 & 144.78 & 144.97 & 145.14 & 145.28 & 145.42 & 145.64 & 145.90 & 146.00 & 146.05 & 146.17 & 146.35 & 146.46 \\
\hline Near wall FS & 53.16 & 53.24 & 53.31 & 53.40 & 53.50 & 53.52 & 53.51 & 53.56 & 53.52 & 53.55 & 53.41 & 53.19 & 53.23 & 53.35 & 53.39 & 53.32 & 53.33 \\
\hline$T @ F X \# 1$ & 297.23 & 297.23 & 297.23 & 297.23 & 297.23 & 297.23 & 297.23 & 297.23 & 297.23 & 297.23 & 297.23 & 297.23 & 297.23 & 297.23 & 297.23 & 297.23 & 297.23 \\
\hline$T @ F X \# 2$ & 298.84 & 298.84 & 298.83 & 298.83 & 298.82 & 298.82 & 298.81 & 298.81 & 298.80 & 298.80 & 298.80 & 298.79 & 298.79 & 298.78 & 298.78 & 298.77 & 298.77 \\
\hline$T(F X \# 3$ & 300.89 & 300.88 & 300.88 & 300.87 & 300.87 & 300.86 & 300.86 & 300.85 & 300.85 & 300.85 & 300.84 & 300.84 & 300.83 & 300.83 & 300.82 & 300.82 & 300.81 \\
\hline$T(F X \# 4$ & 302.95 & 302.94 & 302.94 & 302.93 & 302.93 & 302.92 & 302.92 & 302.91 & 302.91 & 302.90 & 302.90 & 302.89 & 302.89 & 302.88 & 302.88 & 302.87 & 302.87 \\
\hline$T(F X \# 5$ & 305.03 & 305.02 & 305.02 & 305.01 & 305.01 & 305.00 & 305.00 & 304.99 & 304.99 & 304.98 & 304.98 & 304.97 & 304.97 & 304.96 & 30496 & 30495 & 304.95 \\
\hline Wall T & 307.62 & 307.61 & 307.61 & 307.61 & 307.61 & 307.61 & 307.61 & 307.62 & 307.62 & 307.62 & 307.62 & 307.63 & 307.63 & 307.62 & 307.62 & 307.62 & 307.62 \\
\hline
\end{tabular}

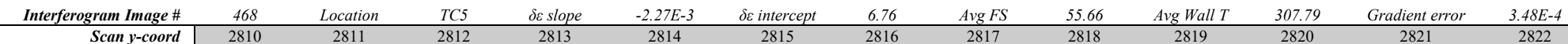




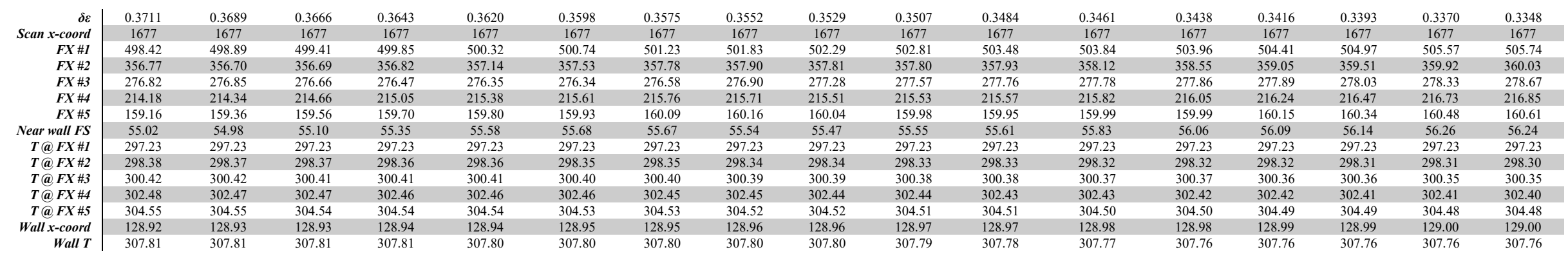

\begin{tabular}{|c|c|c|c|c|c|c|c|c|c|c|c|c|c|c|c|c|c|}
\hline Interferogram Image \# & 468 & Location & TC6 & $\delta \varepsilon$ slope & $-2.12 E-3$ & $\delta \varepsilon$ intercept & 4.32 & $\operatorname{Avg} F S$ & 54.78 & Avg Wall $T$ & 308.20 & Gradient error & $3.48 E-4$ & & & & \\
\hline Scan y-coord & 1996 & 1997 & 1998 & 1999 & 2000 & 2001 & 2002 & 2003 & 2004 & 2005 & 2006 & 2007 & 2008 & 2009 & 2010 & 2011 & 2012 \\
\hline & 0.0828 & 0.0806 & 0.0785 & 0.0764 & 0.0743 & 0.0721 & 0.0700 & 0.0679 & 0.0658 & 0.0637 & 0.0615 & 0.0594 & 0.0573 & 0.0552 & 0.0531 & 0.0509 & 0.0488 \\
\hline Scan $x$-coord & 1657 & 1657 & 1657 & 1657 & 1657 & 1657 & 1657 & 1657 & 1657 & 1657 & 1657 & 1657 & 1657 & 1657 & 1657 & 1657 & 1657 \\
\hline$F X \# 1$ & 672.45 & 675.32 & 677.18 & 678.71 & 697.33 & 696.55 & 700.94 & 696.18 & 697.49 & 698.41 & 699.93 & 710.73 & 736.18 & 717.53 & 719.08 & 725.17 & 685.12 \\
\hline$F X \# 2$ & 396.66 & 396.87 & 396.96 & 397.13 & 397.39 & 397.64 & 397.98 & 398.22 & 398.43 & 398.67 & 399.01 & 399.48 & 399.90 & 400.22 & 400.59 & 400.76 & 400.85 \\
\hline$F X \# 3$ & 301.79 & 301.82 & 302.01 & 302.16 & 302.29 & 302.39 & 302.52 & 302.70 & 302.91 & 303.08 & 303.23 & 303.36 & 303.59 & 303.75 & 303.90 & 304.15 & 304.39 \\
\hline$F X \# 4$ & 235.97 & 236.20 & 236.38 & 236.49 & 236.61 & 236.73 & 236.82 & 236.96 & 237.20 & 237.38 & 237.51 & 237.67 & 237.89 & 238.10 & 238.30 & 238.45 & 238.50 \\
\hline$F X \# 5$ & 179.99 & 180.15 & 180.31 & 180.57 & 180.66 & 181.00 & 181.31 & 181.14 & 181.21 & 181.06 & 180.79 & 180.59 & 180.46 & 180.42 & 180.44 & 180.57 & 180.66 \\
\hline$F X \# 6$ & 124.69 & 124.36 & 124.39 & 125.17 & 126.43 & 126.34 & 126.38 & 126.34 & 125.89 & 125.61 & 125.72 & 126.32 & 126.47 & 126.38 & 126.17 & 126.51 & 126.83 \\
\hline Near wall FS & 55.30 & 55.78 & 55.92 & 55.40 & 54.23 & 54.66 & 54.93 & 54.80 & 55.31 & 55.46 & 55.07 & 54.27 & 53.99 & 54.04 & 54.27 & 54.06 & 53.83 \\
\hline$T @ F X \# 1$ & 297.23 & 297.23 & 297.23 & 297.23 & 297.23 & 297.23 & 297.23 & 297.23 & 297.23 & 297.23 & 297.23 & 297.23 & 297.23 & 297.23 & 297.23 & 297.23 & 297.23 \\
\hline$T @ F X \# 2$ & 297.74 & 297.73 & 297.73 & 297.72 & 297.71 & 297.71 & 297.70 & 297.70 & 297.70 & 297.69 & 297.69 & 297.68 & 297.67 & 297.67 & 297.66 & 297.66 & 297.67 \\
\hline$T(a) F \#$ & 299.82 & 299.82 & 299.82 & 299.81 & 299.81 & 299.80 & 299.80 & 299.79 & 299.79 & 299.79 & 299.78 & 299.78 & 299.77 & 299.77 & 299.76 & 299.76 & 299.76 \\
\hline$T(a) F \# 4$ & 301.88 & 301.87 & 301.87 & 301.86 & 301.86 & 301.85 & 301.85 & 301.85 & 301.84 & 301.84 & 301.83 & 301.83 & 301.82 & 301.82 & 301.81 & 301.81 & 301.81 \\
\hline$T$ (a) $F X \# 5$ & 303.95 & 303.94 & 303.94 & 303.93 & 303.93 & 303.92 & 303.92 & 303.91 & 303.91 & 303.91 & 303.90 & 303.90 & 303.89 & 303.89 & 303.88 & 303.88 & 303.87 \\
\hline$T @ F X \# 6$ & 306.04 & 306.03 & 306.03 & 306.03 & 306.02 & 306.02 & 306.01 & 306.01 & 306.00 & 306.00 & 305.99 & 305.99 & 305.99 & 305.98 & 305.98 & 305.97 & 305.97 \\
\hline Wall $x$-coord & 123.81 & 123.82 & 123.83 & 123.83 & 123.84 & 123.85 & 123.86 & 123.86 & 123.87 & 123.88 & 123.89 & 123.89 & 123.90 & 123.91 & 123.92 & 123.92 & 123.93 \\
\hline Wall $T$ & 308.20 & 308.18 & 308.17 & 308.20 & 308.24 & 308.24 & 308.23 & 308.23 & 308.20 & 308.19 & 308.19 & 308.21 & 308.21 & 308.20 & 308.19 & 308.19 & 308.20 \\
\hline
\end{tabular}

\begin{tabular}{|c|c|c|c|c|c|c|c|c|c|c|c|c|c|c|c|c|c|}
\hline Interferogram Image \# & 468 & Location & $T C 7$ & $\delta \varepsilon$ slope & $-2.17 E-3$ & $\delta \varepsilon$ intercept & 3.36 & $A v g F S$ & 54.54 & Avg Wall $T$ & 308.65 & Gradient error & $3.48 E-4$ & & & & \\
\hline Scan y-coord & 1182 & 1183 & 1184 & 1185 & 1186 & 1187 & 1188 & 1189 & 1190 & 1191 & 1192 & 1193 & 1194 & 1195 & 1196 & 1197 & 1198 \\
\hline & 0.7991 & 0.7969 & 0.7947 & 0.7926 & 0.7904 & 0.7882 & 0.7861 & 0.7839 & 0.7817 & 0.7796 & 0.7774 & 0.7752 & 0.7731 & 0.7709 & 0.7687 & 0.7666 & 0.7644 \\
\hline Scan $x$-coord & 1411 & 1411 & 1411 & 1411 & 1411 & 1411 & 1411 & 1411 & 1411 & 1411 & 1411 & 1411 & 1411 & 1411 & 1411 & 1411 & 1411 \\
\hline$F X \# 1$ & 472.56 & 472.78 & 473.43 & 473.74 & 474.12 & 474.12 & 474.68 & 475.18 & 475.50 & 476.26 & 476.47 & 476.85 & 476.61 & 477.29 & 477.34 & 477.72 & 478.46 \\
\hline $\begin{array}{l}F X+1 \\
F X \# 2\end{array}$ & 340.37 & 340.86 & 341.16 & 341.45 & 341.70 & 341.90 & 342.08 & 342.19 & 342.20 & 342.21 & 342.24 & 342.38 & 342.67 & 342.99 & 343.25 & 343.46 & $\begin{array}{l}470.40 \\
343.58\end{array}$ \\
\hline$F X \# 3$ & 263.05 & 263.26 & 263.40 & 263.51 & 263.68 & 263.89 & 264.08 & 264.14 & 264.25 & 264.33 & 264.44 & 264.55 & 264.69 & 264.82 & 264.94 & 265.07 & 265.21 \\
\hline$F X \# 5$ & 148.22 & 148.27 & 148.30 & 148.36 & 148.35 & 148.38 & 148.38 & 148.47 & 148.36 & 148.35 & 148.41 & 148.36 & 148.39 & 148.51 & 148.66 & 148.83 & 149.00 \\
\hline Near wall FS & 53.91 & 53.95 & 54.02 & 54.10 & 54.20 & 54.22 & 54.28 & 54.32 & 54.45 & 54.58 & 54.66 & 54.89 & 55.17 & 55.26 & 55.16 & 55.02 & 54.92 \\
\hline$T @ F X \# 1$ & 297.23 & 297.23 & 297.23 & 297.23 & 297.23 & 297.23 & 297.23 & 297.23 & 297.23 & 297.23 & 297.23 & 297.23 & 297.23 & 297.23 & 297.23 & 297.23 & 297.23 \\
\hline$T @ F X \# 2$ & 299.14 & 299.14 & 299.13 & 299.13 & 299.12 & 299.12 & 299.12 & 299.11 & 299.11 & 299.10 & 299.10 & 299.09 & 299.09 & 299.08 & 299.08 & 299.08 & 299.07 \\
\hline$T @ F X \# 3$ & 301.20 & 301.19 & 301.19 & 301.18 & 301.18 & 301.17 & 301.17 & 301.17 & 301.16 & 301.16 & 301.15 & 301.15 & 301.14 & 301.14 & 301.13 & 301.13 & 301.13 \\
\hline$T @ F X \# 4$ & 303.26 & 303.26 & 303.25 & 303.25 & 303.24 & 303.24 & 303.23 & 303.23 & 303.23 & 303.22 & 303.22 & 303.21 & 303.21 & 303.20 & 303.20 & 303.19 & 303.19 \\
\hline Wall $x$-coord & 116.68 & 116.69 & 116.70 & 116.71 & 116.72 & 116.73 & 116.74 & 116.75 & 116.76 & 116.77 & 116.78 & 116.79 & 116.80 & 116.81 & 116.82 & 116.83 & 116.84 \\
\hline Wall $T$ & 308.70 & 308.69 & 308.69 & 308.68 & 308.67 & 308.67 & 308.66 & 308.66 & 308.65 & 308.64 & 308.64 & 308.62 & 308.61 & 308.61 & 308.61 & 308.62 & 308.62 \\
\hline
\end{tabular}

\section{B.13 Tabulated Data for Interferogram 469}

\begin{tabular}{|c|c|c|c|c|c|c|c|c|c|c|c|c|c|c|c|c|c|}
\hline ogram Image \# & 469 & Location & $T C 2$ & $\delta \varepsilon$ slope & $-2.48 E-3$ & $\delta \varepsilon$ intercept & 13.82 & $\operatorname{Avg} F S$ & 37.82 & Avg Wall $T$ & 308.16 & Gradient error & $2.30 E-4$ & & & & \\
\hline Scan y-coord & 5266 & 5267 & 5268 & 5269 & 5270 & 5271 & 5272 & 5273 & 5274 & 5275 & 5276 & 5277 & 5278 & 5279 & 5280 & 5281 & 5282 \\
\hline & 0.7668 & 0.7644 & 0.7619 & 0.7594 & 0.7569 & 0.7544 & 0.7520 & 0.7495 & 0.7470 & 0.7445 & 0.7421 & 0.7396 & 0.7371 & 0.7346 & 0.7321 & 0.7297 & 0.7272 \\
\hline Scan $x$-coord & 1021 & 1021 & 1021 & 1021 & 1021 & 1021 & 1021 & 1021 & 1021 & 1021 & 1021 & 1021 & 1021 & 1021 & 1021 & 1021 & 1021 \\
\hline$F X \# I$ & 339.83 & 340.35 & 341.06 & 343.49 & 343.47 & 344.21 & 344.32 & 344.65 & 345.33 & 345.77 & 346.11 & 347.07 & 347.47 & 347.57 & 347.82 & 348.34 & 348.52 \\
\hline$F X \# 2$ & 250.19 & 250.19 & 250.24 & 250.56 & 250.83 & 251.19 & 251.42 & 251.61 & 251.75 & 251.93 & 251.98 & 252.00 & 251.98 & 251.90 & 251.84 & 251.87 & 251.90 \\
\hline$F X \# 3$ & 198.97 & 199.02 & 199.06 & 199.11 & 199.23 & 199.35 & 199.47 & 199.55 & 199.62 & 199.71 & 199.77 & 199.85 & 199.98 & 199.99 & 200.05 & 200.06 & 200.10 \\
\hline$F X \# 4$ & 158.51 & 158.62 & 158.90 & 159.05 & 159.15 & 159.27 & 159.30 & 159.35 & 159.55 & 159.69 & 159.87 & 160.01 & 160.13 & 160.28 & 160.44 & 160.54 & 160.63 \\
\hline$F X \# 5$ & 120.93 & 120.99 & 121.06 & 121.04 & 121.08 & 121.21 & 121.35 & 121.58 & 121.89 & 122.05 & 122.25 & 122.28 & 122.32 & 122.38 & 122.50 & 122.64 & 122.81 \\
\hline Near wall FS & 37.58 & 37.63 & 37.84 & 38.01 & 38.07 & 38.06 & 37.95 & 37.77 & 37.66 & 37.64 & 37.61 & 37.73 & 37.81 & 37.90 & 37.94 & 37.90 & 37.83 \\
\hline$T @ F X \# 1$ & 297.21 & 297.21 & 297.21 & 297.21 & 297.21 & 297.21 & 297.21 & 297.21 & 297.21 & 297.21 & 297.21 & 297.21 & 297.21 & 297.21 & 297.21 & 297.21 & 297.21 \\
\hline$T @ F X \# 2$ & 298.89 & 298.88 & 298.88 & 298.87 & 298.87 & 298.86 & 298.86 & 298.85 & 298.85 & 298.84 & 298.84 & 298.83 & 298.83 & 298.82 & 298.82 & 298.81 & 298.81 \\
\hline$T(F X \# 3$ & 300.92 & 300.91 & 300.91 & 300.90 & 300.89 & 300.89 & 300.88 & 300.88 & 300.87 & 300.87 & 300.86 & 300.86 & 300.85 & 300.85 & 300.84 & 300.84 & 300.83 \\
\hline$T @ F X \# 4$ & 302.96 & 302.96 & 302.95 & 302.94 & 302.94 & 302.93 & 302.93 & 302.92 & 302.92 & 302.91 & 302.91 & 302.90 & 302.90 & 302.89 & 302.89 & 302.88 & 302.88 \\
\hline$T @ F X \# 5$ & 305.03 & 305.03 & 305.02 & 305.01 & 305.01 & 305.00 & 305.00 & 304.99 & 304.99 & 304.98 & 304.98 & 304.97 & 304.97 & 304.96 & 304.96 & 304.95 & 304.95 \\
\hline Wall $x$-coord & 102.46 & 102.46 & 102.46 & 102.46 & 102.46 & 102.45 & 102.45 & 102.45 & 102.45 & 102.45 & 102.44 & 102.44 & 102.44 & 102.44 & 102.44 & 102.43 & 102.43 \\
\hline Wall $T$ & 308.16 & 308.16 & 308.15 & 308.14 & 308.13 & 308.14 & 308.14 & 308.15 & 308.17 & 308.17 & 308.18 & 308.17 & 308.17 & 308.16 & 308.16 & 308.17 & 308.17 \\
\hline
\end{tabular}




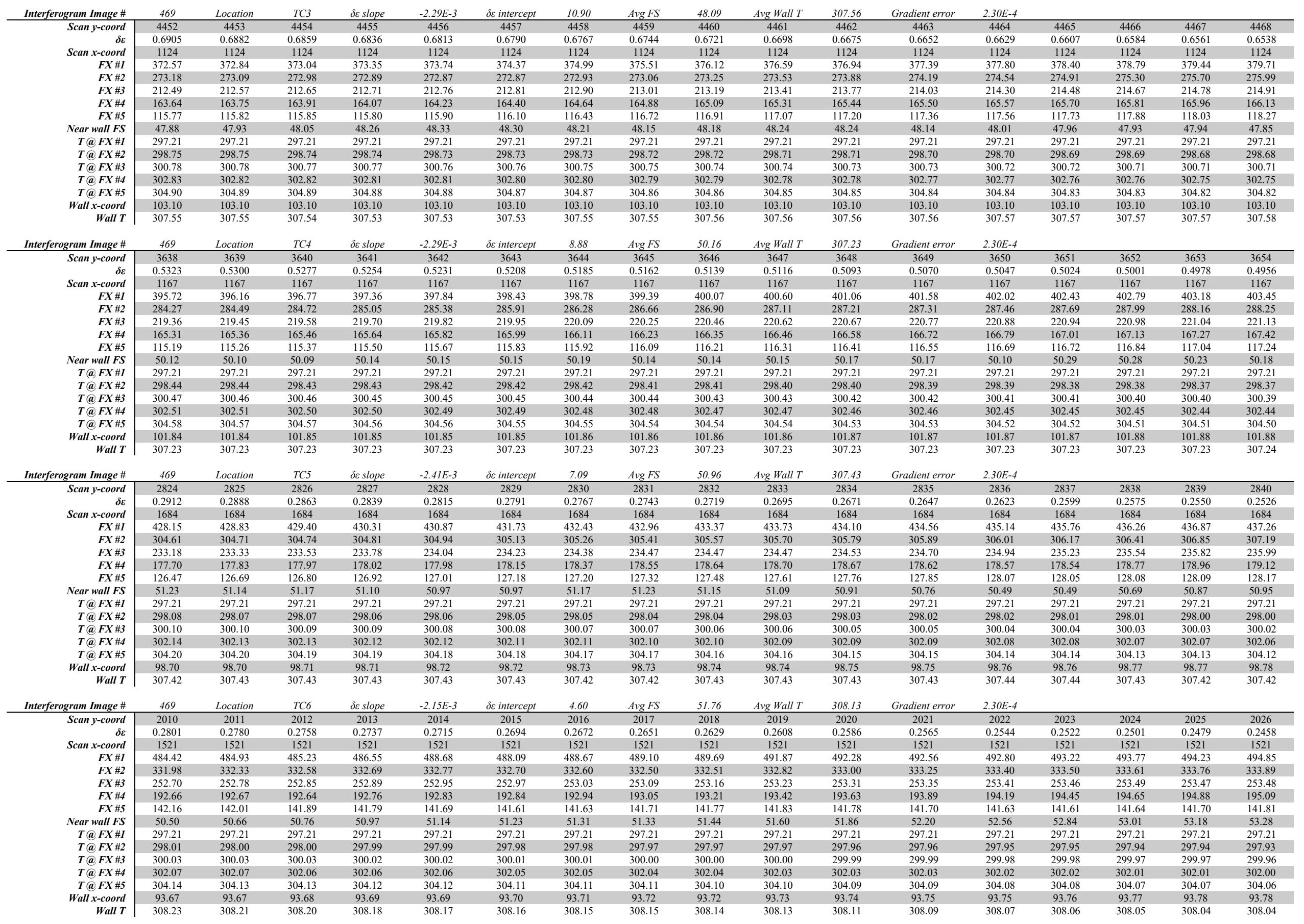




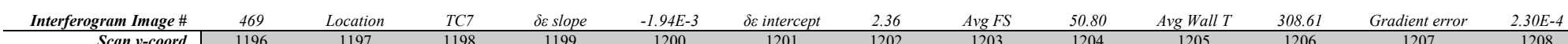

\begin{tabular}{|c|c|c|c|c|c|c|c|c|c|c|c|c|c|c|c|c|c|}
\hline gram Image \# & 469 & Location & IC7 & os slope & $-1.94 \mathrm{E}-3$ & recept & & Avg FS & & Avg Wall T & & terror & & & & & \\
\hline Scan y-coord & 1196 & 1197 & 1198 & 1199 & 1200 & 1201 & 1202 & 1203 & 1204 & 1205 & 1206 & 1207 & 1208 & 1209 & 1210 & 1211 & 1212 \\
\hline & 0.0459 & 0.0440 & 0.0421 & 0.0401 & 0.0382 & 0.0363 & 0.0343 & 0.0324 & 0.0305 & 0.0285 & 0.0266 & 0.0246 & 0.0227 & 0.0208 & 0.0188 & 0.0169 & 0.0150 \\
\hline Scan $x$-coord & 1056 & 1056 & 1056 & 1056 & 1056 & 1056 & 1056 & 1056 & 1056 & 1056 & 1056 & 1056 & 1056 & 1056 & 1056 & 1056 & 1056 \\
\hline$F X \# 1$ & 774.00 & 774.00 & 774.00 & 774.00 & 774.00 & 774.00 & 774.00 & 774.00 & 774.00 & 774.00 & 774.00 & 774.00 & 774.00 & 774.00 & 774.00 & 774.00 & 774.00 \\
\hline$F X \# 2$ & 376.37 & 376.73 & 376.88 & 376.94 & 376.65 & 376.61 & 376.85 & 377.17 & 377.22 & 377.23 & 377.13 & 377.31 & 377.58 & 377.83 & 378.11 & 378.06 & 378.14 \\
\hline$F X \# 3$ & 278.88 & 279.05 & 279.36 & 279.60 & 279.81 & 280.08 & 280.27 & 280.43 & 280.57 & 280.64 & 280.71 & 280.84 & 281.02 & 281.22 & 281.34 & 281.41 & 281.46 \\
\hline$F X \# 4$ & 213.74 & 213.84 & 213.88 & 214.10 & 214.27 & 214.38 & 214.47 & 214.50 & 214.58 & 214.69 & 214.78 & 214.85 & 215.02 & 215.21 & 215.37 & 215.53 & 215.65 \\
\hline$F X \# 5$ & 159.10 & 159.15 & 159.15 & 159.11 & 159.12 & 159.09 & 158.96 & 158.96 & 159.18 & 159.44 & 159.76 & 160.03 & 160.23 & 160.46 & 160.58 & 160.71 & 160.70 \\
\hline$F X \# 6$ & 107.92 & 108.03 & 108.20 & 108.39 & 108.55 & 108.82 & 109.02 & 109.15 & 109.21 & 109.13 & 109.07 & 109.04 & 108.98 & 108.95 & 109.01 & 109.23 & 109.41 \\
\hline Near wall FS & 51.18 & 51.12 & 50.95 & 50.73 & 50.57 & 50.27 & 49.93 & 49.81 & 49.97 & 50.31 & 50.68 & 50.99 & 51.25 & 51.51 & 51.56 & 51.48 & 51.28 \\
\hline$T @ F X \# 1$ & 297.21 & 297.21 & 297.21 & 297.21 & 297.21 & 297.21 & 297.21 & 297.21 & 297.21 & 297.21 & 297.21 & 297.21 & 297.21 & 297.21 & 297.21 & 297.21 & 297.21 \\
\hline$T(F X \# 2$ & 297.37 & 297.36 & 297.36 & 297.36 & 297.35 & 297.35 & 297.35 & 297.34 & 297.34 & 297.33 & 297.33 & 297.33 & 297.32 & 297.32 & 297.32 & 297.31 & 297.31 \\
\hline$T @ F X \# 3$ & 299.45 & 299.44 & 299.44 & 299.43 & 299.43 & 299.43 & 299.42 & 299.42 & 299.41 & 299.41 & 299.41 & 299.40 & 299.40 & 299.40 & 299.39 & 299.39 & 299.38 \\
\hline$T @ F X \# 4$ & 301.48 & 301.48 & 301.47 & 301.47 & 301.47 & 301.46 & 301.46 & 301.45 & 301.45 & 301.45 & 301.44 & 301.44 & 301.43 & 301.43 & 301.43 & 301.42 & 301.42 \\
\hline$T$ @ $F X \# 5$ & 303.54 & 303.53 & 303.53 & 303.53 & 303.52 & 303.52 & 303.51 & 303.51 & 303.51 & 303.50 & 303.50 & 303.49 & 303.49 & 303.49 & 303.48 & 303.48 & 303.47 \\
\hline$T @ F X \# 6$ & 305.62 & 305.61 & 305.61 & 305.61 & 305.60 & 305.60 & 305.59 & 305.59 & 305.59 & 305.58 & 305.58 & 305.57 & 305.57 & 305.57 & 305.56 & 305.56 & 305.55 \\
\hline Wall $x$-coord & 86.75 & 86.76 & 86.77 & 86.78 & 86.79 & 86.79 & 86.80 & 86.81 & 86.82 & 86.83 & 86.84 & 86.85 & 86.86 & 86.87 & 86.88 & 86.89 & 86.90 \\
\hline Wall T & 308.60 & 308.60 & 308.61 & 308.61 & 308.62 & 308.63 & 308.64 & 308.64 & 308.64 & 308.63 & 308.61 & 308.60 & 308.59 & 308.58 & 308.58 & 308.58 & 308.59 \\
\hline
\end{tabular}

\section{B.14 Tabulated Data for Interferogram 470}

\begin{tabular}{|c|c|c|c|c|c|c|c|c|c|c|c|c|c|c|c|c|c|}
\hline Interferogram Image \# & 470 & Location & $T C 2$ & $\delta \varepsilon$ slope & $-2.39 E-3$ & $\delta \varepsilon$ intercept & 13.03 & $\operatorname{Avg} F S$ & 38.12 & Avg Wall $T$ & 308.88 & Gradient error & $2.87 E-4$ & & & & \\
\hline Scan y-coord & 5244 & 5245 & 5246 & 5247 & 5248 & 5249 & 5250 & 5251 & 5252 & 5253 & 5254 & 5255 & 5256 & 5257 & 5258 & 5259 & 5260 \\
\hline & 0.4684 & 0.4660 & 0.4636 & 0.4613 & 0.4589 & 0.4565 & 0.4541 & 0.4517 & 0.4493 & 0.4469 & 0.4445 & 0.4421 & 0.4397 & 0.4373 & 0.4349 & 0.4325 & 0.4301 \\
\hline Scan $x$-coord & 1116 & 1116 & 1116 & 1116 & 1116 & 1116 & 1116 & 1116 & 1116 & 1116 & 1116 & 1116 & 1116 & 1116 & 1116 & 1116 & 1116 \\
\hline$F X \# 1$ & 421.49 & 421.76 & 422.13 & 422.42 & 422.68 & 423.06 & 424.24 & 423.25 & 423.59 & 424.75 & 424.94 & 425.37 & 425.39 & 426.17 & 426.91 & 427.32 & 428.47 \\
\hline$F X \# 2$ & 314.71 & 314.86 & 315.05 & 315.22 & 315.38 & 315.55 & 315.75 & 315.83 & 315.94 & 316.08 & 316.03 & 316.08 & 316.04 & 316.13 & 316.26 & 316.51 & 316.87 \\
\hline$F X \# 3$ & 258.49 & 258.59 & 258.58 & 258.62 & 258.70 & 258.80 & 258.99 & 259.15 & 259.31 & 259.42 & 259.49 & 259.56 & 259.60 & 259.68 & 259.80 & 259.93 & 260.04 \\
\hline$F X \# 5$ & 178.83 & 178.90 & 178.99 & 179.12 & 179.26 & 179.34 & 179.43 & 179.43 & 179.41 & 179.39 & 179.42 & 179.49 & 179.59 & 179.73 & 179.88 & 179.96 & 180.01 \\
\hline Near wall FS & 38.21 & 38.18 & 38.20 & 38.12 & 38.09 & 38.10 & 38.09 & 38.18 & 38.30 & 38.31 & 38.26 & 38.19 & 38.10 & 38.00 & 37.89 & 37.86 & 37.88 \\
\hline$T @ F X \# 1$ & 297.65 & 297.65 & 297.65 & 297.65 & 297.65 & 297.65 & 297.65 & 297.65 & 297.65 & 297.65 & 297.65 & 297.65 & 297.65 & 297.65 & 297.65 & 297.65 & 297.65 \\
\hline$T$ a $F X \# 2$ & 298.78 & 298.77 & 298.77 & 298.76 & 298.76 & 298.75 & 298.75 & 298.74 & 298.74 & 298.73 & 298.73 & 298.72 & 298.72 & 298.71 & 298.71 & 298.70 & 298.70 \\
\hline$T @ F X \# 3$ & 300.81 & 300.81 & 300.80 & 300.80 & 300.79 & 300.79 & 300.78 & 300.78 & 300.77 & 300.77 & 300.76 & 300.76 & 300.75 & 300.75 & 300.74 & 300.74 & 300.73 \\
\hline$T$ @ $F X \# 4$ & 302.86 & 302.86 & 302.85 & 302.85 & 302.84 & 302.84 & 302.83 & 302.83 & 302.82 & 302.82 & 302.81 & 302.81 & 302.80 & 302.80 & 302.79 & 302.79 & 302.78 \\
\hline Wall $x$-coord & 145.17 & 145.16 & 145.16 & 145.16 & 145.16 & 145.15 & 145.15 & 145.15 & 145.15 & 145.14 & 145.14 & 145.14 & 145.14 & 145.13 & 145.13 & 145.13 & 145.13 \\
\hline Wall T & 308.88 & 308.88 & 308.88 & 308.89 & 308.89 & 308.89 & 308.89 & 308.88 & 308.87 & 308.86 & 308.86 & 308.86 & 308.87 & 308.88 & 308.88 & 308.88 & 308.88 \\
\hline Interferogram Image \# & 470 & Location & $T C 3$ & $\delta \varepsilon$ slope & $-2.38 E-3$ & $\delta \varepsilon$ intercept & 10.99 & $\operatorname{Avg} F S$ & 46.99 & Avg Wall $T$ & 308.39 & Gradient error & $2.87 E-4$ & & & & \\
\hline Scan y-coord & 4429 & 4430 & 4431 & 4432 & 4433 & 4434 & 4435 & 4436 & 4437 & 4438 & 4439 & 4440 & 4441 & 4442 & 4443 & 4444 & 4445 \\
\hline & 0.4311 & 0.4287 & 0.4263 & 0.4239 & 0.4215 & 0.4191 & 0.4168 & 0.4144 & 0.4120 & 0.4096 & 0.4072 & 0.4048 & 0.4025 & 0.4001 & 0.3977 & 0.3953 & 0.3929 \\
\hline Scan $x$-coord & 1140 & 1140 & 1140 & 1140 & 1140 & 1140 & 1140 & 1140 & 1140 & 1140 & 1140 & 1140 & 1140 & 1140 & 1140 & 1140 & 1140 \\
\hline$F X \# 1$ & 449.39 & 450.12 & 450.71 & 451.23 & 451.83 & 452.72 & 453.08 & 453.29 & 453.59 & 453.79 & 454.07 & 454.49 & 455.20 & 455.90 & 456.45 & 457.02 & 457.78 \\
\hline$F X \# 2$ & 338.92 & 338.86 & 338.71 & 338.57 & 338.59 & 338.67 & 338.82 & 339.01 & 339.32 & 339.58 & 339.85 & 340.14 & 340.49 & 340.76 & 340.94 & 341.14 & 341.29 \\
\hline$F X \# 3$ & 275.50 & 275.55 & 275.53 & 275.54 & 275.60 & 275.66 & 275.72 & 275.80 & 275.92 & 276.01 & 276.08 & 276.18 & 276.33 & 276.48 & 276.62 & 276.72 & 276.84 \\
\hline$F X \# 4$ & 225.00 & 225.10 & 225.21 & 225.39 & 225.50 & 225.62 & 225.85 & 225.97 & 226.09 & 226.22 & 226.38 & 226.51 & 226.66 & 226.85 & 226.96 & 227.14 & 227.28 \\
\hline$F X \# 5$ & 178.14 & 178.29 & 178.47 & 178.57 & 178.67 & 178.72 & 178.78 & 178.90 & 178.97 & 179.09 & 179.23 & 179.41 & 179.59 & 179.72 & 179.89 & 180.12 & 180.32 \\
\hline Near wall FS & 46.86 & 46.82 & 46.75 & 46.82 & 46.83 & 46.90 & 47.07 & 47.07 & 47.11 & 47.13 & 47.15 & 47.10 & 47.06 & 47.13 & 47.07 & 47.02 & 46.97 \\
\hline$T @ F X \# 3$ & 300.74 & 300.73 & 300.73 & 300.72 & 300.72 & 300.71 & 300.71 & 300.70 & 300.70 & 300.69 & 300.69 & 300.68 & 300.68 & 300.67 & 300.67 & 300.66 & 300.66 \\
\hline$T @ F X \# 4$ & 302.79 & 302.78 & 302.78 & 302.77 & 302.77 & 302.76 & 302.76 & 302.75 & 302.75 & 302.74 & 302.74 & 302.73 & 302.73 & 302.72 & 302.72 & 302.71 & 302.71 \\
\hline$T$ (a) $F X \# 5$ & 304.86 & 304.85 & 304.85 & 304.84 & 304.84 & 304.83 & 304.83 & 304.82 & 304.82 & 304.81 & 304.81 & 304.80 & 304.80 & 304.79 & 304.79 & 304.78 & 304.78 \\
\hline Wall $x$-coord & 146.21 & 146.21 & 146.21 & 146.21 & 146.21 & 146.21 & 146.21 & 146.21 & 146.21 & 146.21 & 146.21 & 146.21 & 146.21 & 146.21 & 146.21 & 146.21 & 146.21 \\
\hline Wall T & 308.39 & 308.39 & 308.40 & 308.40 & 308.39 & 308.39 & 308.38 & 308.38 & 308.38 & 308.38 & 308.38 & 308.38 & 308.39 & 308.39 & 308.39 & 308.40 & 308.40 \\
\hline Interferogram Image \# & 470 & Location & $T C 4$ & $\delta \varepsilon$ slope & $-2.39 E-3$ & $\delta \varepsilon$ intercept & 8.96 & $\operatorname{Avg} F S$ & 52.77 & Avg Wall T & 307.90 & Gradient error & $2.87 E-4$ & & & & \\
\hline Scan y-coord & 3615 & 3616 & 3617 & 3618 & 3619 & 3620 & 3621 & 3622 & 3623 & 3624 & 3625 & 3626 & 3627 & 3628 & 3629 & 3630 & 3631 \\
\hline & 0.3073 & 0.3049 & 0.3025 & 0.3001 & 0.2977 & 0.2953 & 0.2929 & 0.2905 & 0.2881 & 0.2857 & 0.2834 & 0.2810 & 0.2786 & 0.2762 & 0.2738 & 0.2714 & 0.2690 \\
\hline Scan $x$-coord & 1079 & 1079 & 1079 & 1079 & 1079 & 1079 & 1079 & 1079 & 1079 & 1079 & 1079 & 1079 & 1079 & 1079 & 1079 & 1079 & 1079 \\
\hline$F X \# I$ & 486.77 & 487.31 & 488.07 & 488.22 & 488.48 & 488.90 & 489.34 & 490.02 & 490.66 & 491.18 & 491.94 & 492.70 & 493.90 & 494.28 & 494.63 & 494.94 & 495.28 \\
\hline$F X \# 2$ & 356.69 & 356.87 & 357.19 & 357.43 & 357.54 & 357.86 & 358.10 & 358.28 & 358.45 & 358.66 & 358.90 & 359.14 & 359.46 & 359.70 & 359.99 & 360.20 & 360.34 \\
\hline$F X \# 3$ & 285.87 & 285.91 & 286.12 & 286.33 & 286.47 & 286.71 & 286.92 & 287.05 & 287.11 & 287.15 & 287.25 & 287.23 & 287.26 & 287.31 & 287.38 & 287.48 & 287.63 \\
\hline$F X \# 4$ & 228.99 & 229.02 & 229.10 & 229.15 & 229.29 & 229.34 & 229.42 & 229.55 & 229.70 & 229.87 & 229.97 & 230.08 & 230.11 & 230.22 & 230.26 & 230.42 & 230.40 \\
\hline$F X \# 5$ & 175.63 & 175.77 & 175.95 & 176.12 & 176.30 & 176.41 & 176.55 & 176.67 & 176.80 & 177.00 & 177.25 & 177.45 & 177.61 & 177.78 & 177.97 & 178.17 & 178.34 \\
\hline Near wall FS & 53.36 & 53.24 & 53.15 & 53.03 & 52.99 & 52.94 & 52.87 & 52.87 & 52.89 & 52.87 & 52.72 & 52.63 & 52.49 & 52.44 & 52.30 & 52.25 & 52.06 \\
\hline$T @ F X \# 1$ & 297.65 & 297.65 & 297.65 & 297.65 & 297.65 & 297.65 & 297.65 & 297.65 & 297.65 & 297.65 & 297.65 & 297.65 & 297.65 & 297.65 & 297.65 & 297.65 & 297.65 \\
\hline$T @ F X \# 2$ & 298.43 & 298.42 & 298.42 & 298.41 & 298.41 & 298.40 & 298.40 & 298.39 & 298.39 & 298.38 & 298.38 & 298.37 & 298.37 & 298.36 & 298.36 & 298.35 & 298.35 \\
\hline$T @ F X \# 3$ & 300.47 & 300.46 & 300.46 & 300.45 & 300.45 & 300.44 & 300.44 & 300.43 & 300.43 & 300.42 & 300.42 & 300.41 & 300.41 & 300.40 & 300.40 & 300.39 & 300.39 \\
\hline$T @ F X \# 4$ & 302.51 & 302.51 & 302.50 & 302.50 & 302.49 & 302.49 & 302.48 & 302.48 & 302.47 & 302.47 & 302.46 & 302.46 & 302.45 & 302.45 & 302.44 & 302.44 & 302.43 \\
\hline$T(\boldsymbol{C} F X \# 5$ & 304.58 & 304.58 & 304.57 & 304.57 & 304.56 & 304.56 & 304.55 & 304.55 & 304.54 & 304.54 & 304.53 & 304.53 & 304.52 & 304.52 & 304.51 & 304.51 & 304.50 \\
\hline Wall $x$-coord & 145.16 & 145.16 & 145.17 & 145.17 & 145.17 & 145.17 & 145.18 & 145.18 & 145.18 & 145.18 & 145.19 & 145.19 & 145.19 & 145.19 & 145.20 & 145.20 & 145.20 \\
\hline
\end{tabular}


307.88

307.89

307.89

307.89

307.89

307.90

307.90

307.90

307.90

307.91

307.91

307.92

\begin{tabular}{|c|c|c|c|c|c|c|c|c|c|c|c|c|c|c|c|c|c|}
\hline Interferogram Image \# & 470 & Location & $T C 5$ & $\delta \varepsilon$ slope & $-2.09 E-3$ & $\delta \varepsilon$ intercept & 6.09 & $\operatorname{Avg} F S$ & 56.55 & Avg Wall T & 308.16 & Gradient error & $2.87 E-4$ & & & & \\
\hline Scan $y$-coord & 2801 & 2802 & 2803 & 2804 & 2805 & 2806 & 2807 & 2808 & 2809 & 2810 & 2811 & 2812 & 2813 & 2814 & 2815 & 2816 & 2817 \\
\hline & 0.2218 & 0.2197 & 0.2176 & 0.2155 & 0.2134 & 0.2113 & 0.2092 & 0.2071 & 0.2050 & 0.2029 & 0.2008 & 0.1987 & 0.1966 & 0.1946 & 0.1925 & 0.1904 & 0.1883 \\
\hline Scan $x$-coord & 1147 & 1147 & 1147 & 1147 & 1147 & 1147 & 1147 & 1147 & 1147 & 1147 & 1147 & 1147 & 1147 & 1147 & 1147 & 1147 & 1147 \\
\hline$F X \# 1$ & 568.79 & 569.51 & 570.34 & 574.32 & 575.97 & 576.28 & 577.28 & 578.16 & 579.33 & 580.14 & 581.26 & 581.53 & 582.63 & 584.28 & 587.31 & 588.49 & 590.03 \\
\hline$F X \# 2$ & 387.99 & 388.29 & 388.53 & 388.85 & 388.84 & 388.79 & 388.76 & 388.87 & 389.11 & 389.57 & 389.88 & 390.24 & 390.42 & 390.62 & 390.79 & 391.04 & 391.31 \\
\hline$F X \# 3$ & 305.46 & 305.82 & 306.20 & $\begin{array}{l}300.03 \\
30.48\end{array}$ & 306.60 & 306.54 & 306.33 & 306.10 & 305.99 & 306.04 & $\begin{array}{l}307.00 \\
306.25\end{array}$ & 306.65 & 307.04 & 307.34 & 307.50 & 307.43 & 307.31 \\
\hline$F X \# 5$ & 186.18 & 186.46 & 186.66 & 186.81 & 186.97 & 187.13 & 187.20 & 187.24 & 187.36 & 187.51 & 187.63 & 187.61 & 187.59 & 187.48 & 187.39 & 187.31 & 187.34 \\
\hline Near wall FS & 56.72 & 56.50 & 56.36 & 56.23 & 56.07 & 56.05 & 56.25 & 56.58 & 56.72 & 56.70 & 56.49 & 56.40 & 56.45 & 56.61 & 56.87 & 57.09 & 57.32 \\
\hline$T(F X \# 1$ & 297.65 & 297.65 & 297.65 & 297.65 & 297.65 & 297.65 & 297.65 & 297.65 & 297.65 & 297.65 & 297.65 & 297.65 & 297.65 & 297.65 & 297.65 & 297.65 & 297.65 \\
\hline$T @ F X \# 2$ & 298.25 & 298.25 & 298.25 & 298.24 & 298.24 & 298.23 & 298.23 & 298.22 & 298.22 & 298.21 & 298.21 & 298.21 & 298.20 & 298.20 & 298.19 & 298.19 & 298.18 \\
\hline$T @ F X \# 3$ & 300.30 & 300.30 & 300.30 & 300.29 & 300.29 & 300.28 & 300.28 & 300.27 & 300.27 & 300.27 & 300.26 & 300.26 & 300.25 & 300.25 & 300.24 & 300.24 & 300.24 \\
\hline$T(F X \# 4$ & 302.35 & 302.35 & 302.34 & 302.34 & 302.33 & 302.33 & 302.33 & 302.32 & 302.32 & 302.31 & 302.31 & 302.30 & 302.30 & 302.30 & 302.29 & 302.29 & 302.28 \\
\hline Wall $x$-coord & 142.02 & 142.02 & 142.03 & 142.03 & 142.04 & 142.04 & 142.05 & 142.05 & 142.06 & 142.06 & 142.07 & 142.07 & 142.08 & 142.08 & 142.09 & 142.09 & 142.10 \\
\hline Wall T & 308.15 & 308.17 & 308.17 & 308.18 & 308.18 & 308.18 & 308.18 & 308.16 & 308.16 & 308.16 & 308.17 & 308.16 & 308.16 & 308.14 & 308.13 & 308.11 & 308.10 \\
\hline
\end{tabular}

\begin{tabular}{|c|c|c|c|c|c|c|c|c|c|c|c|c|c|c|c|c|c|}
\hline Interferogram Image \# & 470 & Location & TC6 & $\delta \varepsilon$ slope & $-2.18 E-3$ & $\delta \varepsilon$ intercept & 5.25 & $\operatorname{Avg} F S$ & 53.93 & Avg Wall $T$ & 308.59 & Gradient error & $2.87 E-4$ & & & & \\
\hline Scan y-coord & 1987 & 1988 & 1989 & 1990 & 1991 & 1992 & 1993 & 1994 & 1995 & 1996 & 1997 & 1998 & 1999 & 2000 & 2001 & 2002 & 2003 \\
\hline & 0.9157 & 0.9135 & 0.9113 & 0.9091 & 0.9069 & 0.9047 & 0.9026 & 0.9004 & 0.8982 & 0.8960 & 0.8938 & 0.8917 & 0.8895 & 0.8873 & 0.8851 & 0.8829 & 0.8807 \\
\hline Scan $x$-coord & 1258 & 1258 & 1258 & 1258 & 1258 & 1258 & 1258 & 1258 & 1258 & 1258 & 1258 & 1258 & 1258 & 1258 & 1258 & 1258 & 1258 \\
\hline$F X \# 1$ & 427.46 & 427.67 & 427.84 & 427.99 & 428.04 & 428.03 & 428.23 & 428.48 & 428.72 & 429.24 & 429.43 & 429.54 & 429.82 & 430.19 & 430.67 & 430.96 & 431.23 \\
\hline$F X \# 2$ & 331.43 & 331.62 & 331.79 & 331.88 & 331.94 & 331.97 & 332.16 & 332.33 & 332.50 & 332.71 & 332.99 & 333.31 & 333.61 & 333.85 & 334.05 & 334.13 & 334.21 \\
\hline$F X \# 3$ & 263.98 & 264.01 & 264.09 & 264.19 & 264.31 & 264.48 & 264.67 & 264.87 & 264.93 & 265.02 & 265.13 & 265.28 & 265.45 & 265.58 & 265.62 & 265.69 & 265.79 \\
\hline$F X \# 5$ & 150.55 & 150.72 & 151.19 & 151.63 & 152.01 & 152.29 & 152.37 & 152.34 & 152.26 & 152.18 & 152.21 & 152.41 & 152.71 & 152.92 & 153.11 & 153.22 & 153.31 \\
\hline Near wall FS & 55.66 & 55.41 & 54.82 & 54.30 & 53.87 & 53.64 & 53.57 & 53.81 & 54.03 & 54.09 & 54.11 & 54.01 & 53.63 & 53.31 & 53.00 & 52.84 & 52.80 \\
\hline$T @ F X \# 1$ & 297.65 & 297.65 & 297.65 & 297.65 & 297.65 & 297.65 & 297.65 & 297.65 & 297.65 & 297.65 & 297.65 & 297.65 & 297.65 & 297.65 & 297.65 & 297.65 & 297.65 \\
\hline$T @ F X \# 2$ & 299.71 & 299.70 & 299.70 & 299.70 & 299.69 & 299.69 & 299.68 & 299.68 & 299.67 & 299.67 & 299.66 & 299.66 & 299.66 & 299.65 & 299.65 & 299.64 & 299.64 \\
\hline$T @ F X \# 3$ & 301.75 & 301.75 & 301.74 & 301.74 & 301.73 & 301.73 & 301.73 & 301.72 & 301.72 & 301.71 & 301.71 & 301.70 & 301.70 & 301.69 & 301.69 & 301.69 & 301.68 \\
\hline$T @ F X \# 4$ & 303.82 & 303.81 & 303.81 & 303.80 & 303.80 & 303.79 & 303.79 & 303.79 & 303.78 & 303.78 & 303.77 & 303.77 & 303.76 & 303.76 & 303.75 & 303.75 & 303.74 \\
\hline Wall $x$-coord & 136.78 & 136.79 & 136.80 & 136.80 & 136.81 & 136.82 & 136.83 & 136.83 & 136.84 & 136.85 & 136.86 & 136.86 & 136.87 & 136.88 & 136.89 & 136.90 & 136.90 \\
\hline Wall T & 308.55 & 308.55 & 308.57 & 308.59 & 308.60 & 308.61 & 308.61 & 308.60 & 308.59 & 308.58 & 308.58 & 308.58 & 308.59 & 308.60 & 308.61 & 308.61 & 308.61 \\
\hline
\end{tabular}

\begin{tabular}{|c|c|c|c|c|c|c|c|c|c|c|c|c|c|c|c|c|c|}
\hline Interferogram Image \# & 470 & Location & $T C 7$ & $\delta \varepsilon$ slope & $-2.14 E-3$ & $\delta \varepsilon$ intercept & 3.09 & $\operatorname{Avg} F S$ & 56.66 & Avg Wall $T$ & 308.78 & Gradient error & $2.87 E-4$ & & & & \\
\hline Scan $y$-coord & 1172 & 1173 & 1174 & 1175 & 1176 & 1177 & 1178 & 1179 & 1180 & 1181 & 1182 & 1183 & 1184 & 1185 & 1186 & 1187 & 1188 \\
\hline & 0.5761 & 0.5740 & 0.5718 & 0.5697 & 0.5675 & 0.5654 & 0.5632 & 0.5611 & 0.5589 & 0.5568 & 0.5547 & 0.5525 & 0.5504 & 0.5482 & 0.5461 & 0.5439 & 0.5418 \\
\hline Scan $x$-coord & 1341 & 1341 & 1341 & 1341 & 1341 & 1341 & 1341 & 1341 & 1341 & 1341 & 1341 & 1341 & 1341 & 1341 & 1341 & 1341 & 1341 \\
\hline$F X \# 1$ & 508.90 & 509.16 & 509.38 & 509.66 & 510.22 & 511.03 & 511.78 & 512.30 & 512.70 & 513.80 & 514.12 & 514.41 & 515.01 & 515.42 & 515.69 & 515.18 & 516.24 \\
\hline$F X \# 2$ & 366.34 & 366.61 & 367.01 & 367.51 & 367.83 & 368.05 & 368.38 & 368.77 & 369.11 & 369.16 & 369.30 & 369.46 & 369.52 & 369.74 & 369.86 & 370.15 & 370.40 \\
\hline$F X \# 3$ & 287.53 & 287.71 & 287.73 & 287.79 & 287.98 & 288.22 & 288.33 & 288.44 & 288.62 & 288.79 & 288.83 & 288.81 & 288.78 & 288.85 & 289.03 & 289.24 & 289.42 \\
\hline$F X \# 4$ & 225.27 & 225.41 & 225.60 & 225.75 & 225.83 & 225.81 & 225.79 & 225.98 & 226.08 & 226.12 & 226.14 & 226.18 & 226.29 & 226.47 & 226.63 & 226.86 & 227.12 \\
\hline$F X \# 5$ & 168.84 & 168.77 & 168.77 & 168.88 & 169.02 & 169.17 & 169.42 & 169.62 & 169.81 & 169.86 & 169.86 & 169.85 & 169.79 & 169.62 & 169.53 & 169.59 & 169.73 \\
\hline Near wall FS & 56.44 & 56.64 & 56.83 & 56.87 & 56.80 & 56.64 & 56.37 & 56.36 & 56.27 & 56.26 & 56.28 & 56.32 & 56.50 & 56.84 & 57.10 & 57.27 & 57.38 \\
\hline$T @ F X \# 1$ & 297.65 & 297.65 & 297.65 & 297.65 & 297.65 & 297.65 & 297.65 & 297.65 & 297.65 & 297.65 & 297.65 & 297.65 & 297.65 & 297.65 & 297.65 & 297.65 & 297.65 \\
\hline$T(a) F X \# 2$ & 299.03 & 299.03 & 299.02 & 299.02 & 299.01 & 299.01 & 299.00 & 299.00 & 299.00 & 298.99 & 298.99 & 298.98 & 298.98 & 298.97 & 298.97 & 298.97 & 298.96 \\
\hline$T(a) F X \# 3$ & 301.08 & 301.08 & 301.07 & 301.07 & 301.06 & 301.06 & 301.05 & 301.05 & 301.04 & 301.04 & 301.04 & 301.03 & 301.03 & 301.02 & 301.02 & 301.01 & 301.01 \\
\hline$T$ (a) $F X \# 4$ & 303.14 & 303.13 & 303.13 & 303.12 & 303.12 & 303.12 & 303.11 & 303.11 & 303.10 & 303.10 & 303.09 & 303.09 & 303.08 & 303.08 & 303.08 & 303.07 & 303.07 \\
\hline Wall $x$-coord & 129.44 & 129.45 & 129.46 & 129.47 & 129.48 & 129.49 & 129.50 & 129.51 & 129.52 & 129.53 & 129.54 & 129.55 & 129.56 & 129.57 & 129.58 & 129.59 & 129.60 \\
\hline Wall $T$ & 308.80 & 308.79 & 308.78 & 308.77 & 308.78 & 308.78 & 308.79 & 308.80 & 308.80 & 308.80 & 308.79 & 308.79 & 308.77 & 308.75 & 308.74 & 308.73 & 308.73 \\
\hline
\end{tabular}

\section{B.15 Tabulated Data for Interferogram 471}

\begin{tabular}{|c|c|c|c|c|c|c|c|c|c|c|c|c|c|c|c|c|c|}
\hline gram Image \# & 471 & Location & $T C 2$ & $\delta \varepsilon$ slope & $-2.47 E-3$ & $\delta \varepsilon$ intercept & 13.59 & $\operatorname{Avg} F S$ & 183.51 & Avg Wall $T$ & 308.46 & Gradient error & $2.48 E-4$ & & & & \\
\hline Scan $y$-coord & 5341 & 5342 & 5343 & 5344 & 5345 & 5346 & 5347 & 5348 & 5349 & 5350 & 5351 & 5352 & 5353 & 5354 & 5355 & 5356 & 5357 \\
\hline & 0.4026 & 0.4002 & 0.3977 & 0.3952 & 0.3927 & 0.3903 & 0.3878 & 0.3853 & 0.3829 & 0.3804 & 0.3779 & 0.3755 & 0.3730 & 0.3705 & 0.3681 & 0.3656 & 0.3631 \\
\hline Scan $x$-coord & 1090 & 1090 & 1090 & 1090 & 1090 & 1090 & 1090 & 1090 & 1090 & 1090 & 1090 & 1090 & 1090 & 1090 & 1090 & 1090 & 1090 \\
\hline$F X \# 1$ & 438.17 & 438.71 & 438.59 & 440.35 & 440.95 & 441.71 & 448.61 & 449.29 & 449.84 & 444.51 & 445.05 & 445.72 & 446.44 & 446.74 & 447.16 & 447.78 & 448.84 \\
\hline$F X \# 2$ & 321.08 & 321.06 & 320.91 & 321.10 & 321.17 & 321.25 & 321.33 & 321.47 & 321.64 & 321.84 & 321.98 & 322.08 & 322.18 & 322.24 & 322.38 & 322.50 & 322.69 \\
\hline$F X \# 3$ & 262.06 & 262.21 & 262.25 & 262.28 & 262.31 & 262.37 & 262.50 & 262.67 & 262.84 & 263.01 & 263.17 & 263.26 & 263.34 & 263.41 & 263.54 & 263.66 & 263.76 \\
\hline$F X \# 4$ & 220.20 & 220.28 & 220.39 & 220.50 & 220.61 & 220.77 & 220.98 & 221.13 & 221.23 & 221.24 & 221.30 & 221.25 & 221.24 & 221.29 & 221.35 & 221.38 & 221.50 \\
\hline$F X \# 5$ & 182.92 & 183.03 & 183.15 & 183.26 & 183.35 & 183.41 & 183.49 & 183.55 & 183.60 & 183.64 & 183.60 & 183.61 & 183.64 & 183.73 & 183.82 & 183.93 & 184.04 \\
\hline$F X \# 6$ & 0.00 & 0.00 & 0.00 & 0.00 & 0.00 & 0.00 & 0.00 & 0.00 & 0.00 & 0.00 & 0.00 & 0.00 & 0.00 & 0.00 & 0.00 & 0.00 & 0.00 \\
\hline Near wall FS & 182.92 & 183.03 & 183.15 & 183.26 & 183.35 & 183.41 & 183.49 & 183.55 & 183.60 & 183.64 & 183.60 & 183.61 & 183.64 & 183.73 & 183.82 & 183.93 & 184.04 \\
\hline$T @ F X \# 1$ & 297.23 & 297.23 & 297.23 & 297.23 & 297.23 & 297.23 & 297.23 & 297.23 & 297.23 & 297.23 & 297.23 & 297.23 & 297.23 & 297.23 & 297.23 & 297.23 & 297.23 \\
\hline$T @ F X \# 2$ & 298.19 & 298.18 & 298.18 & 298.17 & 298.17 & 298.16 & 298.16 & 298.15 & 298.15 & 298.14 & 298.14 & 298.13 & 298.13 & 298.12 & 298.12 & 298.11 & 298.11 \\
\hline$T @ F X \# 3$ & 300.21 & 300.21 & 300.20 & 300.20 & 300.19 & 300.19 & 300.18 & 300.18 & 300.17 & 300.17 & 300.16 & 300.16 & 300.15 & 300.15 & 300.14 & 300.14 & 300.13 \\
\hline$T(F X \# 4$ & 302.25 & 302.25 & 302.24 & 302.24 & 302.23 & 302.23 & 302.22 & 302.22 & 302.21 & 302.21 & 302.20 & 302.20 & 302.19 & 302.19 & 302.18 & 302.18 & 302.17 \\
\hline
\end{tabular}




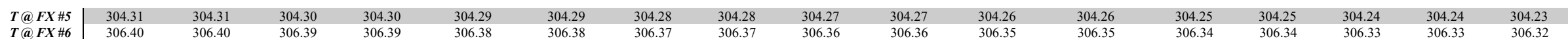

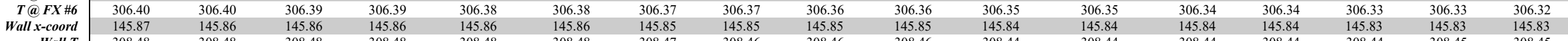

\begin{tabular}{|c|c|c|c|c|c|c|c|c|c|c|c|c|c|c|c|c|c|}
\hline Interferogram Image\# & $4 / 1$ & Location & 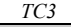 & $\delta$ slope & $2.36 \mathrm{E}-3$ & $\delta \varepsilon$ intercept & 11.05 & & & & & Gradient error & $2.48 \mathrm{E}-4$ & & & & \\
\hline Scan $y$-coord & 4527 & 4528 & 4529 & 4530 & 4531 & 4532 & 4533 & 4534 & 4535 & 4536 & 4537 & $\begin{array}{c}4538 \\
03479\end{array}$ & 4539 & 4540 & 4541 & $\begin{array}{l}4542 \\
03384\end{array}$ & 4543 \\
\hline & $\begin{array}{c}0.3738 \\
936\end{array}$ & $\begin{array}{c}0.3715 \\
936\end{array}$ & $\begin{array}{c}0.3691 \\
936\end{array}$ & $\begin{array}{c}0.3667 \\
936\end{array}$ & $\begin{array}{c}0.3644 \\
936\end{array}$ & $\begin{array}{c}0.3620 \\
936\end{array}$ & $\begin{array}{c}0.3597 \\
936\end{array}$ & $\begin{array}{c}0.3573 \\
936\end{array}$ & $\begin{array}{c}0.3550 \\
936\end{array}$ & $\begin{array}{c}0.3526 \\
936\end{array}$ & $\begin{array}{c}0.3502 \\
936\end{array}$ & $\begin{array}{c}0.3479 \\
936\end{array}$ & $\begin{array}{c}0.3455 \\
936\end{array}$ & $\begin{array}{c}0.3432 \\
936\end{array}$ & $\begin{array}{c}0.3408 \\
936\end{array}$ & $\begin{array}{c}0.3384 \\
936\end{array}$ & $\begin{array}{c}0.3361 \\
936\end{array}$ \\
\hline $\begin{array}{r}\text { Scan } x \text {-coord } \\
F X \# 1\end{array}$ & $\begin{array}{c}936 \\
468.56\end{array}$ & $\begin{array}{c}936 \\
468.91\end{array}$ & $\begin{array}{c}936 \\
469.57\end{array}$ & $\begin{array}{c}936 \\
470.37\end{array}$ & $\begin{array}{c}936 \\
471.26\end{array}$ & $\begin{array}{c}936 \\
471.86\end{array}$ & $\begin{array}{c}936 \\
473.46\end{array}$ & $\begin{array}{c}936 \\
473.87\end{array}$ & $\begin{array}{c}936 \\
474.40\end{array}$ & $\begin{array}{c}936 \\
475.11\end{array}$ & $\begin{array}{c}936 \\
475.33\end{array}$ & $\begin{array}{c}936 \\
475.90\end{array}$ & $\begin{array}{c}936 \\
476.48\end{array}$ & $\begin{array}{c}936 \\
476.40\end{array}$ & $\begin{array}{c}936 \\
473.52\end{array}$ & $\begin{array}{l}936 \\
479.32\end{array}$ & $\begin{array}{l}936 \\
480.13\end{array}$ \\
\hline$F X \# 2$ & 346.65 & 346.53 & 346.52 & 346.41 & 346.42 & 346.47 & 346.65 & 346.78 & 347.00 & 347.35 & 347.76 & 348.02 & 348.29 & 348.50 & 348.65 & 348.98 & 349.06 \\
\hline$F X \# 3$ & 281.33 & 281.41 & 281.45 & 281.49 & 281.55 & 281.61 & 281.63 & 281.66 & 281.72 & 281.76 & 281.86 & 281.93 & 282.02 & 282.14 & 282.21 & 282.25 & 282.33 \\
\hline$F X \# 4$ & 230.74 & 230.89 & 231.02 & 231.14 & 231.21 & 231.30 & 231.43 & 231.49 & 231.58 & 231.76 & 231.93 & 232.13 & 232.40 & 232.55 & 232.79 & 232.93 & 233.06 \\
\hline$F X \# 5$ & 184.82 & 184.94 & 185.04 & 185.17 & 185.26 & 185.34 & 185.37 & 185.42 & 185.49 & 185.63 & 185.83 & 186.02 & 186.13 & 186.23 & 186.35 & 186.51 & 186.70 \\
\hline$F X \# 6$ & 0.00 & 0.00 & 0.00 & 0.00 & 0.00 & 0.00 & 0.00 & 0.00 & 0.00 & 0.00 & 0.00 & 0.00 & 0.00 & 0.00 & 0.00 & 0.00 & 0.00 \\
\hline Near wall FS & 184.82 & 184.94 & 185.04 & 185.17 & 185.26 & 185.34 & 185.37 & 185.42 & 185.49 & 185.63 & 185.83 & 186.02 & 186.13 & 186.23 & 186.35 & 186.51 & 186.70 \\
\hline$T @ F X \# 1$ & 297.23 & 297.23 & 297.23 & 297.23 & 297.23 & 297.23 & 297.23 & 297.23 & 297.23 & 297.23 & 297.23 & 297.23 & 297.23 & 297.23 & 297.23 & 297.23 & 297.23 \\
\hline$T$ (@) $F X \# 2$ & 298.09 & 298.08 & 298.08 & 298.07 & 298.07 & 298.06 & 298.06 & 298.05 & 298.05 & 298.04 & 298.04 & 298.03 & 298.03 & 298.02 & 298.02 & 298.01 & 298.01 \\
\hline$T(F X \# 3$ & 300.11 & 300.11 & 300.10 & 300.10 & 300.09 & 300.09 & 300.08 & 300.08 & 300.07 & 300.07 & 300.06 & 300.06 & 300.05 & 300.05 & 300.04 & 300.04 & 300.04 \\
\hline$T(F X \# 4$ & 302.15 & 302.15 & 302.14 & 302.14 & 302.13 & 302.13 & 302.12 & 302.12 & 302.11 & 302.11 & 302.10 & 302.10 & 302.09 & 302.09 & 302.08 & 302.08 & 302.07 \\
\hline$T(\boldsymbol{T}) \boldsymbol{F X} \# 6$ & 306.30 & 306.30 & 306.29 & 306.29 & 306.28 & 306.28 & 306.27 & 306.27 & 306.26 & 306.26 & 306.25 & 306.25 & 306.24 & 306.24 & 306.23 & 306.23 & 306.22 \\
\hline Wall $x$-coord & 146.79 & 146.79 & 146.80 & 146.80 & 146.80 & 146.80 & 146.80 & 146.80 & 146.80 & 146.80 & 146.80 & 146.80 & 146.80 & 146.80 & 146.80 & 146.80 & 146.80 \\
\hline Wall $T$ & 308.03 & 308.03 & 308.03 & 308.03 & 308.03 & 308.03 & 308.02 & 308.02 & 308.02 & 308.02 & 308.02 & 308.02 & 308.02 & 308.02 & 308.01 & 308.01 & 308.02 \\
\hline
\end{tabular}

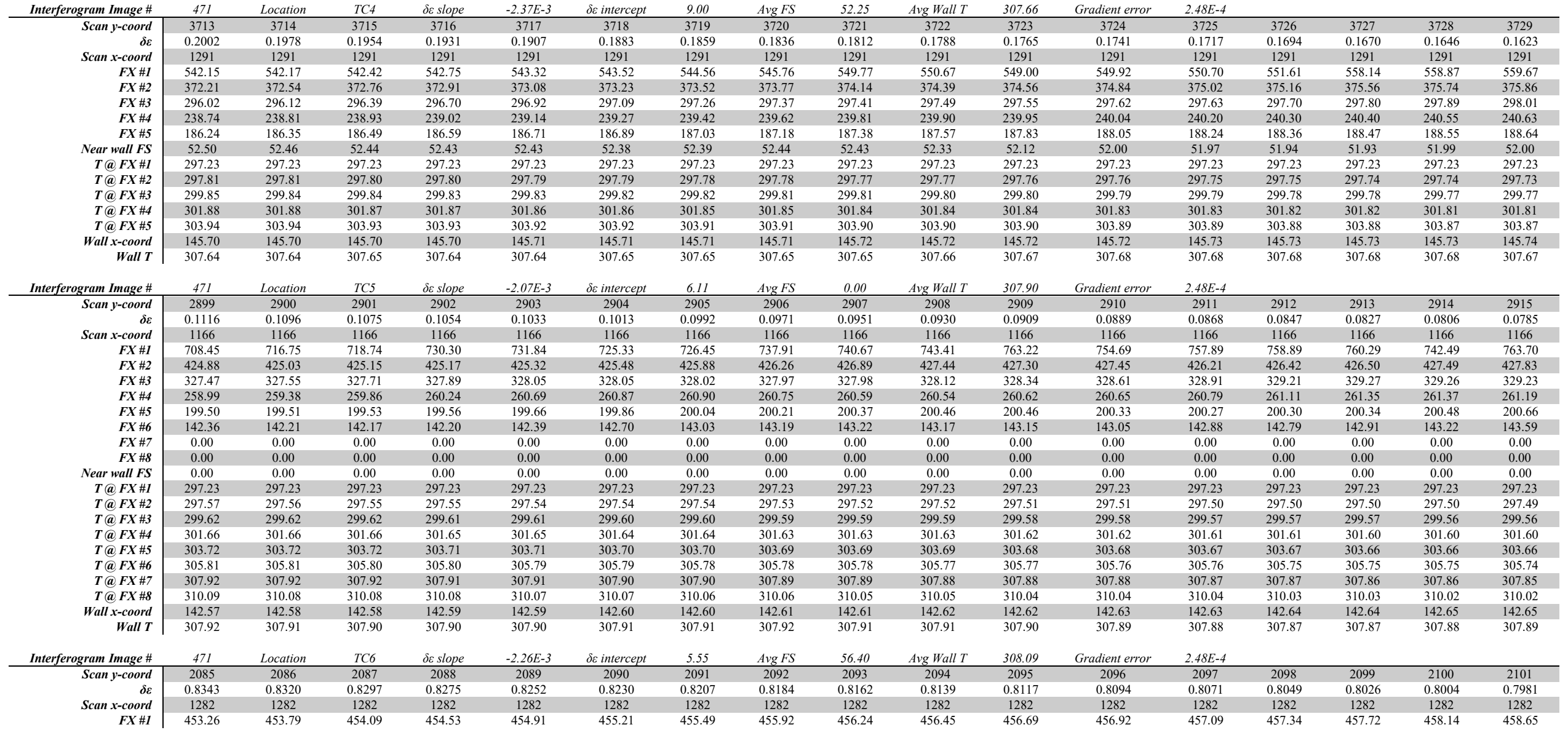




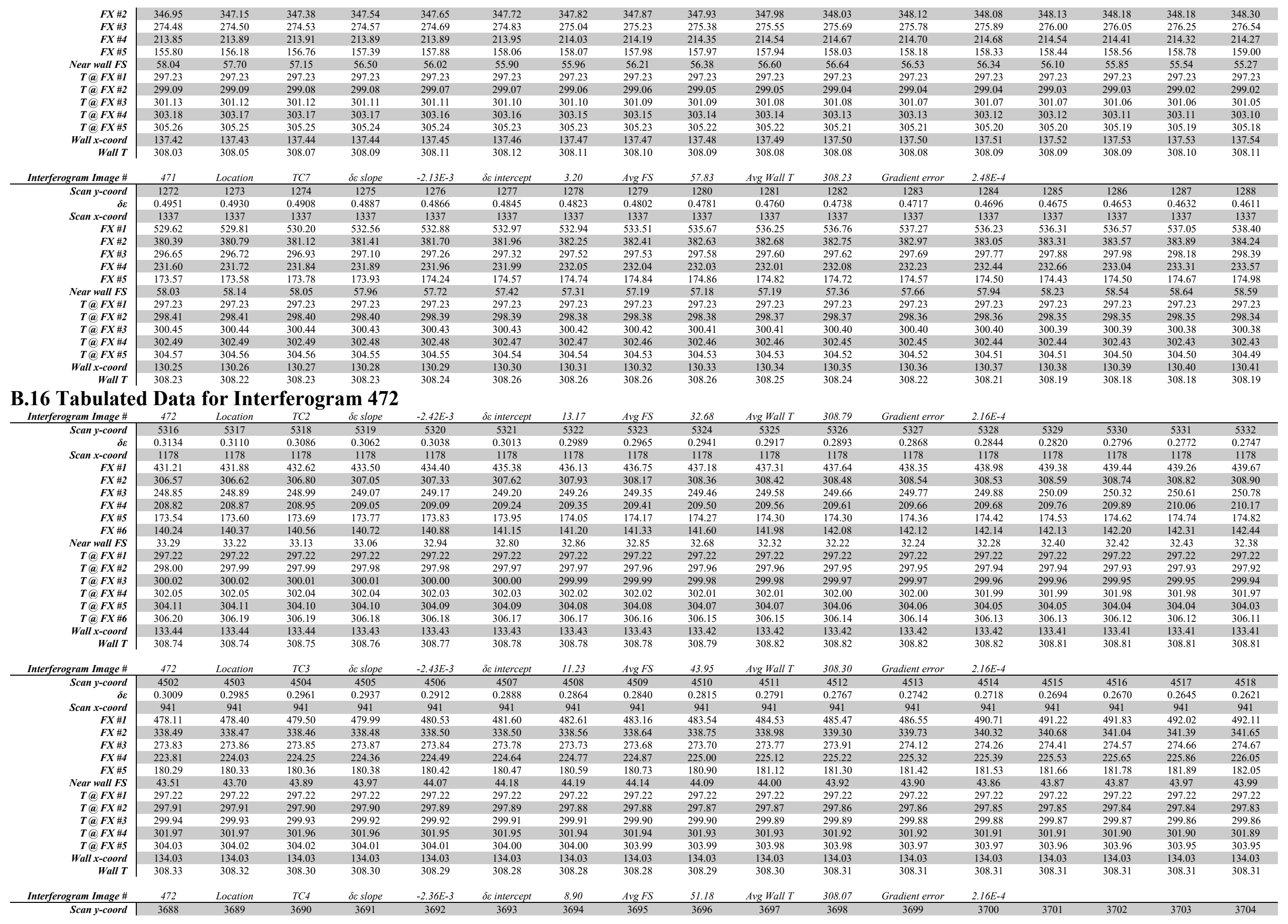




\begin{tabular}{|c|c|c|c|c|c|c|c|c|c|c|c|c|c|c|c|c|c|}
\hline$\delta \varepsilon$ & 0.2070 & 0.2047 & 0.2023 & 0.2000 & 0.1976 & 0.1952 & 0.1929 & 0.1905 & 0.1882 & 0.1858 & 0.1834 & 0.1811 & 0.1787 & 0.1764 & 0.1740 & 0.1717 & 0.1693 \\
\hline Scan $x$-coord & 1307 & 1307 & 1307 & 1307 & 1307 & 1307 & 1307 & 1307 & 1307 & 1307 & 1307 & 1307 & 1307 & 1307 & 1307 & 1307 & 1307 \\
\hline$F X \# I$ & 553.76 & 555.01 & 555.72 & 553.86 & 554.84 & 557.44 & 558.72 & 559.40 & 560.43 & 562.27 & 563.97 & 564.58 & 565.85 & 567.31 & 569.20 & 570.06 & 570.93 \\
\hline$F X \# 2$ & 368.76 & 369.11 & 369.43 & 369.65 & 369.92 & 370.22 & 370.56 & 370.77 & 370.98 & 371.23 & 371.45 & 371.63 & 371.86 & 372.06 & 372.25 & 372.48 & 372.63 \\
\hline$F X \# 3$ & 291.65 & 291.71 & 291.75 & 291.80 & 291.87 & 291.98 & 292.13 & 292.22 & 292.37 & 292.58 & 292.70 & 292.78 & 292.83 & 292.90 & 293.04 & 293.08 & 293.12 \\
\hline$F X \# 4$ & 234.96 & 235.02 & 235.09 & 235.18 & 235.33 & 235.47 & 235.58 & 235.66 & 235.74 & 235.82 & 235.87 & 235.93 & 236.02 & 236.17 & 236.24 & 236.41 & 236.58 \\
\hline$F X \# 5$ & 183.41 & 183.59 & 183.80 & 184.04 & 184.17 & 184.24 & 184.26 & 184.30 & 184.37 & 184.50 & 184.65 & 184.83 & 185.03 & 185.18 & 185.37 & 185.55 & 185.69 \\
\hline Near wall FS & 51.56 & 51.43 & 51.29 & 51.14 & 51.15 & 51.22 & 51.31 & 51.36 & 51.37 & 51.32 & 51.22 & 51.11 & 50.98 & 50.99 & 50.87 & 50.86 & 50.89 \\
\hline$T @ F X \# 1$ & 297.22 & 297.22 & 297.22 & 297.22 & 297.22 & 297.22 & 297.22 & 297.22 & 297.22 & 297.22 & 297.22 & 297.22 & 297.22 & 297.22 & 297.22 & 297.22 & 297.22 \\
\hline$T @ F X \# 2$ & 297.79 & 297.79 & 297.78 & 297.78 & 297.77 & 297.77 & 297.76 & 297.76 & 297.75 & 297.75 & 297.74 & 297.74 & 297.73 & 297.73 & 297.72 & 297.72 & 297.71 \\
\hline$T @ F X \# 3$ & 299.82 & 299.82 & 299.81 & 299.81 & 299.80 & 299.80 & 299.79 & 299.79 & 299.78 & 299.78 & 299.77 & 299.77 & 299.76 & 299.76 & 299.75 & 299.75 & 299.75 \\
\hline$T$ (a) $F X \# 4$ & 301.86 & 301.85 & 301.85 & 301.84 & 301.84 & 301.83 & 301.83 & 301.82 & 301.82 & 301.81 & 301.81 & 301.80 & 301.80 & 301.79 & 301.79 & 301.78 & 301.78 \\
\hline$T @ F X \# 5$ & 303.91 & 303.91 & 303.91 & 303.90 & 303.90 & 303.89 & 303.89 & 303.88 & 303.88 & 303.87 & 303.87 & 303.86 & 303.86 & 303.85 & 303.85 & 303.84 & 303.84 \\
\hline Wall $x$-coord & 132.65 & 132.65 & 132.66 & 132.66 & 132.66 & 132.67 & 132.67 & 132.67 & 132.67 & 132.68 & 132.68 & 132.68 & 132.69 & 132.69 & 132.69 & 132.69 & 132.70 \\
\hline Wall T & 308.05 & 308.06 & 308.07 & 308.08 & 308.08 & 308.07 & 308.07 & 308.06 & 308.06 & 308.06 & 308.07 & 308.07 & 308.08 & 308.08 & 308.09 & 308.09 & 308.09 \\
\hline Interferogram Image \# & 472 & Location & $T C 5$ & $\delta \varepsilon$ slope & $-2.08 E-3$ & $\delta \varepsilon$ intercept & 6.06 & $\operatorname{Avg} F S$ & 56.04 & Avg Wall $T$ & 307.99 & Gradient error & $2.16 E-4$ & & & & \\
\hline Scan y-coord & 2874 & 2875 & 2876 & 2877 & 2878 & 2879 & 2880 & 2881 & 2882 & 2883 & 2884 & 2885 & 2886 & 2887 & 2888 & 2889 & 2890 \\
\hline & 0.0868 & 0.0847 & 0.0826 & 0.0805 & 0.0785 & 0.0764 & 0.0743 & 0.0722 & 0.0702 & 0.0681 & 0.0660 & 0.0639 & 0.0619 & 0.0598 & 0.0577 & 0.0556 & 0.0535 \\
\hline Scan $x$-coord & 1087 & 1087 & 1087 & 1087 & 1087 & 1087 & 1087 & 1087 & 1087 & 1087 & 1087 & 1087 & 1087 & 1087 & 1087 & 1087 & 1087 \\
\hline$F X \# 1$ & 765.00 & 765.00 & 765.00 & 765.00 & 765.00 & 765.00 & 765.00 & 765.00 & 765.00 & 765.00 & 765.00 & 765.00 & 765.00 & 765.00 & 765.00 & 765.00 & 765.00 \\
\hline$F X \# 2$ & 394.81 & 395.08 & 395.36 & 395.47 & 395.45 & 395.39 & 395.33 & 395.37 & 395.63 & 396.01 & 396.55 & 396.94 & 397.22 & 397.55 & 397.88 & 398.00 & 398.13 \\
\hline$F X \# 3$ & 308.68 & 308.84 & 309.01 & 309.23 & 309.44 & 309.68 & 309.81 & 309.86 & 309.81 & 309.74 & 309.73 & 309.81 & 310.11 & 310.53 & 311.02 & 311.34 & 311.54 \\
\hline$F X \# 4$ & 246.41 & 246.44 & 246.46 & 246.53 & 246.63 & 246.72 & 246.85 & 247.06 & 247.23 & 247.30 & 247.36 & 247.42 & 247.43 & 247.43 & 247.41 & 247.60 & 247.76 \\
\hline$F X \# 5$ & 190.27 & 190.42 & 190.52 & 190.65 & 190.87 & 191.05 & 191.19 & 191.27 & 191.30 & 191.37 & 191.46 & 191.55 & 191.61 & 191.64 & 191.67 & 191.63 & 191.55 \\
\hline$F X \# 6$ & 134.13 & 134.40 & 134.81 & 134.93 & 134.88 & 134.53 & 134.65 & 134.76 & 134.76 & 134.96 & 135.59 & 136.06 & 136.17 & 136.01 & 135.76 & 135.54 & 135.45 \\
\hline Near wall FS & 56.14 & 56.02 & 55.71 & 55.73 & 55.99 & 56.52 & 56.54 & 56.50 & 56.53 & 56.40 & 55.87 & 55.48 & 55.44 & 55.64 & 55.92 & 56.09 & 56.10 \\
\hline$T @ F X \# 1$ & 297.22 & 297.22 & 297.22 & 297.22 & 297.22 & 297.22 & 297.22 & 297.22 & 297.22 & 297.22 & 297.22 & 297.22 & 297.22 & 297.22 & 297.22 & 297.22 & 297.22 \\
\hline$T @ F X \# 2$ & 297.46 & 297.46 & 297.45 & 297.45 & 297.44 & 297.44 & 297.44 & 297.43 & 297.43 & 297.42 & 297.42 & 297.41 & 297.41 & 297.41 & 297.40 & 297.40 & 297.39 \\
\hline$T @ F X \# 3$ & 299.53 & 299.52 & 299.52 & 299.51 & 299.51 & 299.51 & 299.50 & 299.50 & 299.49 & 299.49 & 299.48 & 299.48 & 299.48 & 299.47 & 299.47 & 299.46 & 299.46 \\
\hline$T(F X \# 4$ & 301.56 & 301.56 & 301.55 & 301.55 & 301.54 & 301.54 & 301.53 & 301.53 & 301.53 & 301.52 & 301.52 & 301.51 & 301.51 & 301.50 & 301.50 & 301.50 & 301.49 \\
\hline$T @ F X \# 5$ & 303.61 & 303.61 & 303.61 & 303.60 & 303.60 & 303.59 & 303.59 & 303.58 & 303.58 & 303.58 & 303.57 & 303.57 & 303.56 & 303.56 & 303.55 & 303.55 & 303.55 \\
\hline$T @ F X \# 6$ & 305.70 & 305.69 & 305.69 & 305.68 & 305.68 & 305.68 & 305.67 & 305.67 & 305.66 & 305.66 & 305.65 & 305.65 & 305.64 & 305.64 & 305.64 & 305.63 & 305.63 \\
\hline Wall $x$-coord & 129.31 & 129.32 & 129.33 & 129.33 & 129.34 & 129.34 & 129.35 & 129.35 & 129.36 & 129.36 & 129.37 & 129.37 & 129.38 & 129.38 & 129.39 & 129.39 & 129.40 \\
\hline Wall $T$ & 307.99 & 307.99 & 308.01 & 308.01 & 308.00 & 307.98 & 307.98 & 307.98 & 307.97 & 307.98 & 308.00 & 308.01 & 308.01 & 308.00 & 307.99 & 307.97 & 307.96 \\
\hline Interferogram Image \# & 472 & Location & TC6 & $\delta \varepsilon$ slope & $-2.36 E-3$ & $\delta$ s intercept & 5.70 & $\operatorname{Avg} F S$ & 55.95 & Avg Wall $T$ & 308.06 & Gradient error & $2.16 E-4$ & & & & \\
\hline Scan y-coord & 2059 & 2060 & 2061 & 2062 & 2063 & 2064 & 2065 & 2066 & 2067 & 2068 & 2069 & 2070 & 2071 & 2072 & 2073 & 2074 & 2075 \\
\hline & 0.8360 & 0.8336 & 0.8313 & 0.8289 & 0.8266 & 0.8242 & 0.8218 & 0.8195 & 0.8171 & 0.8148 & 0.8124 & 0.8100 & 0.8077 & 0.8053 & 0.8030 & 0.8006 & 0.7982 \\
\hline Scan $x$-coord & 1334 & 1334 & 1334 & 1334 & 1334 & 1334 & 1334 & 1334 & 1334 & 1334 & 1334 & 1334 & 1334 & 1334 & 1334 & 1334 & 1334 \\
\hline$F X \# 1$ & 429.16 & 429.41 & 429.53 & 429.69 & 429.76 & 429.99 & 430.26 & 430.49 & 430.63 & 430.80 & 431.08 & 431.31 & 431.51 & 431.78 & 432.17 & 432.61 & 433.03 \\
\hline 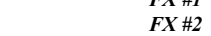 & 327.01 & 327.40 & 327.80 & 328.13 & 328.45 & 328.76 & $\begin{array}{l}450.20 \\
328.94\end{array}$ & 329.12 & $\begin{array}{l}430.03 \\
329.24\end{array}$ & 329.48 & $\begin{array}{l}451.08 \\
329.76\end{array}$ & $\begin{array}{l}\quad 31.01 \\
330.06\end{array}$ & $\begin{array}{l}451.01 \\
330.31\end{array}$ & 330.65 & 330.96 & $\begin{array}{l}452.01 \\
331.23\end{array}$ & 331.34 \\
\hline$F X \# 3$ & 259.63 & 259.54 & 259.36 & 259.30 & 259.33 & 259.34 & 259.33 & 259.29 & 259.32 & 259.36 & 259.44 & 259.59 & 259.84 & 259.97 & 260.17 & 260.35 & 260.51 \\
\hline$F X \# 4$ & 200.47 & 200.43 & 200.43 & 200.37 & 200.29 & 200.16 & 200.04 & 199.90 & 200.01 & 200.14 & 200.18 & 200.39 & 200.51 & 200.60 & 200.61 & 200.65 & 200.76 \\
\hline$F X \# 5$ & 142.19 & 142.28 & 142.63 & 143.15 & 143.79 & 144.44 & 144.76 & 144.82 & 144.69 & 144.61 & 144.60 & 144.75 & 145.10 & 145.41 & 145.65 & 145.84 & 146.07 \\
\hline Near wall FS & 58.28 & 58.14 & 57.80 & 57.22 & 56.49 & 55.73 & 55.27 & 55.08 & 55.33 & 55.53 & 55.59 & 55.63 & 55.41 & 55.20 & 54.96 & 54.81 & 54.68 \\
\hline$T @ F X \# 1$ & 297.22 & 297.22 & 297.22 & 297.22 & 297.22 & 297.22 & 297.22 & 297.22 & 297.22 & 297.22 & 297.22 & 297.22 & 297.22 & 297.22 & 297.22 & 297.22 & 297.22 \\
\hline$T @ F X \# 2$ & 299.07 & 299.07 & 299.06 & 299.06 & 299.05 & 299.05 & 299.04 & 299.04 & 299.03 & 299.03 & 299.02 & 299.02 & 299.01 & 299.01 & 299.00 & 299.00 & 299.00 \\
\hline$T @ F X \# 3$ & 301.10 & 301.10 & 301.09 & 301.09 & 301.08 & 301.08 & 301.07 & 301.07 & 301.06 & 301.06 & 301.05 & 301.05 & 301.04 & 301.04 & 301.03 & 301.03 & 301.02 \\
\hline$T @ F X \# 4$ & 303.15 & 303.15 & 303.14 & 303.14 & 303.13 & 303.13 & 303.12 & 303.12 & 303.11 & 303.11 & 303.10 & 303.10 & 303.09 & 303.09 & 303.08 & 303.08 & 303.07 \\
\hline$T(\boldsymbol{F} X \# 5$ & 305.23 & 305.22 & 305.22 & 305.21 & 305.21 & 305.20 & 305.20 & 305.19 & 305.19 & 305.18 & 305.18 & 305.17 & 305.17 & 305.16 & 305.16 & 305.15 & 305.15 \\
\hline Wall $x$-coord & 124.01 & 124.02 & 124.02 & 124.03 & 124.04 & 124.05 & 124.05 & 124.06 & 124.07 & 124.08 & 124.08 & 124.09 & 124.10 & 124.11 & 124.12 & 124.12 & 124.13 \\
\hline Wall $T$ & 307.99 & 307.99 & 308.00 & 308.02 & 308.05 & 308.08 & 308.09 & 308.09 & 308.08 & 308.07 & 308.06 & 308.06 & 308.07 & 308.08 & 308.09 & 308.09 & 308.10 \\
\hline Interferogram Image \# & 472 & Location & $T C 7$ & $\delta \varepsilon$ slope & $-2.18 E-3$ & $\delta \varepsilon$ intercept & 3.23 & $\operatorname{Avg} F S$ & 159.63 & Avg Wall $T$ & 308.19 & Gradient error & $2.16 E-4$ & & & & \\
\hline Scan y-coord & 1245 & 1246 & 1247 & 1248 & 1249 & 1250 & 1251 & 1252 & 1253 & 1254 & 1255 & 1256 & 1257 & 1258 & 1259 & 1260 & 1261 \\
\hline $\begin{array}{l}\delta c a n y \\
\delta \varepsilon\end{array}$ & 0.5222 & 0.5200 & 0.5178 & 0.5156 & 0.5135 & 0.5113 & 0.5091 & 0.5069 & 0.5048 & 0.5026 & 0.5004 & 0.4982 & 0.4961 & 0.4939 & 0.4917 & 0.4895 & 0.4874 \\
\hline Scan $x$-coord & 1317 & 1317 & 1317 & 1317 & 1317 & 1317 & 1317 & 1317 & 1317 & 1317 & 1317 & 1317 & 1317 & 1317 & 1317 & 1317 & 1317 \\
\hline$F X \# 1$ & 514.42 & 514.88 & 515.04 & 514.32 & 514.73 & 515.27 & 515.74 & 516.48 & 517.27 & 517.55 & 517.89 & 518.19 & 518.55 & 518.78 & 519.12 & 519.53 & 519.93 \\
\hline$F X \# 2$ & 359.88 & 359.86 & 360.10 & 360.56 & 360.77 & 361.08 & 361.45 & 361.97 & 362.49 & 362.86 & 363.05 & 363.31 & 363.59 & 363.90 & 364.09 & 364.24 & 364.39 \\
\hline$F X \# 3$ & 280.01 & 280.17 & 280.35 & 280.40 & 280.48 & 280.62 & 280.80 & 281.00 & 281.10 & 281.07 & 280.95 & 280.98 & 281.12 & 281.29 & 281.58 & 281.79 & 281.93 \\
\hline$F X \# 4$ & 216.48 & 216.67 & 216.88 & 217.02 & 217.11 & 217.13 & 217.16 & 217.16 & 217.08 & 217.07 & 217.13 & 217.23 & 217.39 & 217.59 & 217.89 & 218.16 & 218.42 \\
\hline$F X \# 5$ & 158.34 & 158.29 & 158.37 & 158.59 & 158.87 & 159.12 & 159.49 & 159.92 & 160.22 & 160.44 & 160.49 & 160.47 & 160.38 & 160.29 & 160.19 & 160.10 & 160.14 \\
\hline$F X \# 6$ & $\begin{array}{c}150.00 \\
0.00\end{array}$ & 0.00 & 0.00 & 0.00 & 0.00 & 0.00 & 0.00 & 0.00 & 0.00 & 0.00 & 0.00 & 0.00 & 0.00 & 0.00 & 0.00 & 0.00 & 0.00 \\
\hline Near wall FS & 158.34 & 158.29 & 158.37 & 158.59 & 158.87 & 159.12 & 159.49 & 159.92 & 160.22 & 160.44 & 160.49 & 160.47 & 160.38 & 160.29 & 160.19 & 160.10 & 160.14 \\
\hline$T @ F X \# 1$ & 297.22 & 297.22 & 297.22 & 297.22 & 297.22 & 297.22 & 297.22 & 297.22 & 297.22 & 297.22 & 297.22 & 297.22 & 297.22 & 297.22 & 297.22 & 297.22 & 297.22 \\
\hline$T @ F X \# 2$ & 298.43 & 298.42 & 298.42 & 298.41 & 298.41 & 298.40 & 298.40 & 298.39 & 298.39 & 298.39 & 298.38 & 298.38 & 298.37 & 298.37 & 298.36 & 298.36 & 298.35 \\
\hline$T @ F X \# 3$ & 300.46 & 300.45 & 300.45 & 300.44 & 300.44 & 300.44 & 300.43 & 300.43 & 300.42 & 300.42 & 300.41 & 300.41 & 300.40 & 300.40 & 300.40 & 300.39 & 300.39 \\
\hline$T(F X \# 4$ & 302.50 & 302.50 & 302.49 & 302.49 & 302.48 & 302.48 & 302.48 & 302.47 & 302.47 & 302.46 & $\begin{array}{l}300.41 \\
302.46\end{array}$ & 302.45 & 302.45 & 302.44 & $\begin{array}{l}300.40 \\
302.44\end{array}$ & 302.44 & 302.43 \\
\hline$T$ (a) $F X \# 5$ & 304.57 & 304.57 & 304.56 & 304.56 & 304.55 & 304.55 & 304.54 & 304.54 & 304.53 & 304.53 & 304.53 & 304.52 & 304.52 & 304.51 & 304.51 & 304.50 & 304.50 \\
\hline$T @ F X \# 6$ & 306.67 & 306.66 & 306.66 & 306.65 & 306.65 & 306.64 & 306.64 & 306.63 & 306.63 & 306.62 & 306.62 & 306.62 & 306.61 & 306.61 & 306.60 & 306.60 & 306.59 \\
\hline Wall $x$-coord & 116.74 & 116.75 & 116.76 & 116.77 & 116.78 & 116.80 & 116.81 & 116.82 & 116.83 & 116.84 & 116.85 & 116.86 & 116.87 & 116.88 & 116.89 & 116.90 & 116.91 \\
\hline
\end{tabular}




\section{B.17 Tabulated Data for Interferogram 473}

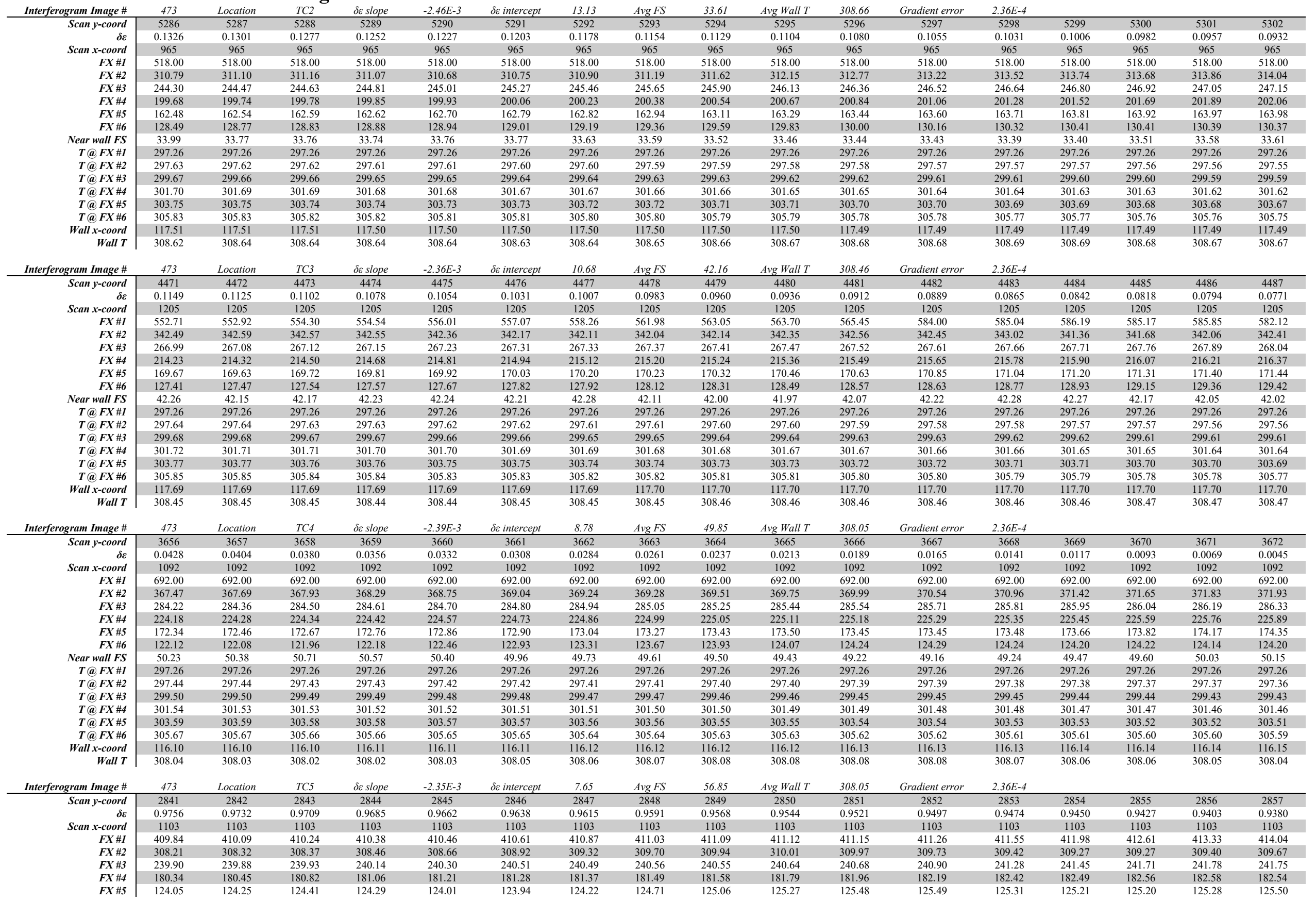




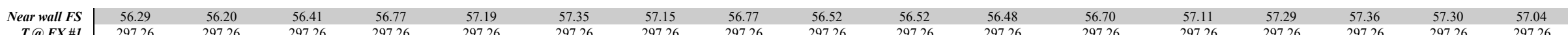

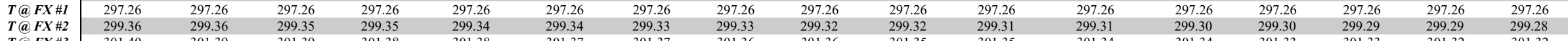

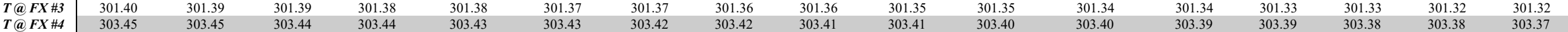

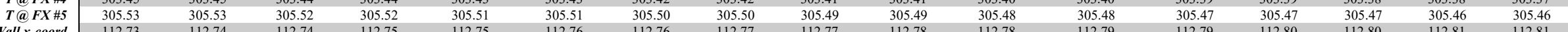

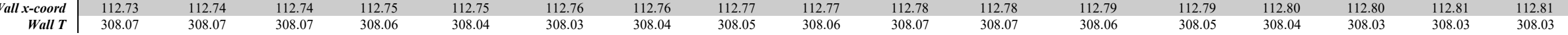

\begin{tabular}{|c|c|c|c|c|c|c|c|c|c|c|c|c|c|c|c|c|c|}
\hline Interferogram Image \# & 473 & Location & TC6 & $\delta \varepsilon$ slope & $-2.32 E-3$ & $\delta \varepsilon$ intercept & 5.40 & $\operatorname{Avg} F S$ & 60.03 & Avg Wall $T$ & 308.01 & Gradient error & $2.36 E-4$ & & & & \\
\hline Scan y-coord & 2026 & 2027 & 2028 & 2029 & 2030 & 2031 & 2032 & 2033 & 2034 & 2035 & 2036 & 2037 & 2038 & 2039 & 2040 & 2041 & 2042 \\
\hline & 0.7093 & 0.7070 & 0.7047 & 0.7024 & 0.7001 & 0.6977 & 0.6954 & 0.6931 & 0.6908 & 0.6885 & 0.6862 & 0.6838 & 0.6815 & 0.6792 & 0.6769 & 0.6746 & 0.6723 \\
\hline Scan $x$-coord & 1282 & 1282 & 1282 & 1282 & 1282 & 1282 & 1282 & 1282 & 1282 & 1282 & 1282 & 1282 & 1282 & 1282 & 1282 & 1282 & 1282 \\
\hline$F X \# 1$ & 449.12 & 449.49 & 449.88 & 450.31 & 450.74 & 451.19 & 451.47 & 451.88 & 452.17 & 452.62 & 453.03 & 453.44 & 453.99 & 454.60 & 455.09 & 455.55 & 455.82 \\
\hline$F X \# 2$ & 333.76 & 333.94 & 334.12 & 334.30 & 334.43 & 334.45 & 334.44 & 334.46 & 334.55 & 334.69 & 334.79 & 334.78 & 334.81 & $\begin{array}{l}434.00 \\
334.91\end{array}$ & 335.01 & $\begin{array}{l}335.14 \\
335.14\end{array}$ & $\begin{array}{l}453.02 \\
33529\end{array}$ \\
\hline$F X \# 3$ & 256.86 & 257.17 & 257.39 & 257.51 & 257.57 & 257.55 & 257.51 & 257.62 & 257.58 & 257.50 & 257.44 & 257.41 & 257.56 & 257.72 & 258.03 & 258.21 & 258.41 \\
\hline$F X \# 5$ & 132.42 & 132.68 & 133.01 & 133.33 & 133.51 & 133.62 & 133.75 & 133.89 & 134.06 & 134.21 & 134.41 & 134.65 & 134.76 & 134.91 & 135.08 & 135.28 & 135.46 \\
\hline Near wall FS & 61.25 & 61.27 & 61.18 & 61.06 & 61.02 & 60.86 & 60.72 & 60.30 & 59.94 & 59.69 & 59.34 & 59.14 & 58.97 & 59.02 & 59.04 & 58.89 & 58.76 \\
\hline$T @ F X \# 1$ & 297.26 & 297.26 & 297.26 & 297.26 & 297.26 & 297.26 & 297.26 & 297.26 & 297.26 & 297.26 & 297.26 & 297.26 & 297.26 & 297.26 & 297.26 & 297.26 & 297.26 \\
\hline$T(F X \# 2$ & 298.86 & 298.86 & 298.85 & 298.85 & 298.84 & 298.84 & 298.83 & 298.83 & 298.83 & 298.82 & 298.82 & 298.81 & 298.81 & 298.80 & 298.80 & 298.79 & 298.79 \\
\hline$T(a) F X \#$ & 300.90 & 300.89 & 300.89 & 300.88 & 300.88 & 300.87 & 300.87 & 300.86 & 300.86 & 300.85 & 300.85 & 300.84 & 300.84 & 300.83 & 300.83 & 300.82 & 300.82 \\
\hline$T(a) \# \#$ & 302.95 & 302.94 & 302.94 & 302.93 & 302.93 & 302.92 & 302.92 & 302.91 & 302.91 & 302.90 & 302.90 & 302.89 & 302.89 & 302.88 & 302.88 & 302.88 & 302.87 \\
\hline Wall $x$-coord & 107.59 & 107.60 & 107.61 & 107.61 & 107.62 & 107.63 & 107.63 & 107.64 & 107.65 & 107.66 & 107.66 & 107.67 & 107.68 & $\begin{array}{l}107.69 \\
107\end{array}$ & 107.69 & 107.70 & 107.71 \\
\hline Wall T & 307.98 & 307.98 & 307.99 & 308.00 & 308.00 & 308.00 & 308.00 & 308.01 & 308.01 & 308.02 & 308.02 & 308.03 & 308.03 & 308.03 & 308.03 & 308.04 & 308.04 \\
\hline
\end{tabular}

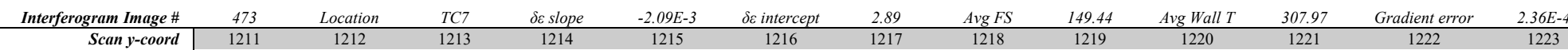

\begin{tabular}{|c|c|c|c|c|c|c|c|c|c|c|c|c|c|c|c|c|c|}
\hline & & & & & & & & & & & & & & & & & \\
\hline Scan y-coord & 1211 & 1212 & 1213 & 1214 & 1215 & 1216 & 1217 & 1218 & 1219 & 1220 & 1221 & 1222 & 1223 & 1224 & 1225 & 1226 & 1227 \\
\hline$\delta \varepsilon$ & 0.3606 & 0.3585 & 0.3564 & 0.3543 & 0.3522 & 0.3502 & 0.3481 & 0.3460 & 0.3439 & 0.3418 & 0.3397 & 0.3376 & 0.3355 & 0.3334 & 0.3314 & 0.3293 & 0.3272 \\
\hline Scan $x$-coord & 1281 & 1281 & 1281 & 1281 & 1281 & 1281 & 1281 & 1281 & 1281 & 1281 & 1281 & 1281 & 1281 & 1281 & 1281 & 1281 & 1281 \\
\hline$F X \# 1$ & 576.80 & 576.26 & 575.98 & 572.97 & 573.60 & 578.37 & 579.29 & 575.37 & 585.33 & 586.12 & 586.95 & 587.04 & 586.63 & 586.93 & 587.97 & 589.42 & 588.96 \\
\hline $\begin{array}{l}F X+1 \\
F X \# 2\end{array}$ & 376.41 & 376.35 & 376.43 & 376.55 & 376.86 & 377.10 & 377.36 & 377.55 & 378.16 & $\begin{array}{l}500.12 \\
378.63\end{array}$ & $\begin{array}{l}379.07 \\
37.07\end{array}$ & 379.37 & $\begin{array}{l}300.05 \\
379.61\end{array}$ & 379.84 & 380.02 & 380.21 & 380.47 \\
\hline$F X \# 3$ & 281.92 & 282.16 & 282.39 & 282.52 & 282.64 & 282.77 & 282.92 & 283.05 & 283.23 & 283.48 & 283.76 & 284.04 & 284.27 & 284.41 & 284.47 & 284.59 & 284.72 \\
\hline$F X \# 4$ & 211.84 & 211.79 & 211.81 & 211.83 & 211.93 & 212.19 & 212.44 & 212.67 & 212.87 & 212.99 & 213.02 & 213.01 & 212.97 & 212.90 & 212.81 & 212.79 & 212.87 \\
\hline$F X \# 5$ & 148.86 & 149.19 & 149.22 & 149.16 & 149.04 & 148.92 & 148.82 & 148.81 & 148.94 & 149.17 & 149.39 & 149.64 & 149.87 & 150.17 & 150.38 & 150.45 & 150.38 \\
\hline$F X \# 6$ & 0.00 & 0.00 & 0.00 & 0.00 & 0.00 & 0.00 & 0.00 & 0.00 & 0.00 & 0.00 & 0.00 & 0.00 & 0.00 & 0.00 & 0.00 & 0.00 & 0.00 \\
\hline Near wall FS & 148.86 & $\begin{array}{l}0.00 \\
149.19\end{array}$ & 149.22 & 149.16 & $\begin{array}{l}0.00 \\
149.04\end{array}$ & 148.92 & 148.82 & 148.81 & 148.94 & 0.00 & 149.39 & $\begin{array}{l}0.00 \\
149.64\end{array}$ & 149.87 & $\begin{array}{l}0.00 \\
150.17\end{array}$ & 150.38 & 150.45 & 150.38 \\
\hline$T @ F X \# 1$ & 297.26 & 297.26 & 297.26 & 297.26 & 297.26 & 297.26 & 297.26 & 297.26 & 297.26 & 297.26 & 297.26 & 297.26 & 297.26 & 297.26 & 297.26 & 297.26 & 297.26 \\
\hline$T @ F X \# 2$ & 298.14 & 298.14 & 298.13 & 298.13 & 298.12 & 298.12 & 298.11 & 298.11 & 298.10 & 298.10 & 298.10 & 298.09 & 298.09 & 298.08 & 298.08 & 298.08 & 298.07 \\
\hline$T$ (a) $F X \# 3$ & 300.18 & 300.18 & 300.17 & 300.17 & 300.17 & 300.16 & 300.16 & 300.15 & 300.15 & 300.14 & 300.14 & 300.14 & 300.13 & 300.13 & 300.12 & 300.12 & 300.12 \\
\hline$T(F X \# 4$ & 302.23 & 302.22 & 302.22 & 302.22 & 302.21 & 302.21 & 302.20 & 302.20 & 302.19 & 302.19 & 302.19 & 302.18 & 302.18 & 302.17 & 302.17 & 302.16 & 302.16 \\
\hline$T(a) F X$ & 304.30 & 304.29 & 304.29 & 304.28 & 304.28 & 304.27 & 304.27 & 304.27 & 304.26 & 304.26 & 304.25 & 304.25 & 304.24 & 304.24 & 304.24 & 304.23 & 304.23 \\
\hline$T @ F X \# 6$ & 306.39 & 306.39 & 306.38 & 306.38 & 306.37 & 306.37 & 306.36 & 306.36 & 306.36 & 306.35 & 306.35 & 306.34 & 306.34 & 306.33 & 306.33 & 306.32 & 306.32 \\
\hline Wall $x$-coord & 100.68 & 100.69 & 100.70 & 100.71 & 100.71 & 100.72 & 100.73 & 100.74 & 100.75 & 100.76 & 100.77 & 100.78 & 100.79 & 100.80 & 100.81 & 100.82 & 100.83 \\
\hline Wall T & 307.99 & 308.01 & 308.01 & 308.00 & 307.98 & 307.96 & 307.95 & 307.94 & 307.93 & 307.94 & 307.95 & 307.96 & 307.97 & 307.98 & 307.99 & 307.99 & 307.98 \\
\hline
\end{tabular}

\section{B.18 Tabulated Data for Interferogram 474}

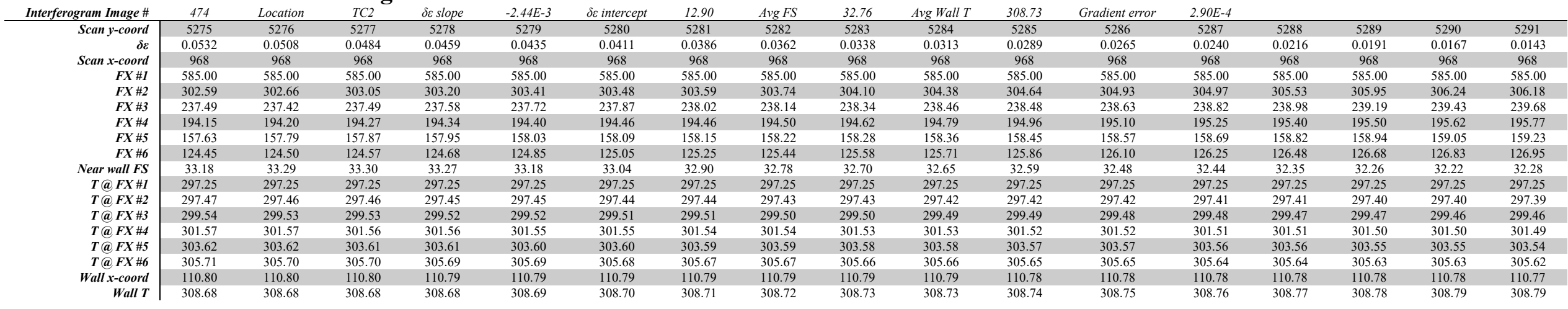

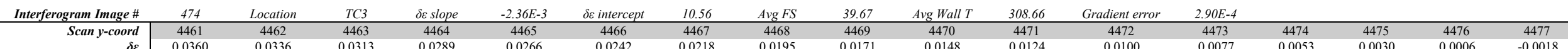

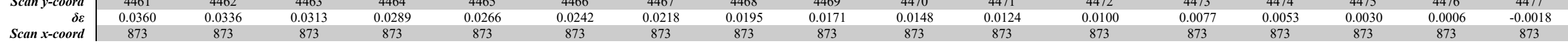




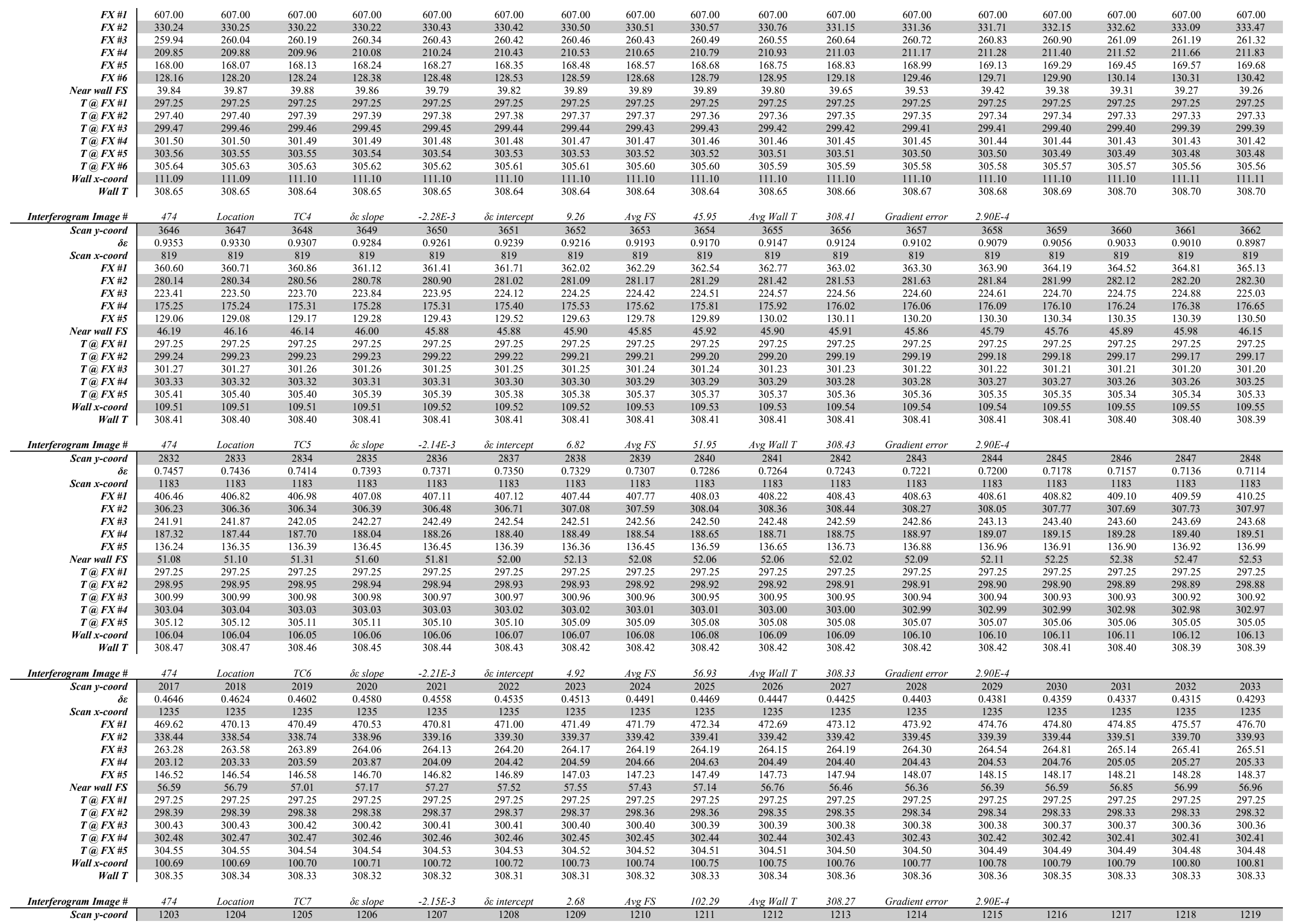




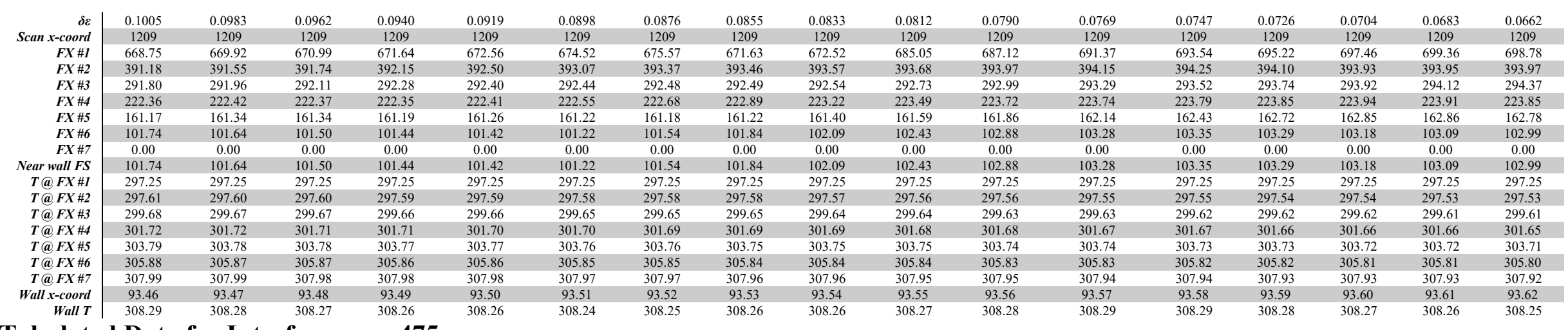

\section{B.19 Tabulated Data for Interferogram 475}

\begin{tabular}{|c|c|c|c|c|c|c|c|c|c|c|c|c|c|c|c|c|c|}
\hline Interferogram Image \# & 475 & Location & $T C 2$ & $\delta \varepsilon$ slope & $-2.45 E-3$ & $\delta \varepsilon$ intercept & 13.16 & $\operatorname{Avg} F S$ & 85.97 & Avg Wall $T$ & 309.12 & Gradient error & $2.03 E-4$ & & & & \\
\hline Scan y-coord & 5257 & 5258 & 5259 & 5260 & 5261 & 5262 & 5263 & 5264 & 5265 & 5266 & 5267 & 5268 & 5269 & 5270 & 5271 & 5272 & 5273 \\
\hline & 0.2819 & 0.2794 & 0.2770 & 0.2745 & 0.2721 & 0.2696 & 0.2672 & 0.2647 & 0.2623 & 0.2598 & 0.2574 & 0.2550 & 0.2525 & 0.2501 & 0.2476 & 0.2452 & 0.2427 \\
\hline Scan $x$-coord & 1229 & 1229 & 1229 & 1229 & 1229 & 1229 & 1229 & 1229 & 1229 & 1229 & 1229 & 1229 & 1229 & 1229 & 1229 & 1229 & 1229 \\
\hline$F X \# 1$ & 418.67 & 419.63 & 420.19 & 422.47 & 421.48 & 422.22 & 423.38 & 424.09 & 424.73 & 426.76 & 426.41 & 426.73 & 427.81 & 443.79 & 444.53 & 445.21 & 445.56 \\
\hline$F X \# 2$ & 276.32 & 276.24 & 276.24 & 276.52 & 276.55 & 276.74 & 276.88 & 277.02 & 277.16 & 277.44 & 277.66 & 277.98 & 278.31 & 278.58 & 278.85 & 279.22 & 279.67 \\
\hline$F X \# 3$ & 215.10 & 215.18 & 215.28 & 215.36 & 215.43 & 215.40 & 215.38 & 215.32 & 215.31 & 215.38 & 215.51 & 215.62 & 215.69 & 215.70 & 215.72 & 215.72 & 215.87 \\
\hline$F X \# 5$ & 136.71 & 136.81 & 136.87 & 136.98 & 137.05 & 137.08 & 137.13 & 137.22 & 137.26 & 137.23 & 137.17 & 137.13 & 137.11 & 137.14 & 137.04 & 137.06 & 137.10 \\
\hline$F X \# 6$ & 104.18 & 104.21 & 104.25 & 104.35 & 104.38 & 104.35 & 104.39 & 104.33 & 104.30 & 104.36 & 104.47 & 104.66 & 104.65 & 104.66 & 0.00 & 0.00 & 0.00 \\
\hline$F X \# 7$ & 0.00 & 0.00 & 0.00 & 0.00 & 0.00 & 0.00 & 0.00 & 0.00 & 0.00 & 0.00 & 0.00 & 0.00 & 0.00 & 0.00 & 0.00 & 0.00 & 0.00 \\
\hline Near wall FS & 104.18 & 104.21 & 104.25 & 104.35 & 104.38 & 104.35 & 104.39 & 104.33 & 104.30 & 104.36 & 104.47 & 104.66 & 104.65 & 104.66 & 0.00 & 0.00 & 0.00 \\
\hline$T @ F X \# 1$ & 297.65 & 297.65 & 297.65 & 297.65 & 297.65 & 297.65 & 297.65 & 297.65 & 297.65 & 297.65 & 297.65 & 297.65 & 297.65 & 297.65 & 297.65 & 297.65 & 297.65 \\
\hline$T$ (a) $F X \# 2$ & 298.37 & 298.37 & 298.36 & 298.36 & 298.35 & 298.35 & 298.34 & 298.34 & 298.33 & 298.33 & 298.32 & 298.32 & 298.31 & 298.30 & 298.30 & 298.29 & 298.29 \\
\hline$T @ F X \# 4$ & 302.44 & 302.43 & 302.43 & 302.42 & 302.42 & 302.41 & 302.41 & 302.40 & 302.40 & 302.39 & 302.39 & 302.38 & 302.38 & 302.37 & 302.37 & 302.36 & 302.36 \\
\hline$T @ F X \# 5$ & 304.50 & 304.50 & 304.49 & 304.49 & 304.48 & 304.48 & 304.47 & 304.47 & 304.46 & 304.46 & 304.45 & 304.45 & 304.44 & 304.44 & 304.43 & 304.43 & 304.42 \\
\hline$T @ F X \# 6$ & 306.59 & 306.59 & 306.58 & 306.58 & $\begin{array}{l}304.48 \\
306.57\end{array}$ & 306.56 & 306.56 & 306.55 & $\begin{array}{l}304.40 \\
306.55\end{array}$ & 306.54 & $\begin{array}{l}304.45 \\
306.54\end{array}$ & 306.53 & 306.53 & $\begin{array}{l}304.44 \\
306.52\end{array}$ & 306.52 & 306.51 & 306.51 \\
\hline$T(\boldsymbol{C} \boldsymbol{F} \# \#$ & 308.71 & 308.70 & 308.70 & 308.69 & 308.69 & 308.68 & 308.68 & 308.67 & 308.67 & 308.66 & 308.66 & 308.65 & 308.65 & 308.64 & 308.66 & 308.65 & 308.65 \\
\hline Wall $x$-coord & 96.58 & 96.58 & 96.58 & 96.58 & 96.58 & 96.58 & 96.57 & 96.57 & 96.57 & 96.57 & 96.57 & 96.57 & 96.56 & 96.56 & 96.56 & 96.56 & 96.56 \\
\hline Wall T & 309.20 & 309.20 & 309.20 & 309.20 & 309.19 & 309.19 & 309.18 & 309.17 & 309.16 & 309.16 & 309.17 & 309.18 & 309.17 & 309.17 & 308.85 & 308.84 & 308.84 \\
\hline Interferogram Image \# & 475 & Location & $T C 3$ & $\delta \varepsilon$ slope & $-2.4 I E-3$ & $\delta \varepsilon$ intercept & 10.96 & $\operatorname{Avg} F S$ & 38.77 & Avg Wall $T$ & 309.24 & Gradient error & $2.03 E-4$ & & & & \\
\hline Scan y-coord & 4443 & 4444 & 4445 & 4446 & 4447 & 4448 & 4449 & 4450 & 4451 & 4452 & 4453 & 4454 & 4455 & 4456 & 4457 & 4458 & 4459 \\
\hline$\delta \varepsilon$ & 0.2666 & 0.2642 & 0.2618 & 0.2594 & 0.2570 & 0.2545 & 0.2521 & 0.2497 & 0.2473 & 0.2449 & 0.2425 & 0.2401 & 0.2377 & 0.2353 & 0.2329 & 0.2305 & 0.2281 \\
\hline Scan $x$-coord & 1327 & 1327 & 1327 & 1327 & 1327 & 1327 & 1327 & 1327 & 1327 & 1327 & 1327 & 1327 & 1327 & 1327 & 1327 & 1327 & 1327 \\
\hline$F X \# I$ & 432.31 & 432.56 & 432.98 & 433.27 & 433.57 & 434.04 & 434.66 & 435.60 & 436.54 & 437.50 & 438.42 & 439.32 & 440.13 & 440.89 & 441.18 & 441.84 & 442.30 \\
\hline$F X \# 2$ & 299.85 & 300.03 & 300.13 & 300.19 & 300.17 & 300.11 & 300.06 & 300.05 & 300.05 & 300.01 & 299.94 & 299.90 & 299.90 & 299.87 & 299.90 & 300.04 & 300.23 \\
\hline $\begin{array}{l}F X \#+4 \\
F X\end{array}$ & 145.98 & 146.01 & 146.00 & 146.01 & 146.07 & 146.10 & 146.15 & 146.18 & 146.21 & 146.13 & $\begin{array}{l}108.50 \\
146.49\end{array}$ & 146.42 & 146.90 & 147.08 & $\begin{array}{l}100.02 \\
147.29\end{array}$ & $\begin{array}{l}100.98 \\
147.52\end{array}$ & 147.72 \\
\hline$F X \# 6$ & 107.12 & 107.23 & 107.26 & 107.20 & 107.20 & 107.27 & 107.37 & 107.48 & 107.58 & 107.74 & 107.91 & 108.10 & 108.29 & 108.40 & 108.46 & 108.47 & 108.53 \\
\hline Near wall FS & 38.86 & 38.78 & 38.73 & 38.81 & 38.88 & 38.83 & 38.78 & 38.70 & 38.63 & 38.60 & 38.58 & 38.55 & 38.61 & 38.68 & 38.83 & 39.05 & 39.19 \\
\hline$T @ F X \# 1$ & 297.65 & 297.65 & 297.65 & 297.65 & 297.65 & 297.65 & 297.65 & 297.65 & 297.65 & 297.65 & 297.65 & 297.65 & 297.65 & 297.65 & 297.65 & 297.65 & 297.65 \\
\hline$T @ F X \# 2$ & 298.36 & 298.35 & 298.35 & 298.34 & 298.34 & 298.33 & 298.33 & 298.33 & 298.32 & 298.32 & 298.31 & 298.31 & 298.30 & 298.30 & 298.29 & 298.29 & 298.28 \\
\hline$T @ F X \# 3$ & 300.38 & 300.38 & 300.37 & 300.37 & 300.37 & 300.36 & 300.36 & 300.35 & 300.35 & 300.34 & 300.34 & 300.33 & 300.33 & 300.32 & 300.32 & 300.31 & 300.31 \\
\hline$T @ F X \# 4$ & 302.42 & 302.42 & 302.41 & 302.41 & 302.40 & 302.40 & 302.39 & 302.39 & 302.38 & 302.38 & 302.37 & 302.37 & 302.36 & 302.36 & 302.36 & 302.35 & 302.35 \\
\hline$T @ F X \# 5$ & 304.49 & 304.48 & 304.48 & 304.47 & 304.47 & 304.46 & 304.46 & 304.45 & 304.45 & 304.44 & 304.44 & 304.43 & 304.43 & 304.42 & 304.42 & 304.41 & 304.41 \\
\hline$T @ F X \# 6$ & 306.58 & 306.57 & 306.57 & 306.56 & 306.56 & 306.55 & 306.55 & 306.54 & 306.54 & 306.53 & 306.53 & 306.52 & 306.52 & 306.51 & 306.51 & 306.50 & 306.50 \\
\hline Wall $x$-coord & 96.99 & 96.99 & 96.99 & 96.99 & 96.99 & 96.99 & 96.99 & 96.99 & 96.99 & 97.00 & 97.00 & 97.00 & 97.00 & 97.00 & 97.00 & 97.00 & 97.00 \\
\hline Wall T & 309.25 & 309.25 & 309.25 & 309.24 & 309.23 & 309.23 & 309.23 & 309.23 & 309.24 & 309.24 & 309.24 & 309.25 & 309.25 & 309.25 & 309.25 & 309.24 & 309.24 \\
\hline Interferogram Image \# & 475 & Location & $T C 4$ & $\delta \varepsilon$ slope & $-2.34 E-3$ & $\delta \varepsilon$ intercept & 8.66 & $\operatorname{Avg} F S$ & 44.97 & Avg Wall $T$ & 308.91 & Gradient error & $2.03 E-4$ & & & & \\
\hline Scan y-coord & 3629 & 3630 & 3631 & 3632 & 3633 & 3634 & 3635 & 3636 & 3637 & 3638 & 3639 & 3640 & 3641 & 3642 & 3643 & 3644 & 3645 \\
\hline$\delta \varepsilon$ & 0.1837 & 0.1814 & 0.1791 & 0.1767 & 0.1744 & 0.1721 & 0.1697 & 0.1674 & 0.1650 & 0.1627 & 0.1604 & 0.1580 & 0.1557 & 0.1534 & 0.1510 & 0.1487 & 0.1464 \\
\hline Scan $x$-coord & 1223 & 1223 & 1223 & 1223 & 1223 & 1223 & 1223 & 1223 & 1223 & 1223 & 1223 & 1223 & 1223 & 1223 & 1223 & 1223 & 1223 \\
\hline$F X \# I$ & 488.88 & 490.48 & 490.20 & 490.41 & 494.20 & 494.88 & 495.20 & 496.10 & 498.49 & 499.35 & 500.38 & 501.33 & 499.71 & 501.40 & 501.47 & 503.74 & 504.54 \\
\hline$F X \# 2$ & 321.28 & 321.55 & 321.78 & 322.03 & 322.35 & 322.51 & 322.71 & 323.01 & 323.28 & 323.47 & 323.69 & 324.02 & 324.35 & 324.79 & 325.15 & 325.41 & 325.53 \\
\hline$F X \# 3$ & 248.72 & 248.80 & 248.93 & 249.15 & 249.32 & 249.48 & 249.61 & 249.72 & 249.84 & 249.97 & 250.13 & 250.29 & 250.37 & 250.41 & 250.41 & 250.41 & 250.47 \\
\hline$F X \# 4$ & 196.31 & 196.40 & 196.48 & 196.57 & 196.71 & 196.81 & 196.89 & 197.01 & 197.14 & 197.22 & 197.30 & 197.35 & 197.41 & 197.44 & 197.47 & 197.53 & 197.63 \\
\hline$F X \# 5$ & 149.28 & 149.46 & 149.56 & 149.64 & 149.71 & 149.77 & 149.82 & 149.87 & 149.97 & 150.12 & 150.38 & 150.61 & 150.75 & 150.81 & 150.82 & 150.87 & 150.93 \\
\hline$F X \# 6$ & 104.24 & 104.27 & 104.38 & 104.50 & 104.58 & 104.73 & 104.94 & 105.13 & 105.25 & 105.33 & 105.46 & 105.63 & 105.73 & 105.79 & 105.85 & 105.95 & 106.05 \\
\hline
\end{tabular}




\begin{tabular}{|c|c|c|c|c|c|c|c|c|c|c|c|c|c|c|c|c|c|}
\hline Near wall $F S$ & 45.04 & 45.19 & 45.17 & 45.14 & 45.13 & 45.04 & 44.87 & 44.74 & 44.72 & 44.79 & 44.91 & 44.98 & 45.02 & 45.02 & 44.97 & 44.92 & 44.88 \\
\hline$T @ F X \# 1$ & 297.65 & 297.65 & 297.65 & 297.65 & 297.65 & 297.65 & 297.65 & 297.65 & 297.65 & 297.65 & 297.65 & 297.65 & 297.65 & 297.65 & 297.65 & 297.65 & 297.65 \\
\hline$T$ @ $F X \# 2$ & 298.16 & 298.16 & 298.15 & 298.15 & 298.14 & 298.14 & 298.13 & 298.13 & 298.12 & 298.12 & 298.11 & 298.11 & 298.10 & 298.10 & 298.10 & 298.09 & 298.09 \\
\hline$T @ F X \# 3$ & 300.19 & 300.19 & 300.18 & 300.18 & 300.17 & 300.17 & 300.16 & 300.16 & 300.15 & 300.15 & 300.14 & 300.14 & 300.14 & 300.13 & 300.13 & 300.12 & 300.12 \\
\hline$T @ F X \# 4$ & 302.23 & 302.23 & 302.22 & 302.22 & 302.21 & 302.21 & 302.20 & 302.20 & 302.19 & 302.19 & 302.18 & 302.18 & 302.17 & 302.17 & 302.16 & 302.16 & 302.15 \\
\hline$T$ (@) $F X \# 5$ & 304.29 & 304.29 & 304.28 & 304.28 & 304.27 & 304.27 & 304.26 & 304.26 & 304.25 & 304.25 & 304.24 & 304.24 & 304.23 & 304.23 & 304.22 & 304.22 & 304.21 \\
\hline$T @ F X \# 6$ & 306.38 & 306.38 & 306.37 & 306.37 & 306.36 & 306.36 & 306.35 & 306.35 & 306.34 & 306.34 & 306.33 & 306.33 & 306.32 & 306.32 & 306.31 & 306.31 & 306.30 \\
\hline Wall $x$-coord & 95.44 & 95.44 & 95.44 & 95.45 & 95.45 & 95.45 & 95.46 & 95.46 & 95.46 & 95.47 & 95.47 & 95.47 & 95.47 & 95.48 & 95.48 & 95.48 & 95.49 \\
\hline Wall $T$ & 308.91 & 308.91 & 308.91 & 308.91 & 308.91 & 308.91 & 308.92 & 308.92 & 308.92 & 308.92 & 308.92 & 308.92 & 308.92 & 308.92 & 308.92 & 308.92 & 308.92 \\
\hline
\end{tabular}

\begin{tabular}{|c|c|c|c|c|c|c|c|c|c|c|c|c|c|c|c|c|c|}
\hline Interferogram Image \# & 475 & Location & TC5 & de slope & $-2.10 E-3$ & $\delta \varepsilon$ intercept & 5.99 & $\operatorname{Avg} F S$ & 50.94 & Avg Wall $T$ & 308.84 & Gradient error & $2.03 E-4$ & & & & \\
\hline Scan y-coord & 2815 & 2816 & 2817 & 2818 & 2819 & 2820 & 2821 & 2822 & 2823 & 2824 & 2825 & 2826 & 2827 & 2828 & 2829 & 2830 & 2831 \\
\hline & 0.0664 & 0.0643 & 0.0622 & 0.0601 & 0.0580 & 0.0559 & 0.0538 & 0.0517 & 0.0496 & 0.0475 & 0.0454 & 0.0433 & 0.0412 & 0.0391 & 0.0370 & 0.0348 & 0.0327 \\
\hline Scan $x$-coord & 1029 & 1029 & 1029 & 1029 & 1029 & 1029 & 1029 & 1029 & 1029 & 1029 & 1029 & 1029 & 1029 & 1029 & 1029 & 1029 & 1029 \\
\hline$F X \# 1$ & 817.00 & 817.00 & 817.00 & 817.00 & 817.00 & 817.00 & 817.00 & 817.00 & 817.00 & 817.00 & 817.00 & 817.00 & 817.00 & 817.00 & 817.00 & 817.00 & 817.00 \\
\hline$F X \# 2$ & 358.84 & 359.15 & 359.57 & 359.97 & 360.33 & 360.64 & 360.91 & 361.11 & 361.38 & 361.61 & 361.85 & 362.06 & 362.14 & 362.24 & 362.25 & 362.35 & 362.64 \\
\hline$F X \# 3$ & 273.02 & 273.08 & 273.23 & 273.34 & 273.37 & 273.39 & 273.32 & 273.23 & 273.31 & 273.45 & 273.71 & 274.00 & 274.31 & 274.46 & 274.42 & 274.33 & 274.25 \\
\hline$F X \# 4$ & 211.35 & 211.57 & 211.64 & 211.70 & 211.81 & 212.11 & 212.35 & 212.50 & 212.70 & 212.94 & 213.06 & 213.12 & 213.24 & 213.51 & 213.89 & 214.06 & 214.16 \\
\hline$F X \# 5$ & 158.31 & 158.30 & 158.20 & 158.28 & 158.46 & 158.72 & 158.86 & 159.02 & 159.20 & 159.25 & 159.41 & 159.49 & 159.62 & 159.84 & 160.06 & 160.21 & 160.38 \\
\hline$F X \# 6$ & 107.15 & 107.51 & 107.75 & 107.87 & 108.06 & 108.19 & 108.19 & 108.14 & 108.33 & 108.52 & 108.68 & 108.68 & 108.68 & 108.64 & 108.48 & 108.37 & 108.41 \\
\hline Near wall FS & 51.16 & 50.79 & 50.45 & 50.41 & 50.40 & 50.53 & 50.67 & 50.87 & 50.86 & 50.73 & 50.73 & 50.81 & 50.93 & 51.20 & 51.58 & 51.84 & 51.96 \\
\hline$T @ F X \# 1$ & 297.65 & 297.65 & 297.65 & 297.65 & 297.65 & 297.65 & 297.65 & 297.65 & 297.65 & 297.65 & 297.65 & 297.65 & 297.65 & 297.65 & 297.65 & 297.65 & 297.65 \\
\hline$T @ F X \# 2$ & 297.82 & 297.82 & 297.82 & 297.81 & 297.81 & 297.80 & 297.80 & 297.80 & 297.79 & 297.79 & 297.78 & 297.78 & 297.77 & 297.77 & 297.77 & 297.76 & 297.76 \\
\hline$T @ F X \# 3$ & 299.91 & 299.90 & 299.90 & 299.90 & 299.89 & 299.89 & 299.88 & 299.88 & 299.87 & 299.87 & 299.87 & 299.86 & 299.86 & 299.85 & 299.85 & 299.84 & 299.84 \\
\hline$T @ F X \# 5$ & 304.01 & 304.00 & 304.00 & 303.99 & 303.99 & 303.98 & 303.98 & 303.97 & 303.97 & 303.97 & 303.96 & 303.96 & 303.95 & 303.95 & 303.94 & 303.94 & 303.94 \\
\hline$T @ F X \# 6$ & 306.09 & 306.09 & 306.08 & 306.08 & 306.07 & 306.07 & 306.06 & 306.06 & 306.06 & 306.05 & 306.05 & 306.04 & 306.04 & 306.03 & 306.03 & 306.03 & 306.02 \\
\hline Wall $x$-coord & 91.93 & 91.94 & 91.94 & 91.95 & 91.95 & 91.96 & 91.96 & 91.97 & 91.97 & 91.98 & 91.98 & 91.99 & 92.00 & 92.00 & 92.01 & 92.01 & 92.02 \\
\hline Wall $T$ & 308.83 & 308.85 & 308.86 & 308.86 & 308.86 & 308.86 & 308.86 & 308.85 & 308.85 & 308.85 & 308.86 & 308.85 & 308.84 & 308.83 & 308.82 & 308.81 & 308.80 \\
\hline
\end{tabular}

Interferogram Image

\begin{tabular}{|c|c|c|c|c|c|c|c|c|c|c|c|c|c|c|c|c|c|}
\hline & $4 / 3$ & Location & $1 C 6$ & $\delta$ s slope & $-2.30 E-3$ & & 0.40 & & & & & Idient error & & & & & \\
\hline Scan y-coord & 2001 & 2002 & 2003 & 2004 & 2005 & 2006 & 2007 & 2008 & 2009 & 2010 & 2011 & 2012 & 2013 & 2014 & 2015 & 2016 & 2017 \\
\hline & 0.7958 & 0.7935 & 0.7912 & 0.7889 & 0.7866 & 0.7843 & 0.7820 & 0.7797 & 0.7774 & 0.7751 & 0.7728 & 0.7705 & 0.7682 & 0.7659 & 0.7636 & 0.7613 & 0.7590 \\
\hline Scan $x$-coord & 1304 & 1304 & 1304 & 1304 & 1304 & 1304 & 1304 & 1304 & 1304 & 1304 & 1304 & 1304 & 1304 & 1304 & 1304 & 1304 & 1304 \\
\hline$F X \# 1$ & 405.29 & 405.45 & 405.59 & 405.94 & 406.44 & 406.89 & 407.27 & 407.61 & 408.00 & 408.39 & 408.90 & 409.48 & 410.13 & 410.76 & 411.07 & 411.30 & 411.57 \\
\hline$F X \# 2$ & 299.10 & 299.25 & 299.46 & 299.71 & 299.98 & 300.28 & 300.68 & 301.03 & 301.33 & 301.61 & 301.82 & 301.92 & 302.10 & 302.17 & 302.34 & 302.56 & 302.76 \\
\hline$F X \# 3$ & 230.40 & 230.62 & 230.75 & 230.79 & 230.78 & 230.78 & 230.73 & 230.67 & 230.57 & 230.46 & 230.43 & 230.48 & 230.45 & 230.58 & 230.63 & 230.81 & 231.00 \\
\hline$F X \# 4$ & 171.58 & 171.76 & 171.95 & 172.14 & 172.28 & 172.41 & 172.36 & 172.38 & 172.21 & 172.07 & 172.07 & 172.02 & 172.04 & 172.08 & 172.13 & 172.24 & 172.34 \\
\hline$F X \# 5$ & 115.15 & 115.32 & 115.30 & 115.37 & 115.43 & 115.63 & 115.70 & 115.94 & 116.06 & 116.07 & 116.28 & 116.40 & 116.55 & 116.64 & 116.73 & 116.88 & 117.18 \\
\hline Near wall $F S$ & 56.43 & 56.44 & 56.65 & 56.77 & 56.85 & 56.78 & 56.65 & 56.44 & 56.15 & 56.00 & 55.78 & 55.62 & 55.49 & 55.44 & 55.41 & 55.35 & 55.16 \\
\hline$T @ F X \# 1$ & 297.65 & 297.65 & 297.65 & 297.65 & 297.65 & 297.65 & 297.65 & 297.65 & 297.65 & 297.65 & 297.65 & 297.65 & 297.65 & 297.65 & 297.65 & 297.65 & 297.65 \\
\hline$T @ F X \# 2$ & 299.41 & 299.41 & 299.40 & 299.40 & 299.39 & 299.39 & 299.39 & 299.38 & 299.38 & 299.37 & 299.37 & 299.36 & 299.36 & 299.35 & 299.35 & 299.34 & 299.34 \\
\hline$T @ F X \# 3$ & 301.45 & 301.44 & 301.44 & 301.43 & 301.43 & 301.42 & 301.42 & 301.41 & 301.41 & 301.41 & 301.40 & 301.40 & 301.39 & 301.39 & 301.38 & 301.38 & 301.37 \\
\hline$T(F X \# 4$ & 303.50 & 303.50 & 303.49 & 303.49 & 303.48 & 303.48 & 303.47 & 303.47 & 303.46 & 303.46 & 303.45 & 303.45 & 303.45 & 303.44 & 303.44 & 303.43 & 303.43 \\
\hline$T @ F X \# 5$ & 305.58 & 305.58 & 305.57 & 305.57 & 305.56 & 305.56 & 305.55 & 305.55 & 305.54 & 305.54 & 305.53 & 305.53 & 305.52 & 305.52 & 305.52 & 305.51 & 305.51 \\
\hline Wall $x$-coord & 86.46 & 86.47 & 86.48 & 86.49 & 86.50 & 86.50 & 86.51 & 86.52 & 86.53 & 86.54 & 86.54 & 86.55 & 86.56 & 86.57 & 86.58 & 86.58 & 86.59 \\
\hline Wall $T$ & 308.76 & 308.76 & 308.75 & 308.75 & 308.74 & 308.75 & 308.75 & 308.76 & 308.76 & 308.76 & 308.77 & 308.77 & 308.77 & 308.77 & 308.77 & 308.77 & 308.78 \\
\hline
\end{tabular}

Interferogram Image

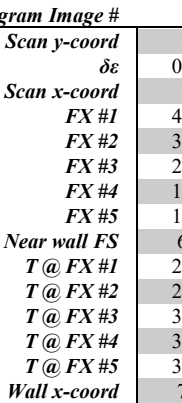

$475 \quad$ Location $\quad 767$

$\delta \varepsilon$ slope

$\begin{array}{ccc}-2.17 E-3 & \delta \varepsilon \text { intercept } & 3.06 \\ 0.4724 & 0.4202 & 0.4680\end{array}$

Avg FS

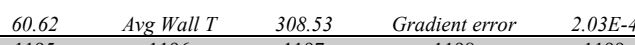

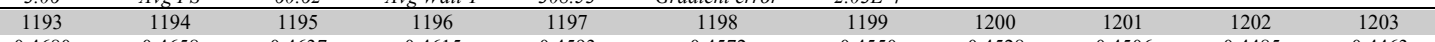

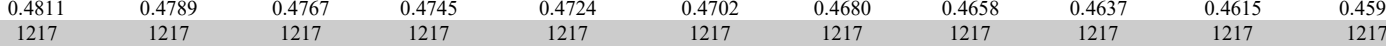

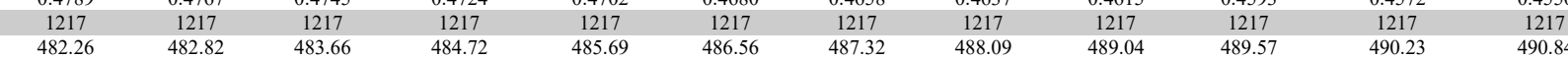

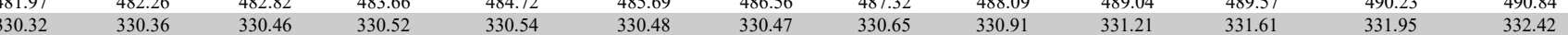

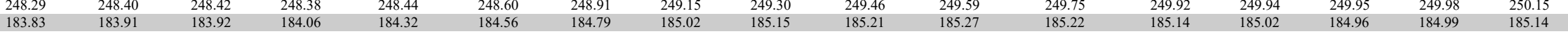
$\begin{array}{lllllllllll}123.01 & 123.20 & 123.29 & 123.41 & 123.38 & 123.32 & 123.31 & 123.41 & 123.76 & 124.13 & 124.43 \\ 60.83 & 60.71 & 60.62 & 60.65 & 60.94 & 61.23 & 61.48 & 61.60 & 61.39 & 61.08 & 60.85\end{array}$

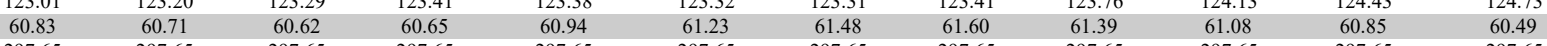

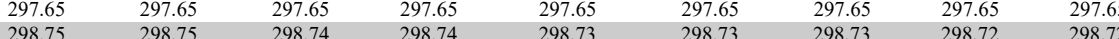

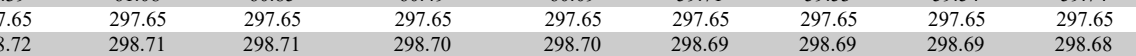

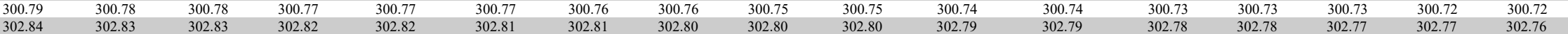

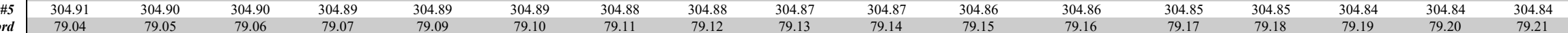

\section{B.20 Tabulated Data for Interferogram 476}

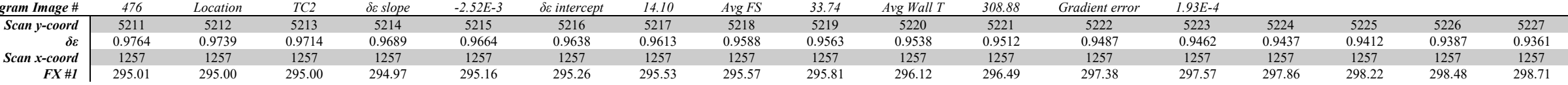




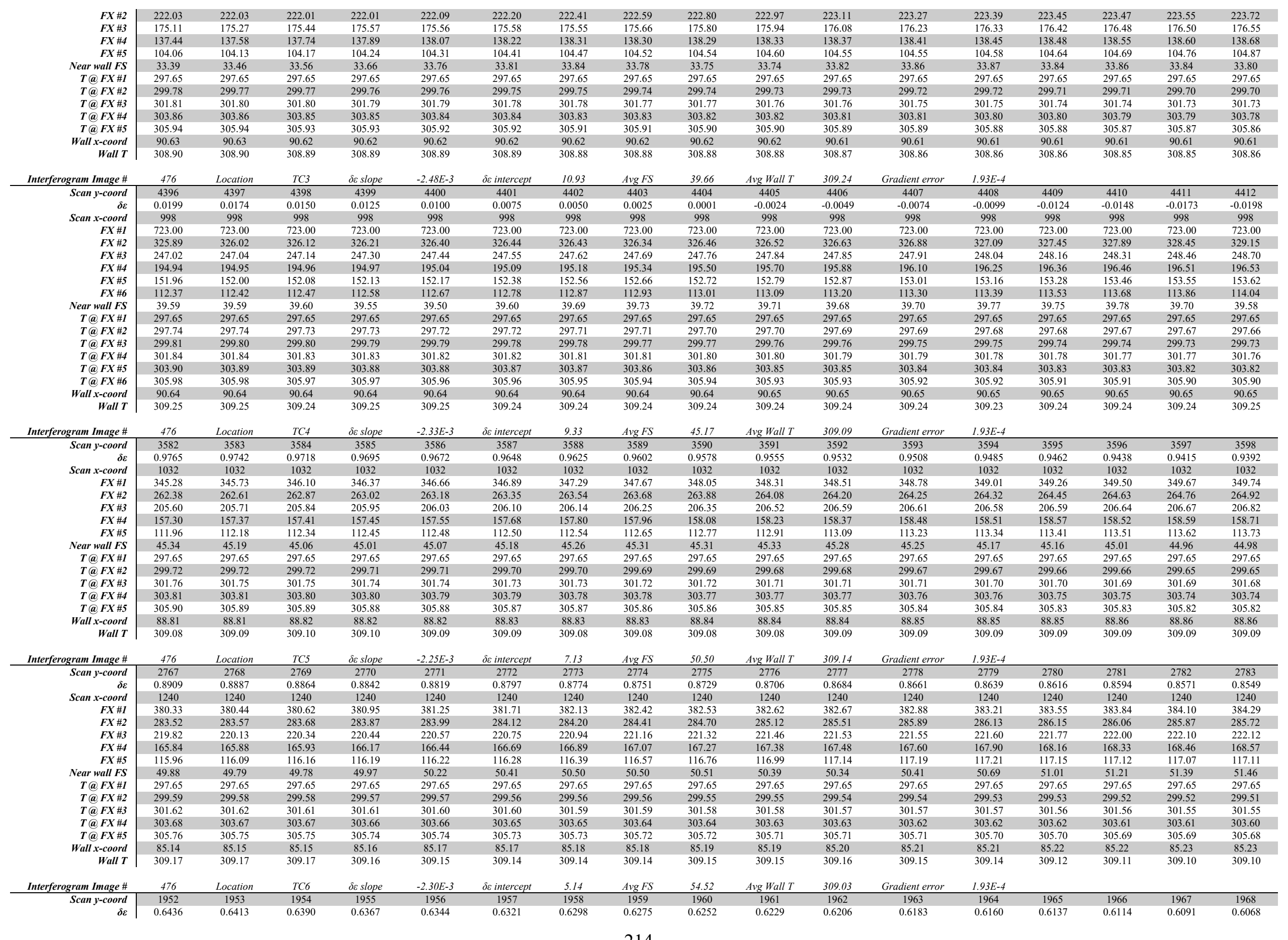




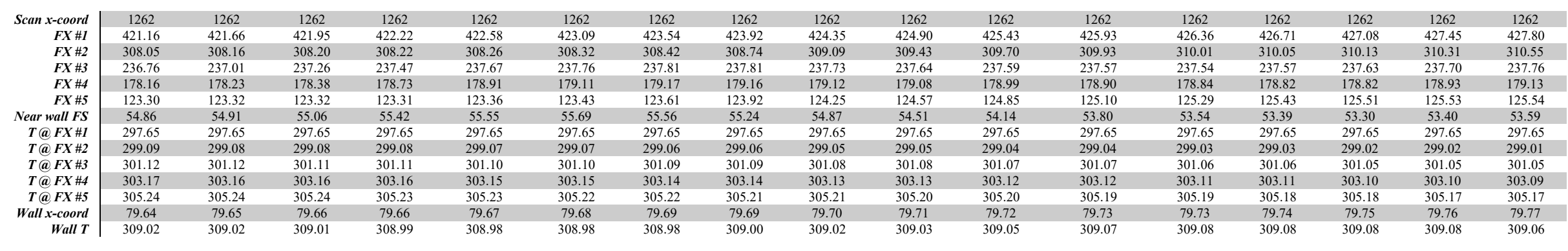

\begin{tabular}{|c|c|c|c|c|c|c|c|c|c|c|c|c|c|c|c|c|c|}
\hline Interferogram Image \# & 476 & Location & $T C 7$ & $\delta \varepsilon$ slope & $-2.20 E-3$ & $\delta \varepsilon$ intercept & 2.84 & $A v g F S$ & 60.37 & Avg Wall T & 308.77 & Gradient error & $1.93 E-4$ & & & & \\
\hline Scan y-coord & 1137 & 1138 & 1139 & 1140 & 1141 & 1142 & 1143 & 1144 & 1145 & 1146 & 1147 & 1148 & 1149 & 1150 & 1151 & 1152 & 1153 \\
\hline & 0.3364 & 0.3342 & 0.3320 & 0.3298 & 0.3276 & 0.3254 & 0.3232 & 0.3210 & 0.3188 & 0.3166 & 0.3143 & 0.3121 & 0.3099 & 0.3077 & 0.3055 & 0.3033 & 0.3011 \\
\hline Scan $x$-coord & 1215 & 1215 & 1215 & 1215 & 1215 & 1215 & 1215 & 1215 & 1215 & 1215 & 1215 & 1215 & 1215 & 1215 & 1215 & 1215 & 1215 \\
\hline$F X \# 1$ & 538.93 & 538.03 & 538.75 & 538.56 & 537.24 & 537.56 & 538.22 & 541.49 & 542.05 & 542.73 & 543.88 & 545.55 & 546.59 & 547.11 & 547.60 & 548.48 & 548.68 \\
\hline$F X \# 2$ & 348.21 & 348.09 & 347.97 & 348.05 & 348.23 & 348.44 & 348.56 & 348.63 & 348.72 & 348.98 & 349.44 & 349.90 & 350.43 & 350.78 & 351.04 & $\begin{array}{l}350.40 \\
351.34\end{array}$ & 351.68 \\
\hline$F X \# 3$ & 258.99 & 259.24 & 259.49 & 259.73 & 259.99 & 260.27 & 260.37 & 260.48 & 260.60 & 260.81 & 261.01 & 261.12 & 261.27 & 261.36 & 261.47 & 261.51 & 261.51 \\
\hline$F X \# 4$ & 193.00 & 192.94 & 192.89 & 192.89 & 192.98 & 193.11 & 193.38 & 193.62 & 193.84 & 194.04 & 194.20 & 194.38 & 194.42 & 194.41 & 194.35 & 194.28 & 194.23 \\
\hline$F X \# 5$ & 132.15 & 132.40 & 132.61 & 132.77 & 132.90 & 132.99 & 132.95 & 132.87 & 132.83 & 132.95 & 133.23 & 133.57 & 133.92 & 134.30 & 134.62 & 134.81 & 134.87 \\
\hline Near wall FS & 60.86 & 60.54 & 60.28 & 60.12 & 60.08 & 60.12 & 60.43 & 60.75 & 61.01 & 61.09 & 60.97 & 60.82 & 60.51 & 60.10 & 59.72 & 59.47 & 59.36 \\
\hline$T @ F X \# 1$ & 297.65 & 297.65 & 297.65 & 297.65 & 297.65 & 297.65 & 297.65 & 297.65 & 297.65 & 297.65 & 297.65 & 297.65 & 297.65 & 297.65 & 297.65 & 297.65 & 297.65 \\
\hline$T(F X \# 2$ & 298.45 & 298.44 & 298.44 & 298.43 & 298.43 & 298.43 & 298.42 & 298.42 & 298.41 & 298.41 & 298.40 & 298.40 & 298.39 & 298.39 & 298.38 & 298.38 & 298.37 \\
\hline$T(F X \# 3$ & 300.48 & 300.48 & 300.47 & 300.47 & 300.47 & 300.46 & 300.46 & 300.45 & 300.45 & 300.44 & 300.44 & 300.43 & 300.43 & 300.43 & 300.42 & 300.42 & 300.41 \\
\hline$T(a) F X \#$ & 302.53 & 302.52 & 302.52 & 302.51 & 302.51 & 302.51 & 302.50 & 302.50 & 302.49 & 302.49 & 302.48 & 302.48 & 302.47 & 302.47 & 302.46 & 302.46 & 302.46 \\
\hline$T @ F X \# 5$ & 304.60 & 304.59 & 304.59 & 304.58 & 304.58 & 304.57 & 304.57 & 304.56 & 304.56 & 304.55 & 304.55 & 304.55 & 304.54 & 304.54 & 304.53 & 304.53 & 304.52 \\
\hline Wall $x$-coord & 72.30 & 72.31 & 72.32 & 72.33 & 72.34 & 72.35 & 72.36 & 72.37 & 72.38 & 72.39 & 72.40 & 72.41 & 72.42 & 72.43 & 72.44 & 72.45 & 72.46 \\
\hline Wall $T$ & 308.75 & 308.77 & 308.78 & 308.78 & 308.78 & 308.78 & 308.76 & 308.74 & 308.73 & 308.73 & 308.73 & 308.75 & 308.76 & 308.79 & 308.81 & 308.82 & 308.82 \\
\hline
\end{tabular}

\section{B.21 Tabulated Data for Interferogram 477}

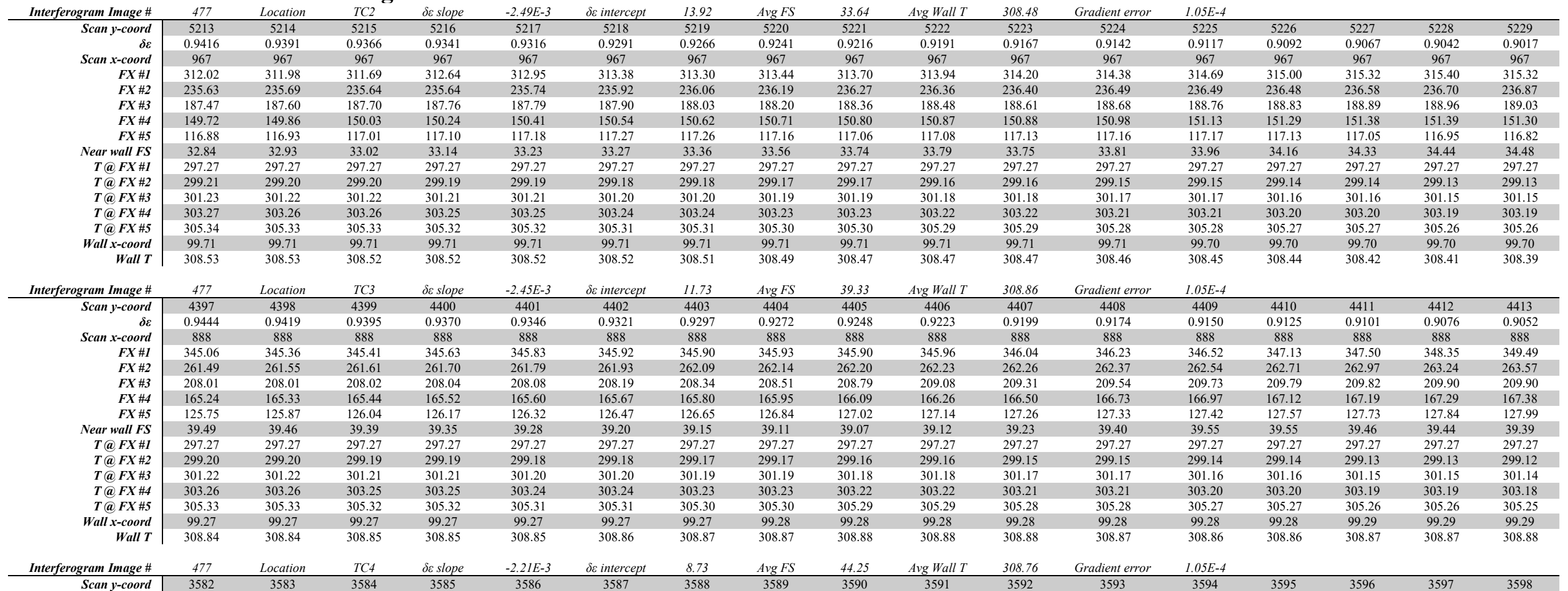




\begin{tabular}{|c|c|c|c|c|c|c|c|c|c|c|c|c|c|c|c|c|c|}
\hline$\delta \varepsilon$ & 0.8023 & 0.8001 & 0.7979 & 0.7957 & 0.7935 & 0.7913 & 0.7890 & 0.7868 & 0.7846 & 0.7824 & 0.7802 & 0.7780 & 0.7758 & 0.7735 & 0.7713 & 0.7691 & 0.7669 \\
\hline Scan $x$-coord & 922 & 922 & 922 & 922 & 922 & 922 & 922 & 922 & 922 & 922 & 922 & 922 & 922 & 922 & 922 & 922 & 922 \\
\hline$F X \# 1$ & 374.32 & 374.72 & 375.19 & 375.50 & 375.84 & 376.03 & 376.30 & 376.63 & 377.02 & 377.46 & 377.85 & 378.25 & 378.57 & 378.99 & 379.44 & 379.94 & 380.28 \\
\hline$F X \# 2$ & 283.25 & 283.48 & 283.67 & 283.86 & 284.12 & 284.37 & 284.60 & 284.80 & 284.92 & 285.06 & 285.23 & 285.44 & 285.52 & 285.52 & 285.55 & 285.65 & 285.84 \\
\hline$F X \# 3$ & $\begin{array}{l}223.89 \\
223.89\end{array}$ & $\begin{array}{l}203.40 \\
223.99\end{array}$ & 224.16 & $\begin{array}{l}224.00 \\
224.23\end{array}$ & 224.31 & $\begin{array}{l}224.37 \\
224.37\end{array}$ & $\begin{array}{l}204.00 \\
224.41\end{array}$ & 224.43 & $\begin{array}{l}204.92 \\
224.53\end{array}$ & $\begin{array}{l}203.00 \\
224.67\end{array}$ & $\begin{array}{l}203.23 \\
224.83\end{array}$ & 224.94 & 225.04 & $\begin{array}{l}203.52 \\
225.10\end{array}$ & 225.16 & $\begin{array}{l}203.05 \\
225.24\end{array}$ & 225.28 \\
\hline$F X \# 4$ & 176.00 & 176.12 & 176.19 & 176.19 & 176.19 & 176.34 & 176.44 & 176.58 & 176.73 & 176.85 & 176.99 & 177.19 & 177.32 & 177.31 & 177.32 & 177.31 & 177.33 \\
\hline$F X \# 5$ & 131.11 & 131.31 & 131.49 & 131.68 & 131.83 & 131.98 & 132.17 & 132.32 & 132.49 & 132.67 & 132.85 & 133.06 & 133.22 & 133.38 & 133.49 & 133.57 & 133.58 \\
\hline Near wall FS & 44.89 & 44.81 & 44.70 & 44.50 & 44.36 & 44.36 & 44.27 & 44.25 & 44.24 & 44.18 & 44.14 & 44.13 & 44.09 & 43.93 & 43.83 & 43.74 & 43.75 \\
\hline$T @ F X \# 1$ & $\begin{array}{r}497.89 \\
297\end{array}$ & $\begin{array}{l}297.27 \\
297.27\end{array}$ & $\begin{array}{l}494.10 \\
297.27\end{array}$ & $\begin{array}{l}49.50 \\
297.27\end{array}$ & $\begin{array}{l}497.30 \\
297.27\end{array}$ & $\begin{array}{l}49.30 \\
297.27\end{array}$ & $\begin{array}{r}497.27 \\
297.27\end{array}$ & $\begin{array}{l}297.25 \\
297.27\end{array}$ & $\begin{array}{r}497.24 \\
297.27\end{array}$ & $\begin{array}{l}49.18 \\
297.27\end{array}$ & $\begin{array}{r}497.14 \\
297.27\end{array}$ & $\begin{array}{l}49.13 \\
297.27\end{array}$ & $\begin{array}{l}497.09 \\
297.27\end{array}$ & $\begin{array}{l}45.93 \\
297.27\end{array}$ & $\begin{array}{r}497.83 \\
297.27\end{array}$ & $\begin{array}{l}49.14 \\
297.27\end{array}$ & $\begin{array}{l}29.15 \\
297.27\end{array}$ \\
\hline $\begin{array}{l}T(a) F X \# 2 \\
T(a) F \# 2\end{array}$ & 298.92 & $\begin{array}{l}297.27 \\
298.92\end{array}$ & $\begin{array}{l}297.27 \\
298.91\end{array}$ & 298.91 & $\begin{array}{l}297.27 \\
298.90\end{array}$ & $\begin{array}{l}297.27 \\
298.90\end{array}$ & $\begin{array}{l}297.27 \\
298.89\end{array}$ & $\begin{array}{l}297.27 \\
298.89\end{array}$ & 298.88 & 298.88 & 298.88 & $\begin{array}{l}297.27 \\
298.87\end{array}$ & 298.87 & 298.86 & 298.86 & 298.85 & $\begin{array}{l}297.27 \\
298.85\end{array}$ \\
\hline$T @ F X \# 3$ & 300.94 & 300.93 & 300.93 & 300.92 & 300.92 & 300.91 & 300.91 & 300.90 & 300.90 & 300.89 & 300.89 & 300.89 & 300.88 & 300.88 & 300.87 & 300.87 & 300.86 \\
\hline$T @ F X \# 4$ & 302.98 & 302.97 & 302.97 & 302.96 & 302.96 & 302.95 & 302.95 & 302.94 & 302.94 & 302.93 & 302.93 & 302.93 & 302.92 & $\begin{array}{l}300.00 \\
302.92\end{array}$ & 302.91 & 302.91 & 302.90 \\
\hline$T @ F X \# 5$ & 305.04 & 305.04 & 305.03 & 305.03 & 305.02 & 305.02 & 305.01 & 305.01 & 305.00 & 305.00 & 305.00 & 304.99 & 304.99 & 304.98 & 304.98 & 304.97 & 304.97 \\
\hline Wall $x$-coord & 97.28 & 97.28 & 97.28 & 97.29 & 97.29 & 97.29 & 97.30 & 97.30 & 97.30 & 97.31 & 97.31 & 97.31 & 97.32 & 97.32 & 97.32 & 97.33 & 97.33 \\
\hline Wall T & 308.71 & 308.72 & 308.73 & 308.74 & 308.75 & 308.75 & 308.76 & 308.76 & 308.76 & 308.77 & 308.77 & 308.78 & 308.78 & 308.79 & 308.80 & 308.80 & 308.80 \\
\hline Interferogram Image \# & 477 & Location & $T C 5$ & $\delta \varepsilon$ slope & $-2.10 E-3$ & $\delta \varepsilon$ intercept & 6.43 & $\operatorname{Avg} F S$ & 49.86 & Avg Wall T & 308.75 & Gradient error & $1.05 E-4$ & & & & \\
\hline Scan y-coord & 2767 & 2768 & 2769 & 2770 & 2771 & 2772 & 27773 & 2774 & 2775 & 2776 & 2777 & 2778 & 2779 & 2780 & 2781 & 2782 & 2783 \\
\hline $\begin{array}{r}\operatorname{sean} y-\operatorname{cora} \\
\delta \varepsilon\end{array}$ & 0.6321 & 0.6300 & $\begin{array}{l}2769 \\
0.6279\end{array}$ & 0.6258 & 0.6237 & 0.6216 & 0.6195 & 0.6174 & 0.6153 & 0.6132 & 0.6111 & 0.6090 & 0.6069 & 0.6048 & 0.6028 & 0.6007 & 0.5986 \\
\hline Scan $x$-coord & 1241 & 1241 & 1241 & 1241 & 1241 & 1241 & 1241 & 1241 & 1241 & 1241 & 1241 & 1241 & 1241 & 1241 & 1241 & 1241 & 1241 \\
\hline$F X \# 1$ & 427.73 & 428.10 & 428.49 & 428.99 & 429.56 & $\begin{array}{l}1240 \\
430.09\end{array}$ & 430.48 & 430.62 & 430.70 & 430.80 & 431.05 & 431.24 & 431.51 & 431.79 & $\begin{array}{l}1241 \\
431.91\end{array}$ & 432.03 & $\begin{array}{l}1321 \\
432.20\end{array}$ \\
\hline$F X \# 2$ & 311.72 & 311.92 & $\begin{array}{l}420.49 \\
312.19\end{array}$ & 312.51 & 312.78 & 312.95 & $\begin{array}{l}450.40 \\
313.15\end{array}$ & $\begin{array}{l}450.02 \\
313.30\end{array}$ & 313.48 & $\begin{array}{l}450.00 \\
313.81\end{array}$ & 314.24 & $\begin{array}{r}31.24 \\
314.68\end{array}$ & 314.95 & 315.09 & $\begin{array}{ll}451.91 \\
315.04\end{array}$ & 314.87 & 314.67 \\
\hline $\begin{array}{l}F X \# 2 \\
F X \# 3\end{array}$ & 244.29 & 244.52 & 244.59 & $\begin{array}{l}312.51 \\
244.63\end{array}$ & 244.60 & $\begin{array}{r}312.93 \\
244.65\end{array}$ & $\begin{array}{l}313.13 \\
244.84\end{array}$ & 245.03 & $\begin{array}{l}313.48 \\
245.19\end{array}$ & $\begin{array}{l}313.81 \\
245.23\end{array}$ & $\begin{array}{l}314.24 \\
245.28\end{array}$ & $\begin{array}{r}114.68 \\
245.26\end{array}$ & $\begin{array}{r}134.93 \\
245.17\end{array}$ & $\begin{array}{l}315.09 \\
245.12\end{array}$ & $\begin{array}{l}315.04 \\
245.23\end{array}$ & $\begin{array}{l}314.81 \\
245.42\end{array}$ & 245.62 \\
\hline$F X \# 4$ & 189.07 & 189.21 & 189.30 & 189.38 & 189.56 & 189.84 & 190.17 & 190.48 & 190.64 & 190.84 & 191.08 & 191.29 & 191.46 & 191.63 & 191.89 & 192.17 & 192.30 \\
\hline$F X \# 5$ & 139.85 & 140.16 & 140.47 & 140.66 & 140.71 & $\begin{array}{l}107.04 \\
140.69\end{array}$ & 140.72 & 140.76 & 140.79 & 140.82 & 140.93 & 141.03 & 141.06 & 141.02 & 141.00 & 140.96 & 140.98 \\
\hline Near wall FS & $\begin{array}{l}139.83 \\
49.22\end{array}$ & $\begin{array}{l}140.10 \\
49.05\end{array}$ & $\begin{array}{l}140.47 \\
48.83\end{array}$ & $\begin{array}{l}140.00 \\
48.72\end{array}$ & 48.85 & $\begin{array}{l}140.09 \\
49.15\end{array}$ & 49.45 & $\begin{array}{l}140.70 \\
49.72\end{array}$ & $\begin{array}{l}140.85 \\
49.85\end{array}$ & $\begin{array}{l}140.02 \\
50.02\end{array}$ & $\begin{array}{l}140.93 \\
50.15\end{array}$ & $\begin{array}{l}141.03 \\
50.26\end{array}$ & 50.40 & $\begin{array}{l}41.02 \\
50.61\end{array}$ & $\begin{array}{l}141.00 \\
50.88\end{array}$ & $\begin{array}{l}140.90 \\
51.21\end{array}$ & $\begin{array}{l}140.98 \\
51.32\end{array}$ \\
\hline$T @ F X \# 1$ & 297.27 & 297.27 & 297.27 & 297.27 & 297.27 & 297.27 & 297.27 & 297.27 & 297.27 & 297.27 & 297.27 & 297.27 & 297.27 & 297.27 & 297.27 & 297.27 & 297.27 \\
\hline$T @ F X \# 3$ & 300.62 & 300.62 & 300.61 & 300.61 & 300.61 & 300.60 & 300.60 & 300.59 & 300.59 & 300.58 & 300.58 & 300.58 & 300.57 & 300.57 & 300.56 & 300.56 & 300.56 \\
\hline$T$ (a) $F X \# 4$ & $\begin{array}{l}3002.62 \\
302.66\end{array}$ & $\begin{array}{l}3002.62 \\
302.65\end{array}$ & 302.65 & $\begin{array}{l}3002.61 \\
302.65\end{array}$ & 302.64 & $\begin{array}{l}500.60 \\
302.64\end{array}$ & $\begin{array}{l}500.60 \\
302.63\end{array}$ & 302.63 & $\begin{array}{l}3002.59 \\
302.62\end{array}$ & 302.62 & $\begin{array}{l}5002.58 \\
302.62\end{array}$ & $\begin{array}{l}300.58 \\
302.61\end{array}$ & 302.61 & 302.60 & $\begin{array}{l}3002.50 \\
302.60\end{array}$ & $\begin{array}{l}5002.56 \\
30259\end{array}$ & 302.59 \\
\hline$T @ F X \# 5$ & 304.72 & 304.72 & 304.71 & 304.71 & 304.70 & 304.70 & 304.70 & 304.69 & $\begin{array}{l}302.02 \\
304.69\end{array}$ & 304.68 & 304.68 & 304.67 & 304.67 & 304.67 & 304.66 & 304.66 & 304.65 \\
\hline Wall $x$-coord & 93.74 & 93.75 & 93.75 & 93.76 & 93.76 & 93.77 & 93.77 & 93.78 & 93.78 & 93.79 & $\begin{array}{l}504.00 \\
93.79\end{array}$ & 93.80 & 93.80 & 93.81 & 93.81 & 93.82 & 93.83 \\
\hline Wall T & 308.77 & 308.79 & 308.80 & 308.81 & 308.80 & 308.79 & 308.77 & 308.76 & 308.75 & 308.74 & 308.73 & $\begin{array}{l}9.80 \\
308.73\end{array}$ & 308.72 & $\begin{array}{l}9.81 \\
308.70\end{array}$ & 308.69 & 308.67 & 308.66 \\
\hline Interferogram Image \# & 477 & Location & TC6 & $\delta \varepsilon$ slope & $-2.23 E-3$ & $\delta \varepsilon$ intercept & 4.72 & $\operatorname{Avg} F S$ & 52.77 & Avg Wall $T$ & 308.73 & Gradient error & $1.05 E-4$ & & & & \\
\hline Scan y-coord & 1952 & 1953 & 1954 & 1955 & 1956 & 1957 & 1958 & 1959 & 1960 & 1961 & 1962 & 1963 & 1964 & 1965 & 1966 & 1967 & 1968 \\
\hline $\begin{array}{l}\text { Scan } y-c o o r a \\
\delta \varepsilon\end{array}$ & 0.3610 & 0.3588 & 0.3565 & 0.3543 & $\begin{array}{l}19300 \\
0.3521\end{array}$ & 0.3498 & 0.3476 & 0.3454 & 0.3431 & $\begin{array}{l}1901 \\
0.3409\end{array}$ & 0.3387 & 0.3364 & 0.3342 & 0.3320 & 0.3298 & 0.3275 & 0.3253 \\
\hline $\begin{array}{l}\text { Scan } x \text {-coord } \\
\text { Ser }\end{array}$ & 1187 & 1187 & 1187 & 1187 & 1187 & 1187 & 1187 & 1187 & 1187 & 1187 & 1187 & 1187 & 1187 & 1187 & 1187 & 1187 & 1187 \\
\hline$F X \# I$ & 500.70 & 501.17 & 501.44 & 501.85 & 502.93 & 503.13 & 503.70 & 504.11 & 504.88 & 505.30 & 505.82 & 506.68 & 507.43 & 508.07 & 508.82 & 509.53 & 509.98 \\
\hline$F X \# 2$ & 344.43 & 344.71 & 344.87 & 345.01 & 345.10 & 345.21 & 345.32 & 345.36 & 345.30 & 345.26 & 345.23 & 345.37 & 345.42 & 345.63 & 345.92 & 346.08 & 346.36 \\
\hline$F X \# 3$ & 264.67 & 264.98 & 265.18 & 265.36 & 265.59 & 265.84 & 266.09 & 266.27 & 266.35 & 266.42 & 266.40 & 266.42 & 266.42 & 266.43 & 266.46 & 266.52 & 266.54 \\
\hline$F X \# 4$ & 204.85 & 205.04 & 205.34 & 205.64 & 206.04 & 206.40 & 206.57 & 206.57 & 206.65 & 206.71 & 206.71 & 206.58 & 206.49 & 206.43 & 206.36 & 206.34 & 206.47 \\
\hline$F X \# 5$ & 151.21 & 151.22 & 151.23 & 151.16 & 151.09 & 151.13 & 151.27 & 151.36 & 151.52 & 151.77 & 151.93 & 152.17 & 152.46 & 152.77 & 152.99 & 153.15 & $\begin{array}{l}20.41 \\
153.11\end{array}$ \\
\hline$F X \# 6$ & 98.56 & 98.22 & 98.01 & 98.00 & 98.10 & 98.49 & 98.69 & 98.92 & 99.09 & 99.13 & 99.18 & 99.34 & $\begin{array}{l}152.40 \\
99.79\end{array}$ & 100.21 & 100.42 & 100.25 & 100.08 \\
\hline Near wall $F S$ & 52.65 & $\begin{array}{l}53.01 \\
53.01\end{array}$ & $\begin{array}{l}93.01 \\
53.22\end{array}$ & 53.17 & $\begin{array}{l}52.10 \\
52.99\end{array}$ & 52.65 & $\begin{array}{l}50.09 \\
52.58\end{array}$ & $\begin{array}{l}52.42 \\
52.44\end{array}$ & 52.43 & 52.65 & 52.76 & 52.83 & 52.67 & 52.56 & 52.57 & 52.89 & 53.03 \\
\hline$T @ F X \# 1$ & 297.27 & 297.27 & 297.27 & 297.27 & 297.27 & 297.27 & 297.27 & 297.27 & 297.27 & 297.27 & 297.27 & 297.27 & 297.27 & 297.27 & 297.27 & 297.27 & 297.27 \\
\hline$T @ F X \# 2$ & 298.06 & 298.05 & 298.05 & 298.04 & 298.04 & 298.04 & 298.03 & 298.03 & 298.02 & 298.02 & 298.01 & 298.01 & 298.00 & 298.00 & 297.99 & 297.99 & 297.99 \\
\hline$T @ F X \# 3$ & 300.07 & 300.06 & 300.06 & 300.06 & 300.05 & 300.05 & 300.04 & 300.04 & 300.03 & 300.03 & 300.02 & 300.02 & 300.01 & 300.01 & 300.01 & 300.00 & 300.00 \\
\hline$T @ F X \# 4$ & 302.10 & 302.09 & 302.09 & 302.09 & 302.08 & 302.08 & 302.07 & 302.07 & 302.06 & 302.06 & 302.05 & 302.05 & 302.04 & 302.04 & 302.03 & 302.03 & 302.03 \\
\hline$T$ (a $F X \# 5$ & 304.15 & 304.15 & 304.14 & 304.14 & 304.14 & 304.13 & 304.13 & 304.12 & 304.12 & 304.11 & 304.11 & 304.10 & 304.10 & 304.09 & 304.09 & 304.09 & 304.08 \\
\hline$T(F X \# 6$ & 306.24 & 306.23 & 306.23 & 306.22 & $\begin{array}{r}304.14 \\
306.22\end{array}$ & 306.21 & 306.21 & 306.20 & 306.20 & 306.20 & $\begin{array}{l}504.11 \\
306.19\end{array}$ & $\begin{array}{l}304.10 \\
306.19\end{array}$ & $\begin{array}{l}304.10 \\
306.18\end{array}$ & 306.18 & 306.17 & 306.17 & 306.16 \\
\hline Wall $x$-coord & 88.66 & 88.67 & 88.68 & 88.68 & 88.69 & 88.70 & 88.70 & 88.71 & 88.72 & 88.73 & 88.73 & 88.74 & 88.75 & 88.75 & 88.76 & 88.77 & 88.78 \\
\hline Wall T & 308.75 & 308.72 & 308.71 & 308.70 & 308.70 & 308.72 & 308.72 & 308.73 & 308.73 & 308.72 & 308.72 & 308.72 & 308.73 & 308.75 & 308.75 & 308.74 & 308.72 \\
\hline Interferogram Image \# & 477 & Location & $T C 7$ & $\delta \varepsilon$ slope & $-2.01 E-3$ & $\delta \varepsilon$ intercept & 2.39 & $\operatorname{Avg} F S$ & 58.36 & Avg Wall T & 308.67 & Gradient error & $1.05 E-4$ & & & & \\
\hline Scan y-coord & 1137 & 1138 & 1139 & CEstope & $\frac{2.01 L-3}{1141}$ & 1142 & 1143 & $\frac{A v S T}{1144}$ & 1145 & 1146 & $\frac{500.07}{1147}$ & 1148 & $\frac{1.0 J E-4}{1149}$ & 1150 & 1151 & 1152 & 1153 \\
\hline $\begin{array}{l}\text { Scan } y-c o o r a \\
\delta \varepsilon\end{array}$ & 0.1098 & 0.1078 & 0.1058 & 0.1038 & 0.1018 & 0.0998 & 0.0978 & 0.0958 & 0.0937 & 0.0917 & 0.0897 & 0.0877 & 0.0857 & 0.0837 & 0.0817 & 0.0797 & 0.0777 \\
\hline Scan $x$-coord & 1462 & 1462 & 1462 & 1462 & 1462 & 1462 & 1462 & 1462 & 1462 & 1462 & 1462 & 1462 & 1462 & 1462 & 1462 & 1462 & 1462 \\
\hline$F X \# 1$ & 814.00 & 814.00 & 814.00 & 814.00 & 814.00 & 814.00 & 814.00 & 814.00 & 814.00 & 814.00 & 814.00 & 814.00 & 814.00 & 814.00 & 814.00 & 814.00 & 814.00 \\
\hline$F X \# 2$ & 394.38 & 394.67 & 395.01 & 395.52 & 395.93 & 396.29 & 396.56 & 396.76 & 396.96 & 397.24 & 397.43 & 397.59 & 397.70 & 397.66 & 397.67 & 397.92 & 398.05 \\
\hline$F X \# 3$ & 292.82 & 292.93 & 293.14 & 293.40 & 293.65 & 293.81 & 293.89 & 293.98 & 294.02 & 294.17 & 294.34 & 294.52 & 294.76 & 294.96 & 295.15 & 295.37 & 295.58 \\
\hline$F X \# 4$ & 223.33 & 223.37 & 223.37 & 223.34 & 223.33 & 223.35 & 223.34 & 223.41 & 223.61 & 224.02 & 224.40 & 224.63 & 224.75 & 224.87 & 224.86 & 224.76 & 224.67 \\
\hline$F X \# 5$ & 163.09 & 163.39 & 163.59 & 163.83 & 163.91 & 163.96 & 164.03 & 164.01 & 163.97 & 164.00 & 164.18 & 164.27 & 164.39 & 164.55 & 164.78 & 165.05 & 165.25 \\
\hline$F X \# 6$ & 104.95 & 105.10 & 105.19 & 105.37 & 105.52 & 105.66 & 105.74 & $\begin{array}{l}104.01 \\
105.73\end{array}$ & 105.67 & $\begin{array}{l}104.00 \\
105.70\end{array}$ & $\begin{array}{l}104.18 \\
105.72\end{array}$ & $\begin{array}{l}104.27 \\
105.79\end{array}$ & $\begin{array}{l}104.59 \\
105.89\end{array}$ & $\begin{array}{l}104.53 \\
106.09\end{array}$ & 106.37 & 106.65 & $\begin{array}{l}105.25 \\
106.89\end{array}$ \\
\hline Near wall FS & 58.14 & 58.29 & 58.40 & 58.46 & 58.39 & 58.30 & 58.29 & 58.29 & 58.30 & 58.30 & 58.45 & 58.48 & 58.50 & 58.45 & 58.40 & 58.40 & 58.36 \\
\hline$T @ F X \# 1$ & 297.27 & 297.27 & 297.27 & 297.27 & 297.27 & 297.27 & 297.27 & 297.27 & 297.27 & $\begin{array}{r}597.30 \\
297.27\end{array}$ & 297.27 & $\begin{array}{r}50.48 \\
297.27\end{array}$ & $\begin{array}{l}58.50 \\
297.27\end{array}$ & $\begin{array}{r}58.45 \\
297.27\end{array}$ & $\begin{array}{r}598.40 \\
297.27\end{array}$ & $\begin{array}{r}598.40 \\
297.27\end{array}$ & $\begin{array}{r}29.07 .27 \\
297\end{array}$ \\
\hline$T @ F X \# 2$ & 297.56 & 297.55 & 297.55 & 297.55 & 297.54 & 297.54 & 297.53 & 297.53 & 297.53 & 297.52 & 297.52 & 297.51 & 297.51 & 297.51 & 297.50 & 297.50 & 297.49 \\
\hline$T @ F X \# 3$ & 299.59 & 299.58 & 299.58 & 299.58 & 299.57 & 299.57 & 299.56 & 299.56 & 299.56 & 299.55 & 299.55 & 299.54 & 299.54 & 299.54 & 299.53 & 299.53 & 299.52 \\
\hline$T @ F X \# 4$ & 301.61 & 301.61 & 301.61 & 301.60 & 301.60 & 301.59 & 301.59 & 301.59 & 301.58 & 301.58 & 301.57 & 301.57 & 301.57 & 301.56 & 301.56 & 301.55 & 301.55 \\
\hline$T @ F X \# 5$ & 303.66 & 303.66 & 303.66 & 303.65 & 303.65 & 303.64 & 303.64 & 303.64 & 303.63 & 303.63 & 303.62 & 303.62 & 303.62 & 303.61 & 303.61 & 303.60 & 303.60 \\
\hline$T @ F X \# 6$ & 305.74 & 305.74 & 305.73 & 305.73 & 305.72 & 305.72 & 305.72 & 305.71 & 305.71 & 305.70 & 305.70 & 305.70 & 305.69 & 305.69 & 305.68 & 305.68 & 305.67 \\
\hline Wall $x$-coord & $\begin{array}{l}505.14 \\
82.04\end{array}$ & $\begin{array}{l}505.14 \\
82.05\end{array}$ & 82.05 & 82.06 & 82.07 & 82.08 & 82.09 & 82.10 & 82.11 & 82.12 & 82.13 & 82.14 & 82.15 & 82.15 & 82.16 & 82.17 & 82.18 \\
\hline
\end{tabular}




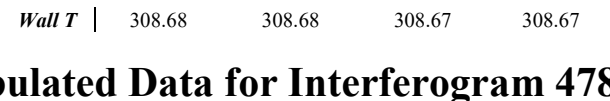

308.68

308.68

308.68

308.67

308.66

308.66

308.65

308.65

308.65

308.65

\section{B.22 Tabulated Data for Interferogram 478}

\begin{tabular}{|c|c|c|c|c|c|c|c|c|c|c|c|c|c|c|c|c|c|}
\hline Interferogram Image \# & 478 & Location & $T C 2$ & $\delta \varepsilon$ slope & $-2.5 I E-3$ & $\delta \varepsilon$ intercept & 13.16 & $\operatorname{Avg} F S$ & 32.13 & Avg Wall $T$ & 308.75 & Gradient error & $1.31 E-4$ & & & & \\
\hline Scan y-coord & 5229 & 5230 & 5231 & 5232 & 5233 & 5234 & 5235 & 5236 & 5237 & 5238 & 5239 & 5240 & 5241 & 5242 & 5243 & 5244 & 5245 \\
\hline & 0.0156 & 0.0131 & 0.0106 & 0.0081 & 0.0056 & 0.0031 & 0.0006 & -0.0020 & -0.0045 & -0.0070 & -0.0095 & -0.0120 & -0.0145 & -0.0170 & -0.0195 & -0.0221 & -0.0246 \\
\hline Scan $x$-coord & 0 & 0 & 0 & 0 & 0 & 0 & 0 & 0 & 0 & 0 & 0 & 0 & 0 & 0 & 0 & 0 & 0 \\
\hline$F X \# 1$ & 640.00 & 640.00 & 640.00 & 640.00 & 640.00 & 640.00 & 640.00 & 640.00 & 640.00 & 640.00 & 640.00 & 640.00 & 640.00 & 640.00 & 640.00 & 640.00 & 640.00 \\
\hline$F X \# 2$ & 287.06 & 286.53 & 286.91 & 287.07 & 287.08 & 287.13 & 287.22 & 287.26 & 287.52 & 288.06 & 288.70 & 289.28 & 289.81 & 290.17 & 290.50 & 290.78 & 291.27 \\
\hline$F X \# 3$ & 220.60 & 220.78 & 220.86 & 221.00 & 221.11 & 221.24 & 221.41 & 221.53 & 221.70 & 221.84 & 221.94 & 221.94 & 221.94 & 221.95 & 222.01 & 222.11 & 222.19 \\
\hline$F X \# 5$ & 138.54 & 138.72 & 138.93 & 139.05 & 139.12 & 139.22 & 139.34 & 139.44 & 139.59 & 139.77 & 139.95 & 140.12 & 140.31 & 140.46 & 140.58 & 140.60 & 140.57 \\
\hline$F X \# 6$ & 106.86 & 107.07 & 107.26 & 107.35 & 107.40 & 107.44 & 107.49 & 107.55 & 107.66 & 107.81 & 107.95 & 107.99 & 107.96 & 107.88 & 107.67 & 107.47 & 107.27 \\
\hline Near wall FS & 31.67 & 31.65 & 31.67 & 31.70 & 31.72 & 31.79 & 31.85 & 31.89 & 31.93 & 31.97 & 32.00 & 32.13 & 32.35 & 32.58 & 32.91 & 33.13 & 33.29 \\
\hline$T @ F X \# 1$ & 297.65 & 297.65 & 297.65 & 297.65 & 297.65 & 297.65 & 297.65 & 297.65 & 297.65 & 297.65 & 297.65 & 297.65 & 297.65 & 297.65 & 297.65 & 297.65 & 297.65 \\
\hline$T @ F X \# 2$ & 297.60 & 297.59 & 297.59 & 297.58 & 297.58 & 297.57 & 297.57 & 297.56 & 297.56 & 297.55 & 297.55 & 297.54 & 297.54 & 297.53 & 297.53 & 297.52 & 297.52 \\
\hline$T(F X \# 3$ & 299.63 & 299.63 & 299.62 & 299.62 & 299.61 & 299.61 & 299.60 & 299.60 & 299.59 & 299.59 & 299.58 & 299.58 & 299.57 & 299.57 & 299.56 & 299.56 & 299.55 \\
\hline $\begin{array}{l}T(a) F X+4 \\
T(F X \# 5\end{array}$ & 303.71 & 303.70 & $\begin{array}{l}301.63 \\
303.69\end{array}$ & $\begin{array}{l}301.64 \\
303.69\end{array}$ & $\begin{array}{l}301.64 \\
303.68\end{array}$ & $\begin{array}{l}301.63 \\
303.68\end{array}$ & $\begin{array}{l}301.63 \\
303.67\end{array}$ & $\begin{array}{l}301.62 \\
303.67\end{array}$ & $\begin{array}{l}301.62 \\
303.66\end{array}$ & $\begin{array}{l}301.61 \\
303.66\end{array}$ & $\begin{array}{l}301.60 \\
303.65\end{array}$ & $\begin{array}{l}301.60 \\
303.65\end{array}$ & 303.64 & $\begin{array}{l}301.59 \\
303.64\end{array}$ & $\begin{array}{l}301.58 \\
303.63\end{array}$ & 303.63 & $\begin{array}{l}300.1 .62 \\
302\end{array}$ \\
\hline$T @ F X \# 6$ & 305.78 & 305.78 & 305.77 & 305.77 & 305.76 & 305.76 & 305.75 & 305.74 & 305.74 & 305.73 & 305.73 & 305.72 & 305.72 & 305.71 & 305.71 & 305.70 & 305.70 \\
\hline Wall $x$-coord & 93.64 & 93.63 & 93.63 & 93.63 & 93.63 & 93.63 & 93.63 & 93.63 & 93.62 & 93.62 & 93.62 & 93.62 & 93.62 & 93.62 & 93.62 & 93.61 & 93.61 \\
\hline Wall T & 308.76 & 308.77 & 308.78 & 308.78 & 308.78 & 308.77 & 308.77 & 308.77 & 308.77 & 308.77 & 308.77 & 308.77 & 308.75 & 308.74 & 308.71 & 308.69 & 308.66 \\
\hline Interferogram Image \# & 478 & Location & $T C 3$ & $\delta \varepsilon$ slope & $-2.47 E-3$ & $\delta \varepsilon$ intercept & 10.96 & $\operatorname{Avg} F S$ & 36.94 & Avg Wall T & 309.20 & Gradient error & $1.31 E-4$ & & & & \\
\hline Scan y-coord & 4413 & 4414 & 4415 & 4416 & 4417 & 4418 & 4419 & 4420 & 4421 & 4422 & 4423 & 4424 & 4425 & 4426 & 4427 & 4428 & 4429 \\
\hline$\delta \varepsilon$ & 0.0695 & 0.0670 & 0.0646 & 0.0621 & 0.0596 & 0.0572 & 0.0547 & 0.0522 & 0.0498 & 0.0473 & 0.0448 & 0.0423 & 0.0399 & 0.0374 & 0.0349 & 0.0325 & 0.0300 \\
\hline Scan $x$-coord & 0 & 0 & 0 & 0 & 0 & 0 & 0 & 0 & 0 & 0 & 0 & 0 & 0 & 0 & 0 & 0 & 0 \\
\hline$F X \# 1$ & 620.00 & 620.00 & 620.00 & 620.00 & 620.00 & 620.00 & 620.00 & 620.00 & 620.00 & 620.00 & 620.00 & 620.00 & 620.00 & 620.00 & 620.00 & 620.00 & 620.00 \\
\hline$F X \# 2$ & 309.71 & 309.82 & 310.04 & 310.22 & 310.35 & 310.57 & 310.83 & 311.06 & 311.20 & 311.27 & 311.32 & 311.43 & 311.55 & 311.63 & 311.74 & 311.90 & 312.10 \\
\hline$F X \# 3$ & 240.30 & 240.35 & 240.40 & 240.46 & 240.52 & 240.61 & 240.70 & 240.83 & 240.96 & 241.07 & 241.23 & 241.41 & 241.58 & 241.68 & 241.69 & 241.72 & 241.77 \\
\hline$F X \# 4$ & 191.96 & 192.17 & 192.28 & 192.46 & 192.55 & 192.64 & 192.81 & 192.94 & 193.02 & 193.10 & 193.16 & 193.25 & 193.38 & 193.45 & 193.56 & 193.55 & 193.58 \\
\hline$F X \# 6$ & 114.97 & 115.10 & 115.20 & 115.31 & 115.38 & 115.45 & 115.57 & 115.65 & 115.71 & 115.81 & 115.84 & 115.93 & 116.03 & 116.15 & 116.27 & 116.37 & 116.44 \\
\hline Near wall FS & 36.74 & 36.73 & 36.78 & 36.90 & 36.96 & 36.95 & 37.02 & 37.04 & 37.04 & 37.00 & 37.01 & 36.98 & 36.99 & 36.98 & 36.96 & 36.96 & 36.96 \\
\hline$T @ F X \# 1$ & 297.65 & 297.65 & 297.65 & 297.65 & 297.65 & 297.65 & 297.65 & 297.65 & 297.65 & 297.65 & 297.65 & 297.65 & 297.65 & 297.65 & 297.65 & 297.65 & 297.65 \\
\hline$T$ (a) $F X \# 2$ & 297.71 & 297.70 & 297.70 & 297.69 & 297.69 & 297.68 & 297.68 & 297.67 & 297.67 & 297.66 & 297.66 & 297.65 & 297.65 & 297.64 & 297.64 & 297.63 & 297.63 \\
\hline$T(F X \# 3$ & 299.74 & 299.73 & 299.73 & 299.72 & 299.72 & 299.71 & 299.71 & 299.70 & 299.70 & 299.69 & 299.69 & 299.68 & 299.68 & 299.67 & 299.67 & 299.66 & 299.66 \\
\hline$T(F X \# 4$ & 301.76 & 301.76 & 301.75 & 301.75 & 301.74 & 301.74 & 301.73 & 301.73 & 301.72 & 301.72 & 301.71 & 301.71 & 301.70 & 301.70 & 301.69 & 301.69 & 301.68 \\
\hline$T @ F X \# 5$ & 303.81 & 303.81 & 303.80 & 303.80 & 303.79 & 303.79 & 303.78 & 303.78 & 303.77 & 303.77 & 303.76 & 303.76 & 303.75 & 303.75 & 303.74 & 303.74 & 303.73 \\
\hline$T @ F X \# 6$ & 305.89 & 305.89 & 305.88 & 305.88 & 305.87 & 305.87 & 305.86 & 305.86 & 305.85 & 305.85 & 305.84 & 305.84 & 305.83 & 305.82 & 305.82 & 305.81 & 305.81 \\
\hline Wall $x$-coord & 93.81 & 93.81 & 93.81 & 93.81 & 93.81 & 93.81 & 93.81 & 93.82 & 93.82 & 93.82 & 93.82 & 93.82 & 93.82 & 93.82 & 93.82 & 93.82 & 93.82 \\
\hline Wall T & 309.21 & 309.21 & 309.21 & 309.21 & 309.21 & 309.21 & 309.20 & 309.20 & 309.20 & 309.20 & 309.20 & 309.20 & 309.20 & 309.20 & 309.20 & 309.20 & 309.20 \\
\hline Interferogram Image \# & 478 & Location & $T C 4$ & $\delta \varepsilon$ slope & $-2.40 E-3$ & $\delta \varepsilon$ intercept & 8.68 & $\operatorname{Avg} F S$ & 41.50 & Avg Wall $T$ & 309.18 & Gradient error & $1.31 E-4$ & & & & \\
\hline Scan y-coord & 3598 & 3599 & 3600 & 3601 & 3602 & 3603 & 3604 & 3605 & 3606 & 3607 & 3608 & 3609 & 3610 & 3611 & 3612 & 3613 & 3614 \\
\hline & 0.0328 & 0.0304 & 0.0280 & 0.0255 & 0.0231 & 0.0207 & 0.0183 & 0.0159 & 0.0135 & 0.0111 & 0.0087 & 0.0063 & 0.0039 & 0.0015 & -0.0009 & -0.0033 & -0.0057 \\
\hline Scan $x$-coord & $\begin{array}{c}0.0520 \\
0\end{array}$ & 0 & 0 & 0 & 0 & 0 & 0 & 0 & 0 & 0 & 0 & 0 & 0 & 0 & 0 & 0 & 0 \\
\hline$F X \# 1$ & 818.00 & 818.00 & 818.00 & 818.00 & 818.00 & 818.00 & 818.00 & 818.00 & 818.00 & 818.00 & 818.00 & 818.00 & 818.00 & 818.00 & 818.00 & 818.00 & 818.00 \\
\hline$F X \# 2$ & 335.22 & 335.40 & 335.55 & 335.59 & 335.71 & 335.66 & 335.88 & 336.09 & 336.29 & 336.35 & 336.61 & 337.34 & 337.82 & 338.34 & 338.85 & 338.73 & 338.84 \\
\hline$F X \# 3$ & 256.80 & 256.98 & 257.18 & 257.33 & 257.43 & 257.51 & 257.65 & 257.79 & 257.96 & 258.08 & 258.20 & 258.41 & 258.52 & 258.63 & 258.72 & 258.83 & 258.93 \\
\hline$F X \# 4$ & 203.43 & 203.58 & 203.66 & 203.69 & 203.67 & 203.71 & 203.77 & 203.86 & 203.92 & 203.99 & 204.14 & 204.33 & 204.48 & 204.70 & 204.84 & 204.94 & 205.04 \\
\hline$F X \# 5$ & $\begin{array}{l}20.43 \\
158.64\end{array}$ & $\begin{array}{l}203.58 \\
158.73\end{array}$ & $\begin{array}{l}203.00 \\
158.80\end{array}$ & $\begin{array}{l}20.09 \\
158.87\end{array}$ & $\begin{array}{l}203.01 \\
158.94\end{array}$ & 159.00 & 159.05 & $\begin{array}{l}203.80 \\
159.09\end{array}$ & $\begin{array}{l}203.92 \\
159.06\end{array}$ & 2039.11 & $\begin{array}{l}204.14 \\
159.17\end{array}$ & $\begin{array}{l}204.33 \\
159.37\end{array}$ & $\begin{array}{l}204.48 \\
159.53\end{array}$ & 159.68 & $\begin{array}{l}204.84 \\
159.86\end{array}$ & $\begin{array}{l}204.94 \\
159.97\end{array}$ & 160.04 \\
\hline$F X \# 6$ & 116.82 & 116.93 & 117.08 & 117.27 & 117.43 & 117.54 & 117.60 & 117.66 & 117.66 & 117.77 & 117.91 & 118.06 & 118.18 & 118.26 & 118.31 & 118.40 & 118.49 \\
\hline Near wall FS & 41.81 & 41.80 & 41.73 & 41.60 & 41.51 & 41.47 & 41.45 & 41.43 & 41.40 & 41.33 & 41.27 & 41.31 & 41.35 & 41.42 & 41.55 & 41.58 & 41.54 \\
\hline$T @ F X \# 1$ & 297.65 & 297.65 & 297.65 & 297.65 & 297.65 & 297.65 & 297.65 & 297.65 & 297.65 & 297.65 & 297.65 & 297.65 & 297.65 & 297.65 & 297.65 & 297.65 & 297.65 \\
\hline$T @ F X \# 2$ & 297.61 & 297.60 & 297.60 & 297.59 & 297.59 & 297.58 & 297.58 & 297.57 & 297.57 & 297.56 & 297.56 & 297.55 & 297.55 & 297.55 & 297.54 & 297.54 & 297.53 \\
\hline$T(F X \# 3$ & 299.66 & 299.65 & 299.65 & 299.64 & 299.64 & 299.64 & 299.63 & 299.63 & 299.62 & 299.62 & 299.61 & 299.61 & 299.60 & 299.60 & 299.59 & 299.59 & 299.58 \\
\hline$T(a) F X \# 4$ & 301.69 & 301.68 & 301.68 & 301.67 & 301.67 & 301.66 & 301.66 & 301.65 & 301.65 & 301.64 & 301.64 & 301.63 & 301.63 & 301.62 & 301.62 & 301.61 & 301.61 \\
\hline$T @ F X \# 5$ & 303.74 & 303.73 & 303.73 & 303.72 & 303.72 & 303.71 & 303.71 & 303.70 & 303.70 & 303.69 & 303.69 & 303.68 & 303.68 & 303.67 & $\begin{array}{l}303.67 \\
303\end{array}$ & 303.66 & 303.66 \\
\hline$T @ F X \# 6$ & 305.81 & 305.81 & 305.80 & 305.80 & 305.79 & 305.79 & 305.78 & 305.78 & 305.77 & 305.77 & 305.76 & 305.76 & 305.75 & 305.75 & 305.74 & 305.74 & 305.73 \\
\hline Wall $x$-coord & 92.04 & 92.04 & 92.04 & 92.05 & 92.05 & 92.05 & 92.06 & 92.06 & 92.06 & 92.07 & 92.07 & 92.07 & 92.08 & 92.08 & 92.08 & 92.09 & 92.09 \\
\hline Wall T & 309.17 & 309.17 & 309.17 & 309.18 & 309.19 & 309.19 & 309.19 & 309.18 & 309.18 & 309.18 & 309.19 & 309.19 & 309.19 & 309.18 & 309.18 & 309.17 & 309.18 \\
\hline Interferogram Image \# & 478 & Location & $T C 5$ & $\delta \varepsilon$ slope & $-2.29 E-3$ & $\delta \varepsilon$ intercept & 7.34 & $\operatorname{Avg} F S$ & 46.46 & Avg Wall $T$ & 309.46 & Gradient error & $1.31 E-4$ & & & & \\
\hline Scan y-coord & 2783 & 2784 & 2785 & 2786 & 2787 & 2788 & 2789 & 2790 & 2791 & 2792 & 2793 & 2794 & 2795 & 2796 & 2797 & 2798 & 2799 \\
\hline$\delta \varepsilon$ & 0.9539 & 0.9516 & 0.9493 & 0.9470 & 0.9447 & 0.9424 & 0.9401 & 0.9378 & 0.9355 & 0.9332 & 0.9309 & 0.9286 & 0.9263 & 0.9240 & 0.9217 & 0.9194 & 0.9171 \\
\hline Scan $x$-coord & 1215 & 1215 & 1215 & 1215 & 1215 & 1215 & 1215 & 1215 & 1215 & 1215 & 1215 & 1215 & 1215 & 1215 & 1215 & 1215 & 1215 \\
\hline$F X \# 1$ & 373.84 & 374.07 & 374.34 & 374.64 & 375.06 & 375.39 & 375.59 & 375.97 & 376.16 & 376.42 & 376.76 & 376.92 & 377.13 & 377.39 & 377.57 & 377.80 & 378.08 \\
\hline$F X \# 2$ & 280.38 & 280.52 & 280.65 & 280.71 & 280.73 & 280.83 & 280.93 & 281.13 & 281.32 & 281.52 & 281.73 & 281.86 & 282.03 & 282.14 & 282.24 & 282.29 & 282.25 \\
\hline$F X \# 3$ & 218.99 & 219.06 & 219.26 & 219.41 & 219.52 & 219.58 & 219.67 & 219.79 & 219.92 & 219.99 & 220.15 & 220.28 & 220.34 & 220.54 & 220.78 & 220.96 & 221.12 \\
\hline
\end{tabular}




\begin{tabular}{|c|c|c|c|c|c|c|c|c|c|c|c|c|c|c|c|c|c|}
\hline$F X \# 4$ & 168.92 & 168.99 & 169.00 & 169.08 & 169.19 & 169.29 & 169.36 & 169.44 & 169.60 & 169.68 & 169.76 & 169.81 & 169.94 & 170.16 & 170.37 & 170.53 & 170.65 \\
\hline$F X \# 5$ & 122.32 & 122.52 & 122.71 & 122.86 & 122.93 & 123.04 & 123.12 & 123.23 & 123.32 & 123.38 & 123.39 & 123.42 & 123.47 & 123.52 & 123.53 & 123.56 & 123.57 \\
\hline Near wall FS & 46.60 & 46.46 & 46.30 & 46.22 & 46.26 & 46.25 & 46.24 & 46.21 & 46.28 & 46.30 & 46.36 & 46.39 & 46.47 & 46.64 & 46.84 & 46.97 & 47.08 \\
\hline$T @ F X \# 1$ & 297.65 & 297.65 & 297.65 & 297.65 & 297.65 & 297.65 & 297.65 & 297.65 & 297.65 & 297.65 & 297.65 & 297.65 & 297.65 & 297.65 & 297.65 & 297.65 & 297.65 \\
\hline$T @ F X \# 2$ & 299.66 & 299.65 & 299.65 & 299.64 & 299.64 & 299.63 & 299.63 & 299.62 & 299.62 & 299.62 & 299.61 & 299.61 & 299.60 & 299.60 & 299.59 & 299.59 & 299.58 \\
\hline$T @ F X \# 3$ & 301.69 & 301.68 & 301.68 & 301.67 & 301.67 & 301.66 & 301.66 & 301.65 & 301.65 & 301.64 & 301.64 & 301.63 & 301.63 & 301.62 & 301.62 & 301.62 & 301.61 \\
\hline$T @ F X \# 4$ & 303.74 & 303.73 & 303.73 & 303.72 & 303.72 & 303.71 & 303.71 & 303.70 & 303.70 & 303.69 & 303.69 & 303.69 & 303.68 & 303.68 & 303.67 & 303.67 & 303.66 \\
\hline$T(\boldsymbol{C}) \boldsymbol{F X}$ & 305.82 & 305.81 & 305.81 & 305.80 & 305.80 & 305.79 & 305.79 & 305.78 & 305.78 & 305.77 & 305.77 & 305.76 & 305.76 & 305.75 & 305.75 & 305.74 & 305.74 \\
\hline Wall $x$-coord & 88.33 & 88.33 & 88.34 & 88.34 & 88.35 & 88.35 & 88.36 & 88.37 & 88.37 & 88.38 & 88.38 & 88.39 & 88.39 & 88.40 & 88.41 & 88.41 & 88.42 \\
\hline Wall T & 309.46 & 309.47 & 309.48 & 309.48 & 309.48 & 309.48 & 309.48 & 309.48 & 309.47 & 309.47 & 309.46 & 309.46 & 309.45 & 309.44 & 309.43 & 309.42 & 309.42 \\
\hline Interferogram Image \# & 478 & Location & TC6 & $\delta \varepsilon$ slope & $-2.35 E-3$ & $\delta \varepsilon$ intercept & 5.34 & $\operatorname{Avg} F S$ & 50.53 & Avg Wall T & 309.37 & Gradient error & $1.31 E-4$ & & & & \\
\hline Scan y-coord & 1967 & 1968 & 1969 & 1970 & 1971 & 1972 & 1973 & 1974 & 1975 & 1976 & 1977 & 1978 & 1979 & 1980 & 1981 & 1982 & 1983 \\
\hline & 0.7206 & 0.7182 & 0.7159 & 0.7135 & 0.7112 & 0.7088 & 0.7065 & 0.7041 & 0.7018 & 0.6995 & 0.6971 & 0.6948 & 0.6924 & 0.6901 & 0.6877 & 0.6854 & 0.6830 \\
\hline Scan $x$-coord & 1246 & 1246 & 1246 & 1246 & 1246 & 1246 & 1246 & 1246 & 1246 & 1246 & 1246 & 1246 & 1246 & 1246 & 1246 & 1246 & 1246 \\
\hline$F X \# I$ & 413.09 & 413.39 & 413.53 & 413.60 & 413.78 & 414.21 & 414.84 & 415.65 & 416.46 & 417.04 & 417.69 & 418.36 & 418.99 & 419.37 & 419.81 & 420.24 & 420.69 \\
\hline$F X \# 2$ & 303.75 & 303.83 & 303.98 & 304.22 & 304.47 & 304.86 & 305.15 & 305.41 & 305.73 & 305.99 & 306.20 & 306.31 & 306.38 & 306.49 & 306.54 & 306.70 & 306.82 \\
\hline$F X \# 3$ & 234.36 & 234.52 & 234.66 & 234.76 & 234.79 & 234.83 & 234.92 & 235.03 & 235.17 & 235.33 & 235.51 & 235.64 & 235.79 & 235.99 & 236.20 & 236.42 & 236.59 \\
\hline$F X \# 4$ & 180.52 & 180.55 & $\begin{array}{l}254.00 \\
180.54\end{array}$ & 180.56 & 180.63 & $\begin{array}{l}234.83 \\
180.85\end{array}$ & $\begin{array}{l}234.92 \\
180.97\end{array}$ & $\begin{array}{l}235.03 \\
181.06\end{array}$ & 181.10 & $\begin{array}{l}2351.03 \\
181.09\end{array}$ & 181.04 & $\begin{array}{l}253.04 \\
180.96\end{array}$ & 180.90 & 180.89 & $\begin{array}{l}230.20 \\
180.91\end{array}$ & $\begin{array}{l}230.42 \\
180.93\end{array}$ & 181.01 \\
\hline$F X \# 5$ & 129.55 & 129.66 & 129.72 & 129.78 & 129.80 & 129.82 & 129.93 & 130.07 & 130.24 & 130.44 & 130.63 & 130.79 & 130.88 & 131.00 & 131.07 & 131.09 & 131.08 \\
\hline Near wall FS & 50.98 & 50.88 & 50.81 & 50.78 & 50.83 & 51.03 & 51.04 & 50.99 & 50.86 & 50.65 & 50.41 & 50.17 & 50.02 & 49.90 & 49.83 & 49.84 & 49.93 \\
\hline$T @ F X \# 1$ & 297.65 & 297.65 & 297.65 & 297.65 & 297.65 & 297.65 & 297.65 & 297.65 & 297.65 & 297.65 & 297.65 & 297.65 & 297.65 & 297.65 & 297.65 & 297.65 & 297.65 \\
\hline$T @ F X \# 2$ & 299.19 & 299.19 & 299.18 & 299.18 & 299.17 & 299.17 & 299.16 & 299.16 & 299.15 & 299.15 & 299.14 & 299.14 & 299.13 & 299.13 & 299.12 & 299.12 & 299.11 \\
\hline$T @ F X \# 3$ & 301.21 & 301.21 & 301.20 & 301.20 & 301.20 & 301.19 & 301.19 & 301.18 & 301.18 & 301.17 & 301.17 & 301.16 & 301.16 & 301.15 & 301.15 & 301.14 & 301.14 \\
\hline$T\left(\begin{array}{c}\mathrm{F} X \# 4 \\
-\end{array}\right.$ & 303.26 & 303.26 & 303.25 & 303.25 & 303.24 & 303.24 & 303.23 & 303.23 & 303.22 & 303.22 & 303.21 & 303.21 & 303.20 & 303.20 & 303.19 & 303.19 & 303.18 \\
\hline$T @ F X \# 5$ & 305.33 & 305.33 & 305.32 & 305.32 & 305.31 & 305.31 & 305.30 & 305.30 & 305.29 & 305.29 & 305.28 & 305.28 & 305.27 & 305.27 & 305.26 & 305.26 & 305.25 \\
\hline Wall $x$-coord & 82.66 & 82.67 & 82.68 & 82.69 & 82.70 & 82.70 & 82.71 & 82.72 & 82.73 & 82.74 & 82.74 & 82.75 & 82.76 & 82.77 & 82.78 & 82.78 & 82.79 \\
\hline Wall T & 309.36 & 309.37 & 309.37 & 309.37 & 309.36 & 309.35 & 309.35 & 309.35 & 309.36 & 309.37 & 309.38 & 309.39 & 309.39 & 309.40 & 309.40 & 309.39 & 309.38 \\
\hline Interferogram Image \# & 478 & Location & $T C 7$ & $\delta \varepsilon$ slope & $-2.21 E-3$ & $\delta \varepsilon$ intercept & 2.98 & $\operatorname{Avg} F S$ & 83.11 & Avg Wall T & 309.23 & Gradient error & $1.31 E-4$ & & & & \\
\hline Scan y-coord & 1152 & 1153 & 1154 & 1155 & 1156 & 1157 & 1158 & 1159 & 1160 & 1161 & 1162 & 1163 & 1164 & 1165 & 1166 & 1167 & 1168 \\
\hline$\delta \varepsilon$ & 0.4327 & 0.4305 & 0.4283 & 0.4261 & 0.4239 & 0.4217 & 0.4195 & 0.4173 & 0.4150 & 0.4128 & 0.4106 & 0.4084 & 0.4062 & 0.4040 & 0.4018 & 0.3996 & 0.3974 \\
\hline Scan $x$-coord & 1285 & 1285 & 1285 & 1285 & 1285 & 1285 & 1285 & 1285 & 1285 & 1285 & 1285 & 1285 & 1285 & 1285 & 1285 & 1285 & 1285 \\
\hline$F X \# 1$ & 507.59 & 508.13 & 508.27 & 509.95 & 513.30 & 511.15 & 512.08 & 513.08 & 515.76 & 514.02 & 514.26 & 514.43 & 515.34 & 514.31 & 514.95 & 515.75 & 516.72 \\
\hline$F X \# 2$ & 337.43 & 337.57 & 337.76 & 337.90 & 338.11 & 338.30 & 338.30 & 338.22 & 338.24 & 338.29 & 338.43 & 338.61 & 338.80 & 339.27 & 339.72 & 340.12 & 340.53 \\
\hline$F X \# 3$ & 254.33 & 254.51 & 254.69 & 254.70 & 254.73 & 254.72 & 254.87 & 255.14 & 255.33 & 255.48 & 255.65 & 255.82 & 256.04 & 256.27 & 256.35 & 256.41 & 256.65 \\
\hline$F X \# 4$ & 193.09 & 193.06 & 193.04 & 193.12 & 193.15 & 193.23 & 193.31 & 193.46 & 193.55 & 193.68 & 193.76 & 193.79 & 193.78 & 193.75 & 193.76 & 193.94 & 194.04 \\
\hline$F X \# 5$ & 135.89 & 136.08 & 136.25 & 136.42 & 136.55 & 136.66 & 136.75 & 136.89 & 136.88 & 136.96 & 137.12 & 137.35 & 137.68 & 137.99 & 138.30 & 138.59 & 138.77 \\
\hline$F X \# 6$ & 82.05 & 82.32 & 82.79 & 82.89 & 82.83 & 82.76 & 82.81 & 82.79 & 82.99 & 83.05 & 83.33 & 83.55 & 83.86 & 83.92 & 83.74 & 83.56 & 83.62 \\
\hline$F \boldsymbol{F} \# 7$ & 0.00 & 0.00 & 0.00 & 0.00 & 0.00 & 0.00 & 0.00 & 0.00 & 0.00 & 0.00 & 0.00 & 0.00 & 0.00 & 0.00 & 0.00 & 0.00 & 0.00 \\
\hline Near wall FS & 82.05 & 82.32 & 82.79 & 82.89 & 82.83 & 82.76 & 82.81 & 82.79 & 82.99 & 83.05 & 83.33 & 83.55 & 83.86 & 83.92 & 83.74 & 83.56 & 83.62 \\
\hline$T @ F X \# 1$ & 297.65 & 297.65 & 297.65 & 297.65 & 297.65 & 297.65 & 297.65 & 297.65 & 297.65 & 297.65 & 297.65 & 297.65 & 297.65 & 297.65 & 297.65 & 297.65 & 297.65 \\
\hline$T @ F X \# 2$ & 298.61 & 298.61 & 298.60 & 298.60 & 298.59 & 298.59 & 298.58 & 298.58 & 298.57 & 298.57 & 298.56 & 298.56 & 298.56 & 298.55 & 298.55 & 298.54 & 298.54 \\
\hline$T(F X \# 3$ & 300.63 & 300.63 & 300.63 & 300.62 & 300.62 & 300.61 & 300.61 & 300.60 & 300.60 & 300.59 & 300.59 & 300.58 & 300.58 & 300.58 & 300.57 & 300.57 & 300.56 \\
\hline$T(F X \# 4$ & 302.67 & 302.67 & 302.67 & 302.66 & 302.66 & 302.65 & 302.65 & 302.64 & 302.64 & 302.63 & 302.63 & 302.62 & 302.62 & 302.62 & 302.61 & 302.61 & 302.60 \\
\hline$T\left(\begin{array}{c}a \\
T\end{array}\right.$ & $\begin{array}{l}502.01 \\
304.74\end{array}$ & $\begin{array}{l}50.01 \\
304.73\end{array}$ & $\begin{array}{l}502.01 \\
304.73\end{array}$ & $\begin{array}{l}502.00 \\
304.73\end{array}$ & $\begin{array}{l}302.00 \\
304.72\end{array}$ & $\begin{array}{l}502.03 \\
304.72\end{array}$ & $\begin{array}{l}502.05 \\
304.71\end{array}$ & $\begin{array}{l}502.04 \\
304.71\end{array}$ & $\begin{array}{l}502.04 \\
304.70\end{array}$ & $\begin{array}{l}302.03 \\
304.70\end{array}$ & $\begin{array}{l}302.03 \\
304.69\end{array}$ & $\begin{array}{l}502.02 \\
304.69\end{array}$ & $\begin{array}{l}302.02 \\
304.68\end{array}$ & $\begin{array}{l}502.02 \\
304.68\end{array}$ & $\begin{array}{l}502.01 \\
304.68\end{array}$ & $\begin{array}{l}502.01 \\
304.67\end{array}$ & $\begin{array}{l}302.00 \\
304.67\end{array}$ \\
\hline$T @ F X \# 6$ & 306.83 & 306.83 & 306.82 & 306.82 & 306.81 & 306.81 & 306.80 & 306.80 & 306.80 & 306.79 & 306.79 & 306.78 & 306.78 & 306.77 & 306.77 & 306.76 & 306.76 \\
\hline$T @ F X \# 7$ & 308.95 & 308.95 & 308.94 & 308.94 & 308.93 & 308.93 & 308.93 & 308.92 & 308.92 & 308.91 & 308.91 & 308.90 & 308.90 & 308.89 & 308.89 & 308.88 & 308.88 \\
\hline Wall $x$-coord & 75.06 & 75.07 & 75.08 & 75.10 & 75.11 & 75.12 & 75.13 & 75.14 & 75.15 & 75.16 & 75.17 & 75.18 & 75.19 & 75.20 & 75.21 & 75.22 & 75.23 \\
\hline Wall T & 309.23 & 309.23 & 309.25 & 309.25 & 309.24 & 309.23 & 309.23 & 309.22 & 309.22 & 309.22 & 309.23 & 309.23 & 309.24 & 309.23 & 309.22 & 309.20 & 309.20 \\
\hline
\end{tabular}

\section{B.23 Tabulated Data for Interferogram 479}

\begin{tabular}{|c|c|c|c|c|c|c|c|c|c|c|c|c|c|c|c|c|c|}
\hline Interferogram Image \# & 479 & Lecation & $T C 2$ & $\delta$ s slope & $-254 E-3$ & $\delta$ s intercept & 13.93 & $\operatorname{Avg} F S$ & 0.00 & Avg Wall T & 308.09 & Gradient error & $1.06 E-4$ & & & & \\
\hline Scan y-coord & 5179 & $\frac{\text { Loction }}{5180}$ & 5181 & $\frac{0 \text { stope }}{5182}$ & $\frac{2.5+L-5}{5183}$ & 5184 & $\frac{1593}{5185}$ & $\frac{4 v g 10}{5186}$ & $\frac{0.00}{5187}$ & $\frac{108}{5188}$ & $\frac{50.0 \%}{5189}$ & 5190 & $\frac{1.00 L-4}{5191}$ & 5192 & 5193 & 5194 & 5195 \\
\hline & 0.7617 & 0.7591 & 0.7566 & 0.7541 & 0.7515 & 0.7490 & 0.7464 & 0.7439 & 0.7413 & 0.7388 & 0.7363 & 0.7337 & 0.7312 & 0.7286 & 0.7261 & 0.7236 & 0.7210 \\
\hline Scan $x$-coord & 873 & 873 & 873 & 873 & 873 & 873 & 873 & 873 & 873 & 873 & 873 & 873 & 873 & 873 & 873 & 873 & \\
\hline$F X \# 1$ & 304.44 & 303.91 & 304.14 & 304.64 & 305.27 & 306.03 & 305.98 & 299.94 & 301.27 & 301.92 & 302.77 & 302.29 & 308.69 & 309.36 & 304.59 & 306.66 & 307.98 \\
\hline$F X \# 2$ & 218.88 & $\begin{array}{l}218.91 \\
218.98\end{array}$ & 219.03 & 219.04 & 219.05 & 219.10 & 219.21 & 219.28 & 219.40 & 219.44 & 219.50 & 219.61 & $\begin{array}{l}519.69 \\
219.69\end{array}$ & 219.76 & 219.87 & $\begin{array}{l}500.00 \\
219.89\end{array}$ & 219.99 \\
\hline$F X \# 3$ & 167.45 & 167.51 & 167.69 & 167.81 & 167.92 & 167.99 & 168.10 & 168.18 & 168.34 & 168.47 & 168.64 & 168.78 & 168.85 & 168.94 & 168.99 & 169.11 & 169.20 \\
\hline$F X \# 4$ & 128.18 & 128.39 & 128.63 & 128.85 & 129.09 & 129.28 & 129.42 & 129.58 & 129.71 & 129.94 & 130.12 & 130.24 & 130.35 & 130.38 & 130.41 & 130.43 & 130.50 \\
\hline$F X \# 5$ & 95.20 & 95.33 & 95.49 & 95.68 & 95.85 & 95.90 & 95.92 & 95.84 & 95.75 & 95.57 & 95.47 & 95.45 & 95.50 & 95.52 & 95.52 & 95.51 & 95.36 \\
\hline$F X \# 6$ & 0.00 & 0.00 & 0.00 & 0.00 & 0.00 & 0.00 & 0.00 & 0.00 & 0.00 & 0.00 & 0.00 & 0.00 & 0.00 & 0.00 & 0.00 & 0.00 & 0.00 \\
\hline$F X \# 7$ & 0.00 & 0.00 & 0.00 & 0.00 & 0.00 & 0.00 & 0.00 & 0.00 & 0.00 & 0.00 & 0.00 & 0.00 & 0.00 & 0.00 & 0.00 & 0.00 & 0.00 \\
\hline Near wall FS & 0.00 & 0.00 & 0.00 & 0.00 & 0.00 & 0.00 & 0.00 & 0.00 & 0.00 & 0.00 & 0.00 & 0.00 & 0.00 & 0.00 & 0.00 & 0.00 & 0.00 \\
\hline$T @ F X \# 1$ & 297.23 & 297.23 & 297.23 & 297.23 & 297.23 & 297.23 & 297.23 & 297.23 & 297.23 & 297.23 & 297.23 & 297.23 & 297.23 & 297.23 & 297.23 & 297.23 & 297.23 \\
\hline$T$ (a) $F X \# 2$ & 298.80 & 298.79 & 298.79 & 298.78 & 298.78 & 298.77 & 298.77 & 298.76 & 298.76 & 298.75 & 298.75 & 298.74 & 298.74 & 298.73 & 298.73 & 298.72 & 298.72 \\
\hline$T$ (a) $F X \# 3$ & 300.81 & 300.81 & 300.80 & 300.79 & 300.79 & 300.78 & 300.78 & 300.77 & 300.77 & 300.76 & 300.76 & 300.75 & 300.75 & 300.74 & 300.74 & 300.73 & 300.73 \\
\hline$T(a) F X \# 4$ & 302.85 & 302.84 & 302.84 & 302.83 & 302.83 & 302.82 & 302.82 & 302.81 & 302.81 & 302.80 & 302.80 & 302.79 & 302.78 & 302.78 & 302.77 & 302.77 & 302.76 \\
\hline$T(a) F X \# 5$ & 304.91 & 304.91 & 304.90 & 304.90 & 304.89 & 304.88 & 304.88 & 304.87 & 304.87 & 304.86 & 304.86 & 304.85 & 304.85 & 304.84 & 304.84 & 304.83 & 304.83 \\
\hline$T(F X \# 6$ & 307.00 & 307.00 & 306.99 & 306.99 & 306.98 & 306.98 & 306.97 & 306.96 & 306.96 & 306.95 & 306.95 & 306.94 & 306.94 & 306.93 & 306.93 & 306.92 & 306.92 \\
\hline$T @ F X \# 7$ & 309.13 & 309.12 & 309.12 & 309.11 & 309.11 & 309.10 & 309.10 & 309.09 & 309.09 & 309.08 & 309.07 & 309.07 & 309.06 & 309.06 & 309.05 & 309.05 & 309.04 \\
\hline Wall $x$-coord & 77.17 & 77.17 & 77.17 & 77.17 & 77.17 & 77.17 & 77.17 & 77.17 & 77.17 & 77.17 & 77.17 & 77.17 & 77.17 & 77.17 & 77.16 & 77.16 & 77.16 \\
\hline \multirow[t]{2}{*}{$\begin{array}{r}\text { Wall X-coora } \\
\text { Wall } T\end{array}$} & 308.15 & 308.15 & 308.15 & 308.15 & 308.16 & 308.15 & 308.14 & 308.12 & 308.10 & 308.07 & 308.05 & 308.04 & 308.04 & 308.03 & 308.03 & 308.02 & 308.00 \\
\hline & & & & & & & & 218 & & & & & & & & & \\
\hline
\end{tabular}




\begin{tabular}{|c|c|c|c|c|c|c|c|c|c|c|c|c|c|c|c|c|c|}
\hline Interferogram Image \# & 479 & Location & $T C 3$ & $\delta \varepsilon$ slope & $-2.44 E-3$ & $\delta \varepsilon$ intercept & 11.45 & $A v g F S$ & 38.80 & Avg Wall T & 308.58 & Gradient error & $1.06 E-4$ & & & & \\
\hline Scan y-coord & 4364 & 4365 & 4366 & 4367 & 4368 & 4369 & 4370 & 4371 & 4372 & 4373 & 4374 & 4375 & 4376 & 4377 & 4378 & 4379 & 4380 \\
\hline & 0.7842 & 0.7818 & 0.7794 & 0.7769 & 0.7745 & 0.7720 & 0.7696 & 0.7671 & 0.7647 & 0.7622 & 0.7598 & 0.7574 & 0.7549 & 0.7525 & 0.7500 & 0.7476 & 0.7451 \\
\hline Scan $x$-coord & 929 & 929 & 929 & 929 & 929 & 929 & 929 & 929 & 929 & 929 & 929 & 929 & 929 & 929 & 929 & 929 & 929 \\
\hline$F X \# 1$ & 329.94 & 330.36 & 330.72 & 330.94 & 331.03 & 331.02 & 331.17 & 331.26 & 331.10 & 331.31 & 331.27 & 330.99 & 331.02 & 331.00 & 331.20 & 331.96 & 333.01 \\
\hline$F X \# 2$ & 241.05 & 241.04 & 241.05 & 241.09 & 241.15 & 241.30 & 241.48 & 241.67 & 241.81 & 241.91 & 242.00 & 242.08 & 242.19 & 242.29 & 242.48 & 242.75 & 242.97 \\
\hline$F X \# 3$ & 186.76 & 186.88 & 186.89 & 186.85 & 186.85 & 186.92 & 187.08 & 187.22 & 187.44 & 187.58 & 187.74 & $\begin{array}{l}272.06 \\
187.95\end{array}$ & 188.11 & 188.27 & 188.38 & 188.54 & 188.69 \\
\hline$F X \# 5$ & 104.90 & 104.94 & 105.04 & 105.17 & 105.32 & 105.46 & 105.63 & 105.84 & 106.04 & 106.18 & 106.30 & 106.40 & 106.55 & 106.68 & 106.78 & 106.84 & 106.95 \\
\hline Near wall FS & 38.83 & 38.90 & 38.92 & 38.89 & 38.86 & 38.84 & 38.77 & 38.67 & 38.60 & 38.60 & 38.63 & 38.73 & 38.75 & 38.82 & 38.88 & 38.97 & 38.96 \\
\hline$T @ F X \# 1$ & 297.23 & 297.23 & 297.23 & 297.23 & 297.23 & 297.23 & 297.23 & 297.23 & 297.23 & 297.23 & 297.23 & 297.23 & 297.23 & 297.23 & 297.23 & 297.23 & 297.23 \\
\hline$T @ F X \# 2$ & 298.84 & 298.84 & 298.83 & 298.83 & 298.83 & 298.82 & 298.82 & 298.81 & 298.81 & 298.80 & 298.80 & 298.79 & 298.79 & 298.78 & 298.78 & 298.77 & 298.77 \\
\hline$T @ F X \# 3$ & 300.86 & 300.85 & 300.85 & 300.84 & 300.84 & 300.83 & 300.83 & 300.82 & 300.82 & 300.81 & 300.81 & 300.80 & 300.80 & 300.80 & 300.79 & 300.79 & 300.78 \\
\hline$T @ F X \# 4$ & 302.90 & 302.89 & 302.89 & 302.88 & 302.88 & 302.87 & 302.87 & 302.86 & 302.86 & 302.85 & 302.85 & 302.84 & 302.84 & 302.83 & 302.83 & 302.82 & 302.82 \\
\hline Wall $x$-coord & 76.86 & 76.86 & 76.86 & 76.87 & 76.87 & 76.87 & 76.87 & 76.87 & 76.87 & 76.87 & 76.88 & 76.88 & 76.88 & 76.88 & 76.88 & 76.88 & 76.88 \\
\hline Wall T & 308.57 & 308.56 & 308.56 & 308.56 & 308.57 & 308.57 & 308.58 & 308.59 & 308.59 & 308.60 & 308.60 & 308.59 & 308.59 & 308.59 & 308.59 & 308.59 & 308.59 \\
\hline Interferogram Image \# & 479 & Location & $T C 4$ & $\delta \varepsilon$ slope & $-2.30 E-3$ & $\delta \varepsilon$ intercept & 8.87 & $A v g F S$ & 42.36 & Avg Wall T & 308.65 & Gradient error & $1.06 E-4$ & & & & \\
\hline Scan y-coord & 3548 & 3549 & 3550 & 3551 & 3552 & 3553 & 3554 & 3555 & 3556 & 3557 & 3558 & 3559 & 3560 & 3561 & 3562 & 3563 & 3564 \\
\hline$\delta \varepsilon$ & 0.7096 & 0.7073 & 0.7050 & 0.7027 & 0.7004 & 0.6981 & 0.6958 & 0.6935 & 0.6912 & 0.6889 & 0.6866 & 0.6843 & 0.6820 & 0.6797 & 0.6774 & 0.6751 & 0.6728 \\
\hline Scan $x$-coord & 935 & 935 & 935 & 935 & 935 & 935 & 935 & 935 & 935 & 935 & 935 & 935 & 935 & 935 & 935 & 935 & $\begin{array}{l}0.0120 \\
935\end{array}$ \\
\hline$F X \# 1$ & 354.72 & 354.94 & 355.12 & 355.52 & 355.91 & 356.36 & 356.81 & 357.06 & 357.28 & 357.87 & 358.28 & 359.12 & 359.59 & 360.37 & 360.92 & 361.42 & 362.04 \\
\hline$F X \# 2$ & 259.14 & 259.25 & 259.39 & 259.65 & 259.89 & 260.15 & 260.40 & 260.66 & 260.87 & 261.04 & 261.18 & 261.34 & 261.47 & 261.62 & 261.72 & 261.77 & 261.79 \\
\hline$F X \# 3$ & 199.58 & 199.71 & 199.89 & 200.02 & 200.08 & 200.15 & 200.22 & 200.34 & 200.46 & 200.58 & 200.66 & 200.73 & 200.84 & 200.94 & 201.06 & 201.16 & 201.20 \\
\hline$F X \# 4$ & 152.78 & 152.90 & 152.97 & 153.09 & 153.28 & 153.31 & 153.40 & 153.42 & 153.56 & 153.65 & 153.78 & 153.90 & 154.00 & 154.11 & 154.21 & 154.33 & 154.47 \\
\hline$F X \# 5$ & 110.19 & 110.28 & 110.40 & 110.50 & 110.62 & 110.82 & 111.05 & 111.21 & 111.30 & 111.38 & 111.48 & 111.60 & 111.69 & 111.85 & 112.05 & 112.23 & 112.37 \\
\hline Near wall FS & 42.59 & 42.61 & 42.58 & 42.58 & 42.66 & 42.49 & 42.36 & 42.21 & 42.26 & 42.27 & 42.29 & 42.31 & 42.31 & 42.26 & 42.16 & 42.09 & 42.10 \\
\hline$T @ F X \# 3$ & 300.71 & 300.70 & 300.70 & 300.69 & 300.69 & 300.68 & 300.68 & 300.67 & 300.67 & 300.67 & 300.66 & 300.66 & 300.65 & 300.65 & 300.64 & 300.64 & 300.63 \\
\hline$T(\mathbb{F} F \# 4$ & 302.74 & 302.74 & 302.73 & 302.73 & 302.73 & 302.72 & 302.72 & 302.71 & 302.71 & 302.70 & 302.70 & 302.69 & 302.69 & 302.68 & 302.68 & 302.67 & 302.67 \\
\hline$T @ F X \# 5$ & 304.81 & 304.80 & 304.80 & 304.79 & 304.79 & 304.78 & 304.78 & 304.77 & 304.77 & 304.76 & 304.76 & 304.75 & 304.75 & 304.75 & 304.74 & 304.74 & 304.73 \\
\hline Wall $x$-coord & 74.87 & 74.87 & 74.87 & 74.88 & 74.88 & 74.88 & 74.89 & 74.89 & 74.89 & 74.90 & 74.90 & 74.90 & 74.91 & 74.91 & 74.91 & 74.92 & 74.92 \\
\hline Wall $T$ & 308.63 & 308.63 & 308.63 & 308.63 & 308.63 & 308.64 & 308.65 & 308.66 & 308.66 & 308.66 & 308.66 & 308.66 & 308.66 & 308.66 & 308.67 & 308.68 & 308.68 \\
\hline Interferogram Image \# & 479 & Location & $T C 5$ & $\delta \varepsilon$ slope & $-2.36 E-3$ & $\delta \varepsilon$ intercept & 7.07 & $\operatorname{Avg} F S$ & 46.81 & Avg Wall $T$ & 308.80 & Gradient error & $1.06 E-4$ & & & & \\
\hline Scan y-coord & 2733 & 2734 & 2735 & 2736 & 2737 & 2738 & 2739 & 2740 & 2741 & 2742 & 2743 & 2744 & 2745 & 2746 & 2747 & 2748 & 2749 \\
\hline & 0.6149 & 0.6125 & 0.6101 & 0.6078 & 0.6054 & 0.6030 & 0.6007 & 0.5983 & 0.5960 & 0.5936 & 0.5912 & 0.5889 & 0.5865 & 0.5841 & 0.5818 & 0.5794 & 0.5771 \\
\hline Scan $x$-coord & 917 & 917 & 917 & 917 & 917 & 917 & 917 & 917 & 917 & 917 & 917 & 917 & 917 & 917 & 917 & 917 & 917 \\
\hline$F X \# 1$ & 398.06 & 398.96 & 399.67 & 400.33 & 400.90 & 401.25 & 401.55 & 401.87 & 402.25 & 402.34 & 402.70 & 403.04 & 403.26 & 403.58 & 403.77 & 404.06 & 404.41 \\
\hline$F X \# 2$ & 284.40 & 284.26 & 284.05 & 283.94 & 284.01 & 284.14 & 284.39 & 284.62 & 284.76 & 284.90 & 284.95 & 284.97 & 285.16 & 285.68 & 286.19 & 286.69 & 286.98 \\
\hline$F X \# 3$ & 216.71 & 216.88 & 217.07 & 217.20 & 217.42 & 217.56 & 217.70 & 217.78 & 217.94 & 218.22 & 218.57 & 218.72 & 218.86 & 218.99 & 219.03 & 218.94 & 218.85 \\
\hline$F X \# 4$ & 164.81 & 164.96 & 165.06 & 165.16 & 165.25 & 165.32 & 165.38 & 165.49 & 165.75 & 166.06 & 166.39 & 166.60 & 166.76 & 166.97 & 167.09 & 167.31 & 167.57 \\
\hline$F X \# 5$ & 118.03 & 118.37 & 118.66 & 118.86 & 119.04 & 119.18 & 119.22 & 119.20 & 119.31 & 119.37 & 119.46 & 119.49 & 119.51 & 119.58 & 119.61 & 119.64 & 119.66 \\
\hline Near wall FS & 46.79 & 46.60 & 46.40 & 46.31 & 46.21 & 46.14 & 46.17 & 46.29 & 46.45 & 46.69 & 46.93 & 47.11 & 47.25 & 47.39 & 47.47 & 47.67 & 47.91 \\
\hline$T @ F X \# 1$ & 297.23 & 297.23 & 297.23 & 297.23 & 297.23 & 297.23 & 297.23 & 297.23 & 297.23 & 297.23 & 297.23 & 297.23 & 297.23 & 297.23 & 297.23 & 297.23 & 297.23 \\
\hline$T @ F X \# 2$ & 298.50 & 298.49 & 298.49 & 298.49 & 298.48 & 298.48 & 298.47 & 298.47 & 298.46 & 298.46 & 298.45 & 298.45 & 298.44 & 298.44 & 298.43 & 298.43 & 298.42 \\
\hline$T @ F X \# 3$ & 300.51 & 300.51 & 300.50 & 300.50 & 300.49 & 300.49 & 300.48 & 300.48 & 300.47 & 300.47 & 300.46 & 300.46 & 300.45 & 300.45 & 300.45 & 300.44 & 300.44 \\
\hline$T @ F X \# 4$ & 302.55 & 302.54 & 302.54 & 302.53 & 302.53 & 302.52 & 302.52 & 302.51 & 302.51 & 302.50 & 302.50 & 302.49 & 302.49 & 302.48 & 302.48 & 302.47 & 302.47 \\
\hline$T$ @ $F X \# 5$ & 304.61 & 304.60 & 304.60 & 304.59 & 304.59 & 304.58 & 304.58 & 304.57 & 304.57 & 304.56 & 304.56 & 304.55 & 304.55 & 304.54 & 304.54 & 304.53 & 304.53 \\
\hline Wall $x$-coord & 71.19 & 71.20 & 71.20 & 71.21 & 71.21 & 71.22 & 71.22 & 71.23 & 71.23 & 71.24 & 71.25 & 71.25 & 71.26 & 71.26 & 71.27 & 71.27 & 71.28 \\
\hline Wall T & 308.79 & 308.81 & 308.82 & 308.83 & 308.84 & 308.84 & 308.84 & 308.83 & 308.82 & 308.80 & 308.79 & 308.78 & 308.77 & 308.76 & 308.75 & 308.74 & 308.72 \\
\hline Interferogram Image \# & 479 & Location & TC6 & $\delta \varepsilon$ slope & $-1.99 E-3$ & $\delta \varepsilon$ intercept & 4.15 & $\operatorname{Avg} F S$ & 49.28 & Avg Wall $T$ & 308.93 & Gradient error & $1.06 E-4$ & & & & \\
\hline Scan y-coord & 1918 & 1919 & 1920 & 1921 & 1922 & 1923 & 1924 & 1925 & 1926 & 1927 & 1928 & 1929 & 1930 & 1931 & 1932 & 1933 & 1934 \\
\hline$F X \# 3$ & 237.93 & 238.30 & 238.64 & 238.97 & 239.18 & 239.43 & 239.57 & 239.73 & 239.87 & 240.00 & 240.13 & 240.16 & 240.15 & 240.15 & 240.19 & 240.23 & 240.25 \\
\hline$F X \# 4$ & 180.59 & 180.69 & 180.75 & 180.86 & 181.17 & 181.40 & 181.80 & 182.11 & 182.28 & 182.43 & 182.51 & 182.55 & 182.56 & 182.60 & 182.57 & 182.48 & 182.43 \\
\hline$F X \# 5$ & 129.83 & 129.96 & 130.21 & 130.29 & 130.37 & 130.26 & 130.25 & 130.25 & 130.21 & 130.20 & 130.29 & 130.50 & 130.68 & 130.99 & 131.27 & 131.53 & 131.79 \\
\hline$F X \# 6$ & 79.93 & 79.84 & 79.91 & 80.13 & 80.44 & 80.74 & 81.09 & 81.35 & 81.50 & 81.48 & 81.54 & 81.62 & 81.81 & 82.08 & 82.41 & 82.55 & 82.66 \\
\hline Near wall FS & 49.90 & 50.12 & 50.30 & 50.16 & 49.93 & 49.51 & 49.17 & 48.90 & 48.71 & 48.72 & 48.75 & 48.87 & 48.87 & 48.91 & 48.86 & 48.99 & 49.13 \\
\hline$T @ F X \# 1$ & 297.23 & 297.23 & 297.23 & 297.23 & 297.23 & 297.23 & 297.23 & 297.23 & 297.23 & 297.23 & 297.23 & 297.23 & 297.23 & 297.23 & 297.23 & 297.23 & 297.23 \\
\hline$T @ F X \# 2$ & 298.01 & 298.01 & 298.01 & 298.00 & 298.00 & 297.99 & 297.99 & 297.99 & 297.98 & 297.98 & 297.97 & 297.97 & 297.97 & 297.96 & 297.96 & 297.95 & 297.95 \\
\hline$T @ F X \# 3$ & 300.03 & 300.02 & 300.02 & 300.01 & 300.01 & 300.01 & 300.00 & 300.00 & 299.99 & 299.99 & 299.99 & 299.98 & 299.98 & 299.97 & 299.97 & 299.97 & 299.96 \\
\hline$T(F X \# 4$ & 302.05 & 302.05 & 302.05 & 302.04 & 302.04 & 302.03 & 302.03 & 302.03 & 302.02 & 302.02 & 302.01 & 302.01 & 302.01 & 302.00 & 302.00 & 301.99 & 301.99 \\
\hline$T(F X \# 5$ & 304.11 & 304.11 & 304.10 & 304.10 & 304.09 & 304.09 & 304.09 & 304.08 & 304.08 & 304.07 & 304.07 & 304.06 & 304.06 & 304.06 & 304.05 & 304.05 & 304.04 \\
\hline$T @ F X \# 6$ & 306.19 & 306.19 & 306.18 & 306.18 & 306.18 & 306.17 & 306.17 & 306.16 & 306.16 & 306.15 & 306.15 & 306.15 & 306.14 & 306.14 & 306.13 & 306.13 & 306.13 \\
\hline
\end{tabular}




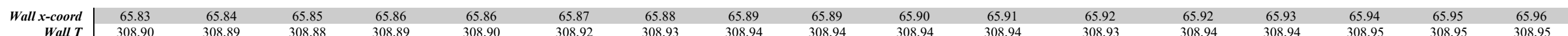

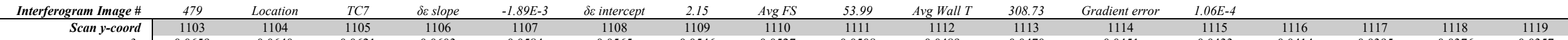

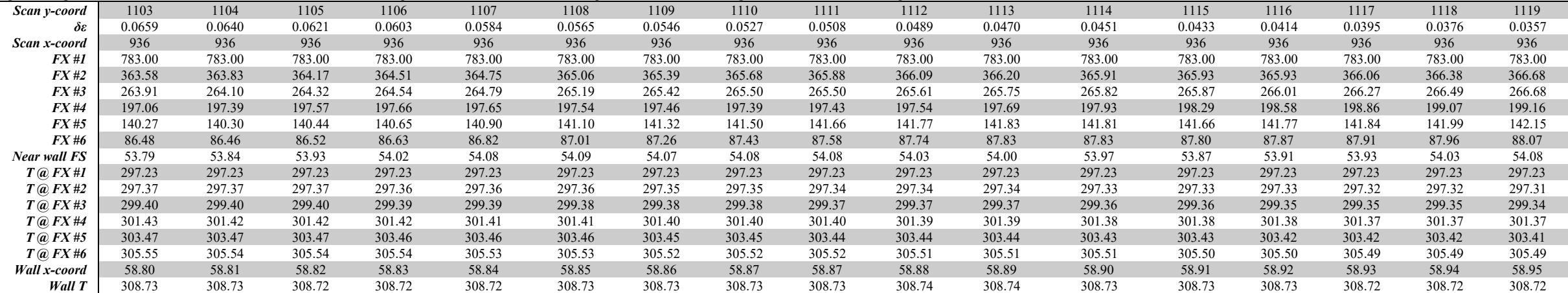

\section{B.24 Tabulated Data for Interferogram 480}

\begin{tabular}{|c|c|c|c|c|c|c|c|c|c|c|c|c|c|c|c|c|c|}
\hline Interferogram Image \# & 480 & Location & $T C 2$ & $\delta \varepsilon$ slope & $-2.41 E-3$ & $\delta \varepsilon$ intercept & 13.15 & $\operatorname{Avg} F S$ & 35.51 & Avg Wall T & 307.88 & Gradient error & $1.38 E-4$ & & & & \\
\hline Scan y-coord & 5252 & 5253 & 5254 & 5255 & 5256 & 5257 & 5258 & 5259 & 5260 & 5261 & 5262 & 5263 & 5264 & 5265 & 5266 & 5267 & 5268 \\
\hline & 0.4786 & 0.4762 & 0.4738 & 0.4714 & 0.4690 & 0.4666 & 0.4642 & 0.4618 & 0.4593 & 0.4569 & 0.4545 & 0.4521 & 0.4497 & 0.4473 & 0.4449 & 0.4425 & 0.4401 \\
\hline $\begin{array}{l}\text { Scan } x \text {-coord } \\
\text { Ser }\end{array}$ & $\begin{array}{l}0.41190 \\
1119\end{array}$ & $\begin{array}{l}0.4119 \\
119\end{array}$ & $\begin{array}{l}0.4119 \\
1119\end{array}$ & 1119 & 1119 & $\begin{array}{l}0.4000 \\
1119\end{array}$ & 1119 & $\begin{array}{l}0.4010 \\
1119\end{array}$ & 1119 & $\begin{array}{l}0.309 \\
1119\end{array}$ & $\begin{array}{l}0.4439 \\
1119\end{array}$ & 1119 & 1119 & 1119 & 1119 & 1119 & $\begin{array}{l}0.4401 \\
1119\end{array}$ \\
\hline$F X \# I$ & 383.75 & 383.51 & 383.72 & 384.19 & 384.89 & 388.69 & 389.75 & 390.81 & 391.29 & 392.14 & 392.73 & 393.51 & 394.25 & 394.87 & 395.38 & 396.04 & 396.28 \\
\hline$F X \# 2$ & 267.57 & 267.58 & 267.65 & 267.63 & 267.68 & 267.97 & 268.17 & 268.39 & 268.63 & 268.83 & 268.99 & 269.14 & 269.30 & 269.57 & 269.87 & 270.04 & 270.19 \\
\hline$F X \# 3$ & 210.13 & 210.25 & 210.44 & 210.55 & 210.64 & 210.77 & 210.89 & 210.97 & 210.99 & 211.06 & 211.12 & 211.16 & 211.19 & 211.34 & 211.47 & 211.53 & 211.61 \\
\hline$F X \# 5$ & 134.88 & 134.82 & 134.78 & 134.68 & 134.56 & 134.48 & 134.44 & 134.43 & 134.44 & 134.29 & 134.12 & 133.96 & 133.90 & 133.90 & 133.98 & 134.12 & 134.20 \\
\hline Near wall FS & 34.20 & 34.37 & 34.58 & 34.85 & 35.13 & 35.35 & 35.52 & 35.58 & 35.62 & 35.74 & 35.90 & 36.06 & 36.13 & 36.18 & 36.18 & 36.16 & 36.13 \\
\hline$T @ F X \# 1$ & 297.23 & 297.23 & 297.23 & 297.23 & $\begin{array}{l}297.13 \\
297.23\end{array}$ & 297.23 & 297.23 & 297.23 & $\begin{array}{r}39.02 \\
297.23\end{array}$ & 297.23 & 297.23 & 297.23 & 297.23 & 297.23 & 297.23 & 297.23 & 297.23 \\
\hline$T @ F X \# 2$ & 298.27 & 298.27 & 298.26 & 298.26 & 298.25 & 298.25 & 298.24 & 298.24 & 298.23 & 298.23 & 298.22 & 298.22 & 298.21 & 298.21 & 298.21 & 298.20 & 298.20 \\
\hline$T @ F X \# 3$ & 300.29 & 300.28 & 300.28 & 300.27 & 300.27 & 300.26 & 300.26 & 300.25 & 300.25 & 300.24 & 300.24 & 300.23 & 300.23 & 300.22 & 300.22 & 300.21 & 300.21 \\
\hline$T @ F X \# 4$ & 302.32 & 302.31 & 302.31 & 302.31 & 302.30 & 302.30 & 302.29 & 302.29 & 302.28 & 302.28 & 302.27 & 302.27 & 302.26 & 302.26 & 302.25 & 302.25 & 302.24 \\
\hline Wall $x$-coord & 109.52 & 109.52 & 109.52 & 109.52 & 109.52 & 109.52 & 109.52 & 109.52 & 109.52 & 109.52 & 109.52 & 109.52 & 109.51 & 109.51 & 109.51 & 109.51 & 109.51 \\
\hline Wall $T$ & 308.01 & 307.99 & 307.98 & 307.95 & 307.93 & 307.91 & 307.90 & 307.89 & 307.88 & 307.86 & 307.84 & 307.82 & 307.81 & 307.80 & 307.80 & 307.81 & 307.81 \\
\hline Interferogram Image \# & 480 & Location & $T C 3$ & $\delta \varepsilon$ slope & $-2.31 E-3$ & $\delta \varepsilon$ intercept & 10.69 & $\operatorname{Avg} F S$ & 40.02 & Avg Wall T & 308.48 & Gradient error & $1.38 E-4$ & & & & \\
\hline Scan y-coord & 4438 & 4439 & 4440 & 4441 & 4442 & 4443 & 4444 & 4445 & 4446 & 4447 & 4448 & 4449 & 4450 & 4451 & 4452 & 4453 & 4454 \\
\hline & 0.4370 & 0.4347 & 0.4324 & 0.4301 & 0.4278 & 0.4255 & 0.4231 & 0.4208 & 0.4185 & 0.4162 & 0.4139 & 0.4116 & 0.4093 & 0.4070 & 0.4047 & 0.4024 & 0.4000 \\
\hline Scan $x$-coord & 1196 & 1196 & 1196 & 1196 & 1196 & 1196 & 1196 & 1196 & 1196 & 1196 & 1196 & 1196 & 1196 & 1196 & 1196 & 1196 & 1196 \\
\hline$F X \# I$ & 426.94 & 428.03 & 430.40 & 430.95 & 431.30 & 431.80 & 431.97 & 431.76 & 431.98 & 431.95 & 432.34 & 432.92 & 433.17 & 435.68 & 436.51 & 437.12 & 437.82 \\
\hline$F X \# 2$ & 299.00 & 299.05 & $\begin{array}{l}4509.40 \\
293\end{array}$ & 299.40 & 299.60 & 299.79 & 299.89 & 300.02 & 300.09 & 300.06 & 300.08 & 300.16 & 300.30 & 300.64 & 300.84 & 300.97 & $\begin{array}{l}301.11 \\
301\end{array}$ \\
\hline$F X \# 3$ & $\begin{array}{l}299.00 \\
235.97\end{array}$ & 236.02 & $\begin{array}{l}299.23 \\
236.10\end{array}$ & 236.17 & 236.21 & 236.26 & $\begin{array}{l}299.89 \\
236.34\end{array}$ & $\begin{array}{l}300.02 \\
236.38\end{array}$ & 236.45 & 236.51 & $\begin{array}{l}300.08 \\
236.60\end{array}$ & $\begin{array}{l}300.10 \\
236.72\end{array}$ & $\begin{array}{l}300.30 \\
236.84\end{array}$ & $\begin{array}{l}300.04 \\
236.90\end{array}$ & $\begin{array}{l}300.84 \\
236.97\end{array}$ & 237.07 & 237.17 \\
\hline$F X \# 4$ & 189.31 & 189.39 & 189.47 & 189.59 & 189.79 & 189.90 & 190.02 & 190.16 & 190.25 & 190.38 & 190.52 & 190.70 & 190.84 & 190.99 & 191.19 & 191.29 & 191.36 \\
\hline$F X \# 5$ & 149.45 & 149.47 & 149.51 & 149.58 & 149.66 & 149.76 & 149.87 & 150.02 & 150.24 & 150.46 & 150.64 & 150.75 & 150.85 & 150.94 & 151.04 & 151.19 & 151.38 \\
\hline Near wall FS & 39.86 & 39.92 & 39.96 & 40.01 & 40.14 & 40.14 & 40.16 & 40.14 & 40.01 & 39.93 & 39.88 & 39.95 & 39.99 & 40.05 & 40.15 & 40.10 & 39.98 \\
\hline$T @ F X \# 3$ & 300.21 & 300.21 & 300.20 & 300.20 & 300.19 & 300.19 & 300.18 & 300.18 & 300.17 & 300.17 & 300.16 & 300.16 & 300.15 & 300.15 & 300.14 & 300.14 & 300.14 \\
\hline$T @ F X \# 4$ & 302.24 & 302.24 & 302.23 & 302.23 & 302.22 & 302.22 & 302.21 & 302.21 & 302.20 & 302.20 & 302.20 & 302.19 & 302.19 & 302.18 & 302.18 & 302.17 & 302.17 \\
\hline $\begin{array}{l}T(a) F X 4 \\
T(a) \# 5\end{array}$ & $\begin{array}{l}302.24 \\
304.30\end{array}$ & $\begin{array}{l}302.24 \\
304.30\end{array}$ & $\begin{array}{l}302.23 \\
304.29\end{array}$ & $\begin{array}{l}302.23 \\
304.29\end{array}$ & $\begin{array}{l}502.22 \\
304.28\end{array}$ & $\begin{array}{l}502.22 \\
304.28\end{array}$ & $\begin{array}{l}302.21 \\
304.27\end{array}$ & $\begin{array}{l}302.21 \\
304.27\end{array}$ & $\begin{array}{l}302.20 \\
304.26\end{array}$ & $\begin{array}{l}302.20 \\
304.26\end{array}$ & $\begin{array}{l}302.20 \\
304.25\end{array}$ & $\begin{array}{l}502.19 \\
304.25\end{array}$ & $\begin{array}{l}302.19 \\
304.24\end{array}$ & $\begin{array}{l}502.18 \\
304.24\end{array}$ & $\begin{array}{l}302.18 \\
304.23\end{array}$ & $\begin{array}{l}302.17 \\
304.23\end{array}$ & $\begin{array}{l}302.17 \\
304.22\end{array}$ \\
\hline Wall $x$-coord & $\begin{array}{l}304.30 \\
109.21\end{array}$ & 109.21 & $\begin{array}{l}304.29 \\
109.22\end{array}$ & $\begin{array}{l}304.29 \\
109.22\end{array}$ & $\begin{array}{r}304.28 \\
109.22\end{array}$ & $\begin{array}{l}304.28 \\
109.22\end{array}$ & 109.22 & $\begin{array}{l}304.27 \\
109.22\end{array}$ & $\begin{array}{l}304.20 \\
109.22\end{array}$ & $\begin{array}{l}304.20 \\
109.23\end{array}$ & $\begin{array}{l}304.25 \\
109.23\end{array}$ & $\begin{array}{l}504.25 \\
109.23\end{array}$ & $\begin{array}{r}004.24 \\
109.23\end{array}$ & $\begin{array}{r}304.24 \\
109.23\end{array}$ & $\begin{array}{l}304.23 \\
109.23\end{array}$ & $\begin{array}{l}304.23 \\
109.23\end{array}$ & $\begin{array}{l}304.22 \\
109.23 \\
\end{array}$ \\
\hline Wall T & 308.49 & 308.48 & 308.48 & 308.47 & 308.47 & 308.47 & 308.47 & 308.47 & 308.48 & 308.49 & 308.50 & 308.50 & 308.50 & 308.49 & 308.49 & 308.49 & 308.50 \\
\hline Interferogram Image \# & 480 & Location & $T C 4$ & $\delta \varepsilon$ slope & $-2.35 E-3$ & $\delta \varepsilon$ intercept & 8.84 & $A v g F S$ & 41.02 & Avg Wall T & 308.86 & Gradient error & $1.38 E-4$ & & & & \\
\hline Scan y-coord & 3623 & 3624 & 3625 & 3626 & 3627 & 3628 & $\begin{array}{l}0.04 \\
629\end{array}$ & 3630 & 3631 & 3632 & 3633 & 3634 & 3635 & 3636 & 3637 & 3638 & 3639 \\
\hline Scan $y$-coora & $\begin{array}{r}3023 \\
0.3170\end{array}$ & $\begin{array}{r}3024 \\
0.3146\end{array}$ & $\begin{array}{l}3023 \\
0.3123\end{array}$ & $\begin{array}{l}3620 \\
0.3099\end{array}$ & 0.3076 & 0.3052 & $\begin{array}{r}3029 \\
0.3029\end{array}$ & $\begin{array}{l}3630 \\
0.3005\end{array}$ & $\begin{array}{r}3631 \\
0.2981\end{array}$ & $\begin{array}{l}3625 \\
0.2958\end{array}$ & $\begin{array}{l}3633 \\
0.2934\end{array}$ & $\begin{array}{c}30.2941 \\
0.2911\end{array}$ & $\begin{array}{l}3635 \\
0.2887\end{array}$ & $\begin{array}{l}36280 \\
0.2864\end{array}$ & 0.2840 & 0.2817 & 0.2793 \\
\hline Scan $x$-coord & 1138 & 1138 & 1138 & 1138 & 1138 & 1138 & 1138 & 1138 & 1138 & 1138 & 1138 & 1138 & 1138 & 1138 & 1138 & 1138 & 1138 \\
\hline$F X \# 1$ & 468.00 & 468.46 & 468.31 & 468.57 & $\begin{array}{l}1100 \\
469.02\end{array}$ & 469.90 & 472.11 & 472.38 & 472.66 & 473.35 & 473.85 & 474.51 & 473.73 & 474.25 & 474.66 & 475.06 & 476.62 \\
\hline$F X \# 2$ & 322.63 & 322.84 & 323.08 & 323.37 & 323.67 & 323.89 & 324.17 & 324.29 & 324.40 & 324.64 & 324.94 & 325.25 & 325.52 & 325.90 & 326.27 & 326.56 & 326.84 \\
\hline$F X \# 3$ & 253.67 & 253.76 & 253.87 & 253.95 & 254.04 & 254.12 & 254.29 & 254.41 & 254.58 & 254.71 & 254.82 & 254.87 & 254.87 & 254.87 & 254.89 & 255.01 & 255.11 \\
\hline$F X \# 4$ & 203.80 & 203.88 & 203.95 & 204.06 & 204.12 & 204.20 & 204.24 & 204.39 & 204.51 & 204.59 & 204.71 & 204.84 & 204.94 & 204.98 & 205.03 & 205.09 & 205.11 \\
\hline FX\#5 & 160.38 & 160.52 & 160.68 & 160.71 & 160.73 & 160.77 & 160.83 & 160.99 & 161.20 & 161.42 & 161.58 & $\begin{array}{l}204.04 \\
161.67\end{array}$ & $\begin{array}{l}204.94 \\
161.68\end{array}$ & 161.70 & 161.72 & 161.78 & 161.81 \\
\hline $\begin{array}{l}F X \# 3 \\
F X \# 6\end{array}$ & $\begin{array}{l}100.38 \\
119.46\end{array}$ & $\begin{array}{l}160.52 \\
119.42\end{array}$ & $\begin{array}{l}160.68 \\
119.45\end{array}$ & $\begin{array}{l}160.91 \\
119.50\end{array}$ & $\begin{array}{l}160.13 \\
119.61\end{array}$ & 1100.71 & 1100.83 & $\begin{array}{l}100.99 \\
120.17\end{array}$ & $\begin{array}{l}161.20 \\
120.26\end{array}$ & $\begin{array}{l}161.42 \\
120.40\end{array}$ & $\begin{array}{l}101.58 \\
120.46\end{array}$ & $\begin{array}{l}101.6 \% \\
120.47\end{array}$ & $\begin{array}{l}101.68 \\
120.54\end{array}$ & $\begin{array}{l}101.10 \\
120.70\end{array}$ & $\begin{array}{l}101.12 \\
120.82\end{array}$ & 120.94 & $\begin{array}{l}161.81 \\
121.01\end{array}$ \\
\hline Near wall FS & 40.92 & 41.10 & 41.23 & 41.21 & 41.12 & 41.00 & 40.90 & 40.82 & 40.94 & 41.02 & 41.12 & 41.20 & 41.14 & 41.00 & 40.90 & 40.84 & 40.80 \\
\hline$T @ F X \# 1$ & 297.23 & 297.23 & 297.23 & 297.23 & 297.23 & 297.23 & 297.23 & 297.23 & 297.23 & 297.23 & 297.23 & 297.23 & 297.23 & 297.23 & 297.23 & 297.23 & 297.23 \\
\hline
\end{tabular}




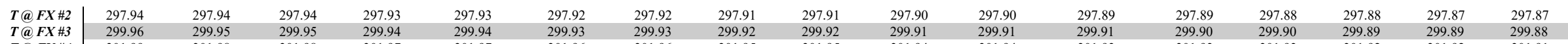

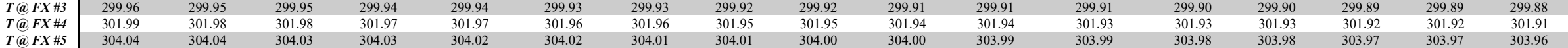

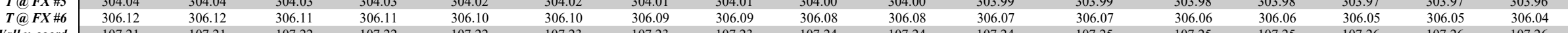

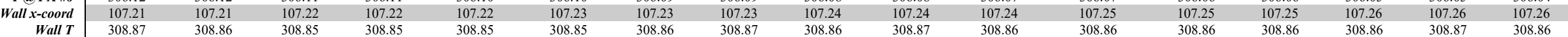

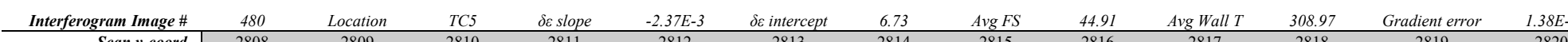

\begin{tabular}{|c|c|c|c|c|c|c|c|c|c|c|c|c|c|c|c|c|c|}
\hline & & & & & & & & & & & & & & & & & \\
\hline Scan y-coord & 2808 & 2809 & 2810 & 2811 & 2812 & 2813 & 2814 & 2815 & 2816 & 2817 & 2818 & 2819 & 2820 & 2821 & 2822 & 2823 & 2824 \\
\hline$\delta \varepsilon$ & 0.0773 & 0.0749 & 0.0726 & 0.0702 & 0.0678 & 0.0654 & 0.0631 & 0.0607 & 0.0583 & 0.0560 & 0.0536 & 0.0512 & 0.0489 & 0.0465 & 0.0441 & 0.0417 & 0.0394 \\
\hline Scan $x$-coord & 1644 & 1644 & 1644 & 1644 & 1644 & 1644 & 1644 & 1644 & 1644 & 1644 & 1644 & 1644 & 1644 & 1644 & 1644 & 1644 & 1644 \\
\hline$F X \# 1$ & 563.88 & 562.42 & 564.95 & 565.78 & 568.66 & 569.52 & 570.48 & 570.13 & 571.09 & 572.08 & 575.66 & 576.67 & 577.25 & 578.80 & 579.71 & 585.93 & 586.94 \\
\hline$F X \# 2$ & 358.04 & 358.24 & 358.67 & 359.03 & 359.33 & 359.36 & 359.28 & 359.12 & 359.15 & 359.41 & 359.93 & 360.40 & 360.77 & 361.01 & 361.10 & 361.24 & 361.49 \\
\hline$F X \# 3$ & 277.29 & 277.35 & 277.32 & 277.26 & 277.25 & 277.33 & 277.45 & 277.63 & 277.90 & 278.21 & 278.34 & 278.43 & 278.48 & 278.61 & 278.76 & 278.97 & 279.06 \\
\hline$F X \# 4$ & 220.63 & 220.71 & 220.75 & 220.90 & 221.21 & 221.63 & 221.97 & 222.20 & 222.34 & 222.37 & 222.38 & 222.46 & 222.58 & 222.70 & 222.86 & 222.99 & 223.08 \\
\hline$F X \# 5$ & 173.83 & 173.82 & 173.84 & 173.88 & 174.02 & 174.13 & 174.28 & 174.35 & 174.37 & 174.36 & 174.33 & 174.33 & 174.40 & 174.53 & 174.67 & 174.72 & 174.70 \\
\hline$F X \# 6$ & 128.49 & 128.60 & 128.74 & 128.87 & 129.04 & 129.14 & 129.31 & 129.44 & 129.54 & 129.51 & 129.46 & 129.49 & 129.62 & 129.73 & 129.86 & 130.01 & 130.20 \\
\hline Near wall FS & 45.35 & 45.22 & 45.11 & 45.02 & 44.99 & 44.99 & 44.97 & 44.91 & 44.83 & 44.85 & 44.87 & 44.84 & 44.77 & 44.80 & 44.81 & 44.71 & 44.50 \\
\hline$T @ F X \# 1$ & 297.23 & 297.23 & 297.23 & 297.23 & 297.23 & 297.23 & 297.23 & 297.23 & 297.23 & 297.23 & 297.23 & 297.23 & 297.23 & 297.23 & 297.23 & 297.23 & 297.23 \\
\hline$T @ F X \# 2$ & 297.53 & 297.52 & 297.52 & 297.51 & 297.51 & 297.50 & 297.50 & 297.49 & 297.49 & 297.48 & 297.48 & 297.47 & 297.47 & 297.46 & 297.46 & 297.45 & 297.45 \\
\hline$T @ F X \# 3$ & 299.54 & 299.54 & 299.53 & 299.53 & 299.52 & 299.52 & 299.51 & 299.51 & 299.51 & 299.50 & 299.50 & 299.49 & 299.49 & 299.48 & 299.48 & 299.47 & 299.47 \\
\hline$T$ (a) $F X \# 4$ & 301.57 & 301.56 & 301.56 & 301.55 & 301.55 & 301.55 & 301.54 & 301.54 & 301.53 & 301.53 & 301.52 & 301.52 & 301.51 & 301.51 & 301.50 & 301.50 & 301.49 \\
\hline$T$ (a) $F X \# 5$ & 303.62 & 303.61 & 303.61 & 303.60 & 303.60 & 303.59 & 303.59 & 303.58 & 303.58 & 303.58 & 303.57 & 303.57 & 303.56 & 303.56 & 303.55 & 303.55 & 303.54 \\
\hline$T @ F X \# 6$ & 305.70 & 305.69 & 305.69 & 305.68 & 305.68 & 305.67 & 305.67 & 305.66 & 305.66 & 305.65 & 305.65 & 305.64 & 305.64 & 305.63 & 305.63 & 305.62 & 305.62 \\
\hline Wall $x$-coord & 103.51 & 103.51 & 103.52 & 103.53 & 103.53 & 103.54 & 103.54 & 103.55 & 103.55 & 103.56 & 103.56 & 103.57 & 103.58 & 103.58 & 103.59 & 103.59 & 103.60 \\
\hline Wall T & 308.96 & 308.96 & 308.97 & 308.97 & 308.97 & 308.97 & 308.98 & 308.98 & 308.98 & 308.97 & 308.96 & 308.96 & 308.96 & 308.96 & 308.96 & 308.97 & 308.98 \\
\hline
\end{tabular}

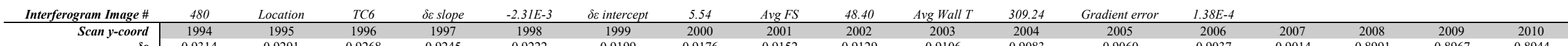

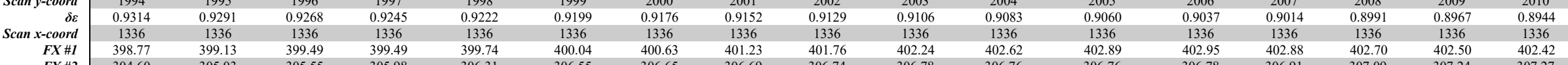

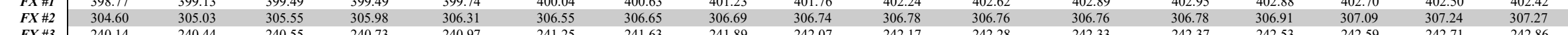

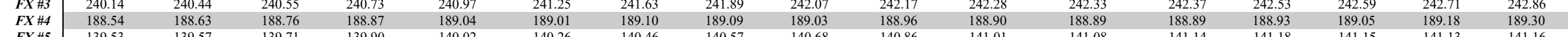

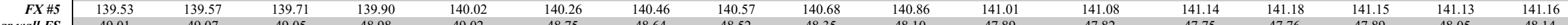

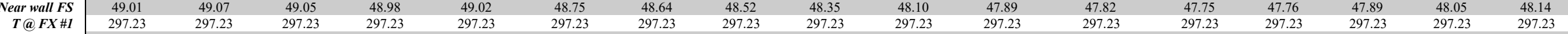

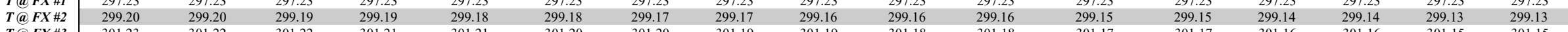

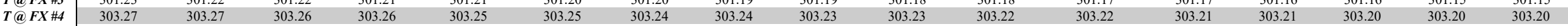

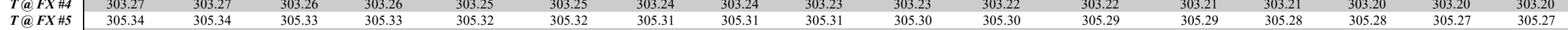

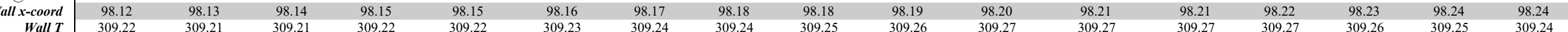

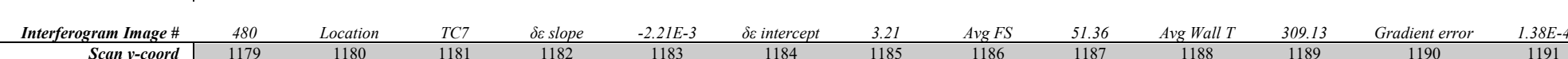

\begin{tabular}{|c|c|c|c|c|c|c|c|c|c|c|c|c|c|c|c|c|c|}
\hline & 800 & Location & & ozstope & $2.21 L-3$ & & & & & & & atent trror & & & & & \\
\hline Scan y-coord & 1179 & 1180 & 1181 & 1182 & 1183 & 1184 & 1185 & 1186 & 1187 & 1188 & 1189 & 1190 & 1191 & 1192 & 1193 & 1194 & 1195 \\
\hline & 0.6072 & 0.6050 & 0.6028 & 0.6006 & 0.5984 & 0.5962 & 0.5940 & 0.5918 & 0.5895 & 0.5873 & 0.5851 & 0.5829 & 0.5807 & 0.5785 & 0.5763 & 0.5741 & 0.5719 \\
\hline Scan $x$-coord & 1302 & 1302 & 1302 & 1302 & 1302 & 1302 & 1302 & 1302 & 1302 & 1302 & 1302 & 1302 & 1302 & 1302 & 1302 & 1302 & 1302 \\
\hline$F X \# 1$ & 485.52 & 485.77 & 486.27 & 486.83 & 487.72 & 488.23 & 488.63 & 489.05 & 489.70 & 490.41 & 491.66 & 492.56 & 493.05 & 493.68 & 494.45 & 494.62 & 494.74 \\
\hline$F X \# 2$ & 339.27 & 339.33 & 339.52 & 339.68 & 339.71 & 339.73 & 339.93 & 340.18 & 340.49 & 340.72 & 340.64 & 340.64 & 340.78 & 341.04 & 341.48 & 341.85 & 342.18 \\
\hline$F X \# 3$ & 261.76 & 261.91 & 261.93 & 261.91 & 261.87 & 261.94 & 262.15 & 262.43 & 262.62 & 262.81 & 262.91 & 263.00 & 263.06 & 263.13 & 263.25 & 263.41 & 263.57 \\
\hline$F X \# 4$ & 202.74 & 202.79 & 202.98 & 203.14 & 203.25 & 203.46 & 203.53 & 203.55 & 203.50 & 203.38 & 203.33 & 203.36 & 203.43 & 203.55 & 203.65 & 203.72 & 203.82 \\
\hline$F X \# 5$ & 148.49 & 148.60 & 148.63 & 148.70 & 148.70 & 148.75 & 148.85 & 149.04 & 149.36 & 149.68 & 149.93 & 150.33 & 150.60 & 150.82 & 150.90 & 150.91 & 150.90 \\
\hline$F X \# 6$ & 97.73 & 97.79 & 97.69 & 97.75 & 97.82 & 98.05 & 98.01 & 97.97 & 98.07 & 98.33 & 98.62 & 98.73 & 98.69 & 98.62 & 98.69 & 98.75 & 98.80 \\
\hline Near wall FS & 50.76 & 50.81 & 50.94 & 50.95 & 50.89 & 50.71 & 50.85 & 51.07 & 51.29 & 51.35 & 51.31 & 51.60 & 51.91 & 52.20 & 52.20 & 52.16 & 52.10 \\
\hline$T @ F X \# 1$ & 297.23 & 297.23 & 297.23 & 297.23 & 297.23 & 297.23 & 297.23 & 297.23 & 297.23 & 297.23 & 297.23 & 297.23 & 297.23 & 297.23 & 297.23 & 297.23 & 297.23 \\
\hline$T @ F X \# 2$ & 298.54 & 298.54 & 298.53 & 298.53 & 298.52 & 298.52 & 298.51 & 298.51 & 298.50 & 298.50 & 298.50 & 298.49 & 298.49 & 298.48 & 298.48 & 298.47 & 298.47 \\
\hline$T$ @ $F X \# 3$ & 300.56 & 300.56 & 300.55 & 300.55 & 300.54 & 300.54 & 300.53 & 300.53 & 300.53 & 300.52 & 300.52 & 300.51 & 300.51 & 300.50 & 300.50 & 300.49 & 300.49 \\
\hline$T @ F X \# 4$ & 302.60 & 302.60 & 302.59 & 302.59 & 302.58 & 302.58 & 302.57 & 302.57 & 302.56 & 302.56 & 302.56 & 302.55 & 302.55 & 302.54 & 302.54 & 302.53 & 302.53 \\
\hline$T @ F X \# 5$ & 304.66 & 304.66 & 304.66 & 304.65 & 304.65 & 304.64 & 304.64 & 304.63 & 304.63 & 304.62 & 304.62 & 304.61 & 304.61 & 304.61 & 304.60 & 304.60 & 304.59 \\
\hline$T @ F X \# 6$ & 306.76 & 306.75 & 306.75 & 306.74 & 306.74 & 306.73 & 306.73 & 306.72 & 306.72 & 306.71 & 306.71 & 306.71 & 306.70 & 306.70 & 306.69 & 306.69 & 306.68 \\
\hline Wall $x$-coord & 91.04 & 91.05 & 91.06 & 91.07 & 91.08 & 91.08 & 91.09 & 91.10 & 91.11 & 91.12 & 91.13 & 91.14 & 91.15 & 91.16 & 91.17 & 91.18 & 91.19 \\
\hline Wall T & 309.16 & 309.15 & 309.14 & 309.14 & 309.14 & 309.14 & 309.14 & 309.13 & 309.13 & 309.13 & 309.14 & 309.14 & 309.13 & 309.12 & 309.12 & 309.11 & 309.11 \\
\hline
\end{tabular}

\section{B.25 Tabulated Data for Interferogram 481}

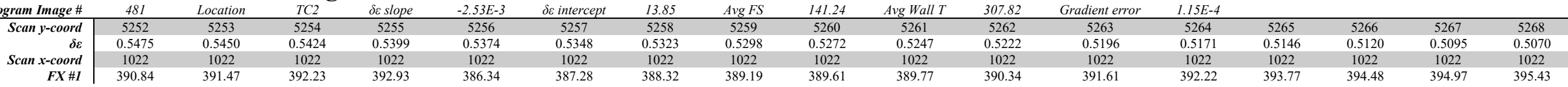




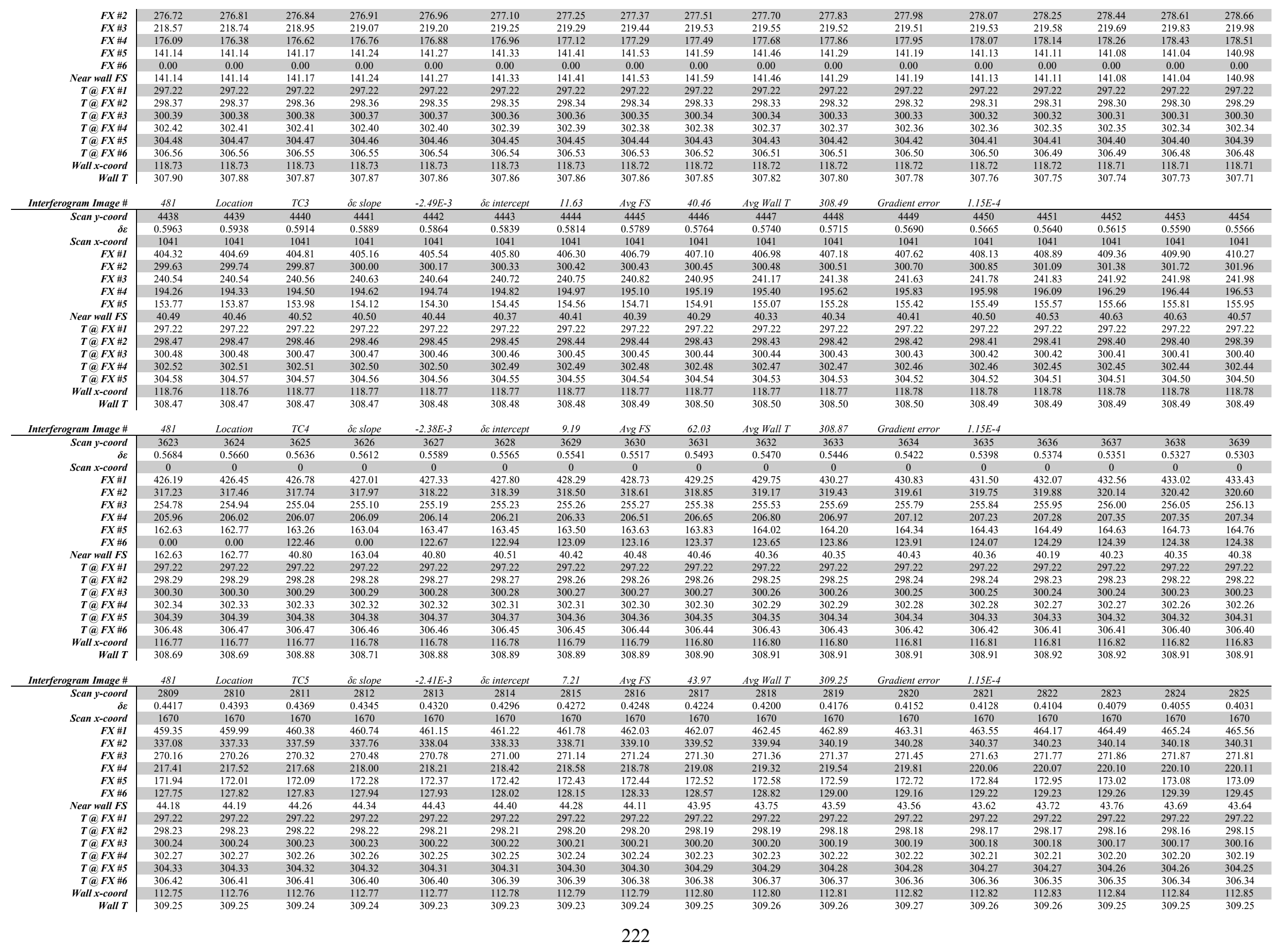




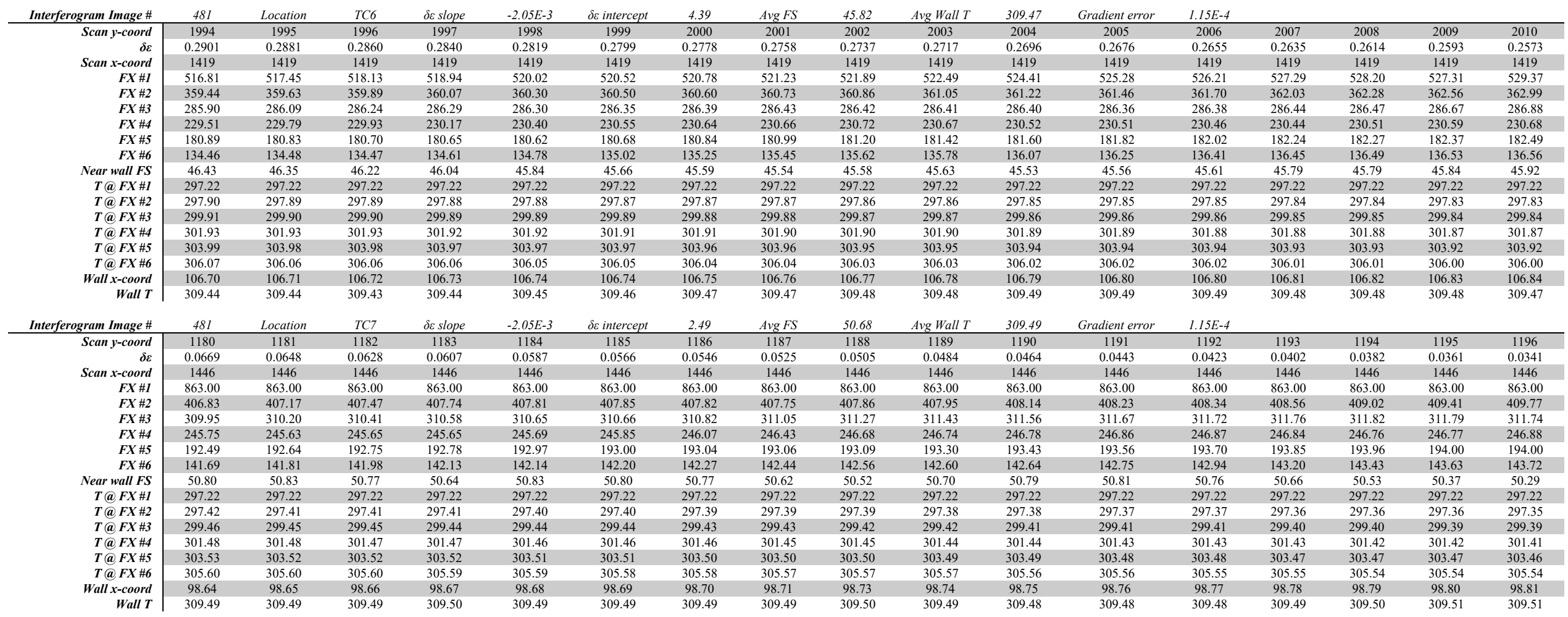

\section{B.26 Tabulated Data for Interferogram 482}

\begin{tabular}{|c|c|c|c|c|c|c|c|c|c|c|c|c|c|c|c|c|c|}
\hline Interferogram Image \# & 482 & Location & $T C 2$ & $\delta \varepsilon$ slope & $-2.54 E-3$ & $\delta \varepsilon$ intercept & 13.86 & $\operatorname{Avg} F S$ & 127.88 & Avg Wall $T$ & 307.77 & Gradient error & $1.36 E-4$ & & & & \\
\hline Scan y-coord & 5216 & 5217 & 5218 & 5219 & 5220 & 5221 & 5222 & 5223 & 5224 & 5225 & 5226 & 5227 & 5228 & 5229 & 5230 & 5231 & 5232 \\
\hline$\delta \varepsilon$ & 0.6296 & 0.6271 & 0.6246 & 0.6220 & 0.6195 & 0.6169 & 0.6144 & 0.6119 & 0.6093 & 0.6068 & 0.6043 & 0.6017 & 0.5992 & 0.5967 & 0.5941 & 0.5916 & 0.5890 \\
\hline Scan $x$-coord & 820 & 820 & 820 & 820 & 820 & 820 & 820 & 820 & 820 & 820 & 820 & 820 & 820 & 820 & 820 & 820 & 820 \\
\hline$F X \# 1$ & 361.27 & 361.34 & 361.19 & 361.44 & 361.75 & 362.53 & 359.81 & 360.66 & 361.79 & 362.94 & 363.82 & 364.84 & 365.22 & 363.45 & 364.02 & 365.46 & 364.67 \\
\hline$F X \# 2$ & 258.07 & 257.99 & 258.07 & 258.20 & 258.43 & 258.64 & 258.78 & 258.86 & 258.91 & 258.95 & 259.11 & 259.23 & 259.36 & 259.63 & 259.72 & 259.59 & 259.72 \\
\hline$F X \# 3$ & 202.87 & 202.99 & 203.09 & 203.26 & 203.50 & 203.69 & 203.83 & 203.84 & 203.86 & 203.91 & 203.91 & 203.99 & 204.14 & 204.28 & 204.34 & 204.43 & 204.54 \\
\hline$F X \# 5$ & 127.02 & 127.16 & 127.27 & 127.28 & 127.38 & 127.48 & 127.63 & 127.84 & 127.96 & 128.06 & 128.19 & 128.28 & 128.34 & 128.39 & 128.51 & 128.60 & 128.64 \\
\hline$F X \# 6$ & 0.00 & 0.00 & 0.00 & 0.00 & 0.00 & 0.00 & 0.00 & 0.00 & 0.00 & 0.00 & 0.00 & 0.00 & 0.00 & 0.00 & 0.00 & 0.00 & 0.00 \\
\hline Near wall FS & 127.02 & 127.16 & 127.27 & 127.28 & 127.38 & 127.48 & 127.63 & 127.84 & 127.96 & 128.06 & 128.19 & 128.28 & 128.34 & 128.39 & 128.51 & 128.60 & 128.64 \\
\hline$T(F X \# 1$ & 297.26 & 297.26 & 297.26 & 297.26 & 297.26 & 297.26 & 297.26 & 297.26 & 297.26 & 297.26 & 297.26 & 297.26 & 297.26 & 297.26 & 297.26 & 297.26 & 297.26 \\
\hline$T @ F X \# 2$ & 298.57 & 298.57 & 298.56 & 298.56 & 298.55 & 298.55 & 298.54 & 298.54 & 298.53 & 298.53 & 298.52 & 298.52 & 298.51 & 298.51 & 298.50 & 298.50 & 298.49 \\
\hline$T @ F X \# 3$ & 300.59 & 300.58 & 300.58 & 300.57 & 300.57 & 300.56 & 300.56 & 300.55 & 300.55 & 300.54 & 300.54 & 300.53 & 300.53 & 300.52 & 300.52 & 300.51 & 300.51 \\
\hline$T @ F X \# 5$ & 304.69 & 304.68 & 304.68 & 304.67 & 304.67 & 304.66 & 304.66 & 304.65 & 304.64 & 304.64 & 304.63 & 304.63 & 304.62 & 304.62 & 304.61 & 304.61 & 304.60 \\
\hline$T(a)=6$ & 306.78 & 306.77 & 306.77 & 306.76 & 306.75 & 306.75 & 306.74 & 306.74 & 306.73 & 306.73 & 306.72 & 306.72 & 306.71 & 306.71 & 306.70 & 306.70 & 306.69 \\
\hline Wall $x$-coord & 110.54 & 110.53 & 110.53 & 110.53 & 110.53 & 110.53 & 110.53 & 110.53 & 110.53 & 110.53 & 110.53 & 110.53 & 110.53 & 110.52 & 110.52 & 110.52 & 110.52 \\
\hline Wall T & 307.79 & 307.79 & 307.79 & 307.77 & 307.77 & 307.77 & 307.77 & 307.78 & 307.78 & 307.78 & 307.78 & 307.78 & 307.77 & 307.77 & 307.76 & 307.76 & 307.76 \\
\hline Interferogram Image \# & 482 & Location & $T C 3$ & $\delta \varepsilon$ slope & $-2.43 E-3$ & $\delta \varepsilon$ intercept & 11.36 & $\operatorname{Avg} F S$ & 39.48 & Avg Wall $T$ & 308.42 & Gradient error & $1.36 E-4$ & & & & \\
\hline Scan y-coord & 4402 & 4403 & 4404 & 4405 & 4406 & 4407 & 4408 & 4409 & 4410 & 4411 & 4412 & 4413 & 4414 & 4415 & 4416 & 4417 & 4418 \\
\hline & 0.6471 & 0.6446 & 0.6422 & 0.6398 & 0.6373 & 0.6349 & 0.6325 & 0.6300 & 0.6276 & 0.6252 & 0.6227 & 0.6203 & 0.6179 & 0.6154 & 0.6130 & 0.6105 & 0.6081 \\
\hline Scan $x$-coord & 907 & 907 & 907 & 907 & 907 & 907 & 907 & 907 & 907 & 907 & 907 & 907 & 907 & 907 & 907 & 907 & 907 \\
\hline$F X \# 1$ & 385.47 & 385.87 & 386.28 & 386.47 & 386.65 & 386.86 & 387.03 & 387.17 & 387.05 & 387.04 & 387.30 & 387.39 & 387.59 & 387.94 & 388.16 & 388.72 & 389.54 \\
\hline$F X \# 2$ & 283.12 & 283.02 & 282.97 & 282.88 & 282.91 & 283.03 & 283.14 & 283.32 & 283.38 & 283.51 & 283.60 & 283.65 & 283.69 & 283.77 & 283.91 & 284.08 & 284.32 \\
\hline$F X \# 3$ & 224.38 & 224.44 & 224.50 & 224.55 & 224.58 & 224.62 & 224.68 & 224.78 & 224.92 & 225.13 & 225.31 & 225.48 & 225.68 & 225.91 & 226.12 & 226.28 & 226.38 \\
\hline$F X \# 4$ & 179.70 & 179.73 & 179.78 & 179.89 & 180.08 & 180.25 & 180.38 & 180.53 & 180.65 & 180.78 & 180.89 & 181.02 & 181.21 & 181.38 & 181.60 & 181.74 & 181.81 \\
\hline
\end{tabular}




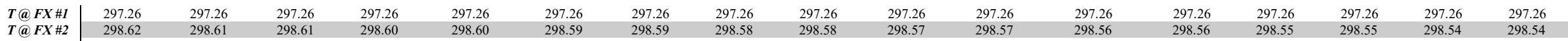

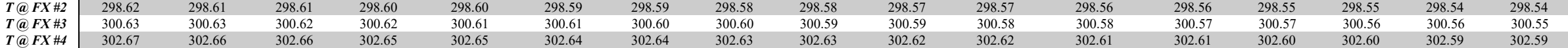

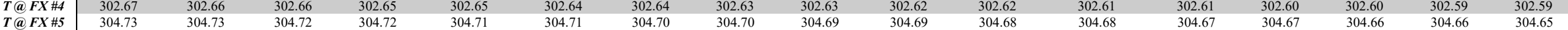

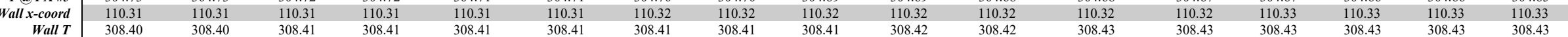

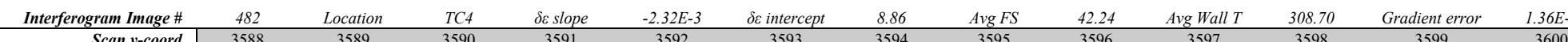

\begin{tabular}{|c|c|c|c|c|c|c|c|c|c|c|c|c|c|c|c|c|c|}
\hline Scan $y$-coord & 3588 & 358 & 3590 & 359 & 3592 & 3593 & 3594 & 3595 & 3596 & 3597 & 3598 & 3599 & 3600 & 3601 & 3602 & 3603 & 3604 \\
\hline & 0.5454 & 0.5431 & 0.5408 & 0.5385 & 0.5361 & 0.5338 & 0.5315 & 0.5292 & 0.5269 & 0.5246 & 0.5222 & 0.5199 & 0.5176 & 0.5153 & 0.5130 & 0.5106 & 0.5083 \\
\hline Scan $x$-coord & 1085 & 1085 & 1085 & 1085 & 1085 & 1085 & 1085 & 1085 & 1085 & 1085 & 1085 & 1085 & 1085 & 1085 & 1085 & 1085 & 1085 \\
\hline$F X \# 1$ & 420.43 & 420.63 & 420.77 & 421.01 & 421.22 & 422.01 & 422.01 & 422.48 & 423.11 & 424.38 & 424.68 & 425.05 & 425.32 & 425.77 & 426.27 & 426.82 & 427.50 \\
\hline$F X \# 2$ & 304.09 & 304.30 & 304.46 & 304.70 & 304.94 & 305.25 & 305.41 & 305.55 & 305.71 & 305.85 & 305.97 & 306.24 & 306.46 & 306.68 & 306.80 & 306.94 & 307.12 \\
\hline$F X \# 3$ & 240.46 & 240.59 & 240.71 & 240.81 & 240.91 & 241.02 & 241.13 & 241.22 & 241.29 & 241.42 & 241.54 & $\begin{array}{l}300.24 \\
241.68\end{array}$ & 241.81 & 241.93 & 242.04 & 242.13 & 242.20 \\
\hline$F X \# 4$ & 192.13 & 192.24 & 192.41 & 192.55 & 192.69 & 192.77 & 192.80 & 192.86 & 193.00 & 193.15 & 193.27 & 193.35 & 193.41 & 193.51 & 193.59 & 193.62 & 193.64 \\
\hline$F X \# 5$ & 149.97 & 150.05 & 150.10 & 150.17 & 150.27 & 150.42 & 150.57 & 150.67 & 150.75 & 150.81 & 150.86 & 150.96 & 151.11 & 151.36 & 151.56 & 151.65 & $\begin{array}{l}151.66 \\
151.64\end{array}$ \\
\hline Near wall FS & 42.16 & 42.19 & 42.31 & 42.38 & 42.42 & 42.35 & 42.24 & 42.19 & 42.25 & 42.34 & 42.42 & 42.39 & 42.30 & 42.15 & 42.04 & 41.97 & 41.98 \\
\hline$T @ F X \# 1$ & $\begin{array}{l}29.10 \\
297.26\end{array}$ & $\begin{array}{l}42.19 \\
297.26\end{array}$ & $\begin{array}{r}42.31 \\
297.26\end{array}$ & $\begin{array}{r}42.38 \\
297.26\end{array}$ & 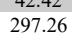 & $\begin{array}{l}29.53 .26 \\
297\end{array}$ & $\begin{array}{r}29.24 \\
297.26\end{array}$ & $\begin{array}{l}29.19 \\
297.26\end{array}$ & $\begin{array}{l}42.25 \\
297.26\end{array}$ & $\begin{array}{l}42.54 \\
297.26\end{array}$ & $\begin{array}{l}\begin{aligned} 42.42 \\
297.26\end{aligned}\end{array}$ & $\begin{array}{l}42.59 \\
297.26\end{array}$ & $\begin{array}{l}\begin{array}{r}42.50 \\
297.26\end{array}\end{array}$ & $\begin{array}{l}29.13 \\
297.26\end{array}$ & 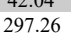 & 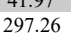 & $\begin{array}{l}41.98 \\
297.26\end{array}$ \\
\hline$T @ F X \# 2$ & 298.43 & 298.43 & 298.42 & 298.42 & 298.42 & 298.41 & 298.41 & 298.40 & 298.40 & 298.39 & 298.39 & 298.38 & 298.38 & 298.37 & 298.37 & 298.36 & 298.36 \\
\hline$T(F X \# 3$ & 300.45 & 300.44 & 300.44 & 300.44 & 300.43 & 300.43 & 300.42 & 300.42 & 300.41 & 300.41 & 300.40 & 300.40 & 300.39 & 300.39 & 300.38 & 300.38 & 300.37 \\
\hline$T(a) F X \#$ & 302.48 & 302.48 & 302.48 & 302.47 & 302.47 & 302.46 & 302.46 & 302.45 & 302.45 & 302.44 & 302.44 & 302.43 & 302.43 & 302.42 & 302.42 & 302.41 & 302.41 \\
\hline$T @ F X \# 5$ & 304.55 & 304.54 & 304.54 & 304.53 & 304.53 & 304.52 & 304.52 & 304.51 & 304.51 & 304.50 & 304.50 & 304.49 & 304.49 & 304.48 & 304.48 & 304.47 & 304.47 \\
\hline Wall $x$-coord & 108.24 & 108.24 & 108.25 & 108.25 & 108.26 & 108.26 & 108.26 & 108.27 & 108.27 & 108.27 & 108.28 & 108.28 & 108.28 & 108.29 & 108.29 & 108.30 & 108.30 \\
\hline Wall T & 308.70 & $\begin{array}{l}10.24 \\
308.70\end{array}$ & $\begin{array}{l}100.25 \\
308.69\end{array}$ & 308.69 & $\begin{array}{l}10.20 \\
308.68\end{array}$ & $\begin{array}{l}100.20 \\
308.69\end{array}$ & $\begin{array}{l}108.26 \\
30870\end{array}$ & $\begin{array}{l}108.27 \\
30870\end{array}$ & $\begin{array}{l}108.21 \\
308.69\end{array}$ & $\begin{array}{l}108.21 \\
308.69\end{array}$ & $\begin{array}{l}108.28 \\
308.68\end{array}$ & $\begin{array}{l}108.28 \\
308.68\end{array}$ & $\begin{array}{l}108.68 \\
308.69\end{array}$ & $\begin{array}{l}108.29 \\
308.70\end{array}$ & $\begin{array}{l}108.29 \\
308.71\end{array}$ & $\begin{array}{l}108.30 \\
308.72\end{array}$ & $\begin{array}{l}10.300 \\
308.71\end{array}$ \\
\hline
\end{tabular}

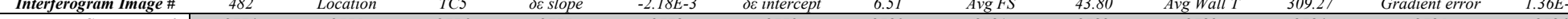

\begin{tabular}{|c|c|c|c|c|c|c|c|c|c|c|c|c|c|c|c|c|c|}
\hline & & & & & & & & & & & & & & & & & \\
\hline Scan y-coord & 2774 & 2775 & 2776 & 2777 & 2778 & 2779 & 2780 & 2781 & 2782 & 2783 & 2784 & 2785 & 2786 & 2787 & 2788 & 2789 & 2790 \\
\hline $\begin{array}{r}\text { Scan } y-c o r a \\
\delta \varepsilon\end{array}$ & 0.4553 & 0.4532 & 0.4510 & 0.4488 & 0.4466 & 0.4444 & 0.4423 & 0.4401 & 0.4379 & 0.4357 & 0.4335 & 0.4314 & 0.4292 & 0.4270 & 0.4248 & 0.4226 & 0.4204 \\
\hline Scan $x$-coord & 1137 & 1137 & 1137 & 1137 & 1137 & 1137 & 1137 & 1137 & 1137 & 1137 & 1137 & 1137 & 1137 & 1137 & 1137 & 1137 & 1137 \\
\hline$F X \# 1$ & 484.62 & 485.94 & 486.25 & 487.15 & 487.65 & 489.99 & 490.29 & 491.29 & 492.06 & 492.74 & 493.56 & 494.21 & 495.10 & 495.84 & 496.39 & 496.86 & 497.39 \\
\hline$F X \# 2$ & 336.33 & 336.57 & 336.81 & 337.24 & 337.53 & 337.84 & 338.06 & 338.28 & 338.49 & 338.67 & 338.98 & 339.28 & 339.67 & 339.67 & 339.81 & 339.88 & 339.79 \\
\hline$F X \# 3$ & 262.76 & 263.00 & 263.13 & 263.27 & 263.32 & 263.35 & 263.48 & 263.70 & 263.98 & 264.18 & 264.29 & 264.33 & 264.26 & 264.20 & 264.21 & 264.37 & 264.56 \\
\hline$F X \# 4$ & 208.63 & 208.72 & 208.76 & 208.75 & 208.77 & 208.92 & 209.22 & 209.56 & 209.78 & 209.92 & 210.10 & 210.25 & 210.50 & 210.70 & 210.96 & 211.14 & 211.31 \\
\hline$F X \# 5$ & 162.92 & 163.02 & 163.17 & 163.31 & 163.43 & 163.46 & 163.47 & 163.48 & 163.48 & 163.46 & 163.61 & 163.66 & 163.85 & 163.94 & $\begin{array}{l}163.99 \\
163.99\end{array}$ & 164.06 & 164.07 \\
\hline$F X \# 6$ & 118.96 & 119.19 & 119.33 & 119.34 & 119.35 & 119.33 & 119.29 & 119.29 & 119.43 & 119.70 & 119.97 & 120.17 & 120.35 & 120.46 & 120.51 & 120.56 & 120.58 \\
\hline Near wall FS & 43.96 & 43.83 & 43.84 & 43.96 & 44.08 & 44.12 & 44.17 & 44.20 & 44.05 & 43.77 & 43.64 & 43.49 & 43.50 & 43.48 & 43.48 & 43.50 & 43.49 \\
\hline$T @ F X \# 1$ & 297.26 & 297.26 & 297.26 & 297.26 & 297.26 & 297.26 & 297.26 & 297.26 & 297.26 & 297.26 & 297.26 & 297.26 & 297.26 & 297.26 & 297.26 & 297.26 & 297.26 \\
\hline$T @ F X \# 2$ & 298.25 & 298.25 & 298.24 & 298.24 & 298.24 & 298.23 & 298.23 & 298.22 & 298.22 & 298.21 & 298.21 & 298.20 & 298.20 & 298.20 & 298.19 & 298.19 & 298.18 \\
\hline$T @ F X \# 3$ & 300.27 & 300.27 & 300.26 & 300.26 & 300.25 & 300.25 & 300.24 & 300.24 & 300.24 & 300.23 & 300.23 & 300.22 & 300.22 & 300.21 & 300.21 & 300.20 & 300.20 \\
\hline$T @ F X \# 4$ & 302.31 & 302.30 & 302.30 & 302.29 & 302.29 & 302.28 & 302.28 & 302.27 & 302.27 & 302.27 & 302.26 & 302.26 & 302.25 & 302.25 & 302.24 & 302.24 & 302.23 \\
\hline$T(\boldsymbol{a}) \# \mathbf{F}$ & 304.36 & 304.36 & 304.36 & 304.35 & 304.35 & 304.34 & 304.34 & 304.33 & 304.33 & 304.32 & 304.32 & 304.31 & 304.31 & 304.31 & 304.30 & 304.30 & 304.29 \\
\hline$T @ F X \# 6$ & 306.45 & 306.45 & 306.44 & 306.44 & 306.43 & 306.43 & 306.42 & 306.42 & 306.41 & 306.41 & 306.41 & 306.40 & 306.40 & 306.39 & 306.39 & 306.38 & 306.38 \\
\hline Wall $x$-coord & 104.33 & 104.34 & 104.35 & 104.35 & 104.36 & 104.36 & 104.37 & 104.38 & 104.38 & 104.39 & 104.39 & 104.40 & 104.41 & 104.41 & 104.42 & 104.42 & 104.43 \\
\hline Wall T & 309.27 & 309.28 & 309.28 & 309.27 & 309.27 & 309.26 & 309.25 & 309.25 & 309.25 & 309.26 & 309.27 & 309.28 & 309.29 & 309.29 & 309.28 & 309.28 & 309.28 \\
\hline
\end{tabular}

\begin{tabular}{|c|c|c|c|c|c|c|c|c|c|c|c|c|c|c|c|c|c|}
\hline Interferogram Image \# & 482 & Location & TC6 & $\delta \varepsilon$ slope & $-2.12 E-3$ & $\delta \varepsilon$ intercept & 4.35 & $\operatorname{Avg} F S$ & 45.63 & Avg Wall T & 309.51 & Gradient error & $1.36 E-4$ & & & & \\
\hline Scan y-coord & 1960 & 1961 & 1962 & 1963 & 1964 & 1965 & 1966 & 1967 & 1968 & 1969 & 1970 & 1971 & 1972 & 1973 & 1974 & 1975 & 1976 \\
\hline$\delta \varepsilon$ & 0.1959 & 0.1938 & 0.1917 & 0.1896 & 0.1874 & 0.1853 & 0.1832 & 0.1811 & 0.1790 & 0.1768 & 0.1747 & 0.1726 & 0.1705 & 0.1684 & 0.1662 & 0.1641 & 0.1620 \\
\hline Scan $x$-coord & 1258 & 1258 & 1258 & 1258 & 1258 & 1258 & 1258 & 1258 & 1258 & 1258 & 1258 & 1258 & 1258 & 1258 & 1258 & 1258 & 1258 \\
\hline$F X \# 1$ & 573.33 & 575.06 & 575.92 & 577.06 & 578.20 & 580.09 & 580.07 & 580.29 & 581.60 & 582.76 & 583.75 & 585.04 & 586.24 & 588.25 & 590.02 & 590.79 & 591.66 \\
\hline$F X \# 2$ & 369.44 & 369.63 & 369.83 & 369.83 & 369.79 & 369.92 & 370.14 & 370.38 & 370.66 & 370.87 & 371.11 & 371.31 & 371.57 & 371.91 & 372.29 & 372.63 & 372.96 \\
\hline$F X \# 3$ & 285.26 & 285.49 & 285.72 & 285.96 & 286.26 & 286.50 & 286.75 & 286.92 & 287.03 & 287.08 & 287.08 & 287.08 & 286.98 & 286.96 & 286.97 & 287.05 & 287.18 \\
\hline$F X \# 4$ & 226.34 & 226.61 & 226.85 & 227.07 & 227.38 & 227.54 & 227.63 & 227.68 & 227.72 & 227.70 & 227.64 & 227.54 & 227.44 & 227.33 & 227.29 & 227.31 & 227.49 \\
\hline$F X \# 5$ & 176.72 & 176.68 & 176.64 & 176.54 & 176.52 & 176.49 & 176.54 & 176.67 & 176.84 & 177.00 & 177.14 & 177.37 & 177.56 & 177.77 & 177.88 & 177.95 & 178.04 \\
\hline$F X \# 6$ & 130.46 & 130.50 & 130.59 & 130.65 & 130.75 & 130.85 & 131.05 & 131.30 & 131.46 & 131.65 & 131.84 & 131.98 & 132.10 & 132.21 & 132.28 & 132.46 & 132.57 \\
\hline Near wall FS & 46.26 & 46.17 & 46.05 & 45.89 & 45.78 & 45.64 & 45.49 & 45.37 & 45.37 & 45.35 & 45.30 & 45.39 & 45.46 & 45.56 & 45.60 & 45.49 & 45.47 \\
\hline$T @ F X \# 1$ & 297.26 & 297.26 & 297.26 & 297.26 & 297.26 & 297.26 & 297.26 & 297.26 & 297.26 & 297.26 & 297.26 & 297.26 & 297.26 & 297.26 & 297.26 & 297.26 & 297.26 \\
\hline$T$ (a) $F X \# 2$ & 297.74 & 297.74 & 297.74 & 297.73 & 297.73 & 297.72 & 297.72 & 297.71 & 297.71 & 297.71 & 297.70 & 297.70 & 297.69 & 297.69 & 297.68 & 297.68 & 297.67 \\
\hline$T(a) \# \#$ & 299.76 & 299.76 & 299.75 & 299.75 & 299.75 & 299.74 & 299.74 & 299.73 & 299.73 & 299.72 & 299.72 & 299.72 & 299.71 & 299.71 & 299.70 & 299.70 & 299.69 \\
\hline$T$ (a) $F X \# 4$ & 301.79 & 301.79 & 301.78 & 301.78 & 301.77 & 301.77 & 301.77 & 301.76 & 301.76 & 301.75 & 301.75 & 301.74 & 301.74 & 301.74 & 301.73 & 301.73 & 301.72 \\
\hline$T @ F X \# 5$ & 303.84 & 303.84 & 303.84 & 303.83 & 303.83 & 303.82 & 303.82 & 303.81 & 303.81 & 303.81 & 303.80 & 303.80 & 303.79 & 303.79 & 303.78 & 303.78 & 303.77 \\
\hline$T @ F X \# 6$ & 305.92 & 305.92 & 305.92 & 305.91 & 305.91 & 305.90 & 305.90 & 305.89 & 305.89 & 305.88 & 305.88 & 305.88 & 305.87 & 305.87 & 305.86 & 305.86 & 305.85 \\
\hline Wall $x$-coord & 98.59 & 98.60 & 98.61 & 98.61 & 98.62 & 98.63 & 98.64 & 98.65 & 98.66 & 98.66 & 98.67 & 98.68 & 98.69 & 98.70 & 98.70 & 98.71 & 98.72 \\
\hline Wall T & 309.48 & 309.48 & 309.49 & 309.49 & 309.49 & 309.50 & 309.51 & 309.52 & 309.52 & 309.52 & 309.53 & 309.53 & 309.53 & 309.52 & 309.52 & 309.53 & 309.53 \\
\hline Interferogram Image \# & 482 & Location & $T C 7$ & $\delta \varepsilon$ slope & $-2.19 E-3$ & $\delta \varepsilon$ intercept & 3.42 & $\operatorname{Avg} F S$ & 50.98 & Avg Wall T & 309.49 & Gradient error & $1.36 E-4$ & & & & \\
\hline Scan y-coord & 1145 & 1146 & 1147 & 1148 & 1149 & 1150 & 1151 & 1152 & 1153 & 1154 & 1155 & 1156 & 1157 & 1158 & 1159 & 1160 & 1161 \\
\hline$\delta \varepsilon$ & 0.9058 & 0.9036 & 0.9014 & 0.8992 & 0.8970 & 0.8948 & 0.8926 & 0.8904 & 0.8882 & 0.8860 & 0.8838 & 0.8816 & 0.8794 & 0.8773 & 0.8751 & 0.8729 & 0.8707 \\
\hline Scan $x$-coord & 1402 & 1402 & 1402 & 1402 & 1402 & 1402 & 1402 & 1402 & 1402 & 1402 & 1402 & 1402 & 1402 & 1402 & 1402 & 1402 & 1402 \\
\hline$F X \# 1$ & 423.89 & 424.09 & 424.41 & 424.91 & 425.44 & 425.91 & 426.25 & 426.54 & 426.66 & 426.97 & 427.41 & 427.83 & 428.11 & 428.36 & 428.42 & 428.38 & 428.28 \\
\hline
\end{tabular}




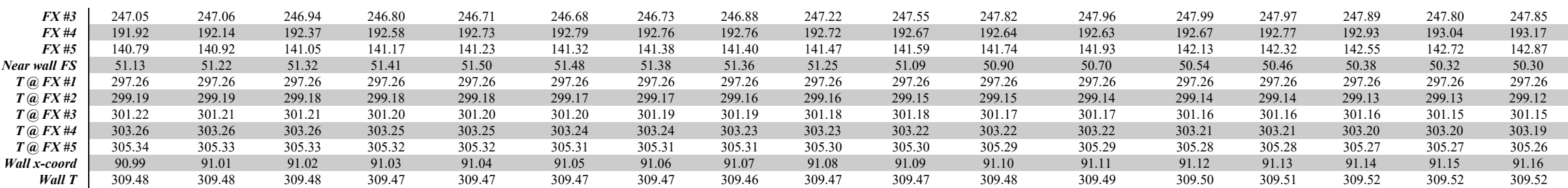

\section{B.27 Tabulated Data for Interferogram 483}

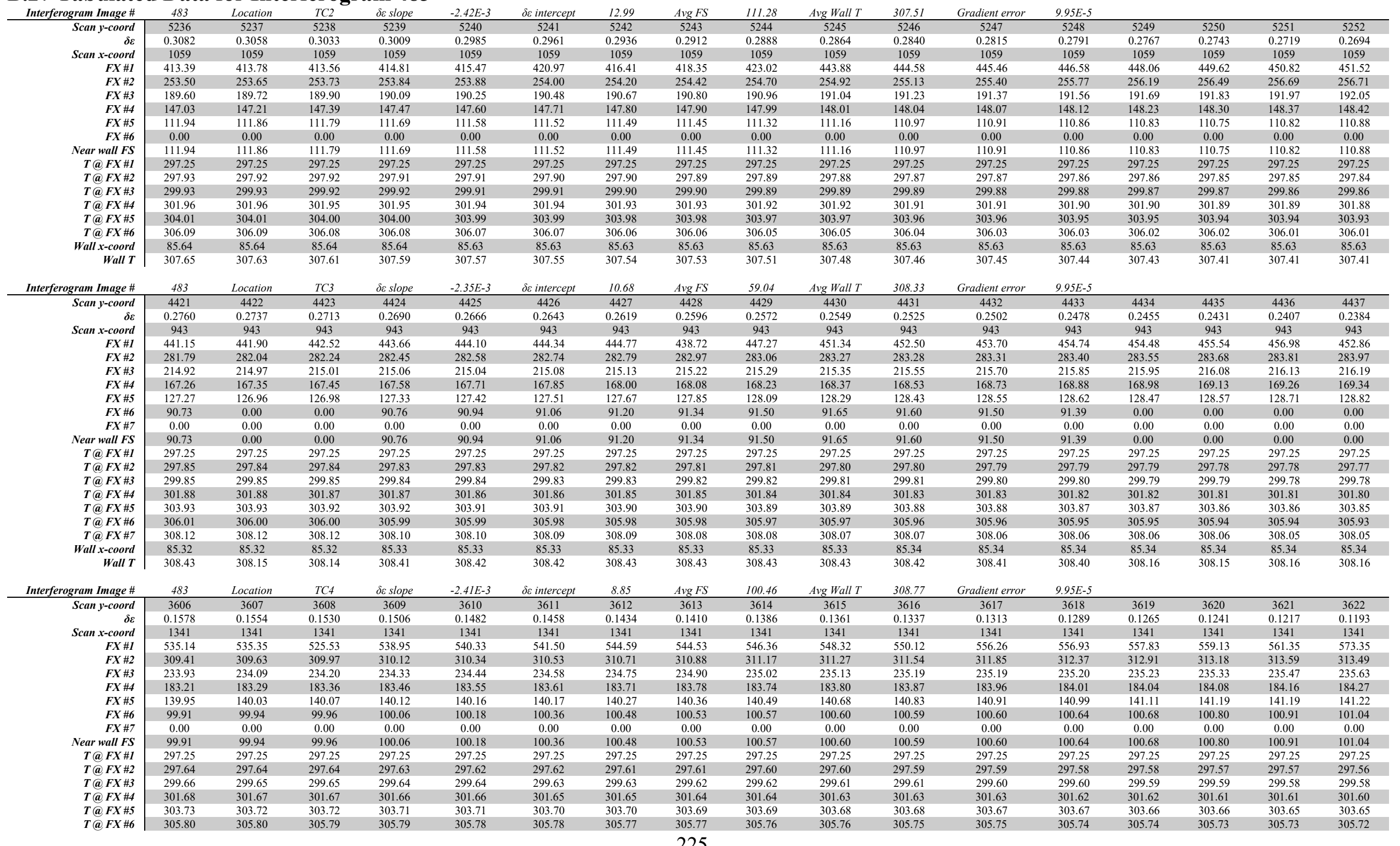




\begin{tabular}{|c|c|c|c|c|c|c|c|c|c|c|c|c|c|c|c|c|c|}
\hline$T @ F X \# 7$ & 307.91 & 307.90 & 307.90 & 307.89 & 307.89 & 307.88 & 307.88 & 307.87 & 307.87 & 307.86 & 307.86 & 307.85 & 307.85 & 307.84 & 307.84 & 307.83 & 307.83 \\
\hline Wall $x$-coord & 83.15 & 83.16 & 83.16 & 83.17 & 83.17 & 83.17 & 83.18 & 83.18 & 83.18 & 83.19 & 83.19 & 83.20 & 83.20 & 83.20 & 83.21 & 83.21 & 83.21 \\
\hline Wall T & 308.79 & 308.78 & 308.78 & 308.78 & 308.78 & 308.79 & 308.79 & 308.79 & 308.78 & 308.78 & 308.77 & 308.76 & 308.75 & 308.75 & 308.75 & 308.75 & 308.76 \\
\hline Interferogram Image \# & 483 & Location & $T C 5$ & $\delta \varepsilon$ slope & $-2.27 E-3$ & $\delta \varepsilon$ intercept & 7.30 & $\operatorname{Avg} F S$ & 43.30 & Avg Wall $T$ & 308.99 & Gradient error & $9.95 E-5$ & & & & \\
\hline Scan y-coord & 2791 & 2792 & 2793 & 2794 & 2795 & 2796 & 2797 & 2798 & 2799 & 2800 & 2801 & 2802 & 2803 & 2804 & 2805 & 2806 & 2807 \\
\hline & 0.9717 & 0.9695 & 0.9672 & 0.9649 & 0.9627 & 0.9604 & 0.9581 & 0.9559 & 0.9536 & 0.9513 & 0.9491 & 0.9468 & 0.9445 & 0.9423 & 0.9400 & 0.9377 & 0.9355 \\
\hline Scan $x$-coord & 866 & 866 & 866 & 866 & 866 & 866 & 866 & 866 & 866 & 866 & 866 & 866 & 866 & 866 & 866 & 866 & 866 \\
\hline$F X \# 1$ & 345.53 & 345.85 & 346.34 & 346.82 & 347.21 & 347.69 & 347.87 & 347.94 & 347.95 & 347.97 & 348.02 & 348.26 & 348.43 & 348.88 & 349.12 & 349.16 & 349.46 \\
\hline$F X \# 2$ & 258.76 & 258.69 & 258.62 & 258.53 & 258.46 & 258.50 & 258.63 & 258.81 & 259.00 & 259.18 & 259.40 & 259.53 & 259.63 & 259.78 & 259.89 & 260.01 & 260.15 \\
\hline$F X \# 3$ & 200.59 & 200.66 & 200.72 & 200.83 & 201.11 & 201.39 & 201.62 & 201.74 & 201.82 & 201.86 & 201.98 & 202.08 & 202.19 & 202.29 & 202.37 & 202.45 & 202.50 \\
\hline$F X \# 4$ & 154.36 & 154.41 & 154.44 & 154.46 & 154.50 & 154.56 & 154.70 & 154.79 & 154.82 & 154.79 & 154.69 & 154.65 & 154.72 & 154.87 & 155.01 & 155.10 & 155.15 \\
\hline$F X \# 5$ & 110.52 & 110.64 & 110.74 & 110.87 & 111.04 & 111.27 & 111.49 & 111.69 & 111.76 & 111.74 & 111.67 & 111.62 & 111.67 & 111.69 & 111.79 & 111.86 & 111.94 \\
\hline Near wall FS & 43.84 & 43.77 & 43.70 & 43.59 & 43.45 & 43.29 & 43.22 & 43.10 & 43.05 & 43.05 & 43.02 & 43.03 & 43.05 & 43.18 & 43.22 & 43.24 & 43.21 \\
\hline$T$ @ $F X \# 2$ & 299.23 & 299.23 & 299.22 & 299.22 & 299.21 & 299.21 & 299.20 & 299.20 & 299.19 & 299.19 & 299.19 & 299.18 & 299.18 & 299.17 & 299.17 & 299.16 & 299.16 \\
\hline$T(F X \# 3$ & 301.25 & 301.25 & 301.24 & 301.24 & 301.23 & 301.23 & 301.22 & 301.22 & 301.21 & 301.21 & 301.20 & 301.20 & 301.20 & 301.19 & 301.19 & 301.18 & 301.18 \\
\hline$T(F X \# 4$ & 303.29 & 303.29 & 303.28 & 303.28 & 303.28 & 303.27 & 303.27 & 303.26 & 303.26 & 303.25 & 303.25 & 303.24 & 303.24 & 303.23 & 303.23 & 303.22 & 303.22 \\
\hline$T @ F X \# 5$ & 305.36 & 305.36 & 305.35 & 305.35 & 305.35 & 305.34 & 305.34 & 305.33 & 305.33 & 305.32 & 305.32 & 305.31 & 305.31 & 305.30 & 305.30 & 305.29 & 305.29 \\
\hline Wall $x$-coord & 79.14 & 79.15 & 79.15 & 79.16 & 79.16 & 79.17 & 79.18 & 79.18 & 79.19 & 79.19 & 79.20 & 79.21 & 79.21 & 79.22 & 79.22 & 79.23 & 79.24 \\
\hline Wall $T$ & 308.96 & 308.97 & 308.97 & 308.97 & 308.98 & 308.99 & 309.00 & 309.01 & 309.01 & 309.01 & 309.00 & 308.99 & 308.99 & 308.98 & 308.98 & 308.97 & 308.97 \\
\hline Interferogram Image \# & 483 & Location & TC6 & $\delta \varepsilon$ slope & $-2.33 E-3$ & $\delta \varepsilon$ intercept & 5.38 & $\operatorname{Avg} F S$ & 43.64 & Avg Wall $T$ & 309.58 & Gradient error & $9.95 E-5$ & & & & \\
\hline Scan y-coord & 1976 & 1977 & 1978 & 1979 & 1980 & 1981 & 1982 & 1983 & 1984 & 1985 & 1986 & 1987 & 1988 & 1989 & 1990 & 1991 & 1992 \\
\hline & 0.7763 & 0.7739 & 0.7716 & 0.7693 & 0.7670 & 0.7646 & 0.7623 & 0.7600 & 0.7576 & 0.7553 & 0.7530 & 0.7507 & 0.7483 & 0.7460 & 0.7437 & 0.7413 & 0.7390 \\
\hline Scan $x$-coord & 1311 & 1311 & 1311 & 1311 & 1311 & 1311 & 1311 & 1311 & 1311 & 1311 & 1311 & 1311 & 1311 & 1311 & 1311 & 1311 & 1311 \\
\hline$F X \# 1$ & 398.95 & 399.25 & 399.61 & 399.94 & 400.40 & 400.94 & 401.53 & 401.88 & 402.13 & 402.09 & 402.29 & 402.65 & 402.85 & 403.12 & 404.27 & 404.42 & 404.50 \\
\hline$F X \# 2$ & 286.93 & 287.33 & 287.78 & 288.29 & 288.81 & 289.20 & 289.48 & 289.60 & 289.65 & 289.61 & 289.64 & 289.66 & 289.68 & 289.69 & 289.82 & 289.95 & 290.13 \\
\hline$F X \# 4$ & 170.56 & 170.69 & 170.79 & 170.93 & 171.00 & 171.07 & 171.16 & 171.22 & 171.21 & 171.16 & 171.15 & 171.03 & 171.07 & 171.10 & 171.16 & 171.36 & 171.53 \\
\hline$F X \# 5$ & 124.49 & 124.52 & 124.56 & 124.70 & 124.80 & 125.02 & 125.18 & 125.29 & 125.39 & 125.46 & 125.52 & 125.61 & 125.69 & 125.80 & 125.90 & 125.94 & 126.05 \\
\hline$F X \# 6$ & 81.19 & 80.95 & 80.82 & 80.83 & 80.95 & 81.18 & 81.47 & 81.68 & 81.87 & 81.97 & 81.95 & 81.94 & 81.96 & 82.09 & 82.29 & 82.44 & 82.47 \\
\hline Near wall FS & 43.29 & 43.58 & 43.74 & 43.88 & 43.85 & 43.85 & 43.71 & 43.61 & 43.52 & 43.48 & 43.57 & 43.67 & 43.73 & 43.72 & 43.62 & 43.50 & 43.58 \\
\hline$T @ F X \# 1$ & 297.25 & 297.25 & 297.25 & 297.25 & 297.25 & 297.25 & 297.25 & 297.25 & 297.25 & 297.25 & 297.25 & 297.25 & 297.25 & 297.25 & 297.25 & 297.25 & 297.25 \\
\hline$T$ T $F X \# 2$ & 298.88 & 298.88 & 298.87 & 298.87 & 298.86 & 298.86 & 298.85 & 298.85 & 298.84 & 298.84 & 298.83 & 298.83 & 298.82 & 298.82 & 298.82 & 298.81 & 298.81 \\
\hline$T$ (a) $F X \# 3$ & 300.90 & 300.89 & 300.89 & 300.88 & 300.88 & 300.87 & 300.87 & 300.86 & 300.86 & 300.85 & 300.85 & 300.85 & 300.84 & 300.84 & 300.83 & 300.83 & 300.82 \\
\hline$T(F X \# 4$ & 302.94 & 302.93 & 302.93 & 302.92 & 302.92 & 302.91 & 302.91 & 302.90 & 302.90 & 302.89 & 302.89 & 302.88 & 302.88 & 302.87 & 302.87 & 302.87 & 302.86 \\
\hline$T\left(\begin{array}{c}\mathrm{F} X \# 5 \\
-\end{array}\right.$ & 305.00 & 305.00 & 304.99 & 304.99 & 304.98 & 304.98 & 304.97 & 304.97 & 304.96 & 304.96 & 304.95 & 304.95 & 304.94 & 304.94 & 304.94 & 304.93 & 304.93 \\
\hline$T(a) F \# \#$ & 307.10 & 307.09 & 307.09 & 307.08 & 307.08 & 307.07 & 307.07 & 307.06 & 307.06 & 307.05 & 307.05 & 307.04 & 307.04 & 307.03 & 307.03 & 307.02 & 307.02 \\
\hline Wall $x$-coord & 73.27 & 73.28 & 73.29 & 73.30 & 73.31 & 73.32 & 73.32 & 73.33 & 73.34 & 73.35 & 73.36 & 73.37 & 73.37 & 73.38 & 73.39 & 73.40 & 73.41 \\
\hline Wall T & 309.61 & 309.59 & 309.57 & 309.57 & 309.57 & 309.57 & 309.58 & 309.59 & 309.59 & 309.59 & 309.59 & 309.58 & 309.58 & 309.58 & 309.58 & 309.58 & 309.58 \\
\hline Interferogram Image \# & 483 & Location & $T C 7$ & $\delta \varepsilon$ slope & $-2.06 E-3$ & $\delta \varepsilon$ intercept & 2.79 & $\operatorname{Avg} F S$ & 47.10 & Avg Wall $T$ & 309.53 & Gradient error & $9.95 E-5$ & & & & \\
\hline Scan y-coord & 1162 & 1163 & 1164 & 1165 & 1166 & 1167 & 1168 & 1169 & 1170 & 1171 & 1172 & 1173 & 1174 & 1175 & 1176 & 1177 & 1178 \\
\hline $\begin{array}{rl}\delta c a n & y-c o s i a \\
\delta \varepsilon\end{array}$ & 0.3991 & 0.3971 & 0.3950 & 0.3929 & 0.3909 & 0.3888 & 0.3868 & 0.3847 & 0.3826 & 0.3806 & 0.3785 & 0.3765 & 0.3744 & 0.3724 & 0.3703 & 0.3682 & 0.3662 \\
\hline Scan $x$-coord & 1232 & 1232 & 1232 & 1232 & 1232 & 1232 & 1232 & 1232 & 1232 & 1232 & 1232 & 1232 & 1232 & 1232 & 1232 & 1232 & 1232 \\
\hline$F X \# 1$ & 531.77 & 532.31 & 529.24 & 529.35 & 529.25 & 529.75 & 530.49 & 531.09 & 531.39 & 533.03 & 543.72 & 545.12 & 547.62 & 548.71 & 550.86 & 551.52 & 550.37 \\
\hline$F X \# 2$ & 328.91 & 328.95 & 329.01 & 329.06 & 328.97 & 328.93 & 328.88 & 329.02 & 329.20 & 329.41 & 329.65 & 329.89 & 330.27 & 330.80 & 331.37 & 331.89 & 332.26 \\
\hline$F X \# 3$ & 245.68 & 245.73 & 245.70 & 245.66 & 245.73 & 245.87 & 246.05 & 246.18 & 246.26 & 246.42 & 246.61 & 246.76 & 246.89 & 247.00 & 247.23 & 247.57 & 247.86 \\
\hline$F X \# 4$ & 187.79 & 187.88 & 188.00 & 188.06 & 188.16 & 188.24 & 188.37 & 188.37 & 188.30 & 188.21 & 188.17 & 188.11 & 188.19 & 188.33 & 188.50 & 188.64 & 188.73 \\
\hline$F X \# 5$ & 137.53 & 137.75 & 137.88 & 137.94 & 137.94 & 137.87 & 137.93 & 138.05 & 138.22 & 138.51 & 138.77 & 138.98 & 139.20 & 139.39 & 139.47 & 139.58 & 139.64 \\
\hline$F X \# 6$ & 90.95 & 91.03 & 91.06 & 91.13 & 91.18 & 91.24 & 91.23 & 91.29 & 91.38 & 91.44 & 91.53 & 91.57 & 91.67 & 91.77 & 91.78 & 91.88 & 91.89 \\
\hline Near wall $F S$ & $\begin{array}{l}96.93 \\
46.58\end{array}$ & 46.71 & $\begin{array}{l}11.00 \\
46.82\end{array}$ & 46.81 & 46.76 & 46.63 & $\begin{array}{l}1.23 \\
46.70\end{array}$ & 46.75 & 46.84 & $\begin{array}{l}19.44 \\
47.07\end{array}$ & 47.25 & 47.41 & 47.54 & 47.61 & 47.69 & 47.70 & 47.75 \\
\hline$T @ F X \# 1$ & 297.25 & 297.25 & 297.25 & 297.25 & 297.25 & 297.25 & 297.25 & 297.25 & 297.25 & 297.25 & 297.25 & 297.25 & 297.25 & 297.25 & 297.25 & 297.25 & 297.25 \\
\hline$T @ F X \# 2$ & 298.11 & 298.11 & 298.10 & 298.10 & 298.09 & 298.09 & 298.09 & 298.08 & 298.08 & 298.07 & 298.07 & 298.06 & 298.06 & 298.06 & 298.05 & 298.05 & 298.04 \\
\hline$T$ (a) $F X \# 3$ & 300.13 & 300.12 & 300.12 & 300.11 & 300.11 & 300.11 & 300.10 & 300.10 & 300.09 & 300.09 & 300.08 & 300.08 & 300.08 & 300.07 & 300.07 & 300.06 & 300.06 \\
\hline Wall $x$-coord & 65.57 & 65.58 & 65.59 & 65.60 & 65.61 & 65.62 & 65.63 & 65.65 & 65.66 & 65.67 & 65.68 & 65.69 & 65.70 & 65.71 & 65.72 & 65.73 & 65.74 \\
\hline Wall T & 309.56 & 309.55 & 309.55 & 309.55 & 309.54 & 309.55 & 309.54 & 309.53 & 309.53 & 309.52 & 309.52 & 309.51 & 309.51 & 309.51 & 309.50 & 309.50 & 309.49 \\
\hline
\end{tabular}

\section{B.28 Tabulated Data for Interferogram 484}

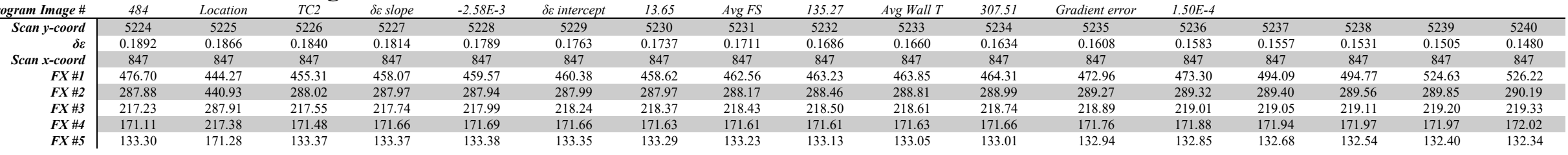




\begin{tabular}{|c|c|c|c|c|c|c|c|c|c|c|c|c|c|c|c|c|c|}
\hline$F X \# 6$ & 0.00 & 0.00 & 0.00 & 0.00 & 0.00 & 0.00 & 0.00 & 0.00 & 0.00 & 0.00 & 0.00 & 0.00 & 0.00 & 0.00 & 0.00 & 0.00 & 0.00 \\
\hline Near wall FS & 133.30 & 171.28 & 133.37 & 133.37 & 133.38 & 133.35 & 133.29 & 133.23 & 133.13 & 133.05 & 133.01 & 132.94 & 132.85 & 132.68 & 132.54 & 132.40 & 132.34 \\
\hline$T @ F X \# 1$ & 297.20 & 297.20 & 297.20 & 297.20 & 297.20 & 297.20 & 297.20 & 297.20 & 297.20 & 297.20 & 297.20 & 297.20 & 297.20 & 297.20 & 297.20 & 297.20 & 297.20 \\
\hline$T @ F X \# 2$ & 297.63 & 297.63 & 297.62 & 297.61 & 297.61 & 297.60 & 297.60 & 297.59 & 297.59 & 297.58 & 297.58 & 297.57 & 297.57 & 297.56 & 297.55 & 297.54 & 297.54 \\
\hline$T @ F X \# 3$ & 299.64 & 299.61 & 299.63 & 299.63 & 299.62 & 299.62 & 299.61 & 299.61 & 299.60 & 299.60 & 299.59 & 299.59 & 299.58 & 299.58 & 299.57 & 299.57 & 299.56 \\
\hline$T(\boldsymbol{C} \boldsymbol{F X} \# 4$ & 301.67 & 301.65 & 301.66 & 301.65 & 301.65 & 301.64 & 301.64 & 301.63 & 301.63 & 301.62 & 301.62 & 301.61 & 301.61 & 301.60 & 301.60 & 301.59 & 301.59 \\
\hline$T @ F X \# 5$ & 303.72 & 303.71 & 303.71 & 303.70 & 303.70 & 303.69 & 303.69 & 303.68 & 303.68 & 303.67 & 303.67 & 303.66 & 303.66 & 303.65 & 303.65 & 303.64 & 303.63 \\
\hline$T @ F X \# 6$ & 305.80 & 305.79 & 305.79 & 305.78 & 305.78 & 305.77 & 305.76 & 305.76 & 305.75 & 305.75 & 305.74 & 305.74 & 305.73 & 305.73 & 305.72 & 305.72 & 305.71 \\
\hline Wall $x$-coord & 101.84 & 101.84 & 101.84 & 101.84 & 101.84 & 101.84 & 101.84 & 101.83 & 101.83 & 101.83 & 101.83 & 101.83 & 101.83 & 101.83 & 101.83 & 101.82 & 101.82 \\
\hline Wall $T$ & 307.52 & 308.92 & 307.50 & 307.49 & 307.48 & 307.48 & 307.47 & 307.46 & 307.44 & 307.43 & 307.42 & 307.40 & 307.38 & 307.36 & 307.34 & 307.32 & 307.31 \\
\hline Interferogram Image \# & 484 & Location & $T C 3$ & $\delta \varepsilon$ slope & $-2.39 E-3$ & $\delta \varepsilon$ intercept & 10.75 & $\operatorname{Avg} F S$ & 38.47 & Avg Wall $T$ & 308.45 & Gradient error & $1.50 E-4$ & & & & \\
\hline Scan y-coord & 4410 & 4411 & 4412 & 4413 & 4414 & 4415 & 4416 & 4417 & 4418 & 4419 & 4420 & 4421 & 4422 & 4423 & 4424 & 4425 & 4426 \\
\hline & 0.2233 & 0.2209 & 0.2185 & 0.2161 & 0.2137 & 0.2113 & 0.2090 & 0.2066 & 0.2042 & 0.2018 & 0.1994 & 0.1970 & 0.1946 & 0.1922 & 0.1899 & 0.1875 & 0.1851 \\
\hline Scan $x$-coord & 992 & 992 & 992 & 992 & 992 & 992 & 992 & 992 & 992 & 992 & 992 & 992 & 992 & 992 & 992 & 992 & 992 \\
\hline$F X \# 1$ & 469.30 & 469.29 & 469.93 & 471.08 & 472.01 & 472.23 & 473.40 & 474.69 & 475.26 & 476.19 & 476.71 & 478.24 & 481.40 & 482.55 & 483.79 & 484.93 & 485.90 \\
\hline$F X \# 2$ & 310.76 & 311.10 & 311.42 & 311.79 & 312.10 & 312.28 & 312.41 & 312.47 & 312.43 & 312.42 & 312.46 & 312.50 & 312.57 & 312.50 & 312.44 & 312.54 & 312.56 \\
\hline$F X \# 3$ & 241.21 & 241.19 & 241.19 & 241.20 & 241.25 & 241.28 & 241.29 & 241.32 & 241.34 & 241.35 & 241.46 & 241.53 & 241.63 & 241.75 & 241.84 & 241.90 & 242.00 \\
\hline $\begin{array}{l}F X \# 4 \\
F X \# 4\end{array}$ & $\begin{array}{l}241.21 \\
190.83\end{array}$ & 190.99 & 191.10 & 191.16 & 191.24 & 191.34 & $\begin{array}{l}241.29 \\
191.41\end{array}$ & $\begin{array}{l}241.32 \\
191.50\end{array}$ & $\begin{array}{l}241.34 \\
191.56\end{array}$ & 191.70 & $\begin{array}{l}241.40 \\
191.76\end{array}$ & 191.83 & $\begin{array}{l}241.03 \\
191.94\end{array}$ & 192.02 & $\begin{array}{l}241.84 \\
192.11\end{array}$ & $\begin{array}{l}241.90 \\
192.17\end{array}$ & 192.24 \\
\hline$F X \# 5$ & 149.06 & 149.12 & 149.24 & 149.24 & 149.21 & 149.18 & 149.15 & 149.21 & 149.29 & 149.37 & 149.47 & 149.58 & 149.71 & 149.86 & 150.01 & 150.17 & 150.34 \\
\hline$F X \# 6$ & 110.50 & 110.57 & 110.55 & 110.52 & 110.51 & 110.56 & 110.61 & 110.74 & 110.80 & 110.92 & 111.11 & 111.37 & 111.58 & 111.72 & 111.79 & 111.74 & 111.70 \\
\hline Near wall FS & 38.57 & 38.55 & 38.68 & 38.72 & 38.69 & 38.62 & 38.53 & 38.47 & 38.48 & 38.45 & 38.36 & 38.22 & 38.13 & 38.15 & 38.22 & 38.43 & 38.64 \\
\hline$T @ F X \# 1$ & 297.20 & 297.20 & 297.20 & 297.20 & 297.20 & 297.20 & 297.20 & 297.20 & 297.20 & 297.20 & 297.20 & 297.20 & 297.20 & 297.20 & 297.20 & 297.20 & 297.20 \\
\hline$T @ F X \# 2$ & 297.72 & 297.71 & 297.71 & 297.70 & 297.70 & 297.69 & 297.69 & 297.68 & 297.68 & 297.67 & 297.67 & 297.66 & 297.66 & 297.65 & 297.65 & 297.64 & 297.64 \\
\hline$T @ F X \# 3$ & 299.73 & 299.72 & 299.72 & 299.72 & 299.71 & 299.71 & 299.70 & 299.70 & 299.69 & 299.69 & 299.68 & 299.68 & 299.67 & 299.67 & 299.66 & 299.66 & 299.65 \\
\hline$T @ F X \# 4$ & 301.76 & 301.75 & 301.75 & 301.74 & 301.74 & 301.73 & 301.73 & 301.72 & 301.72 & 301.71 & 301.71 & 301.70 & 301.70 & 301.69 & 301.69 & 301.68 & 301.68 \\
\hline$T @ F X \# 5$ & 303.81 & 303.80 & 303.80 & 303.79 & 303.79 & 303.78 & 303.78 & 303.77 & 303.77 & 303.77 & 303.76 & 303.76 & 303.75 & 303.75 & 303.74 & 303.74 & 303.73 \\
\hline$T @ F X \# 6$ & 305.89 & 305.88 & 305.88 & 305.87 & 305.87 & 305.86 & 305.86 & 305.85 & 305.85 & 305.84 & 305.84 & 305.83 & 305.83 & 305.82 & 305.82 & 305.81 & 305.81 \\
\hline Wall $x$-coord & 101.93 & 101.93 & 101.93 & 101.93 & 101.93 & 101.93 & 101.94 & 101.94 & 101.94 & 101.94 & 101.94 & 101.94 & 101.94 & 101.94 & 101.94 & 101.95 & 101.95 \\
\hline Wall T & 308.46 & 308.46 & 308.45 & 308.45 & 308.44 & 308.44 & 308.44 & 308.44 & 308.44 & 308.44 & 308.45 & 308.46 & 308.47 & 308.47 & 308.47 & 308.46 & 308.45 \\
\hline Interferogram Image \# & 484 & Location & $T C 4$ & $\delta \varepsilon$ slope & $-2.38 E-3$ & $\delta \varepsilon$ intercept & 8.68 & $\operatorname{Avg} F S$ & 122.88 & Avg Wall $T$ & 308.90 & Gradient error & $1.50 E-4$ & & & & \\
\hline Scan y-coord & 3596 & 3597 & 3598 & 3599 & 3600 & 3601 & 3602 & 3603 & 3604 & 3605 & 3606 & 3607 & 3608 & 3609 & 3610 & 3611 & 3612 \\
\hline & 0.1190 & 0.1166 & 0.1143 & 0.1119 & 0.1095 & 0.1071 & 0.1047 & 0.1024 & 0.1000 & 0.0976 & 0.0952 & 0.0928 & 0.0905 & 0.0881 & 0.0857 & 0.0833 & 0.0809 \\
\hline Scan $x$-coord & 1015 & 1015 & 1015 & 1015 & 1015 & 1015 & 1015 & 1015 & 1015 & 1015 & 1015 & 1015 & 1015 & 1015 & 1015 & 1015 & 1015 \\
\hline$F X \# I$ & 538.93 & 546.44 & 547.00 & 547.16 & 548.36 & 550.85 & 541.29 & 552.71 & 554.72 & 554.72 & 552.91 & 553.67 & 556.85 & 557.73 & 558.79 & 560.01 & 562.13 \\
\hline$F X \# 2$ & 338.25 & 338.65 & 338.97 & 339.18 & 339.34 & 339.47 & 339.63 & 340.00 & 340.21 & 340.41 & 340.52 & 340.59 & 340.79 & 340.93 & 341.26 & 341.52 & 342.00 \\
\hline$F X \# 3$ & 262.08 & 262.16 & 262.22 & 262.35 & 262.50 & 262.65 & 262.81 & 262.96 & 263.09 & 263.11 & 263.12 & 263.18 & 263.21 & 263.31 & 263.39 & 263.53 & 263.66 \\
\hline$F X \# 4$ & 208.52 & 208.53 & 208.60 & 208.79 & 208.96 & 209.08 & 209.19 & 209.24 & 209.31 & 209.32 & 209.35 & 209.35 & 209.41 & 209.47 & 209.56 & 209.71 & 209.84 \\
\hline$F X \# 5$ & 163.68 & 163.70 & 163.76 & 163.80 & 163.84 & 164.01 & 164.16 & 164.21 & 164.33 & 164.40 & 164.49 & 164.59 & 164.69 & 164.78 & 164.90 & 165.02 & 165.11 \\
\hline$F X \# 6$ & 122.16 & 122.32 & 122.51 & 122.64 & 122.70 & 122.75 & 122.83 & 122.85 & 122.85 & 122.87 & 122.95 & 123.07 & 123.13 & 123.19 & 123.25 & 123.34 & 123.48 \\
\hline$F X \# 7$ & 0.00 & 0.00 & 0.00 & 0.00 & 0.00 & 0.00 & 0.00 & 0.00 & 0.00 & 0.00 & 0.00 & 0.00 & 0.00 & 0.00 & 0.00 & 0.00 & 0.00 \\
\hline Near wall FS & 122.16 & 122.32 & 122.51 & 122.64 & 122.70 & 122.75 & 122.83 & 122.85 & 122.85 & 122.87 & 122.95 & 123.07 & 123.13 & 123.19 & 123.25 & 123.34 & 123.48 \\
\hline$T @ F X \# 1$ & 297.20 & 297.20 & 297.20 & 297.20 & 297.20 & 297.20 & 297.20 & 297.20 & 297.20 & 297.20 & 297.20 & 297.20 & 297.20 & 297.20 & 297.20 & 297.20 & 297.20 \\
\hline$T @ F X \# 2$ & 297.50 & 297.50 & 297.49 & 297.49 & 297.48 & 297.48 & 297.47 & 297.47 & 297.46 & 297.46 & 297.45 & 297.45 & 297.44 & 297.44 & 297.43 & 297.43 & 297.42 \\
\hline$T(a) F X 3$ & 299.52 & 299.52 & 299.51 & 299.51 & 299.50 & 299.50 & 299.49 & 299.49 & 299.48 & 299.48 & 299.47 & 299.47 & 299.46 & 299.46 & 299.45 & 299.45 & 299.44 \\
\hline$T @ F X \# 4$ & 301.55 & 301.54 & 301.54 & 301.53 & 301.53 & 301.52 & 301.52 & 301.51 & 301.51 & 301.50 & 301.50 & 301.49 & 301.49 & 301.48 & 301.48 & 301.47 & 301.47 \\
\hline$T @ F X \# 5$ & 303.60 & 303.59 & 303.59 & 303.58 & 303.58 & 303.57 & 303.57 & 303.56 & 303.56 & 303.55 & 303.55 & 303.54 & 303.54 & 303.53 & 303.53 & 303.52 & 303.52 \\
\hline$T(\boldsymbol{F} X \mathbb{F} \# \mathbf{6}$ & 305.67 & 305.67 & 305.66 & 305.66 & 305.65 & 305.65 & 305.64 & 305.64 & 305.63 & 305.63 & 305.62 & 305.62 & 305.61 & 305.61 & 305.60 & 305.60 & 305.59 \\
\hline$T @ F X \# 7$ & 307.78 & 307.77 & 307.77 & 307.76 & 307.76 & 307.75 & 307.75 & 307.74 & 307.74 & 307.73 & 307.73 & 307.72 & 307.72 & 307.71 & 307.71 & 307.70 & 307.70 \\
\hline Wall $x$-coord & 99.96 & 99.96 & 99.97 & 99.97 & 99.97 & 99.98 & 99.98 & 99.98 & 99.99 & 99.99 & 100.00 & 100.00 & 100.00 & 100.01 & 100.01 & 100.01 & 100.02 \\
\hline Wall T & 308.90 & 308.91 & 308.92 & 308.92 & 308.92 & 308.91 & 308.91 & 308.90 & 308.90 & 308.89 & 308.89 & 308.89 & 308.89 & 308.88 & 308.88 & 308.88 & 308.88 \\
\hline Interferogram Image \# & 484 & Location & $T C 5$ & $\delta \varepsilon$ slope & $-2.19 E-3$ & $\delta \varepsilon$ intercept & 6.14 & $\operatorname{Avg} F S$ & 43.97 & Avg Wall $T$ & 309.38 & Gradient error & $1.50 E-4$ & & & & \\
\hline Scan y-coord & 2782 & 2783 & 2784 & 2785 & 2786 & 2787 & 2788 & 2789 & 2790 & 2791 & 2792 & 2793 & 2794 & 2795 & 2796 & 2797 & 2798 \\
\hline & 0.0490 & 0.0468 & 0.0446 & 0.0424 & 0.0402 & 0.0380 & 0.0359 & 0.0337 & 0.0315 & 0.0293 & 0.0271 & 0.0249 & 0.0227 & 0.0205 & 0.0183 & 0.0162 & 0.0140 \\
\hline Scan $x$-coord & 855 & 855 & 855 & 855 & 855 & 855 & 855 & 855 & 855 & 855 & 855 & 855 & 855 & 855 & 855 & 855 & 855 \\
\hline$F X \# 1$ & 850.00 & 850.00 & 850.00 & 850.00 & 850.00 & 850.00 & 850.00 & 850.00 & 850.00 & 850.00 & 850.00 & 850.00 & 850.00 & 850.00 & 850.00 & 850.00 & 850.00 \\
\hline$F X \# 2$ & 366.18 & 366.49 & 366.78 & 367.23 & 367.64 & 367.97 & 368.27 & 368.55 & 368.82 & 368.96 & 369.14 & 369.27 & 369.43 & 369.51 & 369.60 & 369.74 & 370.13 \\
\hline$F X \# 3$ & 282.52 & 282.75 & 282.99 & 283.20 & 283.34 & 283.35 & 283.25 & 283.16 & 283.07 & 283.11 & 283.22 & 283.48 & 283.67 & 283.82 & 283.94 & 284.04 & 284.09 \\
\hline$F X \# 4$ & 224.49 & 224.72 & 224.90 & 225.01 & 225.11 & 225.26 & 225.37 & 225.40 & 225.50 & 225.60 & 225.81 & 225.99 & 226.00 & 226.11 & 226.19 & 226.35 & 226.55 \\
\hline$F X \# 5$ & 177.10 & 177.19 & 177.34 & 177.51 & 177.65 & 177.73 & 177.93 & 177.98 & 178.00 & 178.01 & 178.03 & 178.10 & 178.16 & 178.21 & 178.22 & 178.27 & 178.33 \\
\hline$F X \# 6$ & 132.85 & 133.10 & 133.35 & 133.55 & 133.66 & 133.78 & 133.82 & 133.92 & 134.02 & 134.12 & 134.17 & 134.27 & 134.31 & 134.32 & 134.30 & 134.33 & 134.35 \\
\hline Near wall FS & 44.25 & 44.08 & 43.99 & 43.96 & 43.99 & 43.95 & 44.11 & 44.06 & 43.98 & 43.89 & 43.86 & 43.84 & 43.85 & 43.89 & 43.93 & 43.93 & 43.98 \\
\hline$T @ F X \# 1$ & 297.20 & 297.20 & 297.20 & 297.20 & 297.20 & 297.20 & 297.20 & 297.20 & 297.20 & 297.20 & 297.20 & 297.20 & 297.20 & 297.20 & 297.20 & 297.20 & 297.20 \\
\hline$T @ F X \# 2$ & 297.29 & 297.29 & 297.29 & 297.28 & 297.28 & 297.27 & 297.27 & 297.26 & 297.26 & 297.26 & 297.25 & 297.25 & 297.24 & 297.24 & 297.23 & 297.23 & 297.23 \\
\hline$T(a) F X 3$ & 299.35 & 299.35 & 299.34 & 299.34 & 299.33 & 299.33 & 299.32 & 299.32 & 299.32 & 299.31 & 299.31 & 299.30 & 299.30 & 299.29 & 299.29 & 299.29 & 299.28 \\
\hline$T @ F X \# 4$ & 301.38 & 301.37 & 301.37 & 301.36 & 301.36 & 301.35 & 301.35 & 301.34 & 301.34 & 301.34 & 301.33 & 301.33 & 301.32 & 301.32 & 301.31 & 301.31 & 301.30 \\
\hline$T @ F X \# 5$ & 303.42 & 303.42 & 303.41 & 303.41 & 303.41 & 303.40 & 303.40 & 303.39 & 303.39 & 303.38 & 303.38 & 303.37 & 303.37 & 303.37 & 303.36 & 303.36 & 303.35 \\
\hline$T @ F X \# 6$ & 305.50 & 305.49 & 305.49 & 305.48 & 305.48 & 305.48 & 305.47 & 305.47 & 305.46 & 305.46 & 305.45 & 305.45 & 305.44 & 305.44 & 305.43 & 305.43 & 305.43 \\
\hline \multirow{3}{*}{$\begin{array}{r}\text { Wall } x \text {-coord } \\
\text { Wall T }\end{array}$} & 95.94 & 95.94 & 95.95 & 95.96 & 95.96 & 95.97 & 95.97 & 95.98 & 95.99 & 95.99 & 96.00 & 96.00 & 96.01 & 96.02 & 96.02 & 96.03 & 96.04 \\
\hline & 309.35 & 309.37 & 309.38 & 309.38 & 309.38 & 309.39 & 309.38 & 309.38 & 309.38 & 309.38 & 309.38 & 309.38 & 309.38 & 309.37 & 309.37 & 309.36 & 309.36 \\
\hline & & & & & & & & 227 & & & & & & & & & \\
\hline
\end{tabular}




\begin{tabular}{|c|c|c|c|c|c|c|c|c|c|c|c|c|c|c|c|c|c|}
\hline Interferogram Image \# & 484 & Location & $T C 6$ & $\delta \varepsilon$ slope & $-2.09 E-3$ & $\delta \varepsilon$ intercept & 4.87 & $\operatorname{Avg} F S$ & 44.15 & Avg Wall $T$ & 309.70 & Gradient error & $1.50 E-4$ & & & & \\
\hline Scan y-coord & 1968 & 1969 & 1970 & 1971 & 1972 & 1973 & 1974 & 1975 & 1976 & 1977 & 1978 & 1979 & 1980 & 1981 & 1982 & 1983 & 1984 \\
\hline & 0.7685 & 0.7664 & 0.7644 & 0.7623 & 0.7602 & 0.7581 & 0.7560 & 0.7539 & 0.7518 & 0.7498 & 0.7477 & 0.7456 & 0.7435 & 0.7414 & 0.7393 & 0.7372 & 0.7352 \\
\hline Scan $x$-coord & 1040 & 1040 & 1040 & 1040 & 1040 & 1040 & 1040 & 1040 & 1040 & 1040 & 1040 & 1040 & 1040 & 1040 & 1040 & 1040 & 1040 \\
\hline$F X \# 1$ & 404.96 & 405.12 & 405.26 & 405.49 & 405.83 & 406.20 & 406.72 & 407.25 & 407.68 & 407.98 & 408.07 & 408.22 & 408.43 & 408.74 & 409.45 & 410.10 & 410.85 \\
\hline$F X \# 2$ & 306.88 & 307.05 & 307.25 & 307.49 & 307.83 & 308.21 & 308.64 & 309.06 & 309.41 & 309.73 & 309.88 & 309.97 & 309.92 & 309.90 & 309.84 & 309.76 & 309.79 \\
\hline$F X \# 3$ & 241.94 & 241.98 & 242.03 & 242.22 & 242.42 & 242.60 & 242.78 & 242.97 & 243.10 & 243.30 & 243.54 & 243.76 & 244.04 & 244.28 & 244.53 & 244.67 & 244.79 \\
\hline$F X \# 5$ & 145.41 & 145.47 & 145.55 & 145.64 & 145.67 & 145.68 & 145.78 & 145.85 & 145.96 & 146.16 & 146.20 & 146.21 & 146.36 & 146.45 & 146.53 & 146.60 & 146.63 \\
\hline$F X \# 6$ & 100.82 & 100.69 & 100.71 & 100.85 & 100.99 & 101.30 & 101.62 & 101.93 & 102.14 & 102.29 & 102.35 & 102.38 & 102.57 & 102.71 & 102.74 & 102.76 & 102.71 \\
\hline Near wall FS & 44.59 & 44.78 & 44.84 & 44.79 & 44.68 & 44.38 & 44.17 & 43.92 & 43.82 & 43.87 & 43.86 & 43.82 & 43.79 & 43.74 & 43.79 & 43.84 & 43.92 \\
\hline$T @ F X \# 1$ & 297.20 & 297.20 & 297.20 & 297.20 & 297.20 & 297.20 & 297.20 & 297.20 & 297.20 & 297.20 & 297.20 & 297.20 & 297.20 & 297.20 & 297.20 & 297.20 & 297.20 \\
\hline$T @ F X \# 2$ & 298.81 & 298.81 & 298.81 & 298.80 & 298.80 & 298.79 & 298.79 & 298.78 & 298.78 & 298.78 & 298.77 & 298.77 & 298.76 & 298.76 & 298.76 & 298.75 & 298.75 \\
\hline$T @ F X \# 3$ & 300.83 & 300.83 & 300.82 & 300.82 & 300.82 & 300.81 & 300.81 & 300.80 & 300.80 & 300.80 & 300.79 & 300.79 & 300.78 & 300.78 & 300.77 & 300.77 & 300.77 \\
\hline$T @ F X \# 5$ & 304.94 & 304.94 & 304.93 & 304.93 & 304.93 & 304.92 & 304.92 & 304.91 & 304.91 & 304.90 & 304.90 & 304.89 & 304.89 & 304.89 & 304.88 & 304.88 & 304.87 \\
\hline$T$ (a) $F X \# 6$ & 307.04 & 307.03 & 307.03 & 307.02 & 307.02 & 307.02 & 307.01 & 307.01 & 307.00 & 307.00 & 306.99 & 306.99 & 306.98 & 306.98 & 306.98 & 306.97 & 306.97 \\
\hline Wall $x$-coord & 89.86 & 89.87 & 89.88 & 89.89 & 89.90 & 89.90 & 89.91 & 89.92 & 89.93 & 89.94 & 89.95 & 89.96 & 89.97 & 89.97 & 89.98 & 89.99 & 90.00 \\
\hline Wall $T$ & 309.68 & 309.67 & 309.67 & 309.67 & 309.67 & 309.68 & 309.70 & 309.71 & 309.72 & 309.72 & 309.72 & 309.71 & 309.72 & 309.72 & 309.72 & 309.71 & 309.70 \\
\hline Interferogram Image \# & 484 & Location & $T C 7$ & $\delta \varepsilon$ slope & $-2.25 E-3$ & $\delta \varepsilon$ intercept & 3.18 & $\operatorname{Avg} F S$ & 47.23 & Avg Wall T & 309.83 & Gradient error & $1.50 E-4$ & & & & \\
\hline Scan y-coord & 1155 & 1156 & 1157 & 1158 & 1159 & 1160 & 1161 & 1162 & 1163 & 1164 & 1165 & 1166 & 1167 & 1168 & 1169 & 1170 & 1171 \\
\hline & 0.5807 & 0.5785 & 0.5762 & 0.5740 & 0.5717 & 0.5695 & 0.5672 & 0.5650 & 0.5627 & 0.5605 & 0.5582 & 0.5560 & 0.5537 & 0.5515 & 0.5492 & 0.5470 & 0.5447 \\
\hline Scan $x$-coord & 1294 & 1294 & 1294 & 1294 & 1294 & 1294 & 1294 & 1294 & 1294 & 1294 & 1294 & 1294 & 1294 & 1294 & 1294 & 1294 & 1294 \\
\hline$F X \# 1$ & 489.69 & 490.11 & 490.96 & 490.93 & 492.76 & 493.09 & 493.82 & 494.32 & 495.18 & 495.11 & 496.36 & 497.73 & 499.88 & 499.60 & 501.02 & 501.90 & 501.52 \\
\hline$F X \# 2$ & 336.25 & 336.39 & 336.51 & 336.51 & 336.67 & 336.87 & 337.14 & 337.46 & 337.75 & 337.86 & 337.89 & 338.08 & 338.28 & 338.43 & 338.65 & 338.95 & 339.30 \\
\hline$F X \# 3$ & 258.85 & 258.88 & 258.95 & 259.16 & 259.35 & 259.45 & 259.46 & 259.51 & 259.72 & 259.97 & 260.12 & 260.23 & 260.39 & 260.61 & 260.79 & 260.98 & 261.14 \\
\hline$F X \# 4$ & 202.44 & 202.48 & 202.52 & 202.63 & 202.77 & 202.88 & 203.04 & 203.10 & 203.23 & 203.28 & 203.37 & 203.36 & 203.35 & 203.34 & 203.38 & 203.39 & 203.43 \\
\hline Near wall FS & 46.70 & 46.75 & 46.82 & 46.97 & 47.06 & 47.00 & 46.98 & 46.90 & 46.97 & 47.07 & 47.26 & 47.36 & 47.50 & 47.68 & 47.78 & 47.96 & 48.08 \\
\hline$T @ F X \# 1$ & 297.20 & 297.20 & 297.20 & 297.20 & 297.20 & 297.20 & 297.20 & 297.20 & 297.20 & 297.20 & 297.20 & 297.20 & 297.20 & 297.20 & 297.20 & 297.20 & 297.20 \\
\hline$T @ F X \# 2$ & 298.47 & 298.46 & 298.46 & 298.45 & 298.45 & 298.44 & 298.44 & 298.43 & 298.43 & 298.42 & 298.42 & 298.42 & 298.41 & 298.41 & 298.40 & 298.40 & 298.39 \\
\hline$T @ F X \# 3$ & 300.49 & 300.48 & 300.48 & 300.48 & 300.47 & 300.47 & 300.46 & 300.46 & 300.45 & 300.45 & 300.44 & 300.44 & 300.43 & 300.43 & 300.43 & 300.42 & 300.42 \\
\hline$T @ F X \# 4$ & 302.53 & 302.52 & 302.52 & 302.51 & 302.51 & 302.50 & 302.50 & 302.50 & 302.49 & 302.49 & 302.48 & 302.48 & 302.47 & 302.47 & 302.46 & 302.46 & 302.45 \\
\hline$T @ F X \# 5$ & 304.59 & 304.59 & 304.58 & 304.58 & 304.57 & 304.57 & 304.56 & 304.56 & 304.55 & 304.55 & 304.54 & 304.54 & 304.54 & 304.53 & 304.53 & 304.52 & 304.52 \\
\hline$T @ F X \# 6$ & 306.68 & 306.68 & 306.67 & 306.67 & 306.66 & 306.66 & 306.65 & 306.65 & 306.64 & 306.64 & 306.64 & 306.63 & 306.63 & 306.62 & 306.62 & 306.61 & 306.61 \\
\hline Wall $x$-coord & 81.74 & 81.75 & 81.77 & 81.78 & 81.79 & 81.80 & 81.81 & 81.82 & 81.83 & 81.84 & 81.86 & 81.87 & 81.88 & 81.89 & 81.90 & 81.91 & 81.92 \\
\hline Wall T & 309.85 & 309.85 & 309.86 & 309.85 & 309.85 & 309.85 & 309.85 & 309.84 & 309.84 & 309.83 & 309.82 & 309.82 & 309.82 & 309.82 & 309.81 & 309.80 & 309.80 \\
\hline
\end{tabular}

\section{B.29 Tabulated Data for Interferogram 485}

\begin{tabular}{|c|c|c|c|c|c|c|c|c|c|c|c|c|c|c|c|c|c|}
\hline Interferogram Image \# & 485 & Location & $T C 2$ & $\delta \varepsilon$ slope & $-2.4 I E-3$ & $\delta \varepsilon$ intercept & 12.69 & $A v g F S$ & 0.00 & Avg Wall $T$ & 306.99 & Gradient error & $-1.29 E-4$ & & & & \\
\hline Scan y-coord & 5210 & 5211 & 5212 & 5213 & 5214 & 5215 & 5216 & 5217 & 5218 & 5219 & 5220 & 5221 & 5222 & 5223 & 5224 & 5225 & 5226 \\
\hline$\delta \varepsilon$ & 0.1586 & 0.1562 & 0.1538 & 0.1514 & 0.1490 & 0.1466 & 0.1442 & 0.1418 & 0.1394 & 0.1370 & 0.1346 & 0.1322 & 0.1298 & 0.1274 & 0.1250 & 0.1226 & 0.1201 \\
\hline Scan $x$-coord & 930 & 930 & 930 & 930 & 930 & 930 & 930 & 930 & 930 & 930 & 930 & 930 & 930 & 930 & 930 & 930 & 930 \\
\hline$F X \# 1$ & 503.66 & 505.68 & 508.58 & 510.79 & 507.64 & 513.02 & 514.76 & 513.30 & 525.59 & 527.51 & 531.87 & 529.19 & 532.61 & 530.22 & 530.44 & 531.11 & 531.90 \\
\hline$F X \# 2$ & 302.62 & 302.70 & 302.85 & 303.04 & 302.99 & 302.92 & 303.07 & 303.38 & 303.44 & 303.37 & 303.84 & 304.10 & 304.11 & 304.29 & 304.65 & 305.12 & 305.61 \\
\hline$F X \# 3$ & 234.03 & 234.16 & 234.34 & 234.48 & 234.63 & 234.88 & 235.07 & 235.28 & 235.47 & 235.64 & 235.77 & 235.97 & 236.25 & 236.61 & 236.90 & 237.11 & 237.34 \\
\hline$F X \# 4$ & 189.01 & 189.13 & 189.21 & 189.43 & 189.68 & 189.95 & 190.16 & 190.37 & 190.57 & 190.78 & 191.04 & 191.26 & 191.39 & 191.55 & 191.71 & 191.84 & 191.97 \\
\hline$F X \# 5$ & 152.99 & 153.13 & 153.31 & 153.45 & 153.48 & 153.52 & 153.57 & 153.67 & 153.84 & 153.94 & 153.91 & 153.83 & 153.76 & 153.76 & 153.78 & 153.84 & 153.94 \\
\hline$F X \# 6$ & 0.00 & 0.00 & 0.00 & 0.00 & 0.00 & 0.00 & 0.00 & 0.00 & 0.00 & 0.00 & 0.00 & 0.00 & 0.00 & 0.00 & 0.00 & 0.00 & 0.00 \\
\hline$F X \# 7$ & 0.00 & 0.00 & 0.00 & 0.00 & 0.00 & 0.00 & 0.00 & 0.00 & 0.00 & 0.00 & 0.00 & 0.00 & 0.00 & 0.00 & 0.00 & 0.00 & 0.00 \\
\hline$F X \# 8$ & 0.00 & 0.00 & 0.00 & 0.00 & 0.00 & 0.00 & 0.00 & 0.00 & 0.00 & 0.00 & 0.00 & 0.00 & 0.00 & 0.00 & 0.00 & 0.00 & 0.00 \\
\hline Near wall FS & 0.00 & 0.00 & 0.00 & 0.00 & 0.00 & 0.00 & 0.00 & 0.00 & 0.00 & 0.00 & 0.00 & 0.00 & 0.00 & 0.00 & 0.00 & 0.00 & 0.00 \\
\hline$T(F X \# 1$ & 297.22 & 297.22 & 297.22 & 297.22 & 297.22 & 297.22 & 297.22 & 297.22 & 297.22 & 297.22 & 297.22 & 297.22 & 297.22 & 297.22 & 297.22 & 297.22 & 297.22 \\
\hline$T @ F X \# 2$ & 297.48 & 297.47 & 297.47 & 297.46 & 297.46 & 297.45 & 297.45 & 297.45 & 297.44 & 297.44 & 297.43 & 297.43 & 297.42 & 297.42 & 297.41 & 297.41 & 297.40 \\
\hline$T$ (a) $F X \# 3$ & 299.44 & 299.43 & 299.43 & 299.42 & 299.42 & 299.41 & 299.41 & 299.40 & 299.40 & 299.40 & 299.39 & 299.39 & 299.38 & 299.38 & 299.37 & 299.37 & 299.36 \\
\hline$T @ F X \# 4$ & 301.44 & 301.44 & 301.43 & 301.43 & 301.42 & 301.42 & 301.41 & 301.41 & 301.40 & 301.40 & 301.39 & 301.39 & 301.38 & 301.38 & 301.37 & 301.37 & 301.37 \\
\hline$T$ (a) $F X \# 5$ & 303.48 & 303.47 & 303.47 & 303.46 & 303.46 & 303.45 & 303.45 & 303.44 & 303.44 & 303.43 & 303.43 & 303.42 & 303.42 & 303.41 & 303.41 & 303.40 & 303.40 \\
\hline$T @ F X \# 6$ & 305.54 & 305.54 & 305.53 & 305.53 & 305.52 & 305.52 & 305.51 & 305.51 & 305.50 & 305.50 & 305.49 & 305.49 & 305.48 & 305.48 & 305.47 & 305.47 & 305.46 \\
\hline$T$ @ $F X \# 7$ & 307.62 & 307.61 & 307.61 & 307.60 & 307.60 & 307.59 & 307.59 & 307.58 & 307.58 & 307.57 & 307.57 & 307.56 & 307.56 & 307.55 & 307.55 & 307.54 & 307.54 \\
\hline$T(a) F \#$ & 309.74 & 309.74 & 309.73 & 309.73 & 309.72 & 309.72 & 309.71 & 309.71 & 309.70 & 309.70 & 309.69 & 309.69 & 309.68 & 309.68 & 309.67 & 309.67 & 309.66 \\
\hline Wall $x$-coord & 127.09 & 127.09 & 127.08 & 127.08 & 127.08 & 127.08 & 127.08 & 127.08 & 127.08 & 127.08 & 127.07 & 127.07 & 127.07 & 127.07 & 127.07 & 127.07 & 127.07 \\
\hline Wall T & 307.02 & 307.03 & 307.04 & 307.04 & 307.02 & 307.01 & 307.00 & 307.00 & 307.00 & 307.00 & 306.98 & 306.96 & 306.94 & 306.93 & 306.92 & 306.92 & 306.92 \\
\hline
\end{tabular}

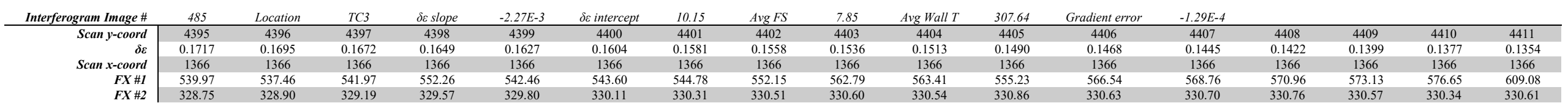




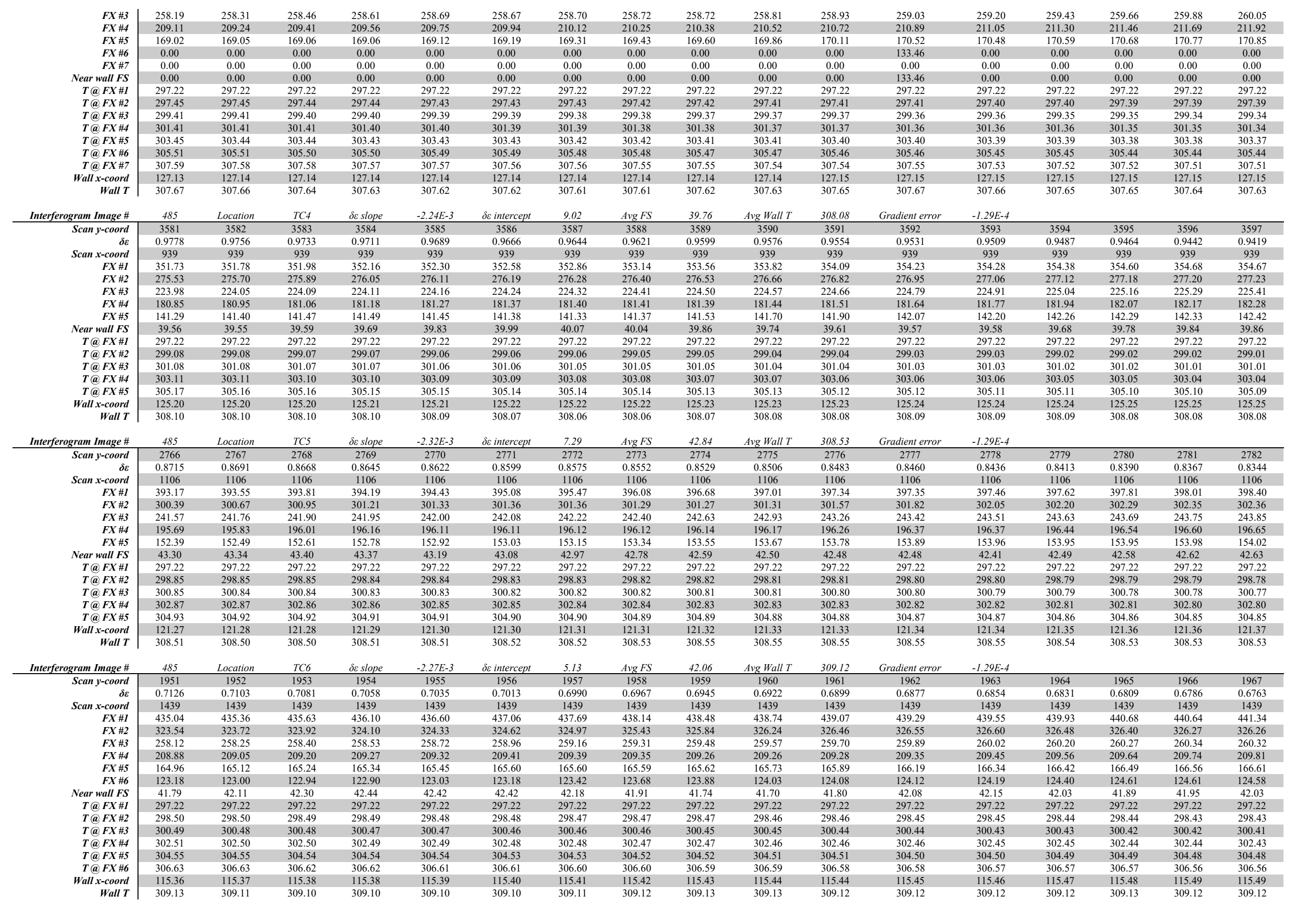




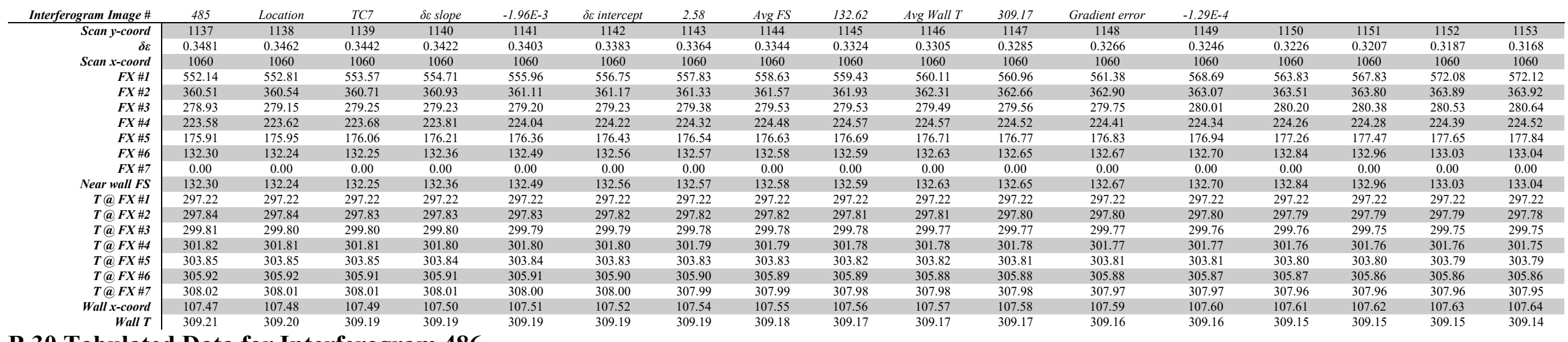

\section{B.30 Tabulated Data for Interferogram 486}

\begin{tabular}{|c|c|c|c|c|c|c|c|c|c|c|c|c|c|c|c|c|c|}
\hline Interferogram Image \# & 486 & Location & $T C 2$ & $\delta \varepsilon$ slope & $-2.49 E-3$ & $\delta \varepsilon$ intercept & 13.09 & $\operatorname{Avg} F S$ & 0.00 & Avg Wall $T$ & 307.12 & Gradient error & $7.92 E-5$ & & & & \\
\hline Scan y-coord & 5215 & 5216 & 5217 & 5218 & 5219 & 5220 & 5221 & 5222 & 5223 & 5224 & 5225 & 5226 & 5227 & 5228 & 5229 & 5230 & 5231 \\
\hline & 0.0951 & 0.0926 & 0.0901 & 0.0876 & 0.0851 & 0.0826 & 0.0801 & 0.0776 & 0.0751 & 0.0726 & 0.0701 & 0.0676 & 0.0651 & 0.0627 & 0.0602 & 0.0577 & 0.0552 \\
\hline Scan $x$-coord & 811 & 811 & 811 & 811 & 811 & 811 & 811 & 811 & 811 & 811 & 811 & 811 & 811 & 811 & 811 & 811 & 811 \\
\hline$F X \# 1$ & 543.32 & 541.97 & 543.29 & 541.42 & 539.43 & 544.12 & 541.89 & 549.79 & 574.81 & 590.84 & 637.19 & 639.63 & 638.06 & 637.82 & 636.86 & 730.41 & 741.48 \\
\hline$F X \# 2$ & 271.06 & 271.52 & 271.20 & 271.51 & 271.54 & 271.76 & 271.67 & 271.41 & 271.59 & 271.48 & 271.28 & 271.25 & 271.68 & 272.19 & 272.25 & 272.12 & 272.52 \\
\hline$F X \# 3$ & 193.67 & 193.97 & 194.28 & 194.49 & 194.67 & 194.85 & 195.06 & 195.23 & 195.44 & 195.67 & 195.91 & 196.18 & 196.38 & 196.44 & 196.52 & 196.66 & 196.82 \\
\hline$F X \# 5$ & 108.01 & 108.32 & 108.56 & 108.70 & 108.87 & 109.04 & 109.17 & 109.22 & 109.30 & 109.36 & 109.43 & 109.43 & 109.43 & 109.42 & 109.34 & 109.21 & 109.14 \\
\hline$F X \# 6$ & 0.00 & 0.00 & 0.00 & 0.00 & 0.00 & 0.00 & 0.00 & 0.00 & 0.00 & 0.00 & 0.00 & 0.00 & 0.00 & 0.00 & 0.00 & 0.00 & 0.00 \\
\hline $\begin{array}{l}F X \# \mathbb{0} \\
F X \# 7\end{array}$ & 0.00 & 0.00 & 0.00 & 0.00 & 0.00 & 0.00 & 0.00 & 0.00 & 0.00 & 0.00 & 0.00 & 0.00 & 0.00 & 0.00 & 0.00 & 0.00 & 0.00 \\
\hline Near wall FS & 0.00 & 0.00 & 0.00 & 0.00 & 0.00 & 0.00 & 0.00 & 0.00 & 0.00 & 0.00 & 0.00 & 0.00 & 0.00 & 0.00 & 0.00 & 0.00 & 0.00 \\
\hline$T @ F X \# 1$ & 297.18 & 297.18 & 297.18 & 297.18 & 297.18 & 297.18 & 297.18 & 297.18 & 297.18 & 297.18 & 297.18 & 297.18 & 297.18 & 297.18 & 297.18 & 297.18 & 297.18 \\
\hline$T @ F X \# 2$ & 297.39 & 297.38 & 297.38 & 297.37 & 297.37 & 297.36 & 297.36 & 297.35 & 297.35 & 297.34 & 297.33 & 297.33 & 297.32 & 297.32 & 297.31 & 297.30 & 297.29 \\
\hline$T @ F X \# 4$ & 301.41 & 301.41 & 301.40 & 301.40 & 301.39 & 301.39 & 301.38 & 301.38 & 301.37 & 301.37 & 301.36 & 301.36 & 301.35 & 301.35 & 301.34 & 301.34 & 301.33 \\
\hline$T @ F X \# 5$ & 303.46 & 303.45 & 303.45 & 303.44 & 303.44 & 303.43 & 303.43 & 303.42 & 303.42 & 303.41 & 303.41 & 303.40 & 303.39 & 303.39 & 303.38 & 303.38 & 303.37 \\
\hline$T @ F X \# 6$ & 305.53 & 305.52 & $\begin{array}{l}305.45 \\
305.52\end{array}$ & 305.51 & 305.51 & 305.50 & 305.50 & $\begin{array}{l}305.42 \\
305.49\end{array}$ & $\begin{array}{l}305.42 \\
305.49\end{array}$ & $\begin{array}{l}305.41 \\
305.48\end{array}$ & $\begin{array}{l}305.41 \\
305.48\end{array}$ & $\begin{array}{l}305.47 \\
30.47\end{array}$ & 305.46 & 305.46 & $\begin{array}{l}305.30 \\
305\end{array}$ & $\begin{array}{l}305.30 \\
305\end{array}$ & 305.44 \\
\hline 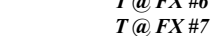 & $\begin{array}{l}305.53 \\
307.63\end{array}$ & $\begin{array}{l}305.52 \\
307.63\end{array}$ & $\begin{array}{l}305.52 \\
307.62\end{array}$ & $\begin{array}{l}305.51 \\
307.62\end{array}$ & $\begin{array}{l}305.51 \\
307.61\end{array}$ & $\begin{array}{l}305.50 \\
307.61\end{array}$ & $\begin{array}{l}305.50 \\
307.60\end{array}$ & $\begin{array}{l}305.49 \\
307.60\end{array}$ & $\begin{array}{l}303.49 \\
307.59\end{array}$ & $\begin{array}{r}305.48 \\
307.58\end{array}$ & $\begin{array}{l}305.48 \\
307.58\end{array}$ & $\begin{array}{l}305.41 \\
307.57\end{array}$ & $\begin{array}{l}300.46 \\
307.57\end{array}$ & $\begin{array}{l}305.46 \\
307.56\end{array}$ & $\begin{array}{l}305.45 \\
307.56\end{array}$ & $\begin{array}{l}305.45 \\
307.55\end{array}$ & $\begin{array}{l}305.44 \\
307.55\end{array}$ \\
\hline Wall $x$-coord & 78.79 & 78.79 & 78.79 & 78.79 & 78.79 & 78.79 & 78.79 & 78.79 & 78.79 & 78.79 & 78.79 & 78.79 & 78.79 & 78.79 & 78.78 & 78.78 & 78.78 \\
\hline Wall T & 307.10 & 307.12 & 307.13 & 307.13 & 307.13 & 307.14 & 307.14 & 307.14 & 307.14 & 307.14 & 307.13 & 307.12 & 307.11 & 307.10 & 307.08 & 307.05 & 307.04 \\
\hline Interferogram Image \# & 486 & Location & $T C 3$ & $\delta \varepsilon$ slope & $-2.45 E-3$ & $\delta \varepsilon$ intercept & 10.91 & $\operatorname{Avg} F S$ & 0.00 & Avg Wall T & 308.06 & Gradient error & $7.92 E-5$ & & & & \\
\hline Scan y-coord & 4401 & 4402 & 4403 & 4404 & 4405 & 4406 & 4407 & 4408 & 4409 & 4410 & 4411 & 4412 & 4413 & 4414 & 4415 & 4416 & 4417 \\
\hline & 0.1418 & 0.1394 & 0.1369 & 0.1345 & 0.1320 & 0.1296 & 0.1271 & 0.1247 & 0.1223 & 0.1198 & 0.1174 & 0.1149 & 0.1125 & 0.1100 & 0.1076 & 0.1051 & 0.1027 \\
\hline Scan $x$-coord & 925 & 925 & 925 & 925 & 925 & 925 & 925 & 925 & 925 & 925 & 925 & 925 & 925 & 925 & 925 & 925 & 925 \\
\hline 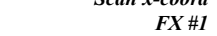 & 515.24 & 515.87 & 518.14 & 519.12 & 520.43 & 521.68 & 522.48 & 519.58 & 523.29 & 530.79 & 531.74 & 531.87 & 533.28 & 534.29 & 546.73 & 540.55 & 545.03 \\
\hline$F X \# 2$ & 293.55 & 293.84 & 294.31 & 294.79 & 295.15 & 295.56 & 295.96 & 296.34 & 296.39 & 296.26 & 296.11 & 296.04 & 295.92 & 296.04 & 293.97 & 294.08 & 294.20 \\
\hline$F X \# 5$ & $\begin{array}{l}105.00 \\
123.89\end{array}$ & 123.90 & 123.87 & 123.86 & 100.44 & $\begin{array}{l}100.00 \\
123.83\end{array}$ & $\begin{array}{l}100.12 \\
123.84\end{array}$ & 100.04 & 123.97 & 124.10 & 124.26 & 124.47 & $\begin{array}{l}124.43 \\
124.64\end{array}$ & 124.85 & 125.06 & 125.28 & 125.45 \\
\hline$F X \#$ & $\begin{array}{l}125.39 \\
84.36\end{array}$ & 84.59 & 84.80 & $\begin{array}{l}84.70 \\
849\end{array}$ & $\begin{array}{c}123.04 \\
0.00\end{array}$ & 84.98 & $\begin{array}{l}115.24 \\
85.20\end{array}$ & 85.46 & 85.58 & 85.73 & $\begin{array}{l}124.200 \\
85.77\end{array}$ & 85.97 & $\begin{array}{l}184.04 \\
86.15\end{array}$ & $\begin{array}{l}14.83 \\
86.26\end{array}$ & $\begin{array}{l}115.00 \\
86.22\end{array}$ & 86.25 & 86.20 \\
\hline$F X \# 7$ & 0.00 & 0.00 & 0.00 & 0.00 & 0.00 & 0.00 & 0.00 & 0.00 & 0.00 & 0.00 & 0.00 & 0.00 & 0.00 & 0.00 & 0.00 & 0.00 & 0.00 \\
\hline $\begin{array}{l}F X \# \# \\
F X \# 8\end{array}$ & 0.00 & 0.00 & 0.00 & 0.00 & 0.00 & 0.00 & $\begin{array}{l}0.00 \\
0.00\end{array}$ & 0.00 & 0.00 & 0.00 & 0.00 & 0.00 & $\begin{array}{l}0.00 \\
0.00\end{array}$ & 0.00 & 0.00 & 0.00 & 0.00 \\
\hline Near wall FS & 0.00 & 0.00 & 0.00 & 0.00 & 0.00 & 0.00 & 0.00 & 0.00 & 0.00 & 0.00 & 0.00 & 0.00 & 0.00 & 0.00 & 0.00 & 0.00 & 0.00 \\
\hline$T @ F X \# I$ & 297.18 & 297.18 & 297.18 & 297.18 & 297.18 & 297.18 & 297.18 & 297.18 & 297.18 & 297.18 & 297.18 & 297.18 & 297.18 & 297.18 & 297.18 & 297.18 & 297.18 \\
\hline$T @ F X \# 2$ & 297.49 & 297.49 & 297.48 & 297.48 & 297.47 & 297.47 & 297.46 & 297.46 & 297.45 & 297.45 & 297.44 & 297.44 & 297.43 & 297.43 & 297.42 & 297.42 & 297.41 \\
\hline$T @ F X \# 3$ & 299.49 & 299.49 & 299.49 & 299.48 & 299.48 & 299.47 & 299.47 & 299.46 & 299.46 & 299.45 & 299.45 & 299.44 & 299.44 & 299.43 & 299.43 & 299.42 & 299.42 \\
\hline$T @ F X \# 4$ & 301.51 & 301.51 & 301.50 & 301.50 & 301.50 & 301.49 & 301.49 & 301.48 & 301.48 & 301.47 & 301.47 & 301.46 & 301.46 & 301.45 & 301.45 & 301.44 & 301.44 \\
\hline $\begin{array}{l}T @ F X \# \# \\
T(a) F \# 5\end{array}$ & $\begin{array}{l}301.51 \\
303.56\end{array}$ & $\begin{array}{l}301.51 \\
303.56\end{array}$ & $\begin{array}{l}301.50 \\
303.55\end{array}$ & $\begin{array}{l}301.50 \\
303.55\end{array}$ & $\begin{array}{l}301.50 \\
303.54\end{array}$ & $\begin{array}{l}301.49 \\
303.54\end{array}$ & $\begin{array}{l}301.49 \\
303.53\end{array}$ & $\begin{array}{l}301.48 \\
303.53\end{array}$ & $\begin{array}{l}301.48 \\
303.52\end{array}$ & $\begin{array}{l}300.41 \\
303.52\end{array}$ & $\begin{array}{l}301.41 \\
303.51\end{array}$ & $\begin{array}{l}301.46 \\
303.50\end{array}$ & $\begin{array}{l}300.46 \\
303.50\end{array}$ & $\begin{array}{l}301.43 \\
303.49\end{array}$ & $\begin{array}{l}301.45 \\
303.49\end{array}$ & $\begin{array}{l}300.44 \\
303.48\end{array}$ & $\begin{array}{l}301.44 \\
303.48\end{array}$ \\
\hline$T @ F X \# 6$ & 305.63 & 305.63 & 305.62 & 305.62 & 305.61 & 305.61 & 305.60 & 305.60 & 305.59 & 305.59 & 305.58 & 305.58 & 305.57 & 305.57 & 305.56 & 305.56 & 305.55 \\
\hline$T @ F X \# 7$ & 307.73 & 307.73 & 307.72 & 307.72 & 307.72 & 307.71 & 307.70 & 307.70 & 307.69 & 307.69 & 307.68 & 307.68 & 307.67 & 307.67 & 307.66 & 307.66 & 307.65 \\
\hline$T @ F X \# 8$ & 309.87 & 309.86 & 309.86 & 309.85 & 309.85 & 309.84 & 309.84 & 309.83 & 309.83 & 309.82 & 309.81 & 309.81 & 309.80 & 309.80 & 309.79 & 309.79 & 309.78 \\
\hline Wall $x$-coord & 78.34 & 78.34 & 78.34 & 78.34 & 78.34 & 78.35 & 78.35 & 78.35 & 78.35 & 78.35 & 78.35 & 78.36 & 78.36 & 78.36 & 78.36 & 78.36 & 78.36 \\
\hline Wall T & 308.05 & 308.06 & 308.07 & 308.06 & 307.81 & 308.07 & 308.08 & 308.09 & 308.09 & 308.09 & 308.09 & 308.09 & 308.10 & 308.10 & 308.09 & 308.08 & 308.07 \\
\hline Interferogram Image \# & 486 & Location & $T C 4$ & $\delta \varepsilon$ slope & $-2.44 E-3$ & $\delta$ s intercept & 8.85 & $\operatorname{Avg} F S$ & 41.50 & Avg Wall $T$ & 308.20 & Gradient error & $7.92 E-5$ & & & & \\
\hline Scan y-coord & 3587 & 3588 & 3589 & 3590 & 3591 & 3592 & 3593 & 3594 & 3595 & 3596 & 3597 & 3598 & 3599 & 3600 & 3601 & 3602 & 3603 \\
\hline & 0.0831 & 0.0806 & 0.0782 & 0.0757 & 0.0733 & 0.0708 & 0.0684 & 0.0660 & 0.0635 & 0.0611 & 0.0586 & 0.0562 & 0.0537 & 0.0513 & 0.0489 & 0.0464 & 0.0440 \\
\hline Scan $x$-coord & 1135 & 1135 & 1135 & 1135 & 1135 & 1135 & 1135 & 1135 & 1135 & 1135 & 1135 & 1135 & 1135 & 1135 & 1135 & 1135 & 1135 \\
\hline
\end{tabular}




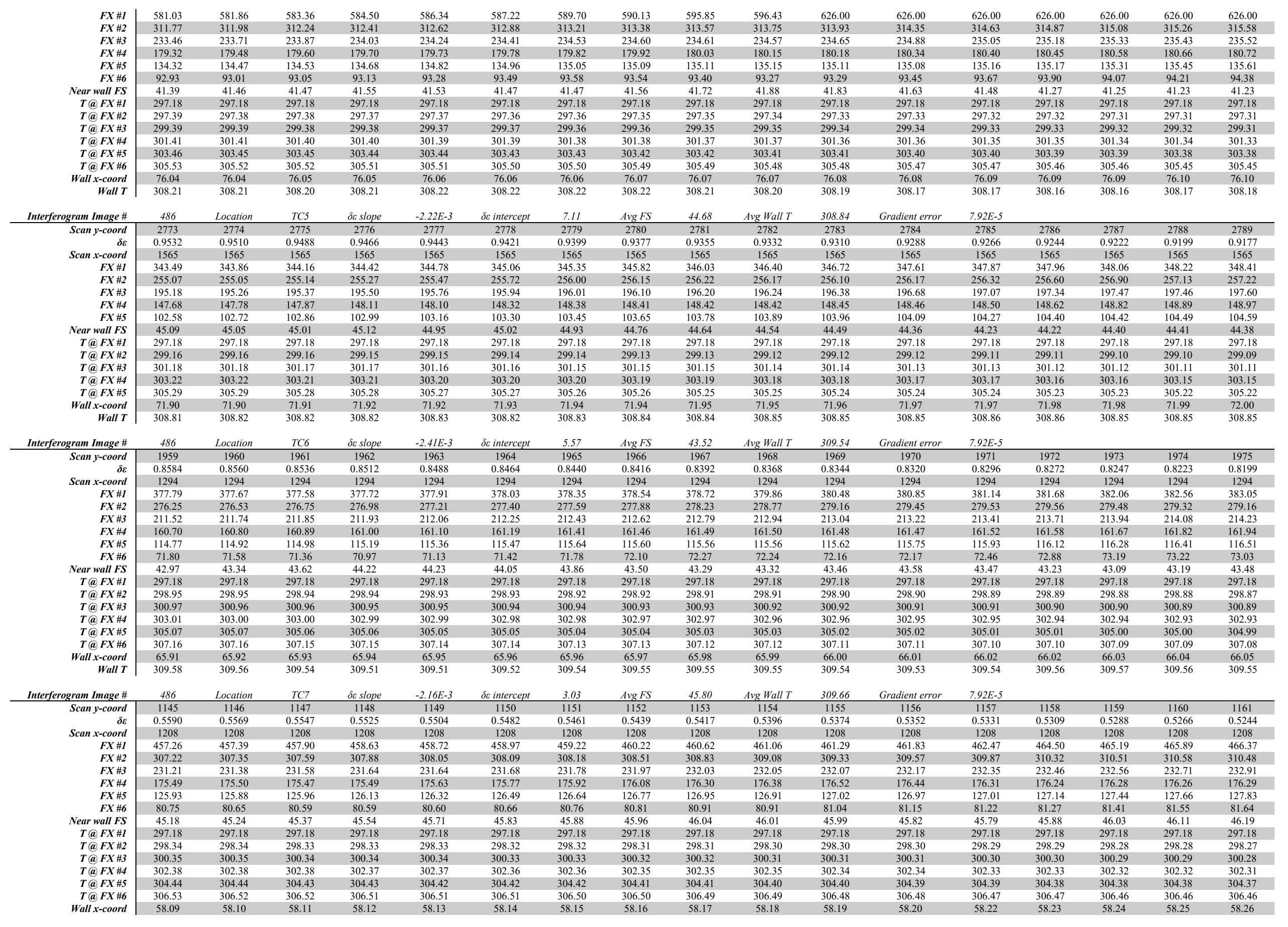




\section{B.31 Tabulated Data for Interferogram 487}

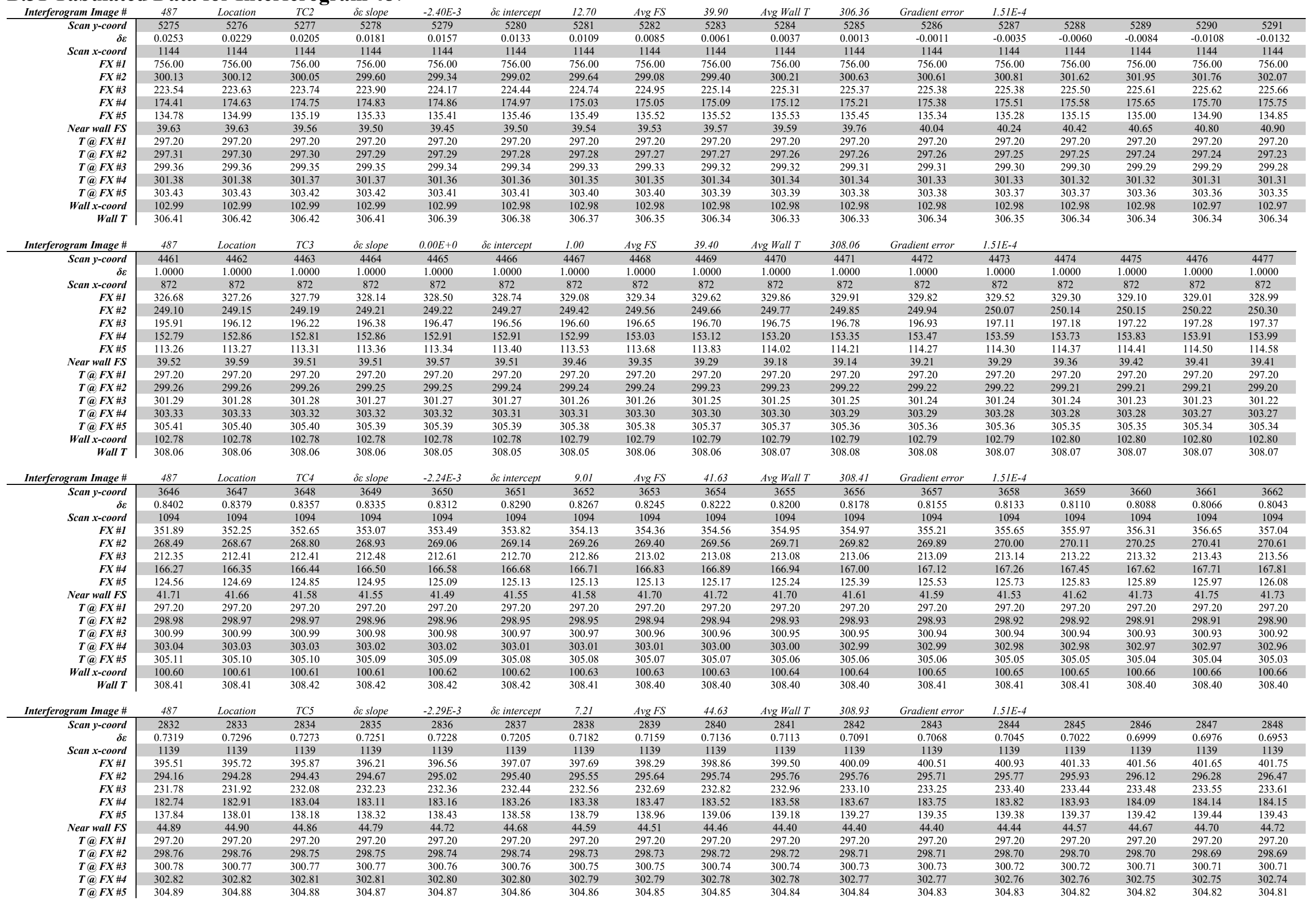




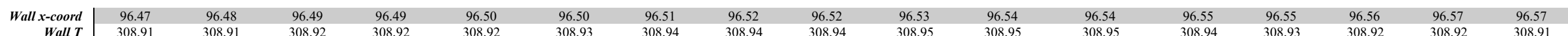

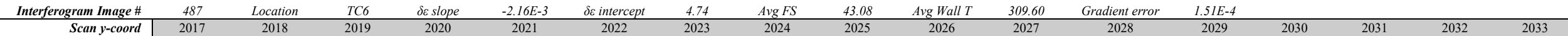

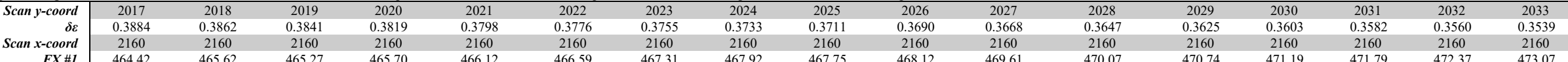

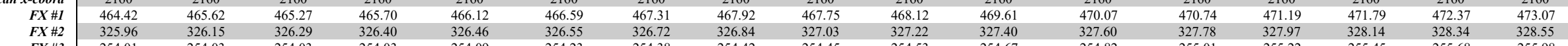

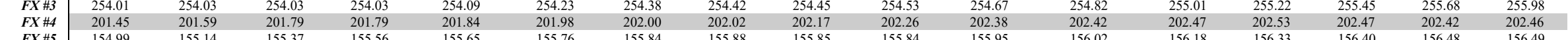

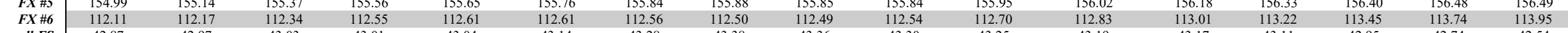

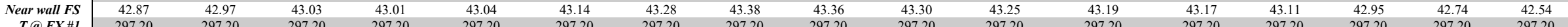

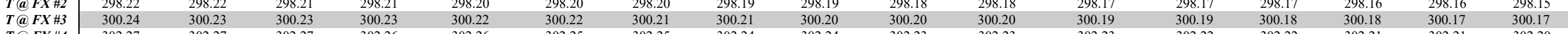

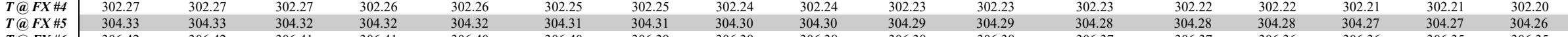

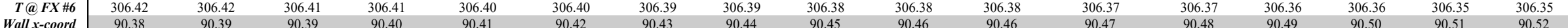

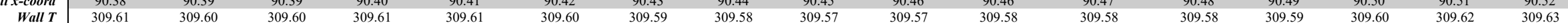

\begin{tabular}{|c|c|c|c|c|c|c|c|c|c|c|c|c|c|c|c|c|c|}
\hline Interferogram Image \# & 487 & Location & $T C 7$ & $\delta \varepsilon$ slope & $-2.18 E-3$ & $\delta \varepsilon$ intercept & 2.73 & $\operatorname{Avg} F S$ & 46.00 & Avg Wall $T$ & 309.72 & Gradient error & $1.51 E-4$ & & & & \\
\hline Scan y-coord & 1203 & 1204 & 1205 & 1206 & 1207 & 1208 & 1209 & 1210 & 1211 & 1212 & 1213 & 1214 & 1215 & 1216 & 1217 & 1218 & 1219 \\
\hline & 0.1089 & 0.1068 & 0.1046 & 0.1024 & 0.1002 & 0.0981 & 0.0959 & 0.0937 & 0.0915 & 0.0893 & 0.0872 & 0.0850 & 0.0828 & 0.0806 & 0.0785 & 0.0763 & 0.0741 \\
\hline Scan $x$-coord & 1668 & 1668 & 1668 & 1668 & 1668 & 1668 & 1668 & 1668 & 1668 & 1668 & 1668 & 1668 & 1668 & 1668 & 1668 & 1668 & 1668 \\
\hline$F X \# 1$ & 765.00 & 765.00 & 765.00 & 765.00 & 765.00 & 765.00 & 765.00 & 765.00 & 765.00 & 765.00 & 765.00 & 765.00 & 765.00 & 765.00 & 765.00 & 765.00 & 765.00 \\
\hline$F X \# 2$ & 372.49 & 372.92 & 373.27 & 373.31 & 373.51 & 373.69 & 374.34 & 374.45 & 374.30 & 374.25 & 374.30 & 374.37 & 374.39 & 374.43 & 374.50 & 374.73 & 374.79 \\
\hline$F X \# 3$ & 280.31 & 280.51 & 280.75 & 280.95 & 281.13 & 281.24 & 281.31 & 281.34 & 281.35 & 281.51 & 281.68 & 281.94 & 282.14 & 282.30 & 282.53 & 282.79 & 282.98 \\
\hline$F X \# 4$ & 219.60 & 219.70 & 219.79 & 219.83 & 219.87 & 219.88 & 219.95 & 220.03 & 220.12 & 220.16 & 220.24 & 220.32 & 220.44 & 220.55 & 220.60 & 220.58 & 220.58 \\
\hline$F X \# 5$ & 168.64 & 168.75 & 168.96 & 169.17 & 169.39 & 169.59 & 169.74 & 169.86 & 170.06 & 170.09 & 170.12 & 170.12 & 170.18 & 170.29 & 170.48 & 170.72 & 170.90 \\
\hline$F X \# 6$ & 123.08 & 123.20 & 123.27 & 123.33 & 123.39 & 123.46 & 123.55 & 123.67 & 123.70 & 123.76 & 123.83 & 124.03 & 124.25 & 124.40 & 124.57 & 124.69 & 124.78 \\
\hline Near wall $F S$ & 45.55 & 45.55 & 45.69 & 45.84 & 46.00 & 46.13 & 46.19 & 46.19 & 46.36 & 46.34 & 46.29 & 46.09 & 45.93 & 45.88 & 45.91 & 46.03 & 46.12 \\
\hline$T @ F X \# 1$ & 297.20 & 297.20 & 297.20 & 297.20 & 297.20 & 297.20 & 297.20 & 297.20 & 297.20 & 297.20 & 297.20 & 297.20 & 297.20 & 297.20 & 297.20 & 297.20 & 297.20 \\
\hline$T$ (a) $F X \# 2$ & 297.55 & 297.55 & 297.54 & 297.54 & 297.53 & 297.53 & 297.52 & 297.52 & 297.52 & 297.51 & 297.51 & 297.50 & 297.50 & 297.49 & 297.49 & 297.49 & 297.48 \\
\hline$T @ F X \# 3$ & 299.60 & 299.59 & 299.59 & 299.58 & 299.58 & 299.57 & 299.57 & 299.57 & 299.56 & 299.56 & 299.55 & 299.55 & 299.54 & 299.54 & 299.54 & 299.53 & 299.53 \\
\hline$T @ F X \# 4$ & 301.63 & 301.62 & 301.62 & 301.61 & 301.61 & 301.60 & 301.60 & 301.59 & 301.59 & 301.59 & 301.58 & 301.58 & 301.57 & 301.57 & 301.56 & 301.56 & 301.55 \\
\hline$T @ F X \# 5$ & 303.68 & 303.67 & 303.67 & 303.66 & 303.66 & 303.66 & 303.65 & 303.65 & 303.64 & 303.64 & 303.63 & 303.63 & 303.62 & 303.62 & 303.62 & 303.61 & 303.61 \\
\hline$T @ F X \# 6$ & 305.76 & 305.75 & 305.75 & 305.74 & 305.74 & 305.73 & 305.73 & 305.72 & 305.72 & 305.72 & 305.71 & 305.71 & 305.70 & 305.70 & 305.69 & 305.69 & 305.68 \\
\hline Wall $x$-coord & 82.33 & 82.34 & 82.35 & 82.36 & 82.37 & 82.39 & 82.40 & 82.41 & 82.42 & 82.43 & 82.44 & 82.45 & 82.46 & 82.47 & 82.49 & 82.50 & 82.51 \\
\hline & & & & & & & & & & & & & & & & & \\
\hline
\end{tabular}

\section{B.32 Tabulated Data for Interferogram 488}

\begin{tabular}{|c|c|c|c|c|c|c|c|c|c|c|c|c|c|c|c|c|c|}
\hline Interferogram Image \# & 488 & Location & $T C 2$ & $\partial \varepsilon$ slope & $-2.53 E-3$ & $\delta \varepsilon$ intercept & 14.14 & Avg FS & 41.26 & Avg Wall T & 306.87 & Gradient error & $1.15 E-4$ & & & & \\
\hline Scan $y$-coord & 5203 & 5204 & 5205 & 5206 & 5207 & 5208 & 5209 & 5210 & 5211 & 5212 & 5213 & 5214 & 5215 & 5216 & 5217 & 5218 & 5219 \\
\hline & 0.9935 & 0.9910 & 0.9884 & 0.9859 & 0.9834 & 0.9809 & 0.9783 & 0.9758 & 0.9733 & 0.9708 & 0.9682 & 0.9657 & 0.9632 & 0.9607 & 0.9581 & 0.9556 & 0.9531 \\
\hline Scan $x$-coord & 1296 & 1296 & 1296 & 1296 & 1296 & 1296 & 1296 & 1296 & 1296 & 1296 & 1296 & 1296 & 1296 & 1296 & 1296 & 1296 & 1296 \\
\hline$F X \# I$ & 312.26 & 312.30 & 312.15 & 311.87 & 311.42 & 311.52 & 311.73 & 312.34 & 312.57 & 312.31 & 312.37 & 312.65 & 313.30 & 314.04 & 314.33 & 314.52 & 314.66 \\
\hline$F X \# 2$ & 231.89 & 231.96 & 232.17 & 232.36 & 232.66 & 232.98 & 233.22 & 233.33 & 233.43 & 233.49 & 233.47 & 233.48 & 233.50 & 233.56 & 233.66 & 233.76 & 233.81 \\
\hline$F X \# 3$ & 181.01 & 181.12 & 181.18 & 181.27 & 181.47 & 181.66 & 181.77 & 181.82 & 181.87 & 181.95 & 182.08 & 182.16 & 182.16 & 182.22 & 182.26 & 182.40 & 182.39 \\
\hline$F X \# 4$ & 140.35 & 140.45 & 140.53 & 140.58 & 140.65 & 140.71 & 140.78 & 140.80 & 140.78 & 140.62 & 140.47 & 140.44 & 140.48 & 140.49 & 140.44 & 140.40 & 140.42 \\
\hline Near wall FS & 40.66 & 40.66 & 40.65 & 40.68 & 40.82 & 40.94 & 40.99 & 41.03 & 41.09 & 41.33 & 41.61 & 41.71 & 41.68 & 41.72 & 41.82 & 41.99 & 41.97 \\
\hline$T(F X \# 1$ & 297.20 & 297.20 & 297.20 & 297.20 & 297.20 & 297.20 & 297.20 & 297.20 & 297.20 & 297.20 & 297.20 & 297.20 & 297.20 & 297.20 & 297.20 & 297.20 & 297.20 \\
\hline$T @ F X \# 2$ & 299.29 & 299.28 & 299.28 & 299.27 & 299.27 & 299.26 & 299.26 & 299.25 & 299.25 & 299.24 & 299.24 & 299.23 & 299.22 & 299.22 & 299.21 & 299.21 & 299.20 \\
\hline$T @ F X \# 3$ & 301.31 & 301.30 & 301.30 & 301.29 & 301.29 & 301.28 & 301.28 & 301.27 & 301.26 & 301.26 & 301.25 & 301.25 & 301.24 & 301.24 & 301.23 & 301.23 & 301.22 \\
\hline$T @ F X \# 4$ & 303.35 & 303.35 & 303.34 & 303.33 & 303.33 & 303.32 & 303.32 & 303.31 & 303.31 & 303.30 & 303.30 & 303.29 & 303.29 & 303.28 & 303.28 & 303.27 & 303.27 \\
\hline Wall $x$-coord & 110.91 & 110.91 & 110.91 & 110.91 & 110.91 & 110.91 & 110.90 & 110.90 & 110.90 & 110.90 & 110.90 & 110.90 & 110.90 & 110.90 & 110.90 & 110.90 & 110.90 \\
\hline Wall T & 306.92 & 306.92 & 306.92 & 306.92 & 306.91 & 306.90 & 306.90 & 306.89 & 306.88 & 306.86 & 306.84 & 306.83 & 306.83 & 306.82 & 306.81 & 306.80 & 306.79 \\
\hline Interferogram Image \# & 488 & Location & $T C 3$ & $\delta \varepsilon$ slope & $-2.44 E-3$ & $\delta \varepsilon$ intercept & 10.79 & $\operatorname{Avg} F S$ & 44.35 & Avg Wall T & 307.63 & Gradient error & $1.15 E-4$ & & & & \\
\hline Scan y-coord & 4388 & 4389 & 4390 & 4391 & 4392 & 4393 & 4394 & 4395 & 4396 & 4397 & 4398 & 4399 & 4400 & 4401 & 4402 & 4403 & 4404 \\
\hline & 0.0730 & 0.0706 & 0.0681 & 0.0657 & 0.0632 & 0.0608 & 0.0584 & 0.0559 & 0.0535 & 0.0510 & 0.0486 & 0.0462 & 0.0437 & 0.0413 & 0.0388 & 0.0364 & 0.0339 \\
\hline Scan $x$-coord & 1097 & 1097 & 1097 & 1097 & 1097 & 1097 & 1097 & 1097 & 1097 & 1097 & 1097 & 1097 & 1097 & 1097 & 1097 & 1097 & 1097 \\
\hline$F X \# 1$ & 679.00 & 679.00 & 679.00 & 679.00 & 679.00 & 679.00 & 679.00 & 679.00 & 679.00 & 679.00 & 679.00 & 679.00 & 679.00 & 679.00 & 679.00 & 679.00 & 679.00 \\
\hline$F X \# 2$ & 329.63 & 330.15 & 330.65 & 331.04 & 331.45 & 331.99 & 332.25 & 332.58 & 332.90 & 333.15 & 333.31 & 333.29 & 333.16 & 333.07 & 333.08 & 332.97 & 332.88 \\
\hline$F X \# 3$ & 252.28 & 252.39 & 252.50 & 252.58 & 252.64 & 252.74 & 252.86 & 252.93 & 253.02 & 253.13 & 253.20 & 253.29 & 253.43 & 253.51 & 253.55 & 253.58 & 253.62 \\
\hline$F X \# 4$ & 198.57 & 198.76 & 198.91 & 199.06 & 199.21 & 199.33 & 199.47 & 199.61 & 199.70 & 199.83 & 199.98 & 200.09 & 200.25 & 200.39 & 200.50 & 200.58 & 200.66 \\
\hline$F X \# 5$ & 154.65 & 154.70 & 154.75 & 154.85 & 154.99 & 155.08 & 155.14 & 155.29 & 155.44 & 155.54 & 155.65 & 155.71 & 155.75 & 155.78 & 155.81 & 155.86 & 155.95 \\
\hline Near wall FS & 43.92 & 44.07 & 44.16 & 44.21 & 44.22 & 44.25 & 44.34 & 44.32 & 44.26 & 44.29 & 44.32 & 44.38 & 44.50 & 44.61 & 44.69 & 44.72 & 44.71 \\
\hline$T @ F X \# 1$ & 297.20 & 297.20 & 297.20 & 297.20 & 297.20 & 297.20 & 297.20 & 297.20 & 297.20 & 297.20 & 297.20 & 297.20 & 297.20 & 297.20 & 297.20 & 297.20 & 297.20 \\
\hline$T @ F X \# 2$ & 297.39 & 297.39 & 297.38 & 297.38 & 297.37 & 297.37 & 297.37 & 297.36 & 297.36 & 297.35 & 297.35 & 297.34 & 297.34 & 297.33 & 297.33 & 297.32 & 297.32 \\
\hline
\end{tabular}




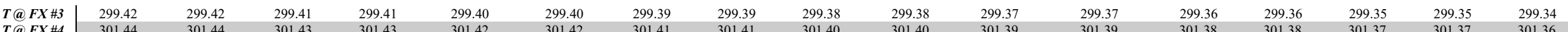

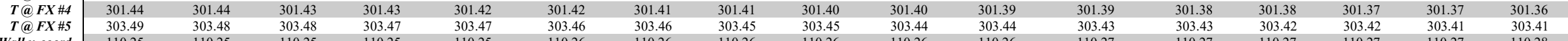

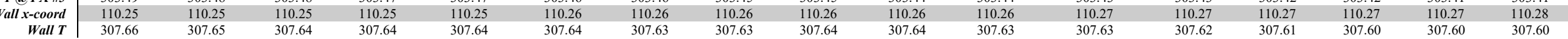

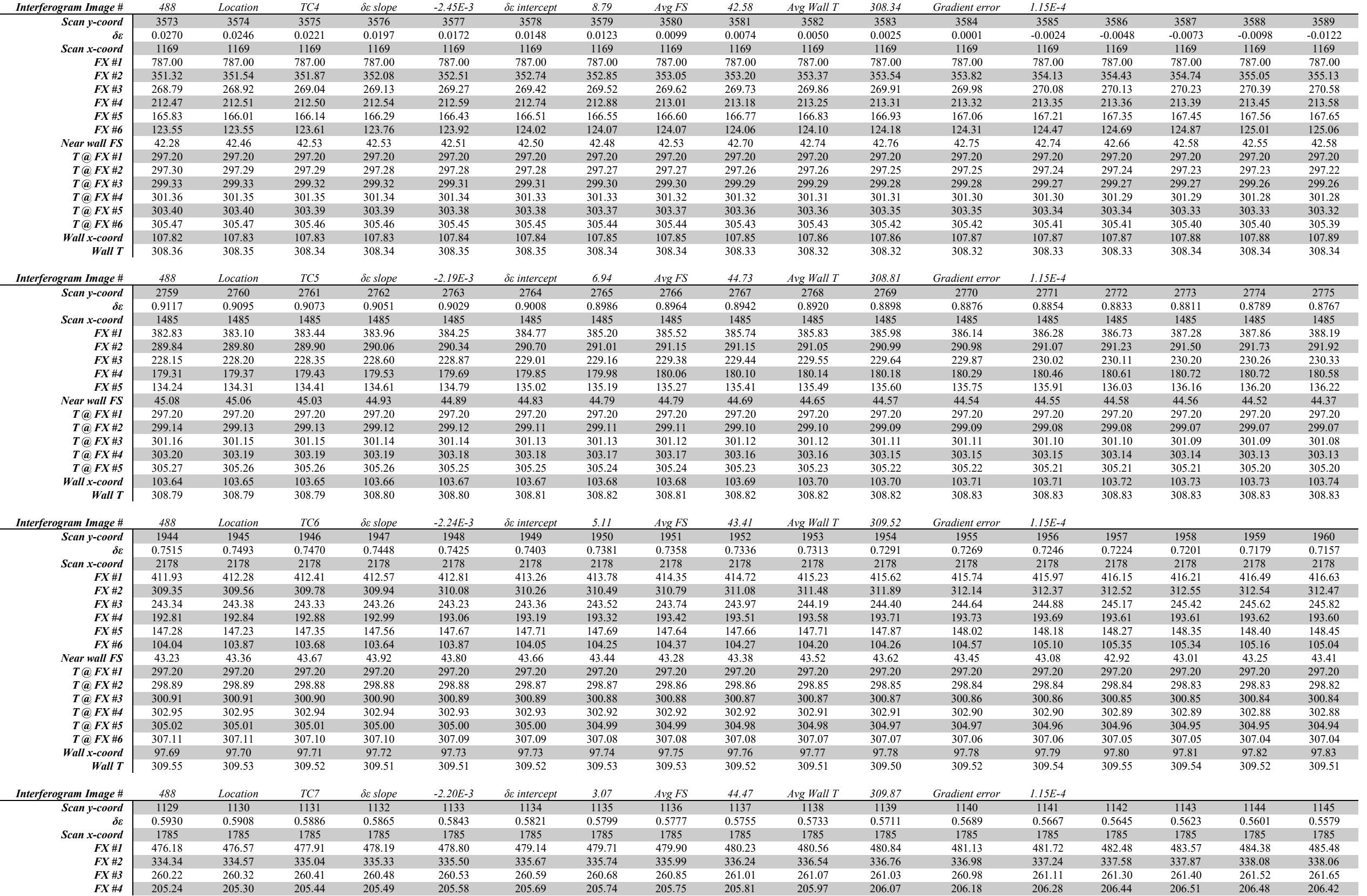




\begin{tabular}{|c|c|c|c|c|c|c|c|c|c|c|c|c|c|c|c|c|c|}
\hline$F X \# 5$ & 155.97 & 155.91 & 155.97 & 15611 & 15626 & 15647 & 15668 & 15682 & 156.95 & 15716 & 15723 & 157.25 & 157.26 & 157.32 & 157.46 & 157.56 & 157.75 \\
\hline$F X \# 6$ & 111.84 & 111.83 & 111.92 & 111.94 & 111.98 & 112.08 & 112.24 & 112.37 & 112.46 & 112.62 & 112.69 & 112.70 & 112.66 & 112.65 & 112.70 & 112.70 & 112.86 \\
\hline Near wall FS & 44.13 & 44.09 & 44.05 & 44.18 & 44.28 & 44.39 & 44.44 & 44.45 & 44.48 & 44.54 & 44.55 & 44.55 & 44.61 & 44.68 & 44.76 & 44.87 & 4489 \\
\hline$T @ F X \# 1$ & 297.20 & 297.20 & 297.20 & 297.20 & 297.20 & 297.20 & 297.20 & 297.20 & 297.20 & 297.20 & 297.20 & 297.20 & 297.20 & 297.20 & 297.20 & 297.20 & 297.20 \\
\hline$T$ (a) $F X \# 2$ & 298.53 & 298.52 & 298.52 & 298.51 & 298.51 & 298.50 & 298.50 & 298.49 & 298.49 & 298.49 & 298.48 & 298.48 & 298.47 & 298.47 & 298.46 & 298.46 & 298.46 \\
\hline$T(a) \# \#$ & 300.54 & 300.54 & 300.53 & 300.53 & 300.53 & 300.52 & 300.52 & 300.51 & 300.51 & 300.50 & 300.50 & 300.49 & 300.49 & 300.49 & 300.48 & 300.48 & 300.47 \\
\hline$T(a) F X \# 4$ & 302.58 & 302.58 & 302.57 & 302.57 & 302.56 & 302.56 & 302.55 & 302.55 & 302.54 & 302.54 & 302.53 & 302.53 & 302.53 & 302.52 & 302.52 & 302.51 & 302.51 \\
\hline$T$ (a) $F X \# 5$ & 304.64 & 304.64 & 304.63 & 304.63 & 304.62 & 304.62 & 304.61 & 304.61 & 304.61 & 304.60 & 304.60 & 304.59 & 304.59 & 304.58 & 304.58 & 304.57 & 304.57 \\
\hline$T @ F X \# 6$ & 306,73 & 306.73 & 306,72 & 306,72 & 306.71 & 306.71 & 306.70 & 306,70 & 306.69 & 306.69 & 306.69 & 306.68 & 306.68 & 306.67 & 306.67 & 306.66 & 306.66 \\
\hline Wall $x$-coord & 89.98 & 89.99 & 90.00 & 90.01 & 90.02 & 90.03 & 90.04 & 90.05 & 90.06 & 90.08 & 90.09 & 90.10 & 90.11 & 90.12 & 90.13 & 90.14 & 90.15 \\
\hline Wall $T$ & 309.90 & 309.89 & 309.89 & 309.89 & 309.88 & 309.88 & 309.88 & 309.88 & 309.88 & 309.88 & 309.88 & 309.87 & 309.86 & 309.86 & 309.85 & 309.84 & 309.85 \\
\hline
\end{tabular}

\section{B.33 Tabulated Data for Interferogram 489}

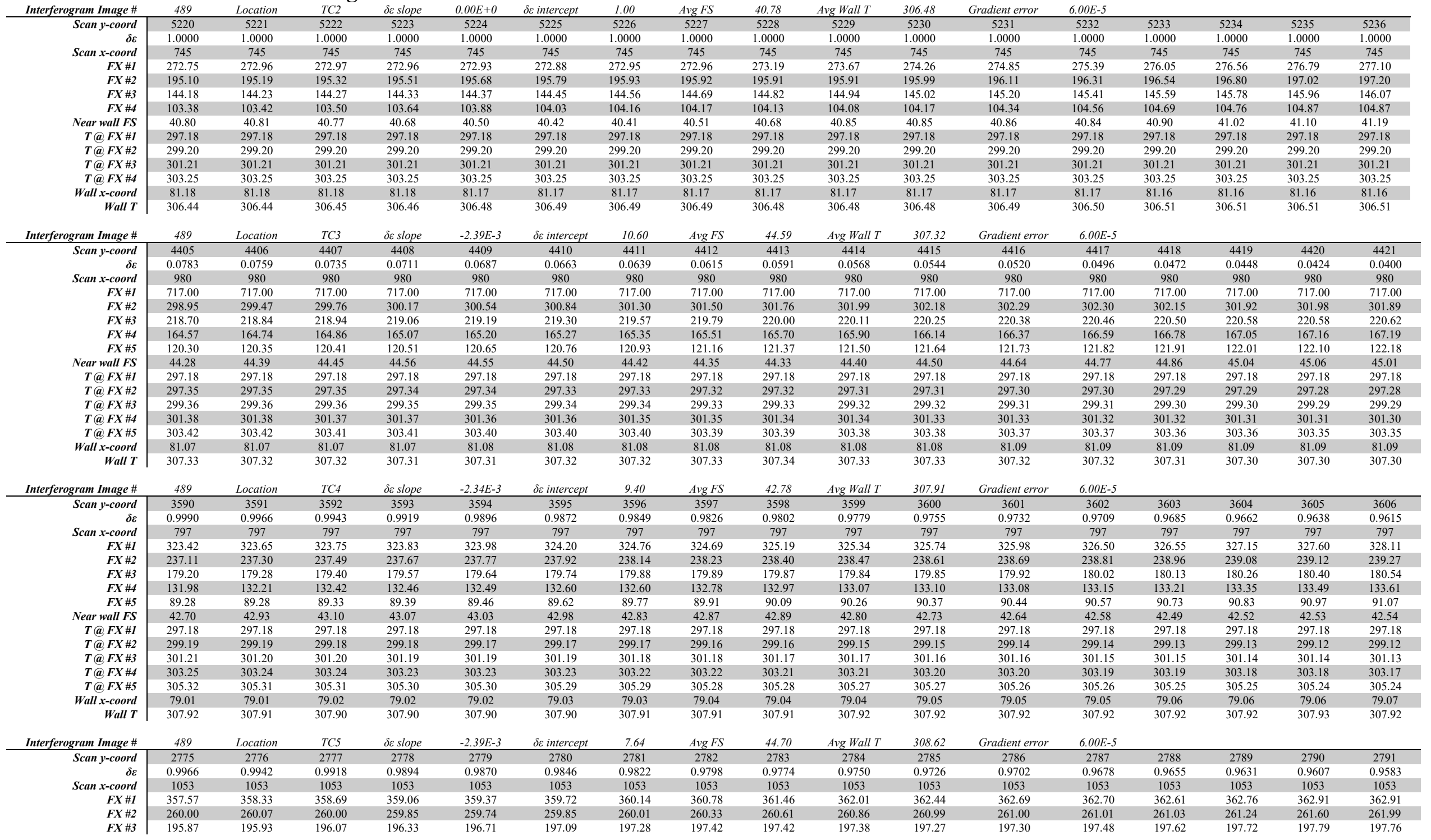




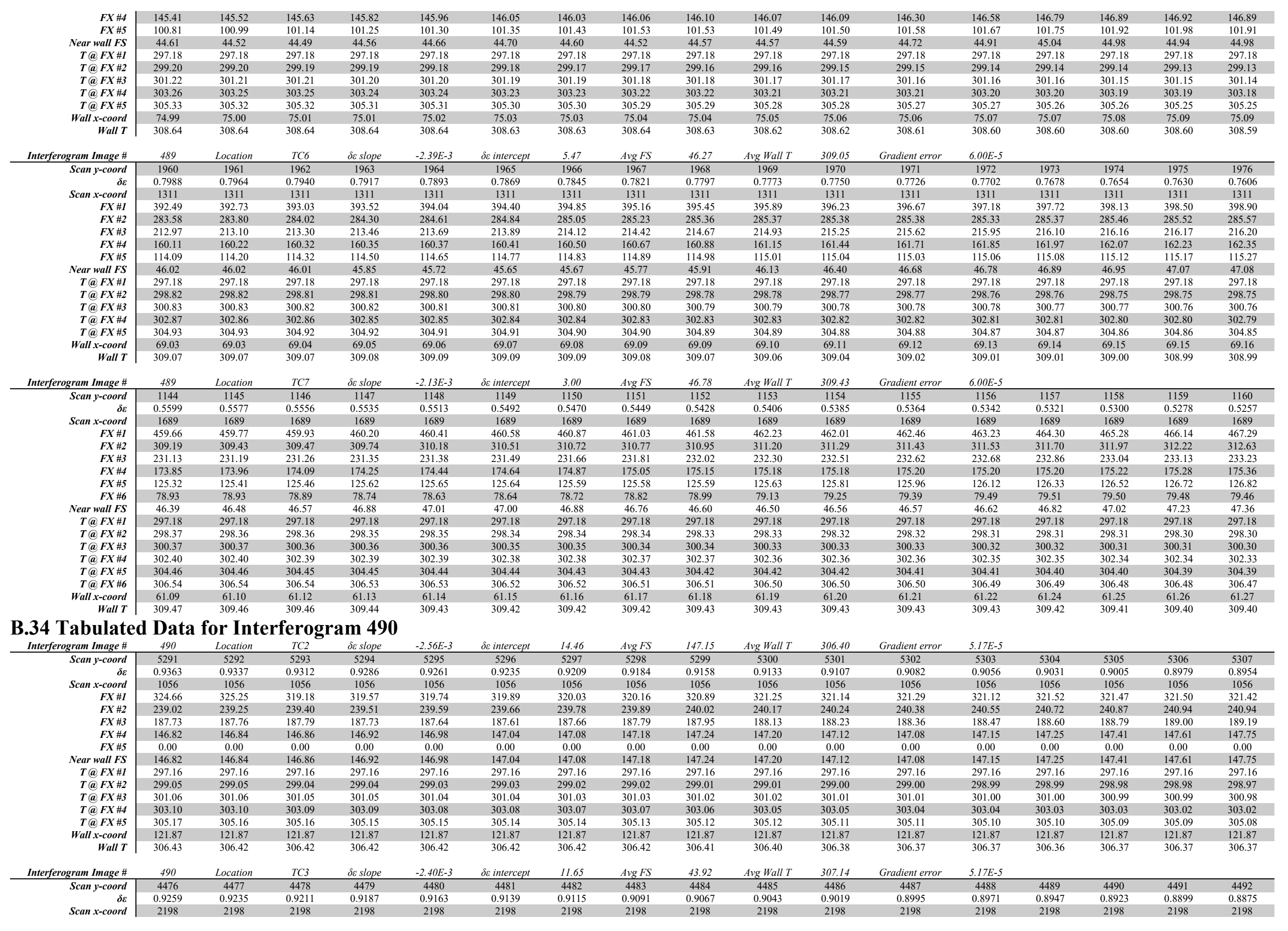




\begin{tabular}{|c|c|c|c|c|c|c|c|c|c|c|c|c|c|c|c|c|c|}
\hline$F X \# I$ & 339.46 & 339.73 & 340.20 & 340.70 & 341.02 & 341.51 & 341.84 & 342.39 & 342.98 & 343.87 & 344.46 & 345.02 & 345.28 & 345.43 & 345.48 & 345.41 & 345.34 \\
\hline$F X \# 2$ & 259.86 & 259.92 & 259.98 & 260.04 & 260.10 & 260.22 & 260.30 & 260.48 & 260.72 & 260.95 & 261.13 & 261.31 & 261.48 & 261.62 & 261.68 & 261.72 & 261.76 \\
\hline$F X \# 3$ & 205.98 & 205.97 & 205.98 & 206.03 & 206.17 & 206.37 & 206.58 & 206.72 & 206.90 & 207.12 & 207.32 & 207.52 & 207.72 & 207.98 & 208.18 & 208.40 & 208.55 \\
\hline$F X \# 4$ & 162.10 & 162.16 & 162.24 & 162.44 & 162.62 & 162.80 & 162.90 & 163.02 & 163.19 & 163.36 & 163.48 & 163.58 & 163.67 & 163.76 & 163.81 & 163.86 & 163.94 \\
\hline Near wall FS & 43.88 & 43.81 & 43.74 & 43.58 & 43.55 & 43.58 & 43.68 & 43.70 & 43.71 & 43.76 & 43.84 & 43.94 & 44.04 & 44.22 & 44.37 & 44.54 & 44.61 \\
\hline$T @ F X \# 1$ & 297.16 & 297.16 & 297.16 & 297.16 & 297.16 & 297.16 & 297.16 & 297.16 & 297.16 & 297.16 & 297.16 & 297.16 & 297.16 & 297.16 & 297.16 & 297.16 & 297.16 \\
\hline$T @ F X \# 2$ & 299.09 & 299.08 & 299.08 & 299.07 & 299.07 & 299.07 & 299.06 & 299.06 & 299.05 & 299.05 & 299.04 & 299.04 & 299.03 & 299.03 & 299.02 & 299.02 & 299.01 \\
\hline$T @ F X \# 3$ & 301.10 & 301.10 & 301.09 & 301.09 & 301.08 & 301.08 & 301.07 & 301.07 & 301.06 & 301.06 & 301.05 & 301.05 & 301.04 & 301.04 & 301.03 & 301.03 & 301.02 \\
\hline$T$ @ $F X \# 4$ & 303.14 & 303.14 & 303.13 & 303.13 & 303.12 & 303.12 & 303.11 & 303.11 & 303.10 & 303.10 & 303.09 & 303.09 & 303.08 & 303.08 & 303.07 & 303.07 & 303.06 \\
\hline Wall $x$-coord & 121.22 & 121.23 & 121.23 & 121.23 & 121.23 & 121.23 & 121.23 & 121.24 & 121.24 & 121.24 & 121.24 & 121.24 & 121.25 & 121.25 & 121.25 & 121.25 & 121.25 \\
\hline Wall $T$ & 307.13 & 307.13 & 307.13 & 307.14 & 307.15 & 307.15 & 307.15 & 307.15 & 307.15 & 307.15 & 307.15 & 307.14 & 307.14 & 307.13 & 307.12 & 307.11 & 307.10 \\
\hline Interferogram Image \# & 490 & Location & $T C 4$ & $\delta \varepsilon$ slope & $-2.31 E-3$ & $\delta \varepsilon$ intercept & 9.37 & $\operatorname{Avg} F S$ & 42.25 & Avg Wall $T$ & 307.84 & Gradient error & $5.17 E-5$ & & & & \\
\hline Scan y-coord & 3662 & 3663 & 3664 & 3665 & 3666 & 3667 & 3668 & 3669 & 3670 & 3671 & 3672 & 3673 & 3674 & 3675 & 3676 & 3677 & 3678 \\
\hline & 0.9110 & 0.9087 & 0.9064 & 0.9041 & 0.9018 & 0.8995 & 0.8972 & 0.8949 & 0.8925 & 0.8902 & 0.8879 & 0.8856 & 0.8833 & 0.8810 & 0.8787 & 0.8764 & 0.8741 \\
\hline Scan $x$-coord & 1157 & 1157 & 1157 & 1157 & 1157 & 1157 & 1157 & 1157 & 1157 & 1157 & 1157 & 1157 & 1157 & 1157 & 1157 & 1157 & 1157 \\
\hline$F X \# 1$ & 365.94 & 366.08 & 366.20 & 366.26 & 366.35 & 366.42 & 366.62 & 366.89 & 367.29 & 367.84 & 367.96 & 368.33 & 368.85 & 369.11 & 369.68 & 370.34 & 370.50 \\
\hline$F X \# 2$ & 277.66 & 277.86 & 278.12 & 278.34 & 278.52 & 278.67 & 278.78 & 278.88 & 278.93 & 278.92 & 279.00 & 279.02 & 279.10 & 279.16 & 279.36 & 279.54 & 279.69 \\
\hline$F X \# 3$ & 220.37 & 220.44 & 220.50 & 220.53 & 220.58 & 220.58 & 220.59 & 220.67 & 220.83 & 221.02 & 221.12 & 221.18 & 221.32 & 221.49 & 221.62 & 221.70 & 221.76 \\
\hline$F X \# 4$ & 173.68 & 173.80 & 173.97 & 174.09 & 174.23 & 174.40 & 174.55 & 174.69 & 174.80 & 174.86 & 174.90 & 174.91 & 175.01 & 175.19 & 175.35 & 175.50 & 175.63 \\
\hline$F X \# 5$ & 131.89 & 132.00 & 132.08 & 132.10 & 132.11 & $\begin{array}{l}132.09 \\
\end{array}$ & 132.09 & 132.10 & 132.18 & 132.32 & 132.44 & 132.55 & 132.74 & 132.92 & 133.06 & 133.22 & 133.36 \\
\hline Near wall FS & 41.79 & 41.80 & 41.88 & 41.99 & 42.11 & 42.31 & 42.46 & 42.59 & 42.62 & 42.54 & 42.46 & 42.36 & 42.27 & 42.27 & 42.29 & 42.29 & 42.27 \\
\hline$T @ F X \# 1$ & 297.16 & 297.16 & 297.16 & 297.16 & 297.16 & 297.16 & 297.16 & 297.16 & 297.16 & 297.16 & 297.16 & 297.16 & 297.16 & 297.16 & 297.16 & 297.16 & 297.16 \\
\hline$T @ F X \# 2$ & 299.00 & 299.00 & 299.00 & 298.99 & 298.99 & 298.98 & 298.98 & 298.97 & 298.97 & 298.96 & 298.96 & 298.95 & 298.95 & 298.94 & 298.94 & 298.94 & 298.93 \\
\hline$T(a) F X$ & 301.02 & 301.01 & 301.01 & 301.00 & 301.00 & 300.99 & 300.99 & 300.98 & 300.98 & 300.97 & 300.97 & 300.96 & 300.96 & 300.96 & 300.95 & 300.95 & 300.94 \\
\hline$T(F X \# 4$ & 303.05 & 303.05 & 303.04 & 303.04 & 303.03 & 303.03 & 303.03 & 303.02 & 303.02 & 303.01 & 303.01 & 303.00 & 303.00 & 302.99 & 302.99 & 302.98 & 302.98 \\
\hline$T @ F X \# 5$ & 305.12 & 305.11 & 305.11 & 305.10 & 305.10 & 305.09 & 305.09 & 305.08 & 305.08 & 305.08 & 305.07 & 305.07 & 305.06 & 305.06 & 305.05 & 305.05 & 305.04 \\
\hline Wall $x$-coord & 118.91 & 118.91 & 118.92 & 118.92 & 118.92 & 118.93 & 118.93 & 118.94 & 118.94 & 118.94 & 118.95 & 118.95 & 118.96 & 118.96 & 118.96 & 118.97 & 118.97 \\
\hline Wall T & 307.86 & 307.86 & 307.86 & 307.85 & 307.85 & 307.84 & 307.83 & 307.82 & 307.82 & 307.83 & 307.83 & 307.83 & 307.84 & 307.84 & 307.84 & 307.84 & 307.85 \\
\hline Interferogram Image \# & 490 & Location & $T C 5$ & $\delta \varepsilon$ slope & $-2.36 E-3$ & $\delta \varepsilon$ intercept & 7.58 & $\operatorname{Avg} F S$ & 44.96 & Avg Wall $T$ & 308.50 & Gradient error & $5.17 E-5$ & & & & \\
\hline Scan $y$-coord & 2847 & 2848 & 2849 & 2850 & 2851 & 2852 & 2853 & 2854 & 2855 & 2856 & 2857 & 2858 & 2859 & 2860 & 2861 & 2862 & 2863 \\
\hline & 0.8623 & 0.8599 & 0.8576 & 0.8552 & 0.8529 & 0.8505 & 0.8481 & 0.8458 & 0.8434 & 0.8411 & 0.8387 & 0.8363 & 0.8340 & 0.8316 & 0.8292 & 0.8269 & 0.8245 \\
\hline Scan $x$-coord & 1211 & 1211 & 1211 & 1211 & 1211 & 1211 & 1211 & 1211 & 1211 & 1211 & 1211 & 1211 & 1211 & 1211 & 1211 & 1211 & 1211 \\
\hline$F X \# 1$ & 412.68 & 412.83 & 412.92 & 413.14 & 413.78 & 413.95 & 414.36 & 414.89 & 415.59 & 416.36 & 417.00 & 417.68 & 418.23 & 418.68 & 419.02 & 419.29 & 419.73 \\
\hline$F X \# 2$ & 304.98 & 304.90 & 304.80 & 304.93 & 305.35 & 305.76 & 306.20 & 306.60 & 306.80 & 306.96 & 307.11 & 307.17 & 307.28 & 307.43 & 307.56 & 307.63 & 307.66 \\
\hline$F X \# 3$ & 240.86 & 240.88 & 240.89 & 240.89 & 240.96 & 241.12 & 241.32 & 241.45 & 241.57 & 241.68 & 241.73 & 241.77 & 241.81 & 241.92 & 242.14 & 242.23 & 242.30 \\
\hline$F X \# 4$ & 190.20 & 190.19 & 190.18 & 190.20 & 190.33 & 190.46 & 190.57 & 190.73 & 190.90 & 190.93 & 190.90 & 190.90 & 190.92 & 190.90 & 190.90 & 190.88 & 190.89 \\
\hline$F X \# 5$ & 144.62 & 144.79 & 144.93 & 145.08 & 145.21 & 145.30 & 145.41 & 145.58 & 145.80 & 145.90 & 145.97 & 146.09 & 146.20 & 146.26 & 146.35 & 146.50 & 146.58 \\
\hline Near wall FS & 45.58 & 45.39 & 45.25 & 45.13 & 45.13 & 45.15 & 45.16 & 45.15 & 45.10 & 45.03 & 44.92 & 44.80 & 44.72 & 44.64 & 44.55 & 44.38 & 44.31 \\
\hline$T @ F X \# 1$ & 297.16 & 297.16 & 297.16 & 297.16 & 297.16 & 297.16 & 297.16 & 297.16 & 297.16 & 297.16 & 297.16 & 297.16 & 297.16 & 297.16 & 297.16 & 297.16 & 297.16 \\
\hline$T$ (a) $F X \# 2$ & 298.91 & 298.90 & 298.90 & 298.89 & 298.89 & 298.88 & 298.88 & 298.87 & 298.87 & 298.87 & 298.86 & 298.86 & 298.85 & 298.85 & 298.84 & 298.84 & 298.83 \\
\hline$T$ (a) $F X \# 3$ & 300.92 & 300.91 & 300.91 & 300.90 & 300.90 & 300.90 & 300.89 & 300.89 & 300.88 & 300.88 & 300.87 & 300.87 & 300.86 & 300.86 & 300.85 & 300.85 & 300.84 \\
\hline$T(F X \# 4$ & 302.96 & 302.95 & 302.95 & 302.94 & 302.94 & 302.93 & 302.93 & 302.92 & 302.92 & 302.91 & 302.91 & 302.90 & 302.90 & 302.89 & 302.89 & 302.88 & 302.88 \\
\hline$T(F X \# 5$ & 305.02 & 305.01 & 305.01 & 305.00 & 305.00 & 304.99 & 304.99 & 304.99 & 304.98 & 304.98 & 304.97 & 304.97 & 304.96 & 304.96 & 304.95 & 304.95 & 304.94 \\
\hline Wall $x$-coord & 114.93 & 114.93 & 114.94 & 114.94 & 114.95 & 114.96 & 114.96 & 114.97 & 114.97 & 114.98 & 114.98 & 114.99 & 115.00 & 115.00 & 115.01 & 115.01 & 115.02 \\
\hline Wall T & 308.47 & 308.48 & 308.49 & 308.49 & 308.49 & 308.49 & 308.49 & 308.49 & 308.50 & 308.50 & 308.50 & 308.51 & 308.51 & 308.51 & 308.51 & 308.52 & 308.52 \\
\hline Interferogram Image \# & 490 & Location & TC6 & $\delta \varepsilon$ slope & $-2.17 E-3$ & $\delta \varepsilon$ intercept & 4.99 & $\operatorname{Avg} F S$ & 43.88 & Avg Wall $T$ & 309.02 & Gradient error & $5.17 E-5$ & & & & \\
\hline Scan y-coord & 2033 & 2034 & 2035 & 2036 & 2037 & 2038 & 2039 & 2040 & 2041 & 2042 & 2043 & 2044 & 2045 & 2046 & 2047 & 2048 & 2049 \\
\hline$\delta \varepsilon$ & 0.5766 & 0.5744 & 0.5722 & 0.5701 & 0.5679 & 0.5657 & 0.5636 & 0.5614 & 0.5592 & 0.5570 & 0.5549 & 0.5527 & 0.5505 & 0.5484 & 0.5462 & 0.5440 & 0.5418 \\
\hline Scan $x$-coord & 2186 & 2186 & 2186 & 2186 & 2186 & 2186 & 2186 & 2186 & 2186 & 2186 & 2186 & 2186 & 2186 & 2186 & 2186 & 2186 & 2186 \\
\hline$F X \# I$ & 464.57 & 464.90 & 465.07 & 465.22 & 465.51 & 466.37 & 467.05 & 467.72 & 468.23 & 468.72 & 468.76 & 468.84 & 469.13 & 469.46 & 469.88 & 470.29 & 470.75 \\
\hline$F X \# 2$ & 333.53 & 333.74 & 333.90 & 333.99 & 334.09 & 334.30 & 334.43 & 334.63 & 334.82 & 335.05 & 335.19 & 335.35 & 335.49 & 335.62 & 335.75 & 335.87 & 335.97 \\
\hline$F X \# 3$ & 261.24 & 261.34 & 261.33 & 261.35 & 261.25 & 261.18 & 261.25 & 261.34 & 261.42 & 261.52 & 261.59 & 261.63 & 261.71 & 261.77 & 261.85 & 261.93 & 262.00 \\
\hline$F X \# 4$ & 206.85 & 207.03 & 207.26 & 207.48 & 207.65 & 207.76 & 207.79 & 207.82 & 207.95 & 208.03 & 208.09 & 208.14 & 208.19 & 208.25 & 208.36 & 208.50 & 208.60 \\
\hline$F X \# 5$ & 160.84 & 160.73 & 160.58 & 160.57 & 160.71 & 160.84 & 161.07 & 161.25 & 161.37 & 161.45 & 161.47 & 161.46 & 161.44 & 161.37 & 161.33 & 161.33 & 161.36 \\
\hline$F X \# 6$ & 116.81 & 116.59 & 116.51 & 116.53 & 116.71 & 117.08 & 117.33 & 117.49 & 117.51 & 117.52 & 117.48 & 117.53 & 117.63 & 117.76 & 117.71 & 117.60 & 117.48 \\
\hline Near wall FS & 44.03 & 44.14 & 44.07 & 44.05 & 44.00 & 43.76 & 43.74 & 43.75 & 43.87 & 43.94 & 43.99 & 43.93 & 43.81 & 43.61 & 43.62 & 43.73 & 43.89 \\
\hline$T @ F X \# 1$ & 297.16 & 297.16 & 297.16 & 297.16 & 297.16 & 297.16 & 297.16 & 297.16 & 297.16 & 297.16 & 297.16 & 297.16 & 297.16 & 297.16 & 297.16 & 297.16 & 297.16 \\
\hline$T(F X \# 2$ & 298.39 & 298.38 & 298.38 & 298.37 & 298.37 & 298.37 & 298.36 & 298.36 & 298.35 & 298.35 & 298.34 & 298.34 & 298.34 & 298.33 & 298.33 & 298.32 & 298.32 \\
\hline$T$ (a) $F X \# 3$ & 300.39 & 300.39 & 300.38 & 300.38 & 300.38 & 300.37 & 300.37 & 300.36 & 300.36 & 300.35 & 300.35 & 300.34 & 300.34 & 300.34 & 300.33 & 300.33 & 300.32 \\
\hline$T$ @ $F X \# 4$ & 302.42 & 302.42 & 302.41 & 302.41 & 302.41 & 302.40 & 302.40 & 302.39 & 302.39 & 302.38 & 302.38 & 302.37 & 302.37 & 302.37 & 302.36 & 302.36 & 302.35 \\
\hline$T(F X \# 5$ & 304.48 & 304.47 & 304.47 & 304.47 & 304.46 & 304.46 & 304.45 & 304.45 & 304.44 & 304.44 & 304.43 & 304.43 & 304.43 & 304.42 & 304.42 & 304.41 & 304.41 \\
\hline$T @ F X \# 6$ & 306.56 & 306.56 & 306.55 & 306.55 & 306.55 & 306.54 & 306.54 & 306.53 & 306.53 & 306.52 & 306.52 & 306.51 & 306.51 & 306.50 & 306.50 & 306.50 & 306.49 \\
\hline Wall $x$-coord & 109.28 & 109.29 & 109.30 & 109.30 & 109.31 & 109.32 & 109.33 & 109.34 & 109.34 & 109.35 & 109.36 & 109.37 & 109.38 & 109.38 & 109.39 & 109.40 & 109.41 \\
\hline Wall T & 309.04 & 309.02 & 309.01 & 309.01 & 309.01 & 309.03 & 309.03 & 309.04 & 309.03 & 309.03 & 309.02 & 309.02 & 309.02 & 309.02 & 309.01 & 309.00 & 308.99 \\
\hline Interferogram Image \# & 490 & Location & $T C 7$ & $\delta \varepsilon$ slope & $-2.14 E-3$ & $\delta \varepsilon$ intercept & 2.95 & $\operatorname{Avg} F S$ & 0.00 & Avg Wall $T$ & 309.31 & Gradient error & $5.17 E-5$ & & & & \\
\hline Scan y-coord & 1218 & 1219 & 1220 & 1221 & 1222 & 1223 & 1224 & 1225 & 1226 & 1227 & 1228 & 1229 & 1230 & 1231 & 1232 & 1233 & 1234 \\
\hline & 0.3373 & 0.3351 & 0.3330 & 0.3308 & 0.3287 & 0.3266 & 0.3244 & 0.3223 & 0.3201 & 0.3180 & 0.3158 & 0.3137 & 0.3115 & 0.3094 & 0.3073 & 0.3051 & 0.3030 \\
\hline Scan $x$-coord & 1749 & 1749 & 1749 & 1749 & 1749 & 1749 & 1749 & 1749 & 1749 & 1749 & 1749 & 1749 & 1749 & 1749 & 1749 & 1749 & 1749 \\
\hline
\end{tabular}




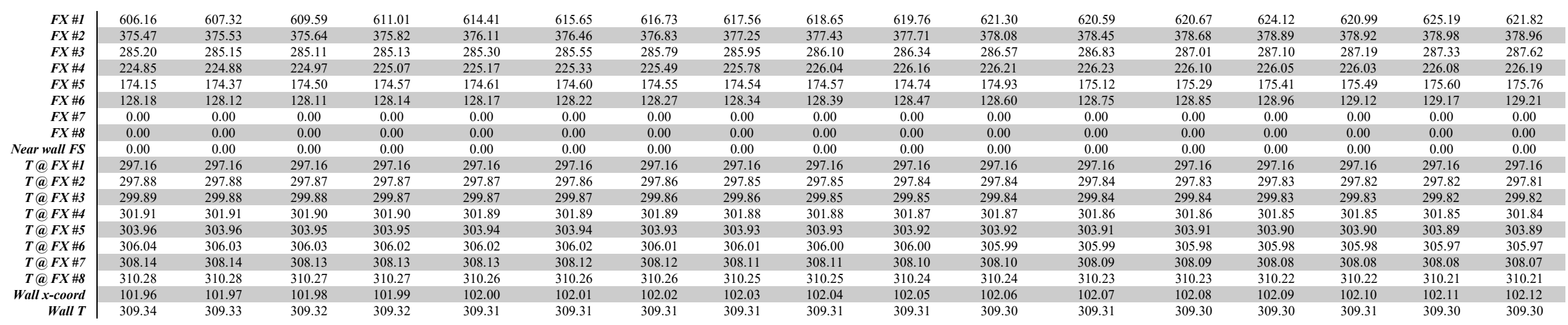

\section{B.35 Tabulated Data for Interferogram 491}

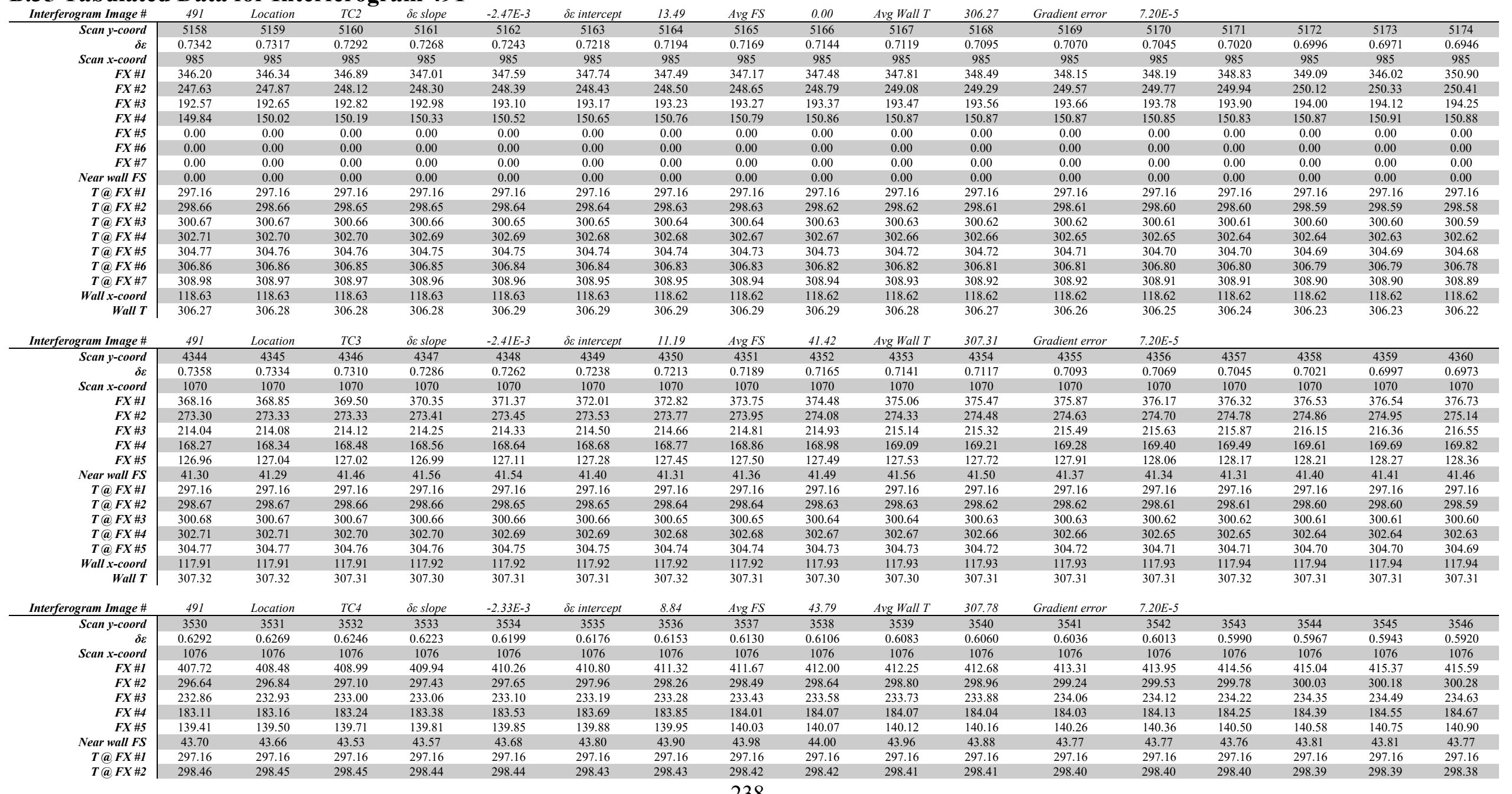




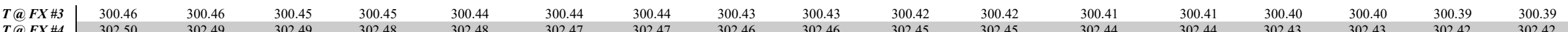

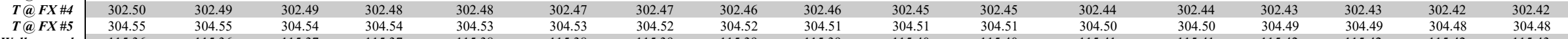

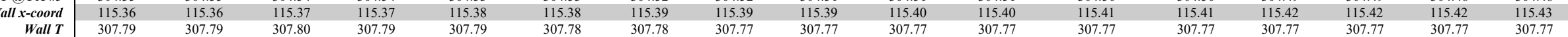

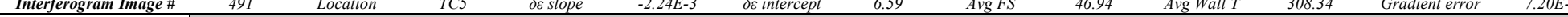

\begin{tabular}{|c|c|c|c|c|c|c|c|c|c|c|c|c|c|c|c|c|c|}
\hline an rage tr & & Location & tej & oz stope & $-2.24 E-3$ & ofmercept & 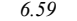 & & & & & Graatent error & & & & & \\
\hline$\overline{c c a n} y$-coord & 2716 & 2717 & 2718 & 2719 & 2720 & 2721 & 2722 & 2723 & 2724 & 2725 & 2726 & (2727 & 2728 & 2729 & 2730 & 2731 & 2732 \\
\hline & 0.5096 & 0.5073 & 0.5051 & 0.5028 & 0.5006 & 0.4984 & 0.4961 & 0.4939 & 0.4917 & 0.4894 & 0.4872 & 0.4849 & 0.4827 & 0.4805 & 0.4782 & 0.4760 & 0.4738 \\
\hline Scan $x$-coord & 1023 & 1023 & 1023 & 1023 & 1023 & 1023 & 1023 & 1023 & 1023 & 1023 & 1023 & 1023 & 1023 & 1023 & 1023 & 1023 & 1023 \\
\hline$F X \# 1$ & 468.21 & 468.85 & 469.38 & 470.11 & 470.88 & 471.63 & 472.44 & 472.86 & 473.36 & 473.84 & 474.39 & 475.09 & 476.76 & 476.73 & 477.77 & 478.61 & 479.28 \\
\hline$F X \# 2$ & 328.78 & 328.92 & 329.22 & 329.77 & 330.30 & 330.58 & 330.62 & 330.62 & 330.56 & 330.59 & 330.72 & 330.79 & 331.24 & 331.23 & 331.45 & 331.68 & 331.89 \\
\hline$F X \# 3$ & 256.38 & 256.37 & 256.40 & 256.47 & 256.62 & 256.71 & 256.80 & 256.93 & 257.06 & 257.10 & 257.12 & 257.12 & 257.28 & 257.39 & 257.55 & 257.61 & 257.76 \\
\hline$F X \# 4$ & 202.15 & 202.25 & 202.44 & 202.63 & 202.80 & 202.93 & 203.08 & 203.16 & 203.23 & 203.25 & 203.29 & 203.36 & 203.25 & 203.11 & 203.08 & 203.15 & 203.30 \\
\hline$F X \# 5$ & 154.65 & 154.89 & 155.12 & 155.36 & 155.57 & 155.71 & 155.85 & 156.05 & 156.28 & 156.43 & 156.55 & 156.58 & 156.63 & 156.63 & 156.66 & 156.71 & 156.79 \\
\hline Near wall FS & 47.50 & 47.36 & 47.33 & 47.26 & 47.23 & 47.22 & 47.23 & 47.11 & 46.95 & 46.82 & 46.74 & 46.78 & 46.63 & 46.48 & 46.41 & 46.44 & 46.51 \\
\hline$T @ F X \# 1$ & 297.16 & 297.16 & 297.16 & 297.16 & 297.16 & 297.16 & 297.16 & 297.16 & 297.16 & 297.16 & 297.16 & 297.16 & 297.16 & 297.16 & 297.16 & 297.16 & 297.16 \\
\hline$T @ F X \# 2$ & 298.21 & 298.21 & 298.20 & 298.20 & 298.19 & 298.19 & 298.18 & 298.18 & 298.17 & 298.17 & 298.17 & 298.16 & 298.16 & 298.15 & 298.15 & 298.14 & 298.14 \\
\hline$T @ F X \# 3$ & 300.22 & 300.21 & 300.21 & 300.20 & 300.20 & 300.19 & 300.19 & 300.19 & 300.18 & 300.18 & 300.17 & 300.17 & 300.16 & 300.16 & 300.15 & 300.15 & 300.14 \\
\hline$T$ @ $F X \# 4$ & 302.25 & 302.24 & 302.24 & 302.23 & 302.23 & 302.22 & 302.22 & 302.21 & 302.21 & 302.20 & 302.20 & 302.20 & 302.19 & 302.19 & 302.18 & 302.18 & 302.17 \\
\hline$T(F X \# 5$ & 304.30 & 304.30 & 304.29 & 304.29 & 304.28 & 304.28 & 304.27 & 304.27 & 304.26 & 304.26 & 304.25 & 304.25 & 304.25 & 304.24 & 304.24 & 304.23 & 304.23 \\
\hline Wall $x$-coord & 110.98 & 110.98 & 110.99 & 111.00 & 111.00 & 111.01 & 111.02 & 111.02 & 111.03 & 111.04 & 111.04 & 111.05 & 111.05 & 111.06 & 111.07 & 111.07 & 111.08 \\
\hline & & & & & & & & & & & & & & & & & \\
\hline
\end{tabular}

Wall T

\begin{tabular}{|c|c|c|c|c|c|c|c|c|c|c|c|c|c|c|c|c|c|}
\hline Interferogram Image \# & 491 & Location & TC6 & SE slope & $-2.17 E-3$ & $\delta$ s intercept & 4.35 & $\operatorname{Avg} F S$ & 45.25 & Avg Wall T & 309.03 & Gradient error & $7.20 E-5$ & & & & \\
\hline Scan $y$-coord & 1901 & 1902 & 1903 & 1904 & 1905 & 1906 & 1907 & 1908 & 1909 & 1910 & 1911 & 1912 & 1913 & 1914 & 1915 & 1916 & 1917 \\
\hline & 0.2252 & 0.2230 & 0.2209 & 0.2187 & 0.2165 & 0.2143 & 0.2122 & 0.2100 & 0.2078 & 0.2057 & 0.2035 & 0.2013 & 0.1992 & 0.1970 & 0.1948 & 0.1927 & 0.1905 \\
\hline Scan $x$-coord & 2059 & 2059 & 2059 & 2059 & 2059 & 2059 & 2059 & 2059 & 2059 & 2059 & 2059 & 2059 & 2059 & 2059 & 2059 & 2059 & 2059 \\
\hline$F X \# I$ & 570.33 & 570.43 & 571.15 & 570.89 & 572.64 & 573.90 & 574.80 & 575.88 & 576.50 & 577.24 & 578.10 & 579.15 & 580.44 & 582.01 & 583.94 & 583.24 & 583.57 \\
\hline$F X \# 2$ & 363.47 & 363.89 & 364.20 & 364.39 & 364.67 & 364.92 & 365.08 & 365.16 & 365.10 & 365.14 & 365.23 & 365.29 & 365.34 & 365.33 & 365.55 & 365.72 & 366.03 \\
\hline$F X \# 3$ & 280.50 & 280.53 & 280.49 & 280.43 & 280.55 & 280.78 & 281.00 & 281.19 & 281.48 & 281.82 & 282.24 & 282.60 & 282.80 & 282.97 & 283.15 & 283.23 & 283.24 \\
\hline$F X \# 4$ & 221.74 & 221.98 & 222.22 & 222.43 & 222.52 & 222.60 & 222.73 & 222.83 & 222.91 & 223.04 & 223.16 & 223.34 & 223.61 & 223.87 & 224.12 & 224.27 & 224.43 \\
\hline$F X \# 5$ & 172.84 & 172.64 & 172.69 & 172.86 & 173.07 & 173.29 & 173.55 & 173.80 & 174.00 & 174.06 & 174.09 & 174.06 & 174.07 & 173.99 & 173.94 & 173.92 & 174.03 \\
\hline$F X \# 6$ & 127.76 & 127.79 & 127.84 & 128.02 & 128.16 & 128.30 & 128.41 & 128.42 & 128.50 & 128.52 & 128.53 & 128.51 & 128.47 & 128.43 & 128.45 & 128.68 & 128.88 \\
\hline Near wall FS & 45.08 & 44.85 & 44.85 & 44.84 & 44.91 & 44.99 & 45.14 & 45.38 & 45.50 & 45.54 & 45.55 & 45.55 & 45.60 & 45.56 & 45.49 & 45.24 & 45.15 \\
\hline$T @ F X \# 1$ & 297.16 & 297.16 & 297.16 & 297.16 & 297.16 & 297.16 & 297.16 & 297.16 & 297.16 & 297.16 & 297.16 & 297.16 & 297.16 & 297.16 & 297.16 & 297.16 & 297.16 \\
\hline$T(F X \# 2$ & 297.71 & 297.71 & 297.71 & 297.70 & 297.70 & 297.69 & 297.69 & 297.68 & 297.68 & 297.68 & 297.67 & 297.67 & 297.66 & 297.66 & 297.65 & 297.65 & 297.64 \\
\hline$T @ F X \# 3$ & 299.72 & 299.71 & 299.71 & 299.71 & 299.70 & 299.70 & 299.69 & 299.69 & 299.68 & 299.68 & 299.68 & 299.67 & 299.67 & 299.66 & 299.66 & 299.65 & 299.65 \\
\hline$T(F X \# 4$ & 301.74 & 301.74 & 301.73 & 301.73 & 301.72 & 301.72 & 301.72 & 301.71 & 301.71 & 301.70 & 301.70 & 301.69 & 301.69 & 301.68 & 301.68 & 301.68 & 301.67 \\
\hline$T$ (a) $F X \# 5$ & 303.79 & 303.79 & 303.78 & 303.78 & 303.77 & 303.77 & 303.76 & 303.76 & 303.76 & 303.75 & 303.75 & 303.74 & 303.74 & 303.73 & 303.73 & 303.72 & 303.72 \\
\hline$T$ @ $F X \# 6$ & 305.87 & 305.86 & 305.86 & 305.85 & 305.85 & 305.84 & 305.84 & 305.84 & 305.83 & 305.83 & 305.82 & 305.82 & 305.81 & 305.81 & 305.80 & 305.80 & 305.79 \\
\hline Wall $x$-coord & 104.75 & 104.76 & 104.77 & 104.78 & 104.79 & 104.79 & 104.80 & 104.81 & 104.82 & 104.83 & 104.84 & 104.85 & 104.86 & 104.86 & 104.87 & 104.88 & 104.89 \\
\hline Wall $T$ & 309.04 & 309.05 & 309.04 & 309.05 & 309.05 & 309.05 & 309.04 & 309.03 & 309.03 & 309.02 & 309.02 & 309.01 & 309.01 & 309.00 & 309.00 & 309.01 & 309.02 \\
\hline Interferogram Image \# & 491 & Location & $T C 7$ & $\delta \varepsilon$ slope & $-2.13 E-3$ & $\delta \varepsilon$ intercept & 3.31 & $\operatorname{Avg} F S$ & 47.75 & Avg Wall $T$ & 309.31 & Gradient error & $7.20 E-5$ & & & & \\
\hline Scan y-coord & 1087 & 1088 & 1089 & 1090 & 1091 & 1092 & 1093 & 1094 & 1095 & 1096 & 1097 & 1098 & 1099 & 1100 & 1101 & 1102 & 1103 \\
\hline & 0.9869 & 0.9848 & 0.9826 & 0.9805 & 0.9784 & 0.9762 & 0.9741 & 0.9720 & 0.9698 & 0.9677 & 0.9656 & 0.9634 & 0.9613 & 0.9592 & 0.9570 & 0.9549 & 0.9528 \\
\hline Scan $x$-coord & 1941 & 1941 & 1941 & 1941 & 1941 & 1941 & 1941 & 1941 & 1941 & 1941 & 1941 & 1941 & 1941 & 1941 & 1941 & 1941 & 1941 \\
\hline$F X \# I$ & 416.01 & 416.25 & 416.51 & 417.08 & 417.69 & 418.13 & 418.43 & 418.73 & 418.85 & 419.14 & 419.43 & 419.84 & 420.10 & 420.13 & 420.01 & 419.57 & 419.40 \\
\hline$F X \# 2$ & 307.55 & 307.47 & 307.46 & 307.57 & 307.75 & 307.89 & 308.04 & 308.30 & 308.46 & 308.58 & 308.76 & 308.87 & 309.03 & 309.15 & 309.36 & 309.49 & 309.67 \\
\hline$F X \# 3$ & 239.47 & 239.65 & 239.90 & 240.16 & 240.52 & 240.95 & 241.18 & 241.28 & 241.28 & 241.20 & 241.07 & 240.97 & 240.89 & 240.95 & 241.12 & 241.42 & 241.72 \\
\hline$F X \# 4$ & 186.76 & 186.87 & 186.91 & 186.94 & 186.95 & 187.03 & 187.15 & 187.34 & 187.60 & 187.81 & 188.08 & 188.23 & 188.34 & 188.45 & 188.50 & 188.53 & 188.58 \\
\hline$T @ F X \# 1$ & 297.16 & 297.16 & 297.16 & 297.16 & 297.16 & 297.16 & 297.16 & 297.16 & 297.16 & 297.16 & 297.16 & 297.16 & 297.16 & 297.16 & 297.16 & 297.16 & 297.16 \\
\hline$T @ F X \# 2$ & 299.23 & 299.23 & 299.22 & 299.22 & 299.21 & 299.21 & 299.20 & 299.20 & 299.20 & 299.19 & 299.19 & 299.18 & 299.18 & 299.17 & 299.17 & 299.17 & 299.16 \\
\hline$T @ F X \# 3$ & 301.25 & 301.24 & 301.24 & 301.23 & 301.23 & 301.23 & 301.22 & 301.22 & 301.21 & 301.21 & 301.20 & 301.20 & 301.20 & 301.19 & 301.19 & 301.18 & 301.18 \\
\hline$T @ F X \# 4$ & 303.29 & 303.29 & 303.28 & 303.28 & 303.27 & 303.27 & 303.26 & 303.26 & 303.26 & 303.25 & 303.25 & 303.24 & 303.24 & 303.23 & 303.23 & 303.22 & 303.22 \\
\hline$T @ F X \# 5$ & 305.36 & 305.36 & 305.35 & 305.35 & 305.34 & 305.34 & 305.33 & 305.33 & 305.32 & 305.32 & 305.32 & 305.31 & 305.31 & 305.30 & 305.30 & 305.29 & 305.29 \\
\hline Wall $x$-coord & 96.70 & 96.71 & 96.72 & 96.73 & 96.74 & 96.75 & 96.76 & 96.77 & 96.79 & 96.80 & 96.81 & 96.82 & 96.83 & 96.84 & 96.85 & 96.86 & 96.87 \\
\hline & & & 309.32 & 309.32 & 309.32 & & 309.31 & 309.31 & 309.31 & & 309,30 & 309.30 & 309.30 & 30930 & 30931 & & \\
\hline
\end{tabular}

\section{B.36 Tabulated Data for Interferogram 492}

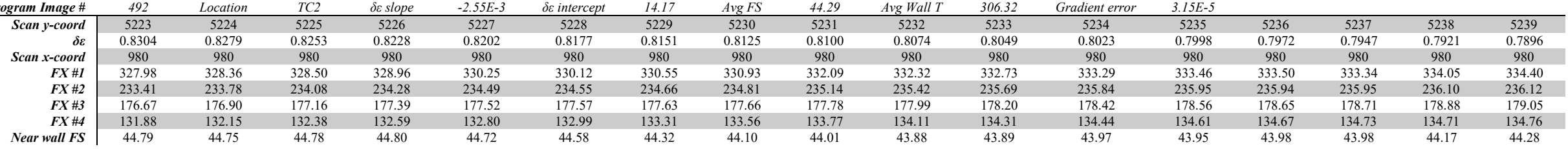




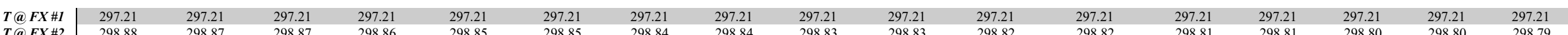

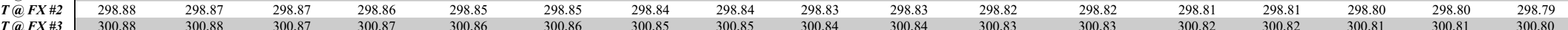

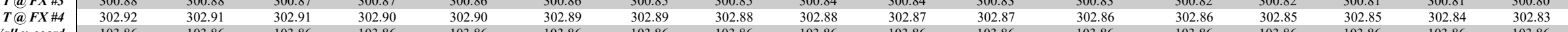

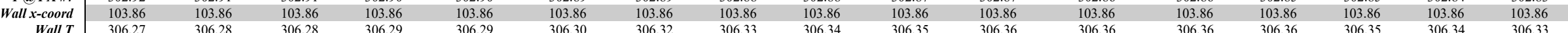

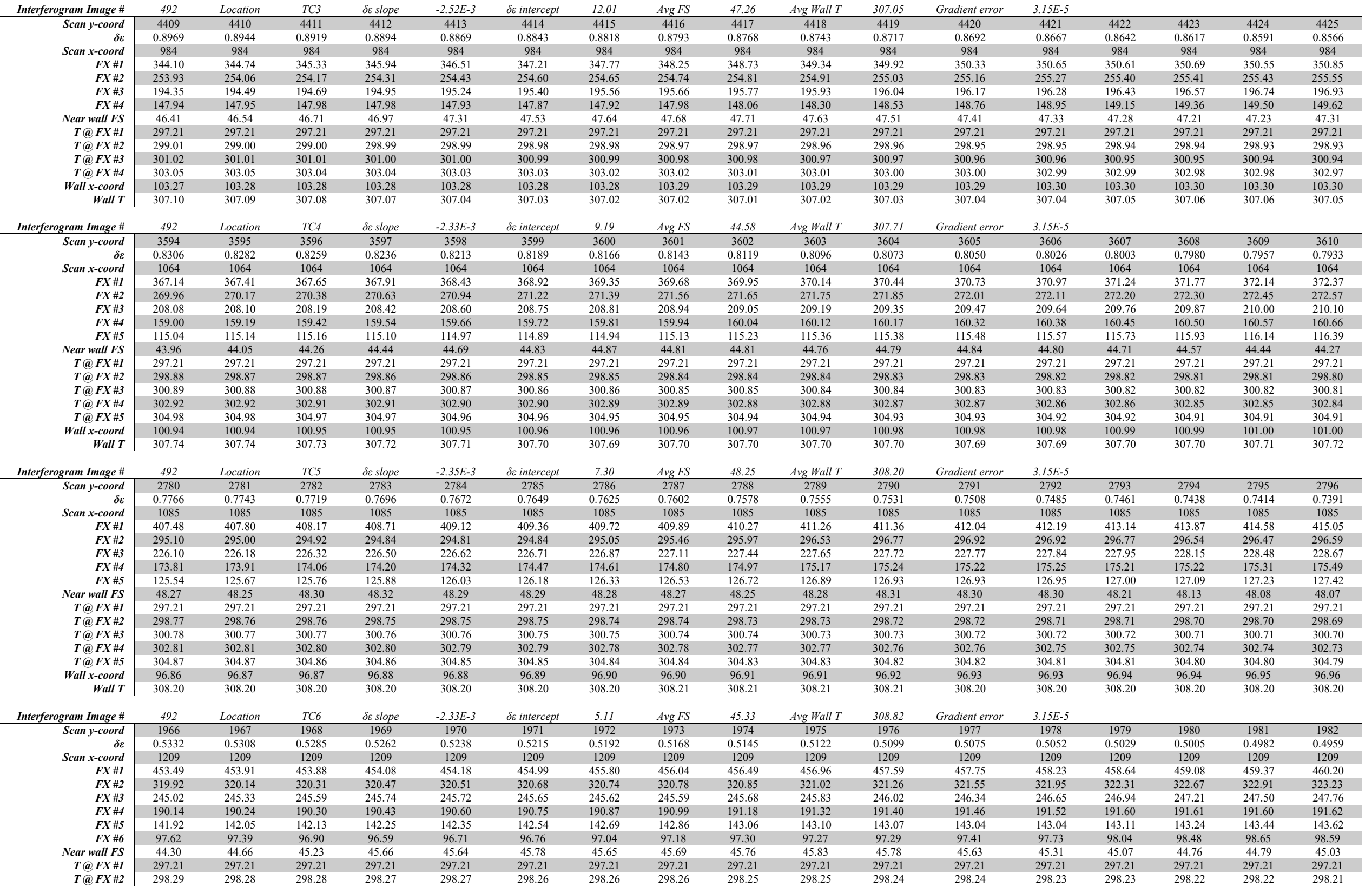




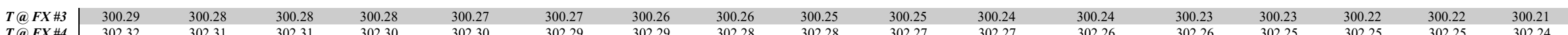

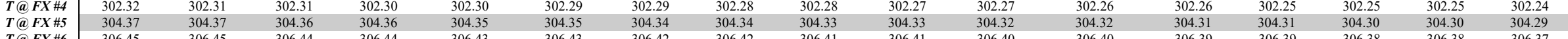

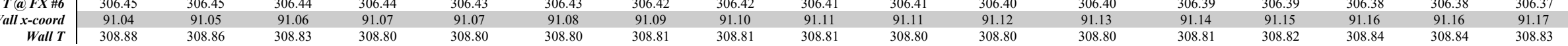

\begin{tabular}{|c|c|c|c|c|c|c|c|c|c|c|c|c|c|c|c|c|c|}
\hline Interferogram Image \# & 492 & Location & $T C 7$ & $\delta \varepsilon$ slope & $-2.19 E-3$ & $\delta$ se intercept & 2.84 & $A v g F S$ & 0.00 & Avg Wall T & 309.21 & Gradient error & 3.15E-5 & & & & \\
\hline Scan y-coord & 1152 & 1153 & 1154 & 1155 & 1156 & 1157 & 1158 & 1159 & 1160 & 1161 & 1162 & 1163 & 1164 & 1165 & 1166 & 1167 & 1168 \\
\hline & 0.3202 & 0.3180 & 0.3158 & 0.3136 & 0.3114 & 0.3092 & 0.3070 & 0.3048 & 0.3027 & 0.3005 & 0.2983 & 0.2961 & 0.2939 & 0.2917 & 0.2895 & 0.2873 & 0.2852 \\
\hline Scan $x$-coord & 1674 & 1674 & 1674 & 1674 & 1674 & 1674 & 1674 & 1674 & 1674 & 1674 & 1674 & 1674 & 1674 & 1674 & 1674 & 1674 & 1674 \\
\hline$F X \# 1$ & 588.19 & 588.54 & 582.80 & 590.34 & 593.86 & 593.71 & 595.22 & 598.38 & 598.04 & 598.24 & 597.56 & 598.02 & 597.08 & 611.69 & 612.52 & 604.35 & 614.62 \\
\hline$F X \# 2$ & 357.64 & 357.55 & 357.71 & 357.98 & 358.46 & 358.64 & 358.88 & 358.97 & 359.09 & 359.31 & 359.64 & 359.90 & 360.13 & 360.40 & 360.51 & 360.72 & 360.98 \\
\hline$F X \# 3$ & 267.33 & 267.34 & 267.43 & 267.53 & 267.67 & 267.85 & 268.02 & 268.07 & 268.12 & 268.25 & 268.53 & 268.68 & 268.72 & 268.79 & 268.89 & 269.04 & 269.23 \\
\hline$F X \# 4$ & 206.46 & 206.47 & 206.45 & 206.45 & 206.43 & 206.47 & 206.57 & 206.67 & 206.82 & 206.94 & 207.10 & 207.27 & 207.38 & 207.39 & 207.43 & 207.40 & 207.35 \\
\hline$F X \# 5$ & 154.34 & 154.34 & 154.36 & 154.38 & 154.44 & 154.60 & 154.82 & 155.01 & 155.26 & 155.38 & 155.48 & 155.56 & 155.57 & 155.61 & 155.68 & 155.76 & 155.90 \\
\hline$F X \# 6$ & 108.27 & 108.25 & 108.20 & 108.14 & 108.06 & 108.11 & 108.15 & 108.21 & 108.32 & 108.39 & 108.46 & 108.58 & 108.67 & 108.74 & 108.85 & 108.91 & 108.93 \\
\hline$F X \# 7$ & 0.00 & 0.00 & 0.00 & 0.00 & 0.00 & 0.00 & 0.00 & 0.00 & 0.00 & 0.00 & 0.00 & 0.00 & 0.00 & 0.00 & 0.00 & 0.00 & 0.00 \\
\hline$F X \# 8$ & 0.00 & 0.00 & 0.00 & 0.00 & 0.00 & 0.00 & 0.00 & 0.00 & 0.00 & 0.00 & 0.00 & 0.00 & 0.00 & 0.00 & 0.00 & 0.00 & 0.00 \\
\hline Near wall FS & 0.00 & 0.00 & 0.00 & 0.00 & 0.00 & 0.00 & 0.00 & 0.00 & 0.00 & 0.00 & 0.00 & 0.00 & 0.00 & 0.00 & 0.00 & 0.00 & 0.00 \\
\hline$T @ F X \# 1$ & 297.21 & 297.21 & 297.21 & 297.21 & 297.21 & 297.21 & 297.21 & 297.21 & 297.21 & 297.21 & 297.21 & 297.21 & 297.21 & 297.21 & 297.21 & 297.21 & 297.21 \\
\hline$T @ F X \# 2$ & 297.88 & 297.87 & 297.87 & 297.86 & 297.86 & 297.85 & 297.85 & 297.85 & 297.84 & 297.84 & 297.83 & 297.83 & 297.82 & 297.82 & 297.81 & 297.81 & 297.81 \\
\hline$T @ F X \# 3$ & 299.88 & 299.87 & 299.87 & 299.86 & 299.86 & 299.85 & 299.85 & 299.84 & 299.84 & 299.84 & 299.83 & 299.83 & 299.82 & 299.82 & 299.81 & 299.81 & 299.81 \\
\hline$T @ F X \# 4$ & 301.90 & 301.89 & 301.89 & 301.88 & 301.88 & 301.88 & 301.87 & 301.87 & 301.86 & 301.86 & 301.85 & 301.85 & 301.84 & 301.84 & 301.84 & 301.83 & 301.83 \\
\hline$T @ F X \# 5$ & 303.95 & 303.94 & 303.94 & 303.93 & 303.93 & 303.92 & 303.92 & 303.91 & 303.91 & 303.91 & 303.90 & 303.90 & 303.89 & 303.89 & 303.88 & 303.88 & 303.87 \\
\hline$T @ F X \# 6$ & 306.02 & 306.02 & 306.01 & 306.01 & 306.00 & 306.00 & 305.99 & 305.99 & 305.99 & 305.98 & 305.98 & 305.97 & 305.97 & 305.96 & 305.96 & 305.95 & 305.95 \\
\hline$T @ F X \# 7$ & 308.13 & 308.12 & 308.12 & 308.11 & 308.11 & 308.10 & 308.10 & 308.09 & 308.09 & 308.08 & 308.08 & 308.08 & 308.07 & 308.07 & 308.06 & 308.06 & 308.05 \\
\hline$T(\boldsymbol{C} F X \# \boldsymbol{B}$ & 310.26 & 310.26 & 310.25 & 310.25 & 310.24 & 310.24 & 310.23 & 310.23 & 310.22 & 310.22 & 310.22 & 310.21 & 310.21 & 310.20 & 310.20 & 310.19 & 310.19 \\
\hline Wall $x$-coord & 83.48 & 83.49 & 83.50 & 83.51 & 83.52 & 83.53 & 83.54 & 83.55 & 83.56 & 83.57 & 83.58 & 83.59 & 83.60 & 83.61 & 83.62 & 83.63 & 83.64 \\
\hline Wall $T$ & 309.26 & 309.25 & 309.24 & 309.23 & 309.22 & 309.22 & 309.21 & 309.20 & 309.20 & 309.20 & 309.19 & 309.19 & 309.20 & 309.19 & $\begin{array}{l}309.19 \\
309\end{array}$ & 309.19 & $\begin{array}{l}309.17 \\
3094\end{array}$ \\
\hline
\end{tabular}

\section{B.37 Tabulated Data for Interferogram 493}

\begin{tabular}{|c|c|c|c|c|c|c|c|c|c|c|c|c|c|c|c|c|c|}
\hline Interferogram Image \# & 493 & Location & $T C 2$ & $\delta \varepsilon$ slope & $-2.38 E-3$ & $\delta \varepsilon$ intercept & 12.85 & $\operatorname{Avg} F S$ & 159.54 & Avg Wall T & 305.94 & Gradient error & $-1.24 E-5$ & & & & \\
\hline Scan y-coord & 5201 & 5202 & 5203 & 5204 & 5205 & 5206 & 5207 & 5208 & 5209 & 5210 & 5211 & 5212 & 5213 & 5214 & 5215 & 5216 & 5217 \\
\hline & 0.4787 & 0.4763 & 0.4739 & 0.4715 & 0.4691 & 0.4668 & 0.4644 & 0.4620 & 0.4596 & 0.4573 & 0.4549 & 0.4525 & 0.4501 & 0.4477 & 0.4454 & 0.4430 & 0.4406 \\
\hline Scan $x$-coord & 1057 & 1057 & 1057 & 1057 & 1057 & 1057 & 1057 & 1057 & 1057 & 1057 & 1057 & 1057 & 1057 & 1057 & 1057 & 1057 & 1057 \\
\hline$F X \# 1$ & 396.53 & 397.42 & 398.09 & 399.10 & 399.94 & 400.54 & 401.44 & 402.15 & 402.58 & 394.02 & 394.35 & 394.87 & 395.84 & 397.52 & 398.76 & 399.60 & 400.99 \\
\hline$F X \# 2$ & 268.06 & 268.23 & 268.42 & 268.38 & 268.33 & 268.39 & 268.33 & 268.29 & 268.40 & 268.54 & 268.69 & 268.88 & 269.08 & 269.35 & 269.58 & 269.82 & 270.06 \\
\hline$F X \# 3$ & 204.21 & 204.32 & 204.47 & 204.54 & 204.63 & 204.70 & 204.79 & 204.86 & 204.94 & 205.05 & 205.17 & 205.26 & 205.33 & 205.35 & 205.44 & 205.55 & 205.74 \\
\hline$F X \# 5$ & 0.00 & 0.00 & 0.00 & 0.00 & 0.00 & 0.00 & 0.00 & 0.00 & 0.00 & 0.00 & 0.00 & 0.00 & 0.00 & 0.00 & 0.00 & 0.00 & 0.00 \\
\hline Near wall FS & 158.45 & 158.66 & 158.88 & 159.09 & 159.29 & 159.45 & 159.57 & 159.62 & 159.65 & 159.67 & 159.75 & 159.80 & 159.92 & 160.03 & 160.07 & 160.12 & 160.17 \\
\hline$T @ F X \# 1$ & 297.20 & 297.20 & 297.20 & 297.20 & 297.20 & 297.20 & 297.20 & 297.20 & 297.20 & 297.20 & 297.20 & 297.20 & 297.20 & 297.20 & 297.20 & 297.20 & 297.20 \\
\hline$T @ F X \# 2$ & 298.14 & 298.14 & 298.13 & 298.13 & 298.12 & 298.12 & 298.11 & 298.11 & 298.10 & 298.10 & 298.09 & 298.09 & 298.08 & 298.08 & 298.07 & 298.07 & 298.07 \\
\hline$T(F X \# 3$ & 300.13 & 300.13 & 300.13 & 300.12 & 300.12 & 300.11 & 300.11 & 300.10 & 300.10 & 300.09 & 300.09 & 300.08 & 300.08 & 300.07 & 300.07 & 300.06 & 300.06 \\
\hline$T @ F X \# 4$ & 302.16 & 302.15 & 302.15 & 302.14 & 302.14 & 302.13 & 302.13 & 302.12 & 302.12 & 302.11 & 302.11 & 302.10 & 302.10 & 302.09 & 302.09 & 302.08 & 302.08 \\
\hline Wall $x$-coord & 120.32 & 120.32 & 120.32 & 120.32 & 120.32 & 120.32 & 120.32 & 120.32 & 120.32 & 120.32 & 120.32 & 120.32 & 120.32 & 120.32 & 120.32 & 120.32 & 120.32 \\
\hline Wall T & 305.91 & 305.92 & 305.93 & 305.94 & 305.95 & 305.95 & 305.96 & 305.95 & 305.95 & 305.94 & 305.94 & 305.93 & 305.93 & 305.94 & 305.93 & 305.93 & 305.92 \\
\hline Interferogram Image \# & 493 & Location & $T C 3$ & $\delta \varepsilon$ slope & $-2.32 E-3$ & $\delta \varepsilon$ intercept & 10.63 & $A v g F S$ & 44.12 & Avg Wall T & 306.95 & Gradient error & $-1.24 E-5$ & & & & \\
\hline Scan y-coord & 4386 & 4387 & 4388 & 4389 & 4390 & 4391 & 4392 & 4393 & 4394 & 4395 & 4396 & 4397 & 4398 & 4399 & 4400 & 4401 & 4402 \\
\hline$\delta \varepsilon$ & 0.4349 & 0.4326 & 0.4302 & 0.4279 & 0.4256 & 0.4233 & 0.4209 & 0.4186 & 0.4163 & 0.4140 & 0.4116 & 0.4093 & 0.4070 & 0.4047 & 0.4023 & 0.4000 & 0.3977 \\
\hline Scan $x$-coord & 1092 & 1092 & 1092 & 1092 & 1092 & 1092 & 1092 & 1092 & 1092 & 1092 & 1092 & 1092 & 1092 & 1092 & 1092 & 1092 & 1092 \\
\hline$F X \# 1$ & 428.19 & 428.66 & 429.18 & 429.30 & 429.86 & 429.80 & 430.46 & 430.75 & 431.06 & 431.38 & 432.12 & 432.45 & 432.47 & 433.30 & 434.40 & 435.48 & 436.07 \\
\hline$F X \# 2$ & 296.51 & 296.55 & 296.61 & 296.66 & 296.82 & 296.96 & 297.15 & 297.31 & 297.41 & 297.49 & 297.49 & 297.65 & 297.63 & 297.72 & 297.86 & 298.05 & 298.27 \\
\hline$F X \# 3$ & 229.35 & 229.28 & 229.25 & 229.32 & 229.47 & 229.60 & 229.73 & 229.88 & 230.07 & 230.26 & 230.46 & 230.70 & 230.91 & 231.08 & 231.23 & 231.34 & 231.46 \\
\hline$F X \# 4$ & 179.63 & 179.75 & 179.88 & 180.01 & 180.12 & 180.23 & 180.34 & 180.43 & 180.53 & 180.65 & 180.79 & 180.94 & 181.02 & 181.06 & 181.13 & 181.21 & 181.28 \\
\hline$F X \# 5$ & 135.66 & 135.71 & 135.76 & 135.84 & 135.94 & 136.06 & 136.16 & 136.23 & 136.24 & 136.30 & 136.44 & 136.61 & 136.82 & 137.01 & 137.21 & 137.40 & 137.61 \\
\hline Near wall FS & 43.98 & 44.04 & 44.12 & 44.17 & 44.18 & 44.17 & 44.19 & 44.20 & 44.29 & 44.35 & 44.35 & 44.33 & 44.20 & 44.05 & 43.92 & 43.80 & 43.67 \\
\hline$T @ F X \# 3$ & 300.05 & 300.04 & 300.04 & 300.03 & 300.03 & 300.02 & 300.02 & 300.01 & 300.01 & 300.00 & 300.00 & 300.00 & 299.99 & 299.99 & 299.98 & 299.98 & 299.97 \\
\hline$T @ F X \# 4$ & 302.07 & 302.06 & 302.06 & 302.05 & 302.05 & 302.04 & 302.04 & 302.03 & 302.03 & 302.02 & 302.02 & 302.02 & 302.01 & 302.01 & 302.00 & 302.00 & 301.99 \\
\hline$T$ (a) $F X \# 5$ & 304.12 & 304.11 & 304.11 & 304.10 & 304.10 & 304.09 & 304.09 & 304.08 & 304.08 & 304.07 & 304.07 & 304.06 & 304.06 & 304.05 & 304.05 & 304.04 & 304.04 \\
\hline Wall $x$-coord & 119.37 & 119.37 & 119.37 & 119.37 & 119.38 & 119.38 & 119.38 & 119.38 & 119.38 & 119.39 & 119.39 & 119.39 & 119.39 & 119.40 & 119.40 & 119.40 & 119.40 \\
\hline Wall $\mathrm{T}$ & 306.96 & 306.96 & 306.95 & 306.95 & 306.95 & 306.95 & 306.95 & 306.95 & 306.94 & 306.94 & 306.94 & 306.94 & 306.95 & 306.96 & 306.97 & 306.97 & 306.98 \\
\hline Interferogram Image \# & 493 & Location & $T C 4$ & $\delta \varepsilon$ slope & $-2.47 E-3$ & $\delta \varepsilon$ intercept & 9.17 & $\operatorname{Avg} F S$ & 152.43 & Avg Wall T & 307.57 & Gradient error & $-1.24 E-5$ & & & & \\
\hline Scan $y$-coord & 3572 & 3573 & 3574 & 3575 & 3576 & 3577 & 3578 & 3579 & 3580 & 3581 & 3582 & 3583 & 3584 & 3585 & 3586 & 3587 & 3588 \\
\hline$\delta \varepsilon$ & 0.3517 & 0.3492 & 0.3467 & 0.3443 & 0.3418 & 0.3393 & 0.3369 & 0.3344 & 0.3319 & 0.3294 & 0.3270 & 0.3245 & 0.3220 & 0.3196 & 0.3171 & 0.3146 & 0.3122 \\
\hline Scan $x$-coord & 1287 & 1287 & 1287 & 1287 & 1287 & 1287 & 1287 & 1287 & 1287 & 1287 & 1287 & 1287 & 1287 & 1287 & 1287 & 1287 & 1287 \\
\hline
\end{tabular}




\begin{tabular}{|c|c|c|c|c|c|c|c|c|c|c|c|c|c|c|c|c|c|}
\hline$F X \# 1$ & 508.72 & 510.05 & 511.27 & 512.11 & 510.69 & 514.07 & 517.06 & 517.58 & 518.34 & 518.69 & 519.06 & 520.23 & 520.98 & 523.75 & 527.12 & 529.80 & 530.81 \\
\hline$F X \# 2$ & 328.72 & 328.79 & 328.90 & 329.08 & 329.25 & 329.51 & 329.86 & 330.14 & 330.35 & 330.57 & 330.91 & 331.36 & 331.76 & 332.07 & 332.45 & 332.78 & 332.95 \\
\hline$F X \# 3$ & 252.30 & 252.42 & 252.45 & 252.55 & 252.62 & 252.78 & 252.86 & 252.97 & 253.09 & 253.25 & 253.38 & 253.46 & 253.55 & 253.68 & 253.74 & 253.86 & 253.96 \\
\hline$F X \# 4$ & 198.30 & 198.40 & 198.48 & 198.57 & 198.64 & 198.78 & 198.90 & 199.00 & 198.99 & 198.94 & 198.91 & 198.89 & 198.91 & 199.00 & 199.12 & 199.25 & 199.38 \\
\hline$F X \# 5$ & 151.16 & 151.29 & 151.53 & 151.68 & 151.81 & 151.93 & 152.07 & 152.27 & 152.41 & 152.57 & 152.75 & 152.98 & 153.16 & 153.28 & 153.38 & 153.47 & 153.57 \\
\hline$F X \# 6$ & 0.00 & 0.00 & 0.00 & 0.00 & 0.00 & 0.00 & 0.00 & 0.00 & 0.00 & 0.00 & 0.00 & 0.00 & 0.00 & 0.00 & 0.00 & 0.00 & 0.00 \\
\hline Near wall FS & 151.16 & 151.29 & 151.53 & 151.68 & 151.81 & 151.93 & 152.07 & 152.27 & 152.41 & 152.57 & 152.75 & 152.98 & 153.16 & 153.28 & 153.38 & 153.47 & 153.57 \\
\hline$T @ F X \# 1$ & 297.20 & 297.20 & 297.20 & 297.20 & 297.20 & 297.20 & 297.20 & 297.20 & 297.20 & 297.20 & 297.20 & 297.20 & 297.20 & 297.20 & 297.20 & 297.20 & 297.20 \\
\hline$T @ F X \# 2$ & 297.89 & 297.88 & 297.88 & 297.87 & 297.87 & 297.86 & 297.86 & 297.85 & 297.85 & 297.84 & 297.84 & 297.83 & 297.83 & 297.82 & 297.82 & 297.81 & 297.81 \\
\hline$T @ F X \# 3$ & 299.88 & 299.87 & 299.87 & 299.86 & 299.86 & 299.85 & 299.85 & 299.84 & 299.84 & 299.83 & 299.83 & 299.82 & 299.82 & 299.81 & 299.81 & 299.80 & 299.80 \\
\hline$T(F X \# 4$ & 301.90 & 301.89 & 301.89 & 301.88 & 301.88 & 301.87 & 301.87 & 301.86 & 301.86 & 301.85 & 301.85 & 301.84 & 301.84 & 301.83 & 301.83 & 301.82 & 301.82 \\
\hline$T(F X \# 5$ & 303.94 & 303.94 & 303.93 & 303.93 & 303.92 & 303.92 & 303.91 & 303.91 & 303.90 & 303.90 & 303.89 & 303.89 & 303.88 & 303.88 & 303.87 & 303.87 & 303.86 \\
\hline$T @ F X \# 6$ & 306.02 & 306.01 & 306.01 & 306.00 & 306.00 & 305.99 & 305.98 & 305.98 & 305.97 & 305.97 & 305.96 & 305.96 & 305.95 & 305.95 & 305.94 & 305.94 & 305.93 \\
\hline Wall $x$-coord & 116.68 & 116.69 & 116.69 & 116.70 & 116.70 & 116.71 & 116.71 & 116.71 & 116.72 & 116.72 & 116.73 & 116.73 & 116.74 & 116.74 & 116.74 & 116.75 & 116.75 \\
\hline Wall T & 307.53 & 307.53 & 307.54 & 307.55 & 307.55 & 307.55 & 307.55 & 307.56 & 307.56 & 307.57 & 307.58 & 307.60 & 307.60 & 307.61 & 307.60 & 307.60 & 307.60 \\
\hline Interferogram Image \# & 493 & Location & TC5 & $\delta \varepsilon$ slope & $-2.47 E-3$ & $\delta \varepsilon$ intercept & 7.07 & $\operatorname{Avg} F S$ & 46.57 & Avg Wall $T$ & 308.28 & Gradient error & $-1.24 E-5$ & & & & \\
\hline Scan y-coord & 2757 & 2758 & 2759 & 2760 & 2761 & 2762 & 2763 & 2764 & 2765 & 2766 & 2767 & 2768 & 2769 & 2770 & 2771 & 2772 & 2773 \\
\hline $\begin{array}{rl}\delta c a n & y-c o r r a \\
\delta \varepsilon\end{array}$ & 0.2500 & 0.2475 & 0.2450 & 0.2426 & 0.2401 & 0.2376 & 0.2352 & 0.2327 & 0.2302 & 0.2277 & 0.2253 & 0.2228 & 0.2203 & 0.2178 & 0.2154 & 0.2129 & 0.2104 \\
\hline Scan $x$-coord & 1663 & 1663 & 1663 & 1663 & 1663 & 1663 & 1663 & 1663 & 1663 & 1663 & 1663 & 1663 & 1663 & 1663 & 1663 & 1663 & 1663 \\
\hline$F X \# 1$ & 612.38 & 614.66 & 616.22 & 616.95 & 617.90 & 618.99 & 620.38 & 621.58 & 626.19 & 626.59 & 628.04 & 629.55 & 632.07 & 633.32 & 634.60 & 635.79 & 637.81 \\
\hline$F X \# 2$ & 367.74 & 367.82 & 367.96 & 368.27 & 368.74 & 369.30 & 369.90 & 370.36 & 370.85 & 371.28 & 371.62 & 371.78 & 371.97 & 372.25 & 372.35 & 372.72 & 373.00 \\
\hline$F X \# 3$ & 278.13 & 278.27 & 278.37 & 278.37 & 278.28 & 278.23 & 278.25 & 278.30 & 278.43 & 278.64 & 278.92 & 279.11 & 279.31 & 279.44 & 279.56 & 279.60 & 279.71 \\
\hline$F X \# 4$ & 217.71 & 217.82 & 217.89 & 217.96 & 218.13 & 218.25 & 218.35 & 218.45 & 218.71 & 218.90 & 218.98 & 219.04 & 219.01 & 218.90 & 218.73 & 218.67 & 218.70 \\
\hline$F X \# 5$ & 166.99 & 167.24 & 167.54 & 167.77 & 167.92 & 167.98 & 168.04 & 168.14 & 168.39 & 168.57 & 168.75 & 168.92 & 169.08 & 169.18 & 169.26 & 169.26 & 169.23 \\
\hline$F X \# 6$ & 120.57 & 120.76 & 120.93 & 121.08 & 121.17 & 121.25 & 121.48 & 121.78 & 122.06 & 122.18 & 122.22 & 122.37 & 122.41 & 122.45 & 122.52 & 122.59 & 122.69 \\
\hline Near wall FS & 46.42 & 46.48 & 46.61 & 46.69 & 46.75 & 46.73 & 46.56 & 46.37 & 46.33 & 46.39 & 46.53 & 46.55 & 46.68 & 46.72 & 46.74 & 46.67 & 46.54 \\
\hline$T @ F X \# 1$ & 297.20 & 297.20 & 297.20 & $\begin{array}{l}29.07 .20 \\
29\end{array}$ & 297.20 & 297.20 & $\begin{array}{l}49.50 \\
297.20\end{array}$ & 297.20 & 297.20 & 297.20 & 297.20 & $\begin{array}{l}290.53 \\
290\end{array}$ & $\begin{array}{l}46.68 \\
297.20\end{array}$ & $\begin{array}{l}46 . / 2 \\
297.20\end{array}$ & $\begin{array}{l}46 . / 4 \\
297.20\end{array}$ & $\begin{array}{r}46.67 \\
297.20\end{array}$ & $\begin{array}{l}46.54 \\
297.20\end{array}$ \\
\hline$T @ F X \# 2$ & 297.68 & 297.68 & 297.67 & 297.67 & 297.66 & 297.66 & 297.65 & 297.65 & 297.64 & 297.64 & 297.63 & 297.63 & 297.62 & 297.62 & 297.62 & 297.61 & 297.61 \\
\hline$T$ (a) $F X \# 3$ & 299.67 & 299.66 & 299.66 & 299.65 & 299.65 & 299.65 & 299.64 & 299.64 & 299.63 & 299.63 & 299.62 & 299.62 & 299.61 & 299.61 & 299.60 & 299.60 & 299.59 \\
\hline$T(F X \# 4$ & 301.69 & 301.68 & 301.67 & 301.67 & 301.66 & 301.66 & 301.65 & 301.65 & 301.64 & 301.64 & 301.63 & 301.63 & 301.62 & 301.62 & 301.61 & 301.61 & 301.60 \\
\hline$T(F X \# 5$ & 303.73 & 303.72 & 303.72 & 303.71 & 303.71 & 303.70 & 303.70 & 303.69 & 303.69 & 303.68 & 303.68 & 303.67 & 303.67 & 303.66 & 303.66 & 303.65 & 303.65 \\
\hline$T @ F X \# 6$ & 305.80 & 305.79 & 305.79 & 305.78 & 305.78 & 305.77 & 305.77 & 305.76 & 305.76 & 305.75 & 305.75 & 305.74 & 305.74 & 305.73 & 305.73 & 305.72 & 305.72 \\
\hline Wall $x$-coord & $\begin{array}{l}50.80 \\
112.26\end{array}$ & 112.27 & $\begin{array}{l}305.19 \\
112.28\end{array}$ & $\begin{array}{l}503.18 \\
112.28\end{array}$ & $\begin{array}{l}303.18 \\
112.29\end{array}$ & 112.30 & 112.30 & $\begin{array}{l}505.10 \\
112.31\end{array}$ & $\begin{array}{l}503.10 \\
112.32\end{array}$ & $\begin{array}{l}505.13 \\
112.32\end{array}$ & $\begin{array}{l}305.13 \\
112.33\end{array}$ & $\begin{array}{l}305.14 \\
112.34\end{array}$ & $\begin{array}{l}503.14 \\
112.34\end{array}$ & $\begin{array}{l}305.13 \\
112.35\end{array}$ & $\begin{array}{l}305.13 \\
112.36\end{array}$ & $\begin{array}{l}305.12 \\
112.36\end{array}$ & $\begin{array}{l}0.12 .37 \\
112.37\end{array}$ \\
\hline Wall T & 308.27 & 308.28 & 308.28 & 308.28 & 308.28 & 308.27 & 308.28 & 308.29 & 308.30 & 308.30 & 308.29 & 308.29 & 308.29 & 308.28 & 308.28 & 308.28 & 308.28 \\
\hline Interferogram Image \# & 493 & Location & ТС6 & $\delta \varepsilon$ slope & $-2.12 E-3$ & $\delta \varepsilon$ intercept & 4.19 & $\operatorname{Avg} F S$ & 47.79 & Avg Wall T & 308.76 & Gradient error & $-1.24 E-5$ & & & & \\
\hline Scan y-coord & 1942 & 1943 & 1944 & 1945 & 1946 & 1947 & 1948 & 1949 & 1950 & 1951 & 1952 & 1953 & 1954 & 1955 & 1956 & 1957 & 1958 \\
\hline $\begin{array}{rl}\delta c a n & y-c o s i a \\
\delta \varepsilon\end{array}$ & 0.0717 & 0.0695 & 0.0674 & 0.0653 & 0.0632 & 0.0611 & 0.0589 & 0.0568 & 0.0547 & 0.0526 & 0.0505 & 0.0484 & $\begin{array}{l}194462 \\
0.0462\end{array}$ & 0.0441 & 0.0420 & 0.0399 & 0.0378 \\
\hline Scan $x$-coord & 1990 & 1990 & 1990 & 1990 & 1990 & 1990 & 1990 & 1990 & 1990 & 1990 & 1990 & 1990 & 1990 & 1990 & 1990 & 1990 & 1990 \\
\hline$F X \# 1$ & 1019.85 & 1024.79 & 1030.24 & 1019.12 & 1032.07 & 1051.96 & 1048.56 & 1039.48 & 1044.59 & 1049.04 & 1094.01 & 1048.52 & 1099.95 & 1062.25 & 1053.76 & 1060.67 & 1067.63 \\
\hline$F X \# 2$ & 396.34 & 396.41 & 396.33 & 396.25 & 396.07 & 396.07 & 396.23 & 396.66 & 397.20 & 397.90 & $\begin{array}{l}1048.60 \\
398.60\end{array}$ & 399.21 & 399.86 & 400.46 & 401.00 & 401.30 & $\begin{array}{l}1061.63 \\
401.43\end{array}$ \\
\hline$F X \# 3$ & 301.20 & 301.18 & 301.13 & 301.08 & 301.20 & 301.33 & 301.51 & 301.82 & 302.08 & 302.29 & 302.51 & 302.74 & 302.88 & 303.05 & 303.35 & 303.58 & 303.85 \\
\hline$F X \# 4$ & 234.81 & 234.94 & 235.15 & 235.41 & 235.72 & 235.92 & 236.15 & 236.25 & 236.31 & 236.44 & 236.48 & 236.62 & 236.80 & 237.03 & 237.26 & 237.49 & 237.70 \\
\hline$F X \# 5$ & 182.86 & 182.74 & 182.69 & 182.63 & 182.63 & 182.73 & 182.86 & 183.02 & 183.19 & 183.41 & 183.54 & 183.66 & 183.76 & 183.82 & 183.82 & 183.73 & 183.63 \\
\hline$F X \# 6$ & 134.87 & 134.93 & 135.10 & 135.26 & 135.39 & 135.44 & 135.49 & 135.53 & 135.56 & 135.57 & 135.57 & 135.58 & 135.57 & 135.58 & 135.55 & 135.62 & 135.75 \\
\hline Near wall $F S$ & 47.99 & 47.82 & 47.58 & 47.36 & 47.25 & 47.29 & 47.37 & 47.49 & 47.63 & 47.85 & 47.97 & 48.08 & 48.19 & 48.24 & 48.26 & 48.10 & 47.88 \\
\hline$T @ F X \# 1$ & 297.20 & 297.20 & 297.20 & 297.20 & 297.20 & 297.20 & 297.20 & 297.20 & 297.20 & 297.20 & 297.20 & 297.20 & 297.20 & 297.20 & 297.20 & 297.20 & 297.20 \\
\hline$T @ F X \# 2$ & 297.33 & 297.33 & 297.32 & 297.32 & 297.32 & 297.31 & 297.31 & 297.30 & 297.30 & 297.29 & 297.29 & 297.29 & 297.28 & 297.28 & 297.27 & 297.27 & 297.27 \\
\hline$T$ (a) $F X \# 3$ & 299.31 & 299.31 & 299.30 & 299.30 & 299.29 & 299.29 & 299.28 & 299.28 & 299.28 & 299.27 & 299.27 & 299.26 & 299.26 & 299.25 & 299.25 & 299.25 & 299.24 \\
\hline$T @ F X \# 4$ & 301.32 & 301.32 & 301.31 & 301.31 & 301.30 & 301.30 & 301.29 & 301.29 & 301.29 & 301.28 & 301.28 & 301.27 & 301.27 & 301.26 & 301.26 & 301.26 & 301.25 \\
\hline$T(F X \# 5$ & 303.36 & 303.35 & 303.35 & 303.34 & 303.34 & 303.34 & 303.33 & 303.33 & 303.32 & 303.32 & 303.31 & 303.31 & 303.31 & 303.30 & 303.30 & 303.29 & 303.29 \\
\hline$T @ F X \# 6$ & 305.42 & 305.42 & 305.41 & 305.41 & 305.41 & 305.40 & 305.40 & 305.39 & 305.39 & 305.38 & 305.38 & 305.37 & 305.37 & 305.37 & 305.36 & 305.36 & 305.35 \\
\hline Wall $x$-coord & $\begin{array}{l}505.42 \\
106.11\end{array}$ & $\begin{array}{l}505.42 \\
106.12\end{array}$ & $\begin{array}{l}505.41 \\
106.13\end{array}$ & $\begin{array}{l}505.41 \\
106.14\end{array}$ & $\begin{array}{l}505.41 \\
106.14\end{array}$ & $\begin{array}{l}30.40 \\
106.15\end{array}$ & $\begin{array}{l}305.40 \\
106.16\end{array}$ & 106.17 & 106.18 & $\begin{array}{l}505.08 \\
106.19\end{array}$ & $\begin{array}{l}305.30 \\
106.20\end{array}$ & $\begin{array}{l}305.37 \\
106.21\end{array}$ & $\begin{array}{l}305.31 \\
106.21\end{array}$ & $\begin{array}{l}305.37 \\
106.22\end{array}$ & $\begin{array}{l}505.30 \\
106.23\end{array}$ & $\begin{array}{l}505.30 \\
106.24\end{array}$ & $\begin{array}{l}505.35 \\
106.25\end{array}$ \\
\hline Wall T & 308.77 & 308.77 & 308.78 & 308.79 & 308.80 & 308.79 & 308.79 & 308.78 & 308.77 & 308.76 & 308.75 & 308.75 & 308.74 & 308.73 & 308.73 & 308.73 & 308.74 \\
\hline Interferogram Image \# & 493 & Location & $T C 7$ & $\delta \varepsilon$ slope & $-2.09 E-3$ & $\delta \varepsilon$ intercept & 3.16 & $\operatorname{Avg} F S$ & 124.94 & Avg Wall $T$ & 309.07 & Gradient error & $-1.24 E-5$ & & & & \\
\hline Scan y-coord & 1128 & 1129 & 1130 & 1131 & 1132 & 1133 & 1134 & 1135 & 1136 & 1137 & 1138 & 1139 & 1140 & 1141 & 1142 & 1143 & 1144 \\
\hline & 0.7988 & 0.7967 & 0.7946 & 0.7925 & 0.7904 & 0.7883 & 0.7862 & 0.7841 & 0.7820 & 0.7799 & 0.7778 & 0.7757 & 0.7736 & 0.7715 & 0.7695 & 0.7674 & 0.7653 \\
\hline Scan $x$-coord & 1877 & $\begin{array}{l}0.1907 \\
1877\end{array}$ & $\begin{array}{l}0.9740 \\
1877\end{array}$ & 1877 & $\begin{array}{l}0.9074 \\
1877\end{array}$ & 1877 & $\begin{array}{l}0.1802 \\
1877\end{array}$ & $\begin{array}{l}0.1841 \\
1877\end{array}$ & 1877 & 1877 & 1877 & 1877 & $\begin{array}{l}0.1730 \\
1877\end{array}$ & 1877 & $\begin{array}{l}0.1895 \\
1877-25\end{array}$ & $\begin{array}{l}0.1877 \\
1877\end{array}$ & $\begin{array}{l}0.1853 \\
1877\end{array}$ \\
\hline$F X \# 1$ & 460.88 & 460.86 & 460.93 & 460.97 & 460.91 & 461.03 & 461.54 & 462.19 & 463.14 & 464.11 & 464.66 & 464.95 & 465.20 & 465.39 & 465.42 & 465.44 & 465.41 \\
\hline$F X \# 2$ & 329.17 & 329.25 & 329.27 & 329.36 & 329.51 & 329.61 & 329.76 & 329.96 & 330.37 & 330.93 & 331.26 & 331.41 & 331.55 & 331.79 & 332.16 & 332.42 & 332.71 \\
\hline$F X \# 3$ & 255.11 & 255.06 & 255.02 & 255.15 & 255.42 & 255.77 & 256.16 & 256.56 & 256.81 & 256.94 & 256.86 & 256.71 & 256.48 & 256.41 & 256.44 & 256.59 & 256.84 \\
\hline$F X \# 4$ & 198.30 & 198.53 & 198.65 & 198.68 & 198.69 & 198.68 & 198.63 & 198.70 & 198.84 & 199.13 & 199.41 & 199.66 & 199.82 & 199.95 & 200.11 & 200.20 & 200.22 \\
\hline$F X \# 5$ & 148.13 & 148.29 & 148.43 & 148.59 & 148.69 & 148.79 & 148.90 & 148.95 & 149.03 & 149.18 & 149.33 & 149.61 & 149.70 & 149.81 & 149.77 & 149.89 & 150.27 \\
\hline$F X \# 6$ & 0.00 & 0.00 & 0.00 & 0.00 & 0.00 & 0.00 & 0.00 & 0.00 & 0.00 & 0.00 & 0.00 & 102.93 & 102.88 & 102.71 & 0.00 & 0.00 & 102.89 \\
\hline $\begin{array}{l}\text { Near wall } F S \\
F S\end{array}$ & 148.13 & 148.29 & $\begin{array}{l}0.00 \\
148.43\end{array}$ & $\begin{array}{l}0.00 \\
148.59\end{array}$ & $\begin{array}{l}0.00 \\
148.69\end{array}$ & $\begin{array}{l}0.00 \\
148.79\end{array}$ & $\begin{array}{l}0.00 \\
148.90\end{array}$ & $\begin{array}{l}0.00 \\
148.95\end{array}$ & $\begin{array}{l}0.00 \\
149.03\end{array}$ & $\begin{array}{l}0.00 \\
149.18\end{array}$ & $\begin{array}{l}0.00 \\
149.33\end{array}$ & $\begin{array}{l}102.93 \\
46.68\end{array}$ & $\begin{array}{l}102.80 \\
46.82\end{array}$ & $\begin{array}{r}102.11 \\
47.10\end{array}$ & $\begin{array}{l}0.00 \\
149.77\end{array}$ & $\begin{array}{l}0.00 \\
149.89\end{array}$ & $\begin{array}{r}102.89 \\
47.38\end{array}$ \\
\hline$T @ F X \# 1$ & 297.20 & 297.20 & 297.20 & 297.20 & 297.20 & 297.20 & 297.20 & 297.20 & 297.20 & 297.20 & 297.20 & 297.20 & 297.20 & 297.20 & 297.20 & 297.20 & 297.20 \\
\hline$T @ F X \# 2$ & 298.77 & 298.76 & 298.76 & 298.75 & 298.75 & 298.75 & 298.74 & 298.74 & 298.73 & 298.73 & 298.73 & 298.72 & 298.72 & 298.71 & 298.71 & 298.70 & 298.70 \\
\hline$T @ F X \# 3$ & 300.77 & 300.77 & 300.76 & 300.76 & 300.75 & 300.75 & 300.74 & 300.74 & 300.74 & 300.73 & 300.73 & 300.72 & 300.72 & 300.72 & 300.71 & 300.71 & 300.70 \\
\hline$T$ (a) $F X \# 4$ & 302.80 & 302.80 & 302.79 & 302.79 & 302.78 & 302.78 & 302.77 & 302.77 & 302.77 & 302.76 & 302.76 & 302.75 & 302.75 & 302.74 & 302.74 & 302.74 & 302.73 \\
\hline$T(F X \# 5$ & 304.86 & 304.85 & 304.85 & 304.85 & 304.84 & 304.84 & 304.83 & 304.83 & 304.82 & 304.82 & 304.81 & 304.81 & 304.81 & 304.80 & 304.80 & 304.79 & 304.79 \\
\hline
\end{tabular}




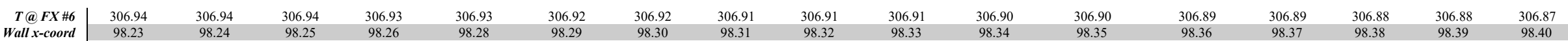

\section{B.38 Tabulated Data for Interferogram 494}

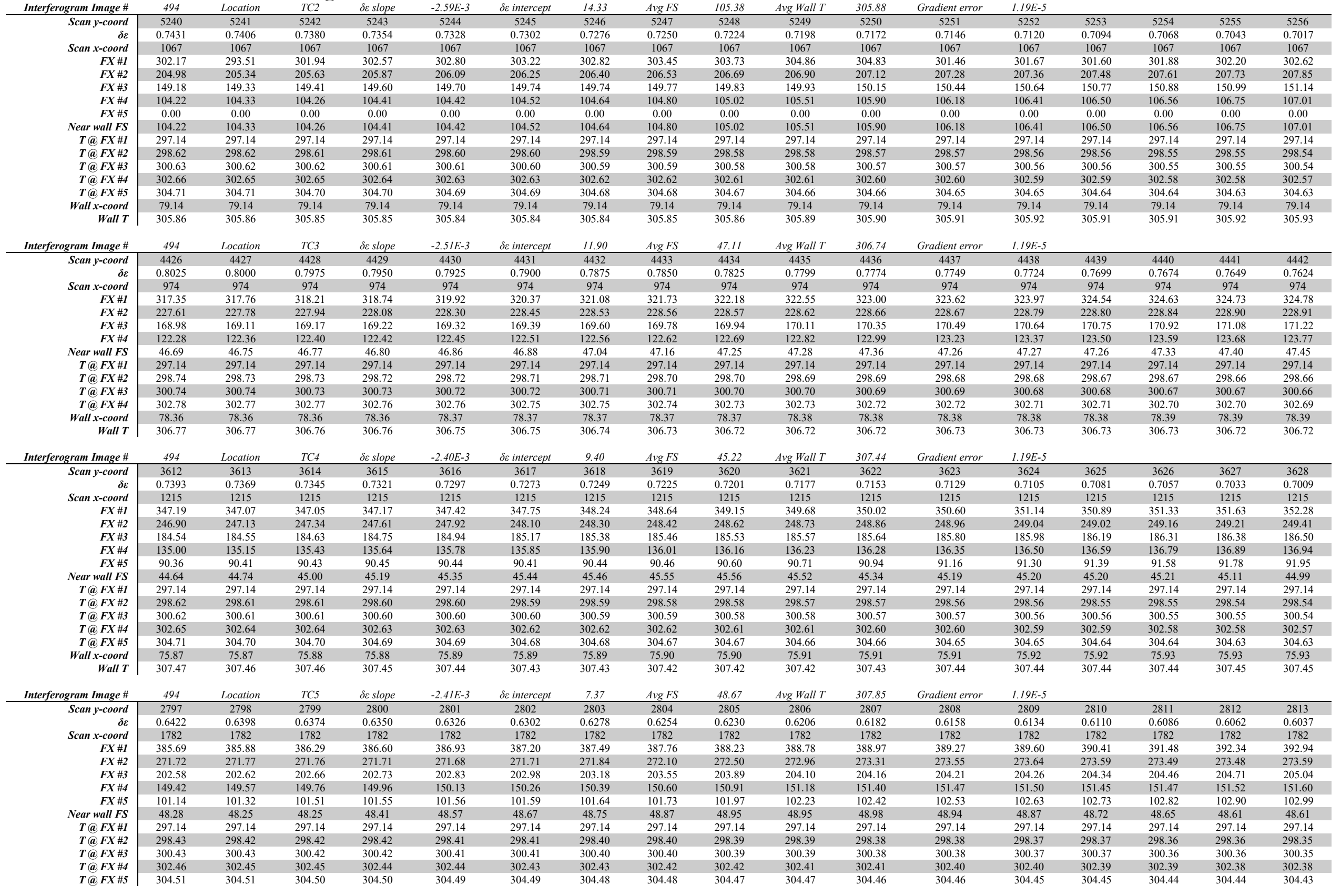




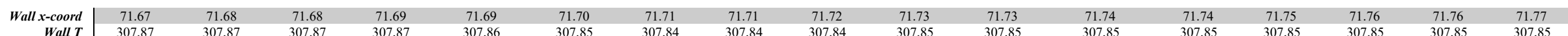

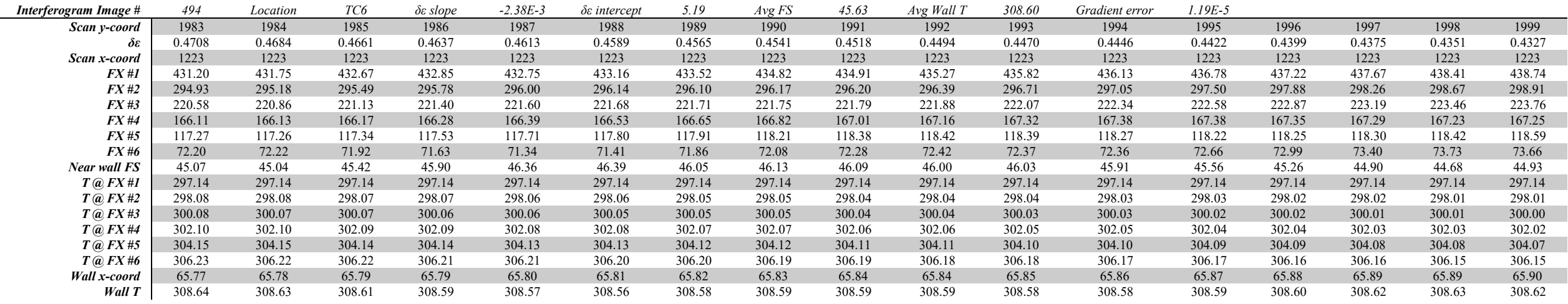

Interferogram Image

\begin{tabular}{|c|c|c|c|c|c|c|c|c|c|c|c|c|c|c|c|c|c|}
\hline gram Image \# & 494 & Location & $T C 7$ & $\delta \varepsilon$ slope & $-2.26 E-3$ & $\delta \varepsilon$ intercept & 2.92 & $\operatorname{Avg} F S$ & 47.78 & Avg Wall $T$ & 308.92 & Gradient error & $1.19 E-5$ & & & & \\
\hline Scan y-coord & 1168 & 1169 & 1170 & 1171 & 1172 & 1173 & 1174 & 1175 & 1176 & 1177 & 1178 & 1179 & 1180 & 1181 & 1182 & 1183 & 1184 \\
\hline$\delta \varepsilon$ & 0.2808 & 0.2786 & 0.2763 & 0.2740 & 0.2718 & 0.2695 & 0.2673 & 0.2650 & 0.2628 & 0.2605 & 0.2582 & 0.2560 & 0.2537 & 0.2515 & 0.2492 & 0.2470 & 0.2447 \\
\hline Scan $x$-coord & 1722 & 1722 & 1722 & 1722 & 1722 & 1722 & 1722 & 1722 & 1722 & 1722 & 1722 & 1722 & 1722 & 1722 & 1722 & 1722 & 1722 \\
\hline$F X \# 1$ & 578.92 & 579.68 & 580.71 & 576.82 & 577.42 & 578.10 & 589.66 & 590.44 & 590.79 & 591.77 & 592.69 & 594.14 & 595.93 & 597.72 & 598.93 & 600.01 & 603.62 \\
\hline$F X \# 2$ & 333.42 & 333.36 & 333.36 & 333.28 & 333.53 & 333.75 & 334.06 & 334.39 & 334.60 & 334.68 & 334.94 & 335.22 & 335.54 & 335.72 & 336.06 & 336.17 & 336.45 \\
\hline$F X \# 3$ & 242.46 & 242.43 & 242.38 & 242.37 & 242.50 & 242.68 & 243.00 & 243.24 & 243.31 & 243.29 & 243.31 & 243.53 & 243.77 & 243.92 & 243.97 & 244.01 & 244.13 \\
\hline$F X \# 4$ & 181.22 & 181.33 & 181.37 & 181.38 & 181.36 & 181.32 & 181.32 & 181.31 & 181.34 & 181.41 & 181.55 & 181.70 & 181.89 & 182.14 & 182.36 & 182.63 & 182.68 \\
\hline$F X \# 5$ & 128.90 & 128.94 & 128.89 & 128.82 & 128.79 & 128.71 & 128.78 & 128.84 & 128.90 & 129.12 & 129.26 & 129.50 & 129.63 & 129.74 & 129.82 & 130.01 & 130.09 \\
\hline$F X \# 6$ & 81.23 & 81.28 & 81.31 & 81.38 & 81.39 & 81.33 & 81.26 & 81.30 & 81.36 & 81.38 & 81.38 & 81.41 & 81.49 & 81.59 & 81.70 & 81.80 & 81.93 \\
\hline Near wall FS & 47.67 & 47.67 & 47.58 & 47.44 & 47.39 & 47.38 & 47.53 & 47.53 & 47.54 & 47.74 & 47.88 & 48.09 & 48.14 & 48.15 & 48.12 & 48.20 & 48.16 \\
\hline$T @ F X \# 1$ & 297.14 & 297.14 & 297.14 & 297.14 & 297.14 & 297.14 & 297.14 & 297.14 & 297.14 & 297.14 & 297.14 & 297.14 & 297.14 & 297.14 & 297.14 & 297.14 & 297.14 \\
\hline$T @ F X \# 2$ & 297.71 & 297.71 & 297.70 & 297.70 & 297.69 & 297.69 & 297.68 & 297.68 & 297.67 & 297.67 & 297.67 & 297.66 & 297.66 & 297.65 & 297.65 & 297.64 & 297.64 \\
\hline$T @ F X \# 3$ & 299.70 & 299.70 & 299.69 & 299.69 & 299.69 & 299.68 & 299.68 & 299.67 & 299.67 & 299.66 & 299.66 & 299.65 & 299.65 & 299.64 & 299.64 & 299.64 & 299.63 \\
\hline$T @ F X \# 4$ & 301.72 & 301.72 & 301.71 & 301.71 & 301.70 & 301.70 & 301.69 & 301.69 & 301.68 & 301.68 & 301.68 & 301.67 & 301.67 & 301.66 & 301.66 & 301.65 & 301.65 \\
\hline$T$ @ $F X \# 5$ & 303.77 & 303.76 & 303.76 & 303.75 & 303.75 & 303.74 & 303.74 & 303.73 & 303.73 & 303.72 & 303.72 & 303.71 & 303.71 & 303.71 & 303.70 & 303.70 & 303.69 \\
\hline$T @ F X \# 6$ & 305.84 & 305.83 & 305.83 & 305.82 & 305.82 & 305.82 & 305.81 & 305.81 & 305.80 & 305.80 & 305.79 & 305.79 & 305.78 & 305.78 & 305.77 & 305.77 & 305.76 \\
\hline Wall $x$-coord & 58.16 & 58.17 & 58.18 & 58.19 & 58.20 & 58.21 & 58.22 & 58.23 & 58.24 & 58.25 & 58.26 & 58.27 & 58.28 & 58.29 & 58.30 & 58.31 & 58.32 \\
\hline Wall T & 308.96 & 308.95 & 308.95 & 308.95 & 308.95 & 308.94 & 308.93 & 308.93 & 308.92 & 308.91 & 308.91 & 308.90 & 308.90 & 308.89 & 308.89 & 308.89 & 308.89 \\
\hline
\end{tabular}

\section{B.39 Tabulated Data for Interferogram 495}

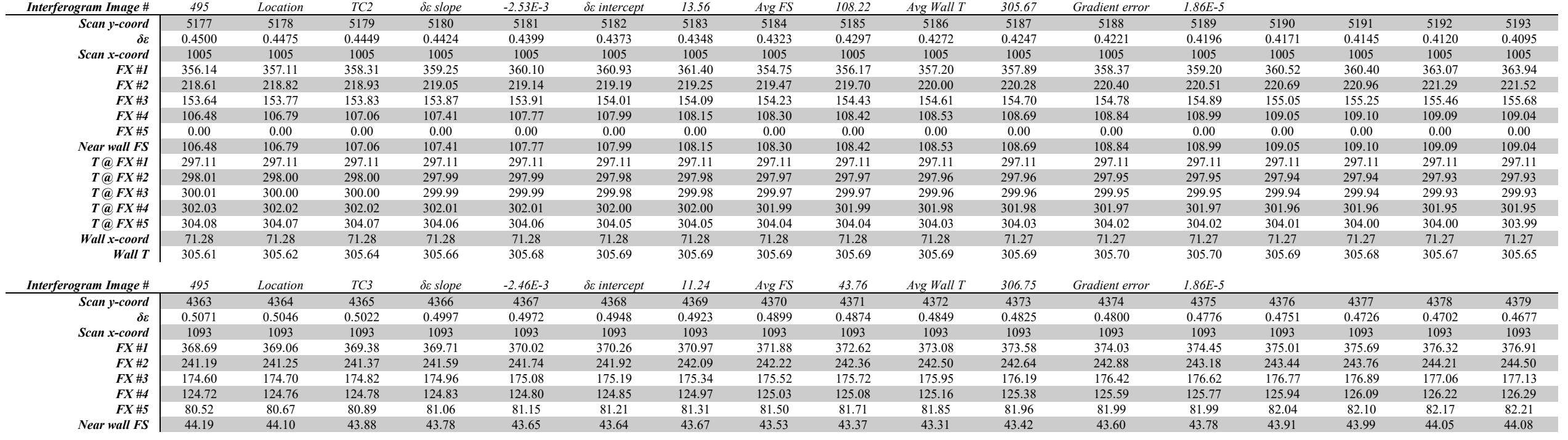




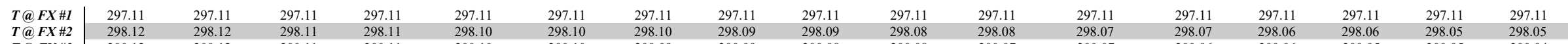

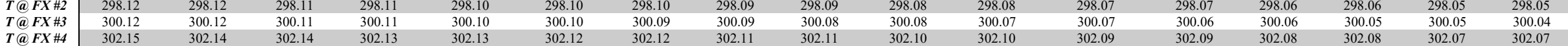

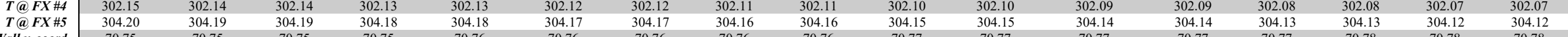

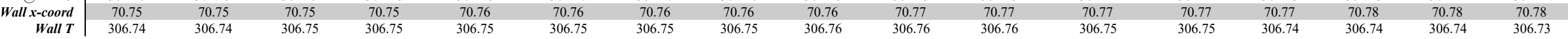

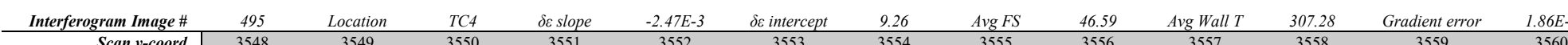

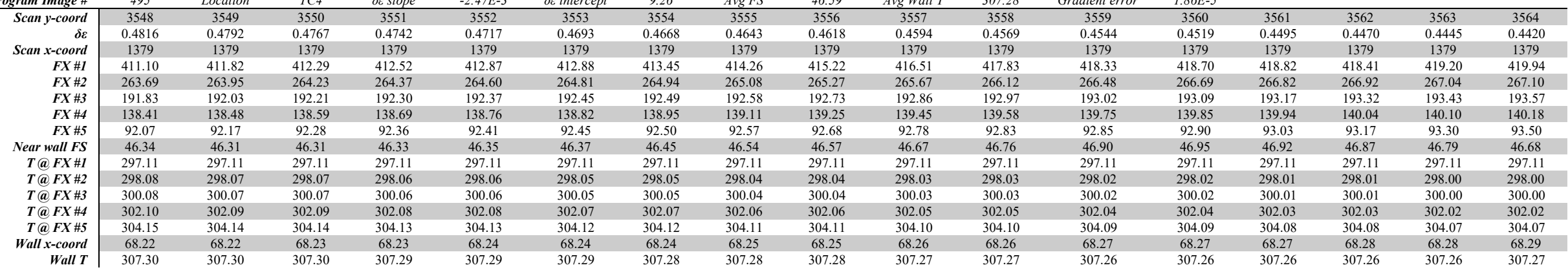

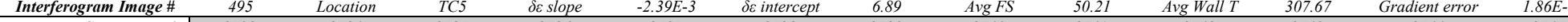

\begin{tabular}{|c|c|c|c|c|c|c|c|c|c|c|c|c|c|c|c|c|c|}
\hline gram image \# & 493 & Location & $1 C 5$ & $\delta$ slope & $-2.59 \mathrm{E}-3$ & $\delta \varepsilon$ intercept & 0.09 & Avg FS & & Avg Wall T & & Gradient error & $1.86 \mathrm{E}-9$ & & & & \\
\hline Scan y-coord & 2733 & 2734 & 2735 & 2736 & 2737 & 2738 & 2739 & 2740 & 2741 & 2742 & 2743 & 2744 & 2745 & 2746 & 2747 & 2748 & 2749 \\
\hline & 0.3492 & 0.3468 & 0.3444 & 0.3420 & 0.3396 & 0.3372 & 0.3348 & 0.3324 & 0.3300 & 0.3276 & 0.3252 & 0.3228 & 0.3205 & 0.3181 & 0.3157 & 0.3133 & 0.3109 \\
\hline Scan $x$-coord & 1667 & 1667 & 1667 & 1667 & 1667 & 1667 & 1667 & 1667 & 1667 & 1667 & 1667 & 1667 & 1667 & 1667 & 1667 & 1667 & 1667 \\
\hline$F X \# 1$ & 472.65 & 473.66 & 474.70 & 475.69 & 477.23 & 478.05 & 480.39 & 484.75 & 485.67 & 486.47 & 487.16 & 487.76 & 488.57 & 489.29 & 489.89 & 490.63 & 491.62 \\
\hline$F X \# 2$ & 293.72 & 293.84 & 294.21 & 294.63 & 295.10 & 295.39 & 295.65 & 296.17 & 296.44 & 296.64 & 296.75 & 296.99 & 297.21 & 297.40 & 297.60 & 297.74 & 297.86 \\
\hline$F X \# 3$ & 212.47 & 212.58 & 212.74 & 212.88 & 213.08 & 213.26 & 213.46 & 213.76 & 213.94 & 214.03 & 214.08 & 214.22 & 214.37 & 214.54 & 214.63 & 214.71 & 214.77 \\
\hline$F X \# 4$ & 155.09 & 155.31 & 155.52 & 155.73 & 155.93 & 156.04 & 156.16 & 156.29 & 156.44 & 156.55 & 156.65 & 156.76 & 156.81 & 156.82 & 156.89 & 157.06 & 157.27 \\
\hline$F X \# 5$ & 105.08 & 105.19 & 105.32 & 105.48 & 105.57 & 105.64 & 105.79 & 106.01 & 106.20 & 106.33 & 106.48 & 106.63 & 106.69 & 106.69 & 106.79 & 106.91 & 106.96 \\
\hline Near wall FS & 50.01 & 50.12 & 50.20 & 50.24 & 50.36 & 50.40 & 50.37 & 50.29 & 50.24 & 50.22 & 50.17 & 50.13 & 50.12 & 50.13 & 50.10 & 50.16 & 50.31 \\
\hline$T @ F X \# 1$ & 297.11 & 297.11 & 297.11 & 297.11 & 297.11 & 297.11 & 297.11 & 297.11 & 297.11 & 297.11 & 297.11 & 297.11 & 297.11 & 297.11 & 297.11 & 297.11 & 297.11 \\
\hline$T @ F X \# 2$ & 297.82 & 297.82 & 297.81 & 297.81 & 297.80 & 297.80 & 297.79 & 297.79 & 297.78 & 297.78 & 297.77 & 297.77 & 297.76 & 297.76 & 297.75 & 297.75 & 297.74 \\
\hline$T @ F X \# 3$ & 299.82 & 299.81 & 299.81 & 299.80 & 299.80 & 299.79 & 299.79 & 299.78 & 299.78 & 299.77 & 299.77 & 299.76 & 299.76 & 299.75 & 299.75 & 299.74 & 299.74 \\
\hline$T @ F X \# 4$ & 301.84 & 301.83 & 301.83 & 301.82 & 301.82 & 301.81 & 301.81 & 301.80 & 301.80 & 301.79 & 301.79 & 301.78 & 301.78 & 301.77 & 301.77 & 301.76 & 301.76 \\
\hline$T @ F X \# 5$ & 303.88 & 303.88 & 303.87 & 303.87 & 303.86 & 303.86 & 303.85 & 303.85 & 303.84 & 303.84 & 303.83 & 303.83 & 303.82 & 303.82 & 303.81 & 303.81 & 303.80 \\
\hline Wall $x$-coord & 63.69 & 63.70 & 63.70 & 63.71 & 63.72 & 63.72 & 63.73 & 63.74 & 63.74 & 63.75 & 63.76 & 63.76 & 63.77 & 63.78 & 63.78 & 63.79 & 63.80 \\
\hline Wall T & 307.67 & 307.67 & 307.67 & 307.67 & 307.66 & 307.66 & 307.66 & 307.66 & 307.67 & 307.67 & 307.67 & 307.67 & 307.67 & 307.67 & 307.67 & 307.66 & 307.66 \\
\hline
\end{tabular}

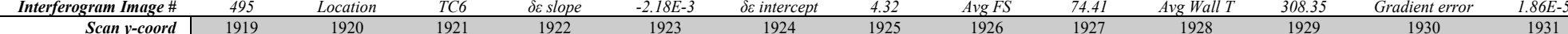

\begin{tabular}{|c|c|c|c|c|c|c|c|c|c|c|c|c|c|c|c|c|c|}
\hline & & & & & & & & & & & & & & & & & \\
\hline Scan $y$-coord & 1919 & 1920 & 1921 & 1922 & 1923 & 1924 & 1925 & 1926 & 1927 & 1928 & 1929 & 1930 & 1931 & 1932 & 1933 & 1934 & 1935 \\
\hline$\delta \varepsilon$ & 0.1395 & 0.1373 & 0.1351 & 0.1329 & 0.1308 & 0.1286 & 0.1264 & 0.1242 & 0.1221 & 0.1199 & 0.1177 & 0.1155 & 0.1134 & 0.1112 & 0.1090 & 0.1068 & 0.1046 \\
\hline Scan $x$-coord & 2029 & 2029 & 2029 & 2029 & 2029 & 2029 & 2029 & 2029 & 2029 & 2029 & 2029 & 2029 & 2029 & 2029 & 2029 & 2029 & 2029 \\
\hline$F X \# 1$ & 568.99 & 569.87 & 569.73 & 569.25 & 569.07 & 569.39 & 570.43 & 572.41 & 573.13 & 575.12 & 576.20 & 578.03 & 579.76 & 583.71 & 596.11 & 583.89 & 583.69 \\
\hline$F X \# 2$ & 322.77 & 323.06 & 323.28 & 323.52 & 323.69 & 323.84 & 323.91 & 324.00 & 324.15 & 324.45 & 324.71 & 324.93 & 325.18 & 325.38 & 325.48 & 325.94 & 326.26 \\
\hline$F X \# 3$ & 233.81 & 233.76 & 233.70 & 233.62 & 233.66 & 233.74 & 234.00 & 234.27 & 234.55 & 234.78 & 235.08 & 235.35 & 235.60 & 235.73 & 235.81 & 235.85 & 235.93 \\
\hline$F X \# 4$ & 172.13 & 172.43 & 172.73 & 172.92 & 172.98 & 173.08 & 173.12 & 173.10 & 173.11 & 173.16 & 173.26 & 173.47 & 173.62 & 173.78 & 173.90 & 174.05 & 174.18 \\
\hline$F X \# 5$ & 120.82 & 120.88 & 121.02 & 121.20 & 121.37 & 121.58 & 121.78 & 121.95 & 122.04 & 122.09 & 122.12 & 122.21 & 122.23 & 122.29 & 122.43 & 122.59 & 122.74 \\
\hline$F X \# 6$ & 72.71 & 72.75 & 72.97 & 73.35 & 73.96 & 74.46 & 74.68 & 74.69 & 74.58 & 74.59 & 74.66 & 74.82 & 75.07 & 75.24 & 75.32 & 75.42 & 75.68 \\
\hline$F X \# 7$ & 0.00 & 0.00 & 0.00 & 0.00 & 0.00 & 0.00 & 0.00 & 0.00 & 0.00 & 0.00 & 0.00 & 0.00 & 0.00 & 0.00 & 0.00 & 0.00 & 0.00 \\
\hline Near wall FS & 72.71 & 72.75 & 72.97 & 73.35 & 73.96 & 74.46 & 74.68 & 74.69 & 74.58 & 74.59 & 74.66 & 74.82 & 75.07 & 75.24 & 75.32 & 75.42 & 75.68 \\
\hline$T$ @ $F X \# 1$ & 297.11 & 297.11 & 297.11 & 297.11 & 297.11 & 297.11 & 297.11 & 297.11 & 297.11 & 297.11 & 297.11 & 297.11 & 297.11 & 297.11 & 297.11 & 297.11 & 297.11 \\
\hline$T$ (a) $F X \# 2$ & 297.41 & 297.41 & 297.40 & 297.40 & 297.39 & 297.39 & 297.39 & 297.38 & 297.38 & 297.37 & 297.37 & 297.36 & 297.36 & 297.36 & 297.35 & 297.35 & 297.34 \\
\hline$T @ F X \# 3$ & 299.40 & 299.40 & 299.39 & 299.39 & 299.38 & 299.38 & 299.38 & 299.37 & 299.37 & 299.36 & 299.36 & 299.35 & 299.35 & 299.35 & 299.34 & 299.34 & 299.33 \\
\hline$T @ F X \# 4$ & 301.42 & 301.41 & 301.41 & 301.40 & 301.40 & 301.39 & 301.39 & 301.39 & 301.38 & 301.38 & 301.37 & 301.37 & 301.36 & 301.36 & 301.35 & 301.35 & 301.35 \\
\hline$T @ F X \# 5$ & 303.46 & 303.45 & 303.45 & 303.44 & 303.44 & 303.43 & 303.43 & 303.43 & 303.42 & 303.42 & 303.41 & 303.41 & 303.40 & 303.40 & 303.39 & 303.39 & 303.39 \\
\hline$T @ F X \# 6$ & 305.53 & 305.52 & 305.52 & 305.51 & 305.51 & 305.50 & 305.50 & 305.49 & 305.49 & 305.49 & 305.48 & 305.48 & 305.47 & 305.47 & 305.46 & 305.46 & 305.45 \\
\hline$T @ F X \# 7$ & 307.62 & 307.62 & 307.61 & 307.61 & 307.60 & 307.60 & 307.60 & 307.59 & 307.59 & 307.58 & 307.58 & 307.57 & 307.57 & 307.56 & 307.56 & 307.55 & 307.55 \\
\hline Wall $x$-coord & 57.17 & 57.18 & 57.19 & 57.20 & 57.21 & 57.21 & 57.22 & 57.23 & 57.24 & 57.25 & 57.26 & 57.27 & 57.28 & 57.29 & 57.30 & 57.31 & 57.32 \\
\hline Wall $T$ & 308.30 & 308.30 & 308.30 & 308.32 & 308.35 & 308.37 & 308.37 & 308.37 & 308.35 & 308.35 & 308.35 & 308.35 & 308.36 & 308.36 & 308.36 & 308.36 & 308.37 \\
\hline
\end{tabular}

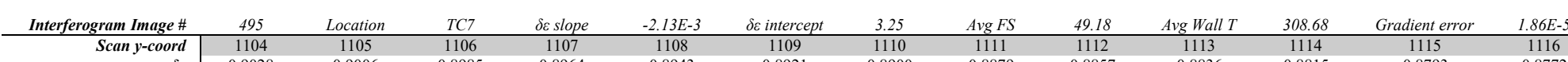

\begin{tabular}{|c|c|c|c|c|c|c|c|c|c|c|c|c|c|c|c|c|c|}
\hline Scan y-coord & 1104 & 1105 & 1106 & 1107 & 1108 & 1109 & 1110 & 1111 & 1112 & 1113 & 1114 & 1115 & 1116 & 1117 & 1118 & 1119 & 1120 \\
\hline & 0.9028 & 0.9006 & 0.8985 & 0.8964 & 0.8943 & 0.8921 & 0.8900 & 0.8879 & 0.8857 & 0.8836 & 0.8815 & 0.8793 & 0.8772 & 0.8751 & 0.8729 & 0.8708 & 0.8687 \\
\hline Scan $x$-coord & 1845 & 1845 & 1845 & 1845 & 1845 & 1845 & 1845 & 1845 & 1845 & 1845 & 1845 & 1845 & 1845 & 1845 & 1845 & 1845 & 1845 \\
\hline$F X \# 1$ & 376.24 & 376.69 & 377.10 & 377.55 & 377.96 & 378.34 & 378.90 & 379.62 & 379.67 & 379.80 & 380.35 & 380.13 & 380.34 & 380,60 & 380.41 & 380.26 & 380.16 \\
\hline
\end{tabular}

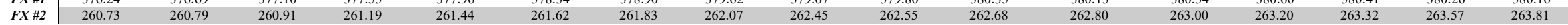




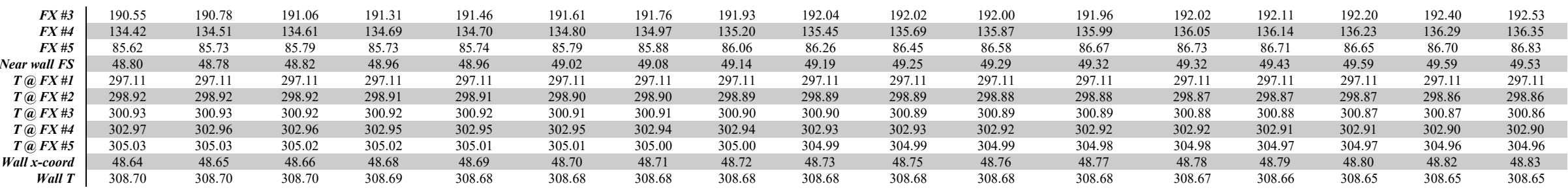

\section{B.40 Tabulated Data for Interferogram 496}

\begin{tabular}{|c|c|c|c|c|c|c|c|c|c|c|c|c|c|c|c|c|c|}
\hline Interferogram Image \# & 496 & Location & $T C 2$ & $\delta \varepsilon$ slope & $-2.59 E-3$ & $\delta \varepsilon$ intercept & 13.98 & $A v g F S$ & 0.00 & Avg Wall T & 305.55 & Gradient error & $-6.94 E-5$ & & & & \\
\hline Scan y-coord & 5208 & 5209 & 5210 & 5211 & 5212 & 5213 & 5214 & 5215 & 5216 & 5217 & 5218 & 5219 & 5220 & 5221 & 5222 & 5223 & 5224 \\
\hline & 0.4686 & 0.4660 & 0.4634 & 0.4608 & 0.4582 & 0.4556 & 0.4530 & 0.4504 & 0.4478 & 0.4452 & 0.4426 & 0.4400 & 0.4374 & 0.4348 & 0.4322 & 0.4297 & 0.4271 \\
\hline Scan $x$-coord & 747 & 747 & 747 & 747 & 747 & 747 & 747 & 747 & 747 & 747 & 747 & 747 & 747 & 747 & 747 & 747 & 747 \\
\hline$F X \# 1$ & 368.41 & 369.02 & 370.00 & 370.99 & 371.91 & 372.79 & 352.43 & 374.38 & 374.85 & 375.33 & 375.56 & 376.17 & 376.68 & 377.36 & 370.48 & 372.84 & 379.39 \\
\hline$F X \# 2$ & 239.20 & 239.48 & 239.64 & 239.78 & 239.90 & 239.90 & 239.96 & 240.14 & 240.29 & 240.45 & 240.65 & 240.83 & 241.10 & 241.33 & 241.54 & 241.82 & 241.98 \\
\hline$F X \# 3$ & 176.42 & 176.58 & 176.67 & 176.76 & 176.85 & 176.93 & 176.99 & 177.04 & 177.15 & 177.28 & 177.45 & 177.60 & 177.70 & 177.79 & 177.89 & 178.05 & 178.22 \\
\hline$F X \# 5$ & 0.00 & 0.00 & 0.00 & 0.00 & 0.00 & 0.00 & 0.00 & 0.00 & 0.00 & 0.00 & 0.00 & 0.00 & 0.00 & 0.00 & 0.00 & 0.00 & 0.00 \\
\hline$F X \# 6$ & 0.00 & 0.00 & 0.00 & 0.00 & 0.00 & 0.00 & 0.00 & 0.00 & 0.00 & 0.00 & 0.00 & 0.00 & 0.00 & 0.00 & 0.00 & 0.00 & 0.00 \\
\hline Near wall FS & 0.00 & 0.00 & 0.00 & 0.00 & 0.00 & 0.00 & 0.00 & 0.00 & 0.00 & 0.00 & 0.00 & 0.00 & 0.00 & 0.00 & 0.00 & 0.00 & 0.00 \\
\hline$T(F X \# 1$ & 297.11 & 297.11 & 297.11 & 297.11 & 297.11 & 297.11 & 297.11 & 297.11 & 297.11 & 297.11 & 297.11 & 297.11 & 297.11 & 297.11 & 297.11 & 297.11 & 297.11 \\
\hline$T @ F X \# 2$ & 298.01 & 298.00 & 298.00 & 297.99 & 297.99 & 297.98 & 297.98 & 297.97 & 297.97 & 297.96 & 297.96 & 297.95 & 297.95 & 297.94 & 297.94 & 297.93 & 297.93 \\
\hline$T @ F X \# 3$ & 299.99 & 299.99 & 299.98 & 299.98 & 299.97 & 299.97 & 299.96 & 299.96 & 299.95 & 299.95 & 299.94 & 299.94 & 299.93 & 299.93 & 299.92 & 299.91 & 299.91 \\
\hline$T @ F X \# 5$ & 304.05 & 304.05 & 304.04 & 304.04 & 304.03 & 304.03 & 304.02 & 304.02 & 304.01 & 304.01 & 304.00 & 304.00 & 303.99 & 303.98 & 303.98 & 303.97 & 303.97 \\
\hline$T @ F X \# 6$ & 306.12 & 306.12 & 306.11 & 306.10 & 306.10 & 306.09 & 306.09 & 306.08 & 306.08 & 306.07 & 306.07 & 306.06 & 306.06 & 306.05 & 306.04 & 306.04 & 306.03 \\
\hline Wall $x$-coord & 95.92 & 95.92 & 95.92 & 95.92 & 95.92 & 95.92 & 95.92 & 95.92 & 95.92 & 95.92 & 95.92 & 95.92 & 95.92 & 95.92 & 95.92 & 95.92 & 95.92 \\
\hline Wall T & 305.51 & 305.51 & 305.52 & 305.53 & 305.54 & 305.55 & 305.56 & 305.57 & 305.57 & 305.58 & 305.57 & 305.57 & 305.57 & 305.57 & 305.57 & 305.55 & 305.55 \\
\hline Interferogram Image \# & 496 & Location & $T C 3$ & $\delta \varepsilon$ slope & $-2.56 E-3$ & $\delta \varepsilon$ intercept & 11.84 & $\operatorname{Avg} F S$ & 55.88 & Avg Wall T & 306.66 & Gradient error & $-6.94 E-5$ & & & & \\
\hline Scan y-coord & 4394 & 4395 & 4396 & 4397 & 4398 & 4399 & 4400 & 4401 & 4402 & 4403 & 4404 & 4405 & 4406 & 4407 & 4408 & 4409 & 4410 \\
\hline & 0.5831 & 0.5805 & 0.5780 & 0.5754 & 0.5728 & 0.5703 & 0.5677 & 0.5652 & 0.5626 & 0.5600 & 0.5575 & 0.5549 & 0.5523 & 0.5498 & 0.5472 & 0.5447 & 0.5421 \\
\hline Scan $x$-coord & 849 & 849 & 849 & 849 & 849 & 849 & 849 & 849 & 849 & 849 & 849 & 849 & 849 & 849 & 849 & 849 & 849 \\
\hline$F X \# I$ & 376.09 & 376.62 & 377.21 & 377.75 & 378.28 & 378.50 & 379.16 & 379.78 & 380.23 & 380.64 & 381.24 & 381.60 & 381.92 & 382.23 & 382.64 & 382.98 & 383.28 \\
\hline$F X \# 2$ & 259.89 & 259.87 & 259.87 & 259.89 & 259.92 & 260.12 & 260.28 & 260.55 & 260.80 & 260.96 & 261.17 & 261.36 & 261.53 & 261.77 & 262.02 & 262.32 & 262.67 \\
\hline$F X \# 3$ & 195.33 & 195.51 & 195.69 & 195.80 & 195.96 & 196.13 & 196.22 & 196.35 & 196.51 & 196.70 & 196.89 & 197.06 & 197.25 & 197.46 & 197.65 & 197.89 & 198.11 \\
\hline$F X \# 4$ & 146.30 & 146.39 & 146.17 & 146.19 & 146.49 & 146.53 & 146.54 & 146.60 & 146.74 & 146.85 & 146.95 & 147.06 & 147.18 & 147.41 & 147.55 & 147.69 & 147.81 \\
\hline$T @ F X \# 1$ & 297.11 & 297.11 & 297.11 & 297.11 & 297.11 & 297.11 & 297.11 & 297.11 & 297.11 & 297.11 & 297.11 & 297.11 & 297.11 & 297.11 & 297.11 & 297.11 & 297.11 \\
\hline$T @ F X \# 2$ & 298.23 & 298.22 & 298.22 & 298.21 & 298.21 & 298.20 & 298.20 & 298.19 & 298.19 & 298.18 & 298.18 & 298.17 & 298.17 & 298.16 & 298.16 & 298.15 & 298.15 \\
\hline$T @ F X \# 3$ & 300.22 & 300.21 & 300.21 & 300.20 & 300.20 & 300.19 & 300.19 & 300.18 & 300.18 & 300.17 & 300.17 & 300.16 & 300.16 & 300.15 & 300.15 & 300.14 & 300.13 \\
\hline$T @ F X \# 4$ & 302.24 & 302.23 & 302.23 & 302.22 & 302.22 & 302.21 & 302.21 & 302.20 & 302.19 & 302.19 & 302.18 & 302.18 & 302.17 & 302.17 & 302.16 & 302.16 & 302.15 \\
\hline$T(F X \# 5$ & 304.28 & 304.28 & 304.27 & 304.27 & 304.26 & 304.26 & 304.25 & 304.25 & 304.24 & 304.24 & 304.23 & 304.23 & 304.22 & 304.22 & 304.21 & 304.20 & 304.20 \\
\hline Wall $x$-coord & 94.96 & 94.96 & 94.96 & 94.97 & 94.97 & 94.97 & 94.97 & 94.97 & 94.98 & 94.98 & 94.98 & 94.98 & 94.99 & 94.99 & 94.99 & 94.99 & 94.99 \\
\hline Wall $T$ & 306.68 & 306.68 & 306.39 & 306.38 & 306.69 & 306.70 & 306.71 & 306.71 & 306.71 & 306.71 & 306.71 & 306.71 & 306.71 & 306.70 & 306.69 & 306.68 & 306.68 \\
\hline Interferogram Image \# & 496 & Location & $T C 4$ & $\delta \varepsilon$ slope & $-2.48 E-3$ & $\delta \varepsilon$ intercept & 9.51 & $\operatorname{Avg} F S$ & 45.80 & Avg Wall T & 307.26 & Gradient error & $-6.94 E-5$ & & & & \\
\hline Scan y-coord & 3579 & 3580 & 3581 & 3582 & 3583 & 3584 & 3585 & 3586 & 3587 & 3588 & 3589 & 3590 & 3591 & 3592 & 3593 & 3594 & 3595 \\
\hline & 0.6326 & 0.6302 & 0.6277 & 0.6252 & 0.6227 & 0.6202 & 0.6178 & 0.6153 & 0.6128 & 0.6103 & 0.6078 & 0.6053 & 0.6029 & 0.6004 & 0.5979 & 0.5954 & 0.5929 \\
\hline Scan $x$-coord & 1536 & 1536 & 1536 & 1536 & 1536 & 1536 & 1536 & 1536 & 1536 & 1536 & 1536 & 1536 & 1536 & 1536 & 1536 & 1536 & 1536 \\
\hline$F X \# 1$ & 395.19 & 395.56 & 396.30 & 396.97 & 397.48 & 397.90 & 398.38 & 398.84 & 399.73 & 399.71 & 399.92 & 400.56 & 401.15 & 401.86 & 402.40 & 403.07 & 403.41 \\
\hline$F X \# 2$ & 275.90 & 276.11 & 276.29 & 276.56 & 276.89 & 277.14 & 277.42 & 277.67 & 277.87 & 277.97 & 278.06 & 278.28 & 278.46 & 278.65 & 278.87 & 279.06 & 279.16 \\
\hline$F X \# 3$ & 208.44 & 208.53 & 208.75 & 208.99 & 209.30 & 209.54 & 209.77 & 209.89 & 209.96 & 210.01 & 210.08 & 210.15 & 210.23 & 210.32 & 210.51 & 210.64 & 210.71 \\
\hline$F X \# 4$ & 156.95 & 156.91 & 156.93 & 157.04 & 157.21 & 157.41 & 157.50 & 157.52 & 157.53 & 157.57 & 157.65 & 157.81 & 157.99 & 158.12 & 158.21 & 158.38 & 158.50 \\
\hline$F X \# 5$ & 111.09 & 111.26 & 111.41 & 111.56 & 111.65 & 111.66 & 111.64 & 111.56 & 111.57 & 111.67 & 111.84 & 112.03 & 112.18 & 112.26 & 112.33 & 112.41 & 112.57 \\
\hline Near wall FS & 45.87 & 45.66 & 45.53 & 45.48 & 45.56 & 45.75 & 45.86 & 45.96 & 45.96 & 45.90 & 45.81 & 45.78 & 45.80 & 45.86 & 45.88 & 45.96 & 45.93 \\
\hline$T(F X \# 1$ & 297.11 & 297.11 & 297.11 & 297.11 & 297.11 & 297.11 & 297.11 & 297.11 & 297.11 & 297.11 & 297.11 & 297.11 & 297.11 & 297.11 & 297.11 & 297.11 & 297.11 \\
\hline$T @ F X \# 2$ & 298.28 & 298.28 & 298.27 & 298.27 & 298.26 & 298.26 & 298.25 & 298.25 & 298.24 & 298.24 & 298.23 & 298.23 & 298.22 & 298.22 & 298.21 & 298.21 & 298.20 \\
\hline$T @ F X \# 3$ & 300.27 & 300.26 & 300.26 & 300.25 & 300.25 & 300.24 & 300.24 & 300.23 & 300.23 & 300.22 & 300.22 & 300.21 & 300.21 & 300.20 & 300.20 & 300.20 & 300.19 \\
\hline$T(F X \# 4$ & 302.29 & 302.28 & 302.28 & 302.27 & 302.27 & 302.26 & 302.26 & 302.25 & 302.25 & 302.24 & 302.24 & 302.23 & 302.23 & 302.22 & 302.22 & 302.21 & 302.21 \\
\hline$T(\boldsymbol{F} X \# 5$ & 304.34 & 304.33 & 304.33 & 304.32 & 304.32 & 304.31 & 304.31 & 304.30 & 304.30 & 304.29 & 304.29 & 304.28 & 304.28 & 304.27 & 304.27 & 304.26 & 304.26 \\
\hline Wall $x$-coord & 92.28 & 92.29 & 92.29 & 92.30 & 92.30 & 92.31 & 92.31 & 92.31 & 92.32 & 92.32 & 92.33 & 92.33 & 92.34 & 92.34 & 92.34 & 92.35 & 92.35 \\
\hline Wall $\mathrm{T}$ & 307.27 & 307.27 & 307.28 & 307.28 & 307.28 & 307.27 & 307.26 & 307.25 & 307.24 & 307.24 & 307.25 & 307.25 & 307.25 & 307.25 & 307.25 & 307.24 & 307.24 \\
\hline Interferogram Image \# & 496 & Location & $T C 5$ & $\delta \varepsilon$ slope & $-2.40 E-3$ & $\delta \varepsilon$ intercept & 7.13 & $\operatorname{Avg} F S$ & 49.48 & Avg Wall $T$ & 307.52 & Gradient error & $-6.94 E-5$ & & & & \\
\hline
\end{tabular}




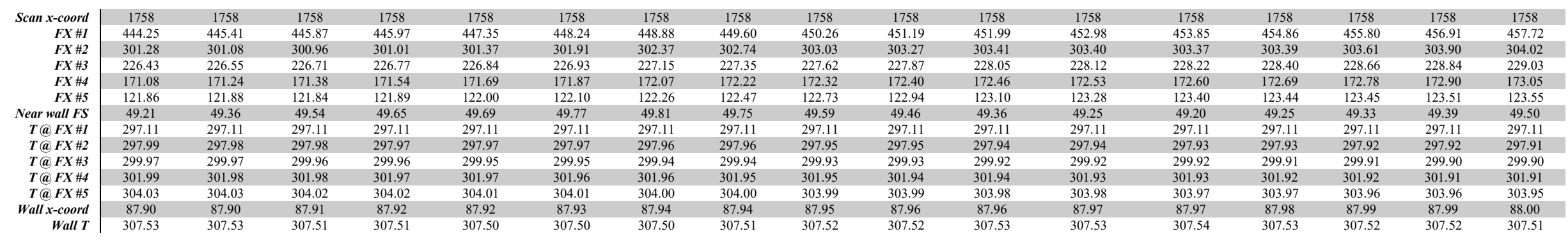

\begin{tabular}{|c|c|c|c|c|c|c|c|c|c|c|c|c|c|c|c|c|c|}
\hline Interferogram Image \# & 490 & Location & 100 & oE slope & $-2.111 \mathrm{E}-3$ & oE intercept & $4.4 \mathrm{~J}$ & Avg $F \mathrm{FS}$ & 09.40 & Avg wall 1 & 308.53 & Gradient error & $-0.94 \mathrm{E}-3$ & & & & \\
\hline Scan $y$-coord & 1950 & 1951 & 1952 & 1953 & 1954 & $\begin{array}{l}1955 \\
03358\end{array}$ & 1956 & $\begin{array}{l}1957 \\
03315\end{array}$ & 1958 & 1959 & $\begin{array}{l}1960 \\
03252\end{array}$ & $\begin{array}{c}1961 \\
03231\end{array}$ & 1962 & $\begin{array}{l}1963 \\
03189\end{array}$ & 1964 & 1965 & 1966 \\
\hline & 0.3463 & 0.3442 & 0.3421 & 0.3400 & 0.3379 & 0.3358 & 0.3336 & 0.3315 & 0.3294 & 0.3273 & 0.3252 & 0.3231 & 0.3210 & 0.3189 & 0.3168 & 0.3147 & 0.3126 \\
\hline Scan $x$-coord & 1614 & 1614 & 1614 & 1614 & 1614 & 1614 & 1614 & 1614 & 1614 & 1614 & 1614 & 1614 & 1614 & 1614 & 1614 & 1614 & 1614 \\
\hline$F X \# 1$ & 549.38 & 547.50 & 548.31 & 550.92 & 553.51 & 552.85 & 554.85 & 556.16 & 557.07 & 558.16 & 559.09 & 560.18 & 562.96 & 563.15 & 563.65 & 562.40 & 563.95 \\
\hline$F X \# 2$ & 333.63 & 333.83 & 334.15 & 334.42 & 334.56 & 334.59 & 334.76 & 334.94 & 335.08 & 335.22 & 335.23 & 335.32 & 335.42 & 335.50 & 335.58 & 556.62 & 335.77 \\
\hline$F X \# 3$ & 248.75 & 248.75 & 248.76 & 248.79 & 248.83 & 248.83 & 248.80 & 248.80 & 248.81 & 248.92 & 249.09 & 249.31 & 249.63 & 250.07 & 250.42 & 335.66 & 250.79 \\
\hline$F X \# 4$ & 188.78 & 188.88 & 189.05 & 189.31 & 189.58 & 189.82 & 189.97 & 190.08 & $\begin{array}{l}240.01 \\
190.21\end{array}$ & 190.34 & 190.43 & 190.64 & 190.72 & 190.78 & 190.86 & 250.68 & 191.06 \\
\hline$F X \# 5$ & 138.62 & 138.58 & 138.57 & 138.66 & 138.72 & 138.86 & 138.96 & 139.13 & 139.32 & 139.57 & 139.70 & 139.78 & 139.78 & 139.83 & 139.91 & 190.93 & 140.12 \\
\hline$F X \# 6$ & 91.97 & 91.77 & 91.50 & 91.30 & 91.20 & 91.47 & 91.80 & 92.13 & 92.34 & 92.38 & 92.41 & 92.46 & 92.58 & 92.78 & 93.11 & 139.99 & 93.24 \\
\hline$F X \# 7$ & 0.00 & 0.00 & 0.00 & 0.00 & 0.00 & 0.00 & 0.00 & 0.00 & 0.00 & 0.00 & 0.00 & 0.00 & 0.00 & 0.00 & 0.00 & 93.26 & 0.00 \\
\hline Near wall FS & 91.97 & 91.77 & 91.50 & 91.30 & 91.20 & 91.47 & 91.80 & 92.13 & 92.34 & 92.38 & 92.41 & 92.46 & 92.58 & 92.78 & 93.11 & 46.72 & 93.24 \\
\hline$T @ F X \# 1$ & 297.11 & 297.11 & 297.11 & 297.11 & 297.11 & 297.11 & 297.11 & 297.11 & 297.11 & 297.11 & 297.11 & 297.11 & 297.11 & 297.11 & 297.11 & 297.11 & 297.11 \\
\hline$T @ F X \# 2$ & 297.72 & 297.71 & 297.71 & 297.71 & 297.70 & 297.70 & 297.69 & 297.69 & 297.69 & 297.68 & 297.68 & 297.67 & 297.67 & 297.66 & 297.66 & 297.66 & 297.65 \\
\hline$T(F X \# 3$ & 299.69 & 299.69 & 299.68 & 299.68 & 299.68 & 299.67 & 299.67 & 299.66 & 299.66 & 299.66 & 299.65 & 299.65 & 299.64 & 299.64 & 299.63 & 299.65 & 299.63 \\
\hline 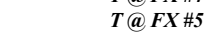 & 303.74 & 303.74 & 303.74 & 303.73 & 303.73 & 303.72 & 303.72 & 303.71 & 303.71 & 303.70 & 303.70 & 303.70 & 303.69 & 303.69 & 303.68 & 303.68 & 303.67 \\
\hline$T @ F X \# 6$ & 305.81 & 305.81 & 305.80 & 305.80 & 305.79 & 305.79 & 305.79 & 305.78 & 305.78 & 305.77 & 305.77 & 305.76 & 305.76 & 305.75 & 305.75 & 305.75 & 305.74 \\
\hline $\boldsymbol{T} @ \boldsymbol{F} \boldsymbol{X} \# 7$ & 307.91 & 307.90 & 307.90 & 307.90 & 307.89 & 307.89 & 307.88 & 307.88 & 307.87 & 307.87 & 307.86 & 307.86 & 307.86 & 307.85 & 307.85 & 307.85 & 307.84 \\
\hline Wall $x$-coord & 81.79 & 81.80 & 81.81 & 81.81 & 81.82 & 81.83 & 81.84 & 81.85 & 81.86 & 81.87 & 81.87 & 81.88 & 81.89 & 81.90 & 81.91 & 81.92 & 81.93 \\
\hline Wall T & 308.37 & 308.35 & 308.33 & 308.32 & 308.30 & 308.31 & 308.32 & 308.34 & 308.34 & 308.34 & 308.33 & 308.33 & 308.33 & 308.34 & 308.35 & 308.35 & 308.34 \\
\hline
\end{tabular}

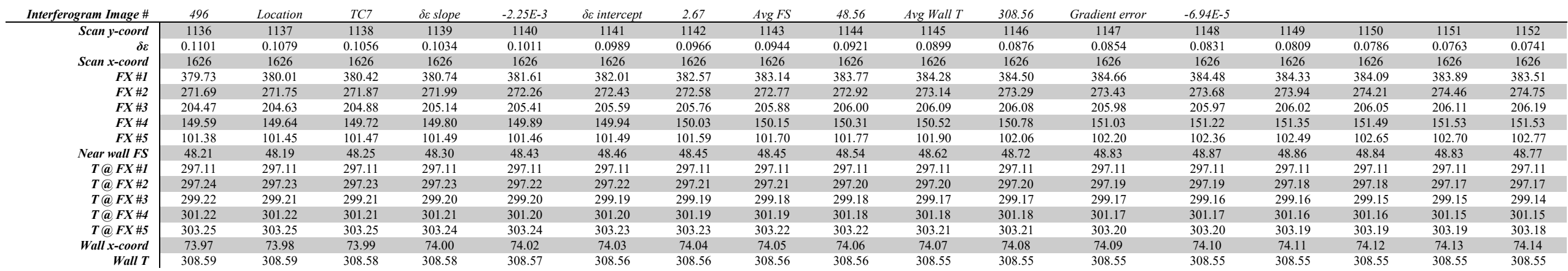

\section{B.41 Tabulated Data for Interferogram 497}

\begin{tabular}{|c|c|c|c|c|c|c|c|c|c|c|c|c|c|c|c|c|c|}
\hline gram Image \# & 497 & Location & $T C 2$ & $\delta \varepsilon$ slope & $-2.60 E-3$ & $\delta \varepsilon$ intercept & 13.76 & $\operatorname{Avg} F S$ & 120.58 & Avg Wall $T$ & 305.27 & Gradient error & $-1.38 E-5$ & & & & \\
\hline Scan y-coord & 5201 & 5202 & 5203 & 5204 & 5205 & 5206 & 5207 & 5208 & 5209 & 5210 & 5211 & 5212 & 5213 & 5214 & 5215 & 5216 & 5217 \\
\hline$\delta \varepsilon$ & 0.2319 & 0.2293 & 0.2267 & 0.2241 & 0.2215 & 0.2189 & 0.2163 & 0.2137 & 0.2111 & 0.2085 & 0.2059 & 0.2033 & 0.2007 & 0.1981 & 0.1955 & 0.1929 & 0.1903 \\
\hline Scan $x$-coord & 672 & 672 & 672 & 672 & 672 & 672 & 672 & 672 & 672 & 672 & 672 & 672 & 672 & 672 & 672 & 672 & 672 \\
\hline$F X \# 1$ & 391.16 & 391.80 & 392.45 & 392.78 & 393.05 & 393.99 & 395.08 & 395.81 & 396.80 & 397.96 & 397.08 & 400.21 & 401.34 & 402.81 & 406.30 & 408.37 & 409.92 \\
\hline$F X \# 2$ & 235.43 & 235.79 & 236.05 & 236.23 & 236.39 & 236.40 & 236.39 & 236.48 & 236.50 & 236.67 & 236.88 & 237.11 & 237.44 & 237.72 & 238.07 & 238.37 & 238.68 \\
\hline$F X \# 3$ & 167.23 & 167.36 & 167.48 & 167.60 & 167.72 & 167.81 & 167.97 & 168.01 & 168.04 & 168.14 & 168.25 & 168.41 & 168.52 & 168.66 & 168.80 & 169.04 & 169.29 \\
\hline$F X \# 4$ & 118.45 & 118.70 & 118.97 & 119.22 & 119.48 & 119.73 & 119.93 & 120.19 & 120.53 & 120.85 & 121.16 & 121.46 & 121.78 & 122.01 & 122.22 & 122.48 & 122.72 \\
\hline$F X \# 5$ & 0.00 & 0.00 & 0.00 & 0.00 & 0.00 & 0.00 & 0.00 & 0.00 & 0.00 & 0.00 & 0.00 & 0.00 & 0.00 & 0.00 & 0.00 & 0.00 & 0.00 \\
\hline Near wall FS & 118.45 & 118.70 & 118.97 & 119.22 & 119.48 & 119.73 & 119.93 & 120.19 & 120.53 & 120.85 & 121.16 & 121.46 & 121.78 & 122.01 & 122.22 & 122.48 & 122.72 \\
\hline$T @ F X \# 1$ & 297.12 & 297.12 & 297.12 & 297.12 & 297.12 & 297.12 & 297.12 & 297.12 & 297.12 & 297.12 & 297.12 & 297.12 & 297.12 & 297.12 & 297.12 & 297.12 & 297.12 \\
\hline$T$ a $F X \# 2$ & 297.57 & 297.57 & 297.56 & 297.56 & 297.55 & 297.55 & 297.54 & 297.54 & 297.53 & 297.53 & 297.52 & 297.52 & 297.51 & 297.51 & 297.50 & 297.50 & 297.49 \\
\hline$T @ F X \# 3$ & 299.56 & 299.56 & 299.55 & 299.54 & 299.54 & 299.53 & 299.53 & 299.52 & 299.52 & 299.51 & 299.51 & 299.50 & 299.50 & 299.49 & 299.49 & 299.48 & 299.48 \\
\hline$T @ F X \# 4$ & 301.57 & 301.57 & 301.56 & 301.56 & 301.55 & 301.55 & 301.54 & 301.54 & 301.53 & 301.53 & 301.52 & 301.52 & 301.51 & 301.51 & 301.50 & 301.50 & 301.49 \\
\hline T@FX\#5 & 303.62 & 303.61 & 303.61 & 303.60 & 303.59 & 303.59 & 303.58 & 303.58 & 303.57 & 303.57 & 303.56 & 303.56 & 303.55 & 303.55 & 303.54 & 303.54 & 303.53 \\
\hline Wall $x$-coord & 81.16 & 81.16 & 81.16 & 81.16 & 81.16 & 81.16 & 81.16 & 81.15 & 81.15 & 81.15 & 81.15 & 81.15 & 81.15 & 81.15 & 81.15 & 81.15 & 81.15 \\
\hline Wall $T$ & 305.18 & 305.19 & 305.20 & 305.21 & 305.22 & 305.23 & 305.23 & 305.24 & 305.26 & 305.28 & 305.30 & 305.31 & 305.33 & 305.33 & 305.34 & 305.35 & 305.35 \\
\hline
\end{tabular}




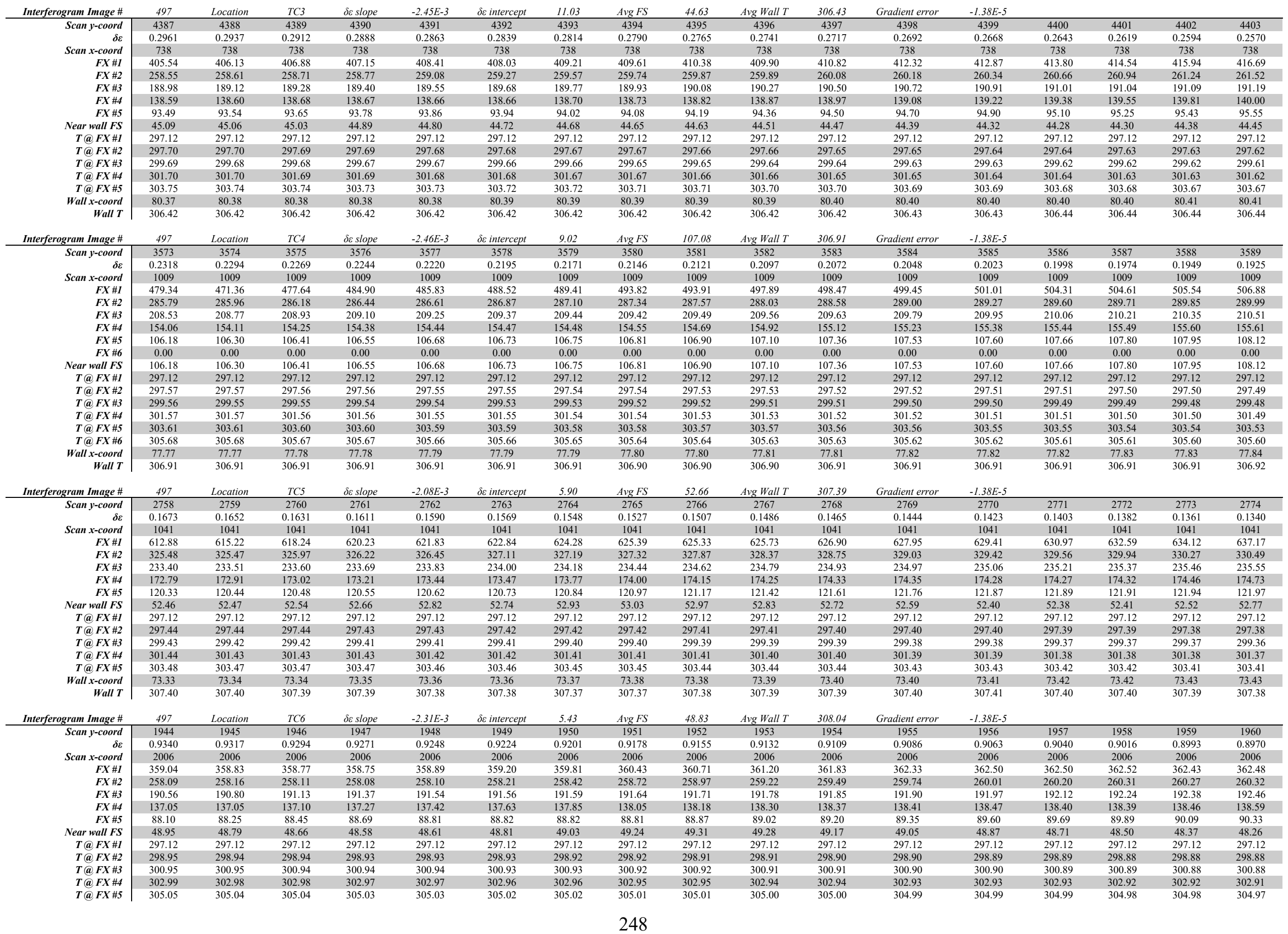




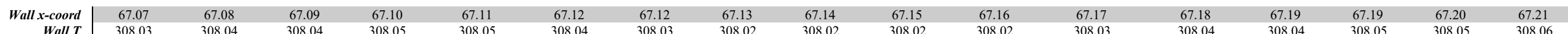

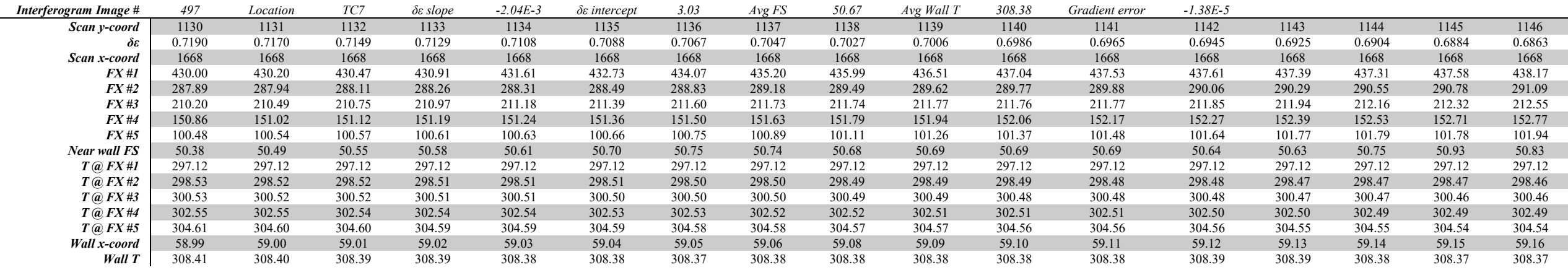

\section{B.42 Tabulated Data for Interferogram 498}

\begin{tabular}{|c|c|c|c|c|c|c|c|c|c|c|c|c|c|c|c|c|c|}
\hline Interferogram Image \# & 498 & Location & $T C 2$ & $\delta \varepsilon$ slope & $-2.48 E-3$ & $\delta \varepsilon$ intercept & 13.06 & $\operatorname{Avg~FS}$ & 47.20 & Avg Wall $T$ & 305.23 & Gradient error & $-2.18 E-5$ & & & & \\
\hline Scan $y$-coord & 5166 & 5167 & 5168 & 5169 & 5170 & 5171 & 5172 & 5173 & 5174 & 5175 & 5176 & 5177 & 5178 & 5179 & 5180 & 5181 & 5182 \\
\hline & 0.2266 & 0.2241 & 0.2217 & 0.2192 & 0.2167 & 0.2142 & 0.2117 & 0.2092 & 0.2067 & 0.2043 & 0.2018 & 0.1993 & 0.1968 & 0.1943 & 0.1918 & 0.1894 & 0.1869 \\
\hline Scan $x$-coord & 1131 & 1131 & 1131 & 1131 & 1131 & 1131 & 1131 & 1131 & 1131 & 1131 & 1131 & 1131 & 1131 & 1131 & 1131 & 1131 & 1131 \\
\hline$F X \# I$ & 507.17 & 499.16 & 508.04 & 507.99 & 507.37 & 506.98 & 507.14 & 503.66 & 498.96 & 493.83 & 494.89 & 496.30 & 515.72 & 504.94 & 520.01 & 519.04 & 504.42 \\
\hline$F X \# 2$ & 489.30 & 266.13 & 474.16 & 491.01 & 491.97 & 472.07 & 490.30 & 471.03 & 471.12 & 268.56 & 268.82 & 269.08 & 495.38 & 269.57 & 503.23 & 508.75 & 485.62 \\
\hline$F X \# 3$ & 462.06 & 190.20 & 266.71 & 466.46 & 466.64 & 267.81 & 469.19 & 268.30 & 268.47 & 190.86 & 191.03 & 191.12 & 477.67 & 191.51 & 493.59 & 483.49 & 270.67 \\
\hline$F X \# 5$ & 266.08 & 0.00 & 138.50 & 190.07 & 190.09 & 139.22 & 190.36 & 139.64 & 139.85 & 0.00 & 0.00 & 0.00 & 191.31 & 0.00 & 269.97 & 191.90 & 142.09 \\
\hline$F X \# 6$ & 190.18 & 0.00 & 0.00 & 138.76 & 138.99 & 0.00 & 139.44 & 0.00 & 0.00 & 0.00 & 0.00 & 0.00 & 141.07 & 0.00 & 191.74 & 141.83 & 0.00 \\
\hline$F \boldsymbol{F} \# 7$ & 138.07 & 0.00 & 0.00 & 0.00 & 0.00 & 0.00 & 0.00 & 0.00 & 0.00 & 0.00 & 0.00 & 0.00 & 0.00 & 0.00 & 141.57 & 0.00 & 0.00 \\
\hline Near wall FS & 52.10 & 0.00 & 0.00 & 138.76 & 138.99 & 0.00 & 139.44 & 0.00 & 0.00 & 0.00 & 0.00 & 0.00 & 141.07 & 0.00 & 50.17 & 141.83 & 0.00 \\
\hline$T @ F X \# 1$ & 297.15 & 297.15 & 297.15 & 297.15 & 297.15 & 297.15 & 297.15 & 297.15 & 297.15 & 297.15 & 297.15 & 297.15 & 297.15 & 297.15 & 297.15 & 297.15 & 297.15 \\
\hline$T$ (a) $F X \# 2$ & 297.58 & 297.58 & 297.57 & 297.57 & 297.56 & 297.56 & 297.55 & 297.55 & 297.54 & 297.54 & 297.53 & 297.53 & 297.52 & 297.52 & 297.51 & 297.51 & 297.50 \\
\hline$T$ (a) $F X \# 4$ & 301.58 & 301.57 & 301.57 & 301.57 & 301.56 & 301.55 & 301.55 & 301.54 & 301.54 & 301.53 & 301.53 & 301.52 & 301.52 & 301.51 & 301.51 & 301.51 & 301.50 \\
\hline$T(a) F \#$ & 303.63 & 303.61 & 303.61 & 303.61 & 303.60 & 303.59 & 303.59 & 303.58 & 303.58 & 303.57 & 303.57 & 303.56 & 303.56 & 303.55 & 303.55 & 303.55 & 303.54 \\
\hline$T(F X \# 6$ & 305.69 & 305.68 & 305.68 & 305.67 & 305.67 & 305.66 & 305.66 & 305.65 & 305.65 & 305.64 & 305.63 & 305.63 & 305.63 & 305.62 & 305.62 & 305.61 & 305.61 \\
\hline$T @ \boldsymbol{T X} \# \mathbf{T}$ & 307.79 & 307.78 & 307.77 & 307.77 & 307.77 & 307.76 & 307.76 & 307.75 & 307.74 & 307.74 & 307.73 & 307.73 & 307.72 & 307.72 & 307.72 & 307.71 & 307.70 \\
\hline Wall $x$-coord & 98.78 & 98.78 & 98.79 & 98.79 & 98.79 & 98.79 & 98.79 & 98.79 & 98.79 & 98.79 & 98.79 & 98.79 & 98.79 & 98.79 & 98.79 & 98.79 & 98.79 \\
\hline Wall $T$ & 305.17 & 305.17 & 305.18 & 305.19 & 305.20 & 305.21 & 305.22 & 305.22 & 305.23 & 305.23 & 305.25 & 305.27 & 305.27 & 305.28 & 305.29 & 305.30 & 305.31 \\
\hline Interferogram Image \# & 498 & Location & $T C 3$ & $\delta \varepsilon$ slope & $-2.43 E-3$ & $\delta \varepsilon$ intercept & 10.86 & $\operatorname{Avg} F S$ & 109.00 & Avg Wall $T$ & 306.40 & Gradient error & $-2.18 E-5$ & & & & \\
\hline Scan y-coord & 4352 & 4353 & 4354 & 4355 & 4356 & 4357 & 4358 & 4359 & 4360 & 4361 & 4362 & 4363 & 4364 & 4365 & 4366 & 4367 & 4368 \\
\hline$\delta \varepsilon$ & 0.2627 & 0.2603 & 0.2578 & 0.2554 & 0.2529 & 0.2505 & 0.2481 & 0.2456 & 0.2432 & 0.2408 & 0.2383 & 0.2359 & 0.2335 & 0.2310 & 0.2286 & 0.2262 & 0.2237 \\
\hline Scan $x$-coord & 1108 & 1108 & 1108 & 1108 & 1108 & 1108 & 1108 & 1108 & 1108 & 1108 & 1108 & 1108 & 1108 & 1108 & 1108 & 1108 & 1108 \\
\hline$F X \# I$ & 481.55 & 482.24 & 483.60 & 484.22 & 484.97 & 485.76 & 491.86 & 488.18 & 489.10 & 489.65 & 491.26 & 503.90 & 502.76 & 504.03 & 505.10 & 505.72 & 506.22 \\
\hline$F X \# 2$ & 290.23 & 290.33 & 290.48 & 290.63 & 290.82 & 291.06 & 291.32 & 291.45 & 291.57 & 291.75 & 292.00 & 478.53 & 292.60 & 292.61 & 292.82 & 293.03 & 293.29 \\
\hline$F X \# 5$ & $\begin{array}{l}10.00 \\
112.15\end{array}$ & 112.24 & 112.29 & 112.36 & 112.40 & 112.50 & 112.56 & 112.71 & 112.84 & 112.95 & 113.09 & 160.06 & 113.32 & 113.45 & 113.63 & 113.78 & 113.92 \\
\hline$F X \# 6$ & 0.00 & 0.00 & 0.00 & 0.00 & 0.00 & 0.00 & 0.00 & 0.00 & 0.00 & 0.00 & 0.00 & 113.19 & 0.00 & 0.00 & 0.00 & 0.00 & 0.00 \\
\hline Near wall FS & 112.15 & 112.24 & 112.29 & 112.36 & 112.40 & 112.50 & 112.56 & 112.71 & 112.84 & 112.95 & 113.09 & 46.87 & 113.32 & 113.45 & 113.63 & 113.78 & 113.92 \\
\hline$T @ F X \# 1$ & 297.15 & 297.15 & 297.15 & 297.15 & 297.15 & 297.15 & 297.15 & 297.15 & 297.15 & 297.15 & 297.15 & 297.15 & 297.15 & 297.15 & 297.15 & 297.15 & 297.15 \\
\hline$T(a) F \# 2$ & 297.65 & 297.65 & 297.64 & 297.64 & 297.63 & 297.63 & 297.62 & 297.62 & 297.61 & 297.61 & 297.60 & 297.60 & 297.60 & 297.59 & 297.59 & 297.58 & 297.58 \\
\hline$T$ (a) $F X \# 3$ & 299.64 & 299.63 & 299.63 & 299.62 & 299.62 & 299.61 & 299.61 & 299.60 & 299.60 & 299.59 & 299.59 & 299.59 & 299.58 & 299.57 & 299.57 & 299.56 & 299.56 \\
\hline$T(a) F X \# 4$ & 301.65 & 301.65 & 301.64 & 301.64 & 301.63 & 301.63 & 301.62 & 301.62 & 301.61 & 301.61 & 301.60 & 301.60 & 301.59 & 301.59 & 301.58 & 301.58 & 301.57 \\
\hline$T(a) F X$ & 303.69 & 303.69 & 303.68 & 303.68 & 303.67 & 303.67 & 303.66 & 303.66 & 303.65 & 303.65 & 303.64 & 303.64 & 303.63 & 303.63 & 303.62 & 303.62 & 303.61 \\
\hline$T @ F X \# 6$ & 305.76 & 305.76 & 305.75 & 305.75 & 305.74 & 305.74 & 305.73 & 305.73 & 305.72 & 305.72 & 305.71 & 305.71 & 305.70 & 305.70 & 305.69 & 305.69 & 305.68 \\
\hline Wall $x$-coord & 97.67 & 97.67 & 97.67 & 97.67 & 97.68 & 97.68 & 97.68 & 97.68 & 97.69 & 97.69 & 97.69 & 97.69 & 97.70 & 97.70 & 97.70 & 97.70 & 97.71 \\
\hline Wall $T$ & 306.41 & 306.40 & 306.40 & 306.40 & 306.39 & 306.39 & 306.39 & 306.39 & 306.39 & 306.39 & 306.39 & 306.39 & 306.39 & 306.39 & 306.40 & 306.40 & 306.40 \\
\hline Interferogram Image \# & 498 & Location & $T C 4$ & $\delta \varepsilon$ slope & $-2.47 E-3$ & $\delta \varepsilon$ intercept & 8.96 & $A v g F S$ & 123.95 & Avg Wall T & 306.87 & Gradient error & $-2.18 E-5$ & & & & \\
\hline Scan y-coord & 3538 & 3539 & 3540 & 3541 & 3542 & 3543 & 3544 & 3545 & 3546 & 3547 & 3548 & 3549 & 3550 & 3551 & 3552 & 3553 & 3554 \\
\hline$\delta \varepsilon$ & 0.2329 & 0.2304 & 0.2280 & 0.2255 & 0.2230 & 0.2206 & 0.2181 & 0.2156 & 0.2132 & 0.2107 & 0.2082 & 0.2058 & 0.2033 & 0.2008 & 0.1984 & 0.1959 & 0.1934 \\
\hline Scan $x$-coord & 1234 & 1234 & 1234 & 1234 & 1234 & 1234 & 1234 & 1234 & 1234 & 1234 & 1234 & 1234 & 1234 & 1234 & 1234 & 1234 & 1234 \\
\hline$F X \# 1$ & 532.89 & 534.14 & 535.34 & 538.18 & 543.82 & 545.24 & 546.57 & 547.79 & 548.60 & 549.94 & 551.42 & 552.79 & 553.83 & 554.91 & 555.91 & 557.08 & 557.60 \\
\hline$F X \# 2$ & 311.84 & 312.08 & 312.28 & 312.38 & 312.55 & 312.69 & 312.94 & 313.34 & 313.72 & 314.16 & 314.66 & 315.06 & 315.52 & 316.00 & 316.39 & 316.60 & 316.80 \\
\hline$F X \# 3$ & 229.37 & 229.54 & 229.77 & 229.99 & 230.16 & 230.22 & 230.30 & 230.35 & 230.34 & 230.33 & 230.45 & 230.67 & 230.90 & 231.06 & 231.27 & 231.45 & 231.59 \\
\hline$F X \# 4$ & 173.14 & 173.28 & 173.32 & 173.31 & 173.28 & 173.30 & 173.38 & 173.49 & 173.60 & 173.75 & 173.93 & 174.15 & 174.33 & 174.44 & 174.49 & 174.49 & 174.50 \\
\hline
\end{tabular}




\begin{tabular}{|c|c|c|c|c|c|c|c|c|c|c|c|c|c|c|c|c|c|}
\hline$F X \# 5$ & 122.98 & 123.08 & 123.15 & 123.23 & 123.34 & 123.52 & 123.74 & 123.95 & 124.10 & 124.18 & 124.26 & 124.37 & 124.49 & 124.59 & 124.67 & 124.70 & 124.80 \\
\hline$F X \# 6$ & 0.00 & 0.00 & 0.00 & 0.00 & 0.00 & 0.00 & 0.00 & 0.00 & 0.00 & 0.00 & 0.00 & 0.00 & 0.00 & 0.00 & 0.00 & 0.00 & 0.00 \\
\hline Near wall FS & 122.98 & 123.08 & 123.15 & 123.23 & 123.34 & 123.52 & 123.74 & 123.95 & 124.10 & 124.18 & 124.26 & 124.37 & 124.49 & 124.59 & 124.67 & 124.70 & 124.80 \\
\hline$T(F X \# 1$ & 297.15 & 297.15 & 297.15 & 297.15 & 297.15 & 297.15 & 297.15 & 297.15 & 297.15 & 297.15 & 297.15 & 297.15 & 297.15 & 297.15 & 297.15 & 297.15 & 297.15 \\
\hline$T @ F X \# 2$ & 297.59 & 297.59 & 297.58 & 297.58 & 297.57 & 297.57 & 297.56 & 297.56 & 297.55 & 297.55 & 297.54 & 297.54 & 297.53 & 297.53 & 297.52 & 297.52 & 297.51 \\
\hline$T @ F X \# 3$ & 299.58 & 299.57 & 299.57 & 299.56 & 299.56 & 299.55 & 299.55 & 299.54 & 299.54 & 299.53 & 299.53 & 299.52 & 299.52 & 299.51 & 299.51 & 299.50 & 299.50 \\
\hline$T(a) F X \#$ & 301.59 & 301.58 & 301.58 & 301.57 & 301.57 & 301.56 & 301.56 & 301.55 & 301.55 & 301.54 & 301.54 & 301.53 & 301.53 & 301.52 & 301.52 & 301.51 & 301.51 \\
\hline$T$ (a) $F X \# 5$ & 303.63 & 303.62 & 303.62 & 303.61 & 303.61 & 303.60 & 303.60 & 303.59 & 303.59 & 303.58 & 303.58 & 303.57 & 303.57 & 303.56 & 303.56 & 303.55 & 303.55 \\
\hline$T @ F X \# 6$ & 305.70 & 305.69 & 305.69 & 305.68 & 305.68 & 305.67 & 305.67 & 305.66 & 305.66 & 305.65 & 305.65 & 305.64 & 305.64 & 305.63 & 305.63 & 305.62 & 305.62 \\
\hline Wall $x$-coord & 94.83 & 94.84 & 94.84 & 94.85 & 94.85 & 94.85 & 94.86 & 94.86 & 94.87 & 94.87 & 94.88 & 94.88 & 94.89 & 94.89 & 94.89 & 94.90 & 94.90 \\
\hline Wall $T$ & 306.86 & 306.86 & 306.86 & 306.86 & 306.86 & 306.86 & 306.87 & 306.88 & 306.88 & 306.88 & 306.87 & 306.87 & 306.87 & 306.86 & 306.86 & 306.86 & 306.86 \\
\hline Interferogram Image \# & 498 & Location & $T C 5$ & $\delta \varepsilon$ slope & $-2.07 E-3$ & $\delta \varepsilon$ intercept & 5.79 & $\operatorname{Avg} F S$ & 0.00 & Avg Wall $T$ & 307.38 & Gradient error & $-2.18 E-5$ & & & & \\
\hline Scan y-coord & 2724 & 2725 & 2726 & 2727 & 2728 & 2729 & 2730 & 2731 & 2732 & 2733 & 2734 & 2735 & 2736 & 2737 & 2738 & 2739 & 2740 \\
\hline & 0.1660 & 0.1639 & 0.1618 & 0.1598 & 0.1577 & 0.1556 & 0.1536 & 0.1515 & 0.1494 & 0.1474 & 0.1453 & 0.1432 & 0.1412 & 0.1391 & 0.1370 & 0.1350 & 0.1329 \\
\hline Scan $x$-coord & 1214 & 1214 & 1214 & 1214 & 1214 & 1214 & 1214 & 1214 & 1214 & 1214 & 1214 & 1214 & 1214 & 1214 & 1214 & 1214 & 1214 \\
\hline$F X \# 1$ & 614.16 & 618.67 & 620.53 & 620.13 & 620.37 & 630.88 & 632.05 & 632.60 & 627.91 & 637.00 & 637.40 & 637.98 & 638.94 & 640.34 & 641.87 & 643.60 & 645.16 \\
\hline$F X \# 2$ & 345.13 & 345.10 & 345.21 & 345.24 & 345.57 & 345.99 & 346.53 & 346.79 & 347.11 & 347.79 & 348.16 & 348.82 & 349.02 & 349.39 & 349.67 & 349.83 & 350.32 \\
\hline$F X \# 3$ & 252.22 & 252.32 & 252.43 & 252.54 & 252.55 & 252.55 & 252.59 & 252.79 & 252.96 & 253.11 & 253.31 & 253.46 & 253.57 & 253.64 & 253.76 & 253.92 & 254.03 \\
\hline$F X \# 4$ & 190.09 & 190.39 & 190.54 & 190.66 & 190.77 & 190.89 & 191.13 & 191.29 & 191.50 & 191.63 & 191.85 & 191.96 & 191.98 & 191.94 & 191.96 & 192.07 & 192.13 \\
\hline$F X \# 5$ & 137.21 & 137.44 & 137.59 & 137.67 & 137.68 & 137.74 & 137.86 & 138.02 & 138.17 & 138.26 & 138.38 & 138.55 & 138.61 & 138.68 & 138.73 & 138.82 & 138.97 \\
\hline$F X \# 6$ & 0.00 & 0.00 & 0.00 & 0.00 & 0.00 & 0.00 & 0.00 & 0.00 & 0.00 & 0.00 & 0.00 & 0.00 & 0.00 & 0.00 & 0.00 & 0.00 & 0.00 \\
\hline$F X \# 7$ & 0.00 & 0.00 & 0.00 & 0.00 & 0.00 & 0.00 & 0.00 & 0.00 & 0.00 & 0.00 & 0.00 & 0.00 & 0.00 & 0.00 & 0.00 & 0.00 & 0.00 \\
\hline Near wall FS & 0.00 & 0.00 & 0.00 & 0.00 & 0.00 & 0.00 & 0.00 & 0.00 & 0.00 & 0.00 & 0.00 & 0.00 & 0.00 & 0.00 & 0.00 & 0.00 & 0.00 \\
\hline$T @ F X \# 1$ & 297.15 & 297.15 & 297.15 & 297.15 & 297.15 & 297.15 & 297.15 & 297.15 & 297.15 & 297.15 & 297.15 & 297.15 & 297.15 & 297.15 & 297.15 & 297.15 & 297.15 \\
\hline$T @ F X \# 2$ & 297.46 & 297.46 & 297.45 & 297.45 & 297.45 & 297.44 & 297.44 & 297.43 & 297.43 & 297.43 & 297.42 & 297.42 & 297.41 & 297.41 & 297.41 & 297.40 & 297.40 \\
\hline$T @ F X \# 3$ & 299.44 & 299.44 & 299.43 & 299.43 & 299.43 & 299.42 & 299.42 & 299.41 & 299.41 & 299.41 & 299.40 & 299.40 & 299.39 & 299.39 & 299.39 & 299.38 & 299.38 \\
\hline$T$ @ $F X \# 4$ & 301.45 & 301.45 & 301.45 & 301.44 & 301.44 & 301.43 & 301.43 & 301.42 & 301.42 & 301.42 & 301.41 & 301.41 & 301.40 & 301.40 & 301.40 & 301.39 & 301.39 \\
\hline$T(a) F=5$ & 303.49 & 303.49 & 303.48 & 303.48 & 303.48 & 303.47 & 303.47 & 303.46 & 303.46 & 303.46 & 303.45 & 303.45 & 303.44 & 303.44 & 303.43 & 303.43 & 303.43 \\
\hline$T(F X \# 6$ & 305.56 & 305.56 & 305.55 & 305.55 & 305.54 & 305.54 & 305.53 & 305.53 & 305.53 & 305.52 & 305.52 & 305.51 & 305.51 & 305.50 & 305.50 & 305.50 & 305.49 \\
\hline$T @ F X \# 7$ & 307.65 & 307.65 & 307.65 & 307.64 & 307.64 & 307.63 & 307.63 & 307.62 & 307.62 & 307.61 & 307.61 & 307.61 & 307.60 & 307.60 & 307.59 & 307.59 & 307.58 \\
\hline Wall $x$-coord & 90.28 & 90.28 & 90.29 & 90.30 & 90.30 & 90.31 & 90.32 & 90.32 & 90.33 & 90.34 & 90.34 & 90.35 & 90.36 & 90.36 & 90.37 & 90.38 & 90.38 \\
\hline Wall $T$ & 307.39 & 307.40 & 307.40 & 307.40 & 307.39 & 307.38 & 307.38 & 307.38 & 307.38 & 307.38 & 307.37 & 307.38 & 307.38 & 307.38 & 307.38 & 307.38 & 307.38 \\
\hline Interferogram Image \# & 498 & Location & $T C 6$ & $\delta \varepsilon$ slope & $-2.07 E-3$ & $\delta \varepsilon$ intercept & 4.83 & $\operatorname{Avg} F S$ & 49.46 & Avg Wall $T$ & 307.94 & Gradient error & $-2.18 E-5$ & & & & \\
\hline Scan y-coord & 1910 & 1911 & 1912 & 1913 & 1914 & 1915 & 1916 & 1917 & 1918 & 1919 & 1920 & 1921 & 1922 & 1923 & 1924 & 1925 & 1926 \\
\hline$\delta \varepsilon$ & 0.8859 & 0.8839 & 0.8818 & 0.8797 & 0.8777 & 0.8756 & 0.8735 & 0.8715 & 0.8694 & 0.8673 & 0.8653 & 0.8632 & 0.8611 & 0.8591 & 0.8570 & 0.8549 & 0.8529 \\
\hline Scan $x$-coord & 1053 & 1053 & 1053 & 1053 & 1053 & 1053 & 1053 & 1053 & 1053 & 1053 & 1053 & 1053 & 1053 & 1053 & 1053 & 1053 & 1053 \\
\hline$F X \# I$ & 373.53 & 373.59 & 373.57 & 373.52 & 373.53 & 373.51 & 373.80 & 374.19 & 374.80 & 375.41 & 376.13 & 376.76 & 377.07 & 377.31 & 377.39 & 377.53 & 377.50 \\
\hline$F X \# 2$ & 275.11 & 275.25 & 275.32 & 275.39 & 275.36 & 275.39 & 275.47 & 275.67 & 275.95 & 276.24 & 276.56 & 276.80 & 277.03 & 277.25 & 277.42 & 277.52 & 277.49 \\
\hline$F X \# 3$ & 208.04 & 208.19 & 208.40 & 208.60 & 208.90 & 209.13 & 209.16 & 209.19 & 209.27 & 209.28 & 209.37 & 209.57 & 209.63 & 209.62 & 209.67 & 209.78 & 209.90 \\
\hline$F X \# 4$ & 154.55 & 154.47 & 154.46 & 154.44 & 154.46 & 154.54 & 154.69 & 154.90 & 155.04 & 155.16 & 155.30 & 155.48 & 155.55 & 155.64 & 155.67 & 155.67 & 155.67 \\
\hline$F X \# 5$ & 104.66 & 104.58 & 104.71 & 104.97 & 105.17 & 105.29 & 105.35 & 105.37 & 105.45 & 105.54 & 105.74 & 105.93 & 106.12 & 106.33 & 106.39 & 106.51 & 106.71 \\
\hline Near wall FS & 49.89 & 49.89 & 49.75 & 49.47 & 49.28 & 49.26 & 49.34 & 49.53 & 49.59 & 49.62 & 49.57 & 49.55 & 49.43 & 49.32 & 49.28 & 49.16 & 48.97 \\
\hline$T$ @ $F X \# 1$ & 297.15 & 297.15 & 297.15 & 297.15 & 297.15 & 297.15 & 297.15 & 297.15 & 297.15 & 297.15 & 297.15 & 297.15 & 297.15 & 297.15 & 297.15 & 297.15 & 297.15 \\
\hline$T @ F X \# 2$ & 298.89 & 298.88 & 298.88 & 298.88 & 298.87 & 298.87 & 298.86 & 298.86 & 298.86 & 298.85 & 298.85 & 298.84 & 298.84 & 298.83 & 298.83 & 298.83 & 298.82 \\
\hline$T @ F X \# 3$ & 300.89 & 300.89 & 300.88 & 300.88 & 300.88 & 300.87 & 300.87 & 300.86 & 300.86 & 300.85 & 300.85 & 300.85 & 300.84 & 300.84 & 300.83 & 300.83 & 300.83 \\
\hline$T$ @ $F X \# 4$ & 302.92 & 302.92 & 302.91 & 302.91 & 302.91 & 302.90 & 302.90 & 302.89 & 302.89 & 302.89 & 302.88 & 302.88 & 302.87 & 302.87 & 302.86 & 302.86 & 302.86 \\
\hline$T @ F X \# 5$ & 304.98 & 304.98 & 304.97 & 304.97 & 304.97 & 304.96 & 304.96 & 304.95 & 304.95 & 304.94 & 304.94 & 304.94 & 304.93 & 304.93 & 304.92 & 304.92 & 304.91 \\
\hline Wall $x$-coord & 84.00 & 84.01 & 84.02 & 84.03 & 84.04 & 84.05 & 84.06 & 84.06 & 84.07 & 84.08 & 84.09 & 84.10 & 84.11 & 84.12 & 84.13 & 84.13 & 84.14 \\
\hline Wall $T$ & 307.93 & 307.93 & 307.93 & 307.94 & 307.95 & 307.95 & 307.95 & 307.94 & 307.94 & 307.93 & 307.94 & 307.94 & 307.95 & 307.95 & 307.95 & 307.95 & 307.96 \\
\hline Interferogram Image \# & 498 & Location & $T C 7$ & $\delta \varepsilon$ slope & $-2.10 E-3$ & $\delta \varepsilon$ intercept & 2.97 & $\operatorname{Avg} F S$ & 51.21 & Avg Wall $T$ & 308.29 & Gradient error & $-2.18 E-5$ & & & & \\
\hline Scan $y$-coord & 1096 & 1097 & 1098 & 1099 & 1100 & 1101 & 1102 & 1103 & 1104 & 1105 & 1106 & 1107 & 1108 & 1109 & 1110 & 1111 & 1112 \\
\hline$\delta \varepsilon$ & 0.6651 & 0.6630 & 0.6609 & 0.6588 & 0.6567 & 0.6546 & 0.6525 & 0.6504 & 0.6483 & 0.6462 & 0.6441 & 0.6420 & 0.6399 & 0.6378 & 0.6357 & 0.6336 & 0.6315 \\
\hline Scan $x$-coord & 1231 & 1231 & 1231 & 1231 & 1231 & 1231 & 1231 & 1231 & 1231 & 1231 & 1231 & 1231 & 1231 & 1231 & 1231 & 1231 & 1231 \\
\hline$F X \# 1$ & 440.03 & 440.33 & 440.68 & 441.19 & 441.57 & 441.84 & 442.27 & 443.19 & 444.19 & 445.17 & 445.83 & 446.55 & 446.98 & 447.35 & 447.38 & 447.32 & 447.14 \\
\hline$F X \# 2$ & 304.42 & 304.51 & 304.50 & 304.46 & 304.46 & 304.47 & 304.62 & 304.81 & 305.17 & 305.47 & 305.63 & 305.71 & 305.87 & 306.15 & 306.42 & 306.67 & 306.80 \\
\hline$F X \# 3$ & 227.83 & 227.89 & 227.93 & 228.10 & 228.35 & 228.54 & 228.79 & 229.08 & 229.37 & 229.55 & 229.65 & 229.65 & 229.62 & 229.61 & 229.67 & 229.87 & 230.05 \\
\hline$F X \# 4$ & 168.66 & 168.88 & 169.07 & 169.19 & 169.22 & 169.29 & 169.40 & 169.45 & 169.53 & 169.61 & 169.72 & 169.94 & 170.18 & 170.38 & 170.54 & 170.71 & 170.81 \\
\hline$F X \# 5$ & 117.67 & 117.74 & 117.79 & 117.88 & 117.93 & 117.90 & 117.94 & 118.04 & 118.25 & 118.50 & 118.75 & 118.92 & 119.12 & 119.31 & 119.43 & 119.44 & 119.46 \\
\hline Near wall FS & 50.99 & 51.14 & 51.28 & 51.31 & 51.29 & 51.39 & 51.45 & 51.41 & 51.28 & 51.11 & 50.97 & 51.02 & 51.06 & 51.08 & 51.11 & 51.27 & 51.35 \\
\hline$T @ F X \# 1$ & 297.15 & 297.15 & 297.15 & 297.15 & 297.15 & 297.15 & 297.15 & 297.15 & 297.15 & 297.15 & 297.15 & 297.15 & 297.15 & 297.15 & 297.15 & 297.15 & 297.15 \\
\hline$T(F X \# 2$ & 298.45 & 298.44 & 298.44 & 298.43 & 298.43 & 298.43 & 298.42 & 298.42 & 298.41 & 298.41 & 298.40 & 298.40 & 298.40 & 298.39 & 298.39 & 298.38 & 298.38 \\
\hline$T(F X \# 3$ & 300.44 & 300.44 & 300.43 & 300.43 & 300.43 & 300.42 & 300.42 & 300.41 & 300.41 & 300.41 & 300.40 & 300.40 & 300.39 & 300.39 & 300.38 & 300.38 & 300.38 \\
\hline$T(F X \# 4$ & 302.47 & 302.46 & 302.46 & 302.46 & 302.45 & 302.45 & 302.44 & 302.44 & 302.43 & 302.43 & 302.43 & 302.42 & 302.42 & 302.41 & 302.41 & 302.40 & 302.40 \\
\hline$T$ @ $F X \# 5$ & 304.52 & 304.52 & 304.51 & 304.51 & 304.50 & 304.50 & 304.50 & 304.49 & 304.49 & 304.48 & 304.48 & 304.47 & 304.47 & 304.46 & 304.46 & 304.46 & 304.45 \\
\hline Wall $x$-coord & 76.01 & 76.02 & 76.03 & 76.04 & 76.05 & 76.06 & 76.08 & 76.09 & 76.10 & 76.11 & 76.12 & 76.13 & 76.14 & 76.15 & 76.16 & 76.17 & 76.18 \\
\hline Wall T & 308.30 & 308.30 & 308.29 & 308.29 & 308.28 & 308.27 & 308.27 & 308.27 & 308.28 & 308.29 & 308.30 & 308.30 & 308.30 & 308.30 & 308.30 & 308.29 & 308.29 \\
\hline \multicolumn{18}{|c|}{ B.43 Tabulated Data for Interferogram 499} \\
\hline Interferogram Image \# & 499 & Location & $T C 2$ & $\delta \varepsilon$ slope & $-2.53 E-3$ & $\delta \varepsilon$ intercept & 13.18 & $\operatorname{Avg} F S$ & 53.24 & Avg Wall $T$ & 305.05 & Gradient error & $-6.94 E-5$ & & & & \\
\hline
\end{tabular}




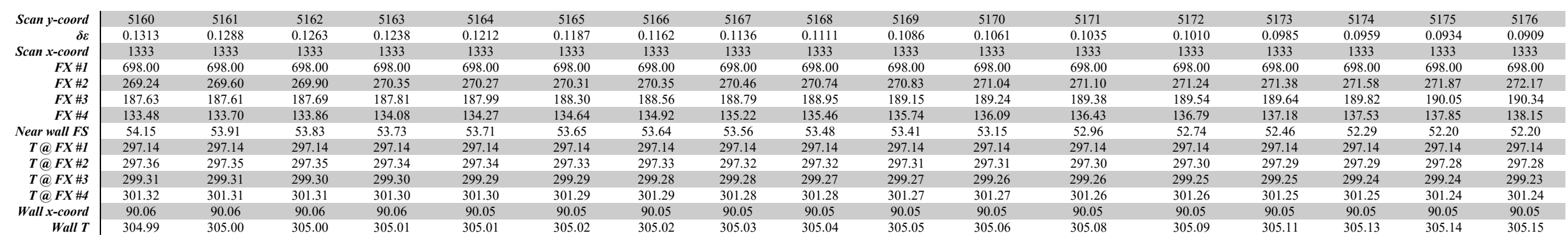

\begin{tabular}{|c|c|c|c|c|c|c|c|c|c|c|c|c|c|c|c|c|c|}
\hline Interferogram 1 mage $\#$ & & Location & & of slope & & & & & & & & & & & & & \\
\hline Scan y-coord & 4345 & 4346 & 4347 & 4348 & 4349 & 4350 & 4351 & 4352 & 4353 & 4354 & 4355 & 4356 & 4357 & 4358 & 4359 & 4360 & 4361 \\
\hline & 0.1675 & 0.1651 & 0.1627 & 0.1602 & 0.1578 & 0.1553 & 0.1529 & 0.1504 & 0.1480 & 0.1455 & 0.1431 & 0.1406 & 0.1382 & 0.1358 & 0.1333 & 0.1309 & 0.1284 \\
\hline Scan $x$-coord & 1456 & 1456 & 1456 & 1456 & 1456 & 1456 & 1456 & 1456 & 1456 & 1456 & 1456 & 1456 & 1456 & 1456 & 1456 & 1456 & 1456 \\
\hline$F X \# 1$ & 574.81 & 576.48 & 570.11 & 571.08 & 571.15 & 573.71 & 584.59 & 586.73 & 587.48 & 589.66 & 593.04 & 602.11 & 603.33 & 605.34 & 607.48 & 609.92 & 611.85 \\
\hline$F X \# 2$ & 295.42 & 295.78 & 296.35 & 296.77 & 296.94 & 297.26 & 297.51 & 297.77 & 298.10 & 297.70 & 298.26 & 298.56 & 299.03 & 298.87 & 299.39 & 299.66 & 299.76 \\
\hline$F X \# 3$ & 211.33 & 211.55 & 211.80 & 212.02 & 212.16 & 212.30 & 212.43 & 212.54 & 212.63 & 212.74 & 212.94 & 213.14 & 213.32 & 213.46 & 213.51 & 213.54 & 213.61 \\
\hline$F X \# 4$ & 154.08 & 154.22 & 154.36 & 154.47 & 154.57 & 154.63 & 154.72 & 154.80 & 154.94 & 155.10 & 155.27 & 155.40 & 155.55 & 155.77 & 155.93 & 156.08 & 156.25 \\
\hline$F X \# 5$ & 105.86 & 105.93 & 106.01 & 106.19 & 106.35 & 106.43 & 106.48 & 106.49 & 106.53 & 106.65 & 106.82 & 106.99 & 107.18 & 107.42 & 107.64 & 107.89 & 108.04 \\
\hline Near wall FS & 48.22 & 48.29 & 48.35 & 48.27 & 48.22 & 48.21 & 48.24 & 48.31 & 48.41 & 48.46 & 48.46 & 48.41 & 48.37 & 48.35 & 48.29 & 48.18 & 48.21 \\
\hline$T @ F X \# 1$ & 297.14 & 297.14 & 297.14 & 297.14 & 297.14 & 297.14 & 297.14 & 297.14 & 297.14 & 297.14 & 297.14 & 297.14 & 297.14 & 297.14 & 297.14 & 297.14 & 297.14 \\
\hline$T @ F X \# 2$ & 297.41 & 297.41 & 297.40 & 297.40 & 297.39 & 297.39 & 297.38 & 297.38 & 297.37 & 297.37 & 297.37 & 297.36 & 297.36 & 297.35 & 297.35 & 297.34 & 297.34 \\
\hline$T @ F X \# 3$ & 299.38 & 299.37 & 299.37 & 299.36 & 299.36 & 299.35 & 299.35 & 299.34 & 299.34 & 299.33 & 299.33 & 299.33 & 299.32 & 299.32 & 299.31 & 299.31 & 299.30 \\
\hline$T @ F X \# 4$ & 301.39 & 301.38 & 301.38 & 301.37 & 301.37 & 301.36 & 301.36 & 301.35 & 301.35 & 301.34 & 301.34 & 301.33 & 301.33 & 301.32 & 301.32 & 301.31 & 301.31 \\
\hline$T \cong F X \# 5$ & 303.42 & 303.42 & 303.41 & 303.41 & 303.40 & 303.40 & 303.39 & 303.39 & 303.38 & 303.38 & 303.37 & 303.37 & 303.36 & 303.36 & 303.35 & 303.35 & 303.34 \\
\hline Wall $T$ & 306.19 & 306.19 & 306.19 & 306.19 & 306.19 & 306.19 & 306.19 & 306.18 & 306.18 & 306.18 & 306.18 & 306.18 & 306.19 & 306.19 & 306.20 & 306.20 & 306.21 \\
\hline Interferogram Image \# & 499 & Location & $T C 4$ & $\delta \varepsilon$ slope & $-2.26 E-3$ & $\delta \varepsilon$ intercept & 8.16 & $\operatorname{Avg} F S$ & 51.47 & Avg Wall $T$ & 306.74 & Gradient error & $-6.94 E-5$ & & & & \\
\hline Scan y-coord & 3531 & 3532 & 3533 & 3534 & 3535 & 3536 & 3537 & 3538 & 3539 & 3540 & 3541 & 3542 & 3543 & 3544 & 3545 & 3546 & 3547 \\
\hline$\delta \varepsilon$ & 0.1674 & 0.1652 & 0.1629 & 0.1606 & 0.1584 & 0.1561 & 0.1538 & 0.1516 & 0.1493 & 0.1470 & 0.1448 & 0.1425 & 0.1403 & 0.1380 & 0.1357 & 0.1335 & 0.1312 \\
\hline Scan $x$-coord & 1787 & 1787 & 1787 & 1787 & 1787 & 1787 & 1787 & 1787 & 1787 & 1787 & 1787 & 1787 & 1787 & 1787 & 1787 & 1787 & 1787 \\
\hline$F X \# I$ & 652.37 & 655.81 & 667.69 & 690.47 & 687.33 & 670.20 & 671.66 & 671.40 & 672.53 & 673.14 & 673.80 & 675.28 & 676.20 & 708.74 & 729.52 & 732.68 & 734.48 \\
\hline$F X \# 2$ & 323.24 & 323.45 & 323.67 & 323.04 & 324.04 & 324.38 & 324.85 & 325.21 & 325.93 & 326.30 & 326.69 & 326.81 & 327.17 & 327.47 & 326.18 & 326.75 & 326.28 \\
\hline$F X \# 3$ & 230.55 & 230.75 & 230.86 & 230.96 & 231.00 & 231.06 & 231.12 & 231.22 & 231.38 & 231.59 & 231.86 & 232.02 & 232.21 & 232.33 & 232.44 & 232.47 & 232.56 \\
\hline$F X \# 4$ & 170.16 & 170.23 & 170.28 & 170.39 & 170.50 & 170.53 & 170.62 & 170.78 & 170.91 & 171.08 & 171.20 & 171.33 & 171.38 & 171.39 & 171.35 & 171.37 & 171.42 \\
\hline$F X \# 5$ & 118.14 & 118.30 & 118.42 & 118.58 & 118.79 & 118.98 & 119.12 & 119.24 & 119.36 & 119.56 & 119.84 & 120.06 & 120.12 & 120.17 & 120.28 & 120.43 & 120.57 \\
\hline Near wall $F S$ & 52.02 & 51.93 & $\begin{array}{l}110.42 \\
51.86\end{array}$ & 51.81 & 51.71 & $\begin{array}{l}110.90 \\
51.55\end{array}$ & 51.50 & 51.54 & 51.55 & 51.52 & 51.36 & 51.28 & 51.26 & 51.22 & 51.07 & 50.93 & 50.85 \\
\hline$T$ (a) FX\#1 & 297.14 & 297.14 & 297.14 & 297.14 & 297.14 & 297.14 & 297.14 & 297.14 & 297.14 & 297.14 & 297.14 & 297.14 & 297.14 & 297.14 & 297.14 & 297.14 & 297.14 \\
\hline$T @ F X \# 3$ & 299.36 & 299.35 & 299.35 & 299.34 & 299.34 & 299.33 & 299.33 & 299.33 & 299.32 & 299.32 & 299.31 & 299.31 & 299.30 & 299.30 & 299.29 & 299.29 & 299.28 \\
\hline$T @ F X \# 4$ & 301.36 & 301.36 & 301.35 & 301.35 & 301.34 & 301.34 & 301.34 & 301.33 & 301.33 & 301.32 & 301.32 & 301.31 & 301.31 & 301.30 & 301.30 & 301.29 & 301.29 \\
\hline$T @ F X \# 5$ & 303.40 & 303.39 & 303.39 & 303.38 & 303.38 & 303.37 & 303.37 & 303.37 & 303.36 & 303.36 & 303.35 & 303.35 & 303.34 & 303.34 & 303.33 & 303.33 & 303.32 \\
\hline Wall $x$-coord & 86.63 & 86.64 & 86.64 & 86.65 & 86.65 & 86.65 & 86.66 & 86.66 & 86.67 & 86.67 & 86.68 & 86.68 & 86.68 & 86.69 & 86.69 & 86.70 & 86.70 \\
\hline Wall T & 306.71 & 306.71 & 306.72 & 306.72 & 306.72 & 306.73 & 306.73 & 306.73 & 306.73 & 306.74 & 306.75 & 306.75 & 306.75 & 306.75 & 306.75 & 306.76 & 306.76 \\
\hline Interferogram Image \# & 499 & Location & $T C 5$ & $\delta \varepsilon$ slope & $-2.25 E-3$ & $\delta \varepsilon$ intercept & 6.16 & $\operatorname{Avg} F S$ & 56.45 & Avg Wall $T$ & 307.08 & Gradient error & $-6.94 E-5$ & & & & \\
\hline Scan y-coord & 2717 & 2718 & 2719 & 2720 & 2721 & 2722 & 2723 & 2724 & 2725 & 2726 & 2727 & 2728 & 2729 & 2730 & 2731 & 2732 & 2733 \\
\hline & 0.0510 & 0.0487 & 0.0465 & 0.0442 & 0.0420 & 0.0397 & 0.0375 & 0.0352 & 0.0330 & 0.0307 & 0.0285 & 0.0262 & 0.0240 & 0.0217 & 0.0195 & 0.0172 & 0.0150 \\
\hline Scan $x$-coord & 2151 & 2151 & 2151 & 2151 & 2151 & 2151 & 2151 & 2151 & 2151 & 2151 & 2151 & 2151 & 2151 & 2151 & 2151 & 2151 & 2151 \\
\hline$F X \# 1$ & 855.00 & 855.00 & 855.00 & 855.00 & 855.00 & 855.00 & 855.00 & 855.00 & 855.00 & 855.00 & 855.00 & 855.00 & 855.00 & 855.00 & 855.00 & 855.00 & 855.00 \\
\hline$F X \# 2$ & 359.60 & 360.03 & 360.50 & 360.91 & 361.23 & 361.60 & 361.69 & 362.02 & 362.24 & 362.68 & 363.13 & 363.40 & 363.52 & 363.69 & 363.93 & 364.19 & 364.46 \\
\hline$F X \# 3$ & 257.89 & 257.92 & 257.89 & 257.89 & 257.89 & 257.94 & 257.97 & 258.13 & 258.30 & 258.51 & 258.64 & 258.75 & 258.88 & 259.05 & 259.25 & 259.43 & 259.68 \\
\hline$F X \# 4$ & 190.55 & 190.61 & 190.66 & 190.80 & 191.01 & 191.23 & 191.27 & 191.53 & 191.65 & 191.84 & 191.84 & 191.86 & 191.85 & 191.88 & 191.99 & 192.15 & 192.35 \\
\hline$F X \# 5$ & 133.80 & 133.95 & 134.10 & 134.24 & 134.36 & 134.42 & 134.58 & 134.77 & 135.04 & 135.20 & 135.39 & 135.56 & 135.68 & 135.76 & 136.14 & 136.17 & 136.21 \\
\hline Near wall FS & 56.75 & 56.66 & 56.56 & 56.56 & 56.64 & 56.82 & 56.69 & 56.77 & 56.61 & 56.64 & 56.45 & 56.30 & 56.17 & 56.12 & 55.84 & 55.97 & 56.14 \\
\hline$T @ F X \# 1$ & 297.14 & 297.14 & 297.14 & 297.14 & 297.14 & 297.14 & 297.14 & 297.14 & 297.14 & 297.14 & 297.14 & 297.14 & 297.14 & 297.14 & 297.14 & 297.14 & 297.14 \\
\hline$T @ F X \# 2$ & 297.15 & 297.15 & 297.14 & 297.14 & 297.14 & 297.13 & 297.13 & 297.12 & 297.12 & 297.11 & 297.11 & 297.10 & 297.10 & 297.10 & 297.09 & 297.09 & 297.08 \\
\hline$T @ F X \# 3$ & 299.10 & 299.10 & 299.09 & 299.09 & 299.08 & 299.08 & 299.07 & 299.07 & 299.07 & 299.06 & 299.06 & 299.05 & 299.05 & 299.04 & 299.04 & 299.03 & 299.03 \\
\hline$T(F X \# 4$ & 301.10 & 301.10 & 301.09 & 301.09 & 301.08 & 301.08 & 301.08 & 301.07 & 301.07 & 301.06 & 301.06 & 301.05 & 301.05 & 301.04 & 301.04 & 301.03 & 301.03 \\
\hline$T @ F X \# 5$ & 303.13 & 303.13 & 303.12 & 303.12 & 303.12 & 303.11 & 303.11 & 303.10 & 303.10 & 303.09 & 303.09 & 303.08 & 303.08 & 303.07 & 303.07 & 303.06 & 303.06 \\
\hline Wall $x$-coord & 82.15 & 82.16 & 82.16 & 82.17 & 82.18 & 82.18 & 82.19 & 82.20 & 82.20 & 82.21 & 82.22 & 82.22 & 82.23 & 82.24 & 82.24 & 82.25 & 82.26 \\
\hline Wall $T$ & 307.07 & 307.07 & 307.08 & 307.08 & 307.07 & 307.06 & 307.07 & 307.07 & 307.08 & 307.08 & 307.09 & 307.09 & 307.10 & 307.10 & 307.12 & 307.11 & 307.10 \\
\hline \multirow{3}{*}{$\begin{array}{r}\mid \text { nterferogram Image \# } \\
\text { Scan } y \text {-coord } \\
\delta \varepsilon\end{array}$} & 499 & Location & TC6 & $\delta \varepsilon$ slope & $-2.38 E-3$ & $\delta \varepsilon$ intercept & 5.41 & $\operatorname{Avg} F S$ & 51.23 & Avg Wall $T$ & 307.97 & Gradient error & $-6.94 E-5$ & & & & \\
\hline & 1902 & 1903 & 1904 & 1905 & 1906 & 1907 & 1908 & 1909 & 1910 & 1911 & 1912 & 1913 & 1914 & 1915 & 16 & 1917 & 1918 \\
\hline & 0.8703 & 0.8679 & 0.8655 & 0.8631 & 0.8608 & 0.8584 & 0.8560 & 0.8536 & 0.8512 & 0.8488 & 0.8465 & 0.8441 & 0.8417 & 0.8393 & & & \\
\hline
\end{tabular}




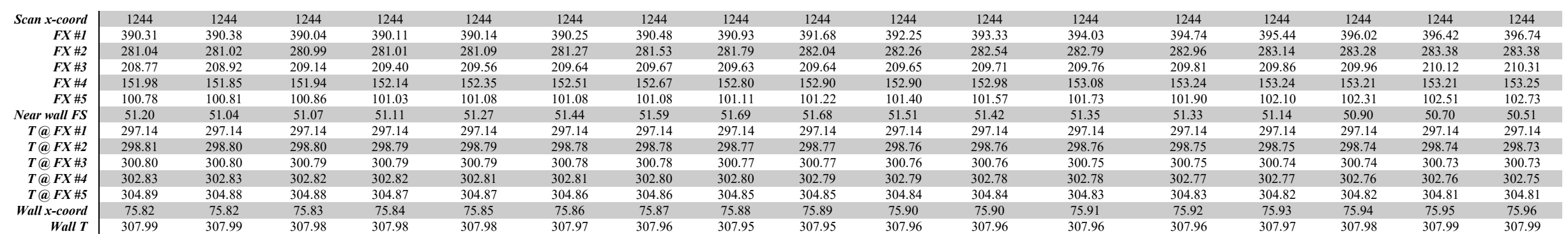

\begin{tabular}{|c|c|c|c|c|c|c|c|c|c|c|c|c|c|c|c|c|c|}
\hline Interferogram Image \# & 499 & Location & $1 C 7$ & $\partial \varepsilon$ slope & $-2.12 E-3$ & $\partial \varepsilon$ intercept & 2.89 & Avg FS & 53.11 & Avg Wall T & 308.11 & Gradient error & 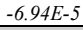 & & & & \\
\hline Scan y-coord & 1088 & 1089 & 1090 & 1091 & 1092 & 1093 & 1094 & 1095 & 1096 & 1097 & 1098 & 1099 & 1100 & 1101 & 1102 & 1103 & 1104 \\
\hline & 0.5863 & 0.5842 & 0.5821 & 0.5799 & 0.5778 & 0.5757 & 0.5736 & 0.5715 & 0.5694 & 0.5672 & 0.5651 & 0.5630 & 0.5609 & 0.5588 & 0.5566 & 0.5545 & 0.5524 \\
\hline Scan $x$-coord & 1434 & 1434 & 1434 & 1434 & 1434 & 1434 & 1434 & 1434 & 1434 & 1434 & 1434 & 1434 & 1434 & 1434 & 1434 & 1434 & 1434 \\
\hline$F X \# I$ & 457.56 & 457.35 & 457.12 & 457.51 & 458.15 & 458.88 & 459.92 & 461.00 & 461.04 & 462.03 & 462.28 & 462.61 & 462.58 & 462.72 & 462.93 & 463.11 & 463.61 \\
\hline$F X \# 2$ & 307.36 & 307.18 & 307.23 & 307.43 & 307.54 & 307.70 & 307.97 & 308.50 & 309.00 & 309.42 & 309.58 & 309.87 & 310.16 & 310.45 & 310.68 & 310.99 & 311.07 \\
\hline$F X \# 3$ & 227.21 & 227.41 & 227.67 & 227.91 & 228.17 & 228.40 & 228.68 & 228.95 & 229.05 & 229.05 & 228.99 & 228.90 & 228.89 & 228.97 & 229.16 & 229.41 & 229.67 \\
\hline$F X \# 4$ & 165.61 & 165.73 & 165.82 & 165.93 & 165.97 & 166.02 & 166.19 & 166.31 & 166.51 & 166.71 & 166.92 & 167.03 & 167.11 & 167.19 & 167.29 & 167.39 & 167.50 \\
\hline$F X \# 5$ & 112.74 & 112.76 & 112.84 & 112.86 & 112.90 & 112.93 & 113.03 & 113.18 & 113.37 & 113.55 & 113.70 & 113.81 & 113.94 & 114.02 & 114.16 & 114.24 & 114.27 \\
\hline Near wall FS & 52.86 & 52.97 & 52.97 & 53.06 & 53.07 & 53.09 & 53.16 & 53.13 & 53.14 & 53.16 & 53.22 & 53.23 & 53.17 & 53.17 & 53.13 & 53.15 & 53.23 \\
\hline$T @ F X \# 1$ & 297.14 & 297.14 & 297.14 & 297.14 & 297.14 & 297.14 & 297.14 & 297.14 & 297.14 & 297.14 & 297.14 & 297.14 & 297.14 & 297.14 & 297.14 & 297.14 & 297.14 \\
\hline$T @ F X \# 2$ & 298.23 & 298.23 & 298.23 & 298.22 & 298.22 & 298.21 & 298.21 & 298.20 & 298.20 & 298.20 & 298.19 & 298.19 & 298.18 & 298.18 & 298.18 & 298.17 & 298.17 \\
\hline$T @ F X \# 3$ & 300.22 & 300.22 & 300.21 & 300.21 & 300.20 & 300.20 & 300.20 & 300.19 & 300.19 & 300.18 & 300.18 & 300.17 & 300.17 & 300.17 & 300.16 & 300.16 & 300.15 \\
\hline$T @ F X \# 4$ & 302.24 & 302.23 & 302.23 & 302.23 & 302.22 & 302.22 & 302.21 & 302.21 & 302.20 & 302.20 & 302.20 & 302.19 & 302.19 & 302.18 & 302.18 & 302.17 & 302.17 \\
\hline$T @ F X \# 5$ & 304.29 & 304.28 & 304.28 & 304.27 & 304.27 & 304.26 & 304.26 & 304.26 & 304.25 & 304.25 & 304.24 & 304.24 & 304.23 & 304.23 & 304.22 & 304.22 & 304.22 \\
\hline Wall T & 308.13 & 308.12 & 308.12 & 308.12 & 308.11 & 308.11 & 308.10 & 308.11 & 308.11 & 308.11 & 308.11 & 308.11 & 308.11 & 308.11 & 308.11 & 308.11 & 308.10 \\
\hline
\end{tabular}

\section{B.44 Tabulated Data for Interferogram 500}

\begin{tabular}{|c|c|c|c|c|c|c|c|c|c|c|c|c|c|c|c|c|c|}
\hline Interferogram Image \# & 500 & Location & $T C 2$ & $\partial \varepsilon$ slope & $-2.58 E-3$ & $\partial \varepsilon$ intercept & 13.50 & Avg FS & 53.04 & Avg Wall T & 304.99 & Gradient error & $-2.47 E-6$ & & & & \\
\hline Scan y-coord & 5176 & 5177 & 5178 & 5179 & 5180 & 5181 & 5182 & 5183 & 5184 & 5185 & 5186 & 5187 & 5188 & 5189 & 5190 & 5191 & 5192 \\
\hline & 0.1198 & 0.1172 & 0.1146 & 0.1120 & 0.1094 & 0.1068 & 0.1043 & 0.1017 & 0.0991 & 0.0965 & 0.0939 & 0.0913 & 0.0888 & 0.0862 & 0.0836 & 0.0810 & 0.0784 \\
\hline Scan $x$-coord & 1503 & 1503 & 1503 & 1503 & 1503 & 1503 & 1503 & 1503 & 1503 & 1503 & 1503 & 1503 & 1503 & 1503 & 1503 & 1503 & 1503 \\
\hline$F X \# I$ & 636.38 & 638.43 & 628.30 & 629.52 & 646.58 & 644.44 & 643.97 & 645.65 & 632.72 & 663.21 & 657.96 & 656.49 & 721.78 & 724.50 & 698.07 & 701.25 & 747.19 \\
\hline$F X \# 2$ & 288.84 & 289.28 & 289.51 & 289.77 & 290.29 & 290.38 & 290.71 & 290.93 & 291.05 & 291.02 & 291.07 & 291.31 & 291.46 & 291.82 & 292.31 & 292.73 & 292.90 \\
\hline$F X \# 3$ & 209.58 & 209.66 & 209.74 & 209.85 & 210.01 & 210.17 & 210.28 & 210.48 & 210.62 & 210.69 & 210.88 & 211.07 & 211.34 & 211.52 & 211.65 & 211.84 & 212.08 \\
\hline$F X \# 4$ & 155.21 & 155.43 & 155.60 & 155.77 & 155.89 & 156.02 & 156.25 & 156.56 & 156.92 & 157.21 & 157.57 & 157.95 & 158.33 & 158.67 & 159.02 & 159.34 & 159.62 \\
\hline Near wall FS & 54.37 & 54.23 & 54.14 & 54.09 & 54.13 & 54.15 & 54.03 & 53.92 & 53.70 & 53.48 & 53.30 & 53.12 & 53.01 & 52.85 & 52.63 & 52.50 & 52.46 \\
\hline$T @ F X \# 1$ & 297.15 & 297.15 & 297.15 & 297.15 & 297.15 & 297.15 & 297.15 & 297.15 & 297.15 & 297.15 & 297.15 & 297.15 & 297.15 & 297.15 & 297.15 & 297.15 & 297.15 \\
\hline$T @ F X \# 2$ & 297.38 & 297.38 & 297.37 & 297.37 & 297.36 & 297.36 & 297.35 & 297.35 & 297.34 & 297.34 & 297.33 & 297.33 & 297.32 & 297.32 & 297.31 & 297.31 & 297.30 \\
\hline$T$ @ $F X \# 3$ & 299.37 & 299.36 & 299.36 & 299.35 & 299.35 & 299.34 & 299.34 & 299.33 & 299.33 & 299.32 & 299.32 & 299.31 & 299.31 & 299.30 & 299.30 & 299.29 & 299.29 \\
\hline$T(\boldsymbol{a}) \mathrm{FX} \# 4$ & 301.38 & 301.38 & 301.37 & 301.37 & 301.36 & 301.36 & 301.35 & 301.34 & 301.34 & 301.33 & 301.33 & 301.32 & 301.32 & 301.31 & 301.31 & 301.30 & 301.30 \\
\hline Wall $x$-coord & 114.89 & 114.89 & 114.89 & 114.90 & 114.90 & 114.90 & 114.90 & 114.90 & 114.90 & 114.90 & 114.90 & 114.90 & 114.90 & 114.90 & 114.90 & 114.90 & 114.91 \\
\hline Wall T & 304.93 & 304.94 & 304.94 & 304.95 & 304.94 & 304.94 & 304.95 & 304.96 & 304.97 & 304.99 & 305.00 & 305.01 & 305.03 & 305.04 & 305.06 & 305.07 & 305.07 \\
\hline Interferogram Image \# & 500 & Location & $T C 3$ & $\delta \varepsilon$ slope & $-2.50 E-3$ & $\delta \varepsilon$ intercept & 11.13 & $A v g F S$ & 110.59 & Avg Wall $T$ & 306.10 & Gradient error & $-2.47 E-6$ & & & & \\
\hline Scan y-coord & 4361 & 4362 & 4363 & 4364 & 4365 & 4366 & 4367 & 4368 & 4369 & 4370 & 4371 & 4372 & 4373 & 4374 & 4375 & 4376 & 4377 \\
\hline & 0.2115 & 0.2090 & 0.2065 & 0.2040 & 0.2015 & 0.1990 & 0.1964 & 0.1939 & 0.1914 & 0.1889 & 0.1864 & 0.1839 & 0.1814 & 0.1789 & 0.1764 & 0.1739 & 0.1714 \\
\hline Scan $x$-coord & 1215 & 1215 & 1215 & 1215 & 1215 & 1215 & 1215 & 1215 & 1215 & 1215 & 1215 & 1215 & 1215 & 1215 & 1215 & 1215 & 1215 \\
\hline$F X \# 1$ & 522.40 & 523.53 & 524.07 & 524.77 & 526.23 & 527.04 & 529.75 & 534.17 & 552.83 & 552.92 & 547.83 & 549.11 & 551.14 & 561.06 & 553.48 & 553.56 & 555.20 \\
\hline$F X \# 2$ & 306.95 & 307.11 & 307.32 & 307.56 & 307.81 & 308.11 & 308.38 & 308.63 & 516.42 & 517.03 & 309.59 & 309.75 & 309.80 & 530.41 & 310.20 & 310.43 & 310.73 \\
\hline$F X \# 3$ & 226.76 & 227.00 & 227.22 & 227.38 & 227.56 & 227.74 & 228.02 & 228.22 & 308.86 & 309.23 & 228.87 & 229.08 & 229.25 & 309.86 & 229.38 & 229.50 & 229.65 \\
\hline$F X \# 4$ & 171.22 & 171.38 & 171.44 & 171.55 & 171.64 & 171.72 & 171.77 & 171.80 & 228.43 & 228.64 & 172.06 & 172.25 & 172.44 & 229.34 & 172.91 & 173.10 & 173.34 \\
\hline$F X \# 5$ & 122.97 & 123.03 & 123.13 & 123.36 & 123.61 & 123.78 & 123.94 & 124.06 & 171.85 & 171.92 & 124.48 & 124.80 & 124.78 & 172.67 & 124.88 & 124.96 & 125.14 \\
\hline$F X \# 6$ & 0.00 & 0.00 & 0.00 & 0.00 & 0.00 & 0.00 & 0.00 & 0.00 & 124.22 & 124.33 & 0.00 & 0.00 & 0.00 & 124.81 & 0.00 & 0.00 & 0.00 \\
\hline Near wall FS & 122.97 & 123.03 & 123.13 & 123.36 & 123.61 & 123.78 & 123.94 & 124.06 & 47.63 & $\begin{array}{l}47.59 \\
\end{array}$ & $\begin{array}{l}124.48 \\
\end{array}$ & 124.80 & 124.78 & 47.86 & $\begin{array}{l}124.88 \\
124\end{array}$ & $\begin{array}{r}124.96 \\
\end{array}$ & 125.14 \\
\hline$T(F X \# 1$ & 297.15 & 297.15 & 297.15 & 297.15 & 297.15 & 297.15 & 297.15 & 297.15 & 297.15 & 297.15 & 297.15 & 297.15 & 297.15 & 297.15 & 297.15 & 297.15 & 297.15 \\
\hline$T @ F X \# 2$ & 297.57 & 297.56 & 297.56 & 297.55 & 297.55 & 297.54 & 297.54 & 297.53 & 297.53 & 297.52 & 297.52 & 297.51 & 297.51 & 297.50 & 297.50 & 297.49 & 297.49 \\
\hline$T$ a $F X \# 4$ & 301.57 & 301.56 & 301.56 & 301.55 & 301.55 & 301.54 & 301.54 & 301.53 & 301.53 & 301.52 & 301.52 & 301.51 & 301.51 & 301.50 & 301.50 & 301.49 & 301.49 \\
\hline$T @ F X \# 5$ & 303.61 & 303.61 & 303.60 & 303.59 & 303.59 & 303.58 & 303.58 & 303.57 & 303.57 & 303.56 & 303.56 & 303.55 & 303.55 & 303.54 & 303.54 & 303.53 & 303.53 \\
\hline$T @ F X \# 6$ & 305.68 & 305.67 & 305.67 & 305.66 & 305.66 & 305.65 & 305.65 & 305.64 & 305.64 & 305.63 & 305.63 & 305.62 & 305.62 & 305.61 & 305.61 & 305.60 & 305.60 \\
\hline Wall $x$-coord & 113.47 & 113.48 & 113.48 & 113.48 & 113.48 & 113.49 & 113.49 & 113.49 & 113.49 & 113.50 & 113.50 & 113.50 & 113.50 & 113.51 & 113.51 & 113.51 & 113.52 \\
\hline Wall $T$ & 306.09 & 306.08 & 306.08 & 306.09 & 306.10 & 306.10 & 306.10 & 306.10 & 306.10 & 306.10 & 306.11 & 306.11 & 306.11 & 306.10 & 306.10 & 306.09 & 306.10 \\
\hline Interferogram Image \# & 500 & Location & $T C 4$ & $\delta \varepsilon$ slope & $-2.52 E-3$ & $\delta \varepsilon$ intercept & 9.16 & $\operatorname{Avg} F S$ & 123.30 & Avg Wall $T$ & 306.59 & Gradient error & $-2.47 E-6$ & & & & \\
\hline Scan y-coord & 3547 & 3548 & 3549 & 3550 & 3551 & 3552 & 3553 & 3554 & 3555 & 3556 & 3557 & 3558 & 3559 & 3560 & 3561 & 3562 & 3563 \\
\hline
\end{tabular}




\begin{tabular}{|c|c|c|c|c|c|c|c|c|c|c|c|c|c|c|c|c|c|}
\hline$\delta \varepsilon$ & 0.2221 & 0.2195 & 0.2170 & 0.2145 & 0.2120 & 0.2095 & 0.2069 & 0.2044 & 0.2019 & 0.1994 & 0.1969 & 0.1943 & 0.1918 & 0.1893 & 0.1868 & 0.1843 & 0.1817 \\
\hline Scan $x$-coord & 1287 & 1287 & 1287 & 1287 & 1287 & 1287 & 1287 & 1287 & 1287 & 1287 & 1287 & 1287 & 1287 & 1287 & 1287 & 1287 & 1287 \\
\hline$F X \# 1$ & 541.55 & 542.76 & 543.93 & 544.39 & 547.17 & 547.41 & 548.24 & 548.38 & 552.55 & 552.63 & 553.64 & 559.40 & 560.28 & 561.17 & 558.78 & 560.32 & 560.97 \\
\hline$F X \# 2$ & 324.79 & 324.95 & 325.15 & 325.31 & 535.75 & 325.74 & 325.97 & 536.32 & 326.67 & 327.03 & 327.46 & 328.00 & 328.58 & 329.08 & 329.28 & 329.47 & 329.59 \\
\hline$F X \# 3$ & 240.70 & 240.88 & 241.01 & 241.17 & 325.49 & 241.45 & 241.61 & 326.31 & 241.88 & 241.94 & 242.00 & 242.07 & 242.19 & 242.29 & 242.43 & 242.64 & 242.81 \\
\hline$F X \# 4$ & 181.86 & 181.90 & 182.01 & 182.23 & 241.33 & 182.73 & 182.86 & 241.78 & 182.97 & 182.99 & 183.01 & 183.05 & 183.18 & 183.31 & 183.54 & 183.80 & 184.03 \\
\hline$F X \# 5$ & 132.40 & 132.61 & 132.73 & 132.77 & 182.50 & 132.72 & 132.75 & 182.94 & 132.85 & 132.96 & 133.14 & 133.31 & 133.44 & 133.51 & 133.56 & 133.63 & 133.77 \\
\hline$F X \# 6$ & 0.00 & 0.00 & 0.00 & 0.00 & 132.77 & 0.00 & 0.00 & 132.78 & 0.00 & 0.00 & 0.00 & 0.00 & 0.00 & 0.00 & 0.00 & 0.00 & 0.00 \\
\hline Near wall FS & 132.40 & 132.61 & 132.73 & 132.77 & 49.73 & 132.72 & 132.75 & 50.16 & 132.85 & 132.96 & 133.14 & 133.31 & 133.44 & 133.51 & 133.56 & 133.63 & 133.77 \\
\hline$T(F X \# 1$ & 297.15 & 297.15 & 297.15 & 297.15 & 297.15 & 297.15 & 297.15 & 297.15 & 297.15 & 297.15 & 297.15 & 297.15 & 297.15 & 297.15 & 297.15 & 297.15 & 297.15 \\
\hline$T @ F X \# 2$ & 297.59 & 297.58 & 297.58 & 297.57 & 297.57 & 297.56 & 297.56 & 297.55 & 297.55 & 297.54 & 297.54 & 297.53 & 297.53 & 297.52 & 297.52 & 297.51 & 297.51 \\
\hline$T(F X \# 3$ & 299.57 & 299.57 & 299.56 & 299.56 & 299.56 & 299.55 & 299.54 & 299.54 & 299.53 & 299.53 & 299.52 & 299.52 & 299.51 & 299.51 & 299.50 & 299.50 & 299.49 \\
\hline$T @ F X \# 4$ & 301.59 & 301.58 & 301.58 & 301.57 & 301.57 & 301.56 & 301.56 & 301.55 & 301.55 & 301.54 & 301.54 & 301.53 & 301.53 & 301.52 & 301.52 & 301.51 & 301.51 \\
\hline$T @ F X \# 5$ & 303.63 & 303.63 & 303.62 & 303.62 & 303.61 & 303.61 & 303.60 & 303.60 & 303.59 & 303.59 & 303.58 & 303.57 & 303.57 & 303.56 & 303.56 & 303.55 & 303.55 \\
\hline$T @ F X \# 6$ & 305.70 & 305.70 & 305.69 & 305.69 & 305.68 & 305.68 & 305.67 & 305.67 & 305.66 & 305.65 & 305.65 & 305.64 & 305.64 & 305.63 & 305.63 & 305.62 & 305.62 \\
\hline Wall $x$-coord & 110.52 & 110.53 & 110.53 & 110.53 & 110.54 & 110.54 & 110.55 & 110.55 & 110.56 & 110.56 & 110.57 & 110.57 & 110.58 & 110.58 & 110.58 & 110.59 & 110.59 \\
\hline Wall T & 306.62 & 306.62 & 306.62 & 306.62 & 306.61 & 306.59 & 306.59 & 306.58 & 306.58 & 306.58 & 306.59 & 306.59 & 306.59 & 306.59 & 306.58 & 306.57 & 306.57 \\
\hline Interferogram Image \# & 500 & Location & $T C 5$ & $\delta \varepsilon$ slope & $-2.17 E-3$ & $\delta \varepsilon$ intercept & 6.14 & $\operatorname{Avg} F S$ & 123.11 & Avg Wall $T$ & 307.06 & Gradient error & $-2.47 E-6$ & & & & \\
\hline Scan y-coord & 2732 & 2733 & 2734 & 2735 & 2736 & 2737 & 2738 & 2739 & 2740 & 2741 & 2742 & 2743 & 2744 & 2745 & 2746 & 2747 & 2748 \\
\hline & 0.2034 & 0.2012 & 0.1990 & 0.1969 & 0.1947 & 0.1925 & 0.1904 & 0.1882 & 0.1860 & 0.1838 & 0.1817 & 0.1795 & 0.1773 & 0.1751 & 0.1730 & 0.1708 & 0.1686 \\
\hline Scan $x$-coord & 1127 & 1127 & 1127 & 1127 & 1127 & 1127 & 1127 & 1127 & 1127 & 1127 & 1127 & 1127 & 1127 & 1127 & 1127 & 1127 & 1127 \\
\hline$F X \# 1$ & 617.16 & 619.64 & 622.13 & 625.29 & 630.06 & 630.79 & 650.15 & 640.06 & 646.38 & 640.59 & 641.05 & 641.62 & 645.16 & 643.88 & 648.90 & 650.13 & 651.29 \\
\hline$F X \# 2$ & 356.28 & 356.35 & 356.44 & 356.62 & 356.85 & 357.09 & 617.74 & 357.63 & 621.55 & 358.29 & 358.54 & 359.00 & 624.96 & 626.42 & 359.93 & 360.21 & 360.59 \\
\hline$F X \# 3$ & 261.76 & 261.79 & 261.85 & 261.88 & 261.93 & 262.02 & 357.46 & 262.34 & 357.96 & 262.93 & 263.28 & 263.49 & 359.46 & 359.82 & 263.84 & 263.98 & 264.13 \\
\hline$F X \# 4$ & 197.73 & 197.87 & 198.03 & 198.15 & 198.34 & 198.57 & 262.15 & 198.96 & 262.64 & 199.30 & 199.46 & 199.70 & 263.60 & 263.66 & 200.28 & 200.37 & 200.47 \\
\hline$F X \# 5$ & 143.13 & 143.21 & 143.34 & 143.46 & 143.66 & 143.82 & 198.74 & 143.99 & 199.12 & 144.22 & 144.40 & 144.55 & 199.99 & 200.18 & 144.93 & 144.92 & 144.84 \\
\hline$F X \# 6$ & 0.00 & 0.00 & 0.00 & 0.00 & 0.00 & 0.00 & 143.91 & 0.00 & 144.12 & 0.00 & 0.00 & 0.00 & 144.70 & 144.83 & 0.00 & 0.00 & 0.00 \\
\hline Near wall FS & 143.13 & 143.21 & 143.34 & 143.46 & 143.66 & 143.82 & 54.84 & 143.99 & 55.00 & 144.22 & 144.40 & 144.55 & 55.28 & 55.34 & 144.93 & 144.92 & 144.84 \\
\hline$T @ F X \# 1$ & 297.15 & 297.15 & 297.15 & 297.15 & 297.15 & 297.15 & 297.15 & 297.15 & 297.15 & 297.15 & 297.15 & 297.15 & 297.15 & 297.15 & 297.15 & 297.15 & 297.15 \\
\hline$T @ F X \# 2$ & 297.55 & 297.55 & 297.54 & 297.54 & 297.53 & 297.53 & 297.53 & 297.52 & 297.52 & 297.51 & 297.51 & 297.50 & 297.50 & 297.49 & 297.49 & 297.49 & 297.48 \\
\hline$T$ (a) $F X \# 3$ & 299.54 & 299.53 & 299.53 & 299.52 & 299.52 & 299.52 & 299.51 & 299.51 & 299.50 & 299.50 & 299.49 & 299.49 & 299.49 & 299.48 & 299.48 & 299.47 & 299.47 \\
\hline$T$ (a) $F X \# 4$ & 301.55 & 301.55 & 301.54 & 301.54 & 301.53 & 301.53 & 301.53 & 301.52 & 301.52 & 301.51 & 301.51 & 301.50 & 301.50 & 301.50 & 301.49 & 301.49 & 301.48 \\
\hline$T(F X \# 5$ & 303.59 & 303.59 & 303.58 & 303.58 & 303.58 & 303.57 & 303.57 & 303.56 & 303.56 & 303.55 & 303.55 & 303.54 & 303.54 & 303.54 & 303.53 & 303.53 & 303.52 \\
\hline$T @ F X \# 6$ & 305.66 & 305.66 & 305.65 & 305.65 & 305.65 & 305.64 & 305.64 & 305.63 & 305.63 & 305.62 & 305.62 & 305.61 & 305.61 & 305.60 & 305.60 & 305.60 & 305.59 \\
\hline Wall $x$-coord & 106.03 & 106.04 & 106.04 & 106.05 & 106.06 & 106.06 & 106.07 & 106.07 & 106.08 & 106.09 & 106.09 & 106.10 & 106.11 & 106.11 & 106.12 & 106.13 & 106.13 \\
\hline Wall $T$ & 307.07 & 307.07 & 307.07 & 307.07 & 307.07 & 307.07 & 307.06 & 307.06 & 307.06 & 307.06 & 307.06 & 307.06 & 307.05 & 307.05 & 307.05 & 307.04 & 307.03 \\
\hline Interferogram Image \# & 500 & Location & $T C 6$ & $\delta \varepsilon$ slope & $-1.97 E-3$ & $\delta \varepsilon$ intercept & 4.70 & $\operatorname{Avg} F S$ & 51.07 & Avg Wall $T$ & 307.53 & Gradient error & $-2.47 E-6$ & & & & \\
\hline Scan y-coord & 1918 & 1919 & 1920 & 1921 & 1922 & 1923 & 1924 & 1925 & 1926 & 1927 & 1928 & 1929 & 1930 & 1931 & 1932 & 1933 & 1934 \\
\hline & 0.9147 & 0.9127 & 0.9107 & 0.9088 & 0.9068 & 0.9048 & 0.9028 & 0.9009 & 0.8989 & 0.8969 & 0.8950 & 0.8930 & 0.8910 & 0.8890 & 0.8871 & 0.8851 & 0.8831 \\
\hline Scan $x$-coord & 971 & 971 & 971 & 971 & 971 & 971 & 971 & 971 & 971 & 971 & 971 & 971 & 971 & 971 & 971 & 971 & 971 \\
\hline$F X \# 1$ & 386.18 & 386.30 & 386.20 & 386.12 & 386.09 & 386.23 & 386.57 & 386.84 & 387.46 & 387.79 & 388.27 & 388.79 & 389.25 & 389.89 & 389.85 & 390.09 & 390.03 \\
\hline$F X \# 2$ & 286.67 & 286.76 & 286.81 & 286.81 & 286.74 & 286.71 & 286.72 & 286.80 & 287.05 & 287.32 & 287.66 & 287.99 & 288.38 & 288.82 & 289.18 & 289.44 & 289.51 \\
\hline$F X \# 3$ & 216.08 & 216.30 & 216.53 & 216.76 & 217.05 & 217.25 & 217.42 & 217.48 & 217.58 & 217.68 & 217.77 & 217.89 & 218.02 & 218.11 & 218.11 & 218.12 & 218.21 \\
\hline$F X \# 4$ & 160.22 & 160.34 & 160.41 & 160.44 & 160.59 & 160.66 & 160.79 & 161.02 & 161.37 & 161.55 & 161.59 & 161.60 & 161.60 & 161.58 & 161.56 & 161.59 & 161.66 \\
\hline$F X \# 5$ & 109.65 & 109.26 & 109.07 & 109.15 & 109.47 & 109.79 & 110.01 & 110.14 & 110.13 & 110.00 & 110.02 & 110.13 & 110.44 & 110.80 & 110.88 & 110.84 & 110.57 \\
\hline Near wall FS & 50.57 & 51.08 & 51.34 & 51.28 & 51.12 & 50.87 & 50.77 & 50.89 & 51.24 & 51.54 & 51.57 & 51.48 & 51.16 & 50.79 & 50.68 & 50.75 & 51.09 \\
\hline$T @ F X \# 1$ & 297.15 & 297.15 & 297.15 & 297.15 & 297.15 & 297.15 & 297.15 & 297.15 & 297.15 & 297.15 & 297.15 & 297.15 & 297.15 & 297.15 & 297.15 & 297.15 & 297.15 \\
\hline$T @ F X \# 2$ & 298.96 & 298.96 & 298.95 & 298.95 & 298.95 & 298.94 & 298.94 & 298.93 & 298.93 & 298.93 & 298.92 & 298.92 & 298.91 & 298.91 & 298.91 & 298.90 & 298.90 \\
\hline$T(F X \# 3$ & 300.97 & 300.96 & 300.96 & 300.96 & 300.95 & 300.95 & 300.94 & 300.94 & 300.94 & 300.93 & 300.93 & 300.92 & 300.92 & 300.92 & 300.91 & 300.91 & 300.90 \\
\hline$T$ (a) $F X \# 4$ & 303.00 & 303.00 & 302.99 & 302.99 & 302.99 & 302.98 & 302.98 & 302.97 & 302.97 & 302.97 & 302.96 & 302.96 & 302.95 & 302.95 & 302.95 & 302.94 & 302.94 \\
\hline$T$ (a) FX\#5 & 305.06 & 305.06 & 305.06 & 305.05 & 305.05 & 305.04 & 305.04 & 305.03 & 305.03 & 305.03 & 305.02 & 305.02 & 305.01 & 305.01 & 305.01 & 305.00 & 305.00 \\
\hline Wall $x$-coord & 100.01 & 100.02 & 100.03 & 100.04 & 100.04 & 100.05 & 100.06 & 100.07 & 100.08 & 100.09 & 100.09 & 100.10 & 100.11 & 100.12 & 100.13 & 100.14 & 100.14 \\
\hline Wall $T$ & 307.55 & 307.53 & 307.51 & 307.51 & 307.52 & 307.53 & 307.54 & 307.54 & 307.53 & 307.52 & 307.51 & 307.51 & 307.53 & 307.54 & 307.54 & 307.53 & 307.51 \\
\hline Interferogram Image \# & 500 & Location & $T C 7$ & $\delta \varepsilon$ slope & $-2.09 E-3$ & $\delta \varepsilon$ intercept & 3.02 & $\operatorname{Avg} F S$ & 53.19 & Avg Wall $T$ & 307.80 & Gradient error & $-2.47 E-6$ & & & & \\
\hline Scan y-coord & 1103 & 1104 & 1105 & 1106 & 1107 & 1108 & 1109 & 1110 & 1111 & 1112 & 1113 & 1114 & 1115 & 1116 & 1117 & 1118 & 1119 \\
\hline & 0.7147 & 0.7127 & 0.7106 & 0.7085 & 0.7064 & 0.7043 & 0.7022 & 0.7001 & 0.6980 & 0.6960 & 0.6939 & 0.6918 & 0.6897 & 0.6876 & 0.6855 & 0.6834 & 0.6814 \\
\hline Scan $x$-coord & 1007 & 1007 & 1007 & 1007 & 1007 & 1007 & 1007 & 1007 & 1007 & 1007 & 1007 & 1007 & 1007 & 1007 & 1007 & 1007 & 1007 \\
\hline$F X \# 1$ & 441.70 & 442.14 & 442.85 & 443.37 & 444.11 & 444.77 & 445.43 & 446.05 & 446.67 & 447.36 & 447.62 & 447.58 & 448.04 & 448.20 & 448.65 & 448.99 & 449.15 \\
\hline$F X \# 2$ & 311.73 & 311.84 & 311.85 & 311.80 & 311.93 & 312.14 & 312.40 & 312.61 & 312.89 & 313.20 & 313.49 & 313.66 & 313.68 & 313.82 & 314.09 & 314.42 & 314.64 \\
\hline$F X \# 3$ & 233.81 & 233.94 & 234.12 & 234.29 & 234.54 & 234.79 & 234.97 & 235.14 & 235.21 & 235.32 & 235.38 & 235.39 & 235.39 & 235.40 & 235.50 & 235.63 & 235.74 \\
\hline$F X \# 4$ & 173.22 & 173.30 & 173.33 & 173.36 & 173.43 & 173.50 & 173.54 & 173.59 & 173.67 & 173.88 & 174.07 & 174.23 & 174.40 & 174.65 & 174.76 & 174.84 & 174.90 \\
\hline$F X \# 5$ & 120.32 & 120.43 & 120.56 & 120.63 & 120.65 & 120.47 & 120.47 & 120.48 & 120.50 & 120.62 & 120.64 & 120.68 & 120.76 & 121.02 & 121.23 & 121.41 & 121.49 \\
\hline Near wall FS & 52.90 & 52.87 & 52.77 & 52.73 & 52.78 & 53.03 & 53.08 & 53.11 & 53.17 & 53.26 & 53.43 & 53.55 & 53.64 & 53.63 & 53.53 & 53.44 & 53.41 \\
\hline$T(F X \# 1$ & 297.15 & 297.15 & 297.15 & 297.15 & 297.15 & 297.15 & 297.15 & 297.15 & 297.15 & 297.15 & 297.15 & 297.15 & 297.15 & 297.15 & 297.15 & 297.15 & 297.15 \\
\hline$T @ F X \# 2$ & 298.56 & 298.56 & 298.56 & 298.55 & 298.55 & 298.54 & 298.54 & 298.53 & 298.53 & 298.53 & 298.52 & 298.52 & 298.51 & 298.51 & 298.51 & 298.50 & 298.50 \\
\hline$T(F X \# 3$ & 300.56 & 300.56 & 300.56 & 300.55 & 300.55 & 300.54 & 300.54 & 300.54 & 300.53 & 300.53 & 300.52 & 300.52 & 300.51 & 300.51 & 300.51 & 300.50 & 300.50 \\
\hline$T @ F X \# 4$ & 302.59 & 302.59 & 302.58 & 302.58 & 302.58 & 302.57 & 302.57 & 302.56 & 302.56 & 302.55 & 302.55 & 302.55 & 302.54 & 302.54 & 302.53 & 302.53 & 302.52 \\
\hline$T$ @ $F X \# 5$ & 304.65 & 304.64 & 304.64 & 304.64 & 304.63 & 304.63 & 304.62 & 304.62 & 304.61 & 304.61 & 304.61 & 304.60 & 304.60 & 304.59 & 304.59 & 304.58 & 304.58 \\
\hline Wall $x$-coord & 92.45 & 92.46 & 92.47 & 92.48 & 92.49 & 92.50 & 92.51 & 92.52 & 92.53 & 92.54 & 92.55 & 92.56 & 92.57 & 92.58 & 92.59 & 92.60 & 92.61 \\
\hline
\end{tabular}




\section{B.45 Tabulated Data for Interferogram 505}

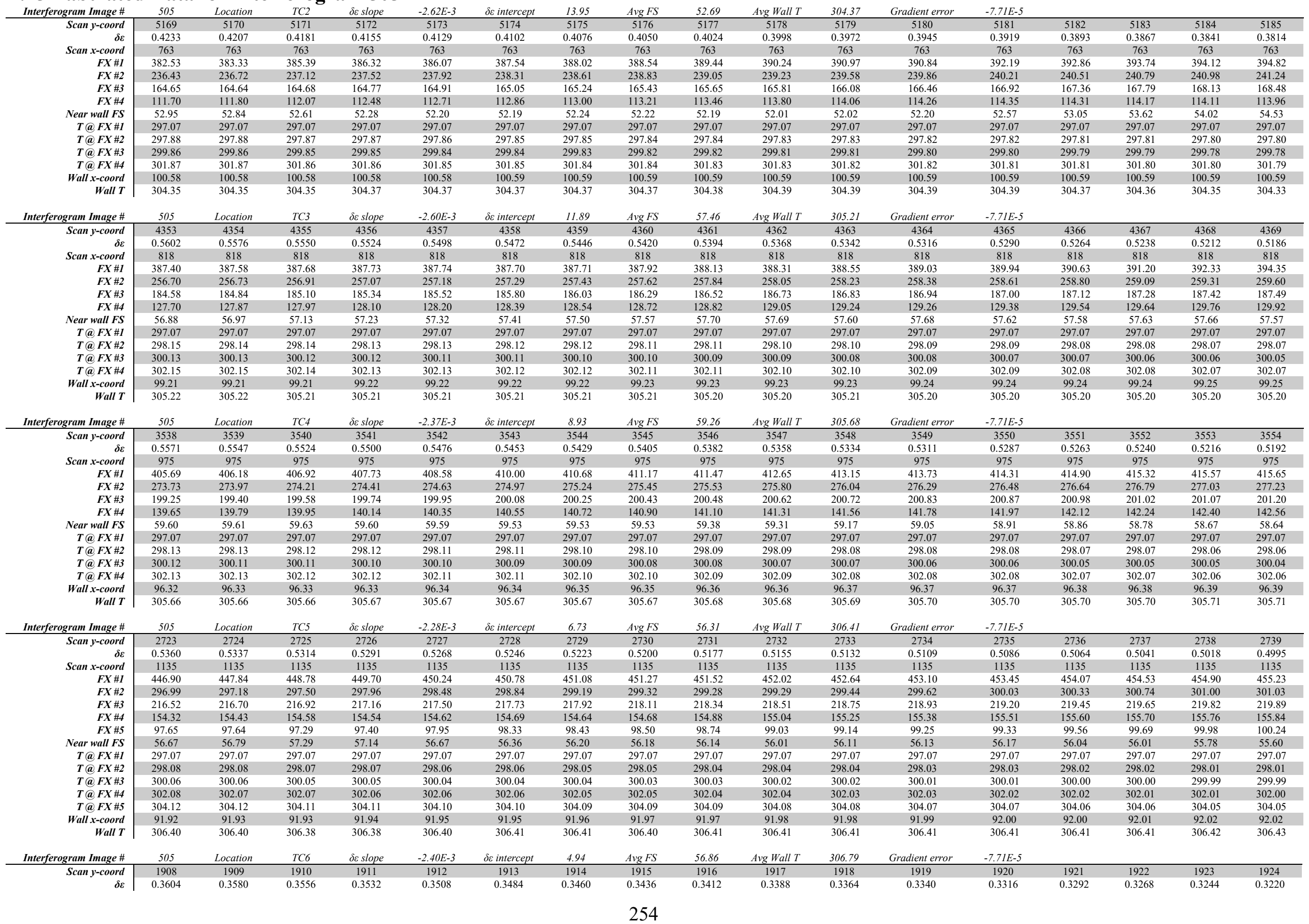




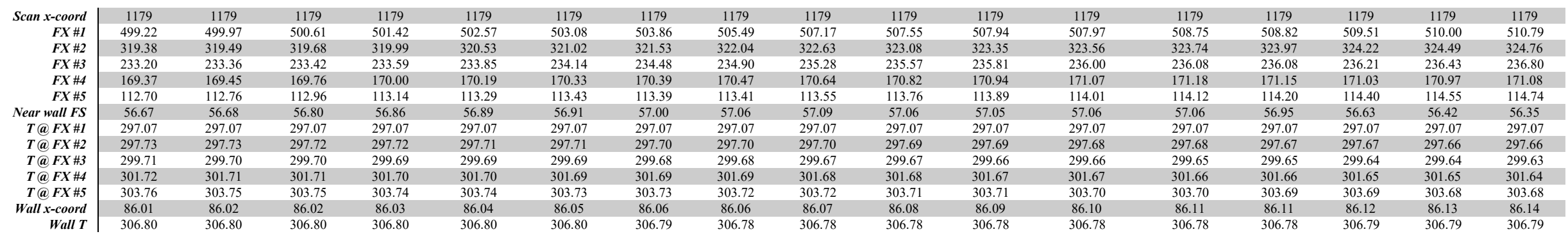

\begin{tabular}{|c|c|c|c|c|c|c|c|c|c|c|c|c|c|c|c|c|c|}
\hline Interferogram Image \# & 505 & Location & $T C 7$ & $\delta \varepsilon$ slope & $-2.31 E-3$ & $\delta$ s intercept & 2.66 & $\operatorname{Avg} F S$ & 60.65 & Avg Wall T & 306.82 & Gradient error & $-7.71 E-5$ & & & & \\
\hline Scan $y$-coord & 1093 & 1094 & 1095 & 1096 & 1097 & 1098 & 1099 & 1100 & 1101 & 1102 & 1103 & 1104 & 1105 & 1106 & 1107 & 1108 & 1109 \\
\hline & 0.1343 & 0.1320 & 0.1297 & 0.1274 & 0.1251 & 0.1228 & 0.1204 & 0.1181 & 0.1158 & 0.1135 & 0.1112 & 0.1089 & 0.1066 & 0.1043 & 0.1019 & 0.0996 & 0.0973 \\
\hline Scan $x$-coord & 1194 & 1194 & 1194 & 1194 & 1194 & 1194 & 1194 & 1194 & 1194 & 1194 & 1194 & 1194 & 1194 & 1194 & 1194 & 1194 & 1194 \\
\hline$F X \# 1$ & 672.22 & 673.71 & 675.23 & 679.62 & 670.58 & 672.18 & 691.57 & 693.68 & 694.41 & 696.14 & 697.24 & 699.13 & 700.13 & 701.73 & 703.43 & 704.78 & 719.87 \\
\hline$F$ & 358.53 & 358.78 & 359.11 & 359.68 & 360.31 & 360.68 & 360.93 & 361.08 & 361.27 & 361.09 & 361.15 & 361.44 & 361.45 & 361.56 & 361.68 & 361.78 & 362.14 \\
\hline$F X \# 3$ & 252.30 & 252.40 & 252.49 & 252.58 & 252.73 & 252.91 & 253.13 & 253.38 & 253.69 & 253.99 & 254.23 & 254.33 & 254.37 & 254.37 & 254.46 & 254.65 & 254.95 \\
\hline$F X \# 4$ & 182.83 & 182.95 & 183.09 & 183.14 & 183.11 & 183.06 & 183.07 & 183.20 & 183.35 & 183.49 & 183.64 & 183.77 & 183.86 & 184.00 & 183.98 & 184.07 & 184.02 \\
\hline$F X \# 5$ & 121.95 & 122.05 & 122.11 & 122.14 & 122.21 & 122.26 & 122.33 & 122.40 & 122.57 & 122.80 & 123.05 & 123.24 & 123.38 & 123.52 & 123.72 & 123.87 & 123.98 \\
\hline Near wall FS & 60.89 & 60.90 & 60.98 & 61.00 & 60.90 & 60.79 & 60.75 & 60.80 & 60.78 & 60.69 & 60.59 & 60.53 & 60.47 & 60.47 & 60.26 & 60.20 & 60.05 \\
\hline$T$ (a) FX \#1 & 297.07 & 297.07 & 297.07 & 297.07 & 297.07 & 297.07 & 297.07 & 297.07 & 297.07 & 297.07 & 297.07 & 297.07 & 297.07 & 297.07 & 297.07 & 297.07 & 297.07 \\
\hline$T(F X \# 2$ & 297.30 & 297.29 & 297.29 & 297.29 & 297.28 & 297.28 & 297.27 & 297.27 & 297.26 & 297.26 & 297.25 & 297.25 & 297.25 & 297.24 & 297.24 & 297.23 & 297.23 \\
\hline$T(a) F X \# 3$ & 299.26 & 299.25 & 299.25 & 299.24 & 299.24 & 299.24 & 299.23 & 299.23 & 299.22 & 299.22 & 299.21 & 299.21 & 299.20 & 299.20 & 299.19 & 299.19 & 299.19 \\
\hline$T(F X \# 4$ & 301.26 & 301.25 & 301.25 & 301.25 & 301.24 & 301.24 & 301.23 & 301.23 & 301.22 & 301.22 & 301.21 & 301.21 & 301.21 & 301.20 & 301.20 & 301.19 & $\begin{array}{l}2991.19 \\
301.19\end{array}$ \\
\hline$T(\boldsymbol{F} X \# 5$ & 303.29 & 303.29 & 303.28 & 303.28 & 303.27 & 303.27 & 303.27 & 303.26 & 303.26 & 303.25 & 303.25 & 303.24 & 303.24 & 303.23 & 303.23 & 303.22 & 303.22 \\
\hline Wall $x$-coord & 78.58 & 78.59 & 78.60 & 78.61 & 78.62 & 78.63 & 78.64 & 78.65 & 78.66 & 78.67 & 78.68 & 78.69 & 78.70 & 78.71 & 78.72 & 78.73 & 78.74 \\
\hline Wall T & 306.82 & 306.82 & 306.82 & 306.81 & 306.81 & 306.81 & 306.81 & 306.80 & 306.80 & 306.81 & 306.82 & 306.82 & 306.82 & 306.82 & 306.83 & 306.83 & 306.83 \\
\hline
\end{tabular}

\section{B.46 Tabulated Data for Interferogram 510}

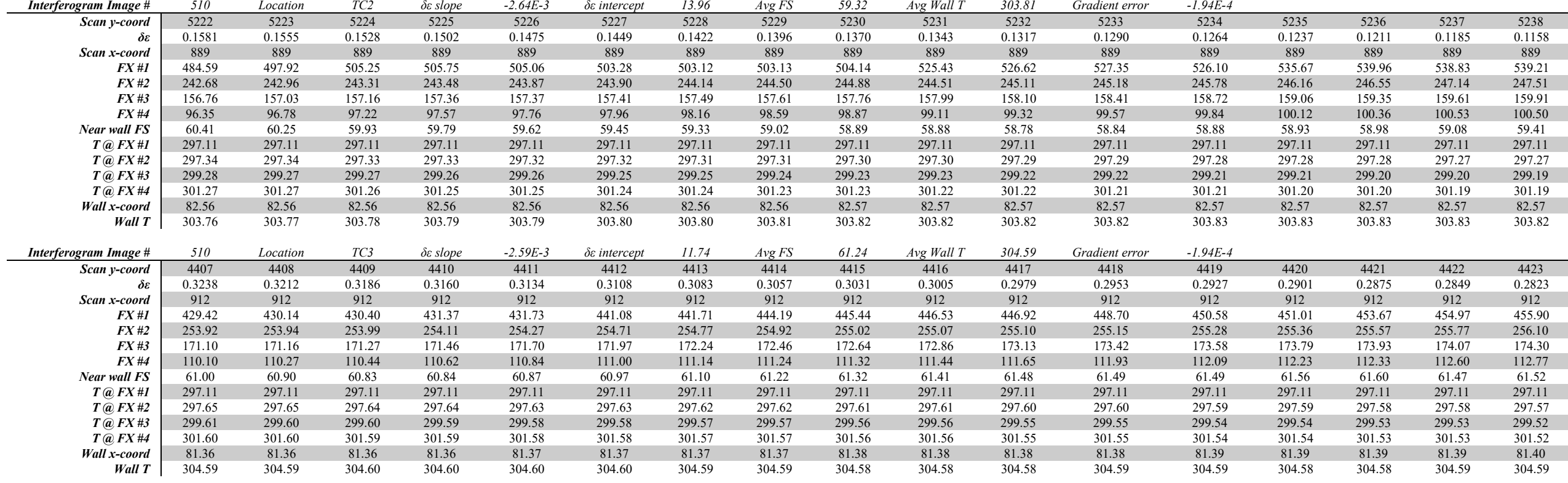

\begin{tabular}{|c|c|c|c|c|c|c|c|c|c|c|c|c|c|c|c|c|c|}
\hline Interferogram Image \# & 510 & Location & $T C 4$ & $\delta \varepsilon$ slope & $-2.53 E-3$ & $\delta \varepsilon$ intercept & 9.46 & $A v g F S$ & 64.34 & Avg Wall T & 305.01 & Gradient error & $-1.94 E-4$ & & & & \\
\hline Scan y-coord & 3593 & 3594 & 3595 & 3596 & 3597 & 3598 & 3599 & 3600 & 3601 & 3602 & 3603 & 3604 & 3605 & 3606 & 3607 & 3608 & 3609 \\
\hline & 0.3831 & 0.3806 & 0.3781 & 0.3755 & 0.3730 & 0.3705 & 0.3680 & 0.3654 & 0.3629 & 0.3604 & 0.3579 & 0.3553 & 0.3528 & 0.3503 & 0.3477 & 0.3452 & 0.3427 \\
\hline Scan $x$-coord & 986 & 986 & 986 & 986 & 986 & 986 & 986 & 986 & 986 & 986 & 986 & 986 & 986 & 986 & 986 & 986 & 986 \\
\hline$F X \# I$ & 442.68 & 443.71 & 444.73 & 446.82 & 446.93 & 447.41 & 448.02 & 448.82 & 450.66 & 451.48 & 451.97 & 452.68 & 453.22 & 453.92 & 454.25 & 454.81 & 455.41 \\
\hline
\end{tabular}




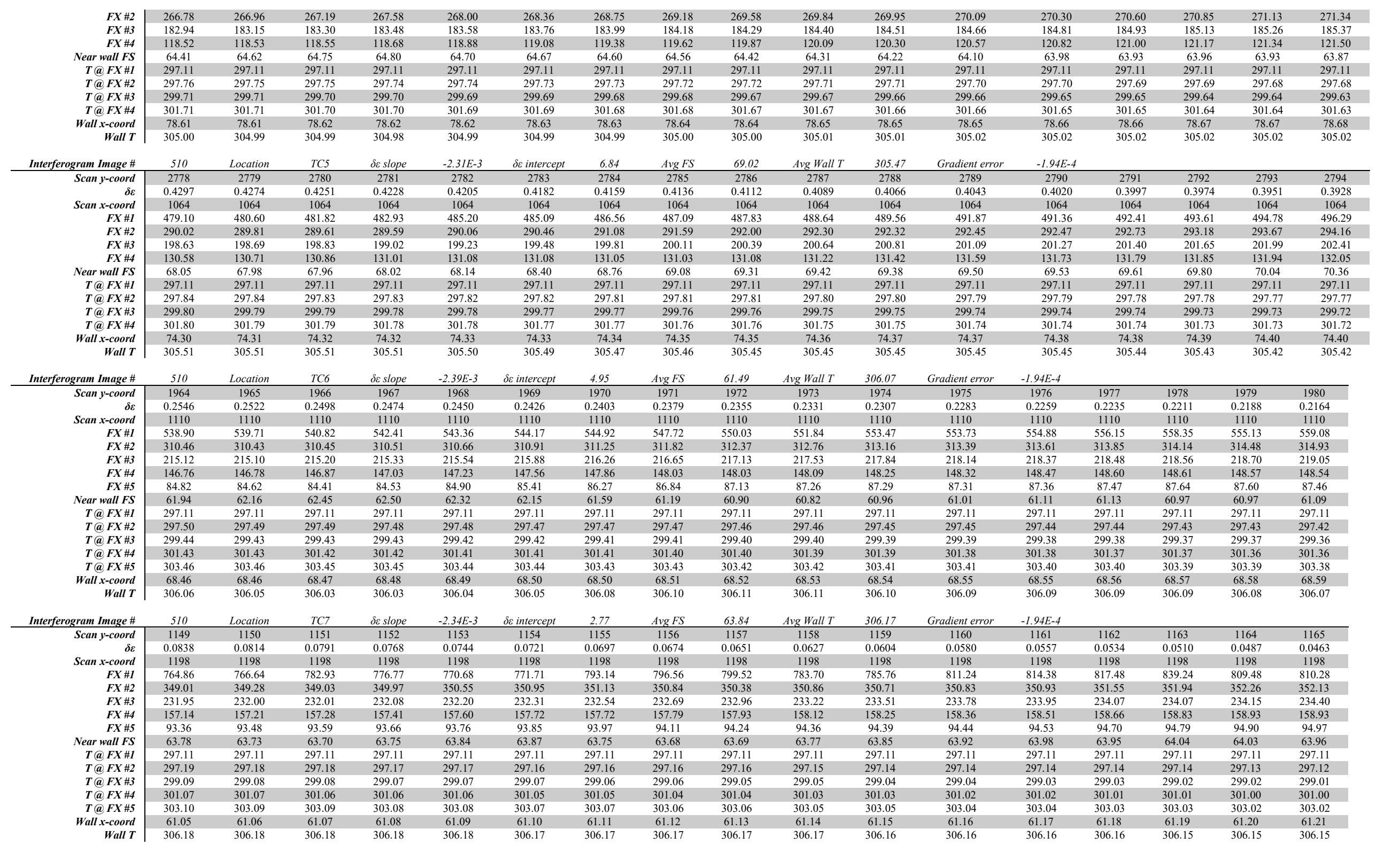




\section{References}

Almeida, F., Naylor, D. and Oosthuizen, P. H. (2008). An interferometric study of free convection in a window with a heated between-panes blind. 3rd Canadian Solar Buildings Research Network Conference, Fredericton.

ANSYS FLUENT (Version 13.0) [computer software], 2010, ANSYS, Inc..

ANSYS FLUENT Theory Guide, 2011, Section 4.13: Near-wall treaments for wall-bounded turbulent flows, ANSYS, Inc., Canonsburg, United States, pp. $119-136$.

ANSYS FLUENT User's Guide, 2010 Chapter 13: Modelling turbulence, ANSYS, Inc., Canonsburg, United States, pp. $642-690$.

Ascione, F., \& Minichiello, F. (2010). Microclimatic control in the museum environment: Air diffusion performance. International Journal of Refrigeration, 33(4), 806-814.

Awbi, H. B. (1998). Calculation of convective heat transfer coefficients of room surfaces for natural convection. Energy and Buildings, 28(2), 219-227.

Balocco, C., \& Grazzini, G. (2009). Numerical simulation of ancient natural ventilation systems of historical buildings. A case study in palermo. Journal of Cultural Heritage, 10(2), 313318.

Bartak, M., Beausoleil-Morrison, I., Clarke, J. A., Denev, J., Drkal, F., Lain, M., et al. (2002). Integrating CFD and building simulation. Building and Environment, 37(8-9), 865-871.

Beausoleil-Morrison, I. (2002). The adaptive conflation of computational fluid dynamics with whole-building thermal simulation. Energy and Buildings, 34(9), 857-871.

Chen, Q. (1995). Comparison of different k- $\varepsilon$ models for indoor air flow computations. Numerical Heat Transfer, Part B: Fundamentals, 28(3), 353-369.

Chen, Q. (2009). Ventilation performance prediction for buildings: A method overview and recent applications. Building and Environment, 44(4), 848-858.

Chen, Q., \& Xu, W. (1998). A zero-equation turbulence model for indoor airflow simulation. Energy and Buildings, 28(2), 137-144.

Cui, Y., Liu, C., Hu, S., \& Yu, X. (2011). The experimental exploration of carbon nanofiber and carbon nanotube additives on thermal behavior of phase change materials. Solar Energy Materials and Solar Cells, 95(4), 1208-1212.

Churchill, S.W., Chu, H.H.S., 1975. Correlating equations for laminar and turbulent free convection from a vertical plate. International Journal of Heat and Mass Transfer 18, 1323-1329. 
Clarke J.A. (1985), Energy simulation in building design, Adam Hilger Ltd, Bristol and Boston.

Coussirat, M., Guardo, A., Jou, E., Egusquiza, E., Cuerva, E., \& Alavedra, P. (2008). Performance and influence of numerical sub-models on the CFD simulation of free and forced convection in double-glazed ventilated façades. Energy and Buildings, 40(10), 1781-1789.

Cunha, A. M., Teixeira, J. C., \& Teixeira, S. F. (2009, January). Computational fluid dynamics applicable to cloth design. ASME 2009 International Mechanical Engineering Congress and Exposition (pp. 233-241). American Society of Mechanical Engineers.

Defraeye, T., Blocken, B., \& Carmeliet, J. (2010). CFD analysis of convective heat transfer at the surfaces of a cube immersed in a turbulent boundary layer. International Journal of Heat and Mass Transfer, 53(1-3), 297-308.

Domingo, J., Barbero, R., Iranzo, A., Cuadra, D., Servert, J., \& Marcos, M. A. (2011). Analysis and optimization of ventilation systems for an underground transport interchange building under regular and emergency scenarios. Tunnelling and Underground Space Technology, 26(1), 179188.

El-Sadi, H., Haghighat, F., \& Fallahi, A. (2010). CFD analysis of turbulent natural ventilation in double-skin fạade: Thermal mass and energy efficiency. Journal of Energy Engineering, 136(3), 68-75.

ElSherbiny, S. M., Raithby, G. D., and Hollands, K. G. T. (1982). Heat transfer by natural convection across vertical and inclined air layers. Journal of Heat Transfer, 104(1), 96-102.

Faye C., McQuinston, Jerald D. Parker and Jeffrey D. Spitler, (2005). Heating, ventilating and air conditioning, analysis and design, 6th Edition, John Wiley \& Sons.

Ferziger, J. H. (1990). Approaches to turbulent flow computation: Applications to flow over obstacles. Journal of Wind Engineering and Industrial Aerodynamics, 35(1-3), 1-19.

Gao, C. F., \& Lee, W. L. (2011). Evaluating the influence of openings configuration on natural ventilation performance of residential units in Hong Kong. Building and Environment, 46(4), 961969.

Gao, J., Zhang, X., \& Zhao, J. N. (2008). Numerical determination of convection coefficients for internal surfaces in buildings dominated by thermally stratified flows. Journal of Building Physics, 31(3), 213-223.

Gunther, E. (2007). Modeling of subcooling and solidification of phase change materials. Modelling and Simulation in Materials Science and Engineering, 15(8), 879-92.

Gunther, E. (2009). Enthalpy of phase change materials as a function of temperature: required accuracy and suitable measurement methods. International Journal of Thermophysics, 30(4), 1257-69. 
Hooff, T. V., \& Blocken, B. (2010). On the effect of wind direction and urban surroundings on natural ventilation of a large semi-enclosed stadium. Computers and Fluids, 39(7), 1146-1155.

Jiang, Y., \& Chen, Q. (2003). Buoyancy-driven single-sided natural ventilation in buildings with large openings. International Journal of Heat and Mass Transfer, 46(6), 973-988.

Kolmogorov, A. N. (1941). The local structure of turbulence in incompressible viscous fluid for very large reynolds numbers. Dokl.Akad.Nauk SSSR, 30, 301-305.

Koskela, H., Häggblom, H., Kosonen, R., \& Ruponen, M. (2010). Air distribution in office environment with asymmetric workstation layout using chilled beams. Building and Environment, 45(9), 1923-1931.

Launder, B. E., \& Spalding, D. B. (1974). The numerical computation of turbulent flows. Computer Methods in Applied Mechanics and Engineering, 3(2), 269-289.

Lien, S. -. J., \& Ahmed, N. A. (2010). Numerical simulation of rooftop ventilator flow. Building and Environment, 45(8), 1808-1815.

Lo, L. J., \& Novoselac, A. (2010). Localized air-conditioning with occupancy control in an open office. Energy and Buildings, 42(7), 1120-1128.

Mehling, H., \& Cabeza, L.F. (2008). Heat and cold storage with PCM: An up to date introduction into basics and applications. Springer.

Morey, S., Coley, D., \& Kershaw, T. (2010). Accessing the thermal mass above suspended ceilings via a perimeter gap: A CFD study of naturally ventilated spaces. International Journal of Ventilation, 9(2), 163-175.

Negra o C.O.R. (1995), Conflation of computational fluid dynamics and building thermal simulation, PhD Thesis, University of Strathclyde, Glasgow UK.

Negrão, C. O. R. (1998). Integration of computational fluid dynamics with building thermal and mass flow simulation. Energy and Buildings, 27(2), 155-165.

Nore, K., Blocken, B., \& Thue, J. V. (2010). On CFD simulation of wind-induced airflow in narrow ventilated facade cavities: Coupled and decoupled simulations and modelling limitations. Building and Environment, 45(8), 1834-1846.

Norton, T., Grant, J., Fallon, R., \& Sun, D. -. (2010). A computational fluid dynamics study of air mixing in a naturally ventilated livestock building with different porous eave opening conditions. Biosystems Engineering, 106(2), 125-137.

Omri, M., \& Galanis, N. (2010). Prediction of 3D airflow and temperature field in an indoor ice rink with radiant heat sources. Building Simulation, , 1-11.

Patankar S.V. (1980), Numerical Heat Transfer and Fluid Flow, McGraw-Hill. 
Plikas, T., Cesta, T., Gunnewiek, L., \& Vanasse, J. (2010). Casthouse ventilation design for the production of air-cooled aluminium sows. International Journal of Ventilation, 9(1), 69-76.

Ploskić, A., \& Holmberg, S. (2011). Low-temperature baseboard heaters with integrated air supply - an analytical and numerical investigation. Building and Environment, 46(1), 176-186.

Posner, J. D., Buchanan, C. R., \& Dunn-Rankin, D. (2003). Measurement and prediction of indoor air flow in a model room. Energy and Buildings, 35(5), 515-526.

Shewen, E., Hollands, K.G.T., and Raithby, G.D. (1996). Heat transfer by natural convection across a vertical air cavity of large aspect ratio. Journal of Heat Transfer, 118(4), 993-995.

Solomon, G. R. (2013). Sub cooling of PCM due to various effects during solidification in a vertical concentric tube thermal storage unit. Applied Thermal Engineering, 52(2), 505-11.

Van Belleghem, M., Steeman, H. -., Steeman, M., Janssens, A., \& De Paepe, M. (2010). Sensitivity analysis of CFD coupled non-isothermal heat and moisture modelling. Building and Environment, 45(11), 2485-2496.

Van Doormaal, J.P. and Raithby, G.D., 1984, G.D. Enhancement of the simple method for predicting incompressible flows, Numerical Heat Transfer, Vol. 7, No. 2, pp. $147-163$.

Versteeg H.K. and Malalasekera W. (1995), An introduction to computational fluid dynamics: the finite volume method, Longman Group.

Voller, V.R., and Prakash, C., (1987), A fixed-grid numerical modeling methodology for convection-diffusion mushy region phase-change problems. International Journal Heat Mass Transfer. Vol. 30, pp. $1709-1720$.

Walsh, P.C. and Leong, W.H., (2004), Effectiveness of several turbulence models in natural convection, International Journal of Methods for Heat and Fluid Flow, Vol. 14, pp. 633 - 648.

Yilmaz, T., \& Fraser, S. M. (2007). Turbulent natural convection in a vertical parallel-plate channel with asymmetric heating. International Journal of Heat and Mass Transfer, 50(13-14), 2612-2623.

Yuan, X., Moser, A., \& Suter, P. (1993). Wall functions for numerical simulation of turbulent natural convection along vertical plates. International Journal of Heat and Mass Transfer, 36(18), 4477-4485.

Zhai, Z., \& Chen, Q. (2003). Solution characters of iterative coupling between energy simulation and CFD programs. Energy and Buildings, 35(5), 493-505.

Zhai, Z., Chen, Q., Haves, P., \& Klems, J. H. (2003). On approaches to couple energy simulation and computational fluid dynamics programs. Building and Environment, 37(8-9), 857864. 
Zhang, R., Zhang, Y., Lam, K. P., \& Archer, D. H. (2010). A prototype mesh generation tool for CFD simulations in architecture domain. Building and Environment, 45(10), 2253-2262. 